\title{
Finite-element simulation of buoyancy-driven turbulent flows
}

\author{
Dissertation \\ zur Erlangung des Doktorgrades \\ der Mathematisch-Naturwissenschaftlichen Fakultäten \\ der Georg-August-Universität zu Göttingen
}

vorgelegt von

Tobias Knopp

aus

Lübeck

Göttingen 2003 
D7

Referent: Prof. Dr. G. Lube

Korreferent: Prof. Dr. R. Schaback

Tag der mündlichen Prüfung: 4. Juni 2003 


\section{Contents}

$\begin{array}{ll}\text { Preface } & 7\end{array}$

Epitome . . . . . . . . . . . . . . . . . . . . 8

Acknowledgements . . . . . . . . . . . . . . . . . . . 9

\begin{tabular}{ll}
\hline . Turbulence modelling for buoyancy driven flows & 11
\end{tabular}

1. The laminar model 13

1.1. Laminar thermally coupled tlow problems . . . . . . . . . . . . . . . . . 13

1.2. Boundary conditions for thermally coupled flows . . . . . . . . . . 15

1.3. A model for non-isothermal flow problems . . . . . . . . . . . . . . 18

1.4. Modelling turbulent boundary layers using a fully overlapping DDM . . . . 19

2. Fundamentals, modelling and simulation of turbulent flows 23

2.1. Aspects of randomness and statistical description of turbulent flows . . . . 23

2.2 . The scales of turbulent tlows . . . . . . . . . . . . . 26

2.3. Criteria for appraising approaches in CFD . . . . . . . . . . . 27

3. The $k / \epsilon$ turbulence model 29

3.1. The Reynolds averaged Navier-Stokes equations . . . . . . . . . . . . . . . . . 29

3.2. Turbulent-viscosity and gradient-diffusion hypotheses . . . . . . . . . . . . 30

3.3. Production and dissipation of turbulent kinetic energy in RANS models . . 33

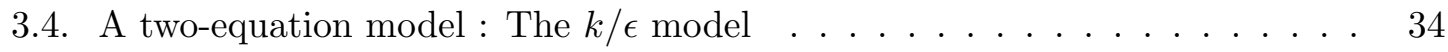

\begin{tabular}{ll}
\hline 4. Large-eddy simulation & 39
\end{tabular}

4.1. Filtering $\ldots \ldots \ldots \ldots \ldots \ldots$

$4.2 . \quad$ Differential filtering . . . . . . . . . . . . . . . . . . . . . 41

4.3. The space averaged non-isothermal Navier-Stokes equations . . . . . . . . . 42

4.4. Modelling the residual stress tensor and the residual fluxes . . . . . . . . . . 44

4.5. System of equations for non-isothermal LES . . . . . . . . . . . . . . . 49

5. Near-wall treatment in turbulence modelling 51

5.1. Fundamentals of turbulent boundary-layer theory . . . . . . . . . . . . . 51

5.2. Boundary-layer equations and singular perturbation methods . . . . . . . . 53

5.3. Algebraic turbulence models for non-isothermal boundary layers . . . . . 59

5.4. Algebraic turbulence models for natural convection boundary layers . . . . 63

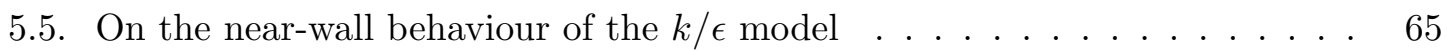

5.6. On LES in the near-wall region . . . . . . . . . . . . . . . . . 66 
$\begin{array}{ll}\text { 6. A computational } k / \epsilon \text { model using wall functions } & \mathbf{6 7}\end{array}$

6.1. A two-domain approach . . . . . . . . . . . . . . . . 67

6.2 . The wall function concept as a fully overlapping DDM . . . . . . . . . 68

6.3. The wall function concept using boundary-layer theory . . . . . . . . . 70

$\begin{array}{ll}\text { 7. A computational LES model } & \mathbf{7 5}\end{array}$

7.1. Wall stress models . . . . . . . . . . . . . . . . . . 77

7.2. Hybrid RANS/LES approaches . . . . . . . . . . . . . . . 80

$\begin{array}{ll}\text { 8. Some analytical results for LES with near wall modelling } & 81\end{array}$

8.1. Some simplifications of the coupled problem . . . . . . . . . . . . . 82

8.2. A separate study of global and local subproblem . . . . . . . . . . . . 84

8.3. The coupled steady state problem . . . . . . . . . . . . . . . . 94

8.4. Some closing remarks . . . . . . . . . . . . . . . 106

II. Numerical solution scheme and numerical tests 111

9. Semidiscretisation in time, decoupling and linearisation 113

9.1. Semidiscretisation in time using the discontinuous Galerkin method . . . . 113

9.2. Semidiscretisation, decoupling, and linearisation for the $k / \epsilon$ model . . . . . 114

9.3. Semidiscretisation, decoupling, and linearisation for the LES model . . . . . 117

9.4. Variational formulation of the arising model problems . . . . . . . . 120

$\begin{array}{lr}\text { 10.Discretisation in space using stabilised FEM } & 121\end{array}$

10.1. Finite-element discretisation for ADR-problems . . . . . . . . . . . . . . 121

10.2. Stabilisation techniques for ADR-problems . . . . . . . . . . . . . . . 121

10.3. Discontinuity capturing for ADR-problems . . . . . . . . . . . . . . . 123

10.4. Finite-element discretisation for Oseen problems . . . . . . . . . . . . . 124

10.5. SUPG- und PSPG-stabilisation for Oseen problems . . . . . . . . . . . . . . 124

$\begin{array}{lr}\text { 11.Non-overlapping domain decomposition methods } & 127\end{array}$

11.1. The Robin-Robin algorithm for advection-diffusion-reaction problems . . . . 128

11.2. Choice of the interface function in the R-R-algorithm for ADR problems . . 129

11.3. The Robin-Robin algorithm for Oseen type problems . . . . . . . . . . . . 130

12. Turbulent channel flow 133

12.1. Fundamentals of isothermal channel tow . . . . . . . . . . . . 133

12.2. Isothermal channel flow computations using the $k / \epsilon$ model . . . . . . . . . 136

12.3. Quasi a priori testing of the SGS model . . . . . . . . . . . . . . 138

13. Turbulent natural convection in an air filled square cavity 151

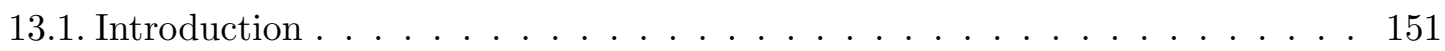

13.2. Description of the flow configuration . . . . . . . . . . . . 152

13.3 . Testing the wall iteration concept . . . . . . . . . . . . . . . . . 154

13.4. A posteriori testing for the $k / \epsilon$ model without DDM . . . . . . . . . . 157 
13.5. A posteriori testing for the $k / \epsilon$ model with DDM . . . . . . . . . . . . . . 160

13.6. Appraisal of the $k / \epsilon$ model predictions $\ldots \ldots \ldots \ldots$. . . . . . . . . . . . 166

13.7. First results for the posteriori testing using LES $\ldots \ldots \ldots$. . . . . . 167

$\begin{array}{lr}14 . \text { Summary and future prospects } & 175\end{array}$

$\begin{array}{lr}\text { III. Appendix } & 177\end{array}$

A. Mathematical tools for residual stress modelling in LES $\quad 179$

A.1. Fourier transformation, convolution and distributions . . . . . . . . . . . 179

A.2. Closure approximations for LES . . . . . . . . . . . . . . . 183

$\begin{array}{ll}\text { B. Some mathematical tools for the Navier-Stokes equations } & 187\end{array}$

B.1. Functional analytic fundamentals . . . . . . . . . . . . . . . . . 187

B.2. Analytical results for some turbulence models . . . . . . . . . . . . . . 191

$\begin{array}{ll}\text { C. Turbulent boundary-layer theory } & 193\end{array}$

C.1. Natural convection turbulent boundary layers . . . . . . . . . . . . . . . 193

C.2. Forced convection boundary-layer equations in non-dimensional form . . . . 195

C.3. The universal log law by Prandtl and van Karman . . . . . . . . . . . . . 196

C.4. A non-isothermal wall law for forced convection problems by Neitzke . . . . 197

$\begin{array}{ll}\text { D. Nomenclature } & 199\end{array}$

$\begin{array}{lr}\text { Bibliography } & 206\end{array}$

$\begin{array}{lr}\text { Curriculum vitae } & 219\end{array}$ 


\section{Preface}

Turbulent flows driven or significantly affected by buoyancy occur in a variety of problems including building ventilation, cooling of electrical equipment, and environmental science. The fundamental mathematical model are the non-isothermal Navier-Stokes equations, governing the time-evolution of velocity $\tilde{\boldsymbol{u}}$, pressure $\tilde{p}$, and temperature $\tilde{T}$. The phenomenon of turbulence reveals that their solutions can become very complex if a critical parameter, e.g., the Reynolds number or the Rayleigh number, becomes large. A proper numerical resolution of the random motion of all scales of $\tilde{\boldsymbol{u}}, \tilde{p}$, and $\tilde{T}$ (called Direct Numerical Simulation) is feasible only for a very limited number of flows. Thus the major task in turbulence modelling is to reduce the complexity of the Navier-Stokes equations in a manner which is appropriate to the needs of science and engineering. The goal is to develop models that are computationally simpler than the Navier-Stokes equations but "whose predictions are close to those of the Navier-Stokes equations". In this thesis we pursue two strategies: The first approach is a statistical approach which is based on a statistical averaging procedure for the Navier-Stokes equations. The objective is to obtain a set of equations for the statistical mean values for $\tilde{\boldsymbol{u}}, \tilde{p}$, and $\tilde{T}$, which requires an empirical modelling of the terms involving statistical fluctuations. The second approach is called large-eddy simulation (LES). The idea of LES is to apply a spatial averaging filter to the Navier-Stokes equations in order to extract the large-scale structures of $\tilde{\boldsymbol{u}}, \tilde{p}$, and $\tilde{T}$, and to attenuate their small-scale structures. Then only the random motion of the large scales is resolved and the effects of the small scales on the large scales are modelled.

This thesis is involved into a longlasting cooperation with the Institute for Thermodynamics and Building Energy Systems at Dresden University of Technology. A major result of this cooperation is our research code PARALlELNS, see e.g. [Mue99] and [KLGR02]. PARALLELNS is intended for the numerical solution of indoor-air flow problems, see e.g. [Gri01]. The building blocks of this code are the $k / \epsilon$ model (which is a statistical turbulence model), an improved wall-function concept for the treatment of the near-wall region, and a stabilised finite-element method together with an iterative substructuring method as a domain decomposition method for the numerical solution process.

The first objective of this thesis is a critical review of the theoretical background of these building blocks. Both the turbulence model and the numerical solution scheme used in PARALLELNS are described in a manner which is more convenient to mathematicians than the presentations in engineering textbooks. Secondly, the aim is to investigate the accuracy of our research code. The near-wall treatment in PARALLELNS conceived by [Nei99] had not yet been assessed by reference with experimental data from other research groups. We will investigate a natural convection flow in an air filled cavity. For this test case KARAYIANNIS ET AL. (see [TKO0a] and [AKO2]) provided widely accepted experimental data. Moreover the accuracy of the domain decomposition method for this three-dimensional test case has to be investigated, since the numerical tests in [Mue.9. ] are restricted to two-dimensional problems. 
The $k / \epsilon$ model is the most widespread turbulence model, but it suffers from several well-known deficiencies. Thus an additional objective of this thesis is to recommend alternative turbulence models which are amenable for use in PARALLELNS. A successful improvement of the standard $k / \epsilon$ model is the so called $k-\epsilon-\overline{v^{2}}$ model, which was devised by Durbin, see [Dur91]. However, this model requires resolving the near-wall region, which is infeasible for three-dimensional problems of practical relevance. Therefore we study LES, which has the additional advantage of being much closer to the NavierStokes equations than statistical turbulence models. Modern advances in computer power have allowed LES to become more and more interesting for engineering applications, see, e.g., the current projects in Prof. Dr. Lars Davidson's research group at the Department of Thermo and Fluid Dynamics at Chalmers University of Technology Göteborg (http://www.tfd.chalmers.se/ lada/projects/proind.html) and the homepage of the Flow Physics and Computation Division at the Department of Mechanical Engineering at Stanford University (http://www-fpc.stanford.edu/). The objective of this thesis is not to devise new LES models but to review current models in order to employ them in PARALLELNS. LES models are often referred to as residual stress models. Three residual stress models have been studied in this thesis, viz., the well-known Smagorinsky model, the Iliescu-Layton model (see [IL.98]), and the Galdi-Layton model (see [GL(0)]), including a modification devised by EIDSON, cf. [Eid85]. We describe how these models can be applied in a natural manner in PARALLELNS using the same near-wall strategy as for the $k / \epsilon$ model. We perform an a priori test and show first results from an a posteriori test. An a priori test uses experimental data or data from a DNS to study the residual stress model separately. In an a posteriori test, we perform a computation for a certain flow problem and then compare the calculated statistics (mean values, variances) with the corresponding statistics extracted from experimental data or from a DNS.

The wall function concept applied in PARALLELNS can be viewed as a fully overlapping domain decomposition method, as devised by TIDRIRI and LETALLEC, cf. [LTT99]. Within this approach, a boundary-layer solution is determined in the near-wall region, which satisfies the correct Dirichlet boundary condition at the wall and which is matched with the global solution on an artificial inner boundary. The crucial point is that the boundarylayer information is transferred to the global problem using a suitable friction (Neumann) boundary condition for the global problem. From a mathematical point of view this approach is not yet well understood. During a research stay at the University of Pittsburgh, in close cooperation with Prof. Dr. W. J. Layton some mathematical results for a certain coupling scheme have been obtained, which will be presented in this thesis.

\section{Epitome}

Part I is dedicated to a detailed description of the turbulence models studied in this thesis. In Chapter 1 the laminar case is studied and the wall function procedure is motivated. In Chapter 2 some fundamental results regarding turbulent flows and their modelling are reviewed. Chapter 3 is devoted to the $k / \epsilon$ turbulence model and in Chapter 4 some LES models are described. In Chapter 5 we derive a set of appropriate boundary-layer equations for the near-wall region. In Chapters 6 we present the $k / \epsilon$ model using the wall-function procedure which is implemented in our research code. The corresponding computational 
model for the LES models is described in Chapter 7. Chapter 8 is dedicated to the analysis of a certain wall-function scheme for LES.

Part II is devoted to a description of the numerical solution scheme and to the numerical investigations. In Chapter 9 we study the semidiscretisation in time and the linearisation for both models. The spatial discretisation is considered in Chapter 10. The domain decomposition method will be described in Chapter 11. Numerical tests for the fully developed turbulent channel flow and for a natural convection flow in a closed cavity are studied in Chapters 12 and 13 resp.

In the appendix, some prerequisited material is reviewed. Moreover, some additional results will be presented there, which do not fit well into the thread of principal ideas in the main text.

\section{Acknowledgements}

I am profoundly grateful to many people for their kind assistance and support in writing this thesis. First, I would like to thank my adviser Prof. Dr. G. Lube for providing me the opportunity to pursue a $\mathrm{PhD}$ in his research group und for his continual support. This thesis has benefited immeasurably from his revisions and advices. Moreover I am very grateful to Gerd Rapin for many valuable discussions on numerical analysis and on domain decomposition methods. My sincere thanks are given to Andreas Priesnitz for his kind assistance in administrating the software used for this thesis. I am very grateful to Markus Rösler, Hannes Müller, Ralf Gritzki, and Joachim Seifert (Institute for Thermodynamics and Building Energy Systems at TU Dresden) for their kind assistance regarding our research code PARAllelNS throughout the last years. For his valuable suggestions, for his encouraging stimulus and for his kind support during my research stay in Pittsburgh, I would like to give my genuine thanks to Prof. Dr. W. J. Layton (Department of Mathematics at Pittsburgh University). I would also like to thank him and his family for their kind hospitality during my first days in Pittsburgh. I gratefully acknowledge the hospitality of the University of Pittsburgh and I would like to thank Tony DiGiorno and Drew Porvaznik for their strong help. Similarly, I am very grateful to Adrian Dunca, Traian Iliescu, Dr. habil. Volker John, Dr. Holger Frahnert, Dr. Claus Wagner, Felix Ampofo, and Dr. Shia-Hui Peng for valuable discussions and communications. I would also like to express appreciation to our system administrators Dr. Gerhard Siebrasse, Rolf Wassmann, Joachim Perske, and Klaus Könnecke for their excellent support. Moreover I am very grateful to the Institute for Numerical and Applied Mathematics at Göttingen University for providing me an excellent working environment. I would like to give my sincere thanks to Mrs. Kalz and to my mother for reading over parts of this thesis for correct use of the English language. I would like to apologise to those, who suffered obstructions in available computing resources due to my own numerical tests during the last few months. For the generous financial support, I am very grateful to my sponsor "Graduiertenkolleg für Strömungsinstabilitäten und Turbulenz".

For their terrific friendship I am very grateful to my very best friends Matthias, Markus, Niklas and Florian. Finally, I would like to thank my family. They have supported me with great patience and loving care throughout my life and taught me the right things. 


\section{Part I.}

Turbulence modelling for buoyancy driven flows 



\section{The laminar model}

The first part of this thesis is devoted to turbulence modelling for incompressible buoyancy driven flows. We begin by considering the laminar case, introducing the incompressible non-isothermal Navier-Stokes equations. These are the governing equations for velocity, pressure and temperature in non-isothermal flow problems.

\subsection{Laminar thermally coupled flow problems}

Let $\Omega$ be an open domain of $\mathbb{R}^{d}(d=2,3)$ and $\Gamma$ its (sufficiently regular, at least Lipschitz continuous) boundary. Denote $\tilde{\boldsymbol{u}}$ the velocity field, $\tilde{p}$ the pressure and $\tilde{T}$ the temperature. Note that in the sequel dimensional variables are labelled by a tilde. Then the time evolution of these quantities is described by the following coupled system of partial differential equations:

$$
\begin{aligned}
\tilde{\rho}\left(\frac{\partial \tilde{\boldsymbol{u}}}{\partial \tilde{t}}+(\tilde{\boldsymbol{u}} \cdot \nabla) \tilde{\boldsymbol{u}}\right)-\nabla \cdot(2 \tilde{\mu} \mathbb{S}(\tilde{\boldsymbol{u}}))+\nabla \tilde{p} & =\tilde{\rho} \tilde{\boldsymbol{g}} \\
\nabla \cdot \tilde{\boldsymbol{u}} & =0 \\
\tilde{\rho} \tilde{c}_{p}\left(\frac{\partial \tilde{T}}{\partial \tilde{t}}+(\tilde{\boldsymbol{u}} \cdot \nabla) \tilde{T}\right)-\nabla \cdot(\tilde{\lambda} \nabla \tilde{T}) & =\tilde{\dot{q}}^{V}
\end{aligned}
$$

together with a set of initial and boundary conditions to be discussed later in this section. $\tilde{\mu}$ is the dynamic viscosity of the fluid and $\tilde{\rho}$ its density. $\tilde{\boldsymbol{g}}$ is the gravitational acceleration, $\tilde{c}_{p}$ denotes the specific heat at constant pressure, $\tilde{\lambda}$ (often used alternative symbol: $\tilde{k}$ ) is the thermal conduction coefficient, and $\tilde{\tilde{q}}^{V}$ is a volume specific external heat source. Eq. (1.1) is called momentum equation. We use the symmetric, deviatoric rate-of-strain tensor

$$
\mathbb{S}(\tilde{\boldsymbol{u}})=\frac{\nabla \tilde{\boldsymbol{u}}+\nabla \tilde{\boldsymbol{u}}^{T}}{2}, \quad \nabla^{s} \boldsymbol{u} \equiv 2 \mathbb{S}(\tilde{\boldsymbol{u}}) .
$$

Eq. (1.2) ensures the incompressibility of the fluid and is called equation of continuity. Eq. (1.3) will be referred to as temperature equation or heat transfer equation. Note that in indoor-air flow problems it is reasonable to neglect the dissipation of mechanical work (Joule effect) and heat transfer via radiation in (1.3).

In thermally coupled flow problems, the governing equations are fully coupled. First, as density is temperature sensitive, temperature variations may lead to density gradients. This can result in buoyancy forces due to gravitational forces. These are taken into account by the right hand side term in (1.1). For this reason temperature is referred to as an 'active' scalar in (1.1). Second, the velocity field is the convection field for the temperature in (1.3). The density dependence on the temperature is modelled by using the so-called Boussinesq 
approximation, which consists of two parts. First, it assumes that $\tilde{\rho}(\tilde{T})$ behaves like

$$
\tilde{\rho}(\tilde{T})=\tilde{\rho}_{0}-\tilde{\rho}_{0} \tilde{\beta}_{0}\left(\tilde{T}-\tilde{T}_{0}\right), \text { with } \tilde{\rho}_{0} \equiv \tilde{\rho}\left(\tilde{T}_{0}\right), \tilde{\beta}_{0} \equiv-\left.\frac{1}{\tilde{\rho}}\left(\frac{\partial \tilde{\rho}}{\partial \tilde{T}}\right)\right|_{\tilde{T}=\tilde{T}_{0}}
$$

and $\tilde{\beta}_{0}$ being the volumetric coefficient of thermal expansion. $\tilde{T}_{0}$ is a reference temperature. This equation can be regarded as a Taylor expansion of $\tilde{\rho}$ around $\tilde{T}_{0}$ (while keeping the pressure constant). Second, it assumes that density variations can be neglected in inertial terms, but not when they are multiplied by gravity, see e.g. [DPR(01], p.223.

\section{Remark 1.1}

References concerning the thermodynamical background of the Boussinesq approximation can be found e.g. in [Cod93b], p.3.2. According to [Mue.91] the Boussinesq approximation is accurate enough for temperature differences of about $50 \mathrm{~K}$. This is satisfied in typical indoor-air flow problems.

Now we introduce a reduced pressure

$$
\tilde{p}_{\text {red }} \equiv \tilde{p}-\tilde{\rho}_{0} \tilde{\boldsymbol{g}} \cdot \tilde{\boldsymbol{x}} .
$$

Using the Boussinesq approximation and the reduced pressure, (1.1)-(1.3) can be rearranged to

$$
\begin{aligned}
\tilde{\rho}_{0}\left(\frac{\partial \tilde{\boldsymbol{u}}}{\partial \tilde{t}}+(\tilde{\boldsymbol{u}} \cdot \nabla) \tilde{\boldsymbol{u}}\right)-\nabla \cdot(2 \tilde{\mu} \mathbb{S}(\tilde{\boldsymbol{u}}))+\nabla \tilde{p}_{r e d} & =-\tilde{\rho}_{0} \tilde{\beta}_{0}\left(\tilde{T}-\tilde{T}_{0}\right) \tilde{\boldsymbol{g}} \\
\nabla \cdot \tilde{\boldsymbol{u}} & =0 \\
\tilde{\rho}_{0} \tilde{c}_{p}\left(\frac{\partial \tilde{T}}{\partial \tilde{t}}+(\tilde{\boldsymbol{u}} \cdot \nabla) \tilde{T}\right)-\nabla \cdot(\tilde{\lambda} \nabla \tilde{T}) & =\tilde{\dot{q}}^{V} .
\end{aligned}
$$

It is convenient to write the (non-isothermal) Navier-Stokes equations in a non-dimensional form, i.e. with respect to the following scaled variables:

$$
t \equiv \frac{\tilde{t} \tilde{U}_{s c}}{\tilde{L}}, \quad \boldsymbol{x} \equiv \frac{\tilde{\boldsymbol{x}}}{\tilde{L}}, \quad \boldsymbol{u} \equiv \frac{\tilde{\boldsymbol{u}}}{\tilde{U}_{s c}}, \quad \theta \equiv \frac{\tilde{T}-\tilde{T}_{0}}{\tilde{T}_{d i f f}} .
$$

Here, $\tilde{L}$ is a characteristic length of the problem, $\tilde{T}_{d i f f}$ is a characteristic temperature difference, and $\tilde{U}_{s c}$ is a suitable velocity scale (which will be determined later in this section). Recall that in fluid mechanics the following dimensionless numbers are defined, see e.g. [KC.93]:

$$
\begin{aligned}
& R e \equiv \frac{\tilde{\rho}_{0} \tilde{L} \tilde{U}_{s c}}{\tilde{\mu}}, \quad \text { Reynolds number, } \operatorname{Pr} \equiv \frac{\tilde{c}_{p} \tilde{\mu}}{\tilde{\lambda}}, \quad \text { Prandtl number, } \\
& G r \equiv \frac{\tilde{\beta}_{0}|\tilde{\boldsymbol{g}}| \tilde{\rho}_{0}^{2} \tilde{L}^{3} \tilde{T}_{d i f f}}{\tilde{\mu}^{2}}, \quad \text { Grashof number, } \quad R a \equiv \frac{\tilde{\beta}_{0}|\tilde{\boldsymbol{g}}| \tilde{c}_{p} \tilde{\rho}_{0}^{2} \tilde{L}^{3} \tilde{T}_{d i f f}}{\tilde{\mu} \tilde{\lambda}}, \quad \text { Rayleigh number. }
\end{aligned}
$$

We introduce the thermal diffusivity $\tilde{a} \equiv \tilde{\lambda} /\left(\tilde{c}_{p} \tilde{\rho}_{0}\right)$ and the kinematic viscosity $\tilde{\nu} \equiv \tilde{\mu} / \tilde{\rho}_{0}$. Note that $\operatorname{Pr}=\tilde{\nu} \tilde{a}^{-1}$. The numbers are related by $R a=G r \operatorname{Pr}$. From these relations it 
can be seen that the Prandtl number is a measure for the similarity of the transport of heat and momentum. The Grashof number is the ratio of the buoyancy force to the viscous force.

Depending on the boundary conditions for the momentum equation, there are two different possibilities for choosing a characteristic scaling velocity $\tilde{U}_{s c}$. In the case of so-called forced convection, the fluid motion is enforced by the boundary conditions (see section 1.2). Then we choose $\tilde{U}_{s c}=\|\boldsymbol{u}\|_{\infty, \Gamma}$. In indoor-air flow problems most of the time there is no external force and $\boldsymbol{u}=0$ or a homogeneous Neumann condition is prescribed on the boundary. The only driving forces are due to buoyancy effects. Then physically meaningful choice is $\tilde{U}_{s c}=\left(\tilde{\beta}_{0}|\tilde{\boldsymbol{g}}| \tilde{T}_{d i f f} \tilde{L}\right)^{1 / 2}$, cf. [KC.93], p.408. In both cases the reduced pressure is nondimensionalised with $\tilde{\rho}_{0} \tilde{U}_{s c}^{2}$.

\section{Remark 1.2}

As it will turn out in Section 10.5, an appropriate choice for $\tilde{U}_{s c}$ is essential for the PSPGstabilisation technique in the numerical solution process.

In this thesis dimensionless quantities are chosen in agreement with [Mue.9.], viz.,

$$
\tilde{a} \equiv \frac{\tilde{\lambda}}{\tilde{c}_{p} \tilde{\rho}_{0}}, a \equiv \frac{\tilde{a}}{\tilde{L} \tilde{U}_{s c}}, \boldsymbol{g} \equiv \frac{\tilde{\boldsymbol{g}} \tilde{L}}{\tilde{U}_{s c}^{2}}, c_{p} \equiv \frac{\tilde{\lambda} \tilde{T}_{d i f f}}{\tilde{\rho}_{0} \tilde{a} \tilde{U}_{s c}^{2}}, \dot{q}^{V} \equiv \frac{\tilde{\dot{q}}^{V} \tilde{L}}{\tilde{\rho}_{0} \tilde{U}_{s c}^{3}}, \beta \equiv \tilde{\beta}_{0} \tilde{T}_{d i f f}, \nu \equiv \frac{\tilde{\mu}}{\tilde{\rho}_{0} \tilde{U}_{s c} \tilde{L}}
$$

This yields the following system of equations

$$
\begin{aligned}
\partial_{t} \boldsymbol{u}-\boldsymbol{\nabla} \cdot(2 \nu \mathbb{S}(\boldsymbol{u}))+(\boldsymbol{u} \cdot \boldsymbol{\nabla}) \boldsymbol{u}+\boldsymbol{\nabla} p_{\text {red }} & =-\beta \theta \boldsymbol{g} \\
\boldsymbol{\nabla} \cdot \boldsymbol{u} & =0, \\
\partial_{t} \theta+(\boldsymbol{u} \cdot \boldsymbol{\nabla}) \theta-\boldsymbol{\nabla} \cdot(a \boldsymbol{\nabla} \theta) & =\dot{q}^{V} c_{p}^{-1}
\end{aligned}
$$

\subsection{Boundary conditions for thermally coupled flows}

For specifying the boundary conditions, we introduce two partitions of $\Gamma$ : one for the momentum equation and one for all scalar equations, e.g., the heat transfer equation and a possible additional equation describing contaminant transport.

The first partition of $\Gamma$ is due to the boundary conditions concerning the momentum equation. For this purpose we define the stress tensor

$$
\sigma(\boldsymbol{u}, p)=-p \mathbb{I}+2 \nu \mathbb{S}(\boldsymbol{u}) .
$$

Moreover we suppose that for almost every point $\boldsymbol{x}$ in $\Gamma$ we have a local orthonormal basis $\left\{\boldsymbol{n}(\boldsymbol{x}), \boldsymbol{t}_{j}(\boldsymbol{x}), 1 \leq j \leq d-1\right\}$, where $\left\{\boldsymbol{t}_{j}\right\}_{j=1}^{d-1}$ is a local orthonormal basis for the tangent space of $\Gamma$ in $\boldsymbol{x}$ and $\boldsymbol{n}$ denotes the outer unit normal vector to $\Gamma$ at $\boldsymbol{x}$. Denote

$$
\Gamma_{F}=\left\{x \in \Gamma \mid \boldsymbol{u}=\boldsymbol{u}_{F}, \quad \boldsymbol{u}_{F} \cdot \boldsymbol{n}<0 \text { a.e. in } \Gamma_{F}\right\},
$$

(1.13) $\Gamma_{W}=\left\{x \in \Gamma \mid \boldsymbol{u} \cdot \boldsymbol{n}=0, \quad \chi \boldsymbol{n}^{T} \sigma(\boldsymbol{u}, p) \boldsymbol{t}_{j}=\boldsymbol{\sigma}_{t}(\boldsymbol{u}) \cdot \boldsymbol{t}_{j} \quad 1 \leq j \leq d-1\right\}$,

(1.14) $\Gamma_{N}=\left\{x \in \Gamma \mid \sigma(\boldsymbol{u}, p) \boldsymbol{n}=\boldsymbol{\sigma}_{n}\right\}$ 
which are mutually disjoint and satisfy $\overline{\Gamma_{F}} \cup \overline{\Gamma_{N}} \cup \overline{\Gamma_{W}}=\Gamma$. The quantity $\left.\boldsymbol{n}^{T} \operatorname{Tr}\right|_{\Gamma_{W}} \sigma(\boldsymbol{u}, p)$ is called stress vector, which represents the force that the fluid exerts on the wall. Here $\left.\operatorname{Tr}\right|_{\Gamma_{W}}$ denotes the trace operator, see Chapter B and Remark 8.4. $\Gamma_{F}$ is a forced convection inflow boundary; on $\Gamma_{F}$ a non-zero inflow velocity profile is prescribed. (1.13) describes a general (non-linear) friction law, covering the following situations:

(i) slip with linear friction: $\chi \equiv 1$, and $\boldsymbol{\sigma}_{t}(\boldsymbol{u}) \cdot \boldsymbol{t}_{j} \equiv-\beta_{j} \boldsymbol{u} \cdot \boldsymbol{t}_{j}$,

(ii) wall stress condition: $\chi \equiv 1$, and $\boldsymbol{\sigma}_{t}(\boldsymbol{u}) \cdot \boldsymbol{t}_{j} \equiv \tau_{w} \frac{\boldsymbol{u} \cdot \boldsymbol{t}_{j}}{\left\|\boldsymbol{u} \cdot \boldsymbol{t}_{j}\right\|}\left(\right.$ provided $\boldsymbol{u} \cdot \boldsymbol{t}_{j} \neq 0$ ),

(iii) no-slip condition: $\chi=0$, and $\boldsymbol{\sigma}_{t}(\boldsymbol{u}) \cdot \boldsymbol{t}_{j} \equiv-\boldsymbol{u} \cdot \boldsymbol{t}_{j}$.

Note that in the case of $(i), \boldsymbol{\sigma}_{t}(\boldsymbol{u}) \cdot \boldsymbol{t}_{j}$ depends linearly on the magnitude of $\boldsymbol{u} \cdot \boldsymbol{t}_{j}$ whereas in the case of (ii), only a directional and a so-called phase information of $\boldsymbol{u} \cdot \boldsymbol{t}_{j}$ is used. Due to the definition of $\Gamma_{F}$, even in case (iii) $\Gamma_{F}$ and $\Gamma_{W}$ are disjoint.

Now we explain how different physical situations can be modelled using these types of boundary conditions. Informally spoken, in indoor-air flow simulations the boundary consists of openings and solid impermeable and smooth walls. On the wall, in any case we impose $\boldsymbol{u} \cdot \boldsymbol{n}=0$, being covered by (1.13), (iii). Next openings are studied. There is a wide agreement that $\sigma(\boldsymbol{u}, p) \boldsymbol{n}=\mathbf{0}$ is suitable to model undisturbed outflow. Concerning inflow, we have to distinguish between forced convection and natural convection. In the former case, on a part of the boundary a nonzero inflow velocity is prescribed, i.e. $\Gamma_{F} \neq \emptyset$. Alternatively, inflow can be enforced by imposing a suitable external pressure $\boldsymbol{\sigma}_{n}$ in (1.14). Of course, when selecting (1.14), it is possible that $\boldsymbol{u}=\mathbf{0}$ or $\boldsymbol{u} \cdot \boldsymbol{n}=0$ on parts of $\Gamma_{N}$. In the latter case of natural convection, i.e. $\Gamma_{F}=\emptyset, \sigma_{n}=\mathbf{0}$ in (1.14), the fluid motion is induced by buoyancy forces. It is worth rewriting both cases in the following form:

Forced convection: $\Gamma_{F} \neq \emptyset$ or $\boldsymbol{\sigma}_{n} \neq \mathbf{0}$.

Natural convection: $\Gamma_{F}=\emptyset$ and $\boldsymbol{\sigma}_{n}=\mathbf{0}$.

In most indoor-air flow problems both natural and forced convection have to be considered. This case is also referred to as mixed convection. As pointed out in [KC.93], in mixed convection problems often the forced convection character dominates, in particular if $\mathrm{Gr}$ is small compared to Re. The crucial question is whether the buoyancy force term in the momentum equation is significant or not.

The most general condition describing solid impermeable walls is (1.13). Measurements showed that no-slip is the correct boundary condition on walls for indoor-air flow problems, cf. [Nei99]. However, as it will turn out later, it is useful considering the more general condition (1.13).

A second partition of $\Gamma$ can be defined w.r.t. the sign of $\boldsymbol{u} \cdot \boldsymbol{n}$, where $\boldsymbol{n}$ denotes the outer unit vector normal to $\Gamma$, viz., 


$$
\begin{aligned}
& \Gamma_{-}(\boldsymbol{u})=\{\boldsymbol{x} \in \Gamma \mid \boldsymbol{u} \cdot \boldsymbol{n}<0\} \quad \text { inflow boundary }, \\
& \Gamma_{0}(\boldsymbol{u})=\{\boldsymbol{x} \in \Gamma \mid \boldsymbol{u} \cdot \boldsymbol{n}=0\} \text { "wall" except a set of measure zero , } \\
& \Gamma_{+}(\boldsymbol{u})=\{\boldsymbol{x} \in \Gamma \mid \boldsymbol{u} \cdot \boldsymbol{n}>0\} \text { outflow, }
\end{aligned}
$$

which are mutually disjoint and satisfy $\overline{\Gamma_{-}(\boldsymbol{u})} \cup \overline{\Gamma_{0}(\boldsymbol{u})} \cup \overline{\Gamma_{+}(\boldsymbol{u})}=\Gamma$. Note that $\Gamma_{W}=\Gamma_{0}(\boldsymbol{u})$ (except for a set of measure zero) and $\Gamma_{F} \subset \Gamma_{-}(\boldsymbol{u})$. In Figure 1.1 (from [Gri01], p.98) the situation of an opened window is sketched, which is described by (1.14) with $\boldsymbol{\sigma}_{n}=\mathbf{0}$. Inflow and outflow is a consequence of thermal buoyancy effects. It is worth mentioning that in almost every application the so-called neutral zone, consisting of points located in the opening with $\boldsymbol{u} \cdot \boldsymbol{n}=0$, is of measure zero. A survey on boundary conditions for the

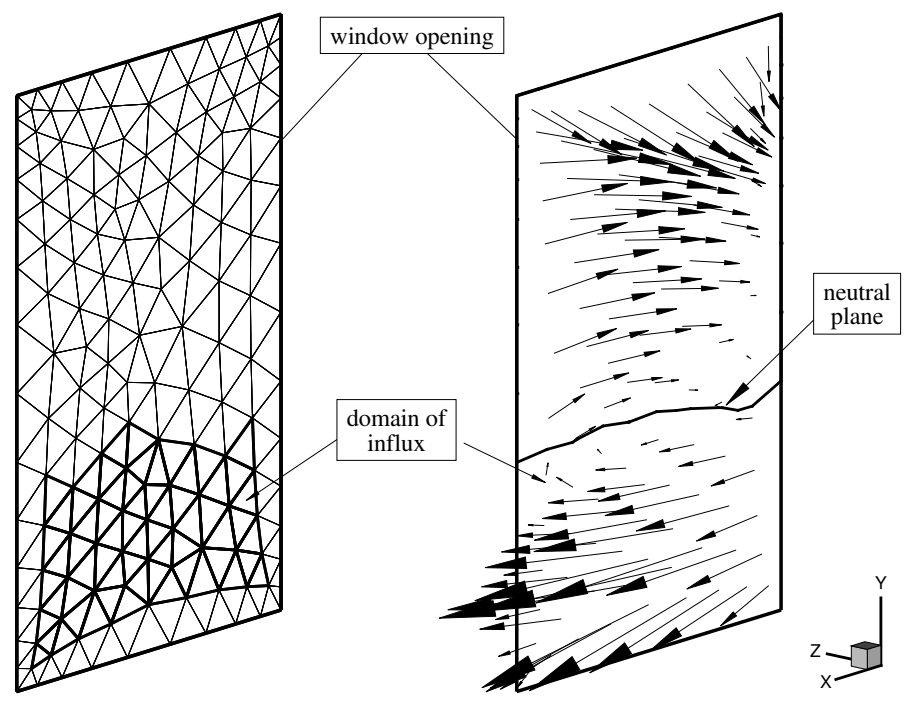

Figure 1.1.: Inflow at outflow regions at an opened window.

isothermal Navier-Stokes equations and further references thereon can be found in [Lia99]. More details on boundary conditions regarding the simulation of indoor-air movement can be found in [Nei99] and [Gri01].

The partition $\Gamma_{-}(\boldsymbol{u}), \Gamma_{0}(\boldsymbol{u})$ and $\Gamma_{+}(\boldsymbol{u})$ is used for imposing boundary conditions for the temperature equation. It seems natural to require

$$
\theta=\theta_{\text {in }} \quad \text { on } \quad \Gamma_{-}(\boldsymbol{u}), \quad a \boldsymbol{\nabla} \theta \cdot \boldsymbol{n}=0 \quad \text { on } \quad \Gamma_{+}(\boldsymbol{u}),
$$

where $\theta_{\text {in }}$ designates the outside (fluid) temperature.

Depending on the physical boundary conditions at the wall, we consider the following sub-partitioning of $\Gamma_{0}(\boldsymbol{u})$, videlicet,

$$
\theta=\theta_{w} \text { on } \Gamma_{W, D}, \quad a \nabla \theta \cdot \boldsymbol{n}=\dot{q} c_{p}^{-1} \quad \text { on } \Gamma_{W, N},
$$

where $\theta_{w}$ denotes the wall temperature and $\dot{q}$ denotes the heat-flux at the wall. Of course, $\Gamma_{W, D} \cap \Gamma_{W, N}=\emptyset, \quad \overline{\Gamma_{W, D}} \cup \overline{\Gamma_{W, N}}=\overline{\Gamma_{W}}$. 


\subsection{A model for non-isothermal flow problems}

Putting together the results of the previous sections we can state our basic model for laminar thermally-coupled flow problems, later referred to as MODEL TNSE (thermally coupled Navier-Stokes equations).

\section{A model for thermally-driven flows}

- Non-isothermal Navier-Stokes equations

$$
\begin{aligned}
\partial_{t} \boldsymbol{u}-\boldsymbol{\nabla} \cdot(2 \nu \mathbb{S}(\boldsymbol{u}))+(\boldsymbol{u} \cdot \boldsymbol{\nabla}) \boldsymbol{u}+\boldsymbol{\nabla} p_{\text {red }} & =-\beta \theta \boldsymbol{g} \\
\boldsymbol{\nabla} \cdot \boldsymbol{u} & =0, \\
\partial_{t} \theta+(\boldsymbol{u} \cdot \boldsymbol{\nabla}) \theta-\boldsymbol{\nabla} \cdot(a \boldsymbol{\nabla} \theta) & =\dot{q}^{V} c_{p}^{-1} .
\end{aligned}
$$

- Boundary conditions

- Momentum Equation

* Forced convection problem:

$$
\boldsymbol{u}=\boldsymbol{u}_{F} \text { on } \Gamma_{F}, \quad \boldsymbol{u}=\mathbf{0} \text { on } \Gamma_{W}, \quad \sigma(\boldsymbol{u}, p) \boldsymbol{n}=\mathbf{0} \text { on } \Gamma_{N}
$$

* Natural convection problem:

$$
\Gamma_{F}=\emptyset, \quad \boldsymbol{u}=\mathbf{0} \text { on } \Gamma_{W}, \quad \sigma(\boldsymbol{u}, p) \boldsymbol{n}=\mathbf{0} \text { on } \Gamma_{N} .
$$

- Heat Equation

$$
\begin{aligned}
& \theta=\theta_{i n} \text { on } \Gamma_{-}(\boldsymbol{u}), \quad \nabla \theta \cdot \boldsymbol{n}=0 \text { on } \Gamma_{+}(\boldsymbol{u}) \\
& \theta=\theta_{w} \text { on } \Gamma_{W, D}, \quad a \nabla \theta \cdot \boldsymbol{n}=\dot{q} c_{p}^{-1} \text { on } \Gamma_{W, N} .
\end{aligned}
$$

Finally we have to prescribe the initial conditions

$$
\boldsymbol{u}=\boldsymbol{u}_{0} \quad, \quad \theta=\theta_{0} \quad \text { in } \Omega \times\{0\}
$$

where the initial condition satisfies $\nabla \cdot \boldsymbol{u}_{0}=0$.

\section{Remark 1.3}

From the point of numerical analysis, the boundary conditions specified in MODEL TNSE can cause severe problems. For example a discontinuity in the boundary condition for $\theta$ occurs, if $\bar{\Gamma}_{-}(\boldsymbol{u}) \cap \bar{\Gamma}_{W, D} \neq \emptyset$ and $\theta_{\text {in }} \neq \theta_{w}$. 


\subsection{Modelling turbulent boundary layers using a fully overlapping DDM}

Most flow problems of interest are wall bounded flows. Surface boundary conditions often cause several problems. In the laminar case, imposing a no-slip condition and the first option in (1.18) on a solid wall, the solutions of velocity and temperature equations can exhibit sharp gradients in the vicinity of the wall, referred to as boundary layers. Moreover, in the turbulent case in the near-wall region the behaviour of the solution is strongly influenced by complicated turbulent processes, being discussed in Chapters 2-7.

There are two major solution strategies for wall-bounded flow problems:

(i) Resolve the near-wall region using a suitable grid refinement technique. In the turbulent case, this is called direct numerical simulation, abbreviated DNS.

(ii) Model the overall effect of the solution in the near-wall region on the flow remote from the wall, i.e., "bridge" the boundary layer. This is called near-wall modelling.

Strategy $(i)$ is not feasible for most high Reynolds resp. Rayleigh number turbulent flows, in particular in complex geometries. However, when studying the physics in the near-wall region, a DNS must be accomplished. On the other hand, in engineering applications, often only the effect of the near-wall behaviour of the solution on the flow remote from the wall is of interest, as proposed in strategy $(i i)$. Moreover, to obtain certain characteristic quantities on the wall, which are of great engineering interest (i.e., so-called surface transfer coefficients), it is not necessary to perform a DNS; they can be determined from the results of the near-wall modelling process immediately.

The most popular near-wall modelling scheme is the so-called wall function concept. The application of this strategy to turbulence modelling is a building block of this thesis being considered in great detail in Chapters 6 and 7 . The wall function method has been used by engineers for more than thirty years. As an introduction, in this section we present the underlying idea from a mathematician's point of view: As devised by TIDRIRI and LETALLEC, cf. [LT T99], we interpret the wall function concept as a fully overlapping domain decomposition method. Following [LTT99], first we consider the case of an advectiondiffusion-reaction problem. After that, some analytical results obtained by LETALLEC and TIDRIRI are resumed. Finally two alternative strategies for applying this method to the Navier-Stokes equations will be presented.

To understand the underlying idea, we start with the instationary advection-diffusion problem of seeking $\phi: \Omega \times(0, \infty) \mapsto \mathbb{R}$, s.t.

$$
\begin{aligned}
\partial_{t} \phi-a \nabla \cdot(\nabla \phi)+(\boldsymbol{u} \cdot \nabla) \phi & =0 & & \text { in } \Omega \times(0, \infty), \\
\phi & =0 & & \text { on } \Gamma \times(0, \infty), \\
\phi(0) & =0 & & \text { in } \Omega .
\end{aligned}
$$

Here, $\Gamma \equiv \partial \Omega$ and we suppose $\nabla \cdot \boldsymbol{u}=0$ in $\Omega$. Moreover we assume that there exists a uniquely determined stationary solution of (1.27)-(1.29) and that the solution of the corresponding backward-Euler scheme converges to this stationary solution as $t \rightarrow \infty$.

Instead of solving (1.27)-(1.29), the following modified problem is studied. Denote $\Omega_{\text {layer }} \subset$ $\Omega$ a suitable neighbourhood of $\Gamma_{W} \equiv \Gamma$, cf. Figure 1.2. Denote $\Gamma_{i} \equiv \partial \Omega_{\text {layer }} \cap \Omega$. Then we 


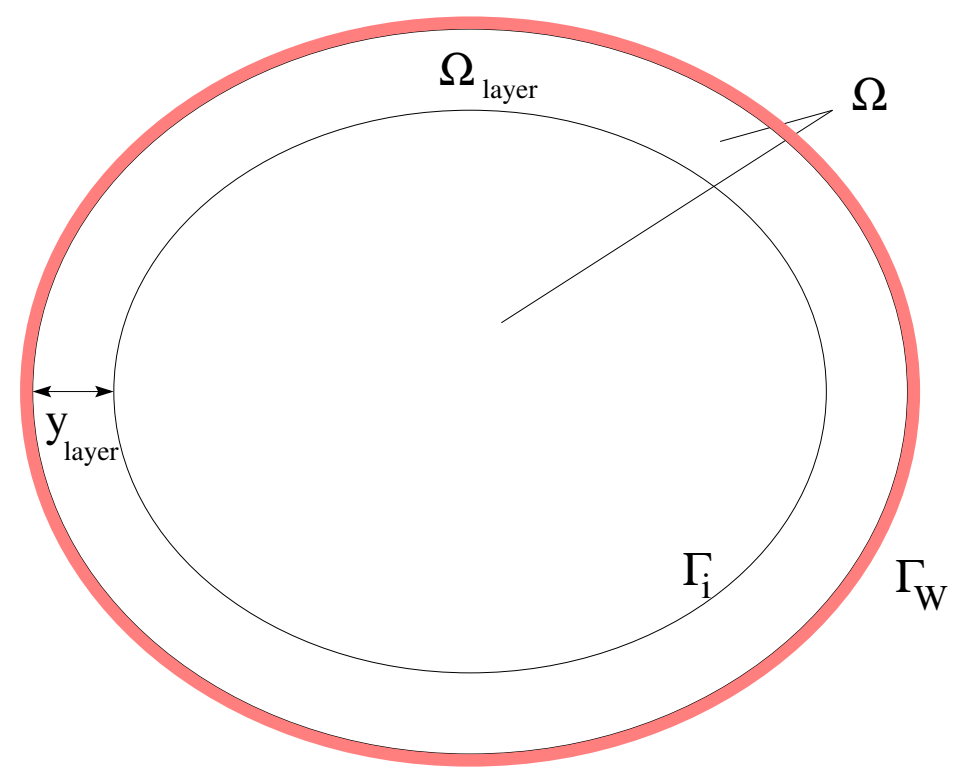

Figure 1.2.: Sketch of fully overlapping DDM.

seek $\Phi: \Omega \times(0, \infty) \mapsto \mathbb{R}$ (the so-called global solution) and $\phi^{B L}: \Omega_{\text {layer }} \times(0, \infty) \mapsto \mathbb{R}$ (the boundary-layer solution or local solution or inner solution) such that

$$
\begin{aligned}
\partial_{t} \Phi-a \nabla \cdot(\nabla \Phi)+(\boldsymbol{u} \cdot \nabla) \Phi & =0 & & \text { in } \Omega \times(0, \infty), \\
a \nabla \Phi \cdot \boldsymbol{n} & =a \nabla \phi^{B L} \cdot \boldsymbol{n} & & \text { on } \Gamma_{W} \times(0, \infty), \\
\partial_{t} \phi^{B L}-a \nabla \cdot\left(\nabla \phi^{B L}\right)+(\boldsymbol{u} \cdot \nabla) \phi^{B L} & =0 & & \text { in } \Omega_{\text {layer }} \times(0, \infty), \\
\phi^{B L}=0 \text { on } \Gamma_{W} \times(0, \infty), \phi^{B L} & =\Phi & & \text { on } \Gamma_{i} \times(0, \infty), \\
\Phi(0)=0 \text { in } \Omega, \quad \phi^{B L}(0) & =0 & & \text { in } \Omega .
\end{aligned}
$$

In (1.32)-(1.34) a solution in the boundary layer is determined. Note that $\phi^{B L}$ satisfies the correct homogeneous Dirichlet condition on $\Gamma_{W}$ and that $\phi^{B L}$ is matched with the global solution on $\Gamma_{i}$. The crucial point is that the boundary-layer information is transferred to the global problem via (1.31) using a friction (Neumann) boundary condition.

Le Tallec and Tidriri now perform a semidiscretization in time using a backward Euler scheme: Within each time step, they consider the following coupled problem: Given a time step width $\triangle t$ and $\Phi^{k}, \phi^{B L, k}$ from the previous time step ( resp. from an initial guess $\Phi^{0}, \phi^{B L, 0}$ if $\left.k=0\right)$ seek $\Phi^{k+1}, \phi^{B L, k+1}$ s.t.

$$
\begin{aligned}
\frac{\phi^{B L, k+1}-\phi^{B L, k}}{\triangle t}-a \nabla \cdot\left(\nabla \phi^{B L, k+1}\right)+(\boldsymbol{u} \cdot \nabla) \phi^{B L, k+1} & =0 & & \text { in } \Omega_{\text {layer }}, \\
\phi^{B L, k+1}=0 \text { on } \Gamma_{W}, \phi^{B L, k+1} & =\Phi^{k+1} & & \text { on } \Gamma_{i}, \\
\frac{\Phi^{k+1}-\Phi^{k}}{\triangle t}-a \nabla \cdot\left(\nabla \Phi^{k+1}\right)+(\boldsymbol{u} \cdot \nabla) \Phi^{k+1} & =0 & & \text { in } \Omega, \\
a \nabla \Phi^{k+1} \cdot \boldsymbol{n}-a \nabla \phi^{B L, k+1} \cdot \boldsymbol{n} & =0 & & \text { on } \Gamma_{W} .
\end{aligned}
$$


The coupled problem (1.35)-(1.38) can be solved using the following fixed point method. Denote a lower index $j$ the iteration cycle. Then Le Tallec and Tidriri studied the following scheme: Given $\Phi^{k}, \phi^{B L, k}$ as the solution of the previous time step and $\Phi_{j}^{k+1}, \phi_{j}^{B L, k+1}$ from the previous iteration step (or as the solution of the previous time step if $j=0$ ), seek $\Phi_{j+1}^{k+1}, \phi_{j+1}^{B L, k+1}$ s.t.

$$
\begin{aligned}
\frac{\phi_{j+1}^{B L, k+1}-\phi^{B L, k}}{\triangle t}-a \nabla \cdot\left(\nabla \phi_{j+1}^{B L, k+1}\right)+(\boldsymbol{u} \cdot \nabla) \phi_{j+1}^{B L, k+1} & =0 & & \text { in } \Omega_{\text {layer }}, \\
\phi_{j+1}^{B L, k+1}=0 \quad \text { on } \Gamma_{W}, \quad \phi_{j+1}^{B L, k+1} & =\Phi_{j}^{k+1} & & \text { on } \Gamma_{i}, \\
\frac{\Phi_{j+1}^{k+1}-\Phi^{k}}{\triangle t}-a \nabla \cdot\left(\nabla \Phi_{j+1}^{k+1}\right)+(\boldsymbol{u} \cdot \nabla) \Phi_{j+1}^{k+1} & =0 & & \text { in } \Omega, \\
a \nabla \Phi_{j+1}^{k+1} \cdot \boldsymbol{n}-a \nabla \phi_{j+1}^{B L, k+1} \cdot \boldsymbol{n} & =0 & & \text { on } \Gamma_{W} .
\end{aligned}
$$

LE TALLEC and TIDRIRI show that $\Phi_{j+1}^{k+1} \rightarrow \Phi^{k+1}, \phi_{j+1}^{B L, k+1} \rightarrow \phi^{B L, k+1}$ linearly as $j \rightarrow \infty$, cf. [LTT T96]. Moreover they can prove that the solution of (1.35)-(1.38) converges linearly in $H^{1}(\Omega)$ to the stationary solution of the problem (1.27)-(1.29) as $k \rightarrow \infty$.

There are (at least) two alternative strategies for applying this method to the Navier-Stokes equations. We restrict ourselves to the isothermal flow problem of seeking $\boldsymbol{u}: \Omega \times(0, \infty) \mapsto$ $\mathbb{R}^{d}, p: \Omega \times(0, \infty) \mapsto \mathbb{R}$, s.t.

$$
\begin{aligned}
\partial_{t} \boldsymbol{u}-\nu \nabla \cdot(\nabla \boldsymbol{u})+(\boldsymbol{u} \cdot \nabla) \boldsymbol{u}+\nabla p & =\boldsymbol{f} & & \text { in } \Omega \times(0, \infty), \\
\nabla \cdot \boldsymbol{u} & =0 & & \text { in } \Omega \times(0, \infty), \\
\boldsymbol{u} & =0 & & \text { on } \Gamma \times(0, \infty), \\
\boldsymbol{u}(0) & =\mathbf{0} & & \text { in } \Omega
\end{aligned}
$$

with given external force $f$. Both approaches can be distinguished by the boundary condition for the global problem, transferring the boundary-layer information to the global solution. However, both are a special case of (1.13).

First we consider the traditional approach, which has been applied in CFD for more than thirty years: Seek $\boldsymbol{u}: \Omega \times(0, \infty) \mapsto \mathbb{R}^{d}, p: \Omega \times(0, \infty) \mapsto \mathbb{R}$ (the global solution) and $\boldsymbol{u}^{B L}: \Omega_{\text {layer }} \times(0, \infty) \mapsto \mathbb{R}^{d}, p^{B L}: \Omega_{\text {layer }} \times(0, \infty) \mapsto \mathbb{R}$ (the boundary-layer solution or local solution or inner solution) such that

$$
\begin{aligned}
\partial_{t} \boldsymbol{u}-\nu \nabla \cdot(\nabla \boldsymbol{u})+(\boldsymbol{u} \cdot \nabla) \boldsymbol{u}+\nabla p & =\boldsymbol{f} & & \text { in } \Omega \times(0, \infty), \\
\boldsymbol{u} \cdot \boldsymbol{n}=0, \quad \boldsymbol{n}^{T} \sigma(\boldsymbol{u}, p) \boldsymbol{t}_{j}-\boldsymbol{n}^{T} \sigma\left(\boldsymbol{u}^{B L}, p^{B L}\right) \boldsymbol{t}_{j} & =0 & & \text { on } \Gamma_{W} \times(0, \infty), \\
\partial_{t} \boldsymbol{u}^{B L}-\nu \nabla \cdot\left(\nabla \boldsymbol{u}^{B L}\right)+\left(\boldsymbol{u}^{B L} \cdot \nabla\right) \boldsymbol{u}^{B L}+\nabla p^{B L} & =\boldsymbol{f} & & \text { in } \Omega_{\text {layer }} \times(0, \infty), \\
\boldsymbol{u}^{B L}=\mathbf{0} \text { on } \Gamma_{W} \times(0, \infty), \boldsymbol{u}^{B L} & =\boldsymbol{u} & & \text { on } \Gamma_{i} \times(0, \infty), \\
\boldsymbol{u}(0)=\mathbf{0} \text { in } \Omega, \boldsymbol{u}^{B L}(0) & =\mathbf{0} & & \text { in } \Omega .
\end{aligned}
$$

TIDRIRI applied the strategy (1.47)-(1.51) to the compressible Navier-Stokes equations, cf. [Tid95]. He gives promising numerical results for complex flow problems, but he does not 
give any analytical results.

Motivated by the work of Liakos, cf. [Lia99], LAYTON and Galdi, see [GL00], we can formulate an alternative approach for coupling global and boundary-layer problem: Seek $\boldsymbol{u}: \Omega \times(0, \infty) \mapsto \mathbb{R}^{d}, p: \Omega \times(0, \infty) \mapsto \mathbb{R}$ and $\boldsymbol{u}^{B L}: \Omega_{\text {layer }} \times(0, \infty) \mapsto \mathbb{R}^{d}, p^{B L}:$ $\Omega_{\text {layer }} \times(0, \infty) \mapsto \mathbb{R}$ such that

$$
\begin{aligned}
\partial_{t} \boldsymbol{u}-\nu \nabla \cdot(\nabla \boldsymbol{u})+(\boldsymbol{u} \cdot \nabla) \boldsymbol{u}+\nabla p & =\boldsymbol{f} & & \text { in } \Omega \times(0, \infty), \\
\boldsymbol{u} \cdot \boldsymbol{n}=0, \quad \beta_{j}\left(\boldsymbol{u}^{B L}, p^{B L}\right) \boldsymbol{u} \cdot \boldsymbol{t}_{j}+\boldsymbol{n}^{T} \sigma(\boldsymbol{u}, p) \boldsymbol{t}_{j} & =0 & & \text { on } \Gamma_{W} \times(0, \infty), \\
\partial_{t} \boldsymbol{u}^{B L}-\nu \nabla \cdot\left(\nabla \boldsymbol{u}^{B L}\right)+\left(\boldsymbol{u}^{B L} \cdot \nabla\right) \boldsymbol{u}^{B L}+\nabla p^{B L} & =\boldsymbol{f} & & \text { in } \Omega_{\text {layer }} \times(0, \infty), \\
\boldsymbol{u}^{B L}=\mathbf{0} \text { on } \Gamma_{W} \times(0, \infty), \boldsymbol{u}^{B L} & =\boldsymbol{u} & & \text { on } \Gamma_{i} \times(0, \infty), \\
\boldsymbol{u}(0)=\mathbf{0} \text { in } \Omega, \boldsymbol{u}^{B L}(0) & =\mathbf{0} & & \text { in } \Omega .
\end{aligned}
$$

Here we additionally have to specify the so-called friction parameters $\beta_{j}\left(\boldsymbol{u}^{B L}, p^{B L}\right)$. Given a specification for $\beta_{j}\left(\boldsymbol{u}^{B L}, p^{B L}\right)$, we obtain a closed system of equations.

Method (1.47)-(1.51) will be the underlying strategy for the computational treatment of flow problems in this thesis, see Chapters 6 and 7. Approach (1.52)-(1.56) is more amenable to the analysis and will be studied in Chaper 8 .

As explained in Section 1.2, both the slip with linear friction and the wall stress boundary condition can be written in terms of (1.13). Thus the general coupling scheme reads: Seek $\boldsymbol{u}: \Omega \times(0, \infty) \mapsto \mathbb{R}^{d}, p: \Omega \times(0, \infty) \mapsto \mathbb{R}$ and $\boldsymbol{u}^{B L}: \Omega_{\text {layer }} \times(0, \infty) \mapsto \mathbb{R}^{d}, p^{B L}$ : $\Omega_{\text {layer }} \times(0, \infty) \mapsto \mathbb{R}$ such that

$$
\begin{aligned}
\partial_{t} \boldsymbol{u}-\nu \nabla \cdot(\nabla \boldsymbol{u})+(\boldsymbol{u} \cdot \nabla) \boldsymbol{u}+\nabla p=\boldsymbol{f} & \text { in } \Omega \times(0, \infty), \\
\boldsymbol{u} \cdot \boldsymbol{n}=0, \boldsymbol{n}^{T} \sigma(\boldsymbol{u}, p) \boldsymbol{t}_{j}=\boldsymbol{\sigma}_{t}\left(\boldsymbol{u}, \boldsymbol{u}^{B L}\right) \cdot \boldsymbol{t}_{j} & \text { on } \Gamma_{W} \times(0, \infty), \\
\partial_{t} \boldsymbol{u}^{B L}-\nu \nabla \cdot\left(\nabla \boldsymbol{u}^{B L}\right)+\left(\boldsymbol{u}^{B L} \cdot \nabla\right) \boldsymbol{u}^{B L}+\nabla p^{B L}=\boldsymbol{f} & \text { in } \Omega_{\text {layer }} \times(0, \infty), \\
\boldsymbol{u}^{B L}=0 \text { on } \Gamma_{W} \times(0, \infty), \boldsymbol{u}^{B L}=\boldsymbol{u} & \text { on } \Gamma_{i} \times(0, \infty), \\
\boldsymbol{u}(0)=\mathbf{0} \text { in } \Omega, \quad \boldsymbol{u}^{B L}(0)=\mathbf{0} & \text { in } \Omega .
\end{aligned}
$$

Thus, in the general case, coupling global and local problem is accomplished via the nonlinear friction law $\boldsymbol{\sigma}_{t}\left(\boldsymbol{u}, \boldsymbol{u}^{B L}\right)$. 


\section{Fundamentals, modelling and simulation of turbulent flows}

The dynamics of non-isothermal fluid flow including all phenomena of turbulence are governed by the non-isothermal Navier-Stokes equations, see MODEL TNSE. However, the solutions to MODEL TNSE can become very complex if the critical parameter like Re resp. $R a$ becomes sufficiently large. Then the turbulent state of motion is simply the phenomenological aspect of this complexity. The complexity of the solution has two aspects, viz., (i) its randomness and (ii) its vast and continuous range of scales. As pointed out by DURBIN, the turbulence problem is how to describe and how to reduce this complexity in a manner which is appropriate to the needs of science and engineering, see [DPR01], p.1.

Depending on how to handle this complexity, there are three levels of description concerning a computational approach to a turbulent flow problem, videlicet,

- Compute the random motion of all scales, which is referred to as direct numerical simulation (abbreviated DNS),

- compute the random motion of the large scale motion (and model the small scale motion), which is referred to as large-eddy simulation (abbreviated LES),

- predict mean flow field, pressure and temperature (in a statistical sense), referred to as statistical turbulence modelling or Reynolds averaged CFD (called RANS),

The first two approaches are called turbulence simulation, because they account for the randomness of an individual realisation of a flow experiment. Their results have to be statistically averaged to obtain a mean flow. In contrast, the output of a RANS computation is already the mean flow.

In Section 2.1 we focus on aspect (i) and consider the random behaviour of turbulent flows, introducing some basic concepts for describing its statistics. In Section 2.2 we study aspect (ii), i.e., the scales of motion in a turbulent flow, and explain the most fundamental process involving eddies of different sizes, viz., the energy cascade. This chapter concludes by reviewing some criteria for appraising turbulence modelling and simulation, resumed e.g. in [Pope00].

\subsection{Aspects of randomness and statistical description of turbulent flows}

A major property of turbulent flows is that they appear to be chaotic or random. This seems to be in contrast to the a priori deterministic nature of MODEL TNSE. Randomness is a consequence of the interaction of (i) the singular perturbation parameter $R e$ resp. $R a$ and (ii) the non-linearity of the Navier-Stokes equations. In a fluid-flow experiment, there are unavoidably inaccuracies and perturbations in initial conditions, boundary conditions (e.g., differential heating, surface roughness) and material properties, i.e. viscosity and thermal diffusivity (due to impurities of the fluid). Because of (i) and (ii) flow is extremely 
sensitive to small perturbations. Thus a single realisation of a fluid flow experiment has some aspects of randomness, its individual eddies seem to develop randomly and irregularly in space and time. Some mathematical understanding can be gained by studying much simpler model problems like the Lorenz equations or the Rayleigh-Bénard convection. However, statistics, like averages, variances and covariances of velocity and temperature, show a reproducible and regular behaviour in space and time. If a flow experiment is repeated with a very small perturbation in the initial conditions, after a certain time the realisations can differ significantly. However, their statistics are (nearly) identical.

Now some basic concepts for the statistical description of turbulent flows will be introduced. We consider an ensemble of $N$ identical flow experiments, whose initial and boundary conditions differ by small random perturbations. Quantities of the $n$-th experiment are labelled by superscript $(n)$. Then velocity resp. pressure and temperature in an individual experiment can be considered as a time-dependent random field resp. as random variables. These quantities can be subdivided into a mean component and into a "turbulent fluctuation" component, viz.,

$$
(\boldsymbol{u}, p)=\left(\langle\boldsymbol{u}\rangle_{E},\langle p\rangle_{E}\right)+\left(\boldsymbol{u}^{\prime}, p^{\prime}\right), \quad \theta=\langle\theta\rangle_{E}+\theta^{\prime} .
$$

Here $\langle\cdot\rangle_{E}$ denotes the ensemble averaging filter. For a time-dependent random variable $\phi$, we define $\langle\phi\rangle_{E}(\boldsymbol{x}, t)$ as

$$
\langle\phi\rangle_{E}(\boldsymbol{x}, t)=\lim _{N \rightarrow \infty} \frac{1}{N} \sum_{n=1}^{N} \phi^{(n)}(\boldsymbol{x}, t) .
$$

Ensemble averaging is a linear operation. Denote $\phi, \psi$ a random variable and let $\lambda$ be non-random. Then we have

$$
\langle\phi+\psi\rangle_{E}=\langle\phi\rangle_{E}+\langle\psi\rangle_{E}, \quad\langle\lambda \phi\rangle_{E}=\lambda\langle\phi\rangle_{E}, \quad\left\langle\langle\phi\rangle_{E}\right\rangle_{E}=\langle\phi\rangle_{E} .
$$

The last equality implies that the fluctuating component has zero mean. It is essential to point out that

$$
\langle\phi \psi\rangle_{E} \neq\langle\phi\rangle_{E}\langle\psi\rangle_{E}
$$

From the fluctuating velocity field we can define the following tensor of the fluctuation velocity covariances, called Reynolds stress tensor

$$
\left(\begin{array}{ccc}
\left\langle u_{1}^{\prime} u_{1}^{\prime}\right\rangle_{E} & \left\langle u_{1}^{\prime} u_{2}^{\prime}\right\rangle_{E} & \left\langle u_{1}^{\prime} u_{3}^{\prime}\right\rangle_{E} \\
\left\langle u_{2}^{\prime} u_{1}^{\prime}\right\rangle_{E} & \left\langle u_{2}^{\prime} u_{2}^{\prime}\right\rangle_{E} & \left\langle u_{2}^{\prime} u_{3}^{\prime}\right\rangle_{E} \\
\left\langle u_{3}^{\prime} u_{1}^{\prime}\right\rangle_{E} & \left\langle u_{3}^{\prime} u_{2}^{\prime}\right\rangle_{E} & \left\langle u_{3}^{\prime} u_{3}^{\prime}\right\rangle_{E}
\end{array}\right) .
$$

Half its trace is called turbulent kinetic energy, denoted $k$, namely,

$$
k=\frac{1}{2} \sum_{i=1}^{d}\left\langle u_{i}^{\prime} u_{i}^{\prime}\right\rangle_{E},
$$


being the mean kinetic energy per unit mass in the fluctuating velocity field.

Now we want to describe the stochastical behaviour of a random field. The only objective of the remaining part of this section is to introduce some definitions, being needed in the following section. A concept of fundamental importance is the so-called $N$-point, $N$-time joint cumulative distribution function (CDF) of the velocity field, see [Pope00], pp.65, which is defined by

$F_{N}\left(\boldsymbol{v}^{(1)}, \boldsymbol{x}^{(1)}, t^{(1)} ; \ldots ; \boldsymbol{v}^{(n)}, \boldsymbol{x}^{(n)}, t^{(n)}\right) \equiv P\left(\left\{\boldsymbol{u}\left(\boldsymbol{x}^{(1)}, t^{(1)}\right)<\boldsymbol{v}^{(1)} ; \ldots ; \boldsymbol{u}\left(\boldsymbol{x}^{(n)}, t^{(n)}\right)<\boldsymbol{v}^{(n)}\right\}\right)$,

where $\boldsymbol{u}<\boldsymbol{v}$ means $u_{i}<v_{i}(1 \leq i \leq d)$ and $P(A)$ denotes the probability of $A$. To completely characterize a random field, this N-point N-time CDF must be determined for all space-time points, which is impossible. However, it turned out that in many applications the complexity reduces considerably, because the flow is statistically stationary, homogeneous and isotropic.

A random field $\boldsymbol{u}(\boldsymbol{x}, t)$ is called statistically stationary, if all $N$-point CDFs are invariant under a shift in time. Similarly, $\boldsymbol{u}(\boldsymbol{x}, t)$ is called statistically homogeneous, if all $N$-point CDFs are invariant under a shift in position. The field $\boldsymbol{u}(\boldsymbol{x}, t)$ is called statistically isotropic, if it is statistically homogeneous and if all $N$-point CDFs are invariant under rotations and reflections of the coordinate system.

Studying the two-point correlation of $\boldsymbol{u}^{\prime}$ in homogeneous isotropic turbulence has been of greatest interest in turbulence research. The two-point correlation is the two-point, onetime autocovariance

$$
R_{i j}(\boldsymbol{r}, \boldsymbol{x}, t) \equiv\left\langle u_{i}^{\prime}(\boldsymbol{x}, t) u_{j}^{\prime}(\boldsymbol{x}+\boldsymbol{r}, t)\right\rangle_{E},
$$

being independent of $\boldsymbol{x}$ because of homogeneity, i.e., $R_{i j}(\boldsymbol{r}, \boldsymbol{x}, t)=R_{i j}(\boldsymbol{r}, t)$. From this, the velocity spectrum tensor $\Phi_{i j}(\boldsymbol{k}, t)$ can be defined via Fourier transform, viz.,

$$
\Phi_{i j}(\boldsymbol{\kappa}, t)=\frac{1}{(2 \pi)^{d}} \int_{\mathbb{R}^{d}} e^{-i \boldsymbol{\kappa} \cdot \boldsymbol{r}} R_{i j}(\boldsymbol{r}, t) d \boldsymbol{r} .
$$

In isotropic turbulence, $R_{i j}$ and $\Phi_{i j}$ depend only on $|\boldsymbol{r}|$ and $|\boldsymbol{\kappa}|$ resp. Then the turbulent kinetic energy $k=\frac{1}{2}\left\langle\boldsymbol{u}^{\prime} \cdot \boldsymbol{u}^{\prime}\right\rangle_{E}$ can be written as

$$
k=\frac{1}{2}\left\langle\boldsymbol{u}^{\prime 2}\right\rangle_{E}=\frac{1}{2} \sum_{i=1}^{d} R_{i i}(\mathbf{0}, t)=\int_{0}^{\infty} \int_{|\boldsymbol{\kappa}|=\kappa} \frac{1}{2} \sum_{i=1}^{d} \Phi_{i i}(\boldsymbol{\kappa}, t) d \sigma d \kappa=\int_{0}^{\infty} E(\kappa, t) d \kappa,
$$

where $E(\kappa, t)$ is called the spectrum of the turbulent kinetic energy and is defined by

$$
E(\kappa, t)=\int_{|\boldsymbol{\kappa}|=\kappa} \frac{1}{2} \sum_{i=1}^{d} \Phi_{i i}(\boldsymbol{\kappa}, t) d \sigma
$$

with $\int \ldots d \sigma$ denoting the $(d-1)$-dimensional surface integral. From the two-point correlation, the following characteristic lengthscale can be defined

$$
L_{11}(\boldsymbol{x}, t)=\frac{1}{R_{11}(0, \boldsymbol{x}, t)} \int_{0}^{\infty} R_{11}\left(\boldsymbol{e}_{\mathbf{1}} r, \boldsymbol{x}, t\right) d r,
$$

where $\boldsymbol{e}_{1}$ denotes the unit vector in the $x_{1}$ direction. 


\subsection{The scales of turbulent flows}

A second characteristic feature of a turbulent flow is its large variety of scales, primarily studied by Richardson (1922) and Kolmogorov (1941). The goal of this section is to provide some physical understanding of the different scales of motion in turbulent flows and the processes among them, being a motivation for the approach of large-eddy simulation. According to Richardson, a turbulent flow can be thought of as a superposition of locally coherent structures, called eddies, of different sizes. Today, the term 'eddy' is used more ambiguously; it is used to characterise the scales of structures in the flow field: Large eddies refer to large structures, small eddies refer to small structures in the flow field, see [DPR01], p.2. A process of fundamental inportance is the so-called energy cascade: By a production mechanism $\mathcal{P}_{r}$, which will be described later, the large eddies are generated. These are unstable and break up into successively smaller and smaller eddies, i.e. their energy is transferred to smaller and smaller scales by inviscid processes. At the smallest scales the energy is dissipated into heat by molecular viscosity. This process is called dissipation of turbulent kinetic energy or simply dissipation. It is described by the quantity $\epsilon \equiv\left\langle 2 \nu \mathbb{S}\left(\boldsymbol{u}^{\prime}\right): \mathbb{S}\left(\boldsymbol{u}^{\prime}\right)\right\rangle_{E}$.

A more detailed and quantitative analysis of high Reynolds number turbulence was given by Kolmogorov. He introduced various length scales and ranges, depending on the eddy size $l$, as pictured in the following schematic diagram (cf. [Pope00], pp. 187): Whereas

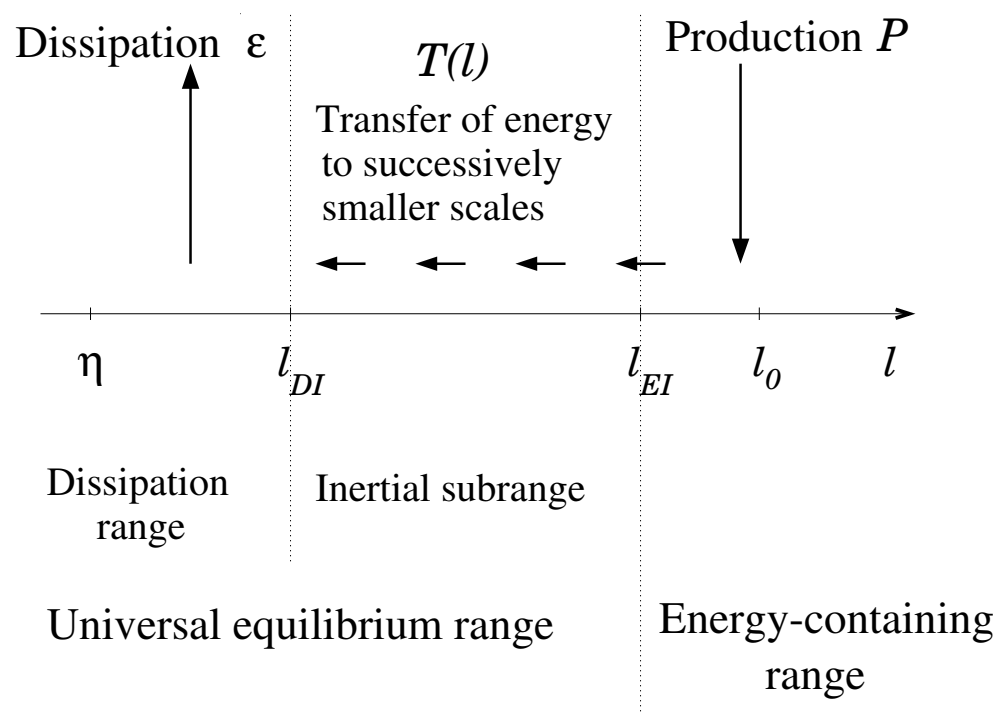

Figure 2.1.: Eddy sizes (on a logarithmic scale) and the energy cascade.

the behaviour of the large eddies $\left(l \approx l_{0}\right)$ depends on the flow problem, the smale-scale motions $\left(l \ll l_{0}\right)$ are locally statistically isotropic according to Kolmogorov's hypothesis of local isotropy. Moreover Kolmogorov's first similarity hypothesis states that the statistics of the small-scale motions $\left(l<l_{E I}\right)$ have a universal form that is uniquely determined by $\epsilon$ and $\nu$. Denote $\mathcal{T}(l)$ the rate of transfer of energy from eddies larger than $l$ to eddies smaller than $l$. Then $\mathcal{T}\left(l_{E I}\right)=\mathcal{T}(l)=\mathcal{T}\left(l_{D I}\right)=\epsilon$ for all $l_{D I} \leq l \leq l_{E I}$ and thus $\epsilon$ is determined by 
the transfer of energy from the largest eddies. Kolmogorov's second similarity hypothesis says that in the inertial subrange the statistics depend only on $\epsilon$.

The characteristic lengthscale in the dissipation range is the so-called Kolmogorov scale $\eta=\left(\nu^{3} / \epsilon\right)^{1 / 4}$. Then the ratio of the largest to smallest scales is of order $R e^{3 / 4}$, which demonstates the vast range of scales.

The question is how turbulent kinetic energy and dissipation are distributed among the eddies of different sizes. Denote $\kappa=2 \pi / l$ the wavenumber corresponding to motions of lengthscale $l$. Then energy and dissipation in the wavenumber range $\left(\kappa_{a}, \kappa_{b}\right)$ are given by

$$
k_{\left(\kappa_{a}, \kappa_{b}\right)}=\int_{\kappa_{a}}^{\kappa_{b}} E(\kappa) d \kappa, \quad \epsilon_{\left(\kappa_{a}, \kappa_{b}\right)}=\int_{\kappa_{a}}^{\kappa_{b}} 2 \nu \kappa^{2} E(\kappa) d \kappa
$$

with $E(\kappa)=E(\kappa, t)$ in statistically stationary turbulence and the energy spectrum function $E(\kappa, t)$ being defined in (2.4). By Kolmogorov's first hypothesis, in the universal equilibrium range $\left(\kappa>2 \pi / l_{E I}\right), E(\kappa)$ is a universal function of $\epsilon$ and $\nu$. In $2 \pi / l_{D I}>\kappa>2 \pi / l_{E I}$ the spectrum is given by

$$
E(\kappa)=C \epsilon^{2 / 3} \kappa^{-5 / 3}
$$

with a universal constant $C=1.5$, see [Pope00], p.231. Abundant physical experiments confirm this law.

To answer the remaining question, the cumulative kinetic energy and the cumulative dissipation have to be introduced

$$
k_{(0, \kappa)}=\int_{0}^{\kappa} E\left(\kappa^{\prime}\right) d \kappa^{\prime} \quad \epsilon_{(0, \kappa)}=\int_{0}^{\kappa} 2 \nu \kappa^{\prime 2} E\left(\kappa^{\prime}\right) d \kappa^{\prime} .
$$

Since $\epsilon_{(0,2 \pi /(60 \eta))}=0.1 \epsilon$, significant dissipation occurs only for $l \leq 60 \eta$. Therefore the demarcation lengthscale between the inertial and dissipative ranges is taken to be $l_{D I}=$ $60 \eta$. Concerning the kinetic energy, if $l_{E I}=1 / 6 L_{11}$ and $\kappa_{E I}=2 \pi / l_{E I}$, cf. (2.5), then $k_{\left(0, \kappa_{E I}\right)}=0.8 k$, i.e. eddies of size $l>l_{E I}$ contain $80 \%$ of the kinetic energy, cf. [Pope00], p.237 and p.241. For this reason, $l>l_{E I}$ is called energy containing range. Thus the bulk of kinetic energy is contained in the large-scale motions, whereas the bulk of dissipation affects the small-scale motions.

\subsection{Criteria for appraising approaches in CFD}

PoPE resumes the following criteria for appraising approaches in CFD, see [Pope00], pp.336, viz.,

- level of description,

- completeness,

- cost and ease of use,

- range of applicability,

- accuracy. 
The level of description specifies which information is provided by the solution of a computation. For example, from a LES we can extract the Reynolds stresses carried by the large scale motion, whereas from a RANS computation, the only quantities obtained are mean values. A model is called complete if there are no unclosed terms in its constituent equations. Both the $k / \epsilon$ model and the LES models studied in this thesis are complete. The criterion concerning cost and ease of use of a model regards its use in a CFD code. We start regarding the cost of a model. Firstly, we have to account for the number of operations needed to perform a computation. Secondly, we have to consider the memory consumption of a computation. Clearly these two points dictate the scale of computer needed, i.e. a supercomputer or a workstation. There has been a tremendous progress in computer architecture in the last decades (see [HP96]). Moreover, the CFD community becomes more and more aware of the need for architecture-friendly algorithms in order to exploit the improvements in computer hardware, see e.g. the URL http://www.math.odu.edu/ keyes/ and in particular [Key00]. Despite these efforts, a DNS for complex flows will be infeasible even with next decades supercomputers.

One aspect of ease of use of a model concerns its numerical properties, e.g., its stability. A further point is regarding the post-processing required to extract the results of interest. In particular, a LES requires ensemble averaging whereas a RANS computation does not. Moreover, the model together with the numerical solution scheme impact the implementation and the data structures required. This determines the ease of code implemention and maintenance for a certain model. Fortunately, even in the CFD community, having relied on Fortran and $\mathrm{C}$ for several decades, the trend is towards object-oriented programming languages. Using an object-oriented programming paradigm facilitates implementing and maintaining complex CFD codes significantly without loss in performance, see e.g. the URL http://www.oonumerics.org/.

Applicability concerns the question whether the model assumptions and requirements are satisfied for a given flow problem. Finally, the accuracy of a model appraises the quality of its predictions by comparison with experimental data. 


\section{The $k / \epsilon$ turbulence model}

In the previous section we introduced the idea of reducing the complexity of a turbulent flow by a statistical approach. The objective of this chapter is to present the so-called $k / \epsilon$ turbulence model. It is the most widely used statistical turbulence model, being incorporated in most commercial CFD codes. The focus will be on the underlying model approximations with emphasis being placed on effects of buoyancy.

\subsection{The Reynolds averaged Navier-Stokes equations}

The starting point is the so-called Reynolds decomposition, cf. (2.1)

$$
(\boldsymbol{u}, p)=\left(\langle\boldsymbol{u}\rangle_{E},\langle p\rangle_{E}\right)+\left(\boldsymbol{u}^{\prime}, p^{\prime}\right), \quad \theta=\langle\theta\rangle_{E}+\theta^{\prime},
$$

where $\langle\cdot\rangle_{E}$ again denotes the ensemble averaging filter, defined in (2.2). For simplicity, in the sequel, ensemble averaged quantities are designated by capital letters. Applying the ensemble-averaging filter to the evolution equations in MODEL TNSE yields the so-called Reynolds averaged Navier-Stokes equations (abbreviated RANS equations)

$$
\begin{aligned}
\partial_{t} \boldsymbol{U}+(\boldsymbol{U} \cdot \nabla) \boldsymbol{U}+\nabla P-\nabla \cdot\left(2 \nu \mathbb{S}(\boldsymbol{U})-\left\langle\boldsymbol{u}^{\prime} \otimes \boldsymbol{u}^{\prime}\right\rangle_{E}\right) & =-\beta \Theta \boldsymbol{g} \\
\nabla \cdot \boldsymbol{U} & =0 \\
\partial_{t} \Theta+(\boldsymbol{U} \nabla) \Theta-\nabla \cdot\left(a \nabla \Theta-\left\langle\boldsymbol{u}^{\prime} \theta^{\prime}\right\rangle_{E}\right) & =\frac{\dot{q}^{V}}{c_{p}} .
\end{aligned}
$$

These are "nearly" the non-isothermal Navier-Stokes equations for the mean values of velocity, pressure and temperature. However, they contain two additional terms of crucial importance. Therein, the velocity covariances $\left\langle\boldsymbol{u}^{\prime} \otimes \boldsymbol{u}^{\prime}\right\rangle_{E}$ appearing in the momentum equation are referred to as Reynolds stresses. They can be interpreted as additional stresses arising from the mean momentum flux due to the fluctuating velocity field. The analogous term in the temperature equation, viz, $\left\langle\boldsymbol{u}^{\prime} \theta^{\prime}\right\rangle$, is called scalar flux. It describes the flux of temperature due to the fluctuating velocity field. POPE generalises and emphasises this observation: In turbulent flows, the rates of mixing of momentum, heat and mass are greatly enhanced, see [Pope00], p.7.

Both fluctuation terms are functions of unknown correlations that cannot be expressed in terms of mean quantities: Because of the non-linearity of the Navier-Stokes equations, the first moment equation contains second moments, the second moment equations will contain third moments, and so forth. Thus, to handle these terms, closure hypotheses are needed. 


\subsection{Turbulent-viscosity and gradient-diffusion hypotheses}

\subsubsection{The RANS equations using the turbulent-viscosity and gradient-diffusion hypotheses}

In 1877, BoussinesQ proposed the so-called eddy-viscosity hypothesis or turbulent-viscosity hypothesis. It assumes the constitutive relation

$$
\left\langle\boldsymbol{u}^{\prime} \otimes \boldsymbol{u}^{\prime}\right\rangle=-2 \nu_{t} \mathbb{S}(\boldsymbol{U})+\frac{2}{3} k I,
$$

where the positive scalar field $\nu_{t}$ is the so-called eddy-viscosity or turbulent viscosity. Sometimes $-\left\langle\boldsymbol{u}^{\prime} \otimes \boldsymbol{u}^{\prime}\right\rangle+\frac{2}{3} k I$ will be referred to as anisotropic Reynolds-stress. The second right hand side term in (3.4) is a normal stress correction which ensures that the traces of both sides equal.

Similarly the gradient-diffusion hypothesis assumes that

$$
\left\langle\boldsymbol{u}^{\prime} \theta^{\prime}\right\rangle=-a_{t} \nabla \Theta,
$$

where $a_{t}$ is the turbulent thermal diffusivity. Moreover we introduce effective viscosity $\nu_{e}$ and effective diffusivity $a_{t}$, viz,

$$
\nu_{e}=\nu+\nu_{t}, \quad a_{e}=a+a_{t} .
$$

Using (3.4), (3.5) and (3.6), the non-isothermal RANS equations (3.1)-(3.3) become

$$
\begin{aligned}
\partial_{t} \boldsymbol{U}+(\boldsymbol{U} \cdot \nabla) \boldsymbol{U}+\nabla\left(P+\frac{2}{3} k\right)-\nabla \cdot\left(2 \nu_{e} \mathbb{S}(\boldsymbol{U})\right) & =-\beta \Theta \boldsymbol{g}, \\
\nabla \cdot \boldsymbol{U} & =0, \\
\partial_{t} \Theta+(\boldsymbol{U} \nabla) \Theta-\nabla \cdot\left(a_{e} \nabla \Theta\right) & =\frac{\dot{q}^{V}}{c_{p}} .
\end{aligned}
$$

Here we should point out a further difficulty. We have two possibilities for treating the term $\frac{2}{3} \nabla k$ arising in (3.4). We could (i) include it in the pressure term or we could (ii) modify the right hand side

$$
\text { (i) } \quad P^{*} \equiv P+\frac{2}{3} k, \quad \text { or } \quad(i i) \boldsymbol{f}^{*} \equiv \boldsymbol{f}-\frac{2}{3} \nabla k .
$$

Case (i) is based on the observation that the stresses due to the term $\frac{2}{3} k I$ are normal stresses that act like pressure forces. But it has the major disadvantage that when using $p^{*}$ as the independent pressure variable, special care must be taken when prescribing boundary conditions involving the physical pressure, cf. [HC(01], p.43.

On the other hand, in case (ii) the right hand side is disturbed. In our field of interest, the study of indoor-air movement, the flow is induced and influenced by temperature differences in a sensitive manner. Consequently we want to avoid contamination of this term by other terms. In our research group therefore strategy (i) was chosen.

The notion that the turbulent motion mixes both momentum and temperature motivates 
the goal to formulate a relationship between the turbulent heat flux and the Reynolds stress tensor which is responsible for that flux. The simplest model is to assume that the scalar flux behaves analogously to the momentum flux. An immediate consequence of this assumption is that there is a constant of proportionality, called turbulent Prandtl number, such that $a_{t}=\operatorname{Pr}_{t}^{-1} \nu_{t}$, which can be rearranged to the more convenient form

$$
\operatorname{Pr}_{t}=\frac{\nu_{t}}{a_{t}}
$$

\section{Remark 3.1}

$P r_{t}$ can depend on many factors that influence the flow field. In particular, $P r_{t}$ is not a constant material property. In indoor-air flow problems, our research group chooses $P r_{t}=0.9$ remote from walls and $P r_{t}=1.15$ in the near-wall region, $P r_{t}$ being smooth in-between. This choice is in agreement with [PS01]. [DPR01], p.53 report $P r_{t}=0.9$ in boundary layers and $P r_{t}=0.7$ in free-shear flows. This again reveals the problem in turbulence modelling that model constants are not physical constants.

So far the closure problem for (3.1)-(3.3) has been reduced to the task of specifying the scalar field $\nu_{t}$. This will be the objective of Section 3.4.

\subsubsection{An appraisal of the turbulent-viscosity hypothesis}

A thorough appraisal of the turbulent-viscosity and gradient-diffusion hypothesis can be found in [Pope00], Section 4.4 and Section 10.1, and in [Wilcox98], Section 3.2 and Chapter 6. According to POPE, the turbulent-viscosity hypothesis can be viewed in two parts, viz., an intrinsic part and a specific part. The intrinsic assumption is that the anisotropic Reynolds-stress $a \equiv\left\langle\boldsymbol{u}^{\prime} \otimes \boldsymbol{u}^{\prime}\right\rangle_{E}-\frac{2}{3} k \mathbb{I}$ at each space-time point $(\boldsymbol{x}, t)$ is determined by the value of the mean rate-of-strain tensor at that space-time point $(\boldsymbol{x}, t)$, i.e., we assume that $a(\boldsymbol{x}, t)=f(\mathbb{S}(\boldsymbol{U})(\boldsymbol{x}, t))$ with some function $f$. The specific assumption is to assume a linear relation.

Obviously, turbulent-viscosity and gradient-diffusion hypothesis are analogous to FOURIER's law and FiCK's law of molecular processes. WILCOX explains why the viscous stress term $2 \nu \mathbb{S}(\boldsymbol{U})$ describes the momentum transfer at the molecular level and that $\nu$ is given by

$$
\nu=\frac{1}{2} v_{t h} l_{m f p},
$$

where $v_{t h}$ is the thermal velocity and $l_{m f p}$ is the mean free path, cf. [Wilcox98]. However, a consideration of the corresponding timescales shows that turbulent processes differ vastly from molecular processes. The timescales corresponding to shear stress and turbulence are $\mathcal{S}^{-1}$ and $k / \epsilon$, resp. The ratio of the molecular timescale to $\mathcal{S}^{-1}$ is very small (e.g. $10^{-10}$ ). Therefore molecular motion adjusts instantaneously to changes in mean straining. But in general, turbulence does not adjust rapidly, because typically $\mathcal{S} k / \epsilon>1$.

Originally, the turbulent-viscosity hypothesis was used with an algebraic model for $\nu_{t}$ by PRANDTL to describe simple shear flows like free-shear flows, e.g., the far wake or the 
mixing-layer, and attached boundary-layer flows, see [Wilcox98], Chapter 3. Surprisingly, using a more sophisticated formula for $\nu_{t}$, complicated two-dimensional flows can also be predicted quite well. The SPALART-ALLMARAS model is a one-equation model conceived for aerodynamic applications, which predicts transonic flows over airfoils including boundarylayer separation successfully. DURBIN developed the $k-\epsilon-\overline{v^{2}}$ model, a successor of the standard $k / \epsilon$ model, which has been applied successfully to complicated two-dimensional flows like jet impingement.

However, there are some situations where all models based on the turbulent-viscosity hypothesis fail inevitably: (i) Flows in ducts where the anisotropy of the Reynolds stresses generates a new component of the mean flow (often referred to as secondary motion), (ii) flows over curved surfaces and flows in rotating fluids and (iii) flows with sudden changes in mean strain rate. Describing the physics of (i), (ii) and (iii) correctly requires more complex models for the Reynolds stresses.

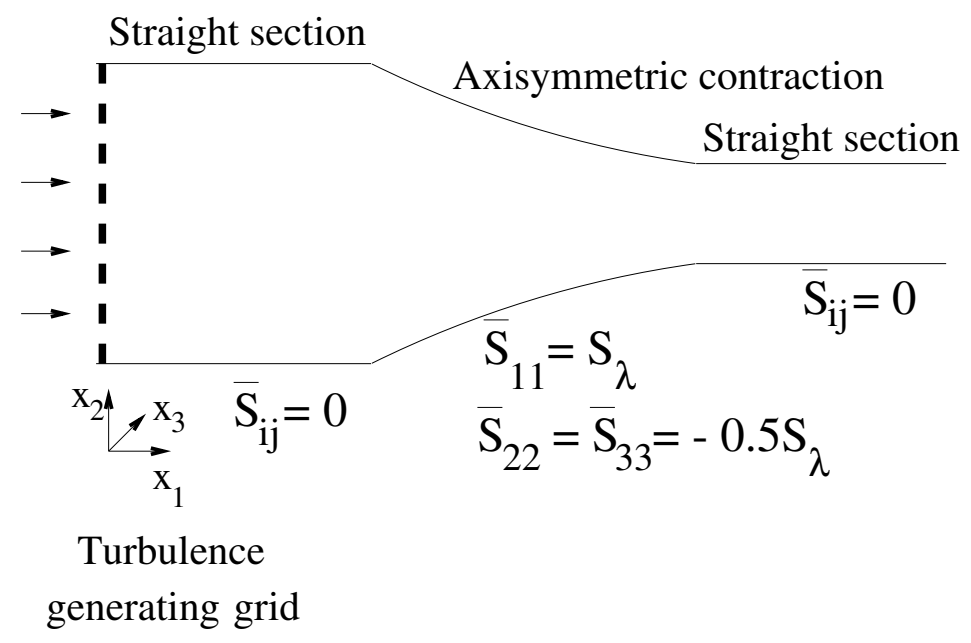

Figure 3.1.: Sketch of Tucker-Reynolds flow experiment.

Ad (i): In order to predict the anisotropy of the normal Reynolds stresses, non-linear constitutive relations (instead of the specific linear assumption $a=\nu_{t} \mathbb{S}(\boldsymbol{U})$ ) have been proposed, see [Wilcox98], Chapter 6.2 and references therein.

Ad (ii): In some situations, the individual components of the Reynolds stress tensor are affected differently by the production of turbulence. For example, in flows over surfaces with convex curvature the component directed toward the centre of curvature will be diminished. Thus a further step is to solve an algebraic equation for the Reynolds stress tensor. Such an equation can be derived from the exact (but unclosed) partial differential equation for the Reynolds stress tensor using some approximations for the unclosed terms and the terms including derivatives of the Reynolds stresses. This approach was originally devised by RoDI and is explained e.g. in [Pope00], pp.448 and [Wilcox98] pp. 282. These so-called algebraic stress models provide a significant improvement for flows with mean streamline curvature.

Ad (iii): On a statistical level, the most complete approach is to consider the partial differential equation for the Reynolds stress tensor. Closure models for unclosed terms lead 
to Reynolds stress models, also called second moment closure models or Reynolds stress transport models. An investigation of the resulting equation in the limit $\|\mathbb{S}(\boldsymbol{U})\| \rightarrow \infty$ reveals that the evolution of the Reynolds stresses at time $t$ depends on the prior history of straining $\int_{0}^{t}\|\mathbb{S}(\boldsymbol{U})\| d t^{\prime}$ (CROW 1968), see [Pope00], p.405. This is in contrast to the intrinsic assumption $a(\boldsymbol{x}, t)=f(\mathbb{S}(\boldsymbol{U})(\boldsymbol{x}, t))$. For example, consider the experiment by TUCKER and REYNOLDS, sketched in Figure 3.1. When the strain is suddenly removed after the axisymmetrical contraction, the intrinsic assumption predicts zero Reynolds stresses. This is in contrast to the experimentally observed relatively slow return to isotropy of the Reynolds stresses, see e.g. [Wilcox98], pp.274, and [Pope00], pp.359.

As a final remark, it is quite interesting that in natural convection boundary layers the turbulent-viscosity hypothesis does not hold in the near-wall region, but the gradientdiffusion hypothesis is satisfied reasonably, see [TN98b].

\subsection{Production and dissipation of turbulent kinetic energy in RANS models}

\subsubsection{Isothermal turbulent flows}

This section is devoted to the processes in turbulent flows that generate and dissipate turbulent kinetic energy. First we consider the isothermal case. The kinetic energy of the fluid per unit mass is $E(\boldsymbol{x}, t)=\boldsymbol{u}(\boldsymbol{x}, t) \cdot \boldsymbol{u}(\boldsymbol{x}, t) / 2$. We can decompose $\langle E(\boldsymbol{x}, t)\rangle_{E}$ into the kinetic energy of the mean flow $\bar{E}=\boldsymbol{U} \cdot \boldsymbol{U} / 2$ and into the turbulent kinetic energy $k=\left\langle\boldsymbol{u}^{\prime} \cdot \boldsymbol{u}^{\prime}\right\rangle_{E} / 2$. Starting from the RANS equations (3.1)-(3.2) and from the corresponding equation for $\boldsymbol{u}^{\prime}$, the following equations can be derived, cf. eq. (5.131)-(5.132) in [Pope00].

$$
\begin{aligned}
\partial_{t} \bar{E}+\boldsymbol{U} \cdot \nabla \bar{E}+\nabla \cdot\left(\left\langle\boldsymbol{u}^{\prime} \otimes \boldsymbol{u}^{\prime}\right\rangle_{E} \boldsymbol{U}+\boldsymbol{U} P-2 \nu \mathbb{S}(\boldsymbol{U}) \boldsymbol{U}\right) & =-\mathcal{P}_{k}-\bar{\epsilon} \\
\partial_{t} k+\boldsymbol{U} \cdot \nabla k+\nabla \cdot\left(\left\langle\boldsymbol{u}^{\prime} \otimes \boldsymbol{u}^{\prime} \boldsymbol{u}^{\prime}\right\rangle_{E}+\left\langle\boldsymbol{u}^{\prime} p\right\rangle_{E}-2 \nu\left\langle\mathbb{S}\left(\boldsymbol{u}^{\prime}\right) \boldsymbol{u}^{\prime}\right\rangle_{E}\right) & =\mathcal{P}_{k}-\epsilon
\end{aligned}
$$

with

$$
\begin{aligned}
\bar{\epsilon} & \equiv 2 \nu \mathbb{S}(\boldsymbol{U}): \mathbb{S}(\boldsymbol{U}), & & \text { (dissipation due to the mean flow) } \\
\epsilon & \equiv 2 \nu\left\langle\mathbb{S}\left(\boldsymbol{u}^{\prime}\right): \mathbb{S}\left(\boldsymbol{u}^{\prime}\right)\right\rangle_{E}, & & \text { (dissipation due to turbulent fluctuations) } \\
\mathcal{P}_{k} & \equiv-\left\langle\boldsymbol{u}^{\prime} \otimes \boldsymbol{u}^{\prime}\right\rangle_{E}: \mathbb{S}(\boldsymbol{U}), & & \text { (production of turbulent kinetic energy). }
\end{aligned}
$$

The last term on the left hand side, i.e. $\nabla \cdot(\ldots)$, in (3.12)-(3.13) is called flux of energy, as it represents the transfer of mean flow kinetic energy resp. turbulent kinetic energy from one region to another. $\mathcal{P}_{k}$ is a sink term in the equation for $\bar{E}$ and a source term in the $k$ equation. $\mathcal{P}_{k}$ describes how kinetic energy is removed from the mean flow and transferred to the fluctuating velocity field. Using the turbulent-viscosity hypothesis, (3.16) becomes

$$
\mathcal{P}_{k}=2 \nu_{t} \mathbb{S}(\boldsymbol{U}): \mathbb{S}(\boldsymbol{U})
$$

\subsubsection{Coupling between buoyancy and turbulence generation}

Now we consider the case of buoyancy driven flows. Then we have to distinguish between two phenomena:

(I) The stabilising effect of stratification. 
(II) A (speculative) additional turbulence generation mechanism due to buoyancy as suggested by the theory of baroclinic vorticity generation.

First we study the effect of stratification on turbulence. In the non-isothermal case, equations for $\bar{E}$ and $k$ can be derived similar to (3.12)-(3.13), see e.g. [DPR01], pp.223. The difference w.r.t. (3.12)-(3.13) is that we have to replace $\mathcal{P}_{k}$ by $\mathcal{P}_{k}+G$. $G$ is often called gravitational production term and is given by

$$
G=-\beta \sum_{i=1}^{d} g_{i}\left\langle u_{i} \theta\right\rangle_{E} .
$$

It is convenient to define the flux Richardson number

$$
R i_{f} \equiv \frac{-G}{\mathcal{P}_{k}},
$$

which is a measure for the stabilising effect of stratification. If $R i_{f}>0$, then turbulence is suppressed; if $R i_{f}<0$, then turbulence is enhanced.

Regarding (II), at the present stage of knowledge, there are two concurring theories regarding an additional coupling mechanism between buoyancy and turbulence generation, being reported briefly by TIESZEN ET AL. in [T(D)B98].

First both perspectives will be reviewed. According to the more traditional theory, the only effect of buoyancy (i.e., density gradients) is to induce vertical momentum. Ascending air requires a transverse inflow. Then turbulence is only due to large-scale instabilities (mean velocity gradients) and the subsequent turbulent energy cascade. The second perspective views buoyancy in terms of the so-called baroclinic vorticity generation (BVG): In a gravitational field, temperature gradients perpendicular (normal) to the direction of gravity tend to result in the generation of vorticity, also referred to as small-scale instabilities. These vortical structures randomly interact with themselves and with the existing turbulence.

Having presented both viewpoints, TIESZEN ET AL. draw the following conclusions regarding the modelling of an additional buoyancy-turbulence interaction. Regarding the traditional perspective, buoyancy acts only on the large lengthscales. In this case, there is no need for modifying the turbulence model under consideration. On the other hand, the BVG theory says that there is an additional interaction between buoyancy and turbulence that has to be modelled.

A relevant situation concerning (II) is a flow along a vertical hot wall. Then in the nearwall region vertical stratification is small compared to the large temperature gradients in cross-stream direction. The observation that the turbulent-viscosity hypothesis does not hold in the near-wall region can be viewed as an indication of the BVG-hypothesis, see [TN.986].

\subsection{A two-equation model : The $k / \epsilon$ model}

\subsubsection{The $k / \epsilon$ model for buoyancy driven flows}

Two-equation models are based on the so-called KOLMOGOROV-PRANDTL relation

$$
\nu_{t}=c u^{*} l_{m}, \text { with } u^{*}=c_{u^{*}} k^{1 / 2} .
$$


(3.20) can be regarded as a formal analogy to (3.11). $l_{m}$ and $u^{*}$ are a suitable lengthscale resp. a suitable velocity scale, being a formal analogy to $l_{m f p}$ and $v_{t h}$ resp. in (3.11). Using dimensional analysis, $l_{m}$ can be expressed using $k$ and $\epsilon$ according to

$$
l_{m}=c_{l_{m}} k^{3 / 2} \epsilon^{-1} .
$$

Combining (3.20) and (3.21) we can compute $\nu_{t}$ from $k$ and $\epsilon$ using the formula

$$
\nu_{t}=C_{\mu} \frac{k^{2}}{\epsilon}, \quad C_{\mu}=0.09 .
$$

Here the value $C_{\mu}=0.09$ is chosen to ensure a correct behaviour in shear flows.

In the $k / \epsilon$ model, $k$ and $\epsilon$ are obtained as solutions of partial differential equations; consequently the model will be finally closed. Using the closure approximation

$$
\left(\left\langle\boldsymbol{u}^{\prime} \otimes \boldsymbol{u}^{\prime} \boldsymbol{u}^{\prime}\right\rangle_{E}+\left\langle\boldsymbol{u}^{\prime} p\right\rangle_{E}-2 \nu\left\langle\mathbb{S}\left(\boldsymbol{u}^{\prime}\right) \boldsymbol{u}^{\prime}\right\rangle_{E}=-\frac{\nu_{t}}{\operatorname{Pr}_{k}} \nabla k,\right.
$$

in (3.13), the following equation for $k$ is obtained (using the further approximation that $P r_{k}=1.0$ equals a constant)

$$
\partial_{t} k+(\boldsymbol{u} \cdot \boldsymbol{\nabla}) k-\boldsymbol{\nabla} \cdot\left(\frac{\nu_{t}}{\operatorname{Pr}_{k}} \nabla k\right)=\mathcal{P}_{k}-\epsilon .
$$

Compared to the $k$ equation, the equation for $\epsilon$ "is best viewed as being entirely empirical" ([Pope00], p.375); it reads (with constants $\mathrm{Pr}_{\epsilon}, C_{1}, C_{2}$ being specified later)

$$
\partial_{t} \epsilon+(\boldsymbol{u} \cdot \nabla) \epsilon-\nabla \cdot\left(\frac{\nu_{t}}{P r_{\epsilon}} \nabla \epsilon\right)+C_{2} \epsilon^{2} k^{-1}=C_{1} \epsilon k^{-1} \mathcal{P}_{k} .
$$

An attempt to a mathematical approach to (3.24) and (3.25) can be found in [MP94]. The standard modification of the $k / \epsilon$ model for buoyancy driven flows is based on simply replacing $\mathcal{P}_{k}$ with $\mathcal{P}_{k}+G$, being defined in (3.16) resp. (3.18). Then for $\mathcal{P}_{k}$ and $G$ the turbulent-viscosity resp. gradient-diffusion assumptions are used. This was originally devised by INCE and LAUNDER, see [L148] , who proposed to replace $\mathcal{P}_{k}$ by

$$
\mathcal{P}_{k}+G, \quad \text { with } \quad G \equiv C_{t} \beta \frac{\nu_{t}}{P r_{t}} \boldsymbol{g} \cdot \nabla \Theta, \quad C_{t}=0.8 .
$$

However, (3.26) can only describe the interaction between stratification and turbulence, see Subsection 3.3. As pointed out in [T(D)B98], p. 294, (3.26) cannot describe the following phenomenon. In a flow along a vertical hot wall, the vertical stratification is small compared to the temperature cross-stream gradient. On the one hand, formula (3.26) implies $G=0$ as temperature gradients are perpendicular to the direction of gravity. On the other hand, BVG theory says that temperature gradients perpendicular to the direction of gravity tend to result in the generation of vorticity. Therefore [T(D)B98] emphasise using the so-called generalized gradient-diffusion hypothesis, originated by DALY AND HARLOW (1970), see [DH70], and applied by INCE AND LAUNDER, cf. [II89], viz.,

$$
G=-\beta c_{\theta} \frac{k}{\epsilon} \sum_{i, j=1}^{d} g_{i}\left[\frac{2}{3} k \delta_{i j}-\nu_{t}\left(\frac{\partial U_{i}}{\partial x_{j}}+\frac{\partial U_{j}}{\partial x_{i}}\right)\right] \frac{\partial \Theta}{\partial x_{j}}
$$


with constant $c_{\theta}$ with standard value $c_{\theta}=0.18$. Numerical tests with our research code revealed that (3.26) and (3.27) give almost the same results due to our near-wall modelling strategy. However, when resolving the near-wall region, (3.27) is reported to be superior to (3.26), see [T()|)B98]. Thus, for practical reasons, we use (3.26). To this end, using (3.26) we arrive at the following system of equations for $\boldsymbol{U}, P, \Theta, k$ and $\epsilon$

$$
\begin{aligned}
\partial_{t} \boldsymbol{U}-\boldsymbol{\nabla} \cdot\left(2 \nu_{e} \mathbb{S}(\boldsymbol{U})\right)+(\boldsymbol{U} \cdot \boldsymbol{\nabla}) \boldsymbol{U}+\boldsymbol{\nabla} P & =-\beta \Theta \boldsymbol{g} \\
\boldsymbol{\nabla} \cdot \boldsymbol{U} & =0 \\
\partial_{t} \Theta+(\boldsymbol{U} \cdot \boldsymbol{\nabla}) \Theta-\boldsymbol{\nabla} \cdot\left(a_{e} \boldsymbol{\nabla} \Theta\right) & =\dot{q}^{V} c_{p}^{-1} \\
\partial_{t} k+(\boldsymbol{U} \cdot \boldsymbol{\nabla}) k-\nabla \cdot\left(\nu_{k} \boldsymbol{\nabla} k\right) & =\mathcal{P}_{k}+G-\epsilon \\
\partial_{t} \epsilon+(\boldsymbol{U} \cdot \boldsymbol{\nabla}) \epsilon-\boldsymbol{\nabla} \cdot\left(\nu_{\epsilon} \boldsymbol{\nabla} \epsilon\right)+C_{2} \epsilon^{2} k^{-1} & =C_{1} \epsilon k^{-1}\left(\mathcal{P}_{k}+G\right)
\end{aligned}
$$

with turbulent-viscosity and thermal diffusivity being related by the turbulent Prandtl number

$$
\nu_{t}=C_{\mu} \frac{k^{2}}{\epsilon}, \quad a=\nu \operatorname{Pr}^{-1}, \quad a_{t}=\nu_{t} \operatorname{Pr}_{t}^{-1}, \text { with } \quad \operatorname{Pr}=0.70, \quad \operatorname{Pr}_{t}=0.9 \quad \text { for air }
$$

with effective viscosities

$$
\nu_{e}=\nu+\nu_{t}, \quad a_{e}=a+a_{t}, \quad \nu_{k}=\nu+\frac{\nu_{t}}{P r_{k}}, \quad \nu_{\epsilon}=\nu+\frac{\nu_{t}}{P r_{\epsilon}}
$$

and with experimentally determined constants (see below)

$$
C_{1}=1.44, \quad C_{2}=1.92, \quad P r_{k}=1.0, \quad P r_{\epsilon}=1.3, \quad C_{t}=0.8, \quad C_{\mu}=0.09,
$$

Production and buoyancy terms $\mathcal{P}_{k}$ and $G$ are defined in (3.17) and (3.26).

It is not possible to determine the empirical constants of the $k / \epsilon$ model from a set of measurements that isolate each term, because the model is not exact. The standard values are rather a compromise for a range of flows. Nevertheless it is worth mentioning that $C_{2}$ determines the decay of homogeneous, isotropic turbulence. The spreading rate of shear layers is controlled by $C_{2}-C_{1}$. Boundary-layer data suggest $C_{1}=1.55$, whereas $C_{1}=1.3$ is appropriately for mixing layer data, see [DPR01], p.181. Discernibly the standard value $C_{1}=1.44$ is a compromise.

\subsubsection{An appraisal of the $k / \epsilon$ model}

A principal limitation of the the $k / \epsilon$ model arises from the underlying turbulent-viscosity hypothesis and its formula for $\nu_{t}$. Instead of the full Reynolds stress tensor only half its trace $k$ is computed. Moreover, in WILcox's opinion, the closure approximation (3.23) for the $k$-equation and much more notably those for the $\epsilon$ equation (given in [Wilcox98] eq.(4.45)), are a "drastic surgery" on the exact equations. Whereas turbulent-viscosity and gradient-diffusion hypotheses have been investigated using various experimental data, the 
terms modeled in the $k$ and $\epsilon$ equation are almost impossible to measure. However, there is hope that DNS studies can help to gain information for suitable closure approximations. A further dispute is on the question whether the lengthscale provided by $\epsilon$ is the correct one for (3.20). For more details, the reader is addressed to [Pope00], Section 10.4 and [Wilcox98], Subsection 4.3.2.

The values of the constants in (3.35) are a compromise, balanced for several basic test cases, e.g. decaying turbulence and behaviour in the log-layer. The standard $k / \epsilon$ model yields acceptable results for the mixing layer and for the plane and radial jet, cf. [Wilcox.98] pp.137. However, the $k / \epsilon$ model erroneously predicts unequal rates for spreading for round and plane jets, a phenomenon referred to as 'round jet-plane jet anomaly'. Of course, the constants can be tuned for a particular flow. It is noteworthy that values for the model constants can be derived from renormalization group (RNG) analysis. Despite its mathematical reasoning, in practice this does not provide a significant improvement to the standard $k / \epsilon$ model, cf. [Wilcox98], p.137.

The main deficiencies of the $k / \epsilon$ model are its poor predictions (i) in the near-wall region and (ii) for flows with strong pressure gradients. The latter is discussed in great detail in [Wilcox98], Chapter 4.6.2. As pointed out in [DPR01], Section 6.2.2, the behaviour of the $k / \epsilon$ model below the log-layer imposes several severe difficulties. First it is not a trivial task to specify meaningful boundary conditions for $\epsilon$ at solid walls. Secondly, in (3.25) the term $C_{2} \epsilon^{2} / k$ behaves like $y^{-2}$ near the wall, with $y$ denoting the distance from the wall, and hence becomes singular. Finally, even if the exact data for $k$ and $\epsilon$ (e.g. from a DNS data base) are substituted into $\nu_{t}=C_{\mu} k^{2} / \epsilon$, the theoretical value $\nu_{t} \equiv-\left\langle u^{\prime} v^{\prime}\right\rangle_{E} /(d U / d y)$ is spuriously overpredicted close to the wall.

These problems gave rise to several of modifications of the $k / \epsilon$ model near solid walls, most noteworthy (a) low Reynolds number models, (b) wall functions (c) two-layer models, (d) the $k-\epsilon-\overline{v^{2}}$ model by DURBIN, for details see [DPR01], Chapter 6.2.2 and references therein. Low Reynolds number models introduce artificial damping functions for damping $\nu_{t}$ near the wall. They are unreliable for flows with significant pressure gradient and cause numerical stiffness problems. Hence this approach is virtually unanimously doomed in the CFD community. Approach (b) has been employed in our research group and will be described in great detail in this thesis. It is computationally attractive since it circumvents resolving the near wall region. The wall function concept can be justified for attached boundary-layer flows with small pressure gradients. In practical applications wall functions are also used when the underlying assumptions do not hold. In flows with massive separation or strong pressure gradients their predictions can be poor. However, such situations do not often occur in indoor-air flow problems. Nevertheless, more accurate approaches are desirable. The strategies (c) and (d) both require a near-wall grid. A two-layer model was devised by CHEN AND PATEL, who proposed to use a suitable one-equation model for $k$ in the near-wall region, which is matched with the $k / \epsilon$ model at a certain artificial boundary in the log-layer.

The $k-\epsilon-\overline{v^{2}}$ model is a four equation model, presented in [Dur9]]. It is based on the idea that it is the cross-stream fluctuation velocity $\overline{v^{\prime 2}}$ that is responsible for turbulent momentum transport in the near-wall region and that $\overline{v^{\prime 2}}$ is suppressed in the proximity of walls. The model adds one advection-diffusion-reaction equation for the scalar $\overline{v^{2}}$ and an 
advection-reaction equation for a scalar $f$ which is motivated from the theory of second moment closure modelling and tries to emulate effects of redistribution of turbulent kinetic energy from the streamwise to the wall-normal component. Very reasonable results have been obtained even for complicated test cases including separation and jet impingement, see [DPR01] . The notion that the model $k-\epsilon-\overline{v^{2}}$ model is significantly superior to the $k / \epsilon$ model in predicting the heat transfer in an axisymmetric turbulent jet impinging on a flat plate, [BPD98], makes this model quite attractive for application in indoor-air flow problems. It is worth mentioning that PIOMELLI ET AL., see [SP02] performed a thorough study of today's most successful near-wall RANS models, including the one-equation Spalart-Allmaras model, the $k / \epsilon$ model with the wall functions of LAM and BrEmHORST, the $k / \omega^{2}$ model of SAFFman and WilCOX, and the $k-\epsilon-\overline{v^{2}}$ model of Durbin for a pulsating flow in [SP02], the latter being the most successful.

Besides the $k / \epsilon$ model, there are other two-equation models, most remarkebly the $k / \omega$ model. It has two well-known advantages over the standard $k / \epsilon$ model. First, it yields reasonable predictions for the mean velocity field throughout the near-wall region provided a suitable near-wall grid is used. Secondly, it gives good results even for flows with strong pressure gradients. Both observations have made this model very interesting for aeronautical flows. However, a more detailed analysis reveals that the propitous predictions for $\nu_{t}$ are just a consequence of underpredicting $k$ and overpredicting $\epsilon$; its success in the near-wall region is not based on physical reasoning. Moreover the model is unreliable for free-shear layers, whose correct predictions are also quite important for indoor-air flow problems, see [DPR01], p.132. Thus concerning future projects, the $k-\epsilon-\overline{v^{2}}$ model seems to be the most promising RANS model for problems involving indoor-air movement. 


\section{Large-eddy simulation}

This chapter is dedicated to large-eddy simulation (LES). LES is an alternative approach for reducing the complexity of turbulent flow problems. As described in Section 2.2, turbulent flows are characterized by a large range of scales, the ratio of the smallest to largest eddies increasing as $R e^{-3 / 4}$. When the turbulent motions of all scales are fully resolved, i.e. in a DNS, the computational efforts for resolving the small scale motions exceed those for resolving the large scale motions by far. Since engineers are primarily interested in the behaviour of the large scale motions, "there is a mismatch between DNS and the objective of determining the mean velocity and energy-containing motions in a turbulent flow", as pointed out by [Pope00], p.357. Thus the idea is to reduce complexity of turbulence by first filtering out the small scale motions using a spatial filter, resolving the random motion of the remaining large eddies. However, again a closure problem arises. Thus the idea of LES is to resolve the large-scale motions and to model the effects of the small-scale motions on the large-scale motions. This approach is supported by the observation that the small-scale motions have, to some extent, a universal behaviour, making them amenable for modelling.

\subsection{Filtering}

The objective of filtering a variable is to extract its large-scale structures and to attenuate its small-scale structures. The filter width $\Delta$ specifies the demarcation line of this scale separation. Such a space-averaging filter $\langle\cdot\rangle_{\Delta}$ should have the following properties:

(F1) Filtering is a linear operation, i.e. $\langle f+\lambda g\rangle_{\Delta}=\langle f\rangle_{\Delta}+\lambda\langle g\rangle_{\Delta}, f, g: \mathbb{R}^{d} \rightarrow \mathbb{R}, \lambda \in \mathbb{R}$.

(F2) Derivatives and averages commute, i.e. $\left\langle\frac{\partial f}{\partial x_{i}}\right\rangle_{\Delta}=\frac{\partial\langle f\rangle_{\Delta}}{\partial x_{i}},\left\langle\frac{\partial f}{\partial t}\right\rangle_{\Delta}=\frac{\partial\langle f\rangle_{\Delta}}{\partial t}$.

The classical filtering technique used in LES is the convolution with a suitable filter function. Let $f(\boldsymbol{x}, t)$ be an instantaneous variable. If $f$ is defined w.r.t. the spatial variable on a bounded domain $\Omega$, then $f$ is extended by zero onto $\mathbb{R}^{d}$. Then its corresponding filtered variable is defined by the convolution integral

$$
\bar{f}(\boldsymbol{x}, t) \equiv\langle f\rangle_{\Delta}=\int_{\mathbb{R}^{d}} g_{\Delta}(\boldsymbol{x}-\boldsymbol{y}) f(\boldsymbol{y}, t) d \boldsymbol{y}, \quad g_{\Delta}(\boldsymbol{x})=\prod_{j=1}^{d} g_{\Delta}^{j}\left(x_{j}\right) .
$$

with $g_{\Delta}$ being a filter function and $\Delta$ denoting the filter width. If $f$ is vector-valued resp. tensor-valued, then filtering has to be understood componentwise. Since $f \equiv 0$ on $\mathbb{R}^{d} \backslash \Omega$, $f \in \mathcal{E}^{\prime} \subset \mathcal{S}^{\prime}$, cf. Section A.1. Given $g_{\Delta} \in \mathcal{S}$, the spatial averaging filter can be interpreted as an operator $\langle\cdot\rangle_{\Delta}$

$$
\langle\cdot\rangle_{\Delta}: \mathcal{S}^{\prime}\left(\mathbb{R}^{d}\right) \mapsto \mathcal{S}\left(\mathbb{R}^{d}\right), \quad\langle\boldsymbol{f}\rangle_{\Delta} \equiv g_{\Delta} * \boldsymbol{f}
$$


If $\Delta=$ const, then the filter defined by (4.1) satisfies (F1) and (F2), see Theorem A.4. $\bar{f}(\boldsymbol{x}, t)$ is the weighted mean value of $f$ with weight function $g_{\Delta}(\boldsymbol{x}-\cdot)$. In the case $\operatorname{supp}\left(g_{\Delta}^{j}\right) \subset[-\Delta, \Delta]$ the averaging is performed over $B_{\Delta}(\boldsymbol{x})$ (ball in the maximum norm). Then velocity, pressure and temperature can be decomposed into a filtered part and a residual part, videlicet,

$$
\begin{aligned}
(\boldsymbol{u}, p)(\boldsymbol{x}, t) & =\overline{(\boldsymbol{u}, p)}(\boldsymbol{x}, t)+\left(\boldsymbol{u}^{\prime}, p^{\prime}\right)(\boldsymbol{x}, t), \quad\left(\boldsymbol{u}^{\prime}, p^{\prime}\right)(\boldsymbol{x}, t) \equiv(\boldsymbol{u}, p)(\boldsymbol{x}, t)-\overline{(\boldsymbol{u}, p)}(\boldsymbol{x}, t) \\
\theta(\boldsymbol{x}, t) & =\bar{\theta}(\boldsymbol{x}, t)+\theta^{\prime}(\boldsymbol{x}, t), \quad \theta^{\prime}(\boldsymbol{x}, t) \equiv \theta(\boldsymbol{x}, t)-\bar{\theta}(\boldsymbol{x}, t)
\end{aligned}
$$

It is worth considering the effect of the filtering operation in the Fourier space. The relation

$$
\hat{\bar{f}} \equiv \mathcal{F}(\bar{f})=\mathcal{F}\left(g_{\Delta} * f\right)=\mathcal{F}\left(g_{\Delta}\right) \mathcal{F}(f) .
$$

shows that all the high wave number components of $f$ are annihilated by convolution with $g_{\Delta}$, if $\mathcal{F}\left(g_{\Delta}\right)(\boldsymbol{\kappa})=0$ for $|\boldsymbol{\kappa}|>\kappa_{c}$, where $\kappa_{c}$ is a cut-off wave number. A filter with such a characteristic is called an ideal low pass filter. If the filter function in wave number space rapidly falls off, a cut-off wave number can also be defined for all practical purposes.

The most popular filtering functions in LES and their corresponding Fourier transforms are, cf. [Pope00], p.563:

1. Box filter

$$
g_{\Delta}^{j}\left(x_{j}\right)=\left\{\begin{array}{ll}
\frac{1}{\Delta}, & \text { if }\left|x_{j}\right| \leq \Delta / 2 \\
0, & \text { if }\left|x_{j}\right|>\Delta / 2 .
\end{array}, \quad \hat{g}_{\Delta}^{j}\left(k_{j}\right)=\frac{\sin \left(\Delta k_{j} / 2\right)}{\Delta k_{j} / 2}\right.
$$

2. Sharp spectral filter

$$
g_{\Delta}^{j}\left(x_{j}\right)=\frac{\sin \left(\pi x_{j} / \Delta\right)}{\pi x_{j}} \quad \hat{g}_{\Delta}^{j}\left(k_{j}\right)=\left\{\begin{array}{ll}
1, & \text { if }\left|k_{j}\right| \leq \pi / \Delta \\
0, & \text { if }\left|k_{j}\right|>\pi / \Delta .
\end{array},\right.
$$

3. Gaussian filter

$$
g_{\Delta}^{j}\left(x_{j}\right)=\sqrt{\frac{\gamma}{\pi \Delta^{2}}} e^{-\frac{\gamma x_{j}^{2}}{\Delta^{2}}}, \quad \hat{g}_{\Delta}^{j}\left(k_{j}\right)=e^{-\frac{\Delta^{2} k_{j}^{2}}{4 \gamma}}, \quad \gamma=6 .
$$

The specification $\gamma=6$ in (4.8) ensures that box filter function and the Gaussian have the same second moment, see [Pope00], p.563. Direct calculation yields the following equations for $\boldsymbol{u}^{\prime}$, cf. [Pope00], p.566,

$$
\hat{\boldsymbol{u}}^{\prime}(\boldsymbol{\kappa}, t) \equiv \mathcal{F}\left(\boldsymbol{u}^{\prime}\right)(\boldsymbol{\kappa}, t)=\left[1-\hat{g}_{\Delta}(\kappa)\right] \hat{\overline{\boldsymbol{u}}}(\boldsymbol{\kappa}, t), \quad \overline{\boldsymbol{u}^{\prime}}(\boldsymbol{x}, t)=\overline{\boldsymbol{u}}(\boldsymbol{x}, t)-\overline{\overline{\boldsymbol{u}}}(\boldsymbol{x}, t)
$$

On the one hand, the Gaussian is reasonably sharp both in physical space and in wavenumber space, see (4.8). On the other hand, since $0<g_{\Delta}(\boldsymbol{x}) \leq 1$ and $0<\hat{g}_{\Delta}(\boldsymbol{\kappa}) \leq 1$ it follows 
that, in principle, filtering with a Gaussian is an invertible operation (although poorly conditioned).

It is worthwhile studying the resolution requirements for the filtered field. Denote $\kappa_{c}=\pi / \Delta$ the cutoff wavenumber. For the Gaussian $\hat{\overline{\boldsymbol{u}}}(\kappa, t)=\hat{g}_{\Delta}(\kappa) \hat{\boldsymbol{u}}(\kappa, t)>0$ (for $\kappa>\kappa_{c}$ ), i.e. despite filtering, $\hat{\overline{\boldsymbol{u}}}(\kappa, t)$ has a non-vanishing contribution for $\kappa>\kappa_{c}$. Equivalently spoken, $\overline{\boldsymbol{u}}$ contains (non-negligible) structures of size smaller than $\Delta$. This suggests to resolve $\overline{\boldsymbol{u}}$ up to $\kappa_{r}$, called the highest resolved mode, with $\kappa_{r}=n \kappa_{c}(n \geq 2)$. In other words, filter width $\Delta$ and the grid size of a numerical calculation $h$ should be related by $\Delta=n h(n \geq 2)$. This intuitive reasoning is supported by numerical analysis, cf. [.II01].

\subsection{Differential filtering}

Explicit filtering is an important issue in LES. In the previous section, filtering was introduced using an integral operator, viz.,

$$
u(t, \boldsymbol{x})=\left(g_{\Delta} * u_{0}\right)(t, \boldsymbol{x}), \quad g_{\Delta}(\boldsymbol{y})=\left(\frac{\gamma}{\pi \Delta^{2}}\right)^{d / 2} \exp \left(-\frac{\gamma \boldsymbol{y}^{2}}{\Delta^{2}}\right) .
$$

In his work on differential filters, GERMANo proposed to approximate this type of integral operator by taking the inverse of an appropriate differential operator, cf. [Ger86]. In the appendix, this approach will be motivated by (A.25). The objective of this section is to describe the relation between the convolution with a gaussian filter and the solution operator of the heat equation. For this purpose we consider the Cauchy-problem of the heat equation: For given $u_{0} \in \mathcal{S}\left(\mathbb{R}^{d}\right)$ find $u: \mathbb{R}^{d} \times \mathbb{R}^{+} \mapsto \mathbb{R}$ s.t.

$$
\begin{aligned}
\frac{\partial u}{\partial s}-a^{2} \nabla^{2} u & =0 & & \text { in } \mathbb{R}^{d} \times \mathbb{R}^{+}, \\
u & =u_{0} & & \text { in } \mathbb{R}^{d} \times\{0\} .
\end{aligned}
$$

Its solution $u(\boldsymbol{x}, s)$ is given by (cf. [Kre89], p.134)

$$
u(\boldsymbol{x}, s)=\left(\mathcal{G} * u_{0}\right)(\boldsymbol{x}, s), \quad \mathcal{G}(\boldsymbol{y}, s)=\left(4 \pi a^{2} s\right)^{-d / 2} \exp \left(-\frac{\|\boldsymbol{y}\|^{2}}{4 a^{2} s}\right),
$$

which holds even for $u_{0} \in \mathcal{S}^{\prime}\left(\mathbb{R}^{d}\right)$. For $s$ small, we can approximate the solution $u(\cdot, s)$ of (4.11)-(4.12) using one step of a backward Euler scheme. Using the notation $u(\cdot, 0)=u_{0}$, the solution $u(\cdot, s)$ of the problem

$$
\frac{u(\boldsymbol{x}, s)-u_{0}(\boldsymbol{x})}{s}-a^{2} \nabla^{2} u(\boldsymbol{x}, s)=0 \quad \text { in } \quad \mathbb{R}^{d} \times \mathbb{R}^{+}
$$

is an approximation to the solution of (4.11)-(4.12). Thus, for fixed $s$, the solution of

$$
\left(-a^{2} s \nabla^{2}+\mathbb{I}\right) u(\boldsymbol{x}, s)=u_{0}(\boldsymbol{x}) \quad \text { in } \quad \mathbb{R}^{d} \times \mathbb{R}^{+}
$$

is an approximation to $u(\cdot, s)$ in (4.13). Comparing (4.13) with (4.10) implies $\Delta^{2} \gamma^{-1}=$ $4 a^{2} s$. 
After this motivation we can describe how to approximate (4.10) using a differential filter: Given $u(\boldsymbol{x}, t)$ ( $t$ being fixed), approximate $\bar{u}(\boldsymbol{x}, t) \equiv\left(g_{\Delta} * u\right)(\boldsymbol{x}, t)$ as solution $\overline{u_{s}}$ of

$$
\left(-\frac{\Delta^{2}}{4 \gamma} \nabla^{2}+\mathbb{I}\right) \overline{u_{s}}(\cdot, t)=u(\cdot, t) \text { in } \mathbb{R}^{d} .
$$

Often $u(\boldsymbol{x}, t)$ is given in $\Omega \times \mathbb{R}^{+}$and $\Omega$ is a domain. Denote $\mathcal{R}(u)$ (a suggestion for) an approximation of $\bar{u}(\boldsymbol{x}, t)$. Then given $u: \Omega \times \mathbb{R}^{+} \mapsto R$, we seek $\mathcal{R}(u): \Omega \times \mathbb{R}^{+} \mapsto \mathbb{R}$ s.t. for each fixed $t$

$$
\left(-\frac{\Delta^{2}}{4 \gamma} \nabla^{2}+\mathbb{I}\right) \mathcal{R}(u)(\cdot, t)=u(\cdot, t) \quad \text { in } \Omega, \quad \nabla \mathcal{R}(u) \cdot \boldsymbol{n}=0 \text { on } \Gamma .
$$

The idea of choosing a Neumann boundary condition in (4.15) is to keep the modelling error near the boundary small. To the author's best knowledge, error estimates of $\left\|g_{\Delta} * u-\mathcal{R}(u)\right\|$ are not available. $\left\|g_{\Delta} * u-\mathcal{R}(u)\right\|$ will be studied numerically in Section 12.3 .

\section{Remark 4.1}

An alternative differential filter based on the solution of a Stokes problem was proposed by LAYTON, see [LL0.3] . A further question is whether the differential filtering scheme can be improved by using a higher order scheme w.r.t. the variable $s$ in (4.11).

\subsection{The space averaged non-isothermal Navier-Stokes equations}

This section is devoted to the evolution equations for $\overline{\boldsymbol{u}}, \bar{p}$ and $\bar{\theta}$ and to the closure problem in non-isothermal LES. Assume $\boldsymbol{u}, \boldsymbol{p}$ and $\boldsymbol{\theta}$ are extended by zero onto $\mathbb{R}^{d}$. Then applying a spatial averaging filter $\langle\cdot\rangle_{\Delta}$ to (1.19)-(1.21) gives (using (F1) and (F2))

$$
\begin{aligned}
\partial_{t} \overline{\boldsymbol{u}}-\boldsymbol{\nabla} \cdot(2 \nu \mathbb{S}(\overline{\boldsymbol{u}})-\overline{\boldsymbol{u} \otimes \boldsymbol{u}}+\overline{\boldsymbol{u}} \otimes \overline{\boldsymbol{u}})+(\overline{\boldsymbol{u}} \cdot \boldsymbol{\nabla}) \overline{\boldsymbol{u}}+\boldsymbol{\nabla} \bar{p} & =-\beta \bar{\theta} \boldsymbol{g}, \\
\boldsymbol{\nabla} \cdot \overline{\boldsymbol{u}} & =0, \\
\partial_{t} \bar{\theta}+(\overline{\boldsymbol{u}} \cdot \boldsymbol{\nabla}) \bar{\theta}-\boldsymbol{\nabla} \cdot(a \boldsymbol{\nabla} \bar{\theta}-\overline{\boldsymbol{u} \theta}+\overline{\boldsymbol{u}} \bar{\theta}) & =\dot{q}^{V} c_{p}^{-1} .
\end{aligned}
$$

\section{Remark 4.2}

Regarding the isothermal Navier-Stokes equations, we can assume the following regularities, cf. e.g. [Tem77]: $\boldsymbol{f} \in L^{2}\left(0, T ;\left(H_{\text {div }}^{1}(\Omega)^{*}\right)^{d}\right), \boldsymbol{u} \in L^{2}\left(0, T ;\left(H_{\text {div }}^{1}(\Omega)\right)^{d}\right), \partial_{t} \boldsymbol{u} \in$ $L^{1}\left(0, T ;\left(H_{\text {div }}^{1}(\Omega)^{*}\right)^{d}\right)$, and $\nabla p \in \mathcal{E}^{\prime}(\Omega \times(0, T))$. Therefore, for each $t \in(0, T), \nabla \boldsymbol{u}$ and $\triangle \boldsymbol{u}$ are at least in $\mathcal{E}^{\prime}(\Omega)$ for each $t \in(0, T)$. Hence, for each $t \in(0, T)$, the expressions on both sides are at least in $\mathcal{S}^{\prime}\left(\mathbb{R}^{d}\right)$ and we can convolve both sides with a function $g_{\Delta} \in \mathcal{S}\left(\mathbb{R}^{d}\right)$. $\diamond$

\section{Remark 4.3}

According to JOHN and LAYTON, the extension of variables onto $\mathbb{R}^{d}$ leads to a commutation error in the Navier-Stokes equations due to a loss in regularity. JoHN and DuncA studied this error in the case of the isothermal Navier-Stokes equations with homogeneous Dirichlet conditions. Extending $\boldsymbol{u}$ by zero in $\mathbb{R}^{d} \backslash \Omega$ gives rise to an additional boundary term both in the Navier-Stokes equations for $\boldsymbol{u}$ in $\mathbb{R}^{d}$ and the space filtered equations. However, in a numerical solution using FEM, this term is negligible small, cf. [.Joh02], pp.10 and pp.14. 
It is worth emphasising that the fields involved in (4.16)-(4.18), i.e $\overline{\boldsymbol{u}}, \bar{p}$, and $\bar{\theta}$ are still random.

Equations (4.16)-(4.18) are unclosed. Similar to the RANS equations, we introduce

$$
\begin{aligned}
\tau^{R} & \equiv \overline{\boldsymbol{u} \otimes \boldsymbol{u}}-\overline{\boldsymbol{u} \otimes \overline{\boldsymbol{u}},} & & \text { residual stress tensor }, \\
k_{r} & \equiv \frac{1}{2} \sum_{i=1}^{d} \tau_{i i}^{R}, & & \text { residual kinetic energy }, \\
\tau^{r} & \equiv \tau^{R}-\frac{2}{d} k_{r} \mathbb{I}, & & \text { anisotropic residual stress tensor }, \\
\boldsymbol{h} & \equiv \overline{\boldsymbol{u} \theta}-\overline{\boldsymbol{u}} \bar{\theta}, & & \text { residual temperature flux } .
\end{aligned}
$$

The isotropic residual stress is included in the modified pressure $\bar{p}_{\text {mod }} \equiv \bar{p}+\frac{2}{d} k_{r}$. The residual stress tensor and the residual temperature flux represent the effects of the residual scales on the filtered scales. They are often referred to as subgrid scale (abbreviated SGS) stresses and fluxes resp. Their modelling will be studied in Section 4.4 .

As described in Section 2.2, the transfer of kinetic energy between filtered and residual scales is an important mechanism in turbulent flows. In order to quantify this, we focus on filtered kinetic energy $\bar{E}(\boldsymbol{x}, t)$, kinetic energy of the filtered velocity field $E_{f}$ and residual kinetic energy $k_{r}$, being defined as

(4.23) $\bar{E} \equiv \frac{1}{2} \overline{\boldsymbol{u} \cdot \boldsymbol{u}}, \quad E_{f} \equiv \frac{1}{2} \overline{\boldsymbol{u}} \cdot \overline{\boldsymbol{u}}, \quad k_{r} \equiv \frac{1}{2} \overline{\boldsymbol{u} \cdot \boldsymbol{u}}-\frac{1}{2} \overline{\boldsymbol{u}} \cdot \overline{\boldsymbol{u}}, \quad$ with $\bar{E}=E_{f}+k_{r}$.

Multiplying (4.16) with $\overline{\boldsymbol{u}}$ we obtain the conservation equation for $E_{f}$, viz.,

$$
\partial_{t} E_{f}+\overline{\boldsymbol{u}} \nabla E_{f}-\nabla \cdot\left[\left(2 \nu \mathbb{S}(\overline{\boldsymbol{u}})-\tau^{r}-\bar{p} \mathbb{I}\right) \overline{\boldsymbol{u}}\right]=-\epsilon_{f}-\mathcal{P}_{r}-\beta \bar{\theta} \boldsymbol{g} \cdot \overline{\boldsymbol{u}},
$$

where

$$
\epsilon_{f} \equiv 2 \nu \mathbb{S}(\overline{\boldsymbol{u}}): \mathbb{S}(\overline{\boldsymbol{u}}), \quad \mathcal{P}_{r} \equiv-\tau^{r}: \mathbb{S}(\overline{\boldsymbol{u}})
$$

The corresponding equation for $k_{r}$ reads (cf. e.g. [PYA96], p.217 or [Sag01], p.37)

$$
\begin{aligned}
\partial_{t} k_{r}+\overline{\boldsymbol{u}} \cdot \nabla k_{r}-\nabla \cdot\left[\left(\bar{E} \overline{\boldsymbol{u}}-\frac{1}{2} \overline{\boldsymbol{u} \cdot \boldsymbol{u u}}\right)+(\bar{p} \overline{\boldsymbol{u}}\right. & \left.-\overline{p \boldsymbol{u}})+\nu \nabla k_{r}+\tau^{r} \overline{\boldsymbol{u}}\right] \\
& =-\epsilon_{\nu}+\mathcal{P}_{r}-\beta \overline{\theta^{\prime} \boldsymbol{u}^{\prime}} \cdot \boldsymbol{g}
\end{aligned}
$$

with

$$
\epsilon_{\nu} \equiv \nu\left(\sum_{i, j=1}^{d} \frac{\overline{\partial u_{i}}}{\partial x_{j}} \frac{\partial u_{i}}{\partial x_{j}}-\sum_{i, j=1}^{d} \frac{\partial \overline{u_{i}}}{\partial x_{j}} \frac{\partial \overline{u_{i}}}{\partial x_{j}}\right) .
$$

The right hand side terms in (4.24) and (4.26) are source or sink terms. $\epsilon_{f}$ describes viscous dissipation directly from the filtered velocity field, being small compared to $\epsilon$, cf. [Pope00], p.588. $\epsilon_{\nu}$ represents the residual kinetic energy dissipated by viscous forces. $\mathcal{P}_{r}$ appears as a sink term in the equation for $E_{f}$ and as a source term in the equation for $k_{r}$. It represents the rate of transfer of energy from the filtered velocity field to the residual motions. In the mean, $\mathcal{P}_{r}>0$, but locally, backscatter can occur with $\mathcal{P}_{r}<0$, i.e. energy transfer from the residual motions to the filtered motions. 


\subsection{Modelling the residual stress tensor and the residual fluxes}

In order to close (4.16)-(4.18), we have to model $\tau^{r}$ and $\boldsymbol{h}$. Concerning the isothermal case, for a survey on closure models see [Pope00], [Sag01] and [Geu01]. A mathematical review can be found in e.g. [Lay02] and [Lay02a]. For detailed numerical tests of the most popular residual stress models see e.g. [VGK.97]. In subsection 4.4.1, we accomplish closure modelling using formal series expansions, also referred to as structural modelling. We will augment this with a functional model in order to explicitely account for the energy cascade, see subsection 4.4.2. Effects of buoyancy are regarded in subsection 4.4.3.

\subsubsection{Structural modelling}

One classical strategy for obtaining models for the residual stress tensor and the residual temperature flux is so-called structural modelling: The underlying idea is to use formal series expansions for the filter kernel in Fourier space. A detailed derivation of the following two models can be found in appendix A.2. Starting point is the decomposition

$$
\begin{aligned}
\overline{\boldsymbol{u} \otimes \boldsymbol{u}} & =\overline{\left(\overline{\boldsymbol{u}}+\boldsymbol{u}^{\prime}\right) \otimes\left(\overline{\boldsymbol{u}}+\boldsymbol{u}^{\prime}\right)}=\overline{\overline{\boldsymbol{u} \otimes \overline{\boldsymbol{u}}}}+\overline{\overline{\boldsymbol{u}} \otimes \boldsymbol{u}^{\prime}}+\overline{\boldsymbol{u}^{\prime} \otimes \overline{\boldsymbol{u}}}+\overline{\boldsymbol{u}^{\prime} \otimes \boldsymbol{u}^{\prime}} \\
\overline{\theta \boldsymbol{u}} & =\overline{\left(\bar{\theta}+\theta^{\prime}\right)\left(\overline{\boldsymbol{u}}+\boldsymbol{u}^{\prime}\right)}=\overline{\bar{\theta} \overline{\boldsymbol{u}}}+\overline{\bar{\theta} \boldsymbol{u}^{\prime}}+\overline{\theta^{\prime} \overline{\boldsymbol{u}}}+\overline{\theta^{\prime} \boldsymbol{u}^{\prime}}
\end{aligned}
$$

with resolved stresses resp. fluxes $\overline{\overline{\boldsymbol{u}} \otimes \overline{\boldsymbol{u}}}$ and $\overline{\bar{\theta}} \overline{\boldsymbol{u}}$, cross stresses resp. fluxes $\overline{\overline{\boldsymbol{u}} \otimes \boldsymbol{u}^{\prime}}, \overline{\boldsymbol{u}^{\prime} \otimes \overline{\boldsymbol{u}}}$, $\overline{\bar{\theta} \boldsymbol{u}^{\prime}}$ and $\overline{\theta^{\prime} \overline{\boldsymbol{u}}}$, and residual stresses resp. fluxes $\overline{\boldsymbol{u}^{\prime} \otimes \boldsymbol{u}^{\prime}}$ and $\overline{\theta^{\prime} \boldsymbol{u}^{\prime}}$. We introduce

$$
\begin{aligned}
\tau^{S G S} & \left.\equiv \overline{\boldsymbol{u}^{\prime} \otimes \boldsymbol{u}^{\prime}}-\frac{1}{d} \sum_{i=1}^{d} \overline{\boldsymbol{u}^{\prime} \otimes \boldsymbol{u}^{\prime}}\right) \mathbb{I}, \quad \operatorname{trace}(A) \equiv \sum_{i=1}^{d} A_{i i} \text { for } A \in \mathbb{R}^{d \times d}, \\
\boldsymbol{h}^{S G S} & \equiv \overline{\boldsymbol{u}^{\prime} \theta^{\prime}} .
\end{aligned}
$$

The so-called Clark model is based on a Taylor approximation of the filter kernel in Fourier space. The Clark model reads

$$
\begin{aligned}
\overline{\boldsymbol{u} \otimes \boldsymbol{u}} & =\overline{\boldsymbol{u}} \otimes \overline{\boldsymbol{u}}+\frac{\Delta^{2}}{12} \nabla \overline{\boldsymbol{u}} \bullet \nabla \overline{\boldsymbol{u}}+\mathcal{O}^{\text {formal }}\left(\Delta^{4}\right) \\
\overline{\theta \boldsymbol{u}} & =\bar{\theta} \overline{\boldsymbol{u}}+\frac{\Delta^{2}}{12} \nabla \bar{\theta} \circ \nabla \overline{\boldsymbol{u}}+\mathcal{O}^{\text {formal }}\left(\Delta^{4}\right)
\end{aligned}
$$

with the notations

$$
(\nabla \boldsymbol{U} \bullet \nabla \boldsymbol{U})_{i j}=\sum_{l=1}^{d} \frac{\partial U_{i}}{\partial x_{l}} \frac{\partial U_{j}}{\partial x_{l}}, \quad(\nabla \Theta \circ \nabla \boldsymbol{U})_{j}=\sum_{l=1}^{d} \frac{\partial \Theta}{\partial x_{l}} \frac{\partial U_{j}}{\partial x_{l}} .
$$

Instead, GALDI and LAYTON proposed using a rational approximation for the filtering kernel, leading to the following model

$$
\begin{aligned}
& \overline{\boldsymbol{u} \otimes \boldsymbol{u}}=\overline{\boldsymbol{u}} \otimes \overline{\boldsymbol{u}}+\frac{\Delta^{2}}{12} \mathcal{R}(\nabla \overline{\boldsymbol{u}} \bullet \nabla \overline{\boldsymbol{u}})+\mathcal{O}^{\text {formal }}\left(\Delta^{4}\right) \\
& \overline{\theta \boldsymbol{u}}=\bar{\theta} \overline{\boldsymbol{u}}+\frac{\Delta^{2}}{12} \mathcal{R}(\nabla \bar{\theta} \circ \nabla \overline{\boldsymbol{u}}) \quad+\mathcal{O}^{\text {formal }}\left(\Delta^{4}\right)
\end{aligned}
$$

with the regularization operator $\mathcal{R}$ defined in (4.15). 


\section{Remark 4.4}

There is an interesting formal connection between the Galdi-Layton model and today's most successful Reynolds-stress model, the so-called elliptic relaxation model devised by DuRBIN, see [Dur93] . Both models use a similar elliptic regularization operator.

\section{Remark 4.5}

The Fourier analysis reveals that the residual stress term and the residual flux term are of formal order $\Delta^{4}$ for both the Clark model and the Galdi-Layton model. Hence their contribution is neglected in both models. Moreover, numerical tests show that for both models the transfer of energy from the resolved scales to the residual scales is too small leading to numerical instabilities for high Reynolds/Rayleigh numbers, see. e.g. [LMK.94], [VGK.97] and [.Joh02]. For these reasons in the following subsection we introduce two additional subgrid scale models which are based on the turbulent-viscosity hypothesis. $\diamond$

\section{Remark 4.6}

The Galdi-Layton model has to be combined with a suitable damping function in order to obtain an appropriate near-wall behaviour.

\subsubsection{Functional modelling part I : Residual stress modelling for isothermal problems}

The SGS models presented in this subsection are based on the turbulent-viscosity hypothesis. The objective of functional modelling is to provide a mechanism to transfer energy from the filtered scales to the residual scales and to dissipate energy at the end of the energy cascade. As pointed out in [VGK.97] this is an issue of major importance in LES. In analogy to PRANDTL's mixing length hypothesis (see (5.31)), SMAGORInsky (1963) proposed

$$
\tau^{r}=-2 \nu_{t} \mathbb{S}(\overline{\boldsymbol{u}}), \quad \text { with } \quad \nu_{t} \equiv\left(C_{S} \Delta\right)^{2} \overline{\mathcal{S}}, \quad \overline{\mathcal{S}} \equiv \sqrt{\mathbb{S}(\overline{\boldsymbol{u}}): \mathbb{S}(\overline{\boldsymbol{u}})} .
$$

From the relation $\mathcal{P}_{r} \approx \epsilon$, LILLY (1967) obtained $C_{S}=0.17$.

\section{Remark 4.7}

Another interpretation is to replace $\tau^{r}$ by $\tau^{S G S}$ in (4.33). This interpretation accounts for the fact that $\tau^{S G S}$ is formally of order $\Delta^{4}$ and is neglected in the structural modelling. However, the resulting effective model is the same.

A fundamental problem of the Smagorinsky model concerns the choice of $C_{S}^{2}$, which is in general flow dependent. Lilly's approach is reviewed in [BFR80]. Using a constant value for $C_{S}^{2}$ might cause excessive dissipation, e.g. in turbulent channel flow and in the turbulent mixing-layer, see [LMK.94], [VGK.97] and references therein. Moreover, in laminar (shear) flow $C_{S}$ must equal zero. In 1991, Germano devised a widespread modification of (4.33), cf. [GPMC.91] , viz., to compute $C_{S}$ in terms of the filtered velocity field.

The Smagorinsky model can be viewed as a so-called turbulent kinetic energy (TKE) model. TKE models are based on the Kolmogorow-Prandtl relation (3.20) with $l_{m}=\Delta$ and $u^{*} \approx$ $\sqrt{k_{r}}$. Here for $u^{*}$ a model for $\sqrt{k_{r}}$ is needed. Note that for the Smagorinsky model applied to high-Reynolds-number turbulence with the filter width in the inertial subrange the choice $l_{m} \sim \Delta$ can be confirmed, cf. [Pope00], pp.587. The Smagorinsky model takes $u^{*} \sim \Delta \overline{\mathcal{S}}$, 
which can be viewed as an algebraic model for $u^{*}$.

The Smagorinsky model is (formally) of second order in $\Delta$, whereas the SGS term in Fourier analysis is of fourth order in $\Delta$. This is a mismatch (at least formally). Moreover, in many flow experiments the Smagorinsky model has been turned out to be too dissipative - that is, it transfers too much energy to the residual motions. This motivates considering a further SGS model.

BARDina (1980) suggested a different choice for $u^{*}$, based on the second identity in (4.9), cf. [BFR80], viz.,

$$
u^{*}=C_{q}|\overline{\boldsymbol{u}} \cdot \overline{\boldsymbol{u}}-\overline{\overline{\boldsymbol{u}}} \cdot \overline{\overline{\boldsymbol{u}}}|^{1 / 2}, \quad \text { with } \quad C_{q}=0.126 .
$$

Similarly, ILIESCU and LAYTON proposed

$$
u^{*}=C_{q} \sqrt{\overline{|\boldsymbol{u}-\overline{\boldsymbol{u}}|^{2}}} \approx C_{q} \sqrt{|\overline{\boldsymbol{u}}-\overline{\overline{\boldsymbol{u}}}|^{2}}=C_{q}|\overline{\boldsymbol{u}}-\overline{\overline{\boldsymbol{u}}}| \quad, \quad \text { with } \quad C_{q}=0.17,
$$

leading to the following model for $\nu_{t}$ :

$$
\nu_{t}=C_{q} \Delta|\overline{\boldsymbol{u}}-\overline{\overline{\boldsymbol{u}}}| \text {. }
$$

The Iliescu-Layton model can be written as (see [IL.98] eq. (2.6))

$$
\nu_{t}=C_{q} \frac{\Delta^{3}}{6}\|\triangle \overline{\boldsymbol{u}}\|^{2}
$$

demonstrating that this model is formally of third order in $\Delta$.

\section{Remark 4.8}

An inherent problem of TKE models is that they are unable to give accurate predictions for $\tau^{r}$ and $\mathcal{P}_{r}$ simultaneously. This stems from the fact that this type of model implies a much too large correlation $\rho_{r}$ between $\tau^{r}$ and $\mathbb{S}(\overline{\boldsymbol{u}})$. Measurements by LIU show $\rho_{r}$ between 0 and 0.2 (cf. [LMK.94]). However, substituting e.g. the Smagorinsky model for $\tau^{r}$ gives a value $\rho_{r} \approx 0.88$ under some assumptions concerning the probability distribution of $\mathbb{S}(\overline{\boldsymbol{u}})$, cf. [Pope00] exercise 13.33. Thus if the Smagorinsky coefficient chosen ensures a reasonable value for $\left\langle\mathcal{P}_{r}\right\rangle_{E}$, then the modelled residual stresses are significantly underpredicted, cf. [Pope00], p.603. This flaw is confirmed by numerical investigations, see e.g. [VGK.97], section 3.2.4. But, as mentioned above, there is wide agreement that the correct prediction of $\mathcal{P}_{r}$ is much more important than the prediction of $\tau^{r}$.

To ensure that the classical Smagorinsky model and the Iliescu Layton model behave reasonable near solid walls, $\nu_{t}$ has to be damped near the wall. For details see Chapter 7. As a final remark, note that both models predict $\nu_{t} \geq 0$ and thus preclude backscattering. $\diamond$

\subsubsection{Functional modelling part II : SGS modelling for non-isothermal problems}

First effects of buoyancy will be neglected. We focus on modelling the residual temperature flux using the gradient-diffusion hypothesis with SGS diffusivity $a_{t}$, viz.,

$$
\boldsymbol{h} \equiv-\bar{\theta} \overline{\boldsymbol{u}}+\overline{\theta \boldsymbol{u}}=-a_{t} \nabla \bar{\theta} .
$$


In analogy to the dynamic Smagorinsky model, the so-called dynamic scalar model uses $a_{t}=C_{a} \Delta^{2} \mathcal{S}$, with $C_{a}$ being determined dynamically, see [PD.98] and references therein. An alternative approach for specifying $a_{t}$ is to relate $a_{t}$ to $\nu_{t}$ by introducing a SGS turbulent Prandtl number $P r_{t} \equiv \nu_{t} a_{t}^{-1}$. A priori, $P r_{t}$ is an unknown scalar field which can be determined dynamically. In this thesis we choose $P r_{t}=0.4$ remote from solid walls. For the treatment of $\mathrm{Pr}_{t}$ in the near-wall region see section 7.1.1. Further details are given in the next subsection.

\subsubsection{Functional modelling part III : SGS modelling for buoyancy driven problems}

Now the effect of thermal stratification on residual stress modelling will be explored. Recall that in the $k / \epsilon$ model, the production term is modified to account for effects of stratification, cf. (3.26). Via the production term $\mathcal{P}_{k}+G$, gravitational production affects $k$ and hence $\nu_{t}=C_{\mu} k^{2} / \epsilon$.

Residual stress modelling for buoyant convection was investigated most remarkebly by Lilly, see [Lil62], and EIDSON, see [Eid85]. EIDSON's reasoning starts by assuming that in buoyant convection the total transfer of energy from the filtered motions to the residual motions is given by

$$
\mathcal{P}_{r}^{\theta} \equiv-\tau^{r}: \mathbb{S}(\overline{\boldsymbol{u}})-\beta \boldsymbol{g} \cdot \boldsymbol{h} .
$$

Equation (4.37) is motivated by LILLY's analysis for the corresponding compressible case, cf. eq. (10)-(11) in [Lil62].

Using the eddy-viscosity assumption $\tau^{r}=-2 \nu_{t} \mathbb{S}(\boldsymbol{u})$ implies that the transfer of energy from the resolved scales to the residual scales is predicted to be $2 \nu_{t} \overline{\mathcal{S}}$. In the isothermal case, $\nu_{t}$ has to be chosen such that $2 \nu_{t} \overline{\mathcal{S}}$ is a reasonable approximation to $\mathcal{P}_{r} \equiv-\tau^{r}: \mathbb{S}(\overline{\boldsymbol{u}})$. Two appropriate choices were discussed in subsection 4.4.2. In the case of buoyant convection, LILLY and later EIDSON suggested to choose $\nu_{t}$ such that $2 \nu_{t} \overline{\mathcal{S}}$ is a suitable approximation to $\mathcal{P}_{r}^{\theta} \equiv-\tau^{r}: \mathbb{S}(\overline{\boldsymbol{u}})-\beta \boldsymbol{g} \cdot \boldsymbol{h}$.

\section{The LILLY-EIDSON model}

Based on the work of Lilly and Eidson, in [PD98] PENG and DAvidson resume the following modified choices for $\nu_{t}$ for buoyancy driven flows

1. LiLLY-EIDSON model

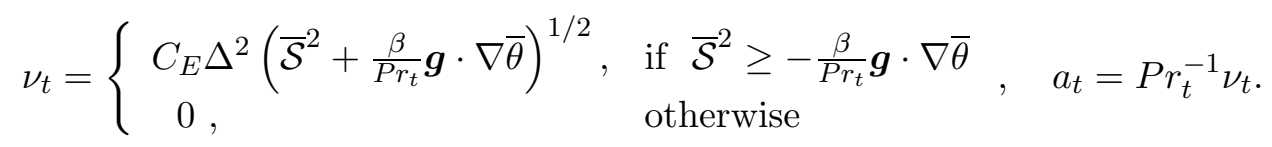

2. Modification by Peng and Davidson:

$$
\nu_{t}=\max \left(C_{E} \Delta^{2} \overline{\mathcal{S}}^{-1}\left(\overline{\mathcal{S}}^{2}+\frac{\beta}{2 P r_{t}} \boldsymbol{g} \cdot \nabla \bar{\theta}\right),-\nu\right), \quad a_{t}=\operatorname{Pr}_{t}^{-1} \nu_{t} .
$$

Unlike (4.38), the modification (4.39) allows negative values of $\nu_{t}$ in the range of $(-\nu, 0)$. Note that the factor two in the denominator of the second term in (4.39) is missing in [PI)98]. Its presence will be motivated below. 
Both in (4.38) and (4.39), $C_{E}$ and $P r_{t}$ have to be determined dynamically. Note that both formulae reduce to the standard Smagorinsky model if $\nabla \bar{\theta}=\mathbf{0}$.

Now the following questions arise :

1. How to determine the two coupled parameters $C_{E}$ and $P r_{t}$ dynamically?

2. Which relation holds between (4.38) and (4.39) ?

3. Do (4.38) and (4.39) provide a suitable approximation to (4.37) ?

Concerning the first question, see [PD.98], [WL.94] and references therein. With respect to the second question, note that for small $x$ the following Taylor expansion holds : $\sqrt{1+x} \approx$ $1+\frac{1}{2} x$. Taking this into account, starting with (4.38) we can obtain (4.39):

$$
\begin{aligned}
& C_{E} \Delta^{2}\left(\overline{\mathcal{S}}^{2}+\frac{\beta}{P r_{t}} \boldsymbol{g} \cdot \nabla \bar{\theta}\right)^{1 / 2}=C_{E} \Delta^{2} \overline{\mathcal{S}}\left(1+\frac{1}{\overline{\mathcal{S}}^{2}} \frac{\beta}{P r_{t}} \boldsymbol{g} \cdot \nabla \bar{\theta}\right)^{1 / 2} \\
& \approx C_{E} \Delta^{2} \overline{\mathcal{S}}\left(1+\frac{1}{2 \overline{\mathcal{S}}^{2}} \frac{\beta}{P r_{t}} \boldsymbol{g} \cdot \nabla \bar{\theta}\right)=C_{E} \Delta^{2} \frac{1}{\overline{\mathcal{S}}}\left(\overline{\mathcal{S}}^{2}+\frac{1}{2} \frac{\beta}{P r_{t}} \boldsymbol{g} \cdot \nabla \bar{\theta}\right)
\end{aligned}
$$

On the physical background and on a numerical comparison of both variants, see [PD.98], [PD01]. To answer the third question, simple calculation yields (using (4.39))

$$
2 \nu_{t} \overline{\mathcal{S}}^{2}=2 C_{E} \Delta^{2} \overline{\mathcal{S}}^{-1}\left(\overline{\mathcal{S}}^{2}+\frac{\beta}{2 P r_{t}} \boldsymbol{g} \cdot \nabla \bar{\theta}\right) \overline{\mathcal{S}}^{2}=2\left(C_{E} \Delta^{2} \overline{\mathcal{S}}\right) \overline{\mathcal{S}}^{2}+\beta \frac{C_{E} \Delta^{2} \overline{\mathcal{S}}}{P r_{t}} \boldsymbol{g} \cdot \nabla \bar{\theta}
$$

This is a consistent approximation to $-\tau^{r}: \mathbb{S}(\overline{\boldsymbol{u}})-\beta \boldsymbol{g} \cdot \boldsymbol{h}$ when taking the eddy-viscosity and the gradient-diffusion hypothesis with the Smagorinski model.

\section{Modification of the Iliescu-Layton SGS model}

Now we modify the ILIESCU-LAYTON model

$$
\nu_{t}=C_{q} \Delta|\overline{\boldsymbol{u}}-\overline{\overline{\boldsymbol{u}}}| .
$$

in order to account for effects of buoyancy.

1. Corresponding to (4.38) it seems natural to propose

$$
\nu_{t}= \begin{cases}C_{q} \Delta|\overline{\boldsymbol{u}}-\overline{\overline{\boldsymbol{u}}}|\left(1+\frac{1}{\overline{\mathcal{S}}^{2}} \frac{\beta}{\operatorname{Pr} t} \boldsymbol{g} \cdot \nabla \bar{\theta}\right)^{1 / 2}, & \text { if } \overline{\mathcal{S}}^{2} \geq-\frac{\beta}{\operatorname{Pr}_{t}} \boldsymbol{g} \cdot \nabla \bar{\theta}, \quad a_{t}=\operatorname{Pr}_{t}^{-1} \nu_{t} . \\ 0, & \text { otherwise }\end{cases}
$$

2. Similarly, to (4.39) the following suggestion corresponds

$$
\nu_{t}=\max \left(C_{q} \Delta|\overline{\boldsymbol{u}}-\overline{\overline{\boldsymbol{u}}}|\left(1+\frac{1}{2 \overline{\mathcal{S}}^{2}} \frac{\beta}{\operatorname{Pr} t} \boldsymbol{g} \cdot \nabla \bar{\theta}\right),-\nu\right), \quad a_{t}=\operatorname{Pr}_{t}^{-1} \nu_{t}
$$


It can be verified easily that (4.40) and (4.41) are related by a Taylor expansion of $\sqrt{1+x}$. Moreover (4.41) is consistent in the sense that

$$
\begin{aligned}
2 \nu_{t} \overline{\mathcal{S}}^{2} & =2 C_{q} \Delta|\overline{\boldsymbol{u}}-\overline{\overline{\boldsymbol{u}}}|\left(1+\frac{1}{2 \overline{\mathcal{S}}^{2}} \frac{\beta}{P r_{t}} \boldsymbol{g} \cdot \nabla \bar{\theta}\right) \overline{\mathcal{S}}^{2} \\
& =2\left(C_{q} \Delta|\overline{\boldsymbol{u}}-\overline{\overline{\boldsymbol{u}}}|\right) \overline{\mathcal{S}}^{2}+\beta \frac{C_{q} \Delta|\overline{\boldsymbol{u}}-\overline{\overline{\boldsymbol{u}}}|}{P r_{t}} \boldsymbol{g} \cdot \nabla \bar{\theta}
\end{aligned}
$$

is a suitable approximation (using the Iliescu-Layton model) to $-\tau^{r}: \mathbb{S}(\overline{\boldsymbol{u}})-\beta \boldsymbol{g} \cdot \boldsymbol{h}$.

\section{Remark 4.9}

Of course, both the turbulent-viscosity SGS model and the Galdi-Layton model contribute to $\mathcal{P}_{r}$. Therefore we should also involve the Galdi-Layton stress tensor into the modifications presented in this subsection. However, as the major contribution to $\mathcal{P}_{r}$ is due to the turbulent-viscosity model, we can neglect the Galdi-Layton stress tensor in our considerations.

\section{Remark 4.10}

It is worth giving some remarks on the model parameters $C$ and $\operatorname{Pr}_{t}$. First it should be emphasised that the SGS turbulent Prandtl number has a different significance and thus a different value than for RANS computations. Based on experimental data EIDSON proposed $P r_{t}=0.4$. Moreover he proposed $C_{E}=0.0441$. PENG and DAVIDSON compared numerical tests with $C_{E}=0.0441, P r_{t}=0.4$ and both coefficients being determined dynamically, see [PD98], [PD01]. They observed that remote from walls the constant values are a reasonable approximation to the dynamically obtained values. On the other hand, near solid walls the dynamically determined values are far from being constant. However, they report that this does not affect the behaviour of the large scale statistics significantly. This point will be considered in more detail in Chapter 7 .

The number of research groups studying buoyancy driven flows using LES is very limited. In virtually all recent papers, a variant of the LILLY-EIDSON model is employed, see e.g. [KD00], [PDOT], [WL.94]. Their focus is on how to determine the model coefficients $C_{E}$ and $P r_{t}$ dynamically.

The Eidson model accounts only for effects of stratification, but not for a (speculative) turbulence generation mechanism due to buoyancy as suggested by the theory of baroclinic vorticity generation, see Section 3.3.2. LES with near-wall resolution might benefit from adding a term similar to (3.27) to the Eidson model.

\subsection{System of equations for non-isothermal LES}

We conclude this chapter by summarizing the system of equations for LES of non-isothermal flow problems. This system will be equipped with suitable boundary conditions in Chapter 7. We seek $\boldsymbol{w}, q$, and $\vartheta$ as an approximation to $\overline{\boldsymbol{u}}, \bar{p}$, and $\bar{\theta}$ as solution of 


$$
\begin{aligned}
\partial_{t} \boldsymbol{w}-\boldsymbol{\nabla} \cdot\left(2\left(\nu_{e} \mathbb{S}(\boldsymbol{w})-\mathcal{A}_{u}(\boldsymbol{w})\right)\right)+(\boldsymbol{w} \nabla) \boldsymbol{w}+\boldsymbol{\nabla} q & =-\beta \vartheta \boldsymbol{g} \\
\boldsymbol{\nabla} \cdot \boldsymbol{w} & =0 \\
\partial_{t} \vartheta-\boldsymbol{\nabla} \cdot\left(a_{e} \boldsymbol{\nabla} \vartheta-\mathcal{A}_{\theta}(\boldsymbol{w}, \vartheta)\right)+(\boldsymbol{w} \boldsymbol{\nabla}) \vartheta & =\dot{q}^{V} c_{p}^{-1}
\end{aligned}
$$

with

$$
\mathcal{A}_{u}(\boldsymbol{w})=\frac{\Delta^{2}}{12} \mathcal{R}(\nabla \boldsymbol{w} \bullet \nabla \boldsymbol{w}), \quad \mathcal{A}_{\theta}(\boldsymbol{w}, \vartheta)=\frac{\Delta^{2}}{12} \mathcal{R}(\nabla \vartheta \circ \nabla \boldsymbol{w})
$$

with the regularization operator $\mathcal{R}$ defined in (4.15). 


\section{Near-wall treatment in turbulence modelling}

This chapter is dedicated to wall bounded flows. Fundamental results of the turbulent boundary-layer theory are reviewed. Moreover, some turbulence models for the near-wall region are discussed. Throughout this chapter we suppose that for almost every point $\boldsymbol{x}$ in $\Gamma$ there exists a local orthonormal basis $\left\{\boldsymbol{n}(\boldsymbol{x}), \boldsymbol{t}_{j}(\boldsymbol{x}), 1 \leq j \leq d-1\right\}$, where $\left\{\boldsymbol{t}_{j}\right\}_{j=1}^{d-1}$ is a local orthonormal basis for the tangent space of $\Gamma$ in $\boldsymbol{x}$ and $\boldsymbol{n}$ denotes the outer unit normal vector to $\Gamma$ at $\boldsymbol{x}$; denote $x \equiv x_{1}, y \equiv x_{2}, z \equiv x_{3}$ streamwise, wall-normal, and spanwise coordinate direction resp.

\subsection{Fundamentals of turbulent boundary-layer theory}

As a starting point we consider a (forced convection) flow over a flat plate, often called a turbulent boundary layer, as sketched in Figure 5.1. In this flow, remote from the plate turbulence is absent and the only source of turbulence is due to the presence of a solid surface, where impermeability and no-slip condition hold. The flow configuration consists of a flat plate located at

$$
\Gamma_{W} \equiv\left\{\boldsymbol{x} \in \mathbb{R}^{3}: y=0, x \geq 0\right\} .
$$

At $x \rightarrow-\infty$ the free-stream velocity is given by $\boldsymbol{u}=U_{\infty} \boldsymbol{e}_{1}$. We assume that due to translational invariance in the $x_{3} \equiv z$ direction the problem is two-dimensional. Measurements and DNS data reveal that there is a neighbourhood of the plate of thickness $\delta_{\text {layer }}^{u}=\delta_{\text {layer }}^{u}(x)$ increasing with $x$, where the Reynolds stresses are significantly large. This region is called the turbulent boundary layer. Remote from the wall, the Reynolds stresses cease and the velocity tends to the free-stream velocity. Figure 5.2 shows the profiles of the Reynolds stresses in a supersonic flat-plate zero-pressure gradient boundary layer from the DNS data of [MAKO1].

The boundary-layer thickness $\delta_{\text {layer }}^{u}(x)$ is generally defined as the value $d_{99}$ of $y$ at which $U(x, y)=0.99 U_{\infty}$ with $U(x, y) \equiv \boldsymbol{U} \cdot \boldsymbol{e}_{1}$. The flow region with $y>d_{99}$ is called the freestream.

In the region between turbulent boundary-layer flow and the irrotational non-turbulent free-stream, the flow is sometimes turbulent and sometimes not, a phenomenon called intermittency. Therefore, strictly speaking, $\delta_{\text {layer }}^{u}$ is a random variable. However, a detailed description of the near-wall turbulent structures goes beyond the scope of this chapter. For details, see e.g [Pope00], Sections 5.5.2 and 7.4.

Now we extend the previous notions to the forced convection non-isothermal boundary layer. Denote $\theta_{w}$ the wall temperature and $\theta_{0}$ the temperature of the free-stream fluid. Then a temperature boundary layer of thickness $\delta_{\text {layer }}^{\theta}$, characterised by a large heat flux vector $\left\langle\boldsymbol{u}^{\prime} \theta^{\prime}\right\rangle_{E}$, can be defined analogously.

Now we make the approximation that $\delta_{\text {layer }}^{u}=\delta_{\text {layer }}^{\theta}=\delta_{\text {layer }}$ for each $\boldsymbol{x}$ in $\Gamma_{W}$, i.e. momentum and temperature boundary layer are assumed to have the same thickness. This 

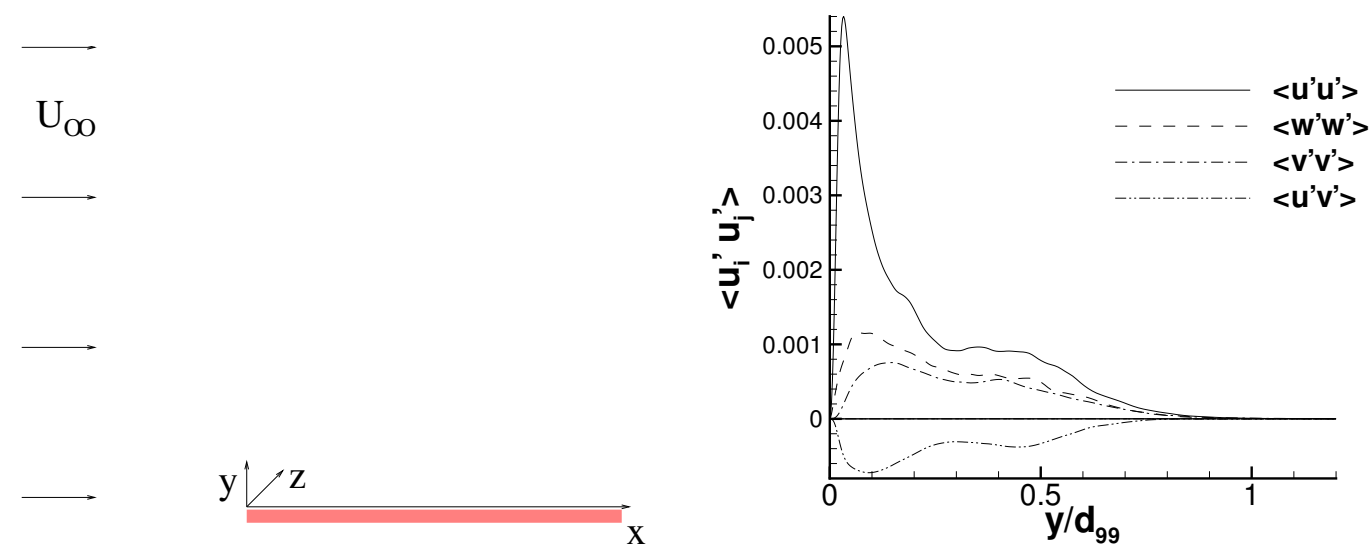

Figure 5.1.: Sketch of flow configuration. Figure 5.2.: Profiles of Reynolds stresses.

was first devised by ECKERT, cf. [Eck50], pp. 158-164. The approximation is reasonable as $\delta_{\text {layer }}^{u} / \delta_{\text {layer }}^{\theta} \approx \sqrt{\nu_{t} / a_{t}}=\sqrt{P r_{t}} \approx 0.95$ for air; it just helps reducing the complexity concerning indices.

We assume that $\delta_{\text {layer }}(\boldsymbol{x})$ is given for each $\boldsymbol{x}$ in $\Gamma_{W}$. Then we can define the turbulent boundary-layer region $\Omega_{\text {layer }}$ and the free-stream region $\Omega_{\text {free }}$ :

$$
\begin{aligned}
\Omega_{\text {layer }} & =\left\{\boldsymbol{x} \in \Omega \mid \operatorname{dist}\left(\boldsymbol{x}, \Gamma_{W}\right)<\delta_{\text {layer }}\right\} \\
\Omega_{\text {free }} & =\Omega \backslash \Omega_{\text {layer }}
\end{aligned}
$$

For each $\boldsymbol{x} \in \Gamma_{W}$ the following characteristic quantities can be defined

$$
\left.\tau_{w} \equiv \nu \nabla \boldsymbol{U}\right|_{\Gamma_{W}} \cdot \boldsymbol{n}, \quad u_{\tau} \equiv \sqrt{\tau_{w}}, \quad c_{f} \equiv \frac{2 \tau_{w}}{U_{\infty}^{2}}
$$

$\tau_{w}$ being the wall shear stress, $u_{\tau}$ being the friction velocity and $c_{f}$ being the skin friction coefficient. In the case of $\partial U_{\infty} / \partial x=0$, the growth of the boundary-layer is given by the formula (cf. [DPR01], p.69)

$$
\frac{d \delta^{*}}{d x}=\frac{c_{f}}{2}, \quad \text { with } \quad \delta^{*} \equiv \int_{0}^{\infty} \frac{U}{U_{\infty}}\left(1-\frac{U}{U_{\infty}}\right) d y
$$

where $\delta^{*}$ is called momentum thickness. Since typically $c_{f}=\mathcal{O}\left(10^{-3}\right)$ the boundary-layer thickness grows very slowly. As $c_{f}$ is unknown a priori, equation (5.4) is unclosed. Using the empirical relation $c_{f} \approx 0.025 R e_{\delta^{*}}$ with $R e_{\delta^{*}}=U_{\infty} \delta^{*} / \nu$ we finally obtain (cf. [DPR0]], p.69)

$$
\frac{\delta^{*}}{x} \approx\left(\frac{5 a}{8}\right)^{4 / 5}\left(\frac{U_{\infty} x}{\nu}\right)^{-1 / 5}, \quad \text { with } a=0.025
$$

demonstrating that $\delta^{*}$ scales with $\nu^{1 / 5}$. In constrast, in the laminar case $\delta^{*} \propto \nu^{1 / 2}$.

Pursuing further the parallel between momentum and temperature boundary layers, the 


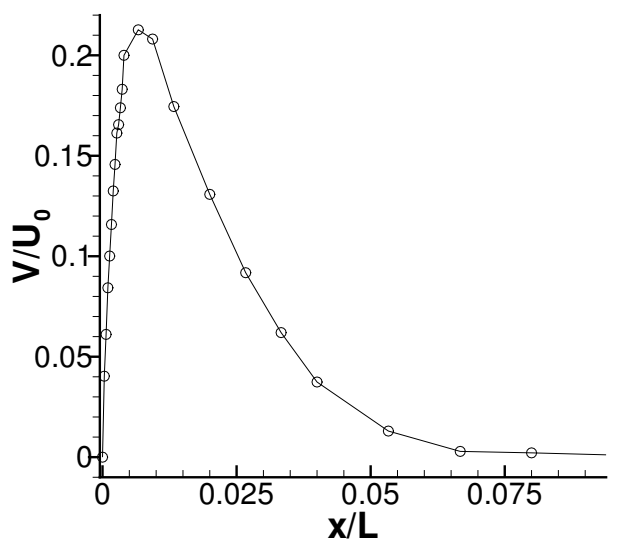

Figure 5.3.: Natural convection: Velocity profile.

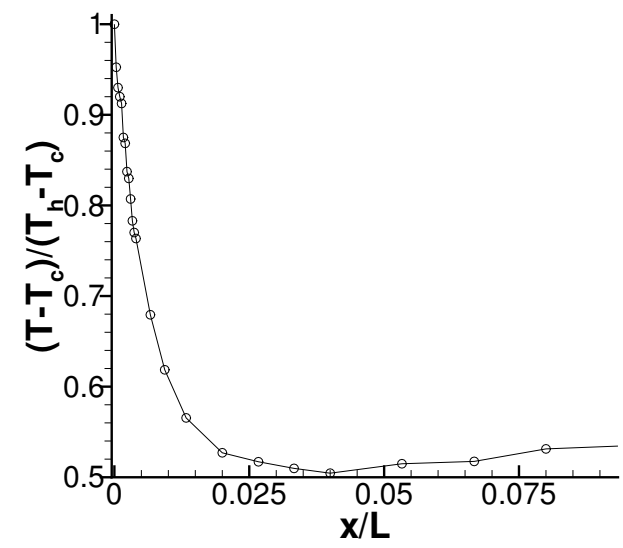

Figure 5.4.: Natural convection: Temperature profile.

enthalpy thickness $\Delta_{2}$ can be introduced measuring the thickness of the temperature boundary layer. Similar to (5.4), the following equation holds

$$
\frac{d \Delta_{2}}{d x}=S t, \quad \text { with } \quad \Delta_{2} \equiv \int_{0}^{\infty} \frac{U}{U_{\infty}}\left(1-\frac{\theta-\theta_{\infty}}{\theta_{w}-\theta_{\infty}}\right) d y, \quad S t \equiv \frac{\dot{q}_{w}}{\left(\theta_{w}-\theta_{\infty}\right) U_{\infty}},
$$

with $\theta_{w}$ and $\theta_{\infty}$ denoting the temperature on the wall resp. in the free stream. The Stanton number $S t$ is the normalized surface heat flux. If $\operatorname{Pr} \approx 1$ and $P r_{t} \approx 1$ then $S t \approx c_{f} / 2$ in a zero pressure gradient forced convection boundary layer.

In natural convection along surfaces, boundary-layer phenomena are also apparent. Figures 5.3-5.4 show velocity and temperature profile for a buoyancy induced flow along a heated vertical plate in a closed cavity recently studied experimentally by TIAM, AMPOFO and KARAYIANNis, cf. [TK00a], [TK00b], and [AK02]. Figures 5.5-5.6 show the turbulence quantities, indicating that turbulent boundary-layer effects are significant only in a narrow strip close to the wall. The substructure of turbulent boundary layers in forced and natural convection flows will be studied in greater detail in Subsections 5.3.1 and 5.4.1. As pointed out by WILCOX, see [Wilcox98], Section 4.6, the turbulent flow over a flat plate is a classical singularly-perturbed problem: The task is to find a boundary-layer solution which satisfies both the no-slip at $y=0$ and the free-stream condition at $y=\delta_{\text {layer }}$. In the next subsection, the asymptotic behaviour in the region very close to the wall will be studied.

\subsection{Boundary-layer equations and singular perturbation methods}

In this subsection we derive simplified differential equations that describe the flow problem approximately in the near-wall region, called boundary-layer equations. This will be accomplished using the method of singular perturbations. To achieve our objective, we will proceed as follows:

1. Derivation of the laminar boundary-layer equations. 


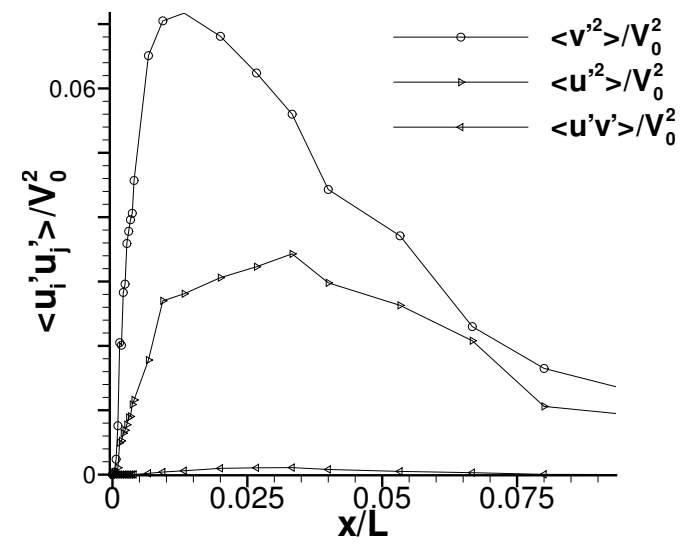

Figure 5.5.: Natural convection: Reynolds stresses.

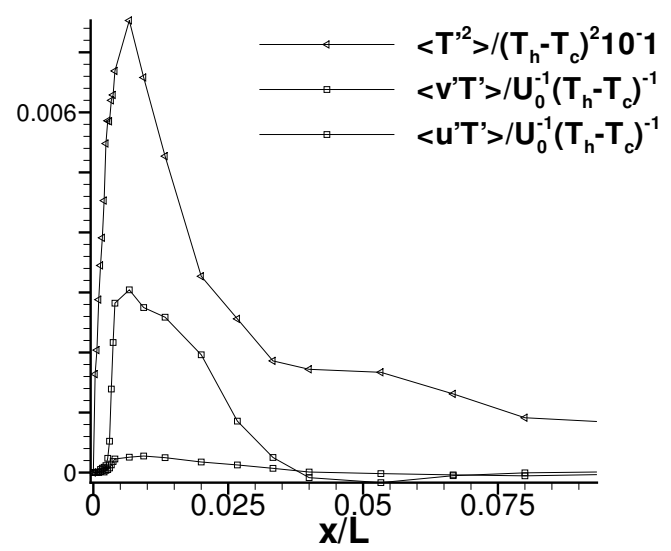

Figure 5.6.: Natural convection: Turbulent heat fluxes.

2. Presentation of the turbulent boundary-layer equations, which are motivated by the laminar boundary-layer equations.

3. Simplification of the three-dimensional turbulent boundary-layer equations to the two-dimensional boundary-layer equations.

4. Simplification of the two-dimensional turbulent boundary-layer equations to the onedimensional boundary-layer equation.

For sake of clarity and simplicity, in step (1)-(3) we restrict ourselves to the isothermal case. Moreover we have to assume that the surface curvature is significantly small. The turbulence in a boundary layer entering a convex (concave) curve is suppressed (amplified), see [DPR01], pp.162. Models based on the eddy-viscosity hypothesis cannot describe such effects. In particular, the predictions near corners can become unreliable. Finally we assume that the processes in the boundary layer are stationary, see e.g. [Pope00], pp. 111.

\subsubsection{The three-dimensional laminar boundary-layer equations}

Throughout this subsection we will deal with two coordinate systems. First, denote $\boldsymbol{e}_{x}$, $\boldsymbol{e}_{y}, \boldsymbol{e}_{z}$ the standard wall fitted coordinate system of streamwise, wall-normal and spanwise direction resp. Second, we suppose that there exists a network of potential lines ( $\phi=$ const) and streamlines $(\psi=$ const), which represent the idealized inviscid wall bounded flow. Then $\boldsymbol{e}_{\phi}$ and $\boldsymbol{e}_{\psi}$ are defined being perpendicular to the lines $(\phi=$ const) and $(\psi=$ const $)$ resp. Obviously, this assumption does not hold for flow separation and recirculation. However, we could derive the laminar boundary-layer equations in almost the same manner in the $\boldsymbol{e}_{x}, \boldsymbol{e}_{y}, \boldsymbol{e}_{z}$ coordinate system, but then the equations involved are more complex. Anyway, at the end, we will present the boundary-layer equations in the standard $\boldsymbol{e}_{x}, \boldsymbol{e}_{y}, \boldsymbol{e}_{z}$ system. As a rough idea, one could imagine that $\boldsymbol{e}_{y} \equiv \boldsymbol{e}_{\psi}$ and $\boldsymbol{e}_{\phi}=\alpha \boldsymbol{e}_{x}+\beta \boldsymbol{e}_{z}$, for some $\alpha, \beta$. Then $\boldsymbol{u}=w_{\phi} \boldsymbol{e}_{\phi}+w_{\psi} \boldsymbol{e}_{\psi}$. 
As mentioned in the previous section, near the wall, i.e. locally in the boundary-layer, flow characteristics vary rapidly in the $\boldsymbol{e}_{\psi}$ direction. Hence we introduce the following local variable, viz., $\psi^{*}=\frac{\psi}{\delta(\nu)}$. Then for the local solution $w_{\phi}, w_{\psi}$ the following ansatz is made

$$
\begin{aligned}
w_{\phi}(\phi, \psi, \nu) & =W_{\phi}^{0}\left(\phi, \psi^{*}, \nu\right)+\delta(\nu) W_{\phi}^{1}\left(\phi, \psi^{*}, \nu\right)+\mathcal{O}\left(\delta^{2}(\nu)\right), \\
w_{\psi}(\phi, \psi, \nu) & =W_{\psi}^{0}\left(\phi, \psi^{*}, \nu\right)+\delta(\nu) W_{\psi}^{1}\left(\phi, \psi^{*}, \nu\right)+\mathcal{O}\left(\delta^{2}(\nu)\right), \\
p(\phi, \psi, \nu) & =P^{0}\left(\phi, \psi^{*}, \nu\right)+\delta(\nu) P^{1}\left(\phi, \psi^{*}, \nu\right)+\mathcal{O}\left(\delta^{2}(\nu)\right) .
\end{aligned}
$$

First we show that $W_{\psi}^{0}=0$. Denote $W_{\phi}\left(\phi, \psi^{*}, \nu\right)=w_{\phi}(\phi, \psi, \nu)$ and $W_{\psi}\left(\phi, \psi^{*}, \nu\right)=$ $w_{\psi}(\phi, \psi, \nu)$. The equation of continuity reads

$$
\frac{\partial w_{\phi}}{\partial \phi}+\frac{\partial w_{\psi}}{\partial \psi}=0 \quad \Longleftrightarrow \quad \frac{\partial W_{\phi}}{\partial \phi}+\frac{1}{\delta(\nu)} \frac{\partial W_{\psi}}{\partial \psi^{*}}=0
$$

Substituting the ansatz the continuity equation becomes

$$
\frac{\partial W_{\phi}^{0}}{\partial \phi}+\delta(\nu) \frac{\partial W_{\phi}^{1}}{\partial \phi}+\mathcal{O}\left(\delta^{2}(\nu)\right)+\frac{1}{\delta(\nu)} \frac{\partial W_{\psi}^{0}}{\partial \psi^{*}}+\frac{\partial W_{\psi}^{1}}{\partial \psi^{*}}+\mathcal{O}(\delta(\nu))=0 .
$$

In order to obtain a nontrivial continuity equation we have to balance the terms with respect to the parameter $\delta(\nu)$. Hence $\frac{\partial W_{\psi}^{0}}{\partial \psi^{*}}=0$. Together with the no-penetration condition $w_{\psi}(\phi, 0)=0$ this implies $W_{\psi}^{0} \equiv 0$ (which follows immediately from the theory of ordinary differential equations). Thus we have deduced that our ansatz can be simplified to

$$
\begin{aligned}
w_{\phi}\left(\phi, \psi^{*}, \nu\right) & =W_{\phi}^{0}\left(\phi, \psi^{*}, \nu\right)+\delta(\nu) W_{\phi}^{1}\left(\phi, \psi^{*}, \nu\right)+\mathcal{O}\left(\delta^{2}(\nu)\right), \\
w_{\psi}\left(\phi, \psi^{*}, \nu\right) & = \\
p(\phi) W_{\psi}^{1}\left(\phi, \psi^{*}, \nu\right)+\mathcal{O}\left(\delta^{2}(\nu)\right), & \\
p\left(\phi, \psi^{*}, \nu\right) & =P^{0}\left(\phi, \psi^{*}, \nu\right)+\delta(\nu) P^{1}\left(\phi, \psi^{*}, \nu\right)+\mathcal{O}\left(\delta^{2}(\nu)\right) .
\end{aligned}
$$

Substituting this ansatz into the momentum equations, the following two observations can be made. First we can deduce $\delta=\mathcal{O}(\sqrt{\nu})$. In the $w_{\phi^{-}}$equation the term $\nu\left(\partial^{2} w_{\phi} / \partial \psi^{2}\right)$ is of order $\nu / \delta^{2}$. As the $w_{\phi}$-equation has to satisfy two boundary conditions, viz., (i) no-slip at $\psi=0$ and (ii) matching the free-stream for $\psi^{*} \rightarrow \infty$, this term must be of order $\mathcal{O}(1)$ and consequently $\delta=\mathcal{O}(\sqrt{\nu})$. Second, the only $\mathcal{O}(1)$ term in the $w_{\psi^{-}}$equation is $\partial P / \partial \psi^{*}$ and therefore the $w_{\psi}$-equation reduces to $\partial P / \partial \psi^{*}=0$. This implies $P=P(\phi)$.

Substituting $\delta=\sqrt{\nu}$ into the $w_{\phi}$-equation and taking into account only terms of order $\mathcal{O}(1)$ we obtain the laminar boundary-layer equations.

In the standard $\boldsymbol{e}_{x}, \boldsymbol{e}_{y}, \boldsymbol{e}_{z}$ coordinate system the equations for $U_{i} \equiv \boldsymbol{U} \cdot \boldsymbol{e}_{i}$ read, cf. e.g. [CT.90],

$$
\sum_{j=1}^{3} \frac{\partial\left(U_{i} U_{j}\right)}{\partial x_{j}}+\frac{\partial P_{\infty}}{\partial x_{i}}=\frac{\partial}{\partial y}\left[\nu \frac{\partial U_{i}}{\partial y}\right], \quad i=1,3
$$

with continuity equation

$$
U_{2}=-\int_{0}^{y} \sum_{i=1,3} \frac{\partial U_{i}\left(y^{\prime}\right)}{\partial x_{i}} d y^{\prime}
$$


and boundary conditions

$$
U_{i}=0 \quad \text { on } \Gamma_{W}, \quad \lim _{y^{+} \rightarrow \infty} U_{i}\left(x_{1}, y^{+}, x_{3}\right)=U_{\infty, i}\left(x_{1}, x_{3}\right)
$$

with $P_{\infty}=P_{\infty}\left(x_{1}, x_{3}\right)$ being the free stream pressure and $U_{\infty}$ being the freestream velocity. Note that $\partial P_{\infty} / \partial x_{i}(\mathrm{i}=1,3)$ can be given in terms of the free-stream velocity by Bernoulli's equation, viz., $-\partial P_{\infty} / \partial x_{i}=\sum_{j=1}^{3} U_{\infty, j} \partial U_{\infty, i} \partial x_{j}$.

\subsubsection{The three-dimensional turbulent boundary-layer equations}

In this subsection we motivate the turbulent boundary-layer equations. We can start with the RANS equations and take into account only the wall-normal derivatives of the Reynolds stresses. Similar to the laminar case the lateral mean momentum equation becomes

$$
\frac{\partial P}{\partial y}+\frac{\partial\left\langle u_{2}^{\prime 2}\right\rangle_{E}}{\partial y}=0
$$

In the free stream $\left(y^{+} \rightarrow \infty\right),\left\langle u_{2}^{\prime 2}\right\rangle_{E}$ is zero, see Figure 5.2. Consequently integration yields $P=P_{\infty}-\left\langle u_{2}^{\prime 2}\right\rangle_{E}$ and therefore

$$
\frac{\partial P}{\partial x_{i}}=\frac{\partial P_{\infty}}{\partial x_{i}}-\frac{\partial\left\langle u_{2}^{\prime 2}\right\rangle_{E}}{\partial x_{i}}, \quad i=1,3 .
$$

Using the approximations devised for the laminar case, the $\boldsymbol{e}_{x^{-}}$and $\boldsymbol{e}_{z}$-momentum equations become when substituting (5.13)

$$
\sum_{j=1}^{3} \frac{\partial\left(U_{i} U_{j}\right)}{\partial x_{j}}+\frac{\partial P_{\infty}}{\partial x_{i}}=\frac{\partial}{\partial y}\left[\nu \frac{\partial U_{i}}{\partial y}\right]-\sum_{j=1}^{3} \frac{\partial\left\langle u_{i}^{\prime} u_{j}^{\prime}\right\rangle_{E}}{\partial x_{j}}+\frac{\partial\left\langle u_{2}^{\prime 2}\right\rangle_{E}}{\partial x_{i}}, \quad i=1,3 .
$$

Taking into account only the derivatives of $\left\langle u_{i}^{\prime} u_{j}^{\prime}\right\rangle_{E}$ in the $\boldsymbol{e}_{y}$-direction in (5.14), we arrive at the turbulent boundary-layer equations, cf. e.g. [CMO0]:

$$
\sum_{j=1}^{3} \frac{\partial\left(U_{i} U_{j}\right)}{\partial x_{j}}+\frac{\partial P_{\infty}}{\partial x_{i}}=\frac{\partial}{\partial y}\left[\nu \frac{\partial U_{i}}{\partial y}\right]-\frac{\partial\left\langle u_{i}^{\prime} u_{2}^{\prime}\right\rangle_{E}}{\partial y}, \quad i=1,3 .
$$

Using the eddy viscosity hypothesis for $\partial\left(\left\langle u_{i}^{\prime} u_{2}^{\prime}\right\rangle_{E}\right) / \partial y$, i.e., $\partial\left(\left\langle u_{i}^{\prime} u_{2}^{\prime}\right\rangle_{E}\right) / \partial y=-\nu_{t} \partial U_{i} / \partial y$, we finally obtain (5.11), (5.12), and

$$
\sum_{j=1}^{3} \frac{\partial\left(U_{i} U_{j}\right)}{\partial x_{j}}+\frac{\partial P_{\infty}}{\partial x_{i}}=\frac{\partial}{\partial y}\left[\left(\nu+\nu_{t}\right) \frac{\partial U_{i}}{\partial y}\right], \quad i=1,3 .
$$

\section{Remark 5.1}

It is worth recalling that in contrast to the laminar case $\left(\delta^{*} \sim \sqrt{\nu}\right)$, in the turbulent case the momentum thickness $\delta^{*}$ scales like $\nu^{1 / 5}$. In the laminar case, it is possible to deduce $\delta \sim \sqrt{\nu}$ using the method of asymptotic expansions. As the RANS equations are unclosed, an empirical closure approximation is necessary. 


\subsubsection{The two-dimensional turbulent boundary-layer equations}

From now on we assume that $\boldsymbol{e}_{x}$ is the dominant direction of the flow. Denote $L, \delta_{\nu}$ and $B$ the characteristic length scales over which flow properties change in the $x, y$ and $z$ direction resp. Since $\delta_{\nu} \ll L$ and $\delta_{\nu} \ll B$ we scale $x, y$ and $z$ differently, viz.,

$$
\xi=\frac{x}{L}, \quad y^{+}=\frac{y}{\delta_{\nu}} \text { with } \delta_{\nu} \equiv \frac{\nu}{u_{\tau}}, \text { and } \eta=\frac{z}{B} .
$$

Then all partial derivatives w.r.t. the scaled variables are at most of order $\mathcal{O}(1)$. First we assume that $L \ll B$. This approximation is reasonable in many applications, e.g. a flow in a channel with large aspect ratio, a flow over an airfoil or a flow along a flat wall in a indoor-air flow problem. Then, in the limit $B \rightarrow \infty$ we obtain $U_{3}=0$. Consequently, (5.16), (5.11), and (5.12), reduce to, cf. [Wilcox98], p.150

$$
\sum_{j=1}^{2} \frac{\partial\left(U_{1} U_{j}\right)}{\partial x_{j}}+\frac{\partial P_{\infty}}{\partial x_{1}}=\frac{\partial}{\partial y}\left[\left(\nu+\nu_{t}\right) \frac{\partial U_{1}}{\partial y}\right], \quad U_{2}=-\int_{0}^{y} \frac{\partial U_{1}\left(y^{\prime}\right)}{\partial x_{1}} d y^{\prime}
$$

with boundary conditions

$$
U_{1}=0 \quad \text { on } \Gamma_{W}, \quad \lim _{y^{+\rightarrow \infty}} U_{1}\left(x_{1}, y^{+}, x_{3}\right)=U_{\infty, 1}\left(x_{1}\right) .
$$

Under all these assumptions and using the same arguments, it is possible to obtain the following corresponding boundary-layer equation for $\Theta$, viz.,

$$
\sum_{j=1}^{2} \frac{\partial\left(U_{j} \Theta\right)}{\partial x_{j}}=\frac{\partial}{\partial y}\left[\left(a+a_{t}\right) \frac{\partial \Theta}{\partial y}\right]+\dot{q}^{V} c_{p}^{-1},
$$

with boundary conditions

$$
\Theta=\Theta_{w} \quad \text { on } \Gamma_{W}, \quad \lim _{y^{+} \rightarrow \infty} \Theta\left(x_{1}, y^{+}, x_{3}\right)=\Theta_{\infty}\left(x_{1}\right),
$$

with $\Theta_{\infty}$ denoting the free-stream temperature, see Section 5.1.

\subsubsection{The one-dimensional non-isothermal boundary-layer equations}

From now on we focus on the non-isothermal case. Equations (5.18)-(5.19) are the starting point for deriving the one-dimensional boundary-layer equations, see e.g. [Wilcox.98], pp.150. For the streamwise and wall normal component of the mean velocity $U$ and $V$ resp., the mean temperature $\Theta$ and turbulent viscosity $\nu_{t}$ WILCOX makes the following ansatz inspired by physical intuition

$$
\begin{aligned}
U & =u_{\tau}\left(U_{0}\left(\xi, y^{+}\right)+\phi_{1} U_{1}\left(\xi, y^{+}\right)+\mathcal{O}\left(\phi_{2}\right)\right), \\
V & =\nu L^{-1}\left(V_{0}\left(\xi, y^{+}\right)+\phi_{1} V_{1}\left(\xi, y^{+}\right)+\mathcal{O}\left(\phi_{2}\right)\right), \\
\Theta & =\Theta_{0}\left(\xi, y^{+}\right)+\phi_{1} \Theta_{1}\left(\xi, y^{+}\right)+\mathcal{O}\left(\phi_{2}\right), \\
\nu_{t} & =\nu\left(n_{0}\left(\xi, y^{+}\right)+\phi_{1} n_{1}\left(\xi, y^{+}\right)+\mathcal{O}\left(\phi_{2}\right)\right), \\
a_{t} & =a\left(m_{0}\left(\xi, y^{+}\right)+\phi_{1} m_{1}\left(\xi, y^{+}\right)+\mathcal{O}\left(\phi_{2}\right)\right),
\end{aligned}
$$

with an asymptotic sequence $\left\{1, \phi_{1}, \phi_{2}, \ldots\right\}, \phi_{j}=\left(\frac{\nu}{u_{\tau} L}\right)^{\alpha j}, 0<\alpha \leq 1$. 


\section{Remark 5.2}

As the following analysis is restricted to the leading-order terms, we are not interested in detailed information regarding $\left\{1, \phi_{1}, \phi_{2}, \ldots\right\}$. As noted in remark 5.1, in the turbulent case it is not possible to deduce information concerning $\left\{1, \phi_{1}, \phi_{2}, \ldots\right\}$ without adding further physical insight. This scaling is based on physical intuition. However it is in full agreement with the ideas presented in subsection 5.2.1. First note that

$$
\frac{\partial U}{\partial x}=\frac{\partial U}{\partial \xi} \frac{\partial \xi}{\partial x}=\frac{1}{L} \frac{\partial U}{\partial \xi}, \quad \frac{\partial^{2} U}{\partial x^{2}}=\frac{1}{L^{2}} \frac{\partial^{2} U}{\partial \xi^{2}}, \quad \frac{\partial U}{\partial y^{+}}=\frac{1}{\delta_{\nu}} \frac{\partial U}{\partial \xi}, \quad \frac{\partial^{2} U}{\partial y^{2}}=\frac{1}{\delta_{\nu}^{2}} \frac{\partial^{2} U}{\partial y^{+^{2}}}
$$

and analogous relations hold for $V$ and $\Theta$. Substituting (5.20)-(5.21) into the equation of continuity yields (taking into account (5.17))

$$
\frac{\partial U}{\partial x}+\frac{\partial V}{\partial y}=\frac{1}{L} \frac{\partial U}{\partial \xi}+\frac{1}{\delta_{\nu}} \frac{\partial V}{\partial y^{+}}=\mathcal{O}\left(\frac{1}{L} u_{\tau}\right)+\mathcal{O}\left(\frac{1}{\delta_{\nu}} \frac{\nu}{L}\right)=\mathcal{O}\left(\frac{u_{\tau}}{L}\right)+\mathcal{O}\left(\frac{u_{\tau}}{L}\right) .
$$

Hence this ansatz ensures a nontrivial continuity equation, as the leading order terms are already balanced with respect to the scaling parameters. Hence the ansatz can be interpreted setting $\delta(\nu)=\frac{\nu}{u_{\tau} L}$ in the laminar asymptotic expansion (5.7)-(5.9). Again it should be emphasised that the relation $u_{\tau}=u_{\tau}(\nu)$ is a priori unknown.

Motivated by equations (5.18)-(5.19), for the further analysis we start with

$$
\begin{aligned}
U \frac{\partial U}{\partial x}+V \frac{\partial U}{\partial y}+\frac{\partial P}{\partial x}-\frac{\partial}{\partial x}\left(\left(\nu+\nu_{t}\right) \frac{\partial U}{\partial x}\right)-\frac{\partial}{\partial y}\left(\left(\nu+\nu_{t}\right) \frac{\partial U}{\partial y}\right) & =-\beta \Theta \boldsymbol{g} \cdot \boldsymbol{e}_{x}, \\
U \frac{\partial \Theta}{\partial x}+V \frac{\partial \Theta}{\partial y}-\frac{\partial}{\partial x}\left(\left(a+a_{t}\right) \frac{\partial \Theta}{\partial x}\right)-\frac{\partial}{\partial y}\left(\left(a+a_{t}\right) \frac{\partial \Theta}{\partial y}\right) & =\dot{q}^{V} c_{p}^{-1} .
\end{aligned}
$$

Neglecting all terms of order $\phi_{i}(i \geq 1)$ we get

$$
\begin{aligned}
\frac{u_{\tau} U_{0}}{L} \frac{u_{\tau} \partial U_{0}}{\partial \xi}+\frac{\nu V_{0}}{L \delta_{\nu}} \frac{u_{\tau} \partial U_{0}}{\partial y^{+}}+\frac{1}{L} \frac{\partial P}{\partial \xi}-\frac{\nu}{L^{2}} \frac{\partial}{\partial \xi}\left(\left(1+n_{0}\right) \frac{u_{\tau} \partial U_{0}}{\partial \xi}\right) & \\
-\frac{\nu}{\delta_{\nu}^{2}} \frac{\partial}{\partial y^{+}}\left(\left(1+n_{0}\right) \frac{u_{\tau} \partial U_{0}}{\partial y^{+}}\right) & =-\beta \Theta \boldsymbol{g} \cdot \boldsymbol{e}_{x}, \\
\frac{u_{\tau} U_{0}}{L} \frac{\partial \Theta_{0}}{\partial \xi}+\frac{\nu V_{0}}{L \delta_{\nu}} \frac{\partial \Theta_{0}}{\partial y^{+}}-\frac{a}{L^{2}} \frac{\partial}{\partial \xi}\left(\left(1+m_{0}\right) \frac{\partial \Theta_{0}}{\partial \xi}\right) & \\
-\frac{a}{\delta_{\nu}^{2}} \frac{\partial}{\partial y^{+}}\left(\left(1+m_{0}\right) \frac{\partial \Theta_{0}}{\partial y^{+}}\right) & =\dot{q}^{V} c_{p}^{-1} .
\end{aligned}
$$

This can be written as

$$
\begin{aligned}
\frac{\tau_{w}}{L} U_{0} \frac{\partial U_{0}}{\partial \xi}+\frac{\tau_{w}}{L} V_{0} \frac{\partial U_{0}}{\partial y^{+}}+\frac{1}{L} \frac{\partial P}{\partial \xi}-\frac{\delta_{\nu} \tau_{w}}{L^{2}} \frac{\partial}{\partial \xi}\left(\left(1+n_{0}\right) \frac{\partial U_{0}}{\partial \xi}\right) & \\
-\frac{\tau_{w}}{\delta_{\nu}} \frac{\partial}{\partial y^{+}}\left(\left(1+n_{0}\right) \frac{\partial U_{0}}{\partial y^{+}}\right) & =-\beta \Theta \boldsymbol{g} \cdot \boldsymbol{e}_{x}, \\
\frac{u_{\tau}}{L} U_{0} \frac{\partial \Theta_{0}}{\partial \xi}+\frac{\nu}{L \delta_{\nu}} V_{0} \frac{\partial \Theta_{0}}{\partial y^{+}}-\frac{a}{L^{2}} \frac{\partial}{\partial \xi}\left(\left(1+m_{0}\right) \frac{\partial \Theta_{0}}{\partial \xi}\right) & \\
-\frac{a}{\delta_{\nu}^{2}} \frac{\partial}{\partial y^{+}}\left(\left(1+m_{0}\right) \frac{\partial \Theta_{0}}{\partial y^{+}}\right) & =\dot{q}^{V} c_{p}^{-1} .
\end{aligned}
$$


For most practical flows $\tau_{w}=\mathcal{O}\left(10^{-3}\right)$ and hence $u_{\tau}=\mathcal{O}\left(10^{-1}\right)$. Now we assume that $\delta_{\nu} \ll L$ and $\tau_{w} \ll L$. Taking into account only terms of order $\mathcal{O}(1)$ and returning back to dimensional variables, we obtain

$$
\begin{aligned}
\frac{d P}{d x}-\frac{\partial}{\partial y}\left(\left(\nu+\nu_{t}\right) \frac{\partial U}{\partial y}\right) & =-\beta \Theta \boldsymbol{g} \cdot \boldsymbol{e}_{x} \\
-\frac{\partial}{\partial y}\left(\left(a+a_{t}\right) \frac{\partial \Theta}{\partial y}\right) & =\dot{q}^{V} c_{p}^{-1} .
\end{aligned}
$$

Now we assume that $\dot{q}^{V}$ vanishes in the boundary layer. The remaining difficulty arises from the pressure gradient. As an immediate consequence of Bernoulli's equation $\left(-d P / d x_{i}=\right.$ $\left.\sum_{j=1}^{3} U_{j} \partial U_{i} \partial x_{j}\right)$, decelerating flow $\left(d U_{\infty} / d x<0\right)$ corresponds to a positive, so-called $a d-$ verse pressure gradient, which can lead to separation of the boundary layer from the surface. But also in attached boundary-layer flows the effect of a pressure gradient on the mean flow and on the Reynolds stresses can be significant. This is described in more details e.g. in [DPR01], pp.66. Moreover, pressure gradients might affect the value of $\operatorname{Pr}_{T}$, see [KC.93], although such effects are small. Nevertheless, in this thesis we restrict ourselves to zero pressure gradient boundary layers. For our purposes this approximation is reasonable since in indoor-air flow problems, buoyance is the driving force of the air movement and pressure gradients are hopefully small. (However, in natural convection in a closed cavity, adverse pressure gradients occur as the flow approaches the corners, see [TODB.98], p.290.) Then (5.25)-(5.26) reduce to the following coupled system of ordinary differential equations:

$$
\begin{aligned}
& -\frac{d}{d y}\left(\left(\nu+\nu_{t}\right) \frac{d U}{d y}\right)=-\beta \Theta \boldsymbol{g} \cdot \boldsymbol{e}_{x}, \\
& -\frac{d}{d y}\left(\left(a+a_{t}\right) \frac{d \Theta}{d y}\right)=0 .
\end{aligned}
$$

It is worthwhile pointing out that in the case $\beta \Theta \boldsymbol{g} \cdot \boldsymbol{e}_{x}=0, \dot{q}^{V}=0$, and $d P / d x=0$, momentum and heat transfer equation look analogously. As shown in Section C.2, when suitably normalized, both have the same profile, an observation called Reynolds analogy.

\subsection{Algebraic turbulence models for non-isothermal boundary layers}

\subsubsection{The substructure of a forced convection turbulent boundary layer}

The total stress is the sum of the viscous stress $\nu \nabla \boldsymbol{U}$ and the Reynolds stress $-\left\langle\boldsymbol{u}^{\prime} \otimes \boldsymbol{u}^{\prime}\right\rangle$. The fundamental observation is that in attached boundary layers the profiles of the viscous and Reynolds stresses are universal, i.e., for different Reynolds numbers their normalized profiles collapse when they are plotted against $y^{+}$. On the basis of the relative magnitude of the stresses and motivated by the profile for $U$ several regions can be distinguished (cf. [Pope00], p. 275), see Table 5.7.

In the literature, the near-wall edge of the $\log$ layer varies from $y^{+}=40$ (Durbin) to $y^{+}=50$ (Pope). An understanding of the names "log-law" and "law of the wake" will 


\begin{tabular}{l|l|l} 
Region & Location & Characteristic property \\
\hline \hline $\begin{array}{l}\text { Near-wall region } \\
\text { (viscous wall region) }\end{array}$ & $y^{+}<40$ & $\begin{array}{l}\text { The viscous contribution to the shear stress } \\
\text { is significant }\end{array}$ \\
\hline Viscous sublayer & $y^{+}<5$ & $\begin{array}{l}\text { The viscous stress highly dominates } \\
\text { the Reynolds shear stress }\end{array}$ \\
\hline Buffer layer & $5<y^{+}<40$ & $\begin{array}{l}\text { large production, large Reynolds stress } \\
\text { anisotropy }\end{array}$ \\
\hline Outer layer & $y^{+}>40$ & $\begin{array}{l}\text { Direct effects of the viscous stresses on } U \\
\text { are negligible }\end{array}$ \\
\hline Log-law region & $y^{+}>40, y / \delta<0.2$ & The log-law holds \\
\hline Defect layer & $0.2<y / \delta<1.0$ & The law of the wake holds \\
\hline
\end{tabular}

Figure 5.7.: Location and defining properties of the near-wall regions and layers.

be postponed to Subsection 5.3.3. A computational method should be able to identify the boundary layer and to distinguish between its subregions. In PARALLELNS the thickness of the boundary layer $\delta_{\text {layer }}$ is estimated before the calculation when the mesh for the numerical computation is chosen. This estimate is checked during the calculation. The sublayers can be identified within the numerical solution process. For this purpose, recall that if using the eddy-viscosity hypothesis, the turbulent viscosity $\nu_{t}$ should be a measure for the magnitude of the Reynolds stresses. Thus, if we have an appropriate model for $\nu_{t}$ in the boundary layer, on the basis of $\nu_{t}$ we can distinguish between its subregions. For this purpose, we review algebraic turbulence models for the boundary layer. Algebraic models are the simplest turbulence models, calculating $\nu_{t}$ from an algebraic expression.

\subsubsection{Algebraic models based on Prandtl's mixing-length hypothesis}

In 1925, PRANDTL proposed his famous mixing-length hypothesis as a relation for $\nu_{t}$ for an isothermal turbulent boundary layer. It is a reasonable model in the region $40 \delta_{\nu} \leq$ $y \leq 0.2 \delta_{\text {layer }}$, later referred to as the log-layer. A quite heuristic derivation of this model will be given in the sequel, cf. [Pope00], p. 289. Therefore we consider a two dimensional boundary-layer flow with $\boldsymbol{U}=U(y) \boldsymbol{e}_{1}$. Then the turbulent-viscosity hypothesis reduces to

$$
\left\langle u^{\prime} v^{\prime}\right\rangle_{E}=-\nu_{t} \frac{d U}{d y}
$$

(3.11) motivates that $\nu_{t}$ is the product of a velocity scale $u^{*}$ and a lengthscale $l_{m}$, viz., $\nu_{t}=u^{*} l_{m}$. Choosing $u^{*}=\left|\left\langle u^{\prime} v^{\prime}\right\rangle_{E}\right|^{1 / 2}$ and substituting this into (5.29) gives

$$
u^{*}=l_{m}\left|\frac{d U}{d y}\right| .
$$

Measurements and DNS data show that in the log-law region (i) $\left\langle u^{\prime} v^{\prime}\right\rangle_{E}$ is approximately constant, viz., $\left|\left\langle u^{\prime} v^{\prime}\right\rangle_{E}\right|^{1 / 2} \approx u_{\tau}$ and (ii) $d U / d y=u_{\tau} /(\kappa y)$. Inserting these two semiempirical relations into (5.30) gives Prandtl's mixing-length hypothesis :

$$
\nu_{t}=u^{*} l_{m}, \quad \text { with } \quad l_{m}=\kappa y, \quad \text { and } \quad u^{*}=l_{m}\left|\frac{d U}{d y}\right|=u_{\tau} .
$$




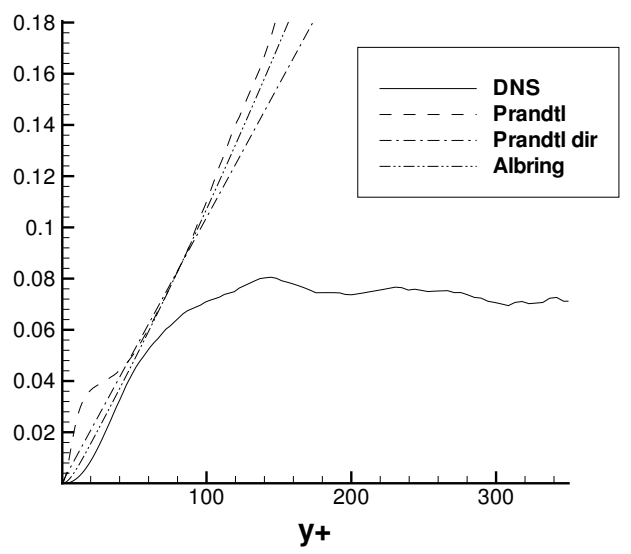

Figure 5.8.: $\nu_{t}$ for simple algebraic models.

Note that (5.31) can be written as

$$
\nu_{t}=u_{\tau} \kappa y
$$

Figure 5.8 provides some illustration. However, this sketch needs some explanation. The solid line shows the profile of $\nu_{t}$ obtained from DNS data for a turbulent channel flow at $R e_{\tau}=395$ : Given DNS data for $U$ and $\left\langle u^{\prime} v^{\prime}\right\rangle_{E}, \nu_{t}$ can be computed numerically using formula (5.29). Secondly, given DNS data for $U$ we can calculate $\nu_{t}$ from Prandtl's relation $\nu_{t}=(\kappa y)^{2} d U / d y$ (dashed line). Next, we can plot $\nu_{t}$ from (5.32) (dot-dashed line). Note that the difference between the second and the third profile is that the latter explicitely uses assumption (ii) whereas the former does not. Finally, from the DNS data for $U$ we can plot $\nu_{t}$ from ALBRING's proposal (5.38) (dot-dot-dashed line), which is covered in the next section. Apparently, $\nu_{t}$ is modelled reasonably in the log-layer $\left(40<y^{+}<80=0.2 \delta\right)$. However, in applications it may appear that we need an extension of our model for $\nu_{t}(i)$ to the near-wall region $y^{+}<40$ or (ii) to the region $0.2<y / \delta<1.0$. An example is the wall function concept presented in the next chapter: An intrinsic objective therein is to estimate the velocity at a certain distance $y_{P}$ from the wall in the boundary layer (to be more precise: at the first node above the wall). On the one hand, when the Reynolds number is large, we only can afford $0.2<y_{P} / \delta<1.0$. On the other hand, in simple test cases we are also interested in the model's behaviour if $y_{P} \nu / u_{\tau}<40$, see also (5.17).

Concerning (i) VAN DRIEST proposed to multiply $l_{m}$ with a suitable damping function $\mathcal{D}\left(y^{+}\right)=1-\exp \left(-y^{+} / A^{+}\right)$, known as the van Driest damping function, viz.,

$$
l_{m}=\delta_{\nu} \kappa y^{+} \mathcal{D}\left(y^{+}\right), \quad \mathcal{D}\left(y^{+}\right)=1-\exp \left(-y^{+} / A^{+}\right), \quad A^{+}=26 .
$$

Then $\nu_{t}$ is effectively multiplied with $\left(\mathcal{D}\left(y^{+}\right)\right)^{2}$. Some heuristic physical support for this modification stems from the fact that the no-penetration condition $v^{\prime}=0$ and the continuity equation imply that asymptotically $u^{\prime} \sim y, v^{\prime} \sim y^{2}$ near the wall and hence $\left\langle u^{\prime} v^{\prime}\right\rangle_{E} \sim y^{3}$. But (5.31) predicts $\left\langle u^{\prime} v^{\prime}\right\rangle_{E} \sim y^{2}$ and thus has to be damped. (5.33) gives $\left\langle u^{\prime} v^{\prime}\right\rangle_{E} \sim y^{4}$ (see [Pope00], Exercise 7.19), which is in much better agreement with DNS data. 
The deviation in the defect layer is much more severe. Figure 5.8 reveals that a constant eddy viscosity is a much better approximation. One modification to accomplish this is to choose $\nu_{t}=0.2 \kappa u_{\tau} d_{99}(y>0.2 \delta)$ (cf. [DPR01], p.116) as originated by CLAUSER (see [Wilcox98], p.73). Alternatively, EsCUDIER proposed to limit $l_{m}$ by setting $l_{m}=$ $\min \left\{\kappa y, 0.09 d_{99}\right\}$. Note that Escudiers modification is quite simple and can be included in both Prandtl's and Albring's model immediately once $d_{99}$ can be estimated. An additional improvement for approaching the freestream from within the boundary layer can be achieved by multiplying $\nu_{t}$ by a so-called intermittency factor $\left[1+5.5\left(0.3 y / d_{99}\right)^{6}\right]^{-1}$, see [DPR01], p.117 or [Wilcox98], pp. 73.

\section{Remark 5.3}

Concerning the isothermal case, a survey of more advanced algebraic turbulence models can be found in [Wilcox.98], Chapters 3.4-3.8.

Nevertheless, one should be aware that all these modifications have been conceived for isothermal boundary layers.

\subsubsection{Forced convection solution in the viscous sublayer and in the log layer}

In this subsection we elaborate on the mean velocity profiles in forced convection. Then $U$ is the solution of the boundary-layer equation (5.27) with zero right hand side. Substituting (5.31) into (5.27) simple integration yields the famous log law (see C.3)

$$
u^{+}\left(y^{+}\right)= \begin{cases}y^{+}, & \text {if } y^{+} \leq 11.06 \\ \frac{1}{\kappa} \ln \left(y^{+}\right)+B, & \text { if } y^{+}>11.06, \quad \text { with } \quad \kappa=0.41, \quad B=5.2\end{cases}
$$

The name log-layer originates from the logarithmic profile of the velocity in that layer. From the definition of $y^{+}$and (5.34), it can be checked during the calculation whether a point with distance $y^{+}$to the wall is located in the viscous sublayer or in the log layer. Another well-established solution is given by REICHARDT'S law

$$
u^{+}=\frac{1}{\kappa} \ln \left(1+0.4 y^{+}\right)+7.8\left[1-\exp \left(-\frac{y^{+}}{11}\right)-\frac{y^{+}}{11} \exp \left(-0.33 y^{+}\right)\right] .
$$

Since the simple algebraic models for $\nu_{t}$ fail in the defect layer, it is evident that the predicted profiles for $U$ deviate from the true profiles. For the boundary layer, in good agreement with experimental data is the modified log-law, cf. [Dea76], viz.,

$$
u^{+}=\frac{1}{\kappa} \ln \left(y^{+}\right)+B+\frac{\Pi}{\kappa} w\left(\frac{y}{d_{99}}\right), \quad w\left(\frac{y}{d_{99}}\right)=2 \sin ^{2}\left(\frac{\pi}{2} \frac{y}{d_{99}}\right)
$$

with $\Pi \approx 0.4757$ for a zero pressure gradient boundary layer. The term $w(\cdot)$ in $(5.36)$ is called wake contribution.

Figure 5.9 visualises the profiles. Apparently the deviation of (5.34), (5.35) and (5.41) from the log-wake law in the defect layer is significant. 

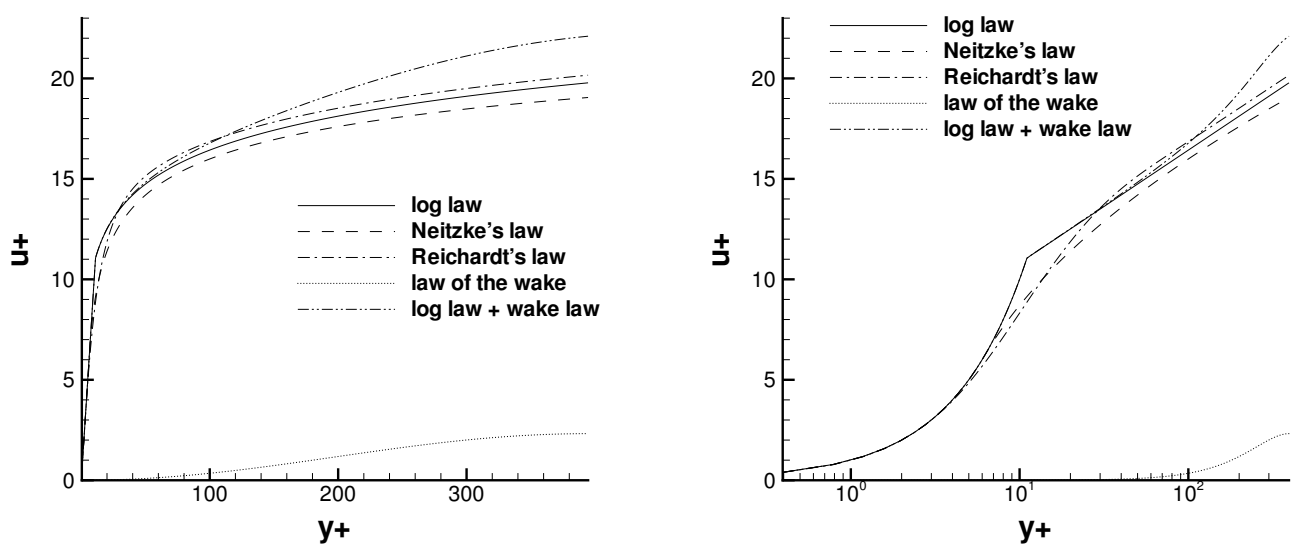

Figure 5.9.: Typical velocity profile in a boundary layer.

\subsection{Algebraic turbulence models for natural convection boundary layers}

\subsubsection{The substructure of a natural convection turbulent boundary layer}

Appropriate experimental data for natural convection turbulent boundary layers are by far less abundant than for the forced convection case. George and CAPP, see [G(779], suggested a two-layer structure: an inner layer and an outer layer are separated by the location of velocity maximum denoted by $y_{\max }$, see Figure 5.3. The inner layer can be divided into a viscous layer next to the wall and a buoyant sublayer. In the viscous sublayer the turbulent fluxes and stresses are negligible small. Therefore this sublayer is also referred to as conductive layer. If $R a$ is sufficiently large, then the logarithmic profile is a reasonable approximation for velocity and temperature, cf. [Nei99]. However, as reported in [TK00a], in their low turbulence natural convection test case $\left(R a=1.58 \times 10^{9}\right)$, the solution does not exhibit a logarithmic region, because $R a$ is not large enough. Finally it should be recalled that density gradients alter the near-wall turbulence compared to the forced convection case, recall Subsection 3.3.2. However, this is still an open problem.

\subsubsection{An algebraic model for boundary layers in buoyancy driven flows}

In buoyancy driven flows, the wall-parallel mean velocity $U$ has a well-defined maximum at wall distance $y_{\max }$ in the boundary layer. Then at $y=y_{\max }$ we get $|d U / d y|=0$ but measurements show $\nu_{t}$ is far from zero there. Hence for buoyance driven flows (5.31)-(5.32) are not an appropriate model for $\nu_{t}$ at least for $y \geq y_{\max }$. Following Neitzke, cf. [Nei99], and using the following scaled variables

$$
y^{+} \equiv \frac{y u_{\tau}}{\nu}, \quad u^{+} \equiv \frac{U}{u_{\tau}}, \quad \theta^{+} \equiv \frac{c_{p} u_{\tau}\left(\Theta_{w}-\Theta\right)}{\dot{q}}
$$


our research group uses the following choice for $\nu_{t}$ suggested by AlBRING, cf. [Alb81]:

$$
\begin{aligned}
\nu+\nu_{t} & =\nu \max \left(1, \frac{R e}{R e_{\min }}\right), \\
a+a_{t} & =\frac{\nu}{P r} \max \left(1, \frac{R e}{R e_{\min }} \frac{P r}{P r_{t}}\right)
\end{aligned}
$$

with $R e=U(y) y \nu^{-1}$, and $R e_{\text {min }}$ being a parameter which has to be determined experimentally. In particular, $R e_{\text {min }}$ depends on $R e$ and on the stratification, see below. Neitzke proposes $\sqrt{R e_{\min }}=6.25$ in neutral stratified boundary layers. We use $\operatorname{Pr}=0.70$ for air. For the non-universal constant $P r_{t}$ we choose $P r_{t}=1.15$ in the boundary layer, which is in agreement with latest papers, cf. [PSOI], Section 3.3. It can be seen from Figure 5.8 that Albring's model exhibits the same deficiencies as Prandtl's model in the isothermal case.

\section{Remark 5.4}

In our research group (5.38)-(5.39) is also used in the case of mixed convection.

As described in Section 3.3.2, the Richardson number is a measure for the stabilising effect of stratification. If $R i_{f}>0$, then turbulence is suppressed; if $R i_{f}<0$, then turbulence is enhanced. In order to account for this effect in boundary layer flows, NEITZKE proposed [Nei99], p.51, for each $\boldsymbol{x} \in \Gamma_{W}$ :

$$
R e_{\min }=R e_{m i n, n} e^{\chi K_{s} R i_{g}}, \text { with } R i_{g} \equiv-\boldsymbol{g} \cdot \boldsymbol{n} \frac{1}{\Theta} \nabla \Theta \cdot \boldsymbol{n}\|\mathbb{S}(\boldsymbol{U})\|_{F}^{-2}, \quad \sqrt{R e_{\min , n}}=6.25 .
$$

Here $\boldsymbol{g}$ is the gravitational acceleration, $\boldsymbol{n}$ is the outer normal vector to $\boldsymbol{x} \in \Gamma_{W}$, and $\chi \in\{0,1\} . R i_{g}$ is called gradient Richardson number. Note that $R i_{f}$ is based on the turbulent fluxes; substituting (3.17) and (3.26) into (3.19) gives $R i_{g}$ which is based on gradient-diffusion and eddy-viscosity hypotheses. If $R i_{g}<0$ then $R e_{\min }<R e_{\min , n}$ and thus $\nu_{t}$, i.e., turbulence, is enhanced; if $R i_{g}>0$ then $\nu_{t}$ is suppressed. Based on numerical tests, NeItZKe suggests $K_{s}=20.0$, see [Nei.9.], Subsection 5.2.4. In agreement with new results from [Sei03] and due to own tests we suggest $K_{s}=25.0, R e_{\min }=20.0$. We use a cut-off technique to ensure that $R e_{\min } \in\left[R e_{\min , c u t}, 70.0\right]$ with $R e_{\min , c u t}=12.0$. Note that $R i_{g}$ can be calculated directly from surface transfer coefficients, viz.,

$$
R i_{g}=g \frac{1}{\Theta} \frac{\partial \Theta}{\partial y}\left(\frac{\partial U}{\partial y}\right)^{-2}=\frac{g \dot{q} P r \nu}{\tau_{w}^{2} c_{p} \Theta}, \quad \text { with } \quad \nu \frac{\partial U}{\partial y}=\tau_{w} \text { and } \quad a \frac{\partial \Theta}{\partial y}=\frac{\dot{q}}{c_{p}}
$$

\subsubsection{Non-isothermal forced convection solution in the sublayer and in the log layer}

Neitzke's proposal (5.38)-(5.39) gives reasonable results also for the forced convection case, see [Nei.99], Section 5.2.1. This is quite important because in practical indoor-air flow problems mixed free and forced convection occurs. Substituting (5.38)-(5.39) into (5.27) 
and (5.28), and integration gives (see C.4)

$$
\begin{aligned}
& u^{+}\left(y^{+}\right)=\left\{\begin{array}{ll}
y^{+}, & \text {if } y^{+} \leq R_{u} \\
R_{u}\left(2 \ln \left(\frac{y^{+}}{R_{u}}\right)+1\right)^{\frac{1}{2}}, & \text { if } y^{+}>R_{u}
\end{array},\right. \\
& \theta^{+}\left(y^{+}\right)=\left\{\begin{array}{ll}
\operatorname{Pr} y^{+}, & \text {if } y^{+} \leq R_{\theta} \\
C_{\theta}\left(2 \ln \left(\frac{y^{+}}{R_{\theta}}\right)+1\right)^{\frac{1}{2},}, & \text { if } y^{+}>R_{\theta}
\end{array},\right.
\end{aligned}
$$

with constants $R_{u}=6.25, \operatorname{Pr}=0.70, R_{\theta}=8.0, C_{\theta}=\operatorname{Pr} R_{\theta}$. From (5.38)-(5.39) resp. from (5.41)-(5.42) it can be checked during the calculation whether a point with distance $y^{+}$to the wall is located in the viscous sublayer or in the log layer.

Substituting (5.31) and $P r_{t}=\nu_{t} / a_{t}$ into (5.28) gives the standard log-law for the temperature for non-isothermal forced convection flows, see C.3. As pointed out in [KC.93], p.274, a streamwise pressure gradient alters the profile in the logarithmic layer significantly, whilst it effects the velocity profile only in the wake region. This underligns the importance of a negligible streamwise pressure gradient.

\subsubsection{Free convection solution in the viscous sublayer and in the log layer}

In the case of natural convection, we have to solve (5.27)-(5.28) using (5.38)-(5.39). It can be confirmed that the analytic solution in the conductive layer is given by

$$
U(y)=\frac{\tau_{w}}{\nu} y-\frac{1}{2} \beta g_{x} \frac{\theta_{w}}{\nu} y^{2}-\frac{\beta g_{x}}{3 \nu a} \frac{\dot{q}}{c_{p}} y^{3}, \quad \Theta(y)=\theta_{w}+\frac{\dot{q}}{a c_{p}} y
$$

with $g_{x}=\boldsymbol{g} \cdot \boldsymbol{e}_{x}$. This theoretical result is supported by the experimental study in [AK02]. The solution procedure in the log-layer has to be accomplished numerically. The wall iteration procedure by Neitzke provides the opportunity to obtain reasonable values for wall surface parameters, even if the first near-wall grid point resides beyond the location of the velocity maximum, see [Nei99], Chapter 5.

Finally, it should be mentioned that experimental data for the Reynolds stresses and for the heat flux vector can be used for testing existing models for $\nu_{t}$ and $a_{t}$ in natural convection problems. Profiles for $\nu_{t}, a_{t}$ and $P r_{t}$, computed from mean flow and temperature gradients and the corresponding fluxes, are shown in [AK02], Figure 11. However, the available experimental data concerning this problem are still very limited.

\subsection{On the near-wall behaviour of the $k / \epsilon$ model}

Using the perturbation techniques described in the previous section, in the log layer, the isothermal RANS equations with the $k / \epsilon$ model, can be written as

$$
\frac{d}{d y}\left(\nu_{t} \frac{d U}{d y}\right)=0
$$




$$
\begin{aligned}
\nu_{t}\left(\frac{d U}{d y}\right)^{2}-\epsilon+\frac{d}{d y}\left(\frac{\nu_{t}}{P r_{k}} \frac{d k}{d y}\right) & =0, \\
C_{1} C_{\mu} k\left(\frac{d U}{d y}\right)^{2}-C_{2} \frac{\epsilon^{2}}{k}+\frac{d}{d y}\left(\frac{\nu_{t}}{P r_{\epsilon}} \frac{d \epsilon}{d y}\right) & =0, \\
\nu_{t} & =C_{\mu} \frac{k^{2}}{\epsilon},
\end{aligned}
$$

cf. Wilcox eq.(4.126) and eq.(4.132). The solution of (5.44)-(5.47) is, cf.[Wilcox.98] eq.(4.133)

$$
U=\frac{u_{\tau}}{\kappa} \ln y+\text { constant }, \quad k=\frac{u_{\tau}^{2}}{\sqrt{C_{\mu}}}, \quad \epsilon=\frac{u_{\tau}^{3}}{\kappa y} .
$$

Based on experimentell results YUAN ET AL. proposed the following modifications:

$$
k^{+} \equiv \frac{k}{u_{\tau}^{2}}=\min \left(\frac{1}{\sqrt{C_{\mu}}} ; 0.05\left(y^{+}\right)^{2}\right), \quad \epsilon^{+} \equiv \frac{\epsilon \nu}{u_{\tau}^{4}}=\frac{0.1+0.003\left(y^{+}\right)^{2}}{1.0+0.00125\left(y^{+}\right)^{3}} .
$$

Obviously these formulae satisfy very close to the wall $k^{+} \sim\left(y^{+}\right)^{2}$, and $\epsilon=\mathcal{O}(1)$, cf. [Pope00], pp. 286., [DPR0T], p.169. Hence (5.49) can be used also in the near-wall region. However, (5.49) becomes erroneous in the defect layer. By definition $2 k=\left\langle u^{\prime 2}\right\rangle_{E}+\left\langle v^{\prime 2}\right\rangle_{E}+$ $\left\langle w^{\prime 2}\right\rangle_{E}$. Figure 5.2 reveals that $k$ ceases when approaching the freestream region in contrast to $(5.49)$.

\subsection{On LES in the near-wall region}

In order to apply LES to the near-wall region, it is essential bearing in mind the physics of turbulence there. Eddies of size $\mathcal{O}\left(\delta_{\nu}\right)$ are subject to fundamental high energetic structures processes and events (e.g. streaks, sweeps and ejections). Moreover the maximum of production of turbulent kinetic energy is located in the buffer layer at $y^{+} \approx 12$, which gives rise to a significant backward energy cascade, cf. [Sag01], p.215. For further details see [Sag01], Section 9.2.1, [DBP01], p.208 and [Pope00], Section 7.4. Regarding turbulent structures in buoyancy driven flows see e.g. [PD(01], Figure 6.

To resolve these very small eddies near the wall, a very fine filter width is necessary. In the wall-normal direction a filter width of $\Delta y \approx \delta_{\nu}$ is recommended, see [Sag01], p.217, [PC.96]. As turbulent flow structures in the free-stream are much larger, it would be desirable to use filters with non-constant filter width. On the problems of non-constant filter width and the commutation error between filtering and differentiation see e.g. [MV02].

Besides the problem of non-constant filter width, there are two major model problems. First, for each $\Delta>0$ the no-slip condition $\boldsymbol{u}=0$ on $\Gamma_{W}$ does not imply $\overline{\boldsymbol{u}}=0$ on $\Gamma_{W}$. Thus a no-slip condition for $\overline{\boldsymbol{u}}$ is only an approximation. A second problem is that classical residual stress models cannot predict backscattering effects accurately, cf. [VGK.97]. Moreover, a correct near-wall scaling of the SGS model is important. Finally, even with today's supercomputers, the computational costs are a major obstacle; LES with near-wall resolution for high Reynolds/Rayleigh-number turbulent flows is still infeasible. 


\section{A computational $k / \epsilon$ model using wall functions}

In this chapter we develop a computational $k / \epsilon$ model for wall-bounded flows, the term "computational" indicating its applicability in a CFD code. For this purpose we combine results from Section 1.4, and Chapters 3 and 5. The $k / \epsilon$ model, introduced in Chapter 3, is a successful model except in a zone adjacent to the wall. In Chapter 5 a much simpler model for boundary-layer flows was reviewed. Now the underlying idea is to couple the global $k / \epsilon$ model with the local boundary-layer model using a fully overlapping DDM by applying the scheme (1.47)-(1.51). This approach is often referred to as wall function procedure.

\subsection{A two-domain approach}

In the previous section we divided the flow region $\Omega$ into the boundary layer $\Omega_{\text {layer }}$ and the free-stream region $\Omega_{\text {free }}$. From a computational point of view, this partitioning is not propitious. As explained in the previous chapter, the $k / \epsilon$ model yields reasonable predictions not only in $\Omega_{\text {free }}$ but also in the log layer, being a subset of $\Omega_{\text {layer }}$. Merely in the sublayer and in the buffer layer the predictions are erroneous. Thus we divide $\Omega$ into $\Omega_{\delta}$ and $\Omega_{\text {outer }}$, being defined as

$$
\Omega_{\delta} \equiv\left\{\boldsymbol{x} \in \Omega \mid \operatorname{dist}\left(\boldsymbol{x}, \Gamma_{W}\right) \leq y_{\delta}\right\}, \quad \Omega_{\text {outer }} \equiv\left\{\boldsymbol{x} \in \Omega \mid \operatorname{dist}\left(\boldsymbol{x}, \Gamma_{W}\right)>y_{\delta}\right\}
$$

Here we have to introduce an artificial curve $\Gamma_{\delta}$, see Figure 6.1. For each $\boldsymbol{x} \in \Gamma_{W}$ denote $y_{\delta} \equiv y_{\delta}(\boldsymbol{x}) \equiv \operatorname{dist}\left(\boldsymbol{x}, \Gamma_{\delta}\right)$. We require that $\Gamma_{\delta}$ is located at most in the log-layer. Thus $\Gamma_{\delta}$ is the curve where the $k / \epsilon$ model prediction for the flow field farther from the surface and a suitable near-wall solution are patched. The idea is to use two different models for $\nu_{t}$ in $\Omega_{\delta}$ and $\Omega_{\text {outer }}$. We start with the following model, later referred to as $k / \epsilon$ MODEL I.

\section{Computational $k / \epsilon$ turbulence model I}

- Non-isothermal RANS equations

$$
\begin{aligned}
\partial_{t} \boldsymbol{U}-\boldsymbol{\nabla} \cdot\left(2 \nu_{e} \mathbb{S}(\boldsymbol{U})\right)+(\boldsymbol{U} \cdot \boldsymbol{\nabla}) \boldsymbol{U}+\boldsymbol{\nabla} p & =-\beta \Theta \boldsymbol{g} & & \text { in } \Omega, \\
\boldsymbol{\nabla} \cdot \boldsymbol{U} & =0 & & \text { in } \Omega, \\
\partial_{t} \Theta+(\boldsymbol{U} \cdot \boldsymbol{\nabla}) \Theta-\boldsymbol{\nabla} \cdot\left(a_{e} \boldsymbol{\nabla} \Theta\right) & =\dot{q}^{V} c_{p}^{-1} & & \text { in } \Omega .
\end{aligned}
$$

- Eddy viscosity model

- In $\Omega_{\text {outer }}: \nu_{t}=C_{\mu} k^{2} \epsilon^{-1}, a_{t}=P r_{t}^{-1} \nu_{t}$, where $k$ and $\epsilon$ are the solution of

$$
\begin{aligned}
\partial_{t} k+(\boldsymbol{U} \cdot \boldsymbol{\nabla}) k-\boldsymbol{\nabla} \cdot\left(\nu_{k} \boldsymbol{\nabla} k\right) & =P_{k}+G-\epsilon & & \text { in } \Omega_{\text {outer }}, \\
\partial_{t} \epsilon+(\boldsymbol{U} \cdot \boldsymbol{\nabla}) \epsilon-\boldsymbol{\nabla} \cdot\left(\nu_{\epsilon} \boldsymbol{\nabla} \epsilon\right)+C_{2} \epsilon^{2} k^{-1} & =C_{1} \epsilon k^{-1}\left(P_{k}+G\right) & & \text { in } \Omega_{\text {outer }} .
\end{aligned}
$$




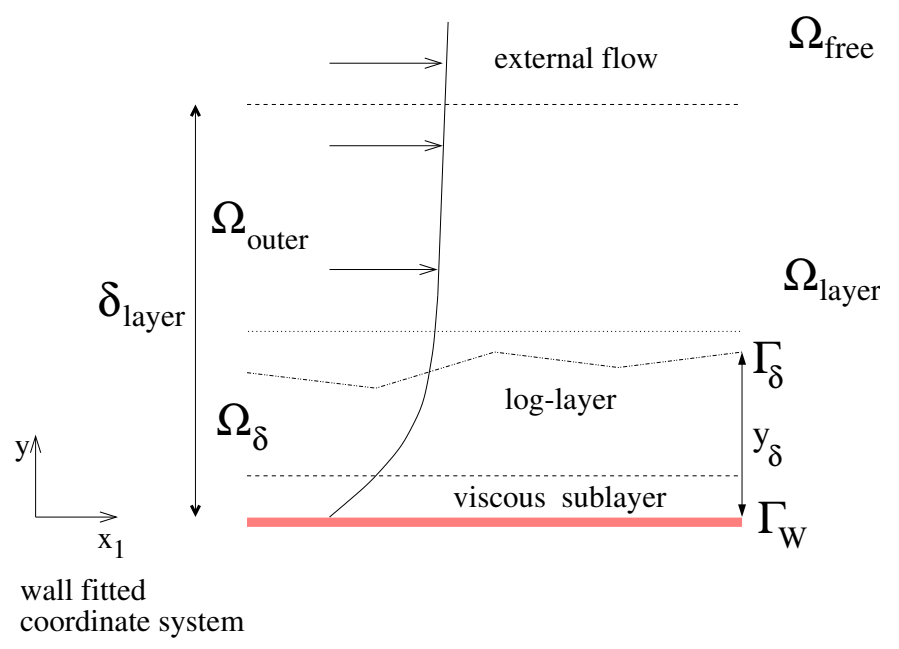

Figure 6.1.: Domain decomposition for wall function procedure.

- In $\Omega_{\delta}: \nu_{t}$ and $a_{t}$ are given by (5.38) and (5.39) resp.

\section{- Boundary conditions}

$$
\begin{gathered}
\boldsymbol{U}=\boldsymbol{U}_{i n} \text { on } \Gamma_{F}, \quad \boldsymbol{U}=\mathbf{0} \text { on } \Gamma_{W}, \quad \sigma(\boldsymbol{U}, P) \cdot \boldsymbol{n}=\mathbf{0} \text { on } \Gamma_{N}, \\
\Theta=\Theta_{i n} \text { on } \Gamma_{-}(\boldsymbol{U}), \quad \Theta=\Theta_{w} \text { on } \Gamma_{W}, \quad a \nabla \Theta \cdot \boldsymbol{n}=0 \text { on } \Gamma_{+}(\boldsymbol{U}), \\
k=1.5\left(T_{u}\|\boldsymbol{U}\|\right)^{2} \text { on } \Gamma_{-}(\boldsymbol{U}), \quad k=C_{\mu}^{-1 / 2} U_{*}^{2} \text { on } \Gamma_{\delta}, \quad \nu_{k} \boldsymbol{\nabla} k \cdot \boldsymbol{n}=0 \text { on } \Gamma_{+}(\boldsymbol{U}), \\
\epsilon=C_{\mu}^{3 / 4} k^{3 / 2} L^{-1} \text { on } \Gamma_{-}(\boldsymbol{U}), \quad \epsilon=U_{*}^{3} /\left(\kappa y_{\delta}\right) \text { on } \Gamma_{\delta}, \quad \nu_{\epsilon} \boldsymbol{\nabla} \epsilon \cdot \boldsymbol{n}=0 \text { on } \Gamma_{+}(\boldsymbol{U}) .
\end{gathered}
$$

Here $\kappa=0.41, T_{u}$ denotes the degree of turbulence and $L$ is a length that is characteristic for this turbulence problem. If $\Gamma_{\delta}$ is partially located in the viscous sublayer, we use (5.49) on $\Gamma_{\delta}$ as boundary condition for $k$ and $\epsilon$.

- together with suitable initial conditions

Note that the boundary condition for $k$ and $\epsilon$ on $\Gamma_{\delta}$ is a consequence of (5.48) when substituting $y=y_{\delta}$. In practice, $\Gamma_{\delta}$ contains all mesh points with minimal positive distance to $\Gamma_{W}$ and the corresponding (d-1)-dimensional simplices from the finite element mesh. It has to be checked during the numerical solution process that $\Gamma_{\delta}$ is located at most in the log-layer.

\subsection{The wall function concept as a fully overlapping DDM}

It seems natural to apply a domain decomposition method (DDM) to this two-domain problem. The idea is to apply the approach from Section 1.4, i.e., a fully overlapping DDM. 
1. In the first step we compute a solution in $\Omega$. This solution has a good accuracy in $\Omega_{\text {outer }}$ and is therefore called the outer solution. But it might be not accurate enough in $\Omega_{\delta}$. For the outer solution at $\Gamma_{W}$ instead of a no-slip condition we impose a mixed boundary condition (1.13) with a traction vector $\boldsymbol{\sigma}_{t}$ that has to be determined during the iterative solution process. The traction vector $\boldsymbol{\sigma}_{t}$ tries to ensure the correct behaviour of the outer solution in the proximity of the wall.

2. In the second step we compute a solution in $\Omega_{\delta}$ by solving a boundary-layer problem in $\Omega_{\delta}$. The boundary-layer solution is often referred to as inner solution, as it is a good solution in the near-wall zone of the boundary layer.

3. In the third step we calculate a new guess for the traction vector such that the inner flow matches the outer flow on $\Gamma_{\delta}$.

First we introduce the following notation: Given $\eta: \Omega \rightarrow \mathbb{R}$ and $\boldsymbol{x} \in \Gamma_{W}$, we write

$$
\left.\eta\right|_{\Gamma_{\delta}} \equiv \eta\left(\boldsymbol{x}-y_{\delta}(\boldsymbol{x}) \boldsymbol{n}\right) .
$$

Thus for each $\boldsymbol{x}^{\prime}$ on $\Gamma_{\delta}$ with $\boldsymbol{x}^{\prime}=\boldsymbol{x}-y_{\delta} \boldsymbol{n}$ we have $\left.\eta\right|_{\Gamma_{\delta}} \equiv \eta\left(\boldsymbol{x}^{\prime}\right)$, which is the value of $\eta$ at the point $\boldsymbol{x}^{\prime}$ corresponding to $\boldsymbol{x} \in \Gamma_{W}$. This implicit relation between $\boldsymbol{x} \in \Gamma_{W}$ and $\boldsymbol{x}^{\prime} \in \Gamma_{\delta}$ will be used throughout this thesis. As an example, we introduce the projection of the velocity onto the plane tangent to the wall,

$$
\boldsymbol{U}_{\delta, t} \equiv \boldsymbol{U}_{\delta}-\left(\boldsymbol{U}_{\delta} \cdot \boldsymbol{n}\right) \boldsymbol{n}, \quad \text { with }\left.\quad \boldsymbol{U}_{\delta} \equiv \boldsymbol{U}\right|_{\Gamma_{\delta}} .
$$

Then we seek an outer solution $\boldsymbol{U}, P, \Theta, k, \epsilon$ in $\Omega \times(0, T)$, an inner solution $\boldsymbol{U}^{B L}, P^{B L}$, $\Theta^{B L}$ in $\Omega_{\delta} \times(0, T)$ (with $\Omega_{\delta} \subset \Omega$ ) and scalars $U_{*}, \dot{q}$ (these are the variables which shall ensure the matching) such that the following equations hold, referred to as $k / \epsilon$ MODEL II:

\section{Computational $k / \epsilon$ turbulence model II}

(1) Compute the outer solution.

- Non-isothermal RANS equations

$$
\begin{aligned}
\partial_{t} \boldsymbol{U}-\boldsymbol{\nabla} \cdot\left(2 \nu_{e} \mathbb{S}(\boldsymbol{U})\right)+(\boldsymbol{U} \cdot \boldsymbol{\nabla}) \boldsymbol{U}+\boldsymbol{\nabla} p=-\beta \Theta \boldsymbol{g} & \text { in } \Omega, \\
\boldsymbol{\nabla} \cdot \boldsymbol{U}=0 & \text { in } \Omega, \\
\partial_{t} \Theta+(\boldsymbol{U} \cdot \boldsymbol{\nabla}) \Theta-\boldsymbol{\nabla} \cdot\left(a_{e} \boldsymbol{\nabla} \Theta\right)=\dot{q}^{V} c_{p}^{-1} & \text { in } \Omega .
\end{aligned}
$$

- Eddy viscosity model: $\nu_{t}$ is given as in $k / \epsilon$ MODEL I.

- Boundary conditions: W.r.t. $k$ and $\epsilon$ see $k / \epsilon$ MODEL I. For $\boldsymbol{U}$ and $\Theta$ we prescribe

$$
\begin{aligned}
\Theta & =\Theta_{i n} \text { on } \Gamma_{-}(\boldsymbol{U}), \quad a \nabla \Theta \cdot \boldsymbol{n}=\dot{q} / c_{p} \text { on } \Gamma_{W}, \quad \nabla \Theta \cdot \boldsymbol{n}=0 \text { on } \Gamma_{+}(\boldsymbol{U}), \\
\boldsymbol{U} & =\boldsymbol{U}_{i n} \text { on } \Gamma_{F}, \quad \sigma(\boldsymbol{U}, P) \boldsymbol{n}=\mathbf{0} \text { on } \Gamma_{N},
\end{aligned}
$$

$$
\boldsymbol{U} \cdot \boldsymbol{n}=0, \quad \boldsymbol{n}^{T} \sigma(\boldsymbol{U}, p) \boldsymbol{t}_{j}=U_{*}{ }^{2} \frac{\boldsymbol{U}_{\delta, t} \cdot \boldsymbol{t}_{j}}{\left\|\boldsymbol{U}_{\delta, t}\right\|}(j=1, \ldots, d-1) \text { on } \Gamma_{W} .
$$


(2) Compute the inner solution and match outer and inner solution

- Non-isothermal RANS equations

$$
\begin{aligned}
\partial_{t} \boldsymbol{U}^{B L}-\boldsymbol{\nabla} \cdot\left(2 \nu_{e} \mathbb{S}\left(\boldsymbol{U}^{B L}\right)\right)+(\boldsymbol{U} \cdot \boldsymbol{\nabla}) \boldsymbol{U}^{B L}+\nabla p^{B L} & =-\beta \Theta^{B L} \boldsymbol{g} & & \text { in } \Omega_{\delta}, \\
\boldsymbol{\nabla} \cdot \boldsymbol{U}^{B L} & =0 & & \text { in } \Omega_{\delta}, \\
\partial_{t} \Theta^{B L}+\left(\boldsymbol{U}^{B L} \cdot \boldsymbol{\nabla}\right) \Theta^{B L}-\boldsymbol{\nabla} \cdot\left(a_{e} \boldsymbol{\nabla} \Theta^{B L}\right) & =\dot{q}^{V} c_{p}^{-1} & & \text { in } \Omega_{\delta} .
\end{aligned}
$$

- Eddy viscosity model : $\nu_{t}$ and $a_{t}$ are given by (5.38) and (5.39) resp.

- Boundary conditions

$$
\begin{aligned}
& \Theta^{B L}=\left.\Theta\right|_{\Gamma_{\delta}} \text { on } \Gamma_{\delta}, \quad \Theta^{B L}=\Theta_{w} \text { on } \Gamma_{W}, \\
& \boldsymbol{U}^{B L}=\left.\boldsymbol{U}\right|_{\Gamma_{\delta}} \text { on } \Gamma_{\delta}, \quad \boldsymbol{U}^{B L}=\mathbf{0} \text { on } \Gamma_{W} \text {. }
\end{aligned}
$$

Matching condition : $U_{*}$ and $\dot{q}$ are given by

$$
\begin{aligned}
\boldsymbol{n}^{T} \sigma\left(\boldsymbol{U}^{B L}, P^{B L}\right) \boldsymbol{t}_{j} & =U_{*}^{2} \frac{\boldsymbol{U}_{\delta, t} \cdot \boldsymbol{t}_{j}}{\left\|\boldsymbol{U}_{\delta, t}\right\|} \quad(j=1, \ldots, d-1) & & \text { on } \Gamma_{W} \\
a \nabla \Theta^{B L} \cdot \boldsymbol{n} & =\dot{q} / c_{p} & & \text { on } \Gamma_{W} .
\end{aligned}
$$

\section{Remark 6.1}

The close relation between $k / \epsilon$ MODEL II and (1.47)-(1.51) is obvious: $k / \epsilon$ MODEL II can be interpreted in the sense of a fully overlapping DDM as introduced in section 1.4. In $\Omega_{\delta}$ the function $\nu_{t}$ is given by (5.38) and (5.39) for both the outer flow and the inner problem. Thus, performing a DDM is originated in a natural manner by the use of two models for computing the turbulent viscosity $\nu_{t}$ in $\Omega_{\delta}$ and $\Omega_{\text {outer }}$.

\section{Remark 6.2}

Note that (6.4) assumes $\boldsymbol{\sigma}_{t}$ being aligned with $\boldsymbol{U}_{\delta, t}$. Moreover, obviously $U_{*}$ is an approximation to $u_{\tau}$. At a two-dimensional separation point $u_{\tau}=0$, see [DPR01], p.128.

\section{Remark 6.3}

A different promising strategy is a so-called hybrid RANS/RANS approach. Then $\Omega$ is divided into $\Omega_{\delta}$ and $\Omega_{\text {outer }}$. In $\Omega_{\delta}$ and $\Omega_{\text {outer }}$ different RANS are used. In contrast to the wall function approach, coupling both subdomains is accomplished at the interface $\Gamma_{\delta}$. For example, for massively separated flows using the SpalarT-Almaras model in $\Omega_{\delta}$ seems reasonable. MENTER proposed to couple the $k / \epsilon$ and the $k / \omega$ models using a blending function, see [Men.94].

\subsection{The wall function concept using boundary-layer theory}

The next step consists in simplifying the model in $\Omega_{\delta}$. In PARALlELNS we use the equations (5.27)-(5.28). Of course, more complex boundary-layer equations like (5.16) can be 
considered, which can be combined with more advanced models for $\nu_{t}$ in $\Omega_{\delta}$, see [CM00]. Thus we focus on the following model, referred to as $k / \epsilon$ MODEL III:

\section{Computational $k / \epsilon$ turbulence model III}

(1) Compute the outer solution: See $k / \epsilon$ MODEL II.

(2) Compute the inner solution and match outer and inner solution

- Non-isothermal RANS equations

$$
\begin{aligned}
-\frac{d}{d y}\left(\left(\nu+\nu_{t}\right) \frac{d U^{B L}}{d y}\right) & =-\beta \Theta^{B L} g_{x} & & \text { in }\left(0, y_{\delta}\right), \\
-\frac{d}{d y}\left(\left(\frac{\nu}{P r}+\frac{\nu_{t}}{P r_{t}}\right) \frac{d \Theta^{B L}}{d y}\right) & =0 & & \text { in }\left(0, y_{\delta}\right) .
\end{aligned}
$$

- Eddy viscosity model: See $k / \epsilon$ MODEL II.

- Boundary conditions:

$$
\begin{array}{ll}
\left.U^{B L}\right|_{y=0}=0, & \left.U^{B L}\right|_{y=y_{\delta}}=U\left(y_{\delta}\right) \equiv \frac{\boldsymbol{U}_{\delta} \cdot \boldsymbol{U}_{\delta, t}}{|| \boldsymbol{U}_{\delta, t}||}, \\
\left.\Theta^{B L}\right|_{y=0}=\Theta_{w}, & \left.\Theta^{B L}\right|_{y=y_{\delta}}=\left.\Theta\left(y_{\delta}\right) \equiv \Theta\right|_{\Gamma_{\delta}} .
\end{array}
$$

(3) Matching condition: $U_{*}$ and $\dot{q}$ are given by

$$
\left.\nu \frac{d U^{B L}}{d y}\right|_{y=0}=U_{*}^{2},\left.\quad a \frac{d \Theta^{B L}}{d y}\right|_{y=0}=\dot{q} / c_{p} \text { on } \Gamma_{W} .
$$

\section{Computational turbulence model IIla for flows significant buoyancy forces}

First we consider the case of significant buoyancy forces. Then for each $\boldsymbol{x} \in \Gamma_{W}$ we have to solve the boundary value problem (6.7)-(6.10) using a shooting method. Therefore for each $\boldsymbol{x} \in \Gamma_{W}$ consider the following initial value problem:

$$
\begin{aligned}
-\frac{d}{d y}\left(\left(\nu+\nu_{t}\right) \frac{d U^{B L}}{d y}\right) & =-\beta \Theta^{B L} g_{x} & & \text { in }\left(0, y_{\delta}\right), \\
-\frac{d}{d y}\left(\left(\frac{\nu}{P r}+\frac{\nu_{t}}{P r_{t}}\right) \frac{d \Theta^{B L}}{d y}\right) & =0 & & \text { in }\left(0, y_{\delta}\right)
\end{aligned}
$$

with the initial conditions

$$
\begin{aligned}
& U^{B L}(y=0)=0,\left.\quad \nu \frac{d U^{B L}}{d y}\right|_{y=0}=r, \\
& \Theta^{B L}(y=0)=\Theta_{w}, \quad a \frac{d \Theta^{B L}}{d y}(y=0)=s .
\end{aligned}
$$


where $(r, s)$ is a parameter in $\mathbb{R} \times \mathbb{R}$. For every given $(r, s)$ in $\mathbb{R} \times \mathbb{R}$ the initial value problem has a unique solution, see C.1. Obviously the solution of the initial value problem is a solution of the boundary value problem, if

$$
\left(\begin{array}{c}
U_{\text {diff }}(r, s) \\
\Theta_{\text {diff }}(r, s)
\end{array}\right)=\left(\begin{array}{c}
0 \\
0
\end{array}\right), \text { with }\left(\begin{array}{c}
U_{\text {diff }}(r, s) \\
\Theta_{\text {diff }}(r, s)
\end{array}\right) \equiv\left(\begin{array}{c}
U^{B L}\left(y_{\delta}\right) \\
\Theta^{B L}\left(y_{\delta}\right)
\end{array}\right)-\left(\begin{array}{c}
U\left(y_{\delta}\right) \\
\Theta\left(y_{\delta}\right)
\end{array}\right) .
$$

$\left(U_{\text {diff }}(r, s), \Theta_{\text {diff }}(r, s)\right)^{T}$ is a function from $\mathbb{R} \times \mathbb{R}$ onto $\mathbb{R} \times \mathbb{R}$. For each pair $(r, s)$ the corresponding value $U_{\text {diff }}(s, r), \Theta_{\text {diff }}(r, s)$ can be computed by solving an initial value problem, see C.1. Consequently we can seek the zero $\left(r_{0}, s_{0}\right)$ of $\left(U_{\text {diff }}, \Theta_{\text {diff }}\right)$ using Newton's method and set $U_{*}{ }^{2} \equiv r_{0}, \dot{q}=c_{p} s_{0}$. Whereas in practice this scheme shows a reasonable convergence behaviour, cf. Section [13.3, a proof for the well-posedness and the convergence of the Newton's method is still an open problem.

Then the $k / \epsilon$ MODEL IIIA reads : Find an outer solution $\boldsymbol{U}, P, \Theta, k, \epsilon$ and an inner solution $\boldsymbol{U}^{B L}, P^{B L}, \Theta^{B L}$ and scalars $U_{*}, \dot{q}$ such that

\section{Computational $k / \epsilon$ turbulence model IIIa}

(1) Compute the outer solution: See $k / \epsilon$ MODEL III.

(2) Compute the inner solution and match outer and inner solution

For each $\boldsymbol{x} \in \Gamma_{W}$ seek the zero $\left(r_{0}, s_{0}\right) \in \mathbb{R} \times \mathbb{R}$ of (6.16) (using Newton's method). Then $U_{*}$ and $\dot{q}$ are given by $U_{*}^{2} \equiv r_{0}, \dot{q}=c_{p} s_{0}$.

\section{Remark 6.4}

It should be mentioned that $k / \epsilon$ MODEL IIIA does not account for effects predicted by BVG theory (see Subsection 3.3.2), because $k / \epsilon$ MODEL IIIA uses a coarse turbulence model for the near-wall region. On the other hand, when using a more sophisticated near-wall turbulence model together with a near-wall resolution technique, numerical tests revealed that (3.27) gives significantly better results than (3.26), see [एODB.98].

\section{Computational turbulence model IIIb for flows with negligible buoyancy forces}

In the case of forced convection we can neglect the buoyancy term in (6.7) and (6.12) resp. Then (6.12)-(6.15) can be solved analytically. The solution depends on the model for $\nu_{t}$ and $a_{t}$. Using (5.38)-(5.39) yields the law of the wall derived by NeITZKe, see C.4. Using (5.31) yields the log law by PrandtL and van Karman, cf. C.3. The profile of the analytical solution depends solely on the parameters $U_{*}$ and $\dot{q}$. Thus $U_{*}$ and $\dot{q}$ can be determined by the requirement that the inner solution matches the corresponding outer solution at $y_{\delta}^{+} \equiv y_{\delta} U_{*} / \nu$, i.e.,

$$
u^{+}\left(y_{\delta}^{+}\right)=u_{\delta}^{+}, \theta^{+}\left(y_{\delta}^{+}\right)=\theta_{\delta}^{+}, \quad \text { with } u_{\delta}^{+} \equiv \frac{U\left(y_{\delta}\right)}{U_{*}}, \theta_{\delta}^{+} \equiv \frac{c_{p} U_{*}\left(\Theta_{W}-\Theta\left(y_{\delta}\right)\right)}{\dot{q}} .
$$

Therein $u^{+}\left(y_{\delta}^{+}\right)$and $\theta^{+}\left(y_{\delta}^{+}\right)$are the (scaled) values of the analytic inner solutions at $y_{\delta}^{+}$ and $u_{\delta}^{+}$and $\theta_{\delta}^{+}$are the (scaled) values of the outer solutions at $y_{\delta}^{+}$. Equation (6.17) can 
be used to compute $\left(U_{*}, \dot{q}\right)$. First, $U_{*}$ is determined by solving the first equation, as $\dot{q}$ does not occur in (5.41). After that $\dot{q}$ can be determined from an algebraic equation.

Then the model reads : Find an outer solution $\boldsymbol{U}, P, \Theta, k, \epsilon$ and scalars $U_{*}, \dot{q}$ such that the following equations hold, which will be referred to as $k / \epsilon$ MODEL IIIB

\section{Computational $k / \epsilon$ turbulence model IIIb}

(1) Compute the outer solution : See $k / \epsilon$ MODEL II.

(2) Compute wall shear stress and heat flux. For each $\boldsymbol{x} \in \Gamma_{W}$

(i) seek $U_{*}$ (using Newton's method) s.t.

$$
u_{\delta}^{+}=\left\{\begin{array}{ll}
y_{\delta}^{+}, & \text {if } y_{\delta}^{+} \leq R_{u} \\
R_{u}\left(2 \ln \left(\frac{y_{\delta}^{+}}{R_{u}}\right)+1\right)^{\frac{1}{2}}, & \text { if } y_{\delta}^{+}>R_{u}
\end{array},\right.
$$

with $u_{\delta}^{+}$given in (6.17), and (ii) given $U_{*}$ from step (i) determine $\dot{q}$ from

$$
\dot{q}=\frac{c_{p} U_{*}\left(\Theta_{W}-\Theta\left(y_{\delta}\right)\right)}{\theta^{+}\left(y_{\delta}^{+}\right)},
$$

with $\theta^{+}\left(y_{\delta}^{+}\right)$given by

$$
\theta^{+}\left(y_{\delta}^{+}\right) \equiv \begin{cases}\operatorname{Pr} y_{\delta}^{+}, & \text {if } y_{\delta}^{+} \leq R_{\Theta} \\ C_{\Theta}\left(2 \ln \left(\frac{y_{\delta}^{+}}{R_{\Theta}}\right)+1\right)^{\frac{1}{2}}, & \text { if } y_{\delta}^{+}>R_{\Theta}\end{cases}
$$

The wall function concept has been conceived for the isothermal case with $y_{\delta}$ located in the log-layer. In the sequel we will focus on this situation and give some mathematical results. Step (2) in model IIIb reads: Given $y_{\delta}$ and $U\left(y_{\delta}\right)$ seek $U_{*}$ s.t.

$$
f\left(U_{*}\right)=0, \quad f(x) \equiv R_{u} S(x)-\frac{U\left(y_{\delta}\right)}{x}, \quad S(x)=\sqrt{2 \ln \left(\frac{y_{\delta} x}{R_{u} \nu}\right)+1} .
$$

Obviously, $f$ is well defined on $D \equiv\left(e^{-1 / 2} R_{u} \nu y_{\delta}^{-1}, \infty\right)$. The function $f$ is twice continuously differentiable on $D$, with

$$
\frac{d f}{d x}=\frac{R_{u}}{S(x) x}+\frac{U\left(y_{\delta}\right)}{x^{2}}, \quad \frac{d^{2} f}{d x^{2}}=-\frac{R_{u}}{x^{2}}\left(\frac{1}{S(x)^{3}}+\frac{1}{S(x)}\right)-\frac{2 U\left(y_{\delta}\right)}{x^{3}} .
$$

Now we study the particular situation $\nu=0.0001, y_{\delta}=0.0625$ and $U\left(y_{\delta}\right)=0.505249$, which appears in a turbulent channel flow at $R e_{\tau}=395$. A plot of $f$ and $d f / d x$ for these values is given in Figures 6.2 and 6.3. Clearly, $f$ has a unique zero in $D$ and $d f / d x$ is strictly positive on $D$. Then Newton's method for the solution of the equation $f(x)=0$ is given by the iterative scheme (which is obviously well defined)

$$
x^{(n+1)}=x^{(n)}-\left[f^{\prime}\left(x^{(n)}\right)\right]^{-1} f\left(x^{(n)}\right)
$$




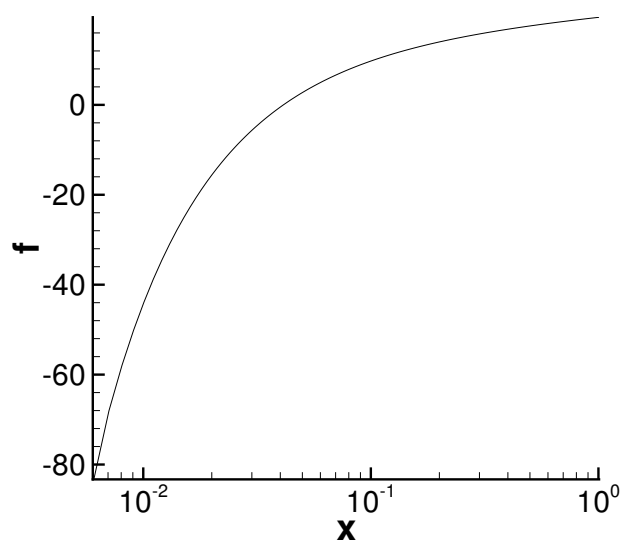

Figure 6.2.: Plot of $f$.

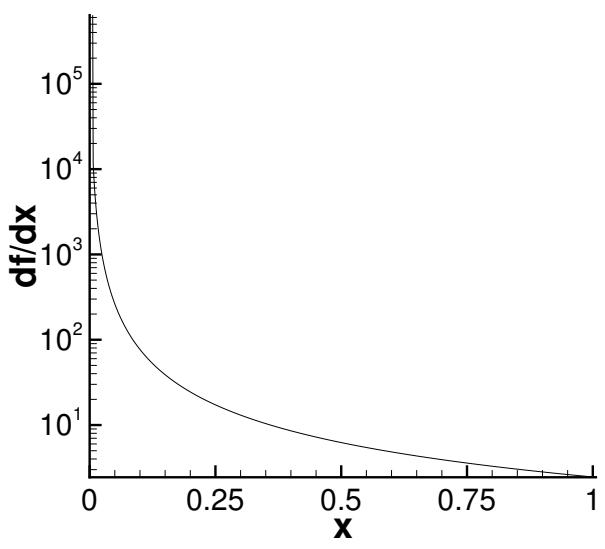

Figure 6.3.: Plot of $d f / d x$.

starting with some $x^{(0)} \in D$. Substituting $(6.18)-(6.19)$ into $(6.20)$ yields the scheme

$$
x^{(n+1)}=x^{(n)}\left(1+S\left(x^{(n)}\right) \frac{U\left(y_{\delta}\right)-R_{u} S\left(x^{(n)}\right) x^{(n)}}{R_{u} x^{(n)}+S\left(x^{(n)}\right) U\left(y_{\delta}\right)}\right) .
$$

Corollary 6.15 in [Kre98] ensures that the scheme (6.21) is locally convergent, i.e., there exists a neighbourhood $B$ of the zero $x_{*}$ such that the Newton iterations converge to $x_{*}$ for all $x^{(0)} \in B$. Computational tests reveal that $B$ is sufficiently large. For turbulent channel flow at $R e_{\tau}=395$ the exact (theoretical) zero is $x_{*}=0.0395$ and convergence of our scheme is obtained for $B=\left(e^{-1 / 2} R_{u} \nu y_{\delta}^{-1}, x_{b}\right)$ with $x_{b} \approx 2 x_{*}$.

In PARAllelNS we start with the initial guess $x^{(0)}=\left(\nu U\left(y_{\delta}\right) y_{\delta}^{-1}\right)^{1 / 2}$. This choice is motivated by $\tau_{w}=x_{*}^{2}$ and $\tau_{w} \equiv \nu d U /\left.d y\right|_{y=0}$ when using the approximation $d U /\left.d y\right|_{y=0} \approx$ $U\left(y_{\delta}\right) y_{\delta}^{-1}$. Of course, a computational method has to check whether $x^{(0)} \in D$ or not.

\subsubsection{Some remarks on the $k / \epsilon$ model using wall functions}

Numerical solutions of $k / \epsilon$ MODEL IIIB are sensitive to the artificial boundary $\Gamma_{\delta}$ where the matching occurs, see [Gri01], pp.32. In particular, $k / \epsilon$ MODEL IIIB yields reasonable results only if $\Gamma_{\delta}$ is sufficiently close to $\Gamma_{W}$. Additional problems can occur if $\Gamma_{\delta}$ is not parallel to $\Gamma_{W}$, e.g. if $\Gamma_{\delta}$ comes from an unstructed grid. Concerning these problems, $k / \epsilon$ MODEL IIIA is significantly superior to $k / \epsilon$ MODEL IIIB, cf.[Gri01], pp.76.

The idea of wall functions is to ensure a correct behaviour in the log law region in attached boundary layers. However, despite using wall functions, the prediction e.g. of the skin friction coefficient can be poor. WILCOX points out that this is not necessarily due to the function concept. This can arise due to shortcomings of the two-equation model itself, cf. [Wilcox98], p.174. A strategy to accounting for pressure gradients in the log layer equations can be found in [Wilcox.98], p.174. Finally it should be recalled that care must be taken regarding separated flows. 


\section{A computational LES model}

The objective of this chapter is similar to that of Chapter 6: We combine a global LES model and a local boundary-layer model using the fully overlapping DDM presented in (1.47)-(1.51). This approach, referred to as stress balance model, has been used by engi-

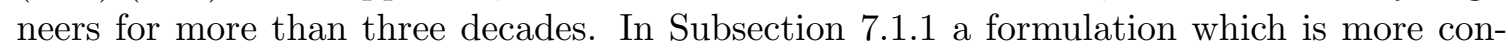
venient to mathematicians will be given. As LES is closer to the Navier-Stokes equations than a RANS model, many efforts have been made to combine the global LES model with a more sophisticated near-wall model. This coupling is called thin boundary-layer equation model and is addressed to in Subsection 7.1.2. A survey of an alternative approach, namely coupling LES with a RANS using a non-overlapping DDM, closes this chapter.

We start with the non-isothermal LES equations equipped with Dirichlet boundary conditions for both velocity and temperature. In the sequel write $\boldsymbol{w}, q$ and $\vartheta$ instead of $\overline{\boldsymbol{u}}, \bar{p}, \bar{\theta}$ because due to the closure hypotheses, $\boldsymbol{w}, q$ and $\vartheta$ are only an approximation to $\overline{\boldsymbol{u}}, \bar{p}, \bar{\theta}$. Note that $\boldsymbol{w}=0$ on $\Gamma_{W}$ is only an approximate boundary condition since obviously $\boldsymbol{u}=0$ does not imply $\overline{\boldsymbol{u}} \neq 0$ on $\Gamma_{W}$, unless the filter width $\Delta$ goes to zero when approaching the wall. The same observation holds for $\vartheta$. Thus we start with LES MODEL I which reads

\section{Computational non-isothermal LES model I}

\section{- Compute residual stress tensor and residual temperature flux}

(i) Compute turbulent viscosity and turbulent diffusivity. Define $\nu_{e}=\nu+\nu_{t}, a_{e}=a+a_{t}$, where $\nu_{t}$ and $a_{t}$ are given in $\Omega$ by

* Smagorinsky model: Given $C_{S} \in\{0.1,0.21\}, \operatorname{Pr}_{t}=0.4$ compute

$$
\nu_{t}=\left(C_{S} \Delta\right)^{2}\left(\max \left\{0 ;\|\mathbb{S}(\boldsymbol{w})\|_{F}^{2}+\frac{\beta}{P r_{t}} \boldsymbol{g} \cdot \nabla \vartheta\right\}\right)^{1 / 2}, \quad a_{t}=\frac{\nu_{t}}{P r_{t}} .
$$

* Iliescu-Layton model: Compute $\overline{\boldsymbol{w}}=\mathcal{R} \boldsymbol{w}$, with regularization operator $\mathcal{R} . \overline{\boldsymbol{w}}_{i} \equiv(\mathcal{R} \boldsymbol{w})_{i}(1 \leq i \leq d)$ is defined as the solution $\overline{\boldsymbol{w}}_{i}$ of

$$
\begin{aligned}
-\frac{\Delta^{2}}{4 \gamma} \triangle \overline{\boldsymbol{w}}_{i}+\overline{\boldsymbol{w}}_{i} & =\boldsymbol{w}_{i} & & \text { in } \Omega, \\
\nabla \overline{\boldsymbol{w}}_{i} \cdot \boldsymbol{n} & =0 & & \text { on } \partial \Omega .
\end{aligned}
$$

Then, given $C_{q} \in\{0.1,0.21\}, P r_{t}=0.4$, compute $\nu_{t}$ and $a_{t}$ $\nu_{t}=C_{q} \Delta\|\boldsymbol{w}-\mathcal{R} \boldsymbol{w}\|\left(\max \left\{0 ; 1+\frac{1}{\|\mathbb{S}(\boldsymbol{w})\|_{F}^{2}} \frac{\beta}{P r_{t}} \boldsymbol{g} \cdot \boldsymbol{\nabla} \vartheta\right\}\right)^{1 / 2}, a_{t}=\frac{\nu_{t}}{P r_{t}}$. 
(ii) Compute Galdi-Layton model.

From the definition of $\mathcal{R}$ in (7.1)-(7.2), for $1 \leq i, j \leq d$ compute

$$
\left(\mathcal{A}_{u}(\boldsymbol{w})\right)_{i j} \equiv \frac{\Delta^{2}}{12} \mathcal{R}\left(\sum_{l=1}^{d} \frac{\partial w_{i}}{\partial x_{l}} \frac{\partial w_{j}}{\partial x_{l}}\right), \quad\left(\mathcal{A}_{\theta}(\boldsymbol{w}, \vartheta)\right)_{j}=\frac{\Delta^{2}}{12} \mathcal{R}\left(\sum_{l=1}^{d} \frac{\partial \vartheta}{\partial x_{l}} \frac{\partial w_{j}}{\partial x_{l}}\right)
$$

(iii) Near-wall damping of residual stress tensor and residual temperature flux. Multiply $\nu_{t}, a_{t},\left(\mathcal{A}_{u}(\boldsymbol{w})\right)_{i j}$ and $\left(\mathcal{A}_{\theta}(\boldsymbol{w}, \vartheta)\right)_{j}$ with $\mathcal{D}\left(y^{+}\right)^{\alpha}, \mathcal{D}\left(y^{+}\right)=1-$ $e^{-y^{+} / 26}$ being the van Driest damping function and $y^{+}$denoting the distance to the closest wall in wall units. We select $\alpha=2$ for Smagorinsky model and Galdi-Layton model; for the Iliescu-Layton model we choose $\alpha=3$.

- Non-isothermal LES-equations

$$
\begin{aligned}
\partial_{t} \boldsymbol{w}-\boldsymbol{\nabla} \cdot\left(2 \nu_{e} \mathbb{S}(\boldsymbol{w})-\mathcal{A}_{u}(\boldsymbol{w})\right)+(\boldsymbol{w} \boldsymbol{\nabla}) \boldsymbol{w}+\boldsymbol{\nabla} q & =-\beta \vartheta \boldsymbol{g} & & \text { in } \Omega \times(0, T), \\
\boldsymbol{\nabla} \cdot \boldsymbol{w} & =0 & & \text { in } \Omega \times(0, T), \\
\partial_{t} \vartheta-\boldsymbol{\nabla} \cdot\left(a_{e} \boldsymbol{\nabla} \vartheta-\mathcal{A}_{\theta}(\boldsymbol{w}, \vartheta)\right)+(\boldsymbol{w} \boldsymbol{\nabla}) \vartheta & =\dot{q}^{V} c_{p}^{-1} & & \text { in } \Omega \times(0, T) .
\end{aligned}
$$

\section{- Boundary conditions}

$$
\begin{aligned}
& \vartheta=\Theta_{w} \quad \text { on } \Gamma_{W, D} \times(0, T), \quad a \boldsymbol{\nabla} \vartheta \cdot \boldsymbol{n}=\dot{q} c_{p}^{-1} \quad \text { on } \Gamma_{W, N} \times(0, T), \\
& \boldsymbol{w}=\mathbf{0} \\
& \text { on } \Gamma_{W} \times(0, T) \text {, }
\end{aligned}
$$

- and a suitable set of initial conditions.

LES MODEL I can be used for practical calculations provided $(i)$ the filter width can be chosen very fine near the wall, i.e. $\Delta \approx \delta_{\nu}$ with $\delta_{\nu}$ denoting the viscous length scale, and (ii) the SGS model (including some near-wall damping if necessary) is valid up to the wall. Concerning LES MODEL I, requirement $(i)$ often cannot be satisfied for problems at higher Rayleigh numbers. Moreover recall the fact that for our purposes, i.e. applications to indoor-air flow problems, only the mean effect of the near-wall turbulent processes on the outer flow has to be captured. In natural convection problems, if constant values for $C_{S}$ and $\mathrm{Pr}_{t}$ are chosen, then the van Driest damping function close to walls has to be used, see e.g. [PD01], [Eid85]. Anyway, the computational costs for the near-wall resolution are too large for most problems of practical relevance.

As a remedy, there are two solution strategies, as distinguished in [Bag98]. The first is to replace the no-slip condition by a mixed boundary condition (see (1.13)), leading to a full-overlapping DDM based on the scheme given in (1.47)-(1.51). This approach will be referred to as wall stress model and is analogous to the method used for the $k / \epsilon$ model. The second strategy is to use the no-slip boundary condition and to couple the LES with a RANS using some kind of non overlapping DDM or by a so-called hybrid method, e.g. by using a blending function; for a survey on this alternative approach see Section 7.2 . Therein also a (moderate) grid refinement near the wall is necessary. In this thesis we will study only the former approach in greater detail. 


\subsection{Wall stress models}

Experimental support for the wall stress model concept can be found e.g. in [BH93]. BROOKE and HANRATTY report that the interaction between the near-wall region and the outer flow region is only weak. Hence there is hope that a wall stress condition can provide enough information of the near-wall turbulence to the outer flow. A survey on wall stress models for the isothermal case can be found e.g. in [Sag01], Section 9.2, in [DBP0I] and in [CM00]. As originated by Schumann and GrötzBach, the idea is to replace (7.4) by a mixed boundary condition of the type

$$
\boldsymbol{w} \cdot \boldsymbol{n}=0, \quad \boldsymbol{n}^{T} \sigma(\boldsymbol{w}, q) \boldsymbol{t}_{j}=W_{*}{ }^{2} \frac{\boldsymbol{v}_{\delta} \cdot \boldsymbol{t}_{j}}{\left\|\boldsymbol{v}_{\delta}\right\|}(j=1, \ldots, d-1) \text { on } \Gamma_{W} .
$$

In (7.5) we still have to determine determine $W_{*}$ and $\boldsymbol{v}_{\delta} /\left\|\boldsymbol{v}_{\delta}\right\|$. Concerning the latter, GRÖTZBACH suggested, cf. [Gro87],

$$
\frac{\boldsymbol{v}_{\delta}}{\left\|\boldsymbol{v}_{\delta}\right\|}=\frac{\boldsymbol{w}_{\delta, t}}{\left\|\left\langle\boldsymbol{w}_{\delta, t}\right\rangle_{E, y=y_{\delta}}\right\|},
$$

using the definition of $\boldsymbol{w}_{\delta, t}$ in (6.3). Moreover $\langle\cdot\rangle_{E, y=y_{\delta}}$ denotes an ensemble average (or time average) over the plane $y=y_{\delta}$. In this thesis, we replace the denominator by simply taking $\left\|\boldsymbol{w}_{\delta, t}\right\|$.

\section{Remark 7.1}

In order to account for physical effects in the near-wall region, there are several modifications of (7.5)-(7.6), most notably the shifted model and the ejection model, devised and tested in [PFMK89]. A recent experimental investigation on these and further models can be found e.g. in [MKP01]].

$W_{*}$ is determined from a boundary-layer solution in $\Omega_{\delta}$. Depending on the boundary-layer model considered, two approaches can be distinguished, viz., stress balance models and thin boundary-layer equation models. Both approaches are explained and appraised in the following two subsections.

\subsubsection{Stress balance models}

Stress balance models for the non-isothermal case use the boundary-layer equations (6.12)(6.13) for determining $W_{*}$ and $\dot{s}$. Note that for $0 \leq y<y_{\max }$ the natural convection solution of (6.12)-(6.13) is reasonably approximated by the forced convection solution (5.41)-(5.42). We require that $\Gamma_{\delta}$ is located in the log layer. Then we can determine $\left(W_{*}, \dot{s}\right)$ by matching $\boldsymbol{w}_{\delta, t}$ and $\vartheta_{\delta}$ from the outer (LES) solution to the modified log law solution (5.41)-(5.42) resp. similar the COMPUTATIONAL $k / \epsilon$ MODEL IIIA. We introduce the following scaled variables

$$
y^{+} \equiv \frac{y W_{*}}{\nu}, \quad w\left(y_{\delta}\right) \equiv \frac{\boldsymbol{w}_{\delta} \cdot \boldsymbol{w}_{\delta, t}}{\left\|\boldsymbol{w}_{\delta, t}\right\|}, \quad w_{\delta}^{+} \equiv \frac{w\left(y_{\delta}\right)}{W_{*}}, \quad \vartheta_{\delta}^{+} \equiv \frac{c_{p} W_{*}\left(\Theta_{0}-\vartheta_{\delta}\right)}{\dot{s}} .
$$

In (7.7), $W_{*}$ and $\dot{s}$ are the LES approximation to $u_{\tau}$ and $\dot{q}$ resp. Then we can propose the following computational model, referred to as LES MODEL II. 


\section{Computational non-isothermal LES model II}

\section{(1) Global LES problem}

- Non-isothermal LES-equations

$$
\begin{aligned}
\partial_{t} \boldsymbol{w}-\boldsymbol{\nabla} \cdot\left(2\left(\nu_{e} \mathbb{S}(\boldsymbol{w})-\mathcal{A}_{u}(\boldsymbol{w})\right)+(\boldsymbol{w} \boldsymbol{\nabla}) \boldsymbol{w}+\boldsymbol{\nabla} q\right. & =-\beta \vartheta \boldsymbol{g} & & \text { in } \Omega \times(0, T), \\
\boldsymbol{\nabla} \cdot \boldsymbol{w} & =0 & & \text { in } \Omega \times(0, T), \\
\partial_{t} \vartheta-\boldsymbol{\nabla} \cdot\left(a_{e} \boldsymbol{\nabla} \vartheta-\mathcal{A}_{\theta}(\boldsymbol{w}, \vartheta)\right)+(\boldsymbol{w} \boldsymbol{\nabla}) \vartheta & =\dot{q}^{V} c_{p}^{-1} & & \text { in } \Omega \times(0, T) .
\end{aligned}
$$

- Subgrid scale model: See LES MODEL I

- Boundary conditions

$$
\begin{aligned}
\left(a \boldsymbol{\nabla}(\vartheta)-\mathcal{A}_{\theta}(\boldsymbol{w}, \vartheta)\right) \cdot \boldsymbol{n} & =\dot{s} c_{p}^{-1} & & \text { on } \Gamma_{W} \times(0, T), \\
\boldsymbol{w} \cdot \boldsymbol{n}=0, \quad \boldsymbol{n}^{T} \sigma_{u}(\boldsymbol{w}, q) \boldsymbol{t}_{j} & =W_{*}{ }^{2} \frac{\boldsymbol{w}_{\delta, t} \cdot \boldsymbol{t}_{j}}{\left\|\boldsymbol{w}_{\delta, t}\right\|} & & \text { on } \Gamma_{W} \times(0, T), \quad(1 \leq j \leq d-1)
\end{aligned}
$$

with

$$
\sigma_{u}(\boldsymbol{w}, q) \equiv \sigma(\boldsymbol{w}, q)-\mathcal{A}_{u}(\boldsymbol{w})
$$

(2) Compute wall shear stress and heat flux.

- Case of negligible buoyancy forces: For each $\boldsymbol{x} \in \Gamma_{W}$ : given $w_{\delta}^{+}$and $\vartheta_{\delta}^{+}$as in (7.7), seek $\left(W_{*}, \dot{s}\right)$ (using Newton's method) s.t.

$$
\begin{aligned}
& w_{\delta}^{+}= \begin{cases}y_{\delta}^{+}, & \text {if } y_{\delta}^{+} \leq R_{u} \\
R_{u}\left(2 \ln \left(\frac{y_{\delta}^{+}}{R_{u}}\right)+1\right)^{\frac{1}{2}}, & \text { if } y_{\delta}^{+}>R_{u}\end{cases} \\
& \vartheta_{\delta}^{+}=\left\{\begin{array}{ll}
\operatorname{Pr} y_{\delta}^{+}, & \text {if } y_{\delta}^{+} \leq R_{\Theta} \\
C_{\Theta}\left(2 \ln \left(\frac{y_{\delta}^{+}}{R_{\Theta}}\right)+1\right)^{\frac{1}{2}}, & \text { if } y_{\delta}^{+}>R_{\Theta}
\end{array} .\right.
\end{aligned}
$$

- Case of significant buoyancy forces: For each $\boldsymbol{x} \in \Gamma_{W}$ proceed analogously to the COMPUTATional $k / \epsilon$ TURBUlence MOdel IIIA.

There are two possible interpretations for this approach. On the one hand, this procedure can be understood as using a very fine filter width in $\Omega_{\delta}$. Then a further problem is that strictly speaking $w_{\delta, t}$ and $\vartheta_{\delta}$ are space averaged quantities. These are matched with the near-wall solution without explicitely ensuring the filter width to be continuous on $\Gamma_{\delta}$. On the other hand, a second interpretation is to match a LES for the outer flow to a RANS model in the near-wall region. The second interpretation is closer to reality, because the simple ordinary differential equations used for the RANS model can hardly bear detailled information about the near-wall turbulence.

Results for a channel flow up to $R e_{\tau}=5000$ using the dynamic model combined with a wall stress model are satisfying, see e.g. [BBP9.5]. 


\subsubsection{Thin boundary-layer equation models}

A more advanced method is to calculate $W_{*}$ from a thin layer equation model. For isothermal flows this strategy was primarily studied by BALARAS ([BBP96]) and СABOT ([Cab96] $)$. In $\Omega_{\delta}$ they consider the following system of equations $\left(x_{1}, y \equiv x_{2}\right.$ and $x_{3}$ denoting the streamwise, wall-normal and spanwise coordinate direction resp.), see also (5.16):

$$
\begin{aligned}
\frac{\partial u_{i}}{\partial t}+\sum_{j=1,3} \frac{\partial\left(u_{i} u_{j}\right)}{\partial x_{j}}+\frac{\partial p_{m}}{\partial x_{i}} & =\frac{\partial}{\partial y}\left[\left(\nu+\nu_{t}\right) \frac{\partial u_{i}}{\partial y}\right], \quad i=1,3 \\
p_{m}=p_{m}\left(x_{1}, x_{3}\right) & =q\left(x_{1}, y_{\delta}, x_{3}\right)
\end{aligned}
$$

with continuity equation

$$
u_{2}=-\int_{0}^{y} \sum_{i=1,3} \frac{\partial u_{i}\left(y^{\prime}\right)}{\partial x_{i}} d y^{\prime}
$$

and boundary conditions

$$
u_{i}=0 \quad \text { on } \Gamma_{W}, \quad u_{i}=w_{i} \quad \text { on } \Gamma_{\delta}
$$

In particular, note that in (7.11) $p_{m}$ is the near-wall pressure from the outer flow, assumed to be independent of $y$ in the inner layer. In (7.10) an algebraic turbulence model is used for $\nu_{t}$. CABOT suggests the following ad hoc damped mixing length hypothesis (with $y^{+}$ being the distance from the wall in wall units and $A^{+}=25$ )

$$
\nu_{t}=\kappa y u_{\tau} D_{C}\left(y^{+}\right), \quad \text { with damping function } \quad D_{C}\left(y^{+}\right)=\left[1-e^{-y^{+} / A^{+}}\right]^{2} .
$$

Finally, the wall stress is determined from

$$
\tau_{w, i}=\left.\nu \frac{\partial u_{i}}{\partial y}\right|_{y=0}
$$

Stress balance model and thin boundary-layer (TBL) model (including its dynamic variant) have been tested for a boundary-layer flow past a trailing-edge by WANG, cf. [Wan00]. He also studied a flow over a circular cylinder, see [W(T)T]. He emphasises the observation that the TBL model can be remarkably improved by determining $\kappa$ in (7.14) dynamically. Numerical investigations on a channel flow and a flow over a backward facing step can be found in [CMO0]. Wang points out that "the total reduction in CPU time, due to both smaller number of grid points and larger time steps, is over $90 \%$ compared to the full LES" ([Wan00], p.243).

Instead of an algebraic model Diurno, Balaras and Piomelli used the SpalartAllmaras (see [SA94]) one-equation model in $\Omega_{\delta}$, cf. [DBP01]. They report good agreement with DNS results and experimental data for a flow over a backward-facing step. 


\subsection{Hybrid RANS/LES approaches}

The second recent strategy in LES for wall bounded flows is based on decomposing $\Omega$ into $\Omega_{\text {outer }}$ and $\Omega_{\delta}$, with $\overline{\Omega_{\text {outer }}} \cup \overline{\Omega_{\delta}}=\bar{\Omega}$ and $\Omega_{\text {outer }} \cap \Omega_{\delta}=\emptyset$. In $\Omega_{\text {outer }}$ a pure LES is used. In $\Omega_{\delta}$ a RANS or a combination of a RANS with a LES model is performed. An example of a simple approach is to couple two algebraic models, e.g. the Baldwin Lomax model and the Smagorinsky model, see [CM02].

A very promising scheme is the so-called detached eddy simulation (DES) turbulence model as proposed in [S.ISA.97]. It was conceived to improve the results for unsteady and massively separated flows. The DES model reduces to the standard SPALART-ALLMARAS model near viscous walls, where the grid is refined and has a large aspect ratio, but acts like a largeeddy simulation model away from the boundary, where the grid is coarser and has an aspect ratio of order one. Results are presented e.g. in [NNWS00].

A further strategy is to use the $k / \omega$ two-equation model in $\Omega_{\delta}$ and a one-equation SGS model in $\Omega_{\text {outer }}$, see [PI01a]. Quemere and SAGAUT studied the hybrid coupling of the $k / \epsilon$ model with LES, see [Que01].

However, as pointed out by BAGGETT in [Bag98], there can be a fundamental problem of artificial near-wall turbulent structures of non-physical origin, when LES and RANS are merged. 


\section{Some analytical results for LES with near wall modelling}

In the previous chapter the concept of a fully overlapping DDM for wall bounded flows using LES as a global model has been studied; the coupling mechanism was given by (1.47)-(1.51). As already mentioned, the accuracy of this method can be improved when using a better model in the near wall region. The most accurate method consists in accomplishing a DNS. Put in other words, if the wall iteration approach using a DNS in the near wall region is not accurate enough, then there is little hope that the method works for coarser boundary-layer models.

In this chapter we study the coupling from a functional analytical point of view. Concerning this issue, boundary condition (1.48) is not suitable. Hence we consider (1.53) (resp. its modification given below) instead.

\section{Remark 8.1}

Throughout this chapter, similar to [Tem77], [GR86] and [Col99] we use $\nabla \boldsymbol{u}$ instead of $\nabla^{s} \boldsymbol{u}$ in the diffusion term of the momentum equation. The reason for this is that we can bound $\nabla^{s} \boldsymbol{u}$ by $\nabla \boldsymbol{u}$ in the $L^{2}$-norm, but we cannot bound $\nabla \boldsymbol{u}$ by $\nabla^{s} \boldsymbol{u}$.

Now we can focus on the following coupling of a global LES with a DNS in the near wall region using a fully overlapping DDM based on (1.57)-(1.61) together with Figure 1.2:

\section{A Model for Coupling LES and DNS}

Seek a global LES solution $\boldsymbol{w}: \Omega \times(0, T) \mapsto \mathbb{R}^{d}, q: \Omega \times(0, T) \mapsto \mathbb{R}$ and a DNS solution in the boundary layer $\boldsymbol{u}: \Omega_{\text {layer }} \times(0, T) \mapsto \mathbb{R}^{d}, p: \Omega_{\text {layer }} \times(0, T) \mapsto \mathbb{R}$, such that

$$
\begin{aligned}
\partial_{t} \boldsymbol{w}-\boldsymbol{\nabla} \cdot\left(\nu_{e} \nabla \boldsymbol{w}-\mathcal{A}_{u}(\boldsymbol{w})\right)+(\boldsymbol{w} \cdot \boldsymbol{\nabla}) \boldsymbol{w}+\boldsymbol{\nabla} q=\overline{\boldsymbol{f}} & \text { in } \Omega \times(0, T), \\
\boldsymbol{\nabla} \cdot \boldsymbol{w}=0 & \text { in } \Omega \times(0, T),
\end{aligned}
$$

$$
\begin{aligned}
\boldsymbol{w} \cdot \boldsymbol{n}=0, \beta_{j}(\delta, \boldsymbol{u}) \boldsymbol{w} \cdot \boldsymbol{t}_{j}+\boldsymbol{n}^{T}\left(\nu_{e} \nabla \boldsymbol{w}-\mathcal{A}_{u}(\boldsymbol{w})-q \mathbb{I}\right) \boldsymbol{t}_{j} & =0 & & \text { on } \Gamma_{W} \times(0, T), \\
\partial_{t} \boldsymbol{u}-\boldsymbol{\nabla} \cdot(\nu \nabla \boldsymbol{u})+(\boldsymbol{u} \cdot \boldsymbol{\nabla}) \boldsymbol{u}+\boldsymbol{\nabla} p & =\boldsymbol{f} & & \text { in } \Omega_{\text {layer }} \times(0, T), \\
\boldsymbol{\nabla} \cdot \boldsymbol{u} & =0 & & \text { in } \Omega_{\text {layer }} \times(0, T),
\end{aligned}
$$

with $\mathcal{A}_{u}(\cdot)$ being defined in (4.45), $\nu_{e}=\nu+\nu_{t}$ and $\beta_{j}$ given by

$$
\beta_{j}(\delta, \boldsymbol{u})=\frac{-\left.\boldsymbol{n}^{T}\left(\nu_{e} \nabla(\overline{\boldsymbol{u}})-\mathcal{A}_{u}(\overline{\boldsymbol{u}})\right)\right|_{\Gamma_{W}} \boldsymbol{t}_{j}}{\left.\overline{\boldsymbol{u}}\right|_{\Gamma_{W}} \cdot \boldsymbol{t}_{j}}, \quad j=1, \ldots, d-1, \quad \overline{\boldsymbol{u}} \equiv g_{\delta} * \boldsymbol{u} .
$$

Before proceeding, it is worthwhile commenting boundary conditions (8.3) and (8.7). 


\section{Remark 8.2}

(i) Note that $\boldsymbol{n}^{T} q \mathbb{I} \boldsymbol{t}_{j}=0$ (appearing e.g. in (8.3)), since $\left\{\boldsymbol{n}, \boldsymbol{t}_{j}\right\}_{j=1}^{d-1}$ forms a local orthonormal basis. Sometimes this term will be omitted for lack of space.

(ii) It should be recalled that $\boldsymbol{w}=\overline{\boldsymbol{u}}$ in $\Omega_{\text {layer }}$ for an ideal LES. Due to the modelling error stemming from the closure model, we can expect only $\boldsymbol{w} \approx \overline{\boldsymbol{u}}$ in $\Omega_{\text {layer. }}$. If $\boldsymbol{w}$ satisfies (8.3) and if $\boldsymbol{w} \approx \overline{\boldsymbol{u}}$ in $\Omega_{\text {layer }}$, then $\overline{\boldsymbol{u}}$ satisfies

$$
\overline{\boldsymbol{u}} \cdot \boldsymbol{n} \approx 0, \beta_{j}(\delta, \boldsymbol{u}) \overline{\boldsymbol{u}} \cdot \boldsymbol{t}_{j}+\boldsymbol{n}^{T}\left(\nu_{e} \nabla \overline{\boldsymbol{u}}-\mathcal{A}_{u}(\overline{\boldsymbol{u}})-\bar{p} \mathbb{I}\right) \boldsymbol{t}_{j} \approx 0 \text { on } \Gamma_{W} \times(0, T) .
$$

Solving this for $\beta_{j}$ gives (8.7). Thus, (8.3) together with (8.7) (using the implicit assumption of LES that $\boldsymbol{w} \approx \overline{\boldsymbol{u}}$ in $\Omega_{\text {layer }}$ ) can be written equivalently as the following condition for $\boldsymbol{w}$ on $\Gamma_{W} \times(0, T)$, viz.,

$$
\boldsymbol{w} \cdot \boldsymbol{n}=0, \frac{\boldsymbol{w} \cdot \boldsymbol{t}_{j}}{\overline{\boldsymbol{u}} \cdot \boldsymbol{t}_{j}} \boldsymbol{n}^{T}\left(\nu_{e} \nabla \overline{\boldsymbol{u}}-\mathcal{A}_{u}(\overline{\boldsymbol{u}})-\bar{p} \mathbb{I}\right) \boldsymbol{t}_{j}=\boldsymbol{n}^{T}\left(\nu_{e} \nabla \boldsymbol{w}-\mathcal{A}_{u}(\boldsymbol{w})-q \mathbb{I}\right) \boldsymbol{t}_{j} .
$$

However, we use (8.3) together with (8.7), since that notation is more convenient. $\diamond$

This chapter is organized as follows:

I Some simplifications of the scheme (8.1)-(8.7) have to be made.

II The global LES and the local DNS problem are studied separately.

III The fully coupled scheme (8.1)-(8.7) is investigated.

Note that the underlying functional analytical basics are reviewed in Appendix B. For readers familiar to the analytical treatment of the Navier Stokes equations, it is not necessary studying Appendix B before delving into the analysis.

\subsection{Some simplifications of the coupled problem}

Before starting with the analysis, we simplify scheme (8.1)-(8.7), namely, we introduce a modified (i.e., smooth) definition for the friction parameters $\beta_{j}$, we consider the corresponding steady state problem, and we simplify the LES model in $\Omega$.

\subsubsection{A modified definition for the friction parameters $\beta_{j}$}

In order to be able doing some analysis, we have to modify (8.7). First we rewrite (8.7):

(a) Given $\boldsymbol{u}: \Omega_{\text {layer }} \times(0, T) \mapsto \mathbb{R}^{d}$, for each $(\boldsymbol{x}, t)$ in $\Gamma_{W} \times(0, T)$, we can evaluate

$$
\eta \equiv \overline{\boldsymbol{u}}(\boldsymbol{x}, t) \cdot \boldsymbol{t}_{j}, \quad \xi \equiv-\boldsymbol{n}^{T}\left[\nu_{e} \nabla(\overline{\boldsymbol{u}})-\mathcal{A}_{u}(\overline{\boldsymbol{u}})\right](\boldsymbol{x}, t) \boldsymbol{t}_{j}
$$

(b) Given $(\xi, \eta) \in \mathbb{R}^{2}$ from step (a), and required $\eta \neq 0$, then $\beta_{j}$ is given by

$$
\beta_{j}=\beta(\xi, \eta) \equiv \xi \eta^{-1} .
$$


In step (b) we introduced the map

$$
\beta: \mathbb{R}^{2} \backslash(\mathbb{R} \times\{0\}) \longrightarrow \mathbb{R}, \quad(\xi, \eta) \longrightarrow \xi \eta^{-1},
$$

which is not amenable to the analysis. The subsequent analysis reveals that the $\beta_{j}$ have to satisfy the following minimal design properties, viz.,

(i) $\beta(\cdot, \cdot)$ is well-defined on $\mathbb{R}^{2}$ and $\beta \geq \beta_{0}>0$ on $\mathbb{R}^{2}$,

(ii) $\beta$ is continuous on $\mathbb{R}^{2}$,

(iii) $\beta$ is Lipschitz continuous on $\mathbb{R}^{2}$, i.e.,

$$
\beta\left(\xi_{2}-\xi_{1}, \eta_{2}-\eta_{1}\right) \leq C_{l c} \max \left\{\left|\xi_{2}-\xi_{1}\right|,\left|\eta_{2}-\eta_{1}\right|\right\}
$$

Clearly, (i)-(iii) are fulfilled when using the following regularisation of $\beta(\cdot, \cdot)$ :

$$
\beta_{j}(\delta, \boldsymbol{u}) \equiv \beta_{j}(\boldsymbol{u}) \equiv \beta_{j}=\beta(\xi, \eta) \equiv \max \left\{\frac{|\xi|}{\sqrt{\eta^{2}+\epsilon^{2}}}, \beta_{0}\right\}, \quad \epsilon>0 .
$$

\subsubsection{The steady state case}

In this thesis we restrict ourselves to the steady state case of (8.1)-(8.6).

\section{A steady state Model for Coupling LES and DNS}

$$
\begin{aligned}
-\boldsymbol{\nabla} \cdot\left(\nu_{e} \nabla \boldsymbol{w}-\mathcal{A}_{u}(\boldsymbol{w})\right)+(\boldsymbol{w} \cdot \boldsymbol{\nabla}) \boldsymbol{w}+\boldsymbol{\nabla} q & =\overline{\boldsymbol{f}} \quad \text { in } \Omega, \\
\boldsymbol{\nabla} \cdot \boldsymbol{w} & =0 \quad \text { in } \Omega,
\end{aligned}
$$

$\boldsymbol{w} \cdot \boldsymbol{n}=0, \quad \beta_{j}(\delta, \boldsymbol{u}) \boldsymbol{w} \cdot \boldsymbol{t}_{j}+\boldsymbol{n}^{T}\left(\nu_{e} \nabla \boldsymbol{w}-\mathcal{A}_{u}(\boldsymbol{w})-q \mathbb{I}\right) \boldsymbol{t}_{j}=0 \quad$ on $\Gamma_{W}$,

$$
\begin{aligned}
& -\boldsymbol{\nabla} \cdot(\nu \nabla \boldsymbol{u})+(\boldsymbol{u} \cdot \boldsymbol{\nabla}) \boldsymbol{u}+\boldsymbol{\nabla} p=\boldsymbol{f} \quad \text { in } \Omega_{\text {layer }}, \\
& \boldsymbol{\nabla} \cdot \boldsymbol{u}=0 \quad \text { in } \Omega_{\text {layer }}, \\
& \boldsymbol{u}=\mathbf{0} \text { on } \Gamma_{W}, \quad \boldsymbol{u}=\boldsymbol{w} \text { on } \Gamma_{i} .
\end{aligned}
$$

We divide this coupled problem (8.11)-(8.16) into two subproblems: A steady state LES problem in $\Omega$ and a steady state NSE problem in $\Omega_{\text {layer }}$.

\section{Steady state LES problem in $\Omega$ :}

For given $\boldsymbol{u}$ in $\boldsymbol{H}^{1}\left(\Omega_{\text {layer }}\right)$ with $\boldsymbol{u}=0$ on $\Gamma_{W}, \nabla \cdot \boldsymbol{u}=0$ in $\Omega_{\text {layer }}$, let $\beta_{j}(\delta, \boldsymbol{u})$ be given by (8.8) and (8.10). Then the steady state LES problem in $\Omega$ reads: Seek $\boldsymbol{w}, q$ such that

$$
\begin{aligned}
& -\boldsymbol{\nabla} \cdot\left(\nu_{e} \nabla \boldsymbol{w}-\mathcal{A}_{u}(\boldsymbol{w})\right)+(\boldsymbol{w} \cdot \boldsymbol{\nabla}) \boldsymbol{w}+\boldsymbol{\nabla} q=\overline{\boldsymbol{f}} \quad \text { in } \Omega, \\
& \boldsymbol{\nabla} \cdot \boldsymbol{w}=0 \quad \text { in } \Omega, \\
& \boldsymbol{w} \cdot \boldsymbol{n}=0, \quad \beta_{j}(\delta, \boldsymbol{u}) \boldsymbol{w} \cdot \boldsymbol{t}_{j}+\boldsymbol{n}^{T}\left(\nu_{e} \nabla \boldsymbol{w}-\mathcal{A}_{u}(\boldsymbol{w})-q \mathbb{I}\right) \boldsymbol{t}_{j}=0 \quad \text { on } \Gamma_{W} .
\end{aligned}
$$


Steady state NSE problem in $\Omega_{\text {layer }}$ :

Given $\boldsymbol{w}$ in $\boldsymbol{H}^{1}(\Omega)$ with $\boldsymbol{w} \cdot \boldsymbol{n}=0$ on $\Gamma_{W}, \nabla \cdot \boldsymbol{w}=0$ in $\Omega$, the steady state NSE problem in $\Omega_{\text {layer }}$ reads: Seek $\boldsymbol{u}, p$ such that

$$
\begin{aligned}
& -\boldsymbol{\nabla} \cdot(\nu \nabla \boldsymbol{u})+(\boldsymbol{u} \cdot \boldsymbol{\nabla}) \boldsymbol{u}+\boldsymbol{\nabla} p=\boldsymbol{f} \quad \text { in } \Omega_{\text {layer }}, \\
& \boldsymbol{\nabla} \cdot \boldsymbol{u}=0 \quad \text { in } \Omega_{\text {layer }}, \\
& \boldsymbol{u}=\mathbf{0} \text { on } \Gamma_{W}, \quad \boldsymbol{u}=\boldsymbol{w} \quad \text { on } \Gamma_{i} .
\end{aligned}
$$

Finally we simplify the LES model in the steady state LES problem in $\Omega$ : We use the following simplest LES model, originated by Kolmogorow:

$$
\mathcal{A}_{u}(\boldsymbol{w}) \equiv 0, \quad \nu_{e}=\nu+\nu_{t}, \quad \nu_{t}=\text { const } .
$$

\section{Remark 8.3}

For the analysis, this simplification is not too restrictive. Emphasis is placed on the mathematical properties of the slip with friction boundary condition. There is hope that the techniques applied in this context can be transferred to the case of a more sophisticated model for $\mathcal{A}_{u}$ and $\nu_{t}$, if (8.17)-(8.19) equipped with homogeneous Dirichlet boundary conditions has a unique solution.

\subsection{A separate study of global and local subproblem}

In this section we study the global LES problem (8.17)-(8.19) and the local DNS problem (8.20)-(8.22) separately. In both subsections, the underlying thread is organized as follows:

(1) Derivation of the corresponding variational formulation.

(2) Definition of Sobolev spaces appropriate to the variational formulation.

(3) Derivation of an a priori estimate for the solution.

(4) Proof of existence of at least one solution. This proof takes three steps:

(a) Using Brouwer's fixed point theorem we show that the finite dimensional Galerkin scheme for step (2) has at least one solution.

(b) From the a priori estimate (step (3)) we show that the sequence of finite dimensional solutions has a subsequence which possesses a weak limit element.

(c) Using standard arguments is can be shown that this limit element solves the variational formulation problem given in step (1).

(5) Proof of uniqueness of the solution. 


\subsubsection{The steady state LES problem in $\Omega$}

First we take under consideration (8.17)-(8.19), starting with the corresponding variational formulation. We define

$$
\begin{aligned}
\mathcal{V}_{2} & \equiv\left\{\boldsymbol{v} \in \boldsymbol{C}^{\infty}(\Omega) \mid \nabla \cdot \boldsymbol{v}=0 \text { in } \Omega, \boldsymbol{v} \cdot \boldsymbol{n}=0 \text { on } \Gamma_{W}\right\}, \\
\boldsymbol{V}_{2} & \equiv\left\{\boldsymbol{v} \in \boldsymbol{H}^{1}(\Omega) \mid \nabla \cdot \boldsymbol{v}=0 \text { in } \Omega, \boldsymbol{v} \cdot \boldsymbol{n}=0 \text { on } \Gamma_{W}\right\} .
\end{aligned}
$$

Multiplying (8.17) with $\boldsymbol{v} \in \mathcal{V}_{2}$, and integration by parts gives

$$
(\boldsymbol{w} \nabla \boldsymbol{w}, \boldsymbol{v})+\left(\nu_{e} \nabla \boldsymbol{w}, \nabla \boldsymbol{v}\right)-\int_{\Gamma_{W}}\left(\nu_{e} \nabla(\boldsymbol{w})-q \mathbb{I}\right) \boldsymbol{n} \boldsymbol{v} d s=(\overline{\boldsymbol{f}}, \boldsymbol{v}) .
$$

The boundary integral over $\Gamma_{W}$ can be rewritten by decomposing the test function $\boldsymbol{v}$ on $\Gamma_{W}$ into $d$ orthonormal components

$$
\boldsymbol{v}=(\boldsymbol{v} \cdot \boldsymbol{n}) \boldsymbol{n}+\sum_{j=1}^{d-1} \boldsymbol{v} \cdot \boldsymbol{t}_{j} \boldsymbol{t}_{j}
$$

Thus (using $\boldsymbol{v} \cdot \boldsymbol{n}=0$ on $\Gamma_{W}$ in the second step)

$$
\begin{aligned}
\int_{\Gamma_{W}} & \left(\nu_{e} \nabla \boldsymbol{w}-q \mathbb{I}\right) \boldsymbol{n} \cdot \boldsymbol{v} d s= \\
\quad= & \int_{\Gamma_{W}}\left(\nu_{e} \nabla \boldsymbol{w}-q \mathbb{I}\right) \boldsymbol{n} \cdot(\boldsymbol{v} \cdot \boldsymbol{n}) \boldsymbol{n} d s+\sum_{j=1}^{d-1} \int_{\Gamma_{W}}\left(\nu_{e} \nabla \boldsymbol{w}-q \mathbb{I}\right) \boldsymbol{n} \cdot \boldsymbol{v} \cdot \boldsymbol{t}_{j} \boldsymbol{t}_{j} d s \\
\quad= & \sum_{j=1}^{d-1} \int_{\Gamma_{W}} \boldsymbol{n}^{T}\left(\nu_{e} \nabla \boldsymbol{w}-q \mathbb{I}\right) \boldsymbol{t}_{j} \boldsymbol{v} \cdot \boldsymbol{t}_{j} d s=-\sum_{j=1}^{d-1}\left\langle\beta_{j} \boldsymbol{w} \cdot \boldsymbol{t}_{j}, \boldsymbol{v} \cdot \boldsymbol{t}_{j}\right\rangle_{\Gamma_{W}} .
\end{aligned}
$$

\section{Remark 8.4}

The boundary integral $\int_{\Gamma_{W}} \sum_{j=1}^{d-1} \boldsymbol{n}^{T}\left(\nu_{e} \nabla \boldsymbol{w}-q \mathbb{I}\right) \boldsymbol{t}_{j} \boldsymbol{v} \cdot \boldsymbol{t}_{j} d s$ can be interpreted as the dual product between $H^{-1 / 2}\left(\Gamma_{W}\right) \times H^{1 / 2}\left(\Gamma_{W}\right)$ provided $\boldsymbol{n}^{T}\left(\nu_{e} \nabla \boldsymbol{w}-q \mathbb{I}\right) \cdot \boldsymbol{t}_{j} \in H^{-1 / 2}\left(\Gamma_{W}\right)$. This can be ensured if $\boldsymbol{w} \in \boldsymbol{H}^{2}(\Omega)$ with $\nabla \cdot \boldsymbol{w}=0$ and $q \in H^{1}(\Omega)$, see [Ver87]. This regularity property is satisfied, e.g., provided $\boldsymbol{f} \in \boldsymbol{H}^{2}(\Omega)$ and $\Omega$ is a bounded $C^{2}$-domain, see e.g. [Soh01], p.173. Care must be taken concerning splitting the boundary integral, which requires that the boundary integral is the inner product in $L^{2}(\Gamma)$.

Hence $(8.25)$ becomes

$$
(\boldsymbol{w} \nabla \boldsymbol{w}, \boldsymbol{v})+\left(\nu_{e} \nabla \boldsymbol{w}, \nabla \boldsymbol{v}\right)+\sum_{j=1}^{d-1}\left\langle\beta_{j} \boldsymbol{w} \cdot \boldsymbol{t}_{j}, \boldsymbol{v} \cdot \boldsymbol{t}_{j}\right\rangle_{\Gamma_{W}}=(\overline{\boldsymbol{f}}, \boldsymbol{v}) .
$$

Using the well-known continuity argument (see e.g. [Tem77], p.160) we arrive at the following variational formulation corresponding to (8.17)-(8.19): 
Steady state LES problem in $\Omega$ : Find $\boldsymbol{w}$ in $\boldsymbol{V}_{2}$, s.t. (8.26) holds for all $\boldsymbol{v}$ in $\boldsymbol{V}_{2}$.

Now we give an a priori estimate for the LES problem in $\Omega$.

\section{Lemma 8.1}

Concerning the steady state LES problem in $\Omega$ the following a priori estimates hold

$$
\begin{aligned}
& \frac{\nu_{e}}{2}\|\nabla \boldsymbol{w}\|_{\boldsymbol{L}^{2}(\Omega)}^{2}+\beta_{0} \sum_{j=1}^{d-1}\left\|\boldsymbol{w} \cdot \boldsymbol{t}_{j}\right\|_{L^{2}\left(\Gamma_{W}\right)}^{2} \leq C_{L E S}^{a p}\|\overline{\boldsymbol{f}}\|_{-1, \Omega}^{2}, \\
& \frac{\nu_{e}}{2} C_{M}^{-2}\|\boldsymbol{w}\|_{1, \Omega}^{2}+\beta_{0} \sum_{j=1}^{d-1}\left\|\boldsymbol{w} \cdot \boldsymbol{t}_{j}\right\|_{L^{2}\left(\Gamma_{W}\right)}^{2} \leq C_{L E S}^{a p}\|\overline{\boldsymbol{f}}\|_{-1, \Omega}^{2} .
\end{aligned}
$$

with the following constants

$$
C_{L E S}^{a p} \equiv \frac{C_{M}^{2}}{2 \nu_{e}}, \quad C_{M} \equiv \sqrt{1+c_{P o i}^{2}(\Omega)} .
$$

\section{Proof:}

We introduce the usual bilinear form $b_{\Omega}(\boldsymbol{u}, \boldsymbol{v}, \boldsymbol{w}) \equiv(\boldsymbol{u} \nabla \boldsymbol{v}, \boldsymbol{w})$. Recall that $b_{\Omega}(\boldsymbol{u}, \boldsymbol{v}, \boldsymbol{v})=0$ for all $\boldsymbol{v}$ in $\boldsymbol{H}^{1}(\Omega)$ if $\nabla \cdot \boldsymbol{u}=0$ and $\boldsymbol{u} \cdot \boldsymbol{n}=0$ on $\partial \Omega$. Setting $\boldsymbol{v}=\boldsymbol{w}$ in (8.26) we obtain

$$
\nu_{e}\|\nabla \boldsymbol{w}\|_{\boldsymbol{L}^{2}(\Omega)}^{2}+\beta_{0} \sum_{j=1}^{d-1}\left\|\boldsymbol{w} \cdot \boldsymbol{t}_{j}\right\|_{L^{2}\left(\Gamma_{W}\right)}^{2} \leq|(\overline{\boldsymbol{f}}, \boldsymbol{w})| \leq\|\overline{\boldsymbol{f}}\|_{-1, \Omega}\|\boldsymbol{w}\|_{1, \Omega} .
$$

Due to (B.14) we have

$$
\|\boldsymbol{w}\|_{1, \Omega}=\left(\|\boldsymbol{w}\|_{\boldsymbol{L}^{2}(\Omega)}^{2}+\|\nabla \boldsymbol{w}\|_{\boldsymbol{L}^{2}(\Omega)}^{2}\right)^{1 / 2} \leq C_{M}\|\nabla \boldsymbol{w}\|_{\boldsymbol{L}^{2}(\Omega)} .
$$

Using the standard estimate

$$
C_{M}\|\overline{\boldsymbol{f}}\|_{-1, \Omega}\|\nabla \boldsymbol{w}\|_{\boldsymbol{L}^{2}(\Omega)} \leq \frac{C_{M}^{2}}{2 \nu_{e}}\|\overline{\boldsymbol{f}}\|_{-1, \Omega}^{2}+\frac{\nu_{e}}{2}\|\nabla \boldsymbol{w}\|_{\boldsymbol{L}^{2}(\Omega)}^{2}
$$

and inserting (8.32) into (8.30) we obtain

$$
\frac{\nu_{e}}{2}\|\nabla \boldsymbol{w}\|_{\boldsymbol{L}^{2}(\Omega)}^{2}+\beta_{0} \sum_{j=1}^{d-1}\left\|\boldsymbol{w} \cdot \boldsymbol{t}_{j}\right\|_{L^{2}\left(\Gamma_{W}\right)}^{2} \leq \frac{C_{M}^{2}}{2 \nu_{e}}\|\overline{\boldsymbol{f}}\|_{-1, \Omega}^{2} .
$$

Finally, from (8.33) we obtain the following a priori estimate for $\|\boldsymbol{w}\|_{1, \Omega}$.

$$
C_{M}^{-2} \frac{\nu_{e}}{2}\|\boldsymbol{w}\|_{1, \Omega}^{2}+\beta_{0} \sum_{j=1}^{d-1}\left\|\boldsymbol{w} \cdot \boldsymbol{t}_{j}\right\|_{L^{2}\left(\Gamma_{W}\right)}^{2} \leq \frac{C_{M}^{2}}{2 \nu_{e}}\|\overline{\boldsymbol{f}}\|_{-1, \Omega}^{2} .
$$




\section{Lemma 8.2}

Let $\overline{\boldsymbol{f}}$ in $L^{2}(\Omega)$ be given. Then there exists at least one solution $\boldsymbol{u}$ in $\boldsymbol{V}_{2}$ and $p$ in $L_{\text {loc }}^{1}(\Omega)$, s.t. (8.26) holds.

\section{Proof:}

The proof proceeds similar to the proof of Theorem 1.2, pp. 164, in [Tem77]. Thus it is assumed that the reader is familiar with this proof and only the necessary modifications will be explained. The first step of the proof in [Tem77] is done using the Galerkin method. Define $\boldsymbol{X}_{m}=\operatorname{span}\left(\boldsymbol{\phi}_{i}\right)_{i=1}^{m} \subset \boldsymbol{V}_{2}$ and seek $\boldsymbol{w}_{m}$ in $\boldsymbol{X}_{m}$ s.t.

$\nu_{e}\left(\nabla \boldsymbol{w}_{m}, \nabla \phi_{k}\right)+b_{\Omega}\left(\boldsymbol{w}_{m}, \boldsymbol{w}_{m}, \phi_{k}\right)+\sum_{j=1}^{d-1}\left\langle\beta_{j} \boldsymbol{w}_{m} \cdot \boldsymbol{t}_{j}, \boldsymbol{\phi}_{k} \cdot \boldsymbol{t}_{j}\right\rangle_{\Gamma_{W}}=\left(\overline{\boldsymbol{f}}, \boldsymbol{\phi}_{k}\right), \quad k=1, \ldots, m$.

The goal is to prove the existence of a solution of (8.35) by applying Lemma 1.4, p.164 in [Tem77], which is a corollary of Brouwer's fixed point theorem. For this purpose we define the operator $P_{m}: \boldsymbol{X}_{m} \mapsto \boldsymbol{X}_{m}$ by

$$
\left(P_{m}(\boldsymbol{w}), \boldsymbol{v}\right)=\nu_{e}(\nabla \boldsymbol{w}, \nabla \boldsymbol{v})+b_{\Omega}(\boldsymbol{w}, \boldsymbol{w}, \boldsymbol{v})+\sum_{j=1}^{d-1}\left\langle\beta_{j} \boldsymbol{w} \cdot \boldsymbol{t}_{j}, \boldsymbol{v} \cdot \boldsymbol{t}_{j}\right\rangle_{\Gamma_{W}}-(\overline{\boldsymbol{f}}, \boldsymbol{v}) .
$$

$P_{m}$ is a well-defined map from $\boldsymbol{X}_{m}$ into itself and continuous. Moreover we have

$$
\begin{aligned}
\left(P_{m}(\boldsymbol{w}), \boldsymbol{w}\right) & =\nu_{e}(\nabla \boldsymbol{w}, \nabla \boldsymbol{w})+\sum_{j=1}^{d-1}\left\langle\beta_{j} \boldsymbol{w} \cdot \boldsymbol{t}_{j}, \boldsymbol{w} \cdot \boldsymbol{t}_{j}\right\rangle_{\Gamma_{W}}-(\overline{\boldsymbol{f}}, \boldsymbol{w}) \\
& \geq \nu_{e}(\nabla \boldsymbol{w}, \nabla \boldsymbol{w})+\sum_{j=1}^{d-1}\left\langle\beta_{j} \boldsymbol{w} \cdot \boldsymbol{t}_{j}, \boldsymbol{w} \cdot \boldsymbol{t}_{j}\right\rangle_{\Gamma_{W}}-\|\overline{\boldsymbol{f}}\|_{-1, \Omega}\|\boldsymbol{w}\|_{1, \Omega} \\
& \geq\|\boldsymbol{w}\|_{1, \Omega}\left(\frac{\nu_{e}}{C_{M}^{2}}\|\boldsymbol{w}\|_{1, \Omega}-\|\overline{\boldsymbol{f}}\|_{-1, \Omega}\right)+\sum_{j=1}^{d-1}\left\langle\beta_{j} \boldsymbol{w} \cdot \boldsymbol{t}_{j}, \boldsymbol{w} \cdot \boldsymbol{t}_{j}\right\rangle_{\Gamma_{W}}
\end{aligned}
$$

Therefore $\left(P_{m}(\boldsymbol{w}), \boldsymbol{w}\right)>0$ for all $\boldsymbol{w}$ with $\|\boldsymbol{w}\|_{1, \Omega}>\frac{C_{M}^{2}}{\nu_{e}}\|\overline{\boldsymbol{f}}\|_{-1, \Omega}$. Note that here we need that at least $\beta_{0} \geq 0$. Then we can apply Lemma 1.4, p.164 in [Tem77], which ensures that, for each $m$, (8.35) has at least one solution, which will be denoted by $\boldsymbol{w}_{m}$.

The final step is to obtain a solution of the infinite dimensional problem from the sequence of finite dimensional solutions $\left(\boldsymbol{w}_{m}\right)_{m}$. According to Lemma 8.1, there exists $C \geq 0$, s.t. $\left\|\boldsymbol{w}_{m}\right\|_{1, \Omega}<C$ for all $m$. Then there exists some $\boldsymbol{w}$ in $\boldsymbol{V}_{2}$ and a subsequence $m_{j}$ s.t.

$$
\boldsymbol{w}_{m_{j}} \longrightarrow \boldsymbol{w} \text { as } j \rightarrow \infty \text { weak in the norm of } \boldsymbol{H}^{1}(\Omega) .
$$

As $\boldsymbol{V}_{2}$ is compact in $\boldsymbol{L}^{2}(\Omega), \boldsymbol{w}_{m_{j}} \longrightarrow \boldsymbol{w}$ strongly in the norm of $\boldsymbol{L}^{2}(\Omega)$. Then it can be shown very similar to [Tem77] (using Lemma 1.5, p.165 in [Tem77]) that $\boldsymbol{w}$ is the solution of (8.26), which completes the proof.

Uniqueness of the solution can be proven similar to Theorem 1.3, p. 167 in [Tem77]. 


\section{Lemma 8.3}

If $d \leq 4$ and if $\nu_{e}$ is "sufficiently large" or $\overline{\boldsymbol{f}}$ "sufficiently small", s.t.

$$
\nu_{e}^{2}>C_{L E S}^{u n}\|\overline{\boldsymbol{f}}\|_{-1, \Omega}, \text { with } C_{L E S}^{u n} \equiv C_{M}^{3} C_{b}
$$

then there exists a unique solution of (8.26).

\section{Proof:}

We proceed similar to the corresponding proof of Theorem 1.3, p. 167 in [Tem77]. Let $\boldsymbol{w}_{1}$, $\boldsymbol{w}_{2}$ denote two different solutions of (8.26) and let $\boldsymbol{w} \equiv \boldsymbol{w}_{1}-\boldsymbol{w}_{2}$. Then $\boldsymbol{w}$ satisfies (cf. [Tem77] eq.(1.40))

$$
\begin{aligned}
& \nu_{e}\|\nabla \boldsymbol{w}\|_{\boldsymbol{L}^{2}(\Omega)}^{2}+\beta_{0} \sum_{j=1}^{d-1}\left\|\boldsymbol{w} \cdot \boldsymbol{t}_{j}\right\|_{L^{2}\left(\Gamma_{W}\right)}^{2} \leq\left|b_{\Omega}\left(\boldsymbol{w}, \boldsymbol{w}_{1}, \boldsymbol{w}\right)\right| \\
& \leq C_{b}\|\boldsymbol{w}\|_{1, \Omega}\left\|\nabla \boldsymbol{w}_{1}\right\|_{\boldsymbol{L}^{2}(\Omega)}\|\boldsymbol{w}\|_{1, \Omega} \leq C_{M}^{2} C_{b} \frac{C_{M}}{\nu_{e}}\|\overline{\boldsymbol{f}}\|_{-1, \Omega}\|\nabla \boldsymbol{w}\|_{\boldsymbol{L}^{2}(\Omega)}^{2}
\end{aligned}
$$

or $\left(\right.$ as $\left.\beta_{0}>0\right)$

$$
\left(\nu_{e}-C_{b} \frac{C_{M}^{3}}{\nu_{e}}\|\overline{\boldsymbol{f}}\|_{-1, \Omega}\right)\|\nabla \boldsymbol{w}\|_{\boldsymbol{L}_{(\Omega)}^{2}}^{2} \leq 0 .
$$

which implies together with (8.37) and (8.31) that $\boldsymbol{w}=0$ and hence $\boldsymbol{w}_{1}=\boldsymbol{w}_{2}$

\section{Remark 8.5}

Discernibly, the lemmata of this subsection require only $\beta_{0} \geq 0$ in (8.10).

\subsubsection{The steady state NSE problem in $\Omega_{i}$}

We proceed considering (8.20)-(8.22). Note that from now on, we write $\Omega_{i}$ instead of $\Omega_{\text {layer }}$. As usual, instead of regarding the non-homogeneous NSE we consider the corresponding homogeneous problem. We define the following spaces

$$
\begin{aligned}
\mathcal{V}_{1} & \equiv\left\{\boldsymbol{v} \in \boldsymbol{C}^{\infty}\left(\Omega_{i}\right) \mid \nabla \cdot \boldsymbol{v}=0 \text { in } \Omega_{i}, \boldsymbol{v}=0 \text { on } \Gamma_{i}, \boldsymbol{v} \cdot \boldsymbol{n}=0 \text { on } \Gamma_{W}\right\}, \\
\boldsymbol{V}_{1} & \equiv\left\{\boldsymbol{v} \in \boldsymbol{H}^{1}\left(\Omega_{i}\right) \mid \nabla \cdot \boldsymbol{v}=0 \text { in } \Omega_{i}, \boldsymbol{v}=0 \text { on } \Gamma_{i}, \boldsymbol{v} \cdot \boldsymbol{n}=0 \text { on } \Gamma_{W}\right\}, \\
\mathcal{V}_{0, W}^{1} & \equiv\left\{\boldsymbol{v} \in \boldsymbol{C}^{\infty}\left(\Omega_{i}\right) \mid \nabla \cdot \boldsymbol{v}=0 \text { in } \Omega_{i}, \boldsymbol{v} \cdot \boldsymbol{n}=0 \text { on } \Gamma_{W}\right\}, \\
\mathrm{V}_{0, W}^{1} & \equiv\left\{\boldsymbol{v} \in \boldsymbol{H}^{1}\left(\Omega_{i}\right) \mid \nabla \cdot \boldsymbol{v}=0 \text { in } \Omega_{i}, \boldsymbol{v} \cdot \boldsymbol{n}=0 \text { on } \Gamma_{W}\right\}, \\
\mathcal{V}_{1,0} & \equiv\left\{\boldsymbol{v} \in \boldsymbol{C}^{\infty}\left(\Omega_{i}\right) \mid \nabla \cdot \boldsymbol{v}=0 \text { in } \Omega_{i}, \boldsymbol{v}=0 \text { on } \Gamma_{i} \cup \Gamma_{W}\right\}, \\
\boldsymbol{V}_{1,0} & \equiv\left\{\boldsymbol{v} \in \boldsymbol{H}^{1}\left(\Omega_{i}\right) \mid \nabla \cdot \boldsymbol{v}=0 \text { in } \Omega_{i}, \boldsymbol{v}=0 \text { on } \Gamma_{i} \cup \Gamma_{W}\right\} .
\end{aligned}
$$

We are looking for a solution of the form

$$
\boldsymbol{u}=\tilde{\boldsymbol{u}}+\boldsymbol{W}
$$

with $\tilde{\boldsymbol{u}}$ in $\boldsymbol{V}_{1,0} . \boldsymbol{W}$ is a suitable extension of $\left.\boldsymbol{w}\right|_{\Gamma_{i}}$ into $\Omega_{i}$, specified in the following lemma. 


\section{Lemma 8.4}

1. Suppose the geometry of Figure 1.8 with $\boldsymbol{y}_{\text {layer }} \geq \boldsymbol{y}_{\text {layer }, 0}>0$ throughout $\Omega_{i}\left(\equiv \Omega_{\text {layer }}\right.$ in Figure 1.2). Then for each $\boldsymbol{v}_{*}$ in $W^{1 / 2,2}\left(\Gamma_{i}\right)$ with $\int_{\Gamma_{i}} \boldsymbol{v}_{*} \cdot \boldsymbol{n} d s=0$ and for each $\alpha>0$ there exists $\boldsymbol{V}$ in $\boldsymbol{H}^{1}\left(\Omega_{i}\right)$ with $\left.\operatorname{Tr}\right|_{\Gamma_{i}} \boldsymbol{V}=\boldsymbol{v}_{*}$ on $\Gamma_{i},\left.\operatorname{Tr}\right|_{\Gamma_{W}} \boldsymbol{V}=0$ on $\Gamma_{W}$, $\nabla \cdot \boldsymbol{V}=0$ in $\Omega_{i}$ and

$$
\begin{aligned}
\left|b_{\Omega_{i}}(\boldsymbol{u}, \boldsymbol{V}, \boldsymbol{u})\right| & \leq \alpha\|\nabla \boldsymbol{u}\|_{\boldsymbol{L}^{2}\left(\Omega_{i}\right)}^{2} \quad \forall \boldsymbol{u} \in \boldsymbol{V}_{1,0}, \\
\|\boldsymbol{V}\|_{1, \Omega_{i}} & \leq C_{1}\left(d, \Omega_{i}\right)\left\|\boldsymbol{v}_{*}\right\|_{1 / 2,2, \Gamma_{i}} .
\end{aligned}
$$

2. Let $\boldsymbol{v}_{*, 1}$ and $\boldsymbol{v}_{*, 2}$ satisfy the assumptions of part 1. Denote $\boldsymbol{V}_{1}$ and $\boldsymbol{V}_{2}$ the corresponding extensions satisfying (8.45) and (8.46). Then the following inequality holds

$$
\left\|\boldsymbol{V}_{1}-\boldsymbol{V}_{2}\right\|_{1, \Omega_{i}} \leq C_{1}\left(d, \Omega_{i}\right)\left\|\boldsymbol{v}_{*, 1}-\boldsymbol{v}_{*, 2}\right\|_{1 / 2,2, \Gamma_{i}} .
$$

\section{Proof:}

Ad 1.: We can apply Lemma 4.2 in [Gal.94II] ch.VIII, pp.27.

Ad 2: We review the proof for part 1. and show that the mapping $\boldsymbol{v}_{*} \mapsto \boldsymbol{V}$ is linear. This will give the assertion.

So let $\boldsymbol{v}_{*, 1}$ and $\boldsymbol{v}_{*, 2}$ in $W^{1 / 2,2}\left(\Gamma_{i}\right)$ satisfy $\int_{\Gamma_{i}} \boldsymbol{v}_{*, 1} \cdot \boldsymbol{n} d s=\int_{\Gamma_{i}} \boldsymbol{v}_{*, 2} \cdot \boldsymbol{n} d s=0$. The construction of the extension takes three steps.

Step (1): due to [Gal94I], exercise III 3.4 (or resp. Lemma 2.2, chapter I in [GR86]), there exist $\phi_{1}, \phi_{2}$ in $\boldsymbol{H}^{1}\left(\Omega_{i}\right)$ s.t. (for $\mathrm{j}=1,2$ )

$$
\begin{aligned}
\nabla \cdot \boldsymbol{\phi}_{j} & =0 \text { in } \Omega_{i}, \\
\boldsymbol{\phi}_{j}=\boldsymbol{v}_{*, j} \quad \text { on } \Gamma_{i}, \quad \boldsymbol{\phi}_{j} & =\mathbf{0} \text { on } \Gamma_{W} \\
\left\|\boldsymbol{\phi}_{j}\right\|_{1, \Omega_{i}} & \leq c\left\|\boldsymbol{v}_{*, j}\right\|_{1 / 2,2, \Gamma_{i}} .
\end{aligned}
$$

First we show that the map $\mathcal{F}_{1}: \boldsymbol{v}_{*, j} \mapsto \boldsymbol{\phi}_{j}$ is linear. Therefore we review the proof given in [GR86]. Let $\boldsymbol{\psi}_{j}(j=1,2)$ be any function of $\boldsymbol{H}^{1}\left(\Omega_{i}\right)$ that satisfies $\boldsymbol{\psi}_{j}=\boldsymbol{v}_{*, j}$ on $\Gamma_{i}$, $\boldsymbol{\psi}_{j}=\mathbf{0}$ on $\Gamma_{W}$. Then according to Green's formula (equation (1.19) in [GR86]):

$$
\int_{\Omega_{i}} \nabla \cdot \boldsymbol{\psi}_{j} d \boldsymbol{x}=\int_{\Gamma_{W} \cup \Gamma_{i}} \boldsymbol{\psi}_{j} \cdot \boldsymbol{n} d \boldsymbol{s}=\int_{\Gamma_{W} \cup \Gamma_{i}} \boldsymbol{v}_{*, j} \cdot \boldsymbol{n} d \boldsymbol{s}=0
$$

Now we use Corollary 2.4, chapter I in [GR86] which states that the operator div is an isomorphism of $\boldsymbol{V}^{\perp} \equiv\left\{\boldsymbol{u} \in \boldsymbol{H}_{0}^{1}\left(\Omega_{i}\right) \mid \nabla \cdot \boldsymbol{u}=0\right\}^{\perp}$ onto $L_{0}^{2}\left(\Omega_{i}\right) \equiv\left\{u \in L^{2}\left(\Omega_{i}\right) \mid \int_{\Omega_{i}} u d \boldsymbol{x}=\right.$ $0\}$. As $\nabla \cdot \boldsymbol{\psi}_{j} \in L_{0}^{2}\left(\Omega_{i}\right)$ (see (8.48)), there exist uniquely determined $\boldsymbol{\zeta}_{j}$ in $\boldsymbol{V}^{\perp}(j=1,2)$ with

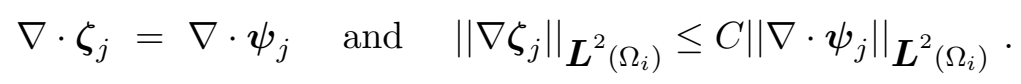

Then we set $\boldsymbol{\phi}_{j} \equiv \boldsymbol{\psi}_{j}-\boldsymbol{\zeta}_{j}(j=1,2)$. Thus so far we have shown that the map $\mathcal{F}_{1}: \boldsymbol{v}_{*, j} \mapsto \boldsymbol{\phi}_{\boldsymbol{j}}$ is linear. Consequently $\phi_{1}-\phi_{2}$ solves

$$
\begin{aligned}
& \nabla \cdot\left(\phi_{1}-\phi_{2}\right)=0 \text { in } \Omega_{i}, \\
& \phi_{1}-\phi_{2}=\boldsymbol{v}_{*, 1}-\boldsymbol{v}_{*, 2} \quad \text { on } \Gamma_{i}, \quad \phi_{1}-\phi_{2}=\mathbf{0} \text { on } \Gamma_{W}
\end{aligned}
$$


Moreover the following estimate holds

$$
\left\|\phi_{1}-\phi_{2}\right\|_{1, \Omega_{i}} \leq c\left\|\boldsymbol{v}_{*, 1}-\boldsymbol{v}_{*, 2}\right\|_{1 / 2,2, \Gamma_{i}} .
$$

Step (2): From now on we restrict ourselves to the three-dimensional case. Moreover we assume that the reader has the textbooks [Gal.94I] and [Gal94II] at hand. The twodimensional case can be handled analogously using the techniques applied in [Gal94II], p.25. According to Exercise 4.1, p.26 in [Gal94II], for $j=1,2$, there exists $\boldsymbol{w}_{j}$ in $\boldsymbol{H}^{2}\left(\Omega_{i}\right)$, s.t. $\phi_{j}=\boldsymbol{\nabla} \times \boldsymbol{w}_{j}$ and $\left\|\boldsymbol{w}_{j}\right\|_{2, \Omega_{i}} \leq C\left\|\boldsymbol{\phi}_{j}\right\|_{1, \Omega_{i}}$, see Exercise 4.1 p.26 in [Gal.94I]]. It can be seen immediately from the proof in [Gal94II], that the map $\mathcal{F}_{2}: \boldsymbol{\phi}_{\boldsymbol{j}} \mapsto \boldsymbol{w}_{j}$ is linear. Therefore we have $\phi_{1}-\phi_{2}=\boldsymbol{\nabla} \times\left(\boldsymbol{w}_{1}-\boldsymbol{w}_{2}\right)$ together with the estimate

$$
\left\|\boldsymbol{w}_{1}-\boldsymbol{w}_{2}\right\|_{2, \Omega_{i}} \leq C\left\|\phi_{1}-\phi_{2}\right\|_{1, \Omega_{i}} .
$$

Step (3): In order to construct an extension satisfying (8.45), we need a suitable "cut-off" function $\psi_{\epsilon}$, see [Gal.94]], Lemma III 6.2. Therefore, denote $y(\boldsymbol{x}) \equiv \operatorname{dist}(\boldsymbol{x}, \partial \Omega)$. Moreover, for any $\epsilon>0$ denote $\gamma(\epsilon)=\exp (-1 / \epsilon)$. According to [Gal.941], Lemma III 6.2, there exists a function $\psi_{\epsilon} \in C^{\infty}(\bar{\Omega})$ such that

(i) $\left|\psi_{\epsilon}(\boldsymbol{x})\right| \leq 1$, for all $\boldsymbol{x} \in \Omega_{i}, \quad$ (ii) $\psi_{\epsilon}(\boldsymbol{x})=1$, if $y(\boldsymbol{x})<\gamma^{2}(\epsilon) /\left(2 \kappa_{1}\right)$,

(iii) $\psi_{\epsilon}(\boldsymbol{x})=0$, if $y(\boldsymbol{x}) \geq 2 \gamma(\epsilon), \quad$ and (iv) $\left|\nabla \psi_{\epsilon}(\boldsymbol{x})\right| \leq \kappa_{2} \epsilon / y(\boldsymbol{x})$, for all $\boldsymbol{x} \in \Omega_{i}$,

with $\kappa_{1}$ and $\kappa_{2}$ being constants introduced in [Gal.941], Lemma III 6.1. To this end, for given $\epsilon>0$ we define

$$
\boldsymbol{V}_{j} \equiv \boldsymbol{\nabla} \times\left(\psi_{\epsilon} \boldsymbol{w}_{j}\right)
$$

and so the map $\mathcal{F}_{3}: \boldsymbol{w}_{j} \mapsto \boldsymbol{V}_{j}$ is linear. Combining step (1) - step (3) reveals that $\mathcal{F}_{3} \circ \mathcal{F}_{2} \circ \mathcal{F}_{1}: \boldsymbol{v}_{*, j} \mapsto \boldsymbol{V}_{j}$ is a linear map. Then from inequality (4.22) in Chapter VIII, see [Gal94II], which states that

$$
\left\|\boldsymbol{V}_{j}\right\|_{1, \Omega_{i}} \leq C_{1}(d, \Omega)\left\|\boldsymbol{v}_{*, j}\right\|_{1 / 2,2, \Gamma_{i}}
$$

we can infer that

$$
\left\|\boldsymbol{V}_{1}-\boldsymbol{V}_{2}\right\|_{1, \Omega_{i}} \leq C_{1}(d, \Omega)\left\|\boldsymbol{v}_{*, 1}-\boldsymbol{v}_{*, 2}\right\|_{1 / 2,2, \Gamma_{i}} .
$$

Let $\boldsymbol{w}$ be the solution of (8.17)-(8.19). Then

$$
0=\int_{\Omega_{i}} \nabla \cdot \boldsymbol{w} d x=\int_{\Gamma_{W}} \boldsymbol{w} \cdot \boldsymbol{n} d s+\int_{\Gamma_{i}} \boldsymbol{w} \cdot \boldsymbol{n} d s=\int_{\Gamma_{i}} \boldsymbol{w} \cdot \boldsymbol{n} d s .
$$


Therefore $\boldsymbol{w}$ satisfies the assumptions of Lemma 8.4. Let $\boldsymbol{W}$ be such an extension of $\boldsymbol{w}$ with

$$
\left|b_{\Omega_{i}}(\boldsymbol{u}, \boldsymbol{W}, \boldsymbol{u})\right| \leq \frac{\nu}{2}|| \nabla \boldsymbol{u} \|_{\boldsymbol{L}^{2}\left(\Omega_{i}\right)}^{2} \quad \forall \boldsymbol{u} \in \boldsymbol{V}_{1,0} .
$$

Using (8.44), (8.20) can be written as

$$
\begin{aligned}
-\boldsymbol{\nabla} \cdot(\nu \nabla \tilde{\boldsymbol{u}})+(\tilde{\boldsymbol{u}} \cdot \boldsymbol{\nabla}) \tilde{\boldsymbol{u}}+(\tilde{\boldsymbol{u}} \cdot \boldsymbol{\nabla}) \boldsymbol{W} & +(\boldsymbol{W} \cdot \boldsymbol{\nabla}) \tilde{\boldsymbol{u}}+\nabla p \\
& =\boldsymbol{f}+\boldsymbol{\nabla} \cdot(\nu \nabla \boldsymbol{W})-(\boldsymbol{W} \cdot \boldsymbol{\nabla}) \boldsymbol{W}
\end{aligned}
$$

Multiplying (8.50) with $\boldsymbol{v}$ in $\mathcal{V}_{1,0}$ and integration by parts gives

$$
\begin{aligned}
\nu(\nabla \tilde{\boldsymbol{u}}, \nabla \boldsymbol{v})+b_{\Omega_{i}}(\tilde{\boldsymbol{u}}, \tilde{\boldsymbol{u}}, \boldsymbol{v})+b_{\Omega_{i}}(\tilde{\boldsymbol{u}}, \boldsymbol{W}, \boldsymbol{v}) & +b_{\Omega_{i}}(\boldsymbol{W}, \tilde{\boldsymbol{u}}, \boldsymbol{v}) \\
& =(\boldsymbol{f}, \boldsymbol{v})+\nu(\nabla \boldsymbol{W}, \nabla \boldsymbol{v})-b_{\Omega_{i}}(\boldsymbol{W}, \boldsymbol{W}, \boldsymbol{v}) .
\end{aligned}
$$

Then the variational form corresponding to (8.20)-(8.22) reads:

Steady state NSE problem in $\Omega_{i}$ : Find $\tilde{\boldsymbol{u}}$ in $\boldsymbol{V}_{1,0}$, s.t. (8.51) holds for all $\boldsymbol{v}$ in $\boldsymbol{V}_{1,0}$.

Now we give an a priori estimate for the steady state NSE problem in $\Omega_{i}$.

\section{Lemma 8.5}

Concerning the steady state NSE problem (8.51) in $\Omega_{i}$ and problem (8.20)-(8.22) the following a priori estimates hold

$$
\frac{\nu}{2}\|\nabla \tilde{\boldsymbol{u}}\|_{\boldsymbol{L}^{2}\left(\Omega_{i}\right)} \leq K_{a}(\boldsymbol{f}, \nu, \boldsymbol{w}), \quad \frac{\nu}{2}\|\nabla \boldsymbol{u}\|_{\boldsymbol{L}^{2}\left(\Omega_{i}\right)} \leq K_{a}(\boldsymbol{f}, \nu, \boldsymbol{w}) .
$$

with

$$
K_{a}(\boldsymbol{f}, \nu, \boldsymbol{w}) \equiv C_{u, 1}^{a p}\|\boldsymbol{f}\|_{-1, \Omega_{i}}+C_{u, 2}^{a p}\|\boldsymbol{w}\|_{1 / 2,2, \Gamma_{i}}+C_{u, 3}^{a p}\|\boldsymbol{w}\|_{1 / 2,2, \Gamma_{i}}^{2} .
$$

and the following constants

$$
\begin{aligned}
C_{u, 1}^{a p} & \equiv C_{P, i}, \quad C_{u, 2}^{a p} \equiv \frac{3}{2} \nu C_{1}\left(d, \Omega_{i}\right), C_{P, i} \equiv \sqrt{1+c_{P o i, 0}^{2}\left(\Omega_{i}\right)} \\
C_{M, i} & \equiv \sqrt{1+c_{P o i}^{2}\left(\Omega_{i}\right)}, \quad C_{u, 3}^{a p} \equiv C_{b} C_{P, i} C_{1}^{2}\left(d, \Omega_{i}\right)
\end{aligned}
$$

\section{Proof:}

In (8.51) we set $\boldsymbol{v}=\tilde{\boldsymbol{u}}$ and obtain (recall that $b_{\Omega_{i}}(\boldsymbol{W}, \tilde{\boldsymbol{u}}, \tilde{\boldsymbol{u}})=b_{\Omega_{i}}(\tilde{\boldsymbol{u}}, \tilde{\boldsymbol{u}}, \tilde{\boldsymbol{u}})=0$ )

$$
\nu\|\nabla \tilde{\boldsymbol{u}}\|_{\boldsymbol{L}_{\left(\Omega_{i}\right)}}+b_{\Omega_{i}}(\tilde{\boldsymbol{u}}, \boldsymbol{W}, \tilde{\boldsymbol{u}})=(\boldsymbol{f}, \tilde{\boldsymbol{u}})+\nu(\nabla \boldsymbol{W}, \nabla \tilde{\boldsymbol{u}})-b_{\Omega_{i}}(\boldsymbol{W}, \boldsymbol{W}, \tilde{\boldsymbol{u}}) .
$$

Using the choice of $\boldsymbol{W}$ and making use of the triangle inequality gives

$$
\frac{\nu}{2}\|\nabla \tilde{\boldsymbol{u}}\|_{\boldsymbol{L}^{2}\left(\Omega_{i}\right)}^{2} \leq|(\boldsymbol{f}, \tilde{\boldsymbol{u}})|+\nu\|\nabla \boldsymbol{W}\|_{\boldsymbol{L}^{2}\left(\Omega_{i}\right)}\|\nabla \tilde{\boldsymbol{u}}\|_{\boldsymbol{L}^{2}\left(\Omega_{i}\right)}+\left|b_{\Omega_{i}}(\boldsymbol{W}, \boldsymbol{W}, \tilde{\boldsymbol{u}})\right| .
$$


Since $\boldsymbol{W}$ in $\boldsymbol{H}^{1}\left(\Omega_{i}\right)$ and $\tilde{\boldsymbol{u}}$ in $\boldsymbol{V}_{1,0}$ we have the following inequality (see (B.4))

$$
\begin{aligned}
\left|b_{\Omega_{i}}(\boldsymbol{W}, \boldsymbol{W}, \tilde{\boldsymbol{u}})\right| & \leq C_{b}\|\boldsymbol{W}\|_{1, \Omega_{i}}\|\nabla \boldsymbol{W}\|_{\boldsymbol{L}^{2}\left(\Omega_{i}\right)}\|\tilde{\boldsymbol{u}}\|_{1, \Omega_{i}} \\
& \leq C_{b} C_{P, i}\|\boldsymbol{W}\|_{1, \Omega_{i}}^{2}\|\nabla \tilde{\boldsymbol{u}}\|_{\boldsymbol{L}^{2}\left(\Omega_{i}\right)} .
\end{aligned}
$$

Substituting the last into the last but one inequality gives

$$
\begin{aligned}
\frac{\nu}{2}\|\nabla \tilde{\boldsymbol{u}}\|_{\boldsymbol{L}^{2}\left(\Omega_{i}\right)}^{2} \leq & C_{P, i}\|\boldsymbol{f}\|_{-1, \Omega_{i}}\|\nabla \tilde{\boldsymbol{u}}\|_{\boldsymbol{L}^{2}\left(\Omega_{i}\right)}+\nu\|\nabla \boldsymbol{W}\|_{\boldsymbol{L}^{2}\left(\Omega_{i}\right)}\|\nabla \tilde{\boldsymbol{u}}\|_{\boldsymbol{L}^{2}\left(\Omega_{i}\right)} \\
& +C_{b} C_{P, i}\|\boldsymbol{W}\|_{1, \Omega_{i}}^{2}\|\nabla \tilde{\boldsymbol{u}}\|_{\boldsymbol{L}^{2}\left(\Omega_{i}\right)}
\end{aligned}
$$

Thus we get the following a priori estimate

$$
\frac{\nu}{2}\|\nabla \tilde{\boldsymbol{u}}\|_{\boldsymbol{L}^{2}\left(\Omega_{i}\right)} \leq C_{P, i}\|\boldsymbol{f}\|_{-1, \Omega_{i}}+\nu\|\nabla \boldsymbol{W}\|_{\boldsymbol{L}^{2}\left(\Omega_{i}\right)}+C_{b} C_{P, i}\|\boldsymbol{W}\|_{1, \Omega_{i}}^{2} .
$$

Using (8.46) gives

$$
\begin{aligned}
\frac{\nu}{2}\|\nabla \tilde{\boldsymbol{u}}\|_{L^{2}\left(\Omega_{i}\right)} \leq C_{P, i}\|\boldsymbol{f}\|_{-1, \Omega_{i}} & +\nu C_{1}\left(d, \Omega_{i}\right)\|\boldsymbol{w}\|_{1 / 2,2, \Gamma_{i}} \\
& +C_{b} C_{P, i} C_{1}^{2}\left(d, \Omega_{i}\right)\|\boldsymbol{w}\|_{1 / 2,2, \Gamma_{i}}^{2} .
\end{aligned}
$$

Then we can get an a priori estimate for $\boldsymbol{u}=\tilde{\boldsymbol{u}}+\boldsymbol{W}$.

$$
\begin{aligned}
\frac{\nu}{2}\|\nabla \boldsymbol{u}\|_{L^{2}\left(\Omega_{i}\right)} & \leq \frac{\nu}{2}\|\nabla \tilde{\boldsymbol{u}}\|_{\boldsymbol{L}_{\left(\Omega_{i}\right)}}+\frac{\nu}{2}\|\nabla \boldsymbol{W}\|_{\boldsymbol{L}^{2}\left(\Omega_{i}\right)} \leq \frac{\nu}{2}\|\nabla \tilde{\boldsymbol{u}}\|_{\boldsymbol{L}^{2}\left(\Omega_{i}\right)}+\frac{\nu}{2}\|\boldsymbol{W}\|_{1, \Omega_{i}} \\
& \leq \frac{\nu}{2}\|\nabla \tilde{\boldsymbol{u}}\|_{\boldsymbol{L}_{\left(\Omega_{i}\right)}}+\frac{\nu}{2} C_{1}\left(d, \Omega_{i}\right)\|\boldsymbol{w}\|_{1 / 2,2, \Gamma_{i}} .
\end{aligned}
$$

Combining the last two inequalities we finally get

$$
\begin{aligned}
\frac{\nu}{2}\|\nabla \boldsymbol{u}\|_{L^{2}\left(\Omega_{i}\right)} \leq & C_{P, i}\|\boldsymbol{f}\|_{-1, \Omega_{i}}+\frac{3 \nu}{2} C_{1}\left(d, \Omega_{i}\right)\|\boldsymbol{w}\|_{1 / 2,2, \Gamma_{i}} \\
& +C_{b} C_{P, i} C_{1}^{2}\left(d, \Omega_{i}\right)\|\boldsymbol{w}\|_{1 / 2,2, \Gamma_{i}}^{2} .
\end{aligned}
$$

\section{Lemma 8.6}

For each given $\boldsymbol{w}$ in $\boldsymbol{V}_{2}$ there exists at least one solution of (8.51).

\section{Proof:}

We can apply Theorem VIII, 4.1, p.32 in [Gal.94II], as all its assumptions are fulfilled.

\section{Lemma 8.7}

Suppose $d \leq 4$. The solution of (8.51) is unique if

$$
\nu^{2}>4 C_{b} C_{P, i}^{2} K_{a}(\boldsymbol{f}, \nu, \boldsymbol{w}) .
$$




\section{Proof:}

Let us suppose there are two solutions $\tilde{\boldsymbol{u}}_{0}=\boldsymbol{u}_{0}-\boldsymbol{W}$ and $\tilde{\boldsymbol{u}}_{1}=\boldsymbol{u}_{1}-\boldsymbol{W}$, where $\boldsymbol{W}$ is given by $(8.49)$. Denote $\tilde{\boldsymbol{u}}=\tilde{\boldsymbol{u}}_{0}-\tilde{\boldsymbol{u}}_{1}$ Then $\tilde{\boldsymbol{u}}_{0}$ and $\tilde{\boldsymbol{u}}_{1}$ resp. fulfill the following equations

$$
\begin{aligned}
& \nu\left(\nabla \tilde{\boldsymbol{u}}_{0}, \nabla \boldsymbol{v}\right)+b_{\Omega_{i}}\left(\tilde{\boldsymbol{u}}_{0}, \tilde{\boldsymbol{u}}_{0}, \boldsymbol{v}\right)+b_{\Omega_{i}}\left(\tilde{\boldsymbol{u}}_{0}, \boldsymbol{W}, \boldsymbol{v}\right)+b_{\Omega_{i}}\left(\boldsymbol{W}, \tilde{\boldsymbol{u}}_{0}, \boldsymbol{v}\right)=(\tilde{\boldsymbol{f}}, \boldsymbol{v}) \\
& \nu\left(\nabla \tilde{\boldsymbol{u}}_{1}, \nabla \boldsymbol{v}\right)+b_{\Omega_{i}}\left(\tilde{\boldsymbol{u}}_{1}, \tilde{\boldsymbol{u}}_{1}, \boldsymbol{v}\right)+b_{\Omega_{i}}\left(\tilde{\boldsymbol{u}}_{1}, \boldsymbol{W}, \boldsymbol{v}\right)+b_{\Omega_{i}}\left(\boldsymbol{W}, \tilde{\boldsymbol{u}}_{1}, \boldsymbol{v}\right)=(\tilde{\boldsymbol{f}}, \boldsymbol{v})
\end{aligned}
$$

where $\tilde{\boldsymbol{f}} \equiv \boldsymbol{f}+\nabla \cdot(\nu \nabla \boldsymbol{W})-\boldsymbol{W} \nabla \boldsymbol{W}$. We set $\boldsymbol{v}=\tilde{\boldsymbol{u}}$ in both equations. Subtracting the second equation from the first one gives (after expanding)

$$
\nu\|\nabla \tilde{\boldsymbol{u}}\|_{\boldsymbol{L}^{2}\left(\Omega_{i}\right)}^{2}=-b_{\Omega_{i}}\left(\tilde{\boldsymbol{u}}, \tilde{\boldsymbol{u}}_{1}, \tilde{\boldsymbol{u}}\right)-b_{\Omega_{i}}(\tilde{\boldsymbol{u}}, \boldsymbol{W}, \tilde{\boldsymbol{u}}) .
$$

Using (8.49) gives

$$
\frac{\nu}{2}|| \nabla \tilde{\boldsymbol{u}} \|_{\boldsymbol{L}^{2}\left(\Omega_{i}\right)}^{2} \leq\left|b_{\Omega_{i}}\left(\tilde{\boldsymbol{u}}, \tilde{\boldsymbol{u}}_{1}, \tilde{\boldsymbol{u}}\right)\right| .
$$

Taking into account (B.4) we get

$$
\begin{aligned}
& \frac{\nu}{2}\|\nabla \tilde{\boldsymbol{u}}\|_{\boldsymbol{L}^{2}\left(\Omega_{i}\right)}^{2} \leq\left|b_{\Omega_{i}}\left(\tilde{\boldsymbol{u}}, \tilde{\boldsymbol{u}}_{1}, \tilde{\boldsymbol{u}}\right)\right| \leq C_{b}\left\|\nabla \tilde{\boldsymbol{u}}_{1}\right\|_{\boldsymbol{L}^{2}\left(\Omega_{i}\right)}\|\tilde{\boldsymbol{u}}\|_{1, \Omega_{i}}^{2} \\
& \leq C_{b} C_{P, i}^{2}\left\|\nabla \tilde{\boldsymbol{u}}_{1}\right\|_{\boldsymbol{L}_{\left(\Omega_{i}\right)}^{2}}\|\nabla \tilde{\boldsymbol{u}}\|_{\boldsymbol{L}^{2}\left(\Omega_{i}\right)}^{2} \leq C_{b} C_{P, i}^{2} \frac{2}{\nu} K_{a}(\boldsymbol{f}, \nu, \boldsymbol{w})\|\nabla \tilde{\boldsymbol{u}}\|_{\boldsymbol{L}^{2}\left(\Omega_{i}\right)}^{2}
\end{aligned}
$$

which can be rearranged to

$$
\left(\frac{\nu}{2}-C_{b} C_{P, i}^{2} \frac{2}{\nu} K_{a}(\boldsymbol{f}, \nu, \boldsymbol{w})\right)\|\nabla \tilde{\boldsymbol{u}}\|_{\boldsymbol{L}^{2}\left(\Omega_{i}\right)}^{2} \leq 0 .
$$

This implies $\tilde{\boldsymbol{u}}=0$, taking into account (B.15) and (8.55).

\section{Remark 8.6}

Before giving the proof, it is worthily commenting the statement of Lemma 8.7. Condition 8.55 is often not satisfied in high Reynolds number applications, i.e. if $\|\boldsymbol{w}\|_{1 / 2,2, \Gamma_{i}}^{2}=\mathcal{O}(1)$ and $\nu \ll 1$. However, in important flow situations this scheme still remains meaningful. Firstly, we consider a flow with separation, see Figure 8.2. Then, by suitably choosing $\Omega_{i}$ we can try to ensure that $\|\boldsymbol{w}\|_{1 / 2,2, \Gamma_{i}}$ is small enough. Secondly, in attached boundary-layer flows typically $\|\boldsymbol{w}\|_{1 / 2,2, \Gamma_{i}}^{2}=\mathcal{O}(1)$. But then we can determine $\beta_{j}$ from a log-law resp. or from a power-law resp. without any need to perform a DNS, see Section 8.4.4.

\section{Remark 8.7}

Instead of the full Navier-Stokes equations, it is also very interesting studying the reduced boundary-layer problem (5.10)-(5.12). The analysis for this problem has been considered by Caussignac and TouzAni. In [CT90] they proved existence and uniqueness of a weak solution of a simplified variant of (5.10)-(5.12). 


\subsection{The coupled steady state problem}

From Lemmata 8.2, 8.3 8.6, and 8.7 we can infer the following Corollary:

\section{Corollary 8.1}

Assume that the assumptions of Lemmata 8.3 and 8.7 hold. Denote

$$
\boldsymbol{V}_{\text {def }}^{2} \equiv\left\{\boldsymbol{w} \in \boldsymbol{V}_{2} \mid \boldsymbol{w} \text { satisfies (8.55) }\right\} .
$$

Then the following operators are well-defined:

$$
\begin{aligned}
& T_{N S \rightarrow L E S}: \boldsymbol{V}_{0, W}^{1} \longrightarrow \boldsymbol{V}_{2}, \boldsymbol{u} \longrightarrow \boldsymbol{w}, \boldsymbol{w} \text { solution of }(8.17)-(8.19) \\
& T_{L E S \rightarrow N S}: \boldsymbol{V}_{d e f}^{2} \longrightarrow \boldsymbol{V}_{0, W}^{1}, \boldsymbol{w} \longrightarrow \boldsymbol{u}, \boldsymbol{u} \text { solution of }(8.20)-(8.22) .
\end{aligned}
$$

\section{Proof:}

The statement follows immediately from Lemmata 8.2, 8.3, 8.6, and 8.7.

An outline of this section reads as follows: Given that $\|\boldsymbol{f}\|_{-1}$ is "sufficiently small" or $\nu$ is "sufficiently large", we can show:

- $T_{L E S \rightarrow N S}$ is continuous on a suitable subset $\boldsymbol{V}_{\text {cont }}^{2} \subset \boldsymbol{V}_{d e f}^{2}$.

- $T_{N S \rightarrow L E S}$ is continuous and compact on $\boldsymbol{V}_{0, W}^{1}$, if $\Gamma_{W}$ is piecewise smooth.

- Using the Schauder Fixed-Point Theorem, we show that $T_{L E S \rightarrow N S} \circ T_{N S \rightarrow L E S}$ and $T_{N S \rightarrow L E S} \circ T_{L E S \rightarrow N S}$ have a fixed point; the fixed point is a solution of the coupled problem (8.11)-(8.16).

- The fixed point is uniquely determined, if an additional assumption regarding $\|\boldsymbol{f}\|_{-1}$ and $\nu$ holds.

\section{Lemma 8.8}

Suppose that the assumptions of Corollary 8.1 hold. We define

$$
\boldsymbol{V}_{\text {cont }}^{2} \equiv\left\{\boldsymbol{w} \in \boldsymbol{V}_{\text {def }}^{2} \mid \boldsymbol{w} \text { satisfies }(8.60)\right\}
$$

with

$$
\frac{3 \nu}{8}>C_{b} \frac{2}{\nu} C_{P, i}^{2} K_{a}(\boldsymbol{f}, \nu, \boldsymbol{w})+C_{b} C_{1}\left(d, \Omega_{i}\right) C_{P, i}\|\boldsymbol{w}\|_{1 / 2,2, \Gamma_{i}} .
$$

Then the operator $\left.T_{L E S \rightarrow N S}\right|_{\boldsymbol{V}_{\text {cont }}^{2}}$ is continuous.

\section{Proof:}

Requiring the assumptions of Lemma 8.7, $T_{L E S \rightarrow N S}$ is well-defined. Let a sequence $\boldsymbol{w}_{n}$ in $\boldsymbol{V}_{\text {def }}^{2}$ be given with $\boldsymbol{w}_{n} \longrightarrow \boldsymbol{w}$ in $\boldsymbol{H}^{1}(\Omega)$. Denote $\boldsymbol{u}_{n} \equiv T_{L E S \rightarrow N S}\left(\boldsymbol{w}_{n}\right)$ and $\boldsymbol{u} \equiv$ $T_{L E S \rightarrow N S}(\boldsymbol{w})$. Then we have to show that $\boldsymbol{u}_{n} \longrightarrow \boldsymbol{u}$ in $\boldsymbol{H}^{1}\left(\Omega_{i}\right)$. 
Based on (8.44) with Lemma 8.4, we write $\boldsymbol{u}_{n}=\tilde{\boldsymbol{u}}_{n}+\boldsymbol{W}_{n}$ and $\boldsymbol{u}=\tilde{\boldsymbol{u}}+\boldsymbol{W}$, where $\boldsymbol{W}_{n}$ and $\boldsymbol{W}$ satisfy (8.49). Since

$$
\begin{aligned}
\left\|\boldsymbol{u}_{n}-\boldsymbol{u}\right\|_{1, \Omega_{i}} & \leq\left\|\tilde{\boldsymbol{u}}_{n}-\tilde{\boldsymbol{u}}\right\|_{1, \Omega_{i}}+\left\|\boldsymbol{W}_{n}-\boldsymbol{W}\right\|_{1, \Omega_{i}} \leq\left\|\tilde{\boldsymbol{u}}_{n}-\tilde{\boldsymbol{u}}\right\|_{1, \Omega_{i}}+C_{1}\left(d, \Omega_{i}\right)\left\|\boldsymbol{w}_{n}-\boldsymbol{w}\right\|_{1 / 2,2, \Gamma_{i}} \\
& \leq\left\|\tilde{\boldsymbol{u}}_{n}-\tilde{\boldsymbol{u}}\right\|_{1, \Omega_{i}}+C_{1}\left(d, \Omega_{i}\right) C_{t r}\left\|\boldsymbol{w}_{n}-\boldsymbol{w}\right\|_{1, \Omega_{i}}
\end{aligned}
$$

we can complete the proof by showing $\left\|\tilde{\boldsymbol{u}}_{n}-\tilde{\boldsymbol{u}}\right\|_{1, \Omega_{i}} \rightarrow 0$ as $n \rightarrow \infty$. Due to (8.51) the following equations hold

$$
\begin{aligned}
\nu\left(\nabla \tilde{\boldsymbol{u}}_{n}, \nabla \boldsymbol{v}\right)+b_{\Omega_{i}}\left(\tilde{\boldsymbol{u}}_{n}, \tilde{\boldsymbol{u}}_{n}, \boldsymbol{v}\right) & +b_{\Omega_{i}}\left(\tilde{\boldsymbol{u}}_{n}, \boldsymbol{W}_{n}, \boldsymbol{v}\right)+b_{\Omega_{i}}\left(\boldsymbol{W}_{n}, \tilde{\boldsymbol{u}}_{n}, \boldsymbol{v}\right) \\
& =(\boldsymbol{f}, \boldsymbol{v})-\nu\left(\nabla \boldsymbol{W}_{n}, \nabla \boldsymbol{v}\right)-b_{\Omega_{i}}\left(\boldsymbol{W}_{n}, \boldsymbol{W}_{n}, \boldsymbol{v}\right) \\
\nu(\nabla \tilde{\boldsymbol{u}}, \nabla \boldsymbol{v})+b_{\Omega_{i}}(\tilde{\boldsymbol{u}}, \tilde{\boldsymbol{u}}, \boldsymbol{v}) & +b_{\Omega_{i}}(\tilde{\boldsymbol{u}}, \boldsymbol{W}, \boldsymbol{v})+b_{\Omega_{i}}(\boldsymbol{W}, \tilde{\boldsymbol{u}}, \boldsymbol{v}) \\
& =(\boldsymbol{f}, \boldsymbol{v})-\nu(\nabla \boldsymbol{W}, \nabla \boldsymbol{v})-b_{\Omega_{i}}(\boldsymbol{W}, \boldsymbol{W}, \boldsymbol{v}) .
\end{aligned}
$$

Denote $\boldsymbol{\phi}_{n} \equiv \tilde{\boldsymbol{u}}_{n}-\tilde{\boldsymbol{u}}$ and $\boldsymbol{\Psi}_{n} \equiv \boldsymbol{W}_{n}-\boldsymbol{W}$. Subtracting the second equation from the first one and setting $\boldsymbol{v}=\phi_{n}$ gives

$$
\begin{aligned}
\nu\left(\nabla \boldsymbol{\phi}_{n}, \nabla \boldsymbol{\phi}_{n}\right) & +b_{\Omega_{i}}\left(\tilde{\boldsymbol{u}}_{n}, \tilde{\boldsymbol{u}}_{n}, \boldsymbol{\phi}_{n}\right)-b_{\Omega_{i}}\left(\tilde{\boldsymbol{u}}, \tilde{\boldsymbol{u}}, \boldsymbol{\phi}_{n}\right)+b_{\Omega_{i}}\left(\tilde{\boldsymbol{u}}_{n}, \boldsymbol{W}_{n}, \boldsymbol{\phi}_{n}\right) \\
& -b_{\Omega_{i}}\left(\tilde{\boldsymbol{u}}, \boldsymbol{W}, \boldsymbol{\phi}_{n}\right)+b_{\Omega_{i}}\left(\boldsymbol{W}_{n}, \tilde{\boldsymbol{u}}_{n}, \boldsymbol{\phi}_{n}\right)-b_{\Omega_{i}}\left(\boldsymbol{W}, \tilde{\boldsymbol{u}}, \boldsymbol{\phi}_{n}\right) \\
& =-\nu\left(\nabla \boldsymbol{\Psi}_{n}, \nabla \boldsymbol{\phi}_{n}\right)-b_{\Omega_{i}}\left(\boldsymbol{W}_{n}, \boldsymbol{W}_{n}, \boldsymbol{\phi}_{n}\right)+b_{\Omega_{i}}\left(\boldsymbol{W}, \boldsymbol{W}, \boldsymbol{\phi}_{n}\right) .
\end{aligned}
$$

Now we proceed using the following transformations:

$$
\begin{aligned}
b_{\Omega_{i}}\left(\tilde{\boldsymbol{u}}_{n}, \tilde{\boldsymbol{u}}_{n}, \boldsymbol{\phi}_{n}\right)-b_{\Omega_{i}}\left(\tilde{\boldsymbol{u}}, \tilde{\boldsymbol{u}}, \boldsymbol{\phi}_{n}\right) & =b_{\Omega_{i}}\left(\boldsymbol{\phi}_{n}, \tilde{\boldsymbol{u}}_{n}, \boldsymbol{\phi}_{n}\right) \\
b_{\Omega_{i}}\left(\tilde{\boldsymbol{u}}_{n}, \boldsymbol{W}_{n}, \boldsymbol{\phi}_{n}\right)-b_{\Omega_{i}}\left(\tilde{\boldsymbol{u}}, \boldsymbol{W}, \boldsymbol{\phi}_{n}\right) & =b_{\Omega_{i}}\left(\boldsymbol{\phi}_{n}, \boldsymbol{W}_{n}, \boldsymbol{\phi}_{n}\right)+b_{\Omega_{i}}\left(\tilde{\boldsymbol{u}}, \boldsymbol{\Psi}_{n}, \boldsymbol{\phi}_{n}\right), \\
b_{\Omega_{i}}\left(\boldsymbol{W}_{n}, \tilde{\boldsymbol{u}}_{n}, \boldsymbol{\phi}_{n}\right)-b_{\Omega_{i}}\left(\boldsymbol{W}, \tilde{\boldsymbol{u}}, \boldsymbol{\phi}_{n}\right) & =b_{\Omega_{i}}\left(\boldsymbol{\Psi}_{n}, \tilde{\boldsymbol{u}}_{n}, \boldsymbol{\phi}_{n}\right) \\
b_{\Omega_{i}}\left(\boldsymbol{W}_{n}, \boldsymbol{W}_{n}, \boldsymbol{\phi}_{n}\right)-b_{\Omega_{i}}\left(\boldsymbol{W}, \boldsymbol{W}, \boldsymbol{\phi}_{n}\right) & =b_{\Omega_{i}}\left(\boldsymbol{\Psi}_{n}, \boldsymbol{W}_{n}, \boldsymbol{\phi}_{n}\right)+b_{\Omega_{i}}\left(\boldsymbol{W}, \boldsymbol{\Psi}_{n}, \boldsymbol{\phi}_{n}\right) .
\end{aligned}
$$

Then the following equation holds

$$
\begin{aligned}
\nu\left(\nabla \boldsymbol{\phi}_{n}, \nabla \boldsymbol{\phi}_{n}\right)= & -b_{\Omega_{i}}\left(\boldsymbol{\phi}_{n}, \tilde{\boldsymbol{u}}_{n}, \boldsymbol{\phi}_{n}\right)-b_{\Omega_{i}}\left(\boldsymbol{\phi}_{n}, \boldsymbol{W}_{n}, \boldsymbol{\phi}_{n}\right)-b_{\Omega_{i}}\left(\tilde{\boldsymbol{u}}, \boldsymbol{\Psi}_{n}, \boldsymbol{\phi}_{n}\right)-b_{\Omega_{i}}\left(\boldsymbol{\Psi}_{n}, \tilde{\boldsymbol{u}}_{n}, \boldsymbol{\phi}_{n}\right) \\
& -\nu\left(\nabla \mathbf{\Psi}_{n}, \nabla \boldsymbol{\phi}_{n}\right)-b_{\Omega_{i}}\left(\boldsymbol{\Psi}_{n}, \boldsymbol{W}_{n}, \boldsymbol{\phi}_{n}\right)-b_{\Omega_{i}}\left(\boldsymbol{W}, \boldsymbol{\Psi}_{n}, \boldsymbol{\phi}_{n}\right)
\end{aligned}
$$

This yields the following inequality

$$
\begin{aligned}
\nu\left(\nabla \boldsymbol{\phi}_{n}, \nabla \boldsymbol{\phi}_{n}\right) \leq & \left|b_{\Omega_{i}}\left(\boldsymbol{\phi}_{n}, \tilde{\boldsymbol{u}}_{n}, \boldsymbol{\phi}_{n}\right)\right|+\left|b_{\Omega_{i}}\left(\boldsymbol{\phi}_{n}, \boldsymbol{W}_{n}, \boldsymbol{\phi}_{n}\right)\right|+\left|b_{\Omega_{i}}\left(\tilde{\boldsymbol{u}}, \boldsymbol{\Psi}_{n}, \boldsymbol{\phi}_{n}\right)\right|+\left|b_{\Omega_{i}}\left(\boldsymbol{\Psi}_{n}, \tilde{\boldsymbol{u}}_{n}, \boldsymbol{\phi}_{n}\right)\right| \\
& +\left|\nu\left(\nabla \boldsymbol{\Psi}_{n}, \nabla \boldsymbol{\phi}_{n}\right)\right|+\left|b_{\Omega_{i}}\left(\boldsymbol{\Psi}_{n}, \boldsymbol{W}_{n}, \boldsymbol{\phi}_{n}\right)\right|+\left|b_{\Omega_{i}}\left(\boldsymbol{W}, \boldsymbol{\Psi}_{n}, \boldsymbol{\phi}_{n}\right)\right| .
\end{aligned}
$$


The following estimates hold

$$
\begin{aligned}
& \left|b_{\Omega_{i}}\left(\boldsymbol{\phi}_{n}, \tilde{\boldsymbol{u}}_{n}, \boldsymbol{\phi}_{n}\right)\right| \leq C_{b}|| \phi_{n}\left\|_{1, \Omega_{i}}^{2} \mid\right\| \nabla \tilde{\boldsymbol{u}}_{n} \|_{L^{2}\left(\Omega_{i}\right)} \\
& \leq C_{b} \frac{2}{\nu} C_{P, i}^{2} K_{a}\left(\boldsymbol{f}, \nu, \boldsymbol{w}_{n}\right)\left\|\nabla \boldsymbol{\phi}_{n}\right\|_{L^{2}\left(\Omega_{i}\right)}^{2} \\
& \left|b_{\Omega_{i}}\left(\boldsymbol{\phi}_{n}, \boldsymbol{W}_{n}, \boldsymbol{\phi}_{n}\right)\right|=\left|b_{\Omega_{i}}\left(\boldsymbol{\phi}_{n}, \boldsymbol{\phi}_{n}, \boldsymbol{W}_{n}\right)\right| \leq C_{b}|| \boldsymbol{\phi}_{n}\left\|_{1, \Omega_{i}}|| \nabla \boldsymbol{\phi}_{n}\right\|_{L^{2}\left(\Omega_{i}\right)}|| \boldsymbol{W}_{n} \|_{1, \Omega_{i}} \\
& \leq C_{b} C_{1}\left(d, \Omega_{i}\right) C_{P, i}\left\|\boldsymbol{w}_{n}\right\|_{1 / 2,2, \Gamma_{i}}\left\|\nabla \phi_{n}\right\|_{L^{2}\left(\Omega_{i}\right)}^{2} \\
& \left|b_{\Omega_{i}}\left(\tilde{\boldsymbol{u}}, \boldsymbol{\Psi}_{n}, \boldsymbol{\phi}_{n}\right)\right|=\left|b_{\Omega_{i}}\left(\tilde{\boldsymbol{u}}, \boldsymbol{\phi}_{n}, \boldsymbol{\Psi}_{n}\right)\right| \leq C_{b}|| \tilde{\boldsymbol{u}}\left\|_{1, \Omega_{i}}|| \boldsymbol{\Psi}_{n}\right\|_{1, \Omega_{i}}|| \nabla \boldsymbol{\phi}_{n} \|_{L^{2}\left(\Omega_{i}\right)} \\
& \leq C_{b} \frac{2}{\nu} K_{a}(\boldsymbol{f}, \nu, \boldsymbol{w}) C_{P, i} C_{1}\left(d, \Omega_{i}\right)\left\|\boldsymbol{w}_{n}-\boldsymbol{w}\right\|_{1 / 2,2, \Gamma_{i}}\left\|\nabla \boldsymbol{\phi}_{n}\right\|_{L^{2}\left(\Omega_{i}\right)} \\
& \leq \frac{8}{\nu^{3}} K_{a}(\boldsymbol{f}, \nu, \boldsymbol{w})^{2} C_{b}^{2} C_{1}^{2}\left(d, \Omega_{i}\right) C_{P, i}^{2}\left\|\boldsymbol{w}_{n}-\boldsymbol{w}\right\|_{1 / 2,2, \Gamma_{i}}^{2}+\frac{\nu}{8}\left\|\nabla \boldsymbol{\phi}_{n}\right\|_{L^{2}\left(\Omega_{i}\right)}^{2} \\
& \left|b_{\Omega_{i}}\left(\boldsymbol{\Psi}_{n}, \tilde{\boldsymbol{u}}_{n}, \boldsymbol{\phi}_{n}\right)\right| \leq \frac{8}{\nu^{3}} K_{a}\left(\boldsymbol{f}, \nu, \boldsymbol{w}_{n}\right)^{2} C_{b}^{2} C_{1}^{2}\left(d, \Omega_{i}\right) C_{P, i}^{2}\left\|\boldsymbol{w}_{n}-\boldsymbol{w}\right\|_{1 / 2,2, \Gamma_{i}}^{2}+\frac{\nu}{8}\left\|\nabla \boldsymbol{\phi}_{n}\right\|_{L^{2}\left(\Omega_{i}\right)}^{2} \\
& \nu\left|\left(\nabla \mathbf{\Psi}_{n}, \nabla \phi_{n}\right)\right| \leq \sqrt{4 \nu}\left\|\nabla \mathbf{\Psi}_{n}\right\|_{L^{2}\left(\Omega_{i}\right)} \sqrt{\frac{\nu}{4}}\left\|\nabla \phi_{n}\right\|_{L^{2}\left(\Omega_{i}\right)} \leq 2 \nu\left\|\nabla \mathbf{\Psi}_{n}\right\|_{L^{2}\left(\Omega_{i}\right)}^{2}+\frac{\nu}{8}\left\|\nabla \phi_{n}\right\|_{L^{2}\left(\Omega_{i}\right)}^{2} \\
& \leq 2 \nu C_{1}^{2}\left(d, \Omega_{i}\right)\left\|\boldsymbol{w}_{n}-\boldsymbol{w}\right\|_{1 / 2,2, \Gamma_{i}}^{2}+\frac{\nu}{8}\left\|\nabla \boldsymbol{\phi}_{n}\right\|_{L^{2}\left(\Omega_{i}\right)}^{2} \\
& \left|b_{\Omega_{i}}\left(\boldsymbol{\Psi}_{n}, \boldsymbol{W}_{n}, \boldsymbol{\phi}_{n}\right)\right| \leq C_{b}|| \boldsymbol{\Psi}_{n}\left\|_{1, \Omega_{i}}|| \boldsymbol{W}_{n}\right\|_{1, \Omega_{i}} C_{P, i}|| \nabla \boldsymbol{\phi}_{n} \|_{L^{2}\left(\Omega_{i}\right)} \\
& \leq C_{b} C_{1}\left(d, \Omega_{i}\right) C_{P, i}\left\|\boldsymbol{w}_{n}-\boldsymbol{w}\right\|_{1 / 2,2, \Gamma_{i}}\left\|\nabla \phi_{n}\right\|_{L^{2}\left(\Omega_{i}\right)} C_{1}\left(d, \Omega_{i}\right)\left\|\boldsymbol{w}_{n}\right\|_{1 / 2,2, \Gamma_{i}} \\
& \leq \frac{2}{\nu} C_{b}^{2} C_{P, i}^{2} C_{1}^{4}\left(d, \Omega_{i}\right)\left\|\boldsymbol{w}_{n}-\boldsymbol{w}\right\|_{1 / 2,2, \Gamma_{i}}^{2}\left\|\boldsymbol{w}_{n}\right\|_{1 / 2,2, \Gamma_{i}}^{2}+\frac{\nu}{8}\left\|\nabla \phi_{n}\right\|_{L^{2}\left(\Omega_{i}\right)}^{2} \\
& \left|b_{\Omega_{i}}\left(\boldsymbol{W}, \boldsymbol{\Psi}_{n}, \boldsymbol{\phi}_{n}\right)\right| \leq \frac{2}{\nu} C_{b}^{2} C_{P, i}^{2} C_{1}^{4}\left(d, \Omega_{i}\right)\left\|\boldsymbol{w}_{n}-\boldsymbol{w}\right\|_{1 / 2,1, \Gamma_{i}}^{2}\|\boldsymbol{w}\|_{1 / 2,2, \Gamma_{i}}^{2}+\frac{\nu}{8}\left\|\nabla \boldsymbol{\phi}_{n}\right\|_{L^{2}\left(\Omega_{i}\right)}^{2} .
\end{aligned}
$$

Putting it all together we arrive at the following inequality for $\phi_{n}$, viz.,

$$
\begin{aligned}
\nu\left\|\nabla \boldsymbol{\phi}_{n}\right\|_{L^{2}\left(\Omega_{i}\right)}^{2} \leq & {\left[\frac{5 \nu}{8}+C_{b} \frac{2}{\nu} C_{P, i}^{2} K_{a}\left(\boldsymbol{f}, \nu, \boldsymbol{w}_{n}\right)+C_{b} C_{1}\left(d, \Omega_{i}\right) C_{P, i}\left\|\boldsymbol{w}_{n}\right\|_{1 / 2,2, \Gamma_{i}}\right]\left\|\nabla \boldsymbol{\phi}_{n}\right\|_{L^{2}\left(\Omega_{i}\right)}^{2} } \\
& +\frac{8}{\nu^{3}} C_{b}^{2} C_{1}^{2}\left(d, \Omega_{i}\right) C_{P, i}^{2}\left[K_{a}(\boldsymbol{f}, \nu, \boldsymbol{w})^{2}+K_{a}\left(\boldsymbol{f}, \nu, \boldsymbol{w}_{n}\right)^{2}\right]\left\|\boldsymbol{w}_{n}-\boldsymbol{w}\right\|_{1 / 2,2, \Gamma_{i}}^{2} \\
& +2 \nu C_{1}^{2}\left(d, \Omega_{i}\right)\left\|\boldsymbol{w}_{n}-\boldsymbol{w}\right\|_{1 / 2,2, \Gamma_{i}}^{2} \\
& +\frac{2}{\nu} C_{b}^{2} C_{P, i}^{2} C_{1}^{4}\left(d, \Omega_{i}\right)\left(\left\|\boldsymbol{w}_{n}\right\|_{1 / 2,2, \Gamma_{i}}^{2}+\|\boldsymbol{w}\|_{1 / 2,2, \Gamma_{i}}^{2}\right)\left\|\boldsymbol{w}_{n}-\boldsymbol{w}\right\|_{1 / 2,2, \Gamma_{i}}^{2} .
\end{aligned}
$$

This can be rearranged to

$$
\begin{aligned}
\left(\frac{3 \nu}{8}-\right. & \left.C_{b} \frac{2}{\nu} C_{P, i}^{2} K_{a}\left(\boldsymbol{f}, \nu, \boldsymbol{w}_{n}\right)-C_{b} C_{1}\left(d, \Omega_{i}\right) C_{P, i}\left\|\boldsymbol{w}_{n}\right\|_{1 / 2,2, \Gamma_{i}}\right)\left\|\nabla \boldsymbol{\phi}_{n}\right\|_{L^{2}\left(\Omega_{i}\right)}^{2} \\
\leq & \frac{8}{\nu^{3}} C_{b}^{2} C_{1}^{2}\left(d, \Omega_{i}\right) C_{P, i}^{2}\left(K_{a}(\boldsymbol{f}, \nu, \boldsymbol{w})^{2}+K_{a}\left(\boldsymbol{f}, \nu, \boldsymbol{w}_{n}\right)^{2}\right)\left\|\boldsymbol{w}_{n}-\boldsymbol{w}\right\|_{1 / 2,2, \Gamma_{i}}^{2} \\
& +2 \nu C_{1}^{2}\left(d, \Omega_{i}\right)\left\|\boldsymbol{w}_{n}-\boldsymbol{w}\right\|_{1 / 2,2, \Gamma_{i}}^{2} \\
& +\frac{2}{\nu} C_{b}^{2} C_{P, i}^{2} C_{1}^{4}\left(d, \Omega_{i}\right)\left(\left\|\boldsymbol{w}_{n}\right\|_{1 / 2,2, \Gamma_{i}}^{2}+\|\boldsymbol{w}\|_{1 / 2,2, \Gamma_{i}}^{2}\right)\left\|\boldsymbol{w}_{n}-\boldsymbol{w}\right\|_{1 / 2,2, \Gamma_{i}}^{2}
\end{aligned}
$$


Since $\boldsymbol{w}_{n} \longrightarrow \boldsymbol{w}$, for each $\epsilon>0$ there exists $N_{0} \in \mathbb{N}$ s.t. $\left(\left\|\boldsymbol{w}_{n}-\boldsymbol{w}\right\|_{1 / 2,2, \Gamma_{i}}\right)<\epsilon$ for all $n>N_{0}$. If $(8.60)$ is satisfied then there exists $N_{1} \in \mathbb{N}$ s.t. the l.h.s. term in (...) in (8.63) is strictly positive for all $n \geq N_{1}$. Therefore $\nabla \phi_{n} \rightarrow 0$ in $L^{2}\left(\Omega_{i}\right)$ and Poincare's inequality implies $\boldsymbol{\phi}_{n} \rightarrow 0$ in $\boldsymbol{H}^{1}\left(\Omega_{i}\right)$ as $n \rightarrow \infty$. Together with (8.61) this gives the assertion.

\section{Remark 8.8}

It is worthily comparing (8.55) and (8.60). Taking into account (8.55), (8.60) can be rewritten as

$$
\begin{aligned}
\frac{3 \nu}{8}>C_{b} & \frac{2}{\nu} C_{P, i}^{2} C_{u, 1}^{a p}\|\boldsymbol{f}\|_{-1, \Omega_{i}}+C_{b} \frac{2}{\nu} C_{P, i}^{2} \nu C_{1}\left(d, \Omega_{i}\right)\|\boldsymbol{w}\|_{1 / 2,2, \Gamma_{i}} \\
& +C_{b} \frac{2}{\nu} C_{P, i}^{2} C_{u, 3}^{a p}\|\boldsymbol{w}\|_{1 / 2,2, \Gamma_{i}}^{2}+C_{b} C_{1}\left(d, \Omega_{i}\right) C_{P, i}\|\boldsymbol{w}\|_{1 / 2,2, \Gamma_{i}} \\
=C_{b} & \frac{2}{\nu} C_{P, i}^{2} C_{u, 1}^{a p}\|\boldsymbol{f}\|_{-1, \Omega_{i}}+C_{b} C_{P, i}\left(2 C_{P, i}+1\right) C_{1}\left(d, \Omega_{i}\right)\|\boldsymbol{w}\|_{1 / 2,2, \Gamma_{i}} \\
& +C_{b} \frac{2}{\nu} C_{P, i}^{2} C_{u, 3}^{a p}\|\boldsymbol{w}\|_{1 / 2,2, \Gamma_{i}}^{2} .
\end{aligned}
$$

Typically, $c_{\text {poi }, 0}\left(\Omega_{i}\right)=\mathcal{O}\left(y_{\text {layer }}\right)$ and $y_{\text {layer }}=\mathcal{O}\left(\nu^{1 / 2}\right)$ in the laminar case and $y_{\text {layer }}=$ $\mathcal{O}\left(\nu^{1 / 5}\right)$ in the turbulent case. Hence, in a first approximation $C_{P, i} \approx 1$, and thus (8.60) is not an essentially stronger "small data" restriction than (8.55).

\section{Lemma 8.9}

Assume that $\Gamma_{W}$ is piecewise $C^{1}$ smooth. Under the assumptions of Corollary 8.1 and that

$$
\nu_{e}-C_{b} C_{M}^{2} K_{b}\left(\boldsymbol{f}, \nu_{e}\right)>0, \quad \text { with } \quad K_{b}\left(\boldsymbol{f}, \nu_{e}\right) \equiv \sqrt{\frac{2}{\nu_{e}} C_{L E S}^{a p}}\|\boldsymbol{f}\|_{-1, \Omega}
$$

the operator $T_{N S \rightarrow L E S}$ is continuous on $\boldsymbol{V}_{0, W}^{1}=\left\{\boldsymbol{v} \in \boldsymbol{H}^{1}\left(\Omega_{i}\right) \mid \nabla \cdot \boldsymbol{v}=0\right.$ in $\Omega_{i}, \boldsymbol{v} \cdot \boldsymbol{n}=$ 0 on $\left.\Gamma_{W}\right\}$.

\section{Proof:}

Given a sequence $\boldsymbol{u}_{n}$ in $\boldsymbol{V}_{0, W}^{1}$ with $\boldsymbol{u}_{n} \longrightarrow \boldsymbol{u}$ in $\boldsymbol{H}^{1}\left(\Omega_{i}\right)$ as $n \rightarrow \infty$. Denote $\boldsymbol{w}_{n} \equiv$ $T_{N S \rightarrow L E S}\left(\boldsymbol{u}_{n}\right)$ and $\boldsymbol{w} \equiv T_{N S \rightarrow L E S}(\boldsymbol{u})$. Then we have to show that $\boldsymbol{w}_{n} \longrightarrow \boldsymbol{w}$ in $\boldsymbol{H}^{1}(\Omega)$ as $n \rightarrow \infty$. The proof takes two steps.

(1) Let $\delta$ be fixed. Given $\boldsymbol{u}_{n} \longrightarrow \boldsymbol{u}$ in $\boldsymbol{H}^{1}\left(\Omega_{i}\right)$, then $\beta_{j}\left(\overline{\boldsymbol{u}}_{n}\right) \longrightarrow \beta_{j}(\overline{\boldsymbol{u}})$ in $L^{\infty}\left(\Gamma_{W}\right)$.

(2) Given $\beta_{j}\left(\overline{\boldsymbol{u}}_{n}\right) \longrightarrow \beta_{j}(\overline{\boldsymbol{u}})$ in $L^{\infty}\left(\Gamma_{W}\right)$, then $\boldsymbol{w}_{n} \longrightarrow \boldsymbol{w}$ in $\boldsymbol{H}^{1}(\Omega)$.

So let $\boldsymbol{u}_{n} \longrightarrow \boldsymbol{u}$ in $\boldsymbol{H}^{1}\left(\Omega_{i}\right)$ be given. First we show that $\overline{\boldsymbol{u}}_{n} \equiv g_{\delta} * \boldsymbol{u}_{n} \longrightarrow \overline{\boldsymbol{u}} \equiv g_{\delta} * \boldsymbol{u}$ (with $g_{\delta}$ being defined in (4.10)) in $C^{m}\left(\bar{\Omega}_{i}\right)$, for each fixed $m$. Denote $\boldsymbol{\alpha}=\left(\alpha_{1}, \ldots, \alpha_{d}\right)$ a multiindex with $|\boldsymbol{\alpha}|=m$. First we extend $\boldsymbol{u}_{n}$ and $\boldsymbol{u}$ by zero onto $\mathbb{R}^{d} \backslash \Omega_{i}$. Then $\boldsymbol{u}_{n}$ and $\boldsymbol{u}$ are at least in $\boldsymbol{E}^{\prime}\left(\mathbb{R}^{d}\right)$. Thus $D^{\boldsymbol{\alpha}}\left(g_{\delta} * \boldsymbol{u}_{n}\right)=\left(D^{\boldsymbol{\alpha}} g_{\delta}\right) * \boldsymbol{u}_{n}, D^{\boldsymbol{\alpha}}\left(g_{\delta} * \boldsymbol{u}\right)=\left(D^{\boldsymbol{\alpha}} g_{\delta}\right) * \boldsymbol{u}$, and 
$D^{\boldsymbol{\alpha}}\left(g_{\delta} * \boldsymbol{u}_{n}\right), D^{\boldsymbol{\alpha}}\left(g_{\delta} * \boldsymbol{u}\right)$ in $C^{\infty}\left(\mathbb{R}^{d}\right)$. cf.(A.2). Then for each fixed $\delta$ and for each $\boldsymbol{\alpha} \in \mathbb{N}_{0}^{d}$ with $|\boldsymbol{\alpha}|=m$

$$
\begin{aligned}
\left\|D^{\boldsymbol{\alpha}}\left(g_{\delta} * \boldsymbol{u}_{n}\right)-D^{\boldsymbol{\alpha}}\left(g_{\delta} * \boldsymbol{u}\right)\right\|_{C^{0}\left(\bar{\Omega}_{i}\right)} & =\left\|\left(D^{\boldsymbol{\alpha}} g_{\delta}\right) * \boldsymbol{u}_{n}-\left(D^{\boldsymbol{\alpha}} g_{\delta}\right) * \boldsymbol{u}\right\|_{C^{0}\left(\bar{\Omega}_{i}\right)} \\
& =\max _{\boldsymbol{x} \in \bar{\Omega}_{i}}\left|\int_{\mathbb{R}^{d}}\left(D^{\boldsymbol{\alpha}} g_{\delta}\right)\left(\boldsymbol{x}-\boldsymbol{x}^{\prime}\right)\left(\boldsymbol{u}_{n}-\boldsymbol{u}\right)\left(\boldsymbol{x}^{\prime}\right) d \boldsymbol{x}^{\prime}\right| \\
& =\max _{\boldsymbol{x} \in \bar{\Omega}_{i}}\left|\int_{\bar{\Omega}_{i}}\left(D^{\boldsymbol{\alpha}} g_{\delta}\right)\left(\boldsymbol{x}-\boldsymbol{x}^{\prime}\right)\left(\boldsymbol{u}_{n}-\boldsymbol{u}\right)\left(\boldsymbol{x}^{\prime}\right) d \boldsymbol{x}^{\prime}\right| \\
& \leq\left\|D^{\boldsymbol{\alpha}} g_{\delta}\right\|_{L^{2}\left(\Omega_{i}\right)}\left\|\boldsymbol{u}_{n}-\boldsymbol{u}\right\|_{L^{2}\left(\Omega_{i}\right)} \rightarrow 0 \text { as } n \rightarrow \infty
\end{aligned}
$$

Thus for each given $m \in \mathbb{N}$ we have $g_{\delta} * \boldsymbol{u}_{n} \longrightarrow g_{\delta} * \boldsymbol{u}$ in $C^{m}\left(\overline{\Omega_{i}}\right)$. Since $\Gamma_{W}$ is piecewise $C^{1}$ smooth, $\overline{\boldsymbol{u}}_{n} \cdot \boldsymbol{t}_{j} \longrightarrow \overline{\boldsymbol{u}} \cdot \boldsymbol{t}_{j}$ and $\boldsymbol{n}^{T} D\left(\overline{\boldsymbol{u}}_{n}\right) \boldsymbol{t}_{j} \longrightarrow \boldsymbol{n}^{T} D(\overline{\boldsymbol{u}}) \boldsymbol{t}_{j}$ both in $L^{\infty}\left(\Gamma_{W}\right)$ Since $\beta(\cdot, \cdot)$ is continuous, $\beta_{j}\left(\boldsymbol{u}_{n}\right) \longrightarrow \beta_{j}(\boldsymbol{u})$ in $L^{\infty}\left(\Gamma_{W}\right)$.

Now we perform the second step : Given $\beta_{j}\left(\overline{\boldsymbol{u}}_{n}\right) \longrightarrow \beta_{j}(\overline{\boldsymbol{u}})$ in $L^{\infty}\left(\Gamma_{W}\right)$, then we have to prove that $\boldsymbol{w}_{n} \longrightarrow \boldsymbol{w}$ in $\boldsymbol{H}^{1}(\Omega)$. For simplicity, we introduce $\beta_{j, n} \equiv \beta_{j}\left(\overline{\boldsymbol{u}}_{n}\right), \beta_{j} \equiv \beta_{j}(\overline{\boldsymbol{u}})$ and $\boldsymbol{\Phi}_{n} \equiv \boldsymbol{w}_{n}-\boldsymbol{w}$. Then $\boldsymbol{w}_{n}$ and $\boldsymbol{w}$ satisfy the following equations, resp., for all $\boldsymbol{v}$ in $\boldsymbol{V}_{2}=\left\{\boldsymbol{v} \in \boldsymbol{H}^{1}(\Omega) \mid \nabla \cdot \boldsymbol{v}=0\right.$ in $\Omega, \boldsymbol{v} \cdot \boldsymbol{n}=0$ on $\left.\Gamma_{W}\right\}$, cf. (8.26):

$$
\begin{array}{r}
b_{\Omega}\left(\boldsymbol{w}_{n}, \boldsymbol{w}_{n}, \boldsymbol{v}\right)+\left(\nu_{e} \nabla \boldsymbol{w}_{n}, \nabla \boldsymbol{v}\right)+\sum_{j=1}^{d-1}\left\langle\beta_{j, n} \boldsymbol{w}_{n} \cdot \boldsymbol{t}_{j}, \boldsymbol{v} \cdot \boldsymbol{t}_{j}\right\rangle_{\Gamma_{W}}=(\overline{\boldsymbol{f}}, \boldsymbol{v}) \\
b_{\Omega}(\boldsymbol{w}, \boldsymbol{w}, \boldsymbol{v})+\left(\nu_{e} \nabla \boldsymbol{w}, \nabla \boldsymbol{v}\right)+\sum_{j=1}^{d-1}\left\langle\beta_{j} \boldsymbol{w} \cdot \boldsymbol{t}_{j}, \boldsymbol{v} \cdot \boldsymbol{t}_{j}\right\rangle_{\Gamma_{W}}=(\overline{\boldsymbol{f}}, \boldsymbol{v}) .
\end{array}
$$

Subtracting (8.66) from (8.65) gives (for all $\boldsymbol{v}$ in $\boldsymbol{V}_{2}$ )

$$
\begin{aligned}
b_{\Omega}\left(\boldsymbol{w}_{n}, \boldsymbol{w}_{n}, \boldsymbol{v}\right) & \left.-b_{\Omega}(\boldsymbol{w}, \boldsymbol{w}, \boldsymbol{v})+\left(\nu_{e} \nabla\left(\boldsymbol{w}_{n}-\boldsymbol{w}\right)\right), \nabla \boldsymbol{v}\right) \\
& +\sum_{j=1}^{d-1}\left\langle\beta_{j, n} \boldsymbol{w}_{n} \cdot \boldsymbol{t}_{j}, \boldsymbol{v} \cdot \boldsymbol{t}_{j}\right\rangle_{\Gamma_{W}}-\sum_{j=1}^{d-1}\left\langle\beta_{j} \boldsymbol{w} \cdot \boldsymbol{t}_{j}, \boldsymbol{v} \cdot \boldsymbol{t}_{j}\right\rangle_{\Gamma_{W}}=0
\end{aligned}
$$

After expanding this can be rewritten as

$$
\begin{aligned}
& b_{\Omega}\left(\boldsymbol{\Phi}_{n}, \boldsymbol{w}_{n}, \boldsymbol{v}\right)+b_{\Omega}\left(\boldsymbol{w}, \boldsymbol{\Phi}_{n}, \boldsymbol{v}\right)+\left(\nu_{e} \nabla \boldsymbol{\Phi}_{n}, \nabla \boldsymbol{v}\right) \\
& +\sum_{j=1}^{d-1}\left\langle\left(\beta_{j, n}-\beta_{j}\right) \boldsymbol{w}_{n} \cdot \boldsymbol{t}_{j}, \boldsymbol{v} \cdot \boldsymbol{t}_{j}\right\rangle_{\Gamma_{W}}+\sum_{j=1}^{d-1}\left\langle\beta_{j} \boldsymbol{\Phi}_{n} \cdot \boldsymbol{t}_{j}, \boldsymbol{v} \cdot \boldsymbol{t}_{j}\right\rangle_{\Gamma_{W}}=0 .
\end{aligned}
$$

Setting $\boldsymbol{v}=\boldsymbol{\Phi}_{\boldsymbol{n}}$ and taking into account $b\left(\boldsymbol{w}, \boldsymbol{\Phi}_{n}, \mathbf{\Phi}_{n}\right)=0\left(\right.$ as $\boldsymbol{w} \cdot \boldsymbol{n}=0$ on $\left.\Gamma_{W}\right)$ gives

$$
\begin{aligned}
\nu_{e}\left\|\nabla \boldsymbol{\Phi}_{n}\right\|_{L^{2}(\Omega)}^{2} & +\sum_{j=1}^{d-1}\left\langle\beta_{j} \boldsymbol{\Phi}_{n} \cdot \boldsymbol{t}_{j}, \boldsymbol{\Phi}_{n} \cdot \boldsymbol{t}_{j}\right\rangle_{\Gamma_{W}} \\
& =-\sum_{j=1}^{d-1}\left\langle\left(\beta_{j, n}-\beta_{j}\right) \boldsymbol{w}_{n} \cdot \boldsymbol{t}_{j}, \boldsymbol{\Phi}_{n} \cdot \boldsymbol{t}_{j}\right\rangle_{\Gamma_{W}}-b_{\Omega}\left(\boldsymbol{\Phi}_{n}, \boldsymbol{w}_{n}, \boldsymbol{\Phi}_{n}\right) .
\end{aligned}
$$


Now we need estimates for the right hand side terms. First, we have

$$
\left|b_{\Omega}\left(\boldsymbol{\Phi}_{n}, \boldsymbol{w}_{n}, \boldsymbol{\Phi}_{n}\right)\right| \leq C_{b}\left\|\nabla \boldsymbol{w}_{n}\right\|_{L^{2}(\Omega)} \mid\left\|\boldsymbol{\Phi}_{n}\right\|_{1, \Omega}^{2} \leq C_{b} C_{M}^{2} K_{b}\left(\boldsymbol{f}, \nu_{e}\right)\left\|\nabla \boldsymbol{\Phi}_{n}\right\|_{L^{2}(\Omega)}^{2},
$$

where we used Lemma 8.1 and (8.64) in the last step. Next we have for each $K>0$

$$
\begin{aligned}
\mid \sum_{j=1}^{d-1}\left\langle\left(\beta_{j, n}\right.\right. & \left.\left.-\beta_{j}\right) \boldsymbol{w}_{n} \cdot \boldsymbol{t}_{\boldsymbol{j}}, \boldsymbol{\Phi}_{n} \cdot \boldsymbol{t}_{\boldsymbol{j}}\right\rangle_{\Gamma_{W}}|\leq| \sum_{j=1}^{d-1}\left\|\beta_{j, n}-\beta_{j}\right\|_{L^{\infty}\left(\Gamma_{W}\right)}\left\langle\boldsymbol{w}_{n} \cdot \boldsymbol{t}_{\boldsymbol{j}}, \boldsymbol{\Phi}_{n} \cdot \boldsymbol{t}_{\boldsymbol{j}}\right\rangle_{\Gamma_{W}} \mid \\
& \leq \sum_{j=1}^{d-1} \frac{1}{2 K}\left\|\boldsymbol{\Phi}_{n} \cdot \boldsymbol{t}_{j}\right\|_{L^{2}\left(\Gamma_{W}\right)}^{2}+\sum_{j=1}^{d-1} \frac{K}{2}\left\|\beta_{j, n}-\beta_{j}\right\|_{L^{\infty}\left(\Gamma_{W}\right)}^{2}\left\|\boldsymbol{w}_{n} \cdot \boldsymbol{t}_{j}\right\|_{L^{2}\left(\Gamma_{W}\right)}^{2} \\
& \leq \sum_{j=1}^{d-1} \frac{1}{2 K}\left\|\boldsymbol{\Phi}_{n} \cdot \boldsymbol{t}_{j}\right\|_{L^{2}\left(\Gamma_{W}\right)}^{2}+\sum_{j=1}^{d-1} \frac{K}{2}\left\|\beta_{j, n}-\beta_{j}\right\|_{L^{\infty}\left(\Gamma_{W}\right)}^{2} C_{t r}^{2} C_{M}^{2} K_{b}\left(\boldsymbol{f}, \nu_{e}\right)^{2}
\end{aligned}
$$

where we used $\left\|\boldsymbol{w}_{n}\right\|_{L^{2}\left(\Gamma_{W}\right)} \leq\left\|\boldsymbol{w}_{n}\right\|_{1 / 2,2, \Gamma_{W}} \leq C_{T r}\left\|\boldsymbol{w}_{n}\right\|_{1, \Omega}$ (cf. [O+t.o99], p.159) and Lemma 8.1 in the last step. Substituting the last two inequalities into (8.67) gives

$$
\begin{aligned}
\left(\nu_{e}-C_{b} C_{M}^{2} K_{b}\left(\boldsymbol{f}, \nu_{e}\right)\right)\left\|\nabla \boldsymbol{\Phi}_{n}\right\|_{L^{2}(\Omega)}^{2} & +\left(\beta_{0}-\frac{1}{2 K}\right) \sum_{j=1}^{d-1}\left\|\boldsymbol{\Phi}_{n} \cdot \boldsymbol{t}_{j}\right\|_{L^{2}\left(\Gamma_{W}\right)}^{2} \\
& \leq C_{t r}^{2} C_{M}^{2} K_{b}\left(\boldsymbol{f}, \nu_{e}\right)^{2} \sum_{j=1}^{d-1} \frac{K}{2}\left\|\beta_{j, n}-\beta_{j}\right\|_{L^{\infty}\left(\Gamma_{W}\right)}^{2} .
\end{aligned}
$$

Now we exploit the fact that $\beta_{0}>0$ and choose $K$, s.t. $\beta_{0}-\frac{1}{2 K} \geq 0$. Then step (1), (8.64), and (B.14) imply that $\Phi_{n} \longrightarrow 0$ as $n \rightarrow \infty$ in the norm of $\boldsymbol{H}^{1}(\Omega)$.

\section{Lemma 8.10}

We assume that $\Omega$ is sufficiently smooth s.t. there exists a continuous linear prolongation operator $\Pi: W^{m, p}(\Omega) \longrightarrow W^{m, p}\left(\mathbb{R}^{d}\right), d=2,3$. Then, under the assumptions of Lemma 8.9 the operator $T_{N S \rightarrow L E S}$ is compact.

\section{Proof:}

We have to show that for each given bounded sequence $\left(\boldsymbol{u}_{n}\right)$ in $\boldsymbol{V}_{0, W}^{1}$, i.e. $\left\|\boldsymbol{u}_{n}\right\|_{1, \Omega_{i}}<C$ for all $n$, the sequence $\left(T_{N S \rightarrow L E S}\left(\boldsymbol{u}_{n}\right)\right)$ contains a subsequence, that converges to some $\boldsymbol{w}$ in $\boldsymbol{V}_{2}$ in the norm of $\boldsymbol{H}^{1}(\Omega)$.

Given a bounded sequence $\left(\boldsymbol{u}_{n}\right)$ in $\boldsymbol{V}_{0, W}^{1}$ with $\left\|\boldsymbol{u}_{n}\right\|_{1, \Omega_{i}}<C$ for all $n$. Then there exists some $\boldsymbol{u}$ in $\boldsymbol{V}_{0, W}^{1}$ and a subsequence $\left(\boldsymbol{u}_{n_{k}}\right)$ s.t. $\boldsymbol{u}_{n_{k}} \rightarrow \boldsymbol{u}$ as $n \rightarrow \infty$ in the weak topology of $\boldsymbol{V}_{0, W}^{1}$. Due to Lemma B.2 we have also $\boldsymbol{u}_{n_{k}} \rightarrow \boldsymbol{u}$ as $n_{k} \rightarrow \infty$ strongly in the norm of $L^{2}(\Omega)$. We define $\beta_{j, n_{k}} \equiv \beta_{j}\left(\boldsymbol{u}_{n_{k}}\right)$ and $\beta_{j} \equiv \beta_{j}(\boldsymbol{u})$. In the proof of Lemma 8.9 it was shown that then $\beta_{n_{k}} \longrightarrow \beta$ as $n_{k} \rightarrow \infty$ in the norm of $L^{\infty}\left(\Gamma_{W}\right)$. Moreover it was shown there that then $T_{N S \rightarrow L E S}\left(\boldsymbol{u}_{n_{k}}\right) \longrightarrow \boldsymbol{w}$ in the norm of $\boldsymbol{V}_{2}$ as $n_{k} \rightarrow \infty$. 
Before proceeding it is worthwhile recalling the Schauder Fixed-Point Theorem, cf. [Zeil], p.57: Let $M$ be a nonempty, closed, bounded, convex subset of a Banach space $X$, and suppose $T: M \longrightarrow M$ is a compact operator. Then $T$ has a fixed-point.

Now we can state a theorem concerning the existence of at least one solution of the steady state model for coupling LES and DNS. In all the preceding steps, all inequalities have been handled very carefully. In contrast, this theorem and its corresponding proof will be presented slightly lax. As will be seen in the proof, figuring out all inequalities involved in the proof is a sisyphus-like task without being necessary.

\section{Theorem 8.1}

If $\nu$ and $\nu_{e}$ are "sufficiently large" and if $\|\boldsymbol{f}\|_{-1, \Omega}$ and $\|\boldsymbol{f}\|_{-1, \Omega_{i}}$ are "sufficiently small", then $T_{N S \rightarrow L E S} \circ T_{L E S \rightarrow N S}$ and $T_{L E S \rightarrow N S} \circ T_{N S \rightarrow L E S}$ have at least one fixed-point.

\section{Proof:}

First we show the existence of a fixed-point of the operator

$$
S_{1} \equiv T_{N S \rightarrow L E S} \circ T_{L E S \rightarrow N S}: V_{0, W}^{1} \supset M_{1} \mapsto M_{1}
$$

with $M_{1}$ to be determined within the proof. The proof takes three steps.

I Show that there is a closed and bounded ball $M_{1}^{\prime} \subset \boldsymbol{V}_{0, W}^{1}$, s.t. $\left.S_{1}\right|_{M_{1}^{\prime}}$ is well-defined and continuous.

II Show that there is a closed and bounded ball $M_{1} \subset M_{1}^{\prime}$, s.t. $S_{1}\left(M_{1}\right) \subset M_{1}$.

III Show that $S_{1}$ is compact.

Then $M_{1}$ is nonempty, closed, bounded and convex. Thus the Schauder Fixed-Point Theorem ensures that there is $\boldsymbol{u} \in M_{1}$, s.t. $S_{1}(\boldsymbol{u})=\boldsymbol{u}$.

Ad I: $T_{N S \rightarrow L E S}$ is well-defined on $\boldsymbol{V}_{0, W}^{1}$. As a consequence of Lemmata 8.8 and 8.9, $S_{1}$ is well-defined and continuous on $M_{1}^{\prime} \subset \boldsymbol{V}_{0, W}^{1}$ provided $T_{N S \rightarrow L E S}\left(M_{1}^{\prime}\right) \subset \boldsymbol{V}_{\text {cont }}^{2}$. Figure 8.1 provides some illustration:

Therefore we have to ensure that $\boldsymbol{w} \in M_{1}^{\prime}$ satisfies the following conditions: First, from (8.55) we have to ensure (i)

$$
\frac{\nu}{2}>C_{b} C_{P, i}^{2} \frac{2}{\nu} K_{a}(\boldsymbol{f}, \nu, \boldsymbol{w})
$$

Second, from (8.60) we need (ii)

$$
\frac{3 \nu}{8}>C_{b} \frac{2}{\nu} C_{P, i}^{2} K_{a}(\boldsymbol{f}, \nu, \boldsymbol{w})+C_{b} C_{1}\left(d, \Omega_{i}\right) C_{P, i}\|\boldsymbol{w}\|_{1 / 2,2, \Gamma_{i}} .
$$




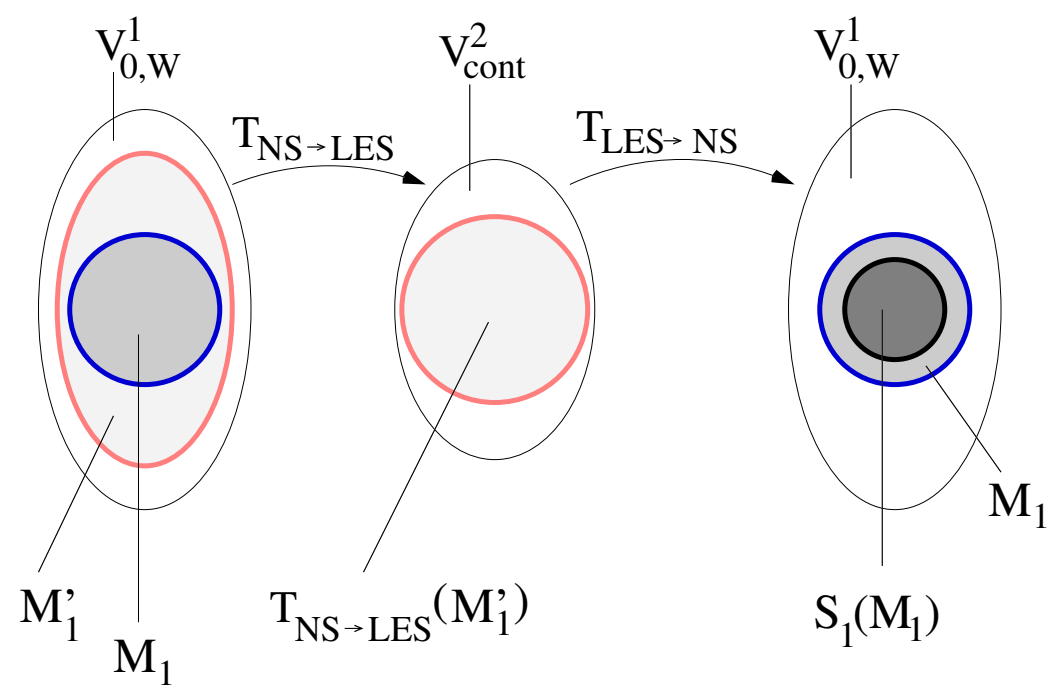

Figure 8.1.: Schematic representation of subspaces.

Due to the priori estimate in Lemma 8.1, we have

$$
\|\boldsymbol{w}\|_{1 / 2,2, \Gamma_{i}} \leq C_{t r} C_{M}\|\nabla \boldsymbol{w}\|_{\boldsymbol{L}^{2}\left(\Omega_{i}\right)} \leq C_{t r} C_{M}\|\nabla \boldsymbol{w}\|_{L^{2}(\Omega)} \leq C_{t r} \frac{C_{M}^{2}}{\nu_{e}}\|\overline{\boldsymbol{f}}\|_{-1, \Omega} .
$$

Thus if $\|\overline{\boldsymbol{f}}\|_{-1 \Omega}^{2}$ is "sufficiently small" and $\nu_{e}$ is "sufficiently large", then we can make $\|\boldsymbol{w}\|_{1 / 2,2, \Gamma_{i}}$ sufficiently small such that (i) and (ii) are satisfied. Therefore we can ensure existence of a closed and bounded ball $M_{1}^{\prime} \subset \boldsymbol{V}_{0, W}^{1}$, s.t. $T_{N S \rightarrow L E S}\left(M_{1}^{\prime}\right) \subset \boldsymbol{V}_{\text {cont }}^{2}$, i.e., $\left.S_{1}\right|_{M_{1}^{\prime}}$ is well-defined.

Ad II: Now we have to show that there is a closed and bounded ball $M_{1} \subset M_{1}^{\prime}$ s.t. $S_{1}\left(M_{1}\right) \subset$ $M_{1}$. As $M_{1}^{\prime}$ is a closed and bounded ball, there is $\rho>0$ s.t. $B(\mathbf{0}, \rho) \subset M_{1}^{\prime}$, with $B(\mathbf{0}, \rho)$ being the ball with radius $\rho$ around the origin. From Lemma 8.5 we know that

$$
\left\|\nabla S_{1}(\boldsymbol{u})\right\|_{\boldsymbol{L}^{2}\left(\Omega_{i}\right)}^{2} \leq \frac{2}{\nu} K_{a}(\boldsymbol{f}, \nu, \boldsymbol{w}) .
$$

We can combine this with (8.69) giving the following estimate

$$
\left\|\nabla S_{1}(\boldsymbol{u})\right\|_{\boldsymbol{L}^{2}\left(\Omega_{i}\right)}^{2} \leq \frac{2}{\nu} C_{u, 1}^{a p}\|\boldsymbol{f}\|_{-1, \Omega_{i}}+C_{u, 2}^{a p} C_{t r} \frac{C_{M}^{2}}{\nu_{e}}\|\overline{\boldsymbol{f}}\|_{-1, \Omega}+C_{u, 3}^{a p} C_{t r}^{2} \frac{C_{M}^{4}}{\nu_{e}^{2}}\|\overline{\boldsymbol{f}}\|_{-1, \Omega}^{2} .
$$

Then we simply require for $f$ and $\nu_{e}$ that the r.h.s. in (8.71) is smaller than $\rho$. Therefore there is a closed and bounded ball $M_{1} \subset M_{1}^{\prime}$ s.t. $S_{1}\left(M_{1}\right) \subset M_{1}$.

Ad III: $S_{1}$ is a compact operator as $T_{L E S \rightarrow N S}$ is continuous and $T_{N S \rightarrow L E S}$ is continuous and compact.

From I-III we can infer that the operator $S_{1}$ has at least one fixed-point. 
Secondly, we show the existence of a fixed-point of the operator

$$
S_{2} \equiv T_{L E S \rightarrow N S} \circ T_{N S \rightarrow L E S}: \boldsymbol{V}_{\text {cont }}^{2} \mapsto \boldsymbol{V}^{2} .
$$

This operator is well-defined, continuous and compact according to Lemmata 8.8, 8.9, and 8.10. From Lemma 8.1 we know the following a priori estimate for $S_{2}(\boldsymbol{w})$, viz.,

$$
\left\|S_{2}(\boldsymbol{w})\right\|_{1, \Omega}^{2} \leq \frac{C_{M}^{4}}{\nu_{e}^{2}}\|\overline{\boldsymbol{f}}\|_{-1, \Omega}^{2} .
$$

Then we require that $\nu$ and $\boldsymbol{f}$ are such that the right hand side is smaller than $\rho$, satifying $B(\mathbf{0}, \rho) \subset \boldsymbol{V}_{\text {cont }}^{2}$. Now we can apply the Schauder Fixed-Point Theorem.

\section{Remark 8.9}

For proving Lemmata 8.9 and 8.10 we need that $\beta(\cdot, \cdot) \geq \beta_{0}>0$ and $\beta(\cdot, \cdot)$ is continuous on $\mathbb{R}^{2}$.

Before giving the proof regarding uniqueness, we need a further result regarding $\beta_{j}(\cdot)$.

Lemma 8.11

Assume $\Gamma_{W}$ is piecewise smooth. Then for all $\boldsymbol{u}_{1}, \boldsymbol{u}_{2}$ in $\boldsymbol{H}^{1}\left(\Omega_{i}\right)$

$$
\left\|\beta_{j}\left(\overline{\boldsymbol{u}}_{1}\right)-\beta_{j}\left(\overline{\boldsymbol{u}}_{2}\right)\right\|_{L^{\infty}\left(\Gamma_{W}\right)} \leq C_{\beta}(\delta)\left\|\boldsymbol{u}_{1}-\boldsymbol{u}_{2}\right\|_{\boldsymbol{H}^{1}\left(\Omega_{i}\right)}, \quad C_{\beta}(\delta)=C_{l c} C \delta^{-5 / 2} .
$$

\section{Proof:}

For given $\overline{\boldsymbol{u}}$ in $C^{\infty}\left(\mathbb{R}^{d}\right)$ and fixed $j=1, \ldots, d-1$ we set $\left.y(\overline{\boldsymbol{u}}) \equiv \operatorname{Tr}\right|_{\Gamma_{W}} \overline{\boldsymbol{u}} \cdot \boldsymbol{t}_{j}$ and $z(\overline{\boldsymbol{u}}) \equiv$ $\left.\boldsymbol{n}^{T} \operatorname{Tr}\right|_{\Gamma_{W}} D(\overline{\boldsymbol{u}}) \boldsymbol{t}_{j}$. As $\Gamma_{W}$ is piecewise smooth, $y(\overline{\boldsymbol{u}})$ and $z(\overline{\boldsymbol{u}})$ are in $L^{\infty}\left(\Gamma_{W}\right)$. So let $\boldsymbol{u}_{1}$, $\boldsymbol{u}_{2}$ in $\boldsymbol{V}_{0, W}^{1}$ be given. Denote $y_{i} \equiv y\left(\overline{\boldsymbol{u}}_{i}\right), z_{i} \equiv z\left(\overline{\boldsymbol{u}}_{i}\right), i=1,2$. Then for each $\boldsymbol{x}$ in $\Gamma_{W}$

$$
\left|\beta\left(y_{1}(\boldsymbol{x}), z_{1}(\boldsymbol{x})\right)-\beta\left(y_{2}(\boldsymbol{x}), z_{2}(\boldsymbol{x})\right)\right| \leq C_{l c} \max \left[\left|y_{1}(\boldsymbol{x})-y_{2}(\boldsymbol{x})\right| ;\left|z_{1}(\boldsymbol{x})-z_{2}(\boldsymbol{x})\right|\right] .
$$

We will use the following embedding result, see [Gri85], p. 27, i.e.,

$$
W^{k+d / p, p}\left(\mathbb{R}^{d}\right) \subset C^{k-1, \alpha}\left(\mathbb{R}^{d}\right), \quad \forall \alpha \in[0,1[, \quad k \in \mathbb{N}, \quad k \geq 1 .
$$

Moreover we use the following estimate, see [.J01], p.271: For $\boldsymbol{f} \in \boldsymbol{L}^{2}(\Omega), \boldsymbol{f}=0$ on $\mathbb{R}^{d} \backslash \Omega$, and $\overline{\boldsymbol{f}} \equiv g_{\delta} * \boldsymbol{f} \in C^{\infty}\left(\mathbb{R}^{d}\right)$ we have

$$
\left\|g_{\delta} * \boldsymbol{f}\right\|_{W^{k, 2}\left(\mathbb{R}^{d}\right)} \leq C\left\|g_{\delta}\right\|_{W^{k, 1}\left(\mathbb{R}^{d}\right)}\|\boldsymbol{f}\|_{L^{2}(\Omega)} \leq C_{3} \delta^{-k}\|\boldsymbol{f}\|_{L^{2}(\Omega)} .
$$

Therefore we obtain

$$
\begin{aligned}
\left\|\beta_{j}\left(\overline{\boldsymbol{u}}_{1}\right)-\beta_{j}\left(\overline{\boldsymbol{u}}_{2}\right)\right\|_{L^{\infty}\left(\Gamma_{W}\right)}= & \max _{\boldsymbol{x} \in \Gamma_{W}}\left|\beta\left(\overline{\boldsymbol{u}}_{1}\right)(\boldsymbol{x})-\beta\left(\overline{\boldsymbol{u}}_{2}\right)(\boldsymbol{x})\right| \\
\leq & C_{l c} \max _{\boldsymbol{x} \in \Gamma_{W}} \max \left[\left|y_{1}(\boldsymbol{x})-y_{2}(\boldsymbol{x})\right| ;\left|z_{1}(\boldsymbol{x})-z_{2}(\boldsymbol{x})\right|\right] \\
= & C_{l c} \max \left[|\operatorname{Tr}|_{\Gamma_{W}}\left(\overline{\boldsymbol{u}}_{1}-\overline{\boldsymbol{u}}_{2}\right) \cdot \boldsymbol{t}_{j} \|_{L^{\infty}\left(\Gamma_{W}\right)},\right. \\
& \left.\left\|\left.\boldsymbol{n}^{T} \operatorname{Tr}\right|_{\Gamma_{W}} \nabla\left(\overline{\boldsymbol{u}}_{1}-\overline{\boldsymbol{u}}_{2}\right) \boldsymbol{t}_{j}\right\|_{L^{\infty}\left(\Gamma_{W}\right)}\right] \\
\leq & C_{l c} \max \left[\left\|\overline{\boldsymbol{u}}_{1}-\overline{\boldsymbol{u}}_{2}\right\|_{L^{\infty}\left(\mathbb{R}^{d}\right)},\left\|\nabla\left(\overline{\boldsymbol{u}}_{1}-\overline{\boldsymbol{u}}_{2}\right)\right\|_{L^{\infty}\left(\mathbb{R}^{d}\right)}\right] \\
= & C_{l c} \max \left[\left\|\overline{\boldsymbol{u}}_{1}-\overline{\boldsymbol{u}}_{2}\right\|_{C^{0}\left(\mathbb{R}^{d}\right)},\left\|\nabla\left(\overline{\boldsymbol{u}}_{1}-\overline{\boldsymbol{u}}_{2}\right)\right\|_{C^{0}\left(\mathbb{R}^{d}\right)}\right] \\
\leq & C_{l c} C \max \left[\left\|\overline{\boldsymbol{u}}_{1}-\overline{\boldsymbol{u}}_{2}\right\|_{W^{5 / 2,2}\left(\mathbb{R}^{d}\right)},\left\|\nabla\left(\overline{\boldsymbol{u}}_{1}-\overline{\boldsymbol{u}}_{2}\right)\right\|_{W^{5 / 2,2}\left(\mathbb{R}^{d}\right)}\right] \\
\leq & C_{l c} C \delta^{-5 / 2}\left\|\boldsymbol{u}_{1}-\boldsymbol{u}_{2}\right\|_{1, \Omega_{i}} .
\end{aligned}
$$




\section{Theorem 8.2}

Suppose that the assumptions of Theorem 8.1 hold. Moreover we assume that there exists $\epsilon>0$ s.t. $\beta_{0}-\epsilon / 2>0$ and

$$
\begin{aligned}
\nu_{e}> & C_{b} \frac{C_{M}^{3}}{\nu_{e}}\|\boldsymbol{f}\|_{-1, \Omega}+\frac{1}{2 \epsilon}(d-1) C_{\beta}^{2}(\delta) C_{t r}^{4} \frac{C_{M}^{6}}{\nu_{e}}\|\boldsymbol{f}\|_{-1, \Omega}^{2}\left[2 C_{1}^{2}\left(d, \Omega_{i}\right)\right. \\
+ & 2 C_{P, i}^{2} K_{2}^{-1}\left(\frac{16}{\nu^{3}} K_{d}\left(\boldsymbol{f}, \nu, \nu_{e}\right)^{2} C_{b}^{2} C_{1}^{2}\left(d, \Omega_{i}\right) C_{P, i}^{2}\right. \\
& \left.\left.+\left(2 \nu C_{1}^{2}\left(d, \Omega_{i}\right)+\frac{2}{\nu} C_{b}^{2} C_{P, i}^{2} C_{1}^{4}\left(d, \Omega_{i}\right) 2 \frac{C_{t r}^{2} C_{M}^{4}}{\nu_{e}^{2}}\|\boldsymbol{f}\|_{-1, \Omega}^{2}\right)\right)\right] .
\end{aligned}
$$

with the following abbreviation

$$
K_{d}\left(\boldsymbol{f}, \nu, \nu_{e}\right) \equiv C_{u, 1}^{a p}\|\boldsymbol{f}\|_{-1, \Omega_{i}}+C_{u, 2}^{a p} \frac{C_{t r} C_{M}^{2}}{\nu_{e}}\|\boldsymbol{f}\|_{-1, \Omega}+C_{u, 3}^{a p} \frac{C_{t r}^{2} C_{M}^{4}}{\nu_{e}^{2}}\|\boldsymbol{f}\|_{-1, \Omega}^{2} .
$$

Then the solution $(\boldsymbol{w}, \boldsymbol{u})$ of (8.11)-(8.16) is unique.

\section{Proof:}

Suppose that there are two solutions $\left(\boldsymbol{w}_{1}, \boldsymbol{u}_{1}\right)$ and $\left(\boldsymbol{w}_{2}, \boldsymbol{u}_{2}\right)$, i.e. $\boldsymbol{w}_{i}=T_{L E S \rightarrow N S} \circ T_{N S \rightarrow L E S}\left(\boldsymbol{w}_{i}\right)$ and $\boldsymbol{u}_{i}=T_{N S \rightarrow L E S} \circ T_{L E S \rightarrow N S}\left(\boldsymbol{u}_{i}\right)$, and $\boldsymbol{u}_{1}=T_{L E S \rightarrow N S}\left(\boldsymbol{w}_{1}\right), \boldsymbol{u}_{2}=T_{L E S \rightarrow N S}\left(\boldsymbol{w}_{2}\right), i=1,2$. Denote $\boldsymbol{w}=\boldsymbol{w}_{1}-\boldsymbol{w}_{2}$ and $\boldsymbol{u}=\boldsymbol{u}_{1}-\boldsymbol{u}_{2}$. Then for all $\boldsymbol{v}$ in $\boldsymbol{V}_{2}$, cf. 8.26),

$$
\begin{aligned}
& \nu_{e}\left(\nabla \boldsymbol{w}_{1}, \nabla \boldsymbol{v}\right)+b_{\Omega}\left(\boldsymbol{w}_{1}, \boldsymbol{w}_{1}, \boldsymbol{v}\right)+\sum_{j=1}^{d-1}\left\langle\beta_{j}\left(T_{L E S \rightarrow N S}\left(\boldsymbol{w}_{1}\right)\right) \boldsymbol{w}_{1} \cdot \boldsymbol{t}_{j}, \boldsymbol{v} \cdot \boldsymbol{t}_{j}\right\rangle_{\Gamma_{W}}=(\overline{\boldsymbol{f}}, \boldsymbol{v}) \\
& \nu_{e}\left(\nabla \boldsymbol{w}_{2}, \nabla \boldsymbol{v}\right)+b_{\Omega}\left(\boldsymbol{w}_{2}, \boldsymbol{w}_{2}, \boldsymbol{v}\right)+\sum_{j=1}^{d-1}\left\langle\beta_{j}\left(T_{L E S \rightarrow N S}\left(\boldsymbol{w}_{2}\right)\right) \boldsymbol{w}_{2} \cdot \boldsymbol{t}_{j}, \boldsymbol{v} \cdot \boldsymbol{t}_{j}\right\rangle_{\Gamma_{W}}=(\overline{\boldsymbol{f}}, \boldsymbol{v}) .
\end{aligned}
$$

Subtracting the second equation from the first one, expanding, and setting $\boldsymbol{v}=\boldsymbol{w}$ gives

$$
\begin{aligned}
\nu_{e}(\nabla \boldsymbol{w}, \nabla \boldsymbol{w}) & +\sum_{j=1}^{d-1}\left\langle\left[\beta_{j}\left(T_{L E S \rightarrow N S}\left(\boldsymbol{w}_{1}\right)\right)-\beta_{j}\left(T_{L E S \rightarrow N S}\left(\boldsymbol{w}_{2}\right)\right)\right] \boldsymbol{w}_{1} \cdot \boldsymbol{t}_{j}, \boldsymbol{w} \cdot \boldsymbol{t}_{j}\right\rangle_{\Gamma_{W}} \\
& +b_{\Omega}\left(\boldsymbol{w}, \boldsymbol{w}_{1}, \boldsymbol{w}\right)+\sum_{j=1}^{d-1}\left\langle\left(\beta_{j}\left(T_{L E S \rightarrow N S}\left(\boldsymbol{w}_{2}\right)\right)\right) \boldsymbol{w} \cdot \boldsymbol{t}_{j}, \boldsymbol{w} \cdot \boldsymbol{t}_{j}\right\rangle_{\Gamma_{W}}=0 .
\end{aligned}
$$

Therefore we arrive at the following estimate

$$
\begin{aligned}
\nu_{e}(\nabla \boldsymbol{w}, \nabla \boldsymbol{w}) & +\sum_{j=1}^{d-1}\left\langle\beta_{j}\left(T_{L E S \rightarrow N S}\left(\boldsymbol{w}_{2}\right)\right) \boldsymbol{w} \cdot \boldsymbol{t}_{j}, \boldsymbol{w} \cdot \boldsymbol{t}_{j}\right\rangle_{\Gamma_{W}} \leq\left|b_{\Omega}\left(\boldsymbol{w}, \boldsymbol{w}_{1}, \boldsymbol{w}\right)\right| \\
& +\sum_{j=1}^{d-1}\left|\left\langle\left[\beta_{j}\left(T_{L E S \rightarrow N S}\left(\boldsymbol{w}_{1}\right)\right)-\beta_{j}\left(T_{L E S \rightarrow N S}\left(\boldsymbol{w}_{2}\right)\right)\right] \boldsymbol{w}_{1} \cdot \boldsymbol{t}_{j}, \boldsymbol{w} \cdot \boldsymbol{t}_{j}\right\rangle_{\Gamma_{W}}\right| .
\end{aligned}
$$


Now the goal is to bound both r.h.s. terms. Regarding the former we have

$$
\left|b_{\Omega}\left(\boldsymbol{w}, \boldsymbol{w}_{1}, \boldsymbol{w}\right)\right| \leq C_{b} \frac{C_{M}^{3}}{\nu_{e}}\|\boldsymbol{f}\|_{-1, \Omega}\|\nabla \boldsymbol{w}\|_{L^{2}(\Omega)}^{2}
$$

Concerning the latter the following estimate holds:

$$
\begin{aligned}
& \sum_{j=1}^{d-1}\left|\left\langle\left[\beta_{j}\left(T_{L E S \rightarrow N S}\left(\boldsymbol{w}_{1}\right)\right)-\beta_{j}\left(T_{L E S \rightarrow N S}\left(\boldsymbol{w}_{2}\right)\right)\right] \boldsymbol{w}_{1} \cdot \boldsymbol{t}_{j}, \boldsymbol{w} \cdot \boldsymbol{t}_{j}\right\rangle_{\Gamma_{W}}\right| \\
& \leq \sum_{j=1}^{d-1}\left\|\beta_{j}\left(T_{L E S \rightarrow N S}\left(\boldsymbol{w}_{1}\right)\right)-\beta_{j}\left(T_{L E S \rightarrow N S}\left(\boldsymbol{w}_{2}\right)\right)\right\|_{L^{\infty}\left(\Gamma_{W}\right)}\left\|\boldsymbol{w}_{1} \cdot \boldsymbol{t}_{j}\right\|_{L^{2}\left(\Gamma_{W}\right)}\left\|\boldsymbol{w} \cdot \boldsymbol{t}_{j}\right\|_{L^{2}\left(\Gamma_{W}\right)} \\
& \leq \frac{1}{2 \epsilon} \sum_{j=1}^{d-1}\left\|\beta_{j}\left(T_{L E S \rightarrow N S}\left(\boldsymbol{w}_{1}\right)\right)-\beta_{j}\left(T_{L E S \rightarrow N S}\left(\boldsymbol{w}_{2}\right)\right)\right\|_{L^{\infty}\left(\Gamma_{W}\right)}^{2}\left\|\boldsymbol{w}_{1} \cdot \boldsymbol{t}_{j}\right\|_{L^{2}\left(\Gamma_{W}\right)}^{2} \\
& \quad+\frac{\epsilon}{2} \sum_{j=1}^{d-1}\left\|\boldsymbol{w} \cdot \boldsymbol{t}_{j}\right\|_{L^{2}\left(\Gamma_{W}\right)}^{2} \\
& \leq \frac{1}{2 \epsilon} \sum_{j=1}^{d-1}\left\|\beta_{j}\left(T_{L E S \rightarrow N S}\left(\boldsymbol{w}_{1}\right)\right)-\beta_{j}\left(T_{L E S \rightarrow N S}\left(\boldsymbol{w}_{2}\right)\right)\right\|_{L^{\infty}\left(\Gamma_{W}\right)}^{2} C_{t r}^{2} \frac{C_{M}^{4}}{\nu_{e}^{2}}\|\boldsymbol{f}\|_{-1, \Omega}^{2} \\
& \quad+\frac{\epsilon}{2} \sum_{j=1}^{d-1}\left\|\boldsymbol{w} \cdot \boldsymbol{t}_{j}\right\|_{L^{2}\left(\Gamma_{W}\right)}^{2},
\end{aligned}
$$

where we bounded the $L^{2}\left(\Gamma_{W}\right)$ norm by the $W^{1 / 2,2, \Gamma_{W}}$ norm, cf. [Otto99], p.159 in the last step. From Lemma 8.11 we know that (for each $j=1, \ldots, d-1$ )

$$
\begin{aligned}
\left\|\beta_{j}\left(T_{L E S \rightarrow N S}\left(\boldsymbol{w}_{1}\right)\right)-\beta_{j}\left(T_{L E S \rightarrow N S}\left(\boldsymbol{w}_{2}\right)\right)\right\|_{L^{\infty}\left(\Gamma_{W}\right)}^{2} & \\
& \leq C_{\beta}^{2}(\delta)\left\|T_{L E S \rightarrow N S}\left(\boldsymbol{w}_{1}\right)-T_{L E S \rightarrow N S}\left(\boldsymbol{w}_{2}\right)\right\|_{1, \Omega_{i}}^{2} .
\end{aligned}
$$

Thus we need an estimate for the term $\left\|T_{L E S \rightarrow N S}\left(\boldsymbol{w}_{1}\right)-T_{L E S \rightarrow N S}\left(\boldsymbol{w}_{2}\right)\right\|_{1, \Omega_{i}}$. We write $T_{L E S \rightarrow N S}\left(\boldsymbol{w}_{k}\right)=\tilde{\boldsymbol{u}}_{k}+\boldsymbol{W}_{k}(k=1,2)$, with $\boldsymbol{W}_{k}$ denoting the Hopf extension. Moreover, we write $\tilde{\boldsymbol{u}} \equiv \tilde{\boldsymbol{u}}_{1}-\tilde{\boldsymbol{u}}_{2}$. Then

$$
\begin{aligned}
\left\|T_{L E S \rightarrow N S}\left(\boldsymbol{w}_{1}\right)-T_{L E S \rightarrow N S}\left(\boldsymbol{w}_{2}\right)\right\|_{1, \Omega_{i}}^{2} & \leq 2\left\|\boldsymbol{W}_{1}-\boldsymbol{W}_{2}\right\|_{1, \Omega_{i}}^{2}+2\left\|\tilde{\boldsymbol{u}}_{1}-\tilde{\boldsymbol{u}}_{2}\right\|_{1, \Omega_{i}}^{2} \\
& \leq 2 C_{1}^{2}\left(d, \Omega_{i}\right)\left\|\boldsymbol{w}_{1}-\boldsymbol{w}_{2}\right\|_{1 / 2,2, \Gamma_{i}}^{2}+2\left\|\tilde{\boldsymbol{u}}_{1}-\tilde{\boldsymbol{u}}_{2}\right\|_{1, \Omega_{i}}^{2} \\
& \leq 2 C_{1}^{2}\left(d, \Omega_{i}\right)\left\|\boldsymbol{w}_{1}-\boldsymbol{w}_{2}\right\|_{1 / 2,2, \Gamma_{i}}^{2}+2 C_{P, i}^{2}\|\nabla \tilde{\boldsymbol{u}}\|_{L^{2}\left(\Omega_{i}\right)}^{2} .
\end{aligned}
$$


Combining the last five inequalities, we arrive at

$$
\begin{aligned}
\nu_{e}\|\nabla \boldsymbol{w}\|_{L^{2}(\Omega)}^{2}+\sum_{j=1}^{d-1}\left\langle\beta_{j}\left(T_{L E S \rightarrow N S}\left(\boldsymbol{w}_{2}\right)\right) \boldsymbol{w} \cdot \boldsymbol{t}_{j}, \boldsymbol{w} \cdot \boldsymbol{t}_{j}\right\rangle_{\Gamma_{W}} \\
(8.76) \leq C_{b} \frac{C_{M}^{3}}{\nu_{e}}\|\boldsymbol{f}\|_{-1, \Omega}\|\nabla \boldsymbol{w}\|_{L^{2}(\Omega)}^{2}+\frac{\epsilon}{2} \sum_{j=1}^{d-1}\left\|\boldsymbol{w} \cdot \boldsymbol{t}_{j}\right\|_{L^{2}\left(\Gamma_{W}\right)}^{2} \\
\quad+\frac{1}{2 \epsilon} \sum_{j=1}^{d-1} C_{\beta}^{2}(\delta)\left(2 C_{1}^{2}\left(d, \Omega_{i}\right)\|\boldsymbol{w}\|_{\left.1 / 2,2, \Gamma_{i}\right)}^{2}+2 C_{P, i}^{2}\|\nabla \tilde{\boldsymbol{u}}\|_{L^{2}\left(\Omega_{i}\right)}^{2}\right) C_{t r}^{2} \frac{C_{M}^{4}}{\nu_{e}^{2}}\|\boldsymbol{f}\|_{-1, \Omega}^{2}
\end{aligned}
$$

So we need an estimate for $\|\nabla \tilde{\boldsymbol{u}}\|_{L^{2}\left(\Omega_{i}\right)}^{2}$. In $(8.63)$, replacing $\boldsymbol{w}_{n}$ by $\boldsymbol{w}_{1}, \boldsymbol{w}$ by $\boldsymbol{w}_{2}$, and $\boldsymbol{\Phi}_{n}$ by $\tilde{\boldsymbol{u}}$, we get

$$
\begin{aligned}
{\left[\frac{3 \nu}{8}-C_{b} \frac{2}{\nu} C_{P, i}^{2}\left[\left.C_{u, 1}^{a p}\|\boldsymbol{f}\|\right|_{-1, \Omega_{i}}+C_{u, 2}^{a p}\left\|\boldsymbol{w}_{1}\right\|_{1 / 2,2, \Gamma_{i}}+C_{u, 3}^{a p}\left\|\boldsymbol{w}_{1}\right\|_{1 / 2,2, \Gamma_{i}}^{2}\right]\right.} \\
\left.\quad-C_{b} C_{1}\left(d, \Omega_{i}\right) C_{P, i}\left\|\boldsymbol{w}_{1}\right\|_{1 / 2,2, \Gamma_{i}}\right]\|\nabla \tilde{\boldsymbol{u}}\|_{L^{2}\left(\Omega_{i}\right)}^{2} \\
\leq \frac{8}{\nu^{3}}\left[C_{u, 1}^{a p}\|\boldsymbol{f}\|_{-1, \Omega_{i}}+C_{u, 2}^{a p}\left\|\boldsymbol{w}_{2}\right\|_{1 / 2,2, \Gamma_{i}}+C_{u, 3}^{a p}\left\|\boldsymbol{w}_{2}\right\|_{1 / 2,2, \Gamma_{i}}^{2}\right]^{2} \\
\quad C_{b}^{2} C_{1}^{2}\left(d, \Omega_{i}\right) C_{P, i}^{2}\|\boldsymbol{w}\|_{1 / 2,2, \Gamma_{i}}^{2} \\
+\frac{8}{\nu^{3}}\left[C_{u, 1}^{a p}\|\boldsymbol{f}\|_{-1, \Omega_{i}}+C_{u, 2}^{a p}\left\|\boldsymbol{w}_{1}\right\|_{1 / 2,2, \Gamma_{i}}+C_{u, 3}^{a p}\left\|\boldsymbol{w}_{1}\right\|_{1 / 2,2, \Gamma_{i}}^{2}\right]^{2} \\
\quad C_{b}^{2} C_{1}^{2}\left(d, \Omega_{i}\right) C_{P, i}^{2}\|\boldsymbol{w}\|_{1 / 2,2, \Gamma_{i}}^{2} \\
+2 \nu C_{1}^{2}\left(d, \Omega_{i}\right)\|\boldsymbol{w}\|_{1 / 2,2, \Gamma_{i}}^{2} \\
\quad+\frac{2}{\nu} C_{b}^{2} C_{P, i}^{2} C_{1}^{4}\left(d, \Omega_{i}\right)\left(\left\|\boldsymbol{w}_{1}\right\|_{1 / 2,2, \Gamma_{i}}^{2}+\left\|\boldsymbol{w}_{2}\right\|_{1 / 2,2, \Gamma_{i}}^{2}\right)\|\boldsymbol{w}\|_{1 / 2,2, \Gamma_{i}}^{2} .
\end{aligned}
$$

Combining trace inequality and the a priori estimate for $\boldsymbol{w}_{i}$ (cf. (8.27)), we obtain

$$
\left\|\boldsymbol{w}_{i}\right\|_{1 / 2,2, \Gamma_{i}}^{2} \leq C_{t r}^{2}\left\|\boldsymbol{w}_{i}\right\|_{1,\left(\Omega \backslash \Omega_{i}\right)}^{2} \leq C_{t r}^{2}\left\|\boldsymbol{w}_{i}\right\|_{1, \Omega}^{2} \leq \frac{C_{t r}^{2} C_{M}^{4}}{\nu_{e}^{2}}\|\boldsymbol{f}\|_{-1, \Omega}^{2} .
$$

Inserting this into (8.77) gives (using (8.73))

$$
\begin{aligned}
& \left(\frac{3 \nu}{8}-C_{b} \frac{2}{\nu} C_{P, i}^{2} K_{d}\left(\boldsymbol{f}, \nu, \nu_{e}\right)-C_{b} C_{1}\left(d, \Omega_{i}\right) C_{P, i} \frac{C_{t r} C_{M}^{2}}{\nu_{e}}\|\boldsymbol{f}\|_{-1, \Omega}\right)\|\nabla \tilde{\boldsymbol{u}}\|_{L^{2}\left(\Omega_{i}\right)}^{2} \\
& \leq \frac{16}{\nu^{3}} K_{d}\left(\boldsymbol{f}, \nu, \nu_{e}\right)^{2} C_{b}^{2} C_{1}^{2}\left(d, \Omega_{i}\right) C_{P, i}^{2}\|\boldsymbol{w}\|_{1 / 2,2, \Gamma_{i}}^{2} \\
& \quad+\left(2 \nu C_{1}^{2}\left(d, \Omega_{i}\right)+\frac{2}{\nu} C_{b}^{2} C_{P, i}^{2} C_{1}^{4}\left(d, \Omega_{i}\right) 2 \frac{C_{t r}^{2} C_{M}^{4}}{\nu_{e}^{2}}\|\boldsymbol{f}\|_{-1, \Omega}^{2}\right)\|\boldsymbol{w}\|_{1 / 2,2, \Gamma_{i}}^{2}
\end{aligned}
$$

Now we define $K_{2}$ as

$$
K_{2} \equiv \frac{3 \nu}{8}-C_{b} C_{1}\left(d, \Omega_{i}\right) C_{P, i} \frac{C_{t r} C_{M}^{2}}{\nu_{e}}\|\boldsymbol{f}\|_{-1, \Omega}-C_{b} \frac{2}{\nu} C_{P, i}^{2} K_{d}\left(\boldsymbol{f}, \nu, \nu_{e}\right) .
$$


Substituting (8.78) and (8.79) into (8.76) and using $\beta_{j} \geq \beta_{0}$, we obtain

$$
\begin{aligned}
& \nu_{e}\|\nabla \boldsymbol{w}\|_{L^{2}(\Omega)}+\sum_{j=1}^{d-1} \beta_{0}\left\|\boldsymbol{w} \cdot \boldsymbol{t}_{j}\right\|_{L^{2}\left(\Gamma_{W}\right)}^{2} \\
& \leq C_{b} \frac{C_{M}^{3}}{\nu_{e}}\|\boldsymbol{f}\|_{-1, \Omega}\|\nabla \boldsymbol{w}\|_{L^{2}(\Omega)}^{2}+\frac{\epsilon}{2} \sum_{j=1}^{d-1}\left\|\boldsymbol{w} \cdot \boldsymbol{t}_{j}\right\|_{L^{2}\left(\Gamma_{W}\right)}^{2} \\
& \quad+\frac{1}{2 \epsilon}(d-1) C_{\beta}^{2}(\delta) C_{t r}^{2} \frac{C_{M}^{4}}{\nu_{e}^{2}}\|\boldsymbol{f}\|_{-1, \Omega}^{2}\left[2 C_{1}^{2}\left(d, \Omega_{i}\right)\|\boldsymbol{w}\|_{1 / 2,2, \Gamma_{i}}^{2}\right. \\
& \quad+2 C_{P, i}^{2} K_{2}^{-1}\left(\frac{16}{\nu^{3}} K_{d}\left(\boldsymbol{f}, \nu, \nu_{e}\right)^{2} C_{b}^{2} C_{1}^{2}\left(d, \Omega_{i}\right) C_{P, i}^{2}\|\boldsymbol{w}\|_{1 / 2,2, \Gamma_{i}}^{2}\right. \\
& \left.\left.\quad+\left(2 \nu C_{1}^{2}\left(d, \Omega_{i}\right)+\frac{2}{\nu} C_{b}^{2} C_{P, i}^{2} C_{1}^{4}\left(d, \Omega_{i}\right) 2 \frac{C_{t r}^{2} C_{M}^{4}}{\nu_{e}^{2}}\|\boldsymbol{f}\|_{-1, \Omega}^{2}\right)\|\boldsymbol{w}\|_{1 / 2,2, \Gamma_{i}}^{2}\right)\right]
\end{aligned}
$$

Finally we apply the following estimate, videlicet,

$$
\|\boldsymbol{w}\|_{1 / 2,2, \Gamma_{i}}^{2} \leq C_{t r}^{2}\|\boldsymbol{w}\|_{1,\left(\Omega \backslash \Omega_{i}\right)}^{2} \leq C_{t r}^{2} C_{M}^{2}\|\nabla \boldsymbol{w}\|_{L^{2}(\Omega)}^{2} .
$$

Substituting this, 8.80 ) can be rearranged to

$$
\left(\nu_{e}-K_{c}\right)\|\nabla \boldsymbol{w}\|_{L^{2}(\Omega)}^{2}+\sum_{j=1}^{d-1}\left(\beta_{0}-\frac{\epsilon}{2}\right)\left\|\boldsymbol{w} \cdot \boldsymbol{t}_{j}\right\|_{L^{2}\left(\Gamma_{W}\right)}^{2} \leq 0
$$

with $K_{2}$ being defined in $(8.79)$ and

$$
\begin{aligned}
K_{c} \equiv & C_{b} \frac{C_{M}^{3}}{\nu_{e}}\|\boldsymbol{f}\|_{-1, \Omega}+\frac{1}{2 \epsilon}(d-1) C_{\beta}^{2}(\delta) C_{t r}^{4} \frac{C_{M}^{6}}{\nu_{e}^{2}}\|\boldsymbol{f}\|_{-1, \Omega}^{2} \quad\left[2 C_{1}^{2}\left(d, \Omega_{i}\right)\right. \\
& +2 C_{P, i}^{2} K_{2}^{-1}\left(\frac{16}{\nu^{3}} K_{d}\left(\boldsymbol{f}, \nu, \nu_{e}\right)^{2} C_{b}^{2} C_{1}^{2}\left(d, \Omega_{i}\right) C_{P, i}^{2}+\left(2 \nu C_{1}^{2}\left(d, \Omega_{i}\right)\right.\right. \\
& \left.\left.\left.+\frac{2}{\nu} C_{b}^{2} C_{P, i}^{2} C_{1}^{4}\left(d, \Omega_{i}\right) 2 \frac{C_{t r}^{2} C_{M}^{4}}{\nu_{e}^{2}}\|\boldsymbol{f}\|_{-1, \Omega}^{2}\right)\right)\right]
\end{aligned}
$$

Due to the assumptions of this theorem, we can find $\epsilon>0$ s.t. $\beta_{0}-\epsilon / 2>0$ and $\nu_{e}-K_{c}>0$. Thus $\boldsymbol{w}=0$, i.e., $\boldsymbol{w}_{1}=\boldsymbol{w}_{2}$. Then $\boldsymbol{u}_{i}=T_{L E S \rightarrow N S}\left(\boldsymbol{w}_{i}\right)$ implies $\boldsymbol{u}_{1}=\boldsymbol{u}_{2}$.

\subsection{Some closing remarks}

In this section the following issues are discussed, viz., the slip with resistance boundary condition for LES, the matching condition on $\Gamma_{i}$ the corresponding steady state problem and its simplification, and finally how to use this coupling scheme in a computational model.

\subsubsection{The slip with resistance boundary condition for LES}

Traditionally, boundary condition (7.5) has been used in LES with near wall modelling. In [GL00] GALDI and LAYTON proposed the following slip with linear friction and no 
penetration boundary condition (with given friction parameters $\beta_{j}$ ):

$$
\begin{aligned}
& \boldsymbol{w} \cdot \boldsymbol{n}=0 \quad \text { on } \Gamma_{W}, \\
& \beta_{j} \boldsymbol{w} \cdot \boldsymbol{t}_{j}+\boldsymbol{n}^{T}\left(\nu_{e} \nabla(\boldsymbol{w})+\mathcal{A}_{u}(\boldsymbol{w})\right) \boldsymbol{t}_{j}=0 \quad \text { on } \quad \Gamma_{W}, \quad 1 \leq j \leq d-1 .
\end{aligned}
$$

As a motivation for this boundary condition, LAYTON refers to the phenomenon of hurricanes, who slip along the ground and thereby loose their energy. Note that in the limit case $\beta_{j} \rightarrow \infty$, the no-slip condition is recovered. In the limit case $\beta_{j} \rightarrow 0$, the free slip condition is obtained.

The parameters $\beta_{j}$ should depend on the filter width $\delta$ and on the flow parameter $R e$ (or, for sake of simplicity, on $\nu$ ). To be consistent, in (8.83) for fixed $\nu$ we must have $\beta_{j}(\delta, \nu) \rightarrow \infty$ as $\delta \rightarrow 0$, since the non-space-filtered velocity field satisfies the no slip condition.

A reasonable specification for $\beta_{j}$ was given in (8.7). We will close this subsection by investigating the following special situation for a flow over a flat plate. Assume the filter width $\delta$ is smaller than the boundary-layer thickness. Denote $y \equiv x_{2}$ the wall normal direction. Denote $x_{1}$ and $x_{3}$ streamwise and spanwise direction resp. Assume that in the near wall region the velocity $\boldsymbol{u}$ is given by $\boldsymbol{u}=U(y) \boldsymbol{t}_{1}$, with $\boldsymbol{t}_{1}$ being directed in the $x_{1}$-direction. Then $\beta_{1}$ is well-defined using (8.7). However, concerning $\beta_{3}$ both numerator and denominator vanish and (8.7) is not well-defined. In this particular situation, as a remedy we suggest $\beta_{3}=\beta_{1}$.

\subsubsection{Some remarks concerning the matching condition on $\Gamma_{i}$}

In this subsection we consider the matching condition on $\Gamma_{i}$, namely (8.6). First we show that $\boldsymbol{u}=\boldsymbol{w}$ on $\Gamma_{i} \times(0, T)$ is in some sense compatible with the continuity equation. Second we develop a more sophisticated matching condition.

\section{Lemma 8.12}

The boundary condition $\boldsymbol{u}=\boldsymbol{w}$ on $\Gamma_{i} \times(0, T)$ (see (8.6)) is compatible with the continuity equations (8.2) and (8.5) in the following sense: The boundary integral of $\boldsymbol{u} \cdot \boldsymbol{n}$ and $\boldsymbol{w} \cdot \boldsymbol{n}$ along $\Gamma_{i}$ vanishes.

\section{Proof:}

Integrating the r.h.s. in (8.2) over $\Omega_{\text {layer }}$ and integration by parts gives (by virtue of (8.3) in the last step)

$$
0=\int_{\Omega_{\text {layer }}} \nabla \cdot \boldsymbol{w} d x=\int_{\Gamma_{W}} \boldsymbol{w} \cdot \boldsymbol{n} d s+\int_{\Gamma_{i}} \boldsymbol{w} \cdot \boldsymbol{n} d s=\int_{\Gamma_{i}} \boldsymbol{w} \cdot \boldsymbol{n} d s
$$

Similarly from (8.5) and (8.6) (i.e. $\boldsymbol{u}=0$ on $\Gamma_{W}$ ) we have

$$
0=\int_{\Omega_{\text {layer }}} \nabla \cdot \boldsymbol{u} d x=\int_{\Gamma_{i}} \boldsymbol{u} \cdot \boldsymbol{n} d s
$$

Therefore both $\boldsymbol{u} \cdot \boldsymbol{n}$ and $\boldsymbol{w} \cdot \boldsymbol{n}$ have zero boundary integral over $\Gamma_{i}$. 
Now we develop a more sophisticated matching on $\Gamma_{i}$. Recall that $\boldsymbol{w}$ is an approximation of $\overline{\boldsymbol{u}}$. Thus it seems natural to provide more information for the matching condition on $\Gamma_{i}$. As an example, write $\boldsymbol{u}=\overline{\boldsymbol{u}}+\boldsymbol{u}^{\prime}$ with $\boldsymbol{w} \approx \overline{\boldsymbol{u}}$. Then a natural matching condition seems to be

$$
\boldsymbol{u}=\boldsymbol{w}+\text { approximation of } \boldsymbol{u}^{\prime} \text { on } \Gamma_{i} .
$$

An approximation of $\boldsymbol{u}^{\prime}$ can be given via a scale similarity argument.

$$
\boldsymbol{u}^{\prime}=\boldsymbol{u}-\overline{\boldsymbol{u}} \approx \overline{\boldsymbol{u}}-\overline{\bar{u}} \approx \boldsymbol{w}-\overline{\boldsymbol{w}} .
$$

This gives the condition

$$
\boldsymbol{u}=\boldsymbol{w}+(\boldsymbol{w}-\overline{\boldsymbol{w}})=2 \boldsymbol{w}-\overline{\boldsymbol{w}} \text { on } \Gamma_{i} .
$$

However, this boundary condition is not compatible in the sense of Lemma 8.12. On the one hand, the integral over the left hand side in (8.88) vanishes, see Lemma 8.12. On the other hand, integrating the right hand side of (8.88) over $\Gamma_{W}$ gives

$$
\int_{\Gamma_{i}}(2 \boldsymbol{w}-\overline{\boldsymbol{w}}) \cdot \boldsymbol{n} d s=-\int_{\Gamma_{i}} \overline{\boldsymbol{w}} \cdot \boldsymbol{n} d s
$$

However, $\int_{\Gamma_{W}} \overline{\boldsymbol{w}} \cdot \boldsymbol{n} d s$ is generally nonzero (although small). Instead we could consider the following matching condition

$$
\boldsymbol{u}=2 \boldsymbol{w}-\overline{\boldsymbol{w}}+\frac{1}{\mu\left(\Gamma_{i}\right)} \int_{\Gamma_{i}} \overline{\boldsymbol{w}} \cdot \boldsymbol{n} d s \quad \text { on } \Gamma_{i} .
$$

By construction, this condition is compatible.

\subsubsection{The steady state case}

In this chapter we restrict ourselves to the isothermal steady state case of (8.1)-(8.6), primarily for the following reason. We recall the model problem considered in section 1.4: Le Tallec and Tidriri seek the stationary solution to the time-dependent problem (1.30)(1.34). Semidiscretization in time by applying a backward-Euler scheme then results in a sequence of coupled stationary problems for each time step. Thus investigating the coupled steady state problem is of mathematical relevance. Of course, in the case of the stationary problem stemming from a backward-Euler scheme, an additional term from the discretized time step appears. In our analysis we neglect this term; however, this term is propitious regarding the analysis. It might be possible improving our results by accounting for this term in the analysis.

For our coupled problem, i.e. (8.1)-(8.6), we cannot expect to obtain similar analytical results as Le Tallec and Tidriri. The reason is the following: The equation corresponding to (1.43) in our case is (8.1). However, there is no exact boundary condition for (8.1). In contrast, for (1.43) (resp. (1.27) for the scalar case) homogeneous Dirichlet conditions can be imposed. Only if $\delta(\boldsymbol{x}) \rightarrow 0$ as $\boldsymbol{x} \rightarrow 0$ we can impose a no slip condition for $\boldsymbol{w}$, but this case is not of practical interest. Moreover, for the same reason, we cannot expect to benefit 
from utilising the analytical techniques used by Le Tallec and Tidriri.

An aim for future research could be to appraise the solution of this coupled scheme. For example, assume that there is a LES model $\mathcal{A}_{u}$, that has the property that the global LES solution $\boldsymbol{w}$ converges to the corresponding global DNS solution $\boldsymbol{u}$ as the filter width $\delta$ goes to zero. Then the question is whether the solution of the coupled scheme converges to $\boldsymbol{u}$ as $\delta \rightarrow 0$.

\subsubsection{Steps towards a computational model}

In this subsection we give some comments on the usage of this coupling method in a computational model. For sake of simplicity, in the analysis we consider the situation sketched in Figure 1.2, i.e., in particular, there is no inflow or outflow boundary. Of course, the aim is to apply the method to more complex flow problems like that shown in figure 8.2. Using a DNS in the near wall region makes the approach computationally expensive.

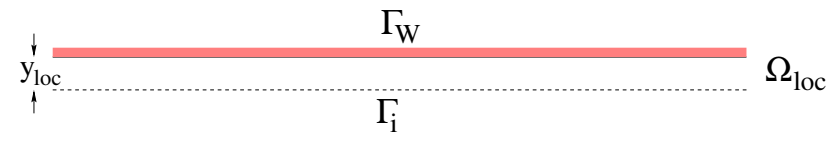

$\Omega$

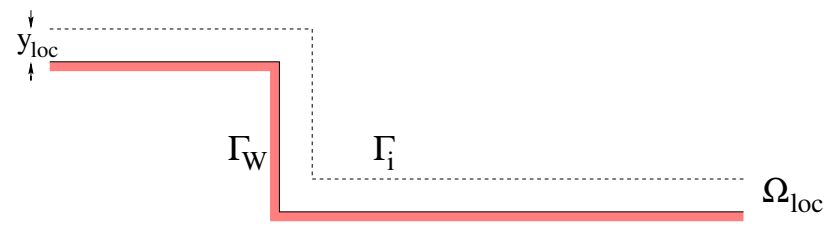

Figure 8.2.: Fully overlapping DDM for a flow over a backward facing step.

The costs of a DNS in the boundary layer can be comparable to those of the global LES. In practical situations it is often not necessary to employ a DNS in $\Omega_{\text {layer }}$, because there are classes of technologically interesting flows for which a certain turbulence model gives quite accurate predictions. Thus again we end up with the task of seeking a simpler boundarylayer solution $\boldsymbol{u}^{B L}$ in the region close to $\Gamma_{W}$.

(1) If we can assume that in the boundary layer the flow $\boldsymbol{u}^{B L}$ is reasonably described by (5.27) with $\Theta=0$, then there are several semi-empirical explicit formulae for $\boldsymbol{u}^{B L}$, e.g. a log-law or a power-law. From these, explicit formulae for $\beta_{j}(\delta, \nu)$ can be derived, see [.MS02], who considered a power-law profile for $\boldsymbol{u}^{B L}$.

(2) A more advanced method is to solve the two dimensional turbulent boundary-layer equations (5.18), i.e. to solve a RANS problem in the boundary layer. For $\nu_{t}$ in (5.18), an algebraic turbulence model or the Spalart-Allmaras model could be used.

In case (2) the space averaged quantities in (8.7) have to be evaluated numerically.

In order to provide some illustration, we consider the situation sketched in Figure 8.2. It 
shows some prototypical flow situations (with unidirectional inflow on the left), for which certain RANS give appropriate results. We can assume that the log-law holds on the upper wall. The log-law also holds on the lower wall until the separation point is reached. However, for predicting the behaviour at the separation point and in the lee of the step, applying the Spalart-Allmaras model seems to be the currently best option. In other situations, e.g. for describing a jet impinging on a flat plate, the $k-\epsilon-\overline{v^{2}}$ model seems to be a suitable choice. 


\section{Part II.}

Numerical solution scheme and numerical tests 



\section{Semidiscretisation in time, decoupling and linearisation}

The second part of this thesis is dedicated to the numerical solution scheme which is applied to the turbulence models presented in Chapters 6 and 7 . This chapter is devoted to the semidiscretisation in time and a subsequent decoupling and linearisation strategy. Finally we will obtain an uncoupled system of linear stationary problems of Oseen-type and of advection-diffusion-reaction-type as underlying basic equations. Describing the variational formulation for these basic equations will conclude this chapter.

\subsection{Semidiscretisation in time using the discontinuous Galerkin method}

In our research group, semidiscretisation in time is performed by the so-called discoutinuous Galerkin method. For this purpose the time interval $(0, T]$ is divided into subsequent subintervals $J_{m} \equiv\left(t_{m-1}, t_{m}\right]$ with splitting points $0=t_{0}<t_{1}<\ldots<t_{M}=T$ and time step widths $\triangle_{m}=t_{m}-t_{m-1}$. Then the discontinuous Galerkin method of order $q$ (abbreviated $D G(q)$ ) seeks an approximate solution (of the corresponding continuous problem), whose restriction to $J_{m}$ is a polynomial in $t$ of degree $q$ with coefficients in $\mathcal{X}$. Here $\mathcal{X}$ is the solution space of the corresponding stationary problem, which is usually a suitable subspace of $H^{1}(\Omega)$ for problems of advection-diffusion-reaction type resp. of $\left(H^{1}(\Omega)\right)^{d} \times L^{2}(\Omega)$ for problems of Navier-Stokes type.

For instationary problems of advection-diffusion-reaction type and of Oseen type, the $D G(0)$ and $D G(1)$ method have been implemented in our research code PARALLELNS; numerical results can be found in [Mue99], [Mue(0)] and [Fis(2)]. For the $k / \epsilon$ model only the $D G(0)$ method is available; for numerical results see e.g. [Gri01]. Therefore in this thesis only the $D G(0)$ method will be considered. It is worthwhile emphasising that the $D G(0)$ method covers the backward Euler method when approximating the r.h.s. integrals by the rectangular rule $\int_{t_{m}-1}^{t_{m}} f(t, \boldsymbol{x}) d t \approx \triangle_{m} f\left(t_{m}, \boldsymbol{x}\right)$. Both schemes are of first order accuracy in time. The $D G(0)$ method is attractive because linearisation and decoupling of our non-linear and coupled systems of equations can be accomplished quite easily in its framework. On the other hand the $D G(0)$ method has the drawback of being only of first order accuracy. We intend to circumvent this shortcoming by using very fine time steps in combination with a time adaptive algorithm, see [Mue(00] and [Fis(2]. As a final remark it is worth pointing out that, to our experience, the presented scheme works reasonable for flow problems for which a (quasi) stationary solution exists. This is satisfied in many indoor-air flow problems of practical interest, but cannot be expected in mixed resp. natural convection flow problems in general.

For the numerical analysis of the $D G(q)$ method we refer to [Tho.97], Chapter 12 and to [Mue00], [Fis(2)]. 


\subsection{Semidiscretisation, decoupling, and linearisation for the $k / \epsilon$ model}

First be consider $k / \epsilon$ MODEL IIIA and $k / \epsilon$ MODEL IIIB.

\subsubsection{Semidiscretisation in time for the $k / \epsilon$ model}

In a first step we semidiscretise $k / \epsilon$ MODEL I, see Section 6.1, w.r.t. the temporal variable using the discontinuous Galerkin method $\mathrm{DG}(0)$. Therefore the time interval $[0, T]$ is divided into subintervals $J_{m} \equiv\left(t_{m-1}, t_{m}\right]$ with splitting points $0=t_{0}<t_{1}<\ldots<t_{M}=T$ and time step widths $\triangle_{m}=t_{m}-t_{m-1} \cdot \boldsymbol{U}^{m}, P^{m}, \Theta^{m}, k^{m}$, and $\epsilon^{m}$ the (time constant) solution in $J_{m}$. Then we arrive at the following scheme:

\section{DG(0) method for the $k / \epsilon$ turbulence model}

Given $\boldsymbol{U}^{m-1}, P^{m-1}, \Theta^{m-1}, k^{m-1}$, and $\epsilon^{m-1}$ from the previous time step (if $m>1$ ) or from the initial condition (if $m=1$ ) seek $\boldsymbol{U}^{m}, P^{m}, \Theta^{m}, k^{m}, \epsilon^{m}$ s.t.

$$
\begin{aligned}
\frac{\boldsymbol{U}^{m}-\boldsymbol{U}^{m-1}}{\triangle_{m}}-\boldsymbol{\nabla} \cdot\left(2 \nu_{e}^{m} \mathbb{S}\left(\boldsymbol{U}^{m}\right)\right)+\left(\boldsymbol{U}^{m} \cdot \boldsymbol{\nabla}\right) \boldsymbol{U}^{m} & =-\boldsymbol{\nabla} P^{m}-\beta \Theta^{m} \boldsymbol{g}, \\
\boldsymbol{\nabla} \cdot \boldsymbol{U}^{m} & =0, \\
\frac{\Theta^{m}-\Theta^{m-1}}{\triangle_{m}}+\left(\boldsymbol{U}^{m} \cdot \boldsymbol{\nabla}\right) \Theta^{m}-\boldsymbol{\nabla} \cdot\left(a_{e}^{m} \boldsymbol{\nabla} \Theta^{m}\right) & =\frac{\left(\dot{q}^{V}\right)^{m}}{c_{p}}, \\
\frac{k^{m}-k^{m-1}}{\triangle_{m}}+\left(\boldsymbol{U}^{m} \cdot \boldsymbol{\nabla}\right) k^{m}-\boldsymbol{\nabla} \cdot\left(\nu_{k}^{m} \nabla k^{m}\right) & =P_{k}^{m}+G^{m}-\epsilon^{m}, \\
\frac{\epsilon^{m}-\epsilon^{m-1}}{\triangle_{m}}+\left(\boldsymbol{U}^{m} \cdot \boldsymbol{\nabla}\right) \epsilon^{m}-\boldsymbol{\nabla} \cdot\left(\nu_{\epsilon}^{m} \nabla \epsilon^{m}\right)+C_{2} \frac{\left(\epsilon^{m}\right)^{2}}{k^{m}} & =C_{1} \frac{\epsilon^{m}}{k^{m}}\left(P_{k}^{m}+G^{m}\right) .
\end{aligned}
$$

Here we used the following abbreviations

$$
\begin{aligned}
& P_{k}^{m}=2 \nu_{t}^{m}\left\|\mathbb{S}\left(\boldsymbol{U}^{m}\right)\right\|_{F}^{2}, \quad G^{m}=C_{t} \beta \frac{\nu_{t}^{m}}{P r_{t}} \boldsymbol{g} \cdot \nabla \Theta^{m}, \quad \nu_{t}^{m}=c_{\mu} \frac{\left(k^{m}\right)^{2}}{\epsilon^{m}}, \\
& \nu_{e}^{m}=\nu+\nu_{t}^{m}, \quad a_{e}^{m}=\frac{\nu}{P r}+\frac{\nu_{t}^{m}}{P r_{t}}, \quad \nu_{k}^{m}=\nu+\frac{\nu_{t}^{m}}{P r_{k}}, \quad \nu_{\epsilon}^{m}=\nu+\frac{\nu_{t}^{m}}{P r_{\epsilon}} .
\end{aligned}
$$

Thus the instationary problem (3.28)-(3.32) is reduced to the sequence of successively solved stationary problems (9.1)-(9.5).

\subsubsection{Iterative decoupling and linearisation for the $k / \epsilon$ model}

Equations (9.1)-(9.5) are highly coupled; moreover, due to the boundary conditions specified in MODEL IIIA and MODEL IIIB an additional coupling occurs. Thus we need a decoupling and linearisation strategy.

We fix $m$ and a time slice $J_{m} \equiv\left(t_{m-1}, t_{m}\right]$. Denote a second upper index $i t_{d l c}$ the decoupling and linearisation cycle.

Given $\boldsymbol{U}^{m, 0}, P^{m, 0}, \Theta^{m, 0}, k^{m, 0}, \epsilon^{m, 0}, U_{*}^{m, 0}, \dot{q}^{m, 0}$ as the solution of the previous time step 
(or from an initial guess or the initial condition):

\section{Decoupling and linearisation scheme for the $k / \epsilon$ model}

(1) Set $i t_{d l c} \longleftarrow 1$.

(2) Set $i \longleftarrow i t_{d l c}$

(3) Update turbulent viscosity and turbulent diffusivities

$$
\begin{aligned}
& \nu_{t}^{m} \longleftarrow C_{\mu} \frac{\left(k^{m, i-1}\right)^{2}}{\epsilon^{m, i-1}}, \quad \nu_{e}^{m} \longleftarrow \nu+\nu_{t}^{m}, \\
& a_{e}^{m} \longleftarrow \frac{\nu}{P r}+\frac{\nu_{t}^{m}}{P r_{t}}, \quad \nu_{k}^{m} \longleftarrow \nu+\frac{\nu_{t}^{m}}{P r_{k}}, \quad \nu_{\epsilon}^{m} \longleftarrow \nu+\frac{\nu_{t}^{m}}{P r_{\epsilon}} .
\end{aligned}
$$

(4) Update boundary conditions for momentum and temperature: For each $\boldsymbol{x} \in \Gamma_{W}$

- If effects of stratification have to be considered: Update $R e_{\min }$ according to

$$
R e_{\min }=6.25 e^{K_{s} R i_{g}}, \text { with } R i_{g}=-\frac{\boldsymbol{g} \cdot \boldsymbol{n} \dot{q}^{m, i-1} \operatorname{Pr} \nu}{\left.\left(U_{*}^{m, i-1}\right)^{4} c_{p} \Theta^{m, i-1}\right|_{\Gamma_{W}}} .
$$

with $\boldsymbol{n}$ being the outer normal vector to $\Gamma_{W}$ in $\boldsymbol{x}$. Use a cut-off technique to ensure $R e_{\text {min,cut }} \leq R e_{\text {min }} \leq 70.0$ with $R e_{\text {min,cut }} \in[2,12]$.

- Define $U\left(y_{\delta}\right)$ and $\Theta\left(y_{\delta}\right)$ by (see also the notation given in (6.3))

$$
U\left(y_{\delta}\right)=\frac{\boldsymbol{U}_{\delta}^{m, i-1} \cdot \boldsymbol{U}_{\delta, t}^{m, i-1}}{\left\|\boldsymbol{U}_{\delta, t}^{m, i-1}\right\|}, \quad \Theta\left(y_{\delta}\right)=\left.\Theta^{m, i-1}\right|_{\Gamma_{\delta}} .
$$

- Seek $\left(U_{*}^{m, i}, \dot{q}^{m, i}\right)$ as specified in step (2) of $k / \epsilon$ MODEL IIIB resp. MOdEL IIIA.

- Check that $\Gamma_{\delta}$ is mostly contained in the log-layer.

(5) Solve the linearised Navier-Stokes equations

$$
\begin{aligned}
& \frac{\boldsymbol{U}^{m, i}-\boldsymbol{U}^{m-1}}{\triangle_{m}}+\left(\boldsymbol{U}^{m, i-1} \cdot \boldsymbol{\nabla}\right) \boldsymbol{U}^{m, i}-\nabla \cdot\left(2 \nu_{e}^{m} \mathbb{S}\left(\boldsymbol{U}^{m, i}\right)\right)+\nabla P^{m, i}=-\beta \Theta^{m, i-1} \boldsymbol{g}, \\
& \boldsymbol{\nabla} \cdot \boldsymbol{U}^{m, i}=0, \\
& \boldsymbol{U}^{m, i}=\boldsymbol{U}_{i n} \text { on } \Gamma_{F}, \quad \sigma\left(\boldsymbol{U}^{m, i}, P^{m, i}\right) \boldsymbol{n}=\mathbf{0} \text { on } \Gamma_{N}, \\
& \boldsymbol{n}^{T} \sigma\left(\boldsymbol{U}^{m, i}, P^{m, i}\right) \boldsymbol{t}_{j}=\left(U_{*}^{m, i}\right)^{2} \frac{\boldsymbol{U}_{\delta, t}^{m, i-1} \cdot \boldsymbol{t}_{j}}{\left\|\boldsymbol{U}_{\delta, t}^{m, i-1}\right\|}, \quad \boldsymbol{U}^{m, i} \cdot \boldsymbol{n}=0 \quad \text { on } \quad \Gamma_{W} \text {. }
\end{aligned}
$$


(6) Solve the temperature equation

$$
\begin{aligned}
\frac{\Theta^{m, i}-\Theta^{m-1}}{\triangle_{m}}+\left(\boldsymbol{U}^{m, i} \cdot \nabla\right) \Theta^{m, i}-\nabla \cdot\left(a_{e}^{m} \nabla \Theta^{m, i}\right) & =\frac{\left(\dot{q}^{V}\right)^{m, i}}{c_{p}} \\
\Theta^{m, i}=\Theta_{i n} \text { on } \Gamma_{-}\left(\boldsymbol{U}^{m, i}\right), & a \nabla \Theta^{m, i} \cdot \boldsymbol{n}=0 \text { on } \Gamma_{+}\left(\boldsymbol{U}^{m, i}\right), \\
a \nabla \Theta^{m, i} \cdot \boldsymbol{n} & =\frac{\dot{q}^{m, i}}{c_{p}} \text { on } \Gamma_{W}
\end{aligned}
$$

(7) Update $P_{k}^{m} \longleftarrow 2 \nu_{t}^{m}\left\|\mathbb{S}\left(\boldsymbol{U}^{m, i}\right)\right\|_{F}^{2}, G^{m} \longleftarrow C_{t} \beta \frac{\nu_{t}^{m}}{P r_{t}} \boldsymbol{g} \cdot \nabla \Theta^{m, i}$, and solve the $k$-equation.

$$
\begin{aligned}
& \frac{k^{m, i}-k^{m-1}}{\triangle_{m}}+\left(\boldsymbol{U}^{m, i} \cdot \nabla\right) k^{m, i}-\nabla \cdot\left(\nu_{k}^{m} \nabla k^{m, i}\right)=P_{k}^{m}+G^{m}-\epsilon^{m, i-1} \\
& k^{m, i}=1.5\left(T_{u}\left\|\boldsymbol{U}^{m, i}\right\|\right)^{2} \quad \text { on } \quad \Gamma_{-}\left(\boldsymbol{U}^{m, i}\right), \quad \nu \nabla k^{m, i} \cdot \boldsymbol{n}=0 \quad \text { on } \quad \Gamma_{+}\left(\boldsymbol{U}^{m, i}\right), \\
& k^{m, i}=\frac{\left(U_{*}^{m, i}\right)^{2}}{C_{\mu}^{1 / 2}} \quad \text { on } \quad \Gamma_{\delta} .
\end{aligned}
$$

(8) Solve the $\epsilon$-equation.

$$
\begin{aligned}
\frac{\epsilon^{m, i}-\epsilon^{m-1}}{\triangle_{m}}+\boldsymbol{U}^{m, i} \cdot \nabla \epsilon^{m, i}-\nabla \cdot\left(\nu_{\epsilon}^{m} \nabla \epsilon^{m, i}\right)+C_{2} \frac{\epsilon^{m, i-1}}{k^{m, i}} \epsilon^{m, i} & =C_{1} \frac{\epsilon^{m, i-1}}{k^{m, i}}\left(P_{k}^{m}+G^{m}\right) \\
\epsilon^{m, i}=C_{\mu}^{3 / 4}\left(k^{m, i}\right)^{3 / 2} L^{-1} \quad \text { on } \quad \Gamma_{-}\left(\boldsymbol{U}^{m, i}\right), \quad \nu \nabla \epsilon^{m, i} \cdot \boldsymbol{n} & =0 \quad \text { on } \quad \Gamma_{+}\left(\boldsymbol{U}^{m, i}\right), \\
\epsilon^{m, i} & =\frac{\left.U_{*}^{m, i}\right)^{3}}{\kappa y_{\delta}} \text { on } \quad \Gamma_{\delta} .
\end{aligned}
$$

(9) Stopping-criterion for linearisation cycle : If $i t_{d l c}<\max _{d l c}$ and if certain stopping criteria for $\left\{\boldsymbol{u}^{m, i}\right\}_{i},\left\{\Theta^{m, i}\right\}_{i},\left\{k^{m, i}\right\}_{i}$, and $\left\{\epsilon^{m, i}\right\}_{i}$ are not yet fulfilled, then set $i t_{d l c} \longleftarrow$ $i t_{d l c}+1$ and goto step (2). Otherwise goto next time step.

\section{Remark 9.1}

If $\Gamma_{\delta}$ is partially located in the viscous sublayer, we use (5.49) on $\Gamma_{\delta}$ for $k^{m, i}$ and $\epsilon^{m, i}$.

\section{Remark 9.2}

Concerning the task of determining $\left(U_{*}^{m, i}\right)$ and $\dot{q}^{m, i}$, our approach is analogous to the fixed point scheme (1.39)-(1.42) and (1.47)-(1.51).

\subsubsection{Arising model problems for the $k / \epsilon$ model}

The iterative scheme requires the solution of the following model problems: First, the linearised equations for $\theta, k$ and $\epsilon$ are advection-diffusion-reaction problems (with nonconstant viscosity) of the general form :

$$
\begin{aligned}
L u \equiv-\nabla \cdot(\nu \nabla u)+(\boldsymbol{b} \cdot \boldsymbol{\nabla}) u+c u & =f & & \text { in } \tilde{\Omega}, \\
u & =g & & \text { on } \tilde{\Gamma}_{D}, \\
\nu \nabla u \cdot \boldsymbol{n} & =h & & \text { on } \tilde{\Gamma}_{N} .
\end{aligned}
$$

The corresponding $\tilde{\Omega}, \tilde{\Gamma}_{D}, \tilde{\Gamma}_{N}, g$ and $h$ can be found in the following table 


\begin{tabular}{|c|c|c|c|c|c|}
\hline Equation & $\tilde{\Omega}$ & $\tilde{\Gamma}_{D}$ & $\tilde{\Gamma}_{N}$ & $g$ & $h$ \\
\hline$\theta$ & $\Omega$ & $\overline{\Gamma_{-}}$ & $\Gamma_{W} \cup \Gamma_{+}$ & $\theta_{\text {in }}$ & $\begin{array}{l}\left.h\right|_{\Gamma_{0}}=\dot{q}^{m, i} / c_{p} \\
\left.h\right|_{\Gamma_{+}}=0\end{array}$ \\
\hline$k$ & $\Omega_{\delta}$ & $\Gamma_{-} \cup \Gamma_{\delta}$ & $\Gamma_{+}$ & $\begin{array}{l}\left.g\right|_{\Gamma_{-}}=1.5\left(T_{u}|| \boldsymbol{U}^{m, i-1}||\right)^{2} \\
\left.g\right|_{\Gamma_{\delta}}=C_{\mu}^{-\frac{1}{2}}\left(U_{*}^{m, i}\right)^{2}\end{array}$ & $h=0$ \\
\hline$\epsilon$ & $\Omega_{\delta}$ & $\Gamma_{-} \cup \Gamma_{\delta}$ & $\Gamma_{+}$ & $\begin{array}{l}\left.g\right|_{\Gamma_{-}}=C_{\mu}^{3 / 4} k^{m, i^{3 / 2}} L^{-1} \\
\left.g\right|_{\Gamma_{\delta}}=\left(U_{*}^{m, i}\right)^{3} /\left(\kappa y_{\delta}\right)\end{array}$ & $h=0$ \\
\hline
\end{tabular}

The other data are given in the following table

\begin{tabular}{|c|c|c|c|c|c|}
\hline Equation & $u$ & $\nu$ & $\boldsymbol{b}$ & $c u$ & $f$ \\
\hline For $\theta$ & $\Theta^{m, i}$ & $a_{e}^{m}$ & $\boldsymbol{U}^{m, i}$ & $\Theta^{m, i} / \triangle_{m}$ & $\frac{\dot{q}^{V}}{c_{p}}+\Theta^{m-1} / \triangle_{m}$ \\
For $k$ & $k^{m, i}$ & $\nu_{k}^{m}$ & $\boldsymbol{U}^{m, i}$ & $k^{m, i} / \triangle_{m}$ & $\begin{array}{c}\left(P_{k}^{m}+G^{m}\right)-\epsilon^{m, i-1} \\
+k^{m-1} / \triangle_{m}\end{array}$ \\
For $\epsilon$ & $\epsilon^{m, i}$ & $\nu_{\epsilon}^{m}$ & $\boldsymbol{U}^{m, i}$ & $\begin{array}{c}C_{2} \frac{\epsilon^{m, i-1}}{k^{m, i}} \epsilon^{m, i} \\
+\epsilon^{m, i} / \triangle_{m}\end{array}$ & $\begin{array}{c}C_{1} \frac{\epsilon^{m, i-1}}{k^{m, i}}\left(P_{k}^{m}+G^{m}\right) \\
+\epsilon^{m-1} / \triangle_{m}\end{array}$ \\
\hline
\end{tabular}

From now on, we simply write $\Omega, \Gamma_{D}$ and $\Gamma_{N}$ and omit the indices of diffusivities and production terms.

The linearised Navier-Stokes equations are an Oseen-type problem with a positive reaction term and non-constant viscosity:

$$
\begin{aligned}
L_{O s}(\boldsymbol{u}, p)=-\nabla \cdot(2 \nu \mathbb{S}(\boldsymbol{u}))+(\boldsymbol{a} \cdot \boldsymbol{\nabla}) \boldsymbol{u}+c \boldsymbol{u}+\boldsymbol{\nabla} p & =\boldsymbol{f} & & \text { in } \Omega, \\
\boldsymbol{\nabla} \cdot \boldsymbol{u} & =0 & & \text { in } \Omega, \\
\boldsymbol{u} & =\boldsymbol{u}_{D} & & \text { on } \Gamma_{D}, \\
\sigma(\boldsymbol{u}, p) \boldsymbol{n} & =\sigma_{n} \boldsymbol{n} & & \text { on } \Gamma_{N}, \\
\boldsymbol{n}^{T} \sigma(\boldsymbol{u}, p) \boldsymbol{t}_{j}=\boldsymbol{\sigma}_{t} \cdot \boldsymbol{t}_{j}(j=1, \ldots, d-1), \boldsymbol{u} \cdot \boldsymbol{n} & =0 & & \text { on } \Gamma_{W} .
\end{aligned}
$$

Comparison with step (5) of the algorithm yields $\Gamma_{D}=\Gamma_{F}, \boldsymbol{u}=\boldsymbol{U}^{m, i}, \nu=\nu_{e}, \boldsymbol{a}=\boldsymbol{U}^{m, i-1}$, $c=1 / \triangle_{m}, p=P^{m, i}, \boldsymbol{f}=-\beta \Theta^{m, i-1} \boldsymbol{g}+1 / \triangle_{m} \boldsymbol{U}^{m-1}$ and $\boldsymbol{\sigma}_{t}=\left(U_{*}^{m, i}\right)^{2} \boldsymbol{U}_{\delta, t}^{m, i-1} \cdot \boldsymbol{t}_{j} /\left\|\boldsymbol{U}_{\delta, t}^{m, i-1}\right\|$.

\subsection{Semidiscretisation, decoupling, and linearisation for the LES model}

\subsubsection{Semidiscretisation in time for the LES model}

Similar to the procedure in the previous section, in this subsection we semidiscretise LES MODEL II (see Section (7.1)) w.r.t. the time variable using the discontinuous Galerkin method DG(0). We divide the time interval $[0, T]$ into subintervals $J_{m} \equiv\left(t_{m-1}, t_{m}\right]$ with splitting points $0=t_{0}<t_{1}<\ldots<t_{M}=T$ and time step widths $\triangle_{m}=t_{m}-t_{m-1}$. Denote $\boldsymbol{w}^{m}, q^{m}$, and $\vartheta^{m}$ the (time constant) solution in $J_{m}$. Then we can state the following 
scheme:

\section{DG(0) method for the LES model}

Given $\boldsymbol{w}^{m-1}, q^{m-1}$, and $\vartheta^{m-1}$ from the previous time step (if $m>1$ ) or from the initial condition (if $m=1$ ) seek $\boldsymbol{w}^{m}, q^{m}, \vartheta^{m}$ s.t.

$$
\begin{aligned}
\frac{\boldsymbol{w}^{m}-\boldsymbol{w}^{m-1}}{\triangle_{m}}-\boldsymbol{\nabla} \cdot\left(2 \nu_{e}^{m} \mathbb{S}\left(\boldsymbol{w}^{m}\right)\right)+\left(\boldsymbol{w}^{m} \cdot \boldsymbol{\nabla}\right) \boldsymbol{w}^{m} & =-\boldsymbol{\nabla} q^{m}-\beta \vartheta^{m} \boldsymbol{g}-\nabla \cdot \mathcal{A}_{u}\left(\boldsymbol{w}^{m}\right), \\
\boldsymbol{\nabla} \cdot \boldsymbol{w}^{m} & =0 \\
\frac{\vartheta^{m}-\vartheta^{m-1}}{\triangle_{m}}+\left(\boldsymbol{w}^{m} \cdot \boldsymbol{\nabla}\right) \vartheta^{m}-\boldsymbol{\nabla} \cdot\left(a_{e}^{m} \boldsymbol{\nabla} \vartheta^{m}\right) & =\frac{\left(\dot{q}^{V}\right)^{m}}{c_{p}}-\nabla \cdot \mathcal{A}_{\theta}\left(\boldsymbol{w}^{m}, \vartheta^{m}\right)
\end{aligned}
$$

with $\nu_{t}$ given by the Smagorinsky model resp. by the Iliescu-Layton model, viz.,

$$
\nu_{t}\left(\boldsymbol{w}^{m}, \vartheta^{m}\right)=\left(C_{S} \Delta\right)^{2}\left(\max \left\{0 ;\left\|\mathbb{S}\left(\boldsymbol{w}^{m}\right)\right\|_{F}^{2}+\frac{\beta}{P r_{t}} \boldsymbol{g} \cdot \nabla \vartheta^{m}\right\}\right)^{1 / 2}, \quad \text { resp. }
$$

$$
\nu_{t}\left(\boldsymbol{w}^{m}, \vartheta^{m}\right)=C_{q} \Delta\left\|\boldsymbol{w}^{m}-\mathcal{R} \boldsymbol{w}^{m}\right\|\left(\max \left\{0 ; 1+\frac{1}{\left\|\mathbb{S}\left(\boldsymbol{w}^{m}\right)\right\|_{F}^{2}} \frac{\beta}{P r_{t}} \boldsymbol{g} \cdot \boldsymbol{\nabla} \vartheta^{m}\right\}\right)^{1 / 2},
$$

with $\mathcal{R}$ being defined in (7.1)-(7.2), together with the following abbreviations

$$
\nu_{e}^{m}=\nu+\nu_{t}^{m}, \quad a_{e}^{m}=\frac{\nu}{P r}+\frac{\nu_{t}^{m}}{P r_{t}} .
$$

Thus the instationary LES MODEL II is reduced to a sequence of stationary problems (9.16)-(9.21).

\subsubsection{Iterative decoupling and linearisation for the LES model}

The system of equations (9.16)-(9.21) is decoupled and linearised as follows: Given $\boldsymbol{w}^{m, 0}$, $q^{m, 0}$, and $\vartheta^{m, 0}$, as the solution of the previous time step (or from an initial guess or the initial condition for $m=0$ )

(1) Set $i t_{d l c} \longleftarrow 1$.

(2) Set $i \longleftarrow i t_{d l c}$

(3) Update $\nu_{e}^{m}, a_{e}^{m}$ using $\boldsymbol{w}^{m, i-1}$ and $\theta^{m, i-1}$ from (9.19) resp. (9.20).

(4) Update $\mathcal{A}_{u}\left(\boldsymbol{w}^{m, i-1}\right), \mathcal{A}_{\theta}\left(\boldsymbol{w}^{m, i-1}, \vartheta^{m, i-1}\right)$ (see LES MODEL I).

(5) Near-wall damping of residual stress tensor and residual temperature flux: Multiply $\nu_{t}^{m}, a_{t}^{m},\left(\mathcal{A}_{u}\left(\boldsymbol{w}^{m, i-1}\right)\right)_{i j}$ and $\left(\mathcal{A}_{\theta}\left(\boldsymbol{w}^{m, i-1}, \vartheta^{m, i-1}\right)\right)_{j}$ with $\mathcal{D}\left(y^{+}\right)^{\alpha}$, where $\mathcal{D}\left(y^{+}\right)=$ $1-e^{-y^{+} / 26}$, and $y^{+}=y W_{*}^{m, i-1} \nu^{-1}$. We select $\alpha=2$ for Smagorinsky model and Galdi-Layton model; for the Iliescu-Layton model we choose $\alpha=3$. 
(6) Update boundary conditions for momentum and temperature: For each $\boldsymbol{x} \in \Gamma_{W}$

- If effects of stratification have to be considered: Update $R e_{\min }$ according to

$$
R e_{\text {min }}=6.25 e^{K_{s} R i_{g}}, \text { with } R i_{g}=-\frac{\boldsymbol{g} \cdot \boldsymbol{n} \dot{s}^{m, i-1} \operatorname{Pr} \nu}{\left.\left(W_{*}^{m, i-1}\right)^{4} c_{p} \vartheta^{m, i-1}\right|_{\Gamma_{W}}}
$$

with $\boldsymbol{n}$ being the outer normal vector to $\Gamma_{W}$ in $\boldsymbol{x}$. Use a cut-off technique to ensure $R e_{\text {min,cut }} \leq R e_{\text {min }} \leq 70.0$ with $R e_{\text {min,cut }} \in[2,12]$.

- Define $w\left(y_{\delta}\right)$ and $\vartheta\left(y_{\delta}\right)$ by

$$
w\left(y_{\delta}\right)=\frac{\boldsymbol{w}_{\delta}^{m, i-1} \cdot \boldsymbol{w}_{\delta, t}^{m, i-1}}{\left\|\boldsymbol{w}_{\delta, t}^{m, i-1}\right\|}, \quad \vartheta\left(y_{\delta}\right)=\left.\vartheta^{m, i-1}\right|_{\Gamma_{\delta}} .
$$

- Seek $\left(W_{*}^{m, i}, \dot{s}^{m, i}\right)$ as specified in step (2) of LES MODEL II.

- Check that $\Gamma_{\delta}$ is mostly contained in the log-layer.

(7) Solve the linearised LES momentum equations

$$
\begin{aligned}
& \frac{\boldsymbol{w}^{m, i}-\boldsymbol{w}^{m-1}}{\triangle_{m}}+\left(\boldsymbol{w}^{m, i-1} \cdot \boldsymbol{\nabla}\right) \boldsymbol{w}^{m, i}-\boldsymbol{\nabla} \cdot\left(2 \nu_{e}^{m} \mathbb{S}\left(\boldsymbol{w}^{m, i}\right)\right)=-\nabla q^{m, i}-\beta \vartheta^{m, i-1} \boldsymbol{g} \\
&-\nabla \cdot \mathcal{A}_{u}\left(\boldsymbol{w}^{m, i-1}\right), \\
& \boldsymbol{\nabla} \cdot \boldsymbol{w}^{m, i}=0, \\
& \boldsymbol{w}^{m, i}=\boldsymbol{w}_{i n} \text { on } \Gamma_{F}, \quad \sigma\left(\boldsymbol{w}^{m, i}, q^{m, i}\right) \cdot \boldsymbol{n}=\mathbf{0} \quad \text { on } \Gamma_{N}, \\
& \boldsymbol{n}^{T} \sigma\left(\boldsymbol{w}^{m, i}, q^{m, i}\right) \boldsymbol{t}_{j}=\left(W_{*}^{m, i}\right)^{2} \frac{\boldsymbol{w}_{\delta, t}^{m, i-1} \cdot \boldsymbol{t}_{j}}{\left\|\boldsymbol{w}_{\delta, t}^{m, i-1}\right\|}, \boldsymbol{w}^{m, i} \cdot \boldsymbol{n}=0 \text { on } \Gamma_{W} .
\end{aligned}
$$

(8) Solve the LES temperature equation

$$
\begin{aligned}
\frac{\vartheta^{m, i}-\vartheta^{m-1}}{\triangle_{m}}+\left(\boldsymbol{w}^{m, i} \cdot \boldsymbol{\nabla}\right) \vartheta^{m, i}-\nabla \cdot\left(a_{e}^{m} \boldsymbol{\nabla} \vartheta^{m, i}\right)= & \frac{\left(\dot{q}^{V}\right)^{m}}{c_{p}} \\
& -\nabla \cdot \mathcal{A}_{\theta}\left(\boldsymbol{w}^{m, i-1}, \vartheta^{m, i-1}\right), \\
\vartheta^{m, i}=\vartheta_{\text {in }} \text { on } \quad \Gamma_{-}\left(\boldsymbol{w}^{m, i}\right), \quad \nabla \vartheta^{m, i} \cdot \boldsymbol{n}= & 0 \text { on } \Gamma_{+}\left(\boldsymbol{w}^{m, i}\right), \\
a \nabla \vartheta^{m, i} \cdot \boldsymbol{n} & =\dot{s}^{m, i} / c_{p} \quad \text { on } \Gamma_{W} .
\end{aligned}
$$

(9) Stopping-criterion for linearisation cycle : If $i t_{d l c}<\max _{d l c}$ and if certain stopping criteria for $\left\{\boldsymbol{w}^{m, i}\right\}_{i}$ and $\left\{\vartheta^{m, i}\right\}_{i}$ are not yet fulfilled, then set $i t_{d l c} \longleftarrow i t_{d l c}+1$ and goto step (2). Otherwise goto next time step.

Again, we have to solve an Oseen-type problem for velocity and pressure, and an advectiondiffusion-reaction problem for the temperature. Moreover we have to solve twelve equations of diffusion-reaction type, viz., six for computing, $\mathcal{A}_{u}\left(\boldsymbol{w}^{m, i-1}\right)$, three for $\mathcal{A}_{\theta}\left(\boldsymbol{w}^{m, i-1}, \vartheta^{m, i-1}\right)$, and three for $\mathcal{R}\left(\boldsymbol{w}^{m, i-1}\right)$. Given $f$, the diffusion-reaction problems seek $u$, s.t.

$$
-\frac{\Delta^{2}}{24} \nabla^{2} u+u=f, \quad \nabla u \cdot \boldsymbol{n}=0 \text { on } \partial \Omega,
$$

i.e., the left hand side for all twelve problems is the same. 


\subsection{Variational formulation of the arising model problems}

In this section a variational formulation of the model problems will be presented. First we consider (9.8)-(9.10). We introduce the following test and ansatz spaces :

$$
V_{T} \equiv\left\{v \in H^{1}(\Omega)|\operatorname{Tr}|_{\Gamma_{D}} v=0\right\}, \quad V_{A} \equiv\left\{v \in H^{1}(\Omega)|\operatorname{Tr}|_{\Gamma_{D}} v=g\right\},
$$

with $\operatorname{Tr}$ denoting the trace operator, see e.g. [Gal.94I], Chapter II 3 or Chapter B. Then the variational form of (9.8)-(9.10) reads:

$$
\text { Find } u \in V_{A}: \quad b_{G}(u, v)=l_{G}(v) \quad \forall v \in V_{T}
$$

with the following bi-/linearforms :

$$
\begin{aligned}
b_{G}(u, v) & =\int_{\Omega} \nu \nabla u \cdot \nabla v d x+\int_{\Omega}((\boldsymbol{b} \cdot \nabla) u+c u) v d x, \\
l_{G}(v) & =\int_{\Omega} f v d x+\int_{\Gamma_{N}} h v d s .
\end{aligned}
$$

The weak form corresponding to (9.11)-(9.15) can be derived very similar to Section 8.2.1, but here we need to stress Remark 8.4. We introduce the following test and ansatz spaces

$$
\begin{aligned}
& \mathbf{V}_{A} \equiv\left\{\boldsymbol{v} \in \boldsymbol{H}_{\text {div }}(\Omega),\left.\operatorname{Tr}\right|_{\Gamma_{D}} \boldsymbol{v}=\boldsymbol{u}_{D} \quad \text { on } \Gamma_{D},\left.\operatorname{Tr}\right|_{\Gamma_{W}} \boldsymbol{v} \cdot \boldsymbol{n}=0 \text { on } \Gamma_{D}\right\}, \\
& \mathbf{V}_{T} \equiv\left\{\boldsymbol{v} \in \boldsymbol{H}_{\text {div }}(\Omega),\left.\operatorname{Tr}\right|_{\Gamma_{D}} \boldsymbol{v}=\mathbf{0} \quad \text { on } \Gamma_{D},\left.\operatorname{Tr}\right|_{\Gamma_{W}} \boldsymbol{v} \cdot \boldsymbol{n}=0 \quad \text { on } \Gamma_{W}\right\} \text {, } \\
& Q \equiv\left\{q \in L^{2}(\Omega), \quad \int_{\Omega} q d x=0\right\},
\end{aligned}
$$

with $\boldsymbol{H}_{d i v}(\Omega)$ being defined in $(\overline{B .9})$. Moreover we define the following bi-/linearforms:

$$
\mathcal{A}(U, V)=a(\boldsymbol{u}, \boldsymbol{v})+b(\boldsymbol{v}, p)-b(\boldsymbol{u}, q), \quad \mathcal{L}(V)=L(\boldsymbol{v}) .
$$

with $U=(\boldsymbol{u}, p), V=(\boldsymbol{v}, q)$ and

$$
\begin{aligned}
a(\boldsymbol{u}, \boldsymbol{v}) & =\int_{\Omega} 2 \nu \mathbb{S}(\boldsymbol{u}): \boldsymbol{\nabla} \boldsymbol{v}+((\boldsymbol{a} \cdot \boldsymbol{\nabla}) \boldsymbol{u}+c \boldsymbol{u}) \cdot \boldsymbol{v} d x \\
b(\boldsymbol{v}, p) & =-\int_{\Omega} p(\boldsymbol{\nabla} \cdot \boldsymbol{v}) d x \\
L(\boldsymbol{v}) & =\int_{\Omega} \boldsymbol{f} \cdot \boldsymbol{v} d x+\int_{\Gamma_{N}} \sigma_{n} \boldsymbol{n} \cdot \boldsymbol{v} d s+\sum_{j=1}^{d-1} \int_{\Gamma_{W}}\left(\boldsymbol{\sigma}_{t} \cdot \boldsymbol{t}_{j}\right)\left(\boldsymbol{v} \cdot \boldsymbol{t}_{j}\right) d s .
\end{aligned}
$$

Now we can state the weak form of the Oseen problem:

$$
\text { Find } U=(\boldsymbol{u}, p) \in \mathbf{V}_{A} \times Q: \quad \mathcal{A}(U, V)=\mathcal{L}(V) \quad \forall V \in \mathbf{V}_{T} \times Q .
$$

\section{Remark 9.3}

In the above formulation, the Dirichlet boundary data have been imposed in a strong way. Let us mention two approaches for imposing the Dirichlet condition in a weak sense. A strategy for imposing $\boldsymbol{u} \cdot \boldsymbol{n}=0$ on $\Gamma_{W}$ in a weak sense by integrating by parts the equation of continuity can be found in [MP94] and in [Mue99]. The weak imposition of the no-slip and no-penetration condition using Lagrange multipliers or as penalty terms can be found in [Lia.99]. 


\section{Discretisation in space using stabilised FEM}

In this chapter we consider the discretisation in space of (9.23) and (9.33). For this purpose we apply a stabilised finite-element method which is also used in our research code PARALLELNS.

\subsection{Finite-element discretisation for ADR-problems}

The computational domain $\Omega$ is now assumed to be polyhedral. Let $\mathcal{T}_{h}$ be a family of admissible and shape-regular triangulations of $\Omega$. $\mathcal{T}_{h}$ is called admissible if the intersection of two triangles belonging to $\mathcal{T}_{h}$ is either empty, a vertex or a whole edge (or a whole facet in $3 \mathrm{~d})$. Shape-regular means that

$$
\exists C>0 \text { such that } \max _{T} \frac{h_{T}}{\rho_{T}} \leq C, \quad \forall T \in \cup_{h} \mathcal{T}_{h}
$$

where $h_{T}$ is the diameter of the minimal ball circumscribed around $T$ and $\rho_{T}$ is the diameter of the maximal ball contained in $T$. On $\mathcal{T}_{h}$ we define the conforming finite-element spaces

$$
X_{h}^{k}:=\left\{v \in C(\bar{\Omega})|v|_{T} \in \Pi_{k}(T) \quad \forall T \in \mathcal{T}_{h}\right\}, \quad k \geq 1
$$

of continuous and piecewise polynomial functions, which are subspaces of $H^{1}(\Omega)$. In the following we restrict ourselves to homogeneous Dirichlet boundary conditions. The Dirichlet boundary conditions are incorporated into the function space by introducing

$$
V_{h} \equiv X_{h}^{k} \cap V_{T}
$$

Then the standard Galerkin method of problem (9.23) reads

$$
\text { Find } u \in V_{h}: \quad b_{G}(u, v)=l_{G}(v) \quad \forall v \in V_{h} .
$$

\subsection{Stabilisation techniques for ADR-problems}

It is well known that the simple Galerkin scheme presented in the previous section fails in the case of dominant advection. In regions where diffusion is small compared to advection, the exact solution can possess inner and/or boundary layers. If the triangulation is not fine enough to resolve these layers, the numerical solution can exhibit unphysical oscillations. To avoid an unaccessable grid refinement, as a remedy a stabilisation technique can be applied. For a survey thereon see [RST96] or [KLR02] and references therein. Our research group uses the so-called streamline-diffusion method (SDFEM), sometimes called StreamlineUpwind/Petrov-Galerkin (SUPG) method, combined with a shock capturing technique. Both will be presented in the following two sections. 


\subsubsection{SUPG-stabilisation for ADR-problems}

The idea is to introduce additional artificial diffusivity in the streamwise direction by adding the term $\sum_{T \in \mathcal{T}_{h}} \delta_{T}(\boldsymbol{b} \cdot \nabla u, \boldsymbol{b} \cdot \nabla v)$ to the bilinear form $b_{G}(\cdot, \cdot)$ in (9.24). We modify this scheme to achive consistency (but see Remark 10.1). Consistency means that the solution of the original problem solves the stabilised problem, given the solution is smooth enough. This is accomplished by adding local weighted residuals tested with $\boldsymbol{b} \cdot \nabla v$, i.e., we add the term $\sum_{T \in \mathcal{T}_{h}} \delta_{T}(L u-f, \boldsymbol{b} \cdot \nabla v)$ to $b_{G}(\cdot, \cdot)$ in (9.24). Recall that $L u$ was defined in (9.8). The choice of $\delta_{T}$ will be postponed to the end of this subsection.

After this motivation, we introduce the following stabilised bi-/linear forms

$$
\begin{gathered}
b_{S G}(u, v)=b_{G}(u, v)+b_{S}(u, v), \quad \text { with } \quad b_{S}(u, v)=\sum_{T \in \mathcal{T}_{h}} \delta_{T}(L u, \boldsymbol{b} \cdot \nabla v)_{T}, \\
l_{S G}(v)=l_{G}(v)+l_{S}(v), \quad \text { with } l_{S}(v)=\sum_{T \in \mathcal{T}_{h}} \delta_{T}(f, \boldsymbol{b} \cdot \nabla v)_{T} .
\end{gathered}
$$

Then the SUPG stabilised discrete formulation of (9.23) reads

$$
\text { Find } u \in V_{h}: \quad b_{S G}(u, v)=l_{S G}(v) \quad \forall v \in V_{h} \text {. }
$$

\section{Remark 10.1}

The above formulation is consistent only for stationary problems. For instationary problems a consistent formulation is obtained by adding the term $\sum_{T \in \mathcal{T}_{h}} \delta_{T}\left(\partial_{t} u+L u-f, \boldsymbol{b} \nabla v\right)$ to $b_{G}(\cdot, \cdot)$ in (9.24). If a $D G(0)$ method is used, then $\partial_{t} u$ vanishes. As a remedy, one could replace the term $\partial_{t} u$ by $\left(u-u_{m-1}\right) / \Delta_{m}$, with $u_{m-1}$ being the solution of the previous time step and $\Delta_{m}$ denoting the time step width.

Finally let us focus on the choice of $\delta_{T}$. An analysis of the one-dimensional finite-difference scheme of singular perturbed problems reveals that stabilisation terms have to be added only in regions where $P e_{T}>1$, with $P e_{T}=h_{T}\|\boldsymbol{b}\|_{\infty, T} \nu^{-1}$ being the mesh Peclet-number, cf. [RST96], p.29. In order to obtain a stable and accurate method, we use

$$
\delta_{T}=\frac{h_{T}^{2}}{2\|\nu\|_{\infty, T} \sqrt{1+P e_{T}^{2}+R_{T}^{2}}}, \quad P e_{T} \equiv \frac{h_{T}\|\boldsymbol{b}\|_{\infty, T}}{\|\nu\|_{\infty, T}}, \quad R_{T} \equiv \frac{h_{T}^{2}}{\Delta_{m}\|\nu\|_{\infty, T}}
$$

with $\Delta_{m}$ denoting the actual time step width.

The choice in (10.2) is motivated by an a priori error analysis for the $D G(q)$ scheme, see [Mue00], Chapter 4. Numerical results can be found in [Mue99], [Mue(00], and in [Fis02]. When applying the second modification described in remark 10.1, the analysis suggests $R_{T} \equiv h_{T}^{2} \Delta_{m}^{-3 / 2}\|\nu\|_{\infty, T}^{-1}$ in (10.2). As shown in [Fis(02], Subsection 5.1.1, this improves the scheme slightly.

Concerning the stationary case, for analytical results of the SUPG method and the choice of $\delta_{T}$ see e.g. [RST96]. 


\subsection{Discontinuity capturing for ADR-problems}

Despite the SUPG-stabilisation, the solution can exhibit small local oscillations (sometimes called over- and under-shoots) in the vicinity of regions, in which the solution has steep gradients. A typical example are the neighbourhood of inner and/or boundary layers of the temperature equation. Moreover, in the non-linear $k / \epsilon$ model, these small oscillations can cause unphysical negative values for $k$ and $\epsilon$, subverting the numerical stability of the model. These oscillations can occur, because the SUPG-method is neither monotone nor monotonicity preserving.

The idea of a discontinuity-capturing/crosswind-dissipation (DC/CD) method is to add artificial diffusivity in crosswind direction in the neighbourhood of layers. A certain class of non-linear shock-capturing methods is presented and studied in great detail by LUBE in [KLRO2].

In our research group we consider a non-linear DC/CD method proposed by CoDINA, see [Cod93] and [CS99]. The artificial viscosity should be proportional to the element residual for consistency and should vanish quickly in regions where the solution is smooth. The amount of artificial diffusivity added on an element $T$ is given by

$$
\nu_{d c}=\frac{1}{2} \xi_{c} h \frac{|L u-f|_{T}}{S_{T}+|\nabla u|_{T}} .
$$

with a suitable constant $S_{T}$. The parameter $\xi_{c}$ is computed elementwise according to

$$
\xi_{c}=\max \left\{0, C_{d c}-\frac{2 \nu}{\left|\boldsymbol{b}^{*}\right|_{T} h}\right\} \quad \text { with } \quad \boldsymbol{b}^{*}=\frac{1}{|\nabla u|_{T}^{2}}(\boldsymbol{b} \cdot \nabla u+c u-f) \nabla u .
$$

Codina proposed to choose $C_{d c}=0.7$ for piecewise linear elements and $C_{d c}=0.35$ for quadratics. Of course, an implementation of (10.3) and (10.4) has to avoid division by zero; for clarity's sake, we omit these details in the presentation.

We restrict ourselves to the case of piecewise linear elements. First we show that $\nu_{d c}$ vanishes if the elementwise residual is zero. For piecewise linear finite-elements, $\boldsymbol{b}^{*}$ is proportional to the residual, because the term $\nabla \cdot(\nu \nabla u)$ vanishes. It follows from (10.4) that $\xi_{c}=0$ on $T$ if $|L u-f|_{T}<2 \nu|\nabla u|_{T} C_{d c}^{-1} h^{-1}$. Numerical tests confirm indeed that the considered DC/CD method adds artificial viscosity only in the vicinity of layers. Moreover, it is worthy mentioning that the sharp gradient of the solution in layers is recovered.

The shock-capturing method proposes to add the following term to the bilinear form (on elements with $\boldsymbol{b} \neq \mathbf{0}$ ):

$$
\begin{gathered}
\sum_{T \in \mathcal{T}_{h}}\left[\nu_{d c}(\nabla u, \nabla v)_{T}+\left(\nu_{s l}-\nu_{d c}\right) \frac{1}{|\boldsymbol{b}|_{T}{ }^{2}}(\boldsymbol{b} \cdot \nabla u, \boldsymbol{b} \cdot \nabla v)_{T}\right], \quad \text { with } \\
\nu_{s l}=\max \left\{0, \nu_{d c}-\nu_{\text {supg }}\right\}, \quad \nu_{\text {supg }}=\frac{\delta_{T}}{|\boldsymbol{b}|_{T}{ }^{2}} .
\end{gathered}
$$

Here $\delta_{T}$ is the parameter from the SUPG stabilisation. The effect of the additional form will be explained at the end of this subsection. Adding the following form

$$
b_{D C}(u, v)=\sum_{T \in \mathcal{T}_{h}}\left[\nu_{d c}(\nabla u, \nabla v)_{T}+\left(\nu_{s l}-\nu_{d c}\right) \frac{1}{|\boldsymbol{b}|_{T}^{2}}(\boldsymbol{b} \cdot \nabla u, \boldsymbol{b} \cdot \nabla v)_{T}\right]
$$


to the bilinear form, the shock-capturing and SUPG stabilised method is

$$
\text { Find } u \in V_{h}: \quad b_{S G}(u, v)+b_{D C}(u, v)=l_{S G}(v) \quad \forall v \in V_{h} .
$$

The form $b_{D C}(\cdot, \cdot)$ adds additional diffusivity in the direction $\mathbb{I}-\boldsymbol{b} \otimes \boldsymbol{b}$, i.e. in crosswind direction. If $\nu_{d c}<\nu_{\text {supg }}$ then $\nu_{s l}=0$. Then the total effect of SUPG and DC/CD is to add an amount $\nu_{\text {supg }}$ of diffusivity in streamwise direction and an amount of $\nu_{d c}$ in the plane given by $\mathbb{I}-\boldsymbol{b} \otimes \boldsymbol{b}$. If $\nu_{d c}>\nu_{\text {supg }}$ then $\nu_{s l}>0$. Then the total effect of SUPG and DC/CD is to add an amount $\nu_{d c}$ of diffusivity isotropically.

Due to (10.3) the scheme is non-linear. We use a simple iteration within the decoupling and linearisation cycle (introduced in Subsection 9.2.2) by computing $\nu_{d c}$ in (10.3) using the solution $u^{m, i-1}$ of the previous iteration cycle, see [KLR02], p.15. Numerical experiments show that one iteration of the $\mathrm{DC} / \mathrm{CD}$ scheme is sufficient to remove crosswind oscillations. Regarding the numerical analysis we refer to the results given by LUBE in [KLRO2]. For further details concerning the implementation and numerical results see [Kno99].

\subsection{Finite-element discretisation for Oseen problems}

Let $\Omega, \mathcal{T}_{h}$, and $X_{h}^{k}$ be given as in the previous section. We introduce the following discrete ansatz and test spaces:

$$
\begin{aligned}
\text { velocity : } & \boldsymbol{V}_{A, h} \equiv\left(X_{h}^{k}\right)^{d} \cap \boldsymbol{V}_{A}, \\
\text { velocity : } & \boldsymbol{V}_{T, h} \equiv\left(X_{h}^{k}\right)^{d} \cap \boldsymbol{V}_{T}, \\
\text { pressure : } & Q_{h} \equiv X_{h}^{l} \cap Q \subset Q .
\end{aligned}
$$

where $\boldsymbol{V}_{A}, \boldsymbol{V}_{T}$ and $Q$ are given in (9.26)-(9.28). Then the discrete variant of the Oseenproblem (9.11) reads:

$$
\text { Find } \boldsymbol{U}=(\boldsymbol{u}, p) \in V_{A, h} \times Q_{h}: \quad \mathcal{A}(\boldsymbol{U}, \boldsymbol{V})=\mathcal{L}(\boldsymbol{V}) \quad \forall \boldsymbol{V} \in V_{T, h} \times Q_{h} .
$$

\subsection{SUPG- und PSPG-stabilisation for Oseen problems}

To avoid oscillations in the case of dominant advection we stabilise our scheme using the SUPG method. We add the term

$$
\sum_{T \in \mathcal{T}_{h}} \delta_{1 u}^{T}\left(L_{O s}(\boldsymbol{u}, p)-\boldsymbol{f},(\boldsymbol{a} \cdot \nabla) \boldsymbol{v}\right)_{T}
$$

to the form $a(\cdot, \cdot)$, see (9.30), with $L_{O s}$ being defined in (9.11).

It is well known that the solution of the pressure exhibits strong unphysical oscillations unless $\boldsymbol{V}_{T, h}$ and $Q_{h}$ satisfy the so-called discrete Babuska-Brezzi (BB) condition for some $\beta$ independent of $h$ :

$$
\inf _{q \in Q_{h} \backslash\{0\}} \sup _{\boldsymbol{v} \in \boldsymbol{V}_{T, h} \backslash\{0\}} \frac{b(\boldsymbol{v}, q)}{\|\boldsymbol{v}\|_{V_{T, h}}\|q\|_{Q_{h}}} \geq \beta>0,
$$


with $b(\cdot, \cdot)$ being defined in (9.31). In our research group we use $k=l=1$, which does not satisfy the discrete BB condition. As a remedy, we use the so-called pressure stabilisation/ Petrov-Galerkin (PSPG) method. The PSPG method consists in adding to the bilinear form $b(\cdot, \cdot)$ the term

$$
\sum_{T \in \mathcal{T}_{h}} \delta_{1 p}^{T}\left(L_{O s}(\boldsymbol{u}, p)-\boldsymbol{f}, \nabla q\right)_{T}
$$

Finally, in the case of small viscosity $\nu$ we stabilise the continuity equation by adding the term

$$
\sum_{T \in \mathcal{T}_{h}} \delta_{2 u}^{T}(\nabla \cdot \boldsymbol{u}, \nabla \cdot \boldsymbol{v})_{T}
$$

Putting all stabilisation terms together, we obtain the following forms

$$
\begin{aligned}
\mathcal{A}_{S G}(\boldsymbol{U}, \boldsymbol{V})= & \mathcal{A}(\boldsymbol{U}, \boldsymbol{V})+\sum_{T \in \mathcal{T}_{h}} \delta_{1 u}^{T}\left(L_{O s}(\boldsymbol{u}, p)(\boldsymbol{a} \cdot \nabla) \boldsymbol{v}\right)_{T} \\
& +\sum_{T \in \mathcal{T}_{h}} \delta_{1 p}^{T}\left(L_{O s}(\boldsymbol{u}, p), \nabla q\right)_{T}+\sum_{T \in \mathcal{T}_{h}} \delta_{2 u}^{T}(\nabla \cdot \boldsymbol{u}, \nabla \cdot \boldsymbol{v})_{T}, \\
\mathcal{L}_{S G}(\boldsymbol{V})= & \mathcal{L}(\boldsymbol{V})+\sum_{T \in \mathcal{T}_{h}} \delta_{1 u}^{T}(\boldsymbol{f},(\boldsymbol{a} \cdot \nabla) \boldsymbol{v})_{T}+\sum_{T \in \mathcal{T}_{h}} \delta_{1 p}^{T}(\boldsymbol{f}, \nabla q)_{T} .
\end{aligned}
$$

Then the stabilised discrete version of the Oseen problem reads:

$$
\text { Find } \boldsymbol{U}=(\boldsymbol{u}, p) \in \boldsymbol{V}_{A, h} \times Q_{h}: \mathcal{A}_{S G}(\boldsymbol{U}, \boldsymbol{V})=\mathcal{L}_{S G}(\boldsymbol{V}) \forall \boldsymbol{V} \in \boldsymbol{V}_{T, h} \times Q_{h} .
$$

The stabilisation parameters are chosen according to

$$
\begin{aligned}
& \delta_{1 u}^{T}=C_{1}^{u} \frac{h_{T}^{2}}{2\|\nu\|_{\infty, T}}\left(1+\left(\frac{h_{T}\|\boldsymbol{a}\|_{\infty, T}}{\|\nu\|_{\infty, T}}\right)^{2}+\left(\frac{h_{T}^{2}}{\|\nu\|_{\infty, T} \Delta_{m}}\right)^{2}\right)^{-\frac{1}{2}}, \\
& \delta_{1 p}^{T}=C_{1}^{p} \frac{h_{T}^{2}}{2\|\nu\|_{\infty, T}}\left(1+\left(\frac{h_{T} U_{r e f}}{\|\nu\|_{\infty, T}}\right)^{2}+\left(\frac{h_{T}^{2}}{\|\nu\|_{\infty, T} \Delta_{m}}\right)^{2}\right)^{-\frac{1}{2}}, \\
& \delta_{2}^{T}=C_{2}\|\nu\|_{\infty, T} \sqrt{1+\left(\frac{h_{T}\|\boldsymbol{a}\|_{\infty, T}}{\|\nu\|_{\infty, T}}\right)^{2}} .
\end{aligned}
$$

For instationary problems, consistency can be ensured similar to the advection-diffusionreaction problem, see Remark 10.1. The choice of the parameters is based on an a priori error analysis for isotropic meshes, cf. e.g. [Mue.97], pp.55. Of particular importance is the choice for $U_{r e f}$ in (10.8), which is due to [Tez.92]. $U_{\text {ref }}$ denotes a suitable reference velocity, for example $U_{r e f}=\|\boldsymbol{a}\|_{\infty, \Omega}$. In indoor-air flow problems we suggest $U_{r e f}=\left\|\boldsymbol{u}^{m, i-1}\right\|_{\infty, \Gamma}$ being the solution for $\boldsymbol{u}$ of the previous iteration step (see Section 9.2.2) in forced convection problems resp. $U_{\text {ref }}=\tilde{U}_{s c}=\left(\tilde{\beta}_{0}|\tilde{\boldsymbol{g}}| \tilde{T}_{d i f f} \tilde{L}\right)^{1 / 2}$ in natural convection problems with a suitable length scale $\tilde{L}$ and temperature difference $\tilde{T}_{d i f f}$. It should be emphasised that 
using $\|\boldsymbol{a}\|_{\infty, T}$ instead of a global scaling velocity $U_{\text {ref }}$ does not lead to a numerically stable method, in particular if anisotropic unstructured meshes are used. However, to the author's knowledge, there is no analytical support for this observation. This is in contrast to the fact that the choice $\delta_{1 u}^{T}=\delta_{1 p}^{T}$ and hence using a local velocity in the formula for $\delta_{1 p}^{T}$ is widespread throughout the literature. For the constants $C_{1}^{u}=C_{1}^{p}=C_{2}=1.0$ is the usual choice.

As a concluding remark we should stress the point that the computational costs for assembling the PSPG terms in a finite-element code are significant. This motivates using discrete spaces that satisfy the discrete $\mathrm{BB}$ condition in future work. 


\section{Non-overlapping domain decomposition methods}

The variational formulations (10.1) and (10.6) of the model problems (9.8)-(9.10) and (9.11)-(9.15) are the starting point for an implementation in a FEM code. Therefore we have to specify finite-element spaces and appropriate quadrature formulas for the numerical approximation of the integrals appearing in the weak formulation. Then from the variational formulation we can determine a linear system, whose solution is the solution of the stabilised discrete variational problem. The arising linear system is very large if a fine grid is chosen, in particular for 3D problems. Today, the trend is towards using a cluster of workstations in scientific computing. If the data segment does not fit into physical memory then due to page faults the performance drops drastically, see [HP96], Chapter 5.

As a remedy, the aim is to divide the flow problem into a set of smaller problems which can be solved in parallel. Then the speed-up is achieved (i) from exploiting the memory hierarchy on a single computer and (ii) from the parallelisation. Its price are the costs for the communication between the processes. A promising strategy are so-called domain decomposition methods (DDM). For a comprehensive review see e.g. [QV99]; for further information visit the homepage of domain decomposition methods at http://www.ddm.org. In this chapter we describe a particular DDM that has been studied in our research group during the last few years. For a detailed presentation we refer to [Otto.9.], [Mue.9.9] and [Mue(0)]. We focus on a non-overlapping DDM. Therefore we consider a non-overlapping partition of $\Omega$ into subdomains $\Omega_{1}, \ldots, \Omega_{N}$ being all of the same regularity as $\Omega$ itself and

$$
\bar{\Omega}=\cup_{k=1}^{N} \bar{\Omega}_{k}, \quad \Omega_{k} \cap \Omega_{j}=\emptyset \quad \forall k \neq j, \quad \forall T \in \mathcal{T}_{h} \quad \exists k \quad: \quad T \subset \Omega_{k} .
$$

The last condition ensures that the partition of $\Omega$ is aligned with the finite-element mesh. Moreover, we set

$$
\Gamma_{k}:=\partial \Omega_{k} \backslash \partial \Omega, \Gamma_{j k}:=\partial \Omega_{j} \cap \partial \Omega_{k}, j \neq k,
$$

where $\Gamma_{k j}$ is identified with $\Gamma_{j k}$.

To obtain a boundary value problem on each subdomain, we have to specify boundary conditions at the interfaces $\Gamma_{j k}$. Lions proposed to apply a transmission condition of Robin type at the interfaces for the Poisson equation, see [Lio.90], in order to enforce (in appropriate trace spaces) continuity of the solution $u$ and of the flux $\nu \nabla u \cdot \boldsymbol{n}$ at the interfaces in the limit of an iterative procedure. This idea was extended to scalar advection-diffusionreaction problems by NATAF, cf. [NR95], and to the linearised Navier-Stokes equations by Lube and Отто, see [L(O)98]. Due to the interchange of Robin interface conditions, this method is sometimes called Robin-Robin algorithm (R-R-algorithm). It belongs to the class of so-called iteration-by-subdomain methods.

In order to avoid technical problems, we assume that the partition $\left\{\Omega_{k}\right\}_{k=1}^{N}$ is stripwise, i.e.

$$
\Gamma_{i j} \neq \Gamma_{k l} \quad \text { implies } \inf _{x \in \Gamma_{i j}, y \in \Gamma_{k l}} \operatorname{dist}(\boldsymbol{x}, \boldsymbol{y})>0
$$




\subsection{The Robin-Robin algorithm for advection-diffusion-reaction problems}

First we consider the Robin-Robin algorithm for problems of advection-diffusion-reaction type, as given in (9.8)-(9.10). To illustrate the idea, first we write the method on the continuous level in a strong formulation, which reads:

For given $u_{k}^{n}$ from iteration step $n$ on each $\Omega_{k}$, seek (in parallel) for $u_{k}^{n+1}$ as the solution of

$$
\begin{aligned}
L u_{k}^{n+1} & =f & & \text { in } \Omega_{k} \\
u_{k}^{n+1} & =0 & & \text { on } \Gamma_{D} \cap \partial \Omega_{k} \\
\nu \nabla u_{k}^{n+1} \cdot \boldsymbol{n}_{k} & =h & & \text { on } \Gamma_{N} \cap \partial \Omega_{k} \\
\Phi_{k}\left(u_{k}^{n+1}\right) & =\theta \Phi_{k}\left(u_{j}^{n}\right)+(1-\theta) \Phi_{k}\left(u_{k}^{n}\right) & & \text { on } \Gamma_{j k}, \quad j=1, \ldots, N, j \neq k
\end{aligned}
$$

with a relaxation parameter $\theta \in(0,1]$. The interface function is given by

$$
\Phi_{k}(u)=\nu \nabla u \cdot \boldsymbol{n}_{k}+\left(-\frac{1}{2} \boldsymbol{b} \cdot \boldsymbol{n}_{k}+z_{k}\right) u .
$$

The choice of $\Phi_{k}(u)$ and in particular the specification of $z_{k}$ are discussed in detail below. Now we give the discrete weak formulation of (11.1)-(11.4), which is implemented in our research code. A detailed derivation of this scheme, which is often referred to as two field formulation, on the continuous level is given in [O+to99] , Section 3.2. For this purpose, let $V_{k, h}, b_{S G}^{k}(\cdot, \cdot)$ and $l_{S G}^{k}(\cdot)$ denote the restrictions of $V_{h}, b_{S G}(\cdot, \cdot)$ and $l_{S G}(\cdot)$ to $\Omega_{k}$, respectively. Let $W_{i j, h}$ be the restriction of $V_{h}$ to the interface part $\Gamma_{i j}$. Furthermore, let $\langle\cdot, \cdot\rangle_{\Gamma_{i j}}$ be the inner product in $L^{2}\left(\Gamma_{i j}\right)$ or, whenever needed, the dual product between $\left(W_{i j, h}\right)^{*}$ and $W_{i j, h}$. Then the fully discretised and stabilised DD method reads :

Parallel computation step :

For $k=1, \ldots, N$, find $u_{k}^{n+1} \in V_{k, h}$ such that $\forall v_{k} \in V_{k, h}$

$$
b_{S G}^{k}\left(u_{k}^{n+1}, v_{k}\right)+\left\langle\left(-\frac{1}{2} \boldsymbol{b} \cdot \boldsymbol{n}_{k}+z_{k}\right) u_{k}^{n+1}, v_{k}\right\rangle_{\Gamma_{k}}=l_{S G}^{k}\left(v_{k}\right)+\sum_{j(\neq k)}\left\langle\Lambda_{j k}^{n}, v_{k}\right\rangle_{\Gamma_{k j}} .
$$

Communication step : For all $k \neq j$, update the Lagrangian multipliers

$$
\left\langle\Lambda_{k j}^{n+1}, \phi\right\rangle_{\Gamma_{k j}}=\left\langle\theta\left(z_{k}+z_{j}\right) u_{k}^{n+1}-\theta \Lambda_{j k}^{n}+(1-\theta) \Lambda_{k j}^{n}, \phi\right\rangle_{\Gamma_{k j}} \quad \forall \phi \in W_{k j, h} .
$$

The analysis of the method for constant viscosity, given in [LMO00a], can be extended easily to the case of non-constant viscosity. If $z_{k}=z_{j}>0$, then the algorithm is wellposed, i.e., all local discrete problems arising throughout the algorithm have a uniquely determined solution. Moreover the sequences $\left\{u_{k}^{n}\right\}_{n}, k=1, \ldots, N$ converge strongly to the restrictions of the global discrete solution to $\Omega_{k}$ with respect to the stabilised energy norm induced by the symmetric part of $b_{S G}^{k}(\cdot, \cdot)$ as $n \rightarrow \infty$. An analysis of the convergence behaviour of the Robin-Robin-algorithm is given by RAPIN in [RL(0)]. Let us review his results briefly. Denote $e_{k}^{n} \equiv u_{k}^{n}-u \mid \Omega_{k}$ the error in $\Omega_{k}$. RAPIN accomplished a Fourier analysis of the two-dimensional case for a problem with constant coefficients. He considers 
$\Omega=(0, L) \times(0,1)$, which is decomposed into $\Omega_{1}=(0, A) \times(0,1)$ and $\Omega_{2}=(A, L) \times(0,1)$. He gives a representation of $e_{k}^{n}(\mathrm{k}=1,2)$ as a series of the form

$$
\begin{aligned}
& e_{1}^{n}(x, y)=\exp \left(\frac{\boldsymbol{b} \cdot(x, y)}{2 \nu}\right) \sum_{l=1}^{\infty} F_{1, l}^{n} \sinh \left(\mu_{l} x\right) \sin (l \pi y), \quad \mu_{l}^{2}=\frac{|\boldsymbol{b}|}{4 \nu^{2}}+\frac{c}{\nu}+l^{2} \pi^{2} \\
& e_{2}^{n}(x, y)=\exp \left(\frac{\boldsymbol{b} \cdot(x, y)}{2 \nu}\right) \sum_{l=1}^{\infty} F_{2, l}^{n} \sinh \left(\mu_{l}(L-x)\right) \sin (l \pi y) .
\end{aligned}
$$

Using $\theta=1$ he gets a formula for the contraction rate $K_{l}$ of the $l$-th Fourier mode of the form $K_{l} \equiv F_{k, l}^{n} / F_{k, l}^{n-2}$. His important result is that $K_{l}$ tends to one as $l \rightarrow \infty$. For this reason the R-R-algorithm cannot converge linearly.

This result is of prime importance regarding the application of the R-R-algorithm to turbulent flow problems. Since turbulent flows are characterised by a variety of small scales, the results in $[\mathrm{RLO0]}]$ suggest that the Robin-Robin-algorithm is not a propitious DDM for a DNS of a turbulent flow problem. However, when employing a turbulence model that resolves only the large scale flow structures, there is hope that the R-R-algorithm does a good job.

\subsection{Choice of the interface function in the R-R-algorithm for ADR problems}

So far, we have not specified the parameter $z_{k}$. It turns out that the parameters $z_{k}$ are important to accelerate the convergence of the scheme. A first suggestion was due to NATAF in [NR.95]. He performed a Fourier analysis for an ADR problem with constant coefficients. He proposed to choose $z_{k}$ such that the first Fourier mode of the error is annihilated after one iteration; his choice was

$$
z_{k}=\frac{1}{2} \sqrt{\left|\boldsymbol{b} \cdot \boldsymbol{n}_{k}\right|^{2}+4 \nu c} .
$$

Recent results by NATAF can be found in [.JRO0].

Another approach has been performed by Lube, Отто and MuelleR. Their choice is based on an a-posteriori error estimate. Their objective was to find a stopping criterion for the iteration process within each DDM cycle. They derived an a posteriori estimate which bounds the error on a subdomain by the difference of the traces of the subdomain solutions on the interface. Hence the convergence on the subdomains can be controlled via the jumps of the discrete DD-solutions across the interface. This result not only gives a stopping criterion. Additionally, it provides information regarding the choice of $z_{k}$, balancing the terms in the a posteriori estimate (for a partitioning of $\Omega$ into two subdomains), proposing

$$
z_{k}=\frac{\left|\boldsymbol{b} \cdot \boldsymbol{n}_{k}\right|}{2}+\max _{j=1,2} \frac{\|\nu\|_{\text {min,j }}}{H_{j}}\left(1+\sqrt{\frac{C_{\infty, j}}{\|\nu\|_{\text {min }, j}}} H_{j}+2 \min \left(\frac{B_{\infty, j}}{\sqrt{\|c \nu\|_{\text {min }, j}}} ; \frac{B_{\infty, j} H_{j}}{\|\nu\|_{\text {min }, j}}\right)\right)
$$

with $H_{j} \approx \operatorname{diam}\left(\Omega_{j}\right), B_{\infty, j} \equiv\|\boldsymbol{b}\|_{0, \infty, \Omega_{j}}, C_{\infty, j} \equiv\|c\|_{0, \infty, \Omega_{j}},\|\phi\|_{m i n, j} \equiv \inf _{\Omega_{j}} \phi(\cdot)$, cf. [LMO00a] and [KLGRO2]. Without the $\min (. .$.$) term in (11.9), (11.8) and (11.9) are$ 
similar. However, numerical tests reveal that the $\min (. .$.$) term can be of crucial importance$ in order to obtain a robust method, in particular if $c=0$. For the symmetric diffusionreaction problem with $\boldsymbol{b}=0$ and constant viscosity, (11.9) reduces to

$$
z_{k}=\max _{j=1,2} \frac{\nu}{H}+\sqrt{C_{\infty, j \nu}}
$$

which is consistent with the results given in [0.99]. For the Poisson equation $(\boldsymbol{b}=0$, $c=0)$ we get $z_{k}=\nu H^{-1}$.

\subsection{The Robin-Robin algorithm for Oseen type problems}

Next we apply the R-R-algorithm to the Oseen problem (10.6). The idea is to guarantee continuity of velocity $\boldsymbol{u}$ and of the Oseen fluxes $\sigma(\boldsymbol{u}, p) \boldsymbol{n}-\frac{1}{2}(\boldsymbol{a} \cdot \boldsymbol{n}) \boldsymbol{u}$ (at least in the limit of an iterative procedure and in appropriate trace spaces). To achieve this goal we impose a transmission condition of Robin type on the interfaces. Then the strong form of the $\mathrm{R}$-R-algorithm for the Oseen problem reads as follows:

For given $\left(\boldsymbol{u}_{k}^{n}, p_{k}^{n}\right)$ from iteration step $n$ on each $\Omega_{k}$, seek (in parallel) for $\left(\boldsymbol{u}_{k}^{n+1}, p_{k}^{n+1}\right)$ with:

$$
\begin{aligned}
L_{O s}\left(\boldsymbol{u}_{k}^{n+1}, p_{k}^{n+1}\right) & =\boldsymbol{f} & & \text { in } \Omega_{k}, \\
\boldsymbol{\nabla} \cdot \boldsymbol{u}_{k}^{n+1} & =0 & & \text { in } \Omega_{k}, \\
\boldsymbol{u}_{k}^{n+1} & =\boldsymbol{u}_{D} & & \text { on } \partial \Omega_{k} \cap \Gamma_{D}, \\
\sigma\left(\boldsymbol{u}_{k}^{n+1}, p_{k}^{n+1}\right) \boldsymbol{n}_{k} & =\sigma_{n} \boldsymbol{n}_{k} & & \text { on } \partial \Omega_{k} \cap \Gamma_{N}, \\
\boldsymbol{n}^{T} \sigma\left(\boldsymbol{u}_{k}^{n+1}, p_{k}^{n+1}\right) \boldsymbol{t}_{\alpha} & =\boldsymbol{\sigma}_{t} \cdot \boldsymbol{t}_{\alpha}, \boldsymbol{u}_{k}^{n+1} \cdot \boldsymbol{n}_{k}=0 & & \text { on } \partial \Omega_{k} \cap \Gamma_{W}, \\
\Phi_{k}\left(\boldsymbol{u}_{k}^{n+1}, p_{k}^{n+1}\right) & =\theta \Phi_{k}\left(\boldsymbol{u}_{j}^{n}, p_{j}^{n}\right)+(1-\theta) \Phi_{k}\left(\boldsymbol{u}_{k}^{n}, p_{k}^{n}\right) & & \text { on } \Gamma_{j k} .
\end{aligned}
$$

Again $\theta \in(0,1]$ is a relaxation parameter. The interface function is given by

$$
\Phi_{k}(\boldsymbol{u}, p)=\sigma(\boldsymbol{u}, p) \boldsymbol{n}_{k}+\left(-\frac{1}{2} \boldsymbol{a} \cdot \boldsymbol{n}_{k}+z_{k}\right) \boldsymbol{u},
$$

where $z_{k}$ is an acceleration parameter, which will be specified later.

The formulation of the corresponding weak problem cannot be accomplished straightforward, see [LMO00C]. As pointed out there, the (possible) discontinuity of the pressure functions across the interface is necessary in the proof of a convergence result based on a two-field formulation. LUBE suggests two solution strategies, viz., $(i)$ to allow continuous pressure and to treat the continuity constraint by additional Lagrange multipliers and $(i i)$ to introduce additional pressure jump terms in the global discrete problem, as described in [RST.96]. Both approaches are described and analyzed in detail in [LMO()0C]. Here we restrict ourselves to the latter strategy, which is studied in [LMO)(b)]. Therein the following modified discrete space for the pressure is chosen

$$
Q^{h}=\left\{q:\left.q\right|_{T} \in P^{l}(T),\left.\quad q\right|_{\Omega_{i}} \in C^{0}\left(\Omega_{i}\right)\right\} \cap Q .
$$

Denote $\Gamma=\cup_{i, j=1}^{N} \Gamma_{i j}$ and denote $E$ the edges of the triangles and [.] the jump across the edge. Then to the global discrete stabilised bilinear form we add the form $\sum_{E \subset \Gamma} \sigma_{E}([p],[q])_{E}$, 
with $(\cdot, \cdot)_{E}$ denoting the $L^{2}$ scalar product over $E$. Moreover, we introduce $\left.\boldsymbol{W}_{i j, h} \equiv \boldsymbol{V}_{h}\right|_{\Gamma_{i j}}$ and its dual space $\boldsymbol{W}_{i j, h}^{*}$. Then the R-R-algorithm for the discrete Oseen problems (for $\theta=1$ for simplicity's sake) reads, cf. Algorithm 2 in [LMO(00)],

\section{Parallel computation step :}

For $k=1, \ldots, N$, find $\boldsymbol{U}_{k}^{n+1}=\left(\boldsymbol{u}_{k}^{n+1}, p_{k}^{n+1}\right) \in \boldsymbol{V}_{k, A, h} \times Q_{k, h}$ such that for all $\boldsymbol{V}_{k}=$ $\left(\boldsymbol{v}_{k}, q_{k}\right) \in \boldsymbol{V}_{k, T, h} \times Q_{k, h}$

$$
\begin{gathered}
\mathcal{A}_{S G}^{k}\left(\boldsymbol{U}_{k}^{n+1}, \boldsymbol{V}_{k}\right)+\left\langle\left(-\frac{1}{2} \boldsymbol{a} \cdot \boldsymbol{n}_{k}+z_{k}\right) \boldsymbol{u}_{k}^{n+1}, \boldsymbol{v}_{k}\right\rangle_{\Gamma_{k}}+\sum_{E \subset \Gamma_{k}} \sigma_{E}\left(p_{k}^{n+1}, q_{k}\right)_{E} \\
=\mathcal{L}_{S G}^{k}\left(\boldsymbol{V}_{k}\right)+\sum_{j(\neq k)}\left\langle\boldsymbol{\Lambda}_{j k}^{n}, \boldsymbol{v}_{k}\right\rangle_{\Gamma_{k j}}+\sum_{j \neq k} \sum_{E \subset \Gamma_{k j}} \sigma_{E}\left(p_{j}^{n}, q_{k}\right)_{E} .
\end{gathered}
$$

Communication step : For all $k \neq j$, update the Lagrangian multipliers

$$
\left\langle\boldsymbol{\Lambda}_{k j}^{n+1}, \phi\right\rangle_{\Gamma_{k j}}=\left\langle\left(z_{k}+z_{j}\right) \boldsymbol{u}_{k}^{n+1}-\boldsymbol{\Lambda}_{j k}^{n}, \phi\right\rangle_{\Gamma_{k j}} \quad \forall \boldsymbol{\phi} \in \boldsymbol{W}_{k j, h} .
$$

For the analysis of this scheme we refer to [LMO(00) $]$. We have to concede that the pressure jump term has not yet been implemented in our research code. This is partially supported by the a posteriori analysis in [LMO00b], which reveals that terms involving $\sigma_{E}$ are not of significant importance. However, the choices $\sigma_{E}=0$ and $\sigma_{E} \neq 0$ have not yet been compared in numerical experiments. Moreover, it is worthwhile mentioning that in the case that the pressure does not appear in the boundary conditions, the subdomain solution for the pressure might oscillate. Then convergence of the solution for the pressure can be accelerated by using a relaxation parameter $\theta<1$.

As in the case of the ADR problem, an a posteriori analysis reveals some information concerning the choice of the interface function $z_{k}$. In this thesis we use (11.9) in agreement with the results in [LMO00b] and [Mue(0)], p.132 and p.140. 


\section{Turbulent channel flow}

The last two chapters of this thesis are dedicated to numerical investigations. Flow simulations based on the turbulence models presented in Part I and on the numerical scheme described in the previous chapters of this part are contaminated by model inaccuracies and numerical errors. This chapter is devoted to an investigation of these errors. To be more precise, the aim is to study

- the accuracy and the convergence behaviour of the wall function procedure,

- the influence of the spatial and temporal discretisation scheme on the solution,

- and the behaviour of the Iliescu-Layton model with differential filtering in wallbounded flows.

An investigation of the DDM is postponed to the next chapter. We choose the fully developed plane channel flow as a test case. The reason for this is twofold. First, fully developed plane channel flow is the simplest wall bounded flow configuration. Second, both experimental and DNS data are abundant for this flow.

\subsection{Fundamentals of isothermal channel flow}

This section is devoted to a presentation of the flow configuration and some fundamental results. A turbulent channel flow is a flow in a rectangular duct of height $2 H$, length $L \gg H$, and aspect ratio $b \gg H$. Channel geometry and flow are sketched in Figure 12.1. It is convenient to characterise the flow using the following Reynolds numbers, viz.,

$$
R e_{H} \equiv(2 H) U_{b u l k} / \nu, \quad R e_{0} \equiv U_{0} H / \nu, \quad R e_{\tau} \equiv \frac{u_{\tau} H}{\nu},
$$

with centerline velocity $U_{0}$ and the bulk velocity $U_{\text {bulk }}$ being defined as

$$
U_{0}=\left.U\right|_{y=H}, \quad U_{b u l k}=\frac{1}{H} \int_{0}^{H} U(y) d y .
$$

Now we simplify the RANS equations for this flow geometry. For time-independent inflow condition resp. force term, the flow is statistically stationary and therefore $\partial(\cdot) / \partial t=0$. Moreover we assume $H \ll B$ and hence (a) the flow is statistically independent of $z$, $\partial(\cdot) / \partial z=0$, and $W \equiv 0$. Thus the flow is steady and two-dimensional, similar to Section 5.2.3. In the vicinity of the entry of the duct at $x=0$, there is a flow-development region. In the fully developed region (large $x), \partial(\cdot) / \partial x=0$ as $H \ll L$, i.e. (b) velocity statistics depend only on $y$, using arguments similar to those in Section 5.2.4. The distance $l_{e l}$ downstream of the entrance where the flow is fully developed is called entrance length. As 


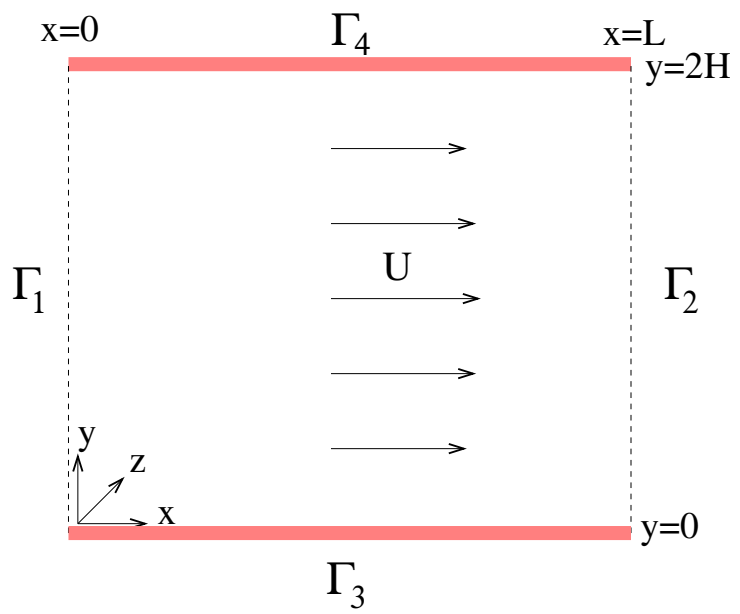

Figure 12.1.: Schematic of channel geometry and flow.

a rule of thumb, $l_{e l}$ is about $60 H$ at $R e_{H}=10^{5}$, see [Wilcox98], p.80.

Thus the full RANS equations (3.1)-(3.2) can be approximated by

$$
\begin{aligned}
\nu \frac{\partial^{2} U}{\partial y^{2}}-\frac{\partial}{\partial y}\left\langle u^{\prime} v^{\prime}\right\rangle_{E}-\frac{\partial P}{\partial x} & =0 \\
-\frac{d\left\langle v^{\prime 2}\right\rangle_{E}}{d y}-\frac{\partial P}{\partial y} & =0
\end{aligned}
$$

bearing discernibly resemblance to (5.25) with $\Theta=0$. Simple integration of (12.4) using $\left.\left\langle v^{\prime 2}\right\rangle_{E}\right|_{y=0}=0$ gives

$$
\left\langle v^{\prime 2}\right\rangle_{E}+P=P_{w}(x), \quad \text { with } \quad P_{w}(x) \equiv P(x, 0,0) .
$$

This implies that the mean axial pressure gradient is uniform across the flow, i.e. $\partial P / \partial x=$ $d P_{w} / d x$, as in the fully developed region derivatives of the Reynolds stresses in the streamline direction can be neglected. Substituting this into (12.3) we obtain

$$
\frac{d \tau}{d y}=\frac{d P_{w}}{d x}=\text { const, } \quad \text { with } \quad \tau(y)=\nu \frac{d U}{d y}-\left\langle u^{\prime} v^{\prime}\right\rangle_{E}
$$

since $\tau=\tau(y)$ and $P_{w}=P_{w}(x) . \tau$ is called total shear stress. It is convenient to express $\tau$ and $P_{w}$ in terms of the wall shear stress $\tau_{w}=\left.\tau\right|_{y=0}$. Then (12.6) can be written as

$$
-\frac{d P_{w}}{d x}=\frac{\tau_{w}}{H}, \quad \tau(y)=\tau_{w}\left(1-\frac{y}{H}\right) .
$$

Note that in a turbulent flow, there is no exact analytical relation between $U_{0}$ and $\tau_{w}$ resp. $U_{b u l k}$ and $\tau_{w}$. For a given flow, they are related by the skin friction coefficients

$$
c_{f}=\frac{\tau_{w}}{\frac{1}{2} U_{0}^{2}}, \quad C_{f}=\frac{\tau_{w}}{\frac{1}{2} U_{b u l k}^{2}},
$$



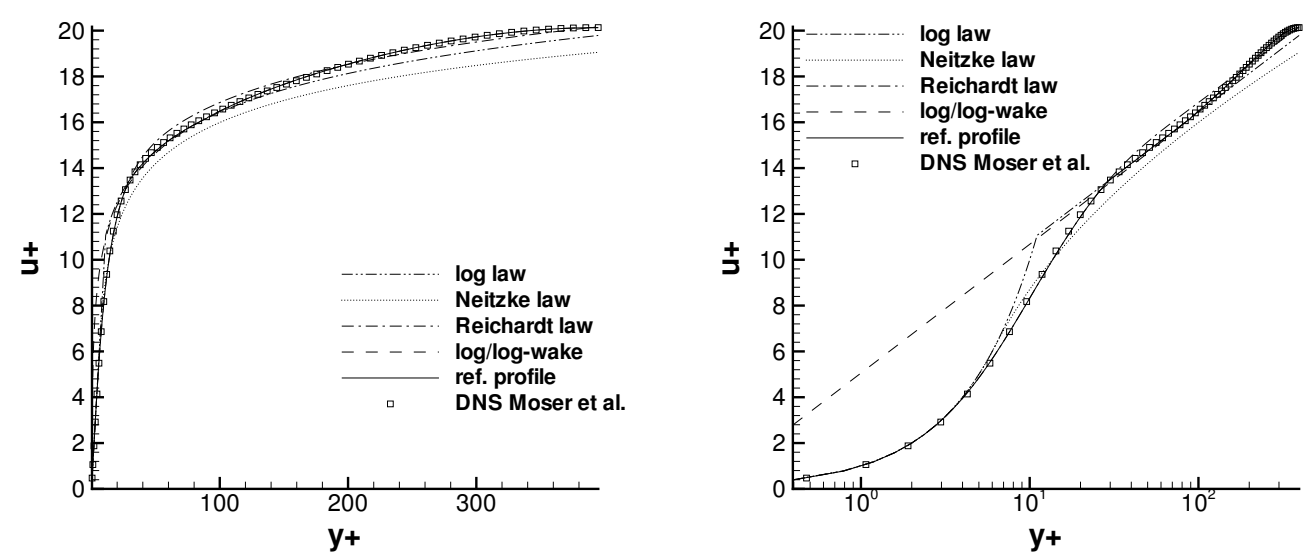

Figure 12.2.: $u^{+}$vs. $y^{+}$(left) and $u^{+}$vs. $\ln \left(y^{+}\right)$(right) for channel flow at $R e_{\tau}=395$.

which are not known a priori in the turbulent case.

From the previous analysis informations regarding the numerical setup can be gained. The aim is to simulate the fully developed region. The homogeneity in the streamwise and spanwise direction suggests imposing periodic boundary conditions in these directions. Then a much smaller computational domain can be used. We choose $\Omega=(0,6) \times(0,2) \times(0,3)$. On the top and bottom walls at $y=0$ and $y=2$, wall functions are used. It should be pointed out that special care must be taken regarding the length of the computational domain in LES. Discernibly from (12.7), the constant pressure gradient drives the flow through the channel. Hence we impose the external force $\boldsymbol{f}=\tau_{w} / H \boldsymbol{e}_{x}$. Then $\tau_{w}$ is determined immediately. In this chapter we focus on a fully developed turbulent channel flow at $R e_{\tau}=395$. For this flow, widely accepted DNS data by Moser ET AL., see [MKM.9.], are available. Note that when specifying $R e_{\tau}$, then (in scaled coordinates) the centre of the channel is located at $H^{+} \equiv u_{\tau} H / \nu=R e_{\tau}$.

Finally we study the mean velocity profile. In Figure 12.2 the approximating velocity profiles by Prandtl/van Karman (5.34), Neitzke (5.41) and Reichardt (5.35) are shown and compared with DNS data of MosER. Moreover we consider the following reference profile $u_{R}=u_{R}^{+} u_{\tau}$, with $u_{R}^{+}$given by

$$
u_{R}^{+}\left(y^{+}\right)= \begin{cases}\frac{1}{\kappa} \ln \left(1+0.4 y^{+}\right)+7.8\left[1-e^{-\frac{y^{+}}{11}}-\frac{y^{+}}{11} e^{-\frac{y^{+}}{3}}\right], & \text { if } y^{+} \leq 30 \\ \frac{1}{\kappa} \ln \left(y^{+}\right)+B, & \text { if } 30 \nu / u_{\tau}<y<0.23 H \\ U_{0} u_{\tau}^{-1}+\kappa^{-1}\left[\ln \left(\frac{y}{H}\right)-2 \Pi+G\left(\frac{y}{H}\right)\right], & \text { if } y>0.23 H .\end{cases}
$$

with wake parameter $\Pi=0.1, G(x) \equiv(1+6 \Pi) x^{2}-(1+4 \Pi) x^{3}$ and $U_{0} / u_{\tau}=20.133$ (from DNS data). The approximation for $y>0.229 H$ was devised in [Dea76] and is used in [C.JB.9. ] as a reference profile. Obviously, for the channel flow under consideration $u_{R}$ is in excellent agreement with the DNS data throughout the channel. 


\subsection{Isothermal channel flow computations using the $k / \epsilon$ model}

The numerical tests for the $k / \epsilon$ model are performed on a structured grid with $49 \times 33 \times 33$ nodes, which are equidistantly distributed in each coordinate direction. Choosing $\nu=10^{-4}$ in a fully developed turbulent channel flow at $R e_{\tau}=395$ implies $u_{\tau}=0.0395$, see (12.1). We start with the initial solution

$$
\begin{aligned}
& \boldsymbol{u}(t=0)=u_{R} \boldsymbol{e}_{x}+\chi \operatorname{rand}\left(\mathcal{O}\left(10^{-4}\right)\right) \\
& k(t=0)=u_{\tau}^{2} \min \left\{\frac{1}{\sqrt{C_{\mu}}} ; 0.05\left(y^{+}\right)^{2}\right\} e^{-y^{+} / 250.0}+\chi \operatorname{rand}\left(\mathcal{O}\left(10^{-6}\right)\right) \\
& \epsilon(t=0)=\frac{u_{\tau}^{4}}{\nu} \frac{0.1+0.003\left(y^{+}\right)^{2}}{1.0+0.00125\left(y^{+}\right)^{3}} e^{-y^{+} / 250.0}+\chi \operatorname{rand}\left(\mathcal{O}\left(10^{-6}\right)\right) .
\end{aligned}
$$

with $\operatorname{rand}\left(\mathcal{O}\left(10^{-n}\right)\right), n \in \mathbb{N}$, denoting a (pseudo) random field with values in $\left[-10^{-n}, 10^{-n}\right]$ resp. $\left[-10^{-n}, 10^{-n}\right]^{d}$. Here $u_{R}=u_{\tau} u_{R}^{+}$, with $u_{R}^{+}$being defined in (12.9). Since for the problem a statistically stationary solution exists, we start with an initial guess for $\boldsymbol{u}, k$ and $\epsilon$ which is close to the RANS solution. Using the parameter $\chi \in\{0,1\}$ we can switch on/off the random perturbation of the initial condition.

\subsubsection{Examination of the wall function approach in periodic channel flow}

First we investigate the wall function procedure and study the predictions for $\tau_{w}$. Recall that $\tau_{w}=u_{\tau}^{2}=(0.0395)^{2}$ is determined immediately from $\boldsymbol{f}=\tau_{w} / H \boldsymbol{e}_{x}$. We use the decoupling and linearisation scheme for the $k / \epsilon$ model described in Section 9.2.2. Therein we choose $\triangle_{m}=0.001$ and perform only one time step and one linearisation cycle. In (12.10)-(12.12) we set $\chi=0$. The result is shown in the following table. Therein, denote $n$ the iteration step and $u_{\tau}^{(n)}$ the corresponding result for $u_{\tau}$.

\begin{tabular}{|l||l|l|l|l|l|l|}
\hline $\mathrm{n}$ & 0 & 1 & 2 & 3 & 4 & 5 \\
\hline $10^{2} \times u_{\tau}^{(n)}$ & 2.843234 & 3.747693 & 4.095170 & 4.127534 & 4.127772 & 4.127772 \\
\hline
\end{tabular}

Figure 12.3.: Convergence history of Newton's method within the wall function procedure.

The deviation from the theoretical value $u_{\tau}=0.0395$ is less than $5 \%$ and stems from the fact that the near-wall profile proposed by NeITZKE and $u_{R}$ differ slightly, see Figure 12.2 . The convergence behaviour is in agreement with e.g. [Kre.98], Chapter 6. It is interesting to examine the influence of the initial guess on our scheme, see also Section 6.3. Denote $B$ the set of all initial guesses $x^{(0)}$ such that $(6.21)$ converges to $u_{\tau}=4.127772 \times 10^{-2}$. Then numerical tests show that $B=\left(6.065307 \times 10^{-3}, 8.279371 \times 10^{-2}\right)$, where the lower bound of $B$ equals the lower bound of $D$. (Recall that $D$ denotes the domain where the Newton's method is well-defined, see Section 6.3.)

\subsubsection{A posteriori testing for the $k / \epsilon$ model}

Now we accomplish an a posteriori testing of the $k / \epsilon$ model, i.e., we compute a solution of the scheme presented in Section 9.2.2. We use time step width $\triangle_{m} \equiv \triangle t=0.01$, end time 
$T=6.0$ and $\max _{d l c}=5$. For the initial solution we select $\chi=1$ in (12.10)-(12.12).

The convergence history for $U_{1}=\boldsymbol{u} \cdot \boldsymbol{e}_{x}, k$ and $\epsilon$ is shown in Figures 12.4, 12.6, and 12.8. In the caption of the figures we use the notation $\left\|f^{n+1}-f^{n}\right\|_{L^{2}} /\left(t^{n+1}-t^{n}\right)$ for a quantity $f$, with $t^{n}=n \triangle t$ and $f^{n}=f\left(\cdot, t_{n}\right)$. Discernibly, two phases can be distinguished in the convergence history. During the first time steps (until $t \approx 0.3$ ) the changes of the solution are relatively large, and the turbulence quantities $k$ and $\epsilon$ converge fast. For $t \gtrsim 0.3$ the convergence of all quantities becomes relatively slow. Plots of the solution (being omitted for lack of space) reveal that the solution is quite good at $t=0.3$. After $t \approx 0.3$ the numerical diffusivity alters the solution significantly. This is well-illustrated in the history of the profiles for $k$, see Figure 12.11, 12.13, and 12.15. This observation is supported by the results in [Mue9.9] and [Fis(2)]. Numerical tests reveal that choosing a finer time step width does not improve the results. Using a much finer grid for the spatial discretisation might improve the results, see below. However, the necessary computer power is not available for testing this in the three-dimensional case. Regarding the effect of the computational time step on turbulence statistics in plane channel flow for a $D N S$ at $R e_{\tau}=180$, it is interesting to mention the results by CHOI AND MoIN, see [CM94]. For a given time step $\Delta t$ the corresponding time step in wall unit is defined by

$$
(\Delta t)^{+} \equiv \Delta t u_{\tau}^{2} / \nu
$$

The viscous time scale in the sublayer is given by the so-called Kolmogorov time scale, $\tau^{+} \equiv$ $\left(u_{\tau}^{4} / \epsilon \nu\right)^{1 / 2} \approx 2.4$ in this case. For $(\Delta t)^{+} \geq 1.6$ they observe laminar flow solutions for both the Crank-Nicolson scheme and the backward Euler scheme. Moreover they report that the turbulent fluctuations $\left\langle u_{i}^{\prime} u_{j}^{\prime}\right\rangle_{E}$ are contamined by a significant error unless $(\Delta t)^{+} \leq 0.4$. These observations emphasise that both time discretisation schemes are too dissipative for large time steps in a DNS.

Secondly, we study the accuracy of the predictions for $U_{1}=\boldsymbol{u} \cdot \boldsymbol{e}_{x}, k$ and $\nu_{t}$, at $t=2.0$, $t=4.0$, and $t=6.0$, see Figures 12.5 12.15. In the plots the solid lines give the DNS results from [MKM99]. The dot-dot-dashed lines are our results of the $k / \epsilon$ model. In the $\log$ layer $50 \lesssim y^{+} \lesssim 140$ the prediction for $\nu_{t}$ is good. For $y^{+} \gtrsim 140, \nu_{t}$ is significantly overpredicted. The well-known failure of the $k / \epsilon$ model in the near-wall region $y^{+} \lesssim 40$ was already described in Section 3.4.2. The velocity profile is clearly underpredicted for $y^{+} \lesssim 120$. This stems both from the numerical diffusivity of the scheme and from the overprediction of $\nu_{t}$ in the near-wall region. Particularly at the first near-wall node, the velocity is significantly underpredicted. Consequently, $\tau_{w}$ is significantly underpredicted because matching is accomplished with a too small value for the velocity of the outer flow. At $t=1.0$ we obtain $\tau_{w}=1.246 \times 10^{-3}$, i.e., $u_{\tau}=0.0353$ (instead of the theoretical value $\left.u_{\tau}=0.0395\right)$. Additional numerical tests have been performed for the corresponding two-dimensional problem on much finer grids using a simple low Reynolds model in the nearwall region, but with a much smaller end time. Our wall function procedure matches the outer flow with the near-wall predictions for $\boldsymbol{u}, k$ and $\nu_{t}$ from (5.41) and (5.49), which are valid also in the viscous sublayer. Moreover low Reynolds models are reliable for the fully developed channel flow. Hence this approach is reasonable. The predictions for $U$ and $\nu_{t}$ are in much better agreement with the DNS data than the results for the three-dimensional calculation presented here. Even in the viscous sublayer the results are good. However, the plots are not shown here for lack of space and for sake of clarity. In three-dimensional 
problems a resolution of the near-wall region is often infeasible and only a relatively coarse mesh can be afforded. Moreover, low Reynolds models are not very reliable in complex flow configurations, see e.g. [DPR01], Chapter 6.

\subsection{Quasi a priori testing of the SGS model}

This section is dedicated to an investigation of our LES model. The aim is to isolate the different constitutive blocks of the LES model, viz., residual stress model (eddy viscosity SGS model, Galdi-Layton model), near-wall damping, explicit filtering technique, and wall function approach. Moreover we attempt to isolate modelling from the numerical scheme (DG(0), SDFEM). Thus we perform an a priori analysis of our LES model. Strictly speaking, we only perform a "quasi a priori testing" rather than a full a priori testing, as will be explained in the sequel.

An a priori test uses DNS (or experimental) data to study directly the accuracy of an LES model. This is a valuable tool for understanding a particular residual stress model. Such an a priori testing for fully developed channel flow and fully developed pipe flow was accomplished e.g. by HärTel et Al., see [HKUF94], Piomelli ET Al., cf. [PYA.96] and Brun AND FRIEDRICH, cf. [BF99]. In this thesis we will compare our results with those presented by BRUn AND FRIEDRICH. They considered a fully developed pipe flow at $R e_{\tau}=180$. As shown in [HKUF94], the quantities of interest (see below) behave quite similarly in channel flow and in pipe flow. Given DNS data $\boldsymbol{u}\left(\cdot, t_{i}\right)$ at time instances $t_{i}(i=1, \ldots, M)$ and a space averaging filter $\langle\cdot\rangle_{\Delta}$, all SGS quantities of interest, e.g. $\mathcal{P}_{r}=-\tau^{r}: \mathbb{S}(\overline{\boldsymbol{u}})$, can be computed numerically. BRUN chose $\left(\Delta_{r}\right)^{+}=28.0=4 h_{x}$ for the filter width in axial direction and $\left(R \Delta_{\phi}\right)^{+}=35.2=4 h_{\phi}$ for the filter width in circumferential direction, $R$ being the radius of the pipe, and $h_{x}$ and $h_{\phi}$ denoting the mesh width in the axial and circumferential direction for the underlying DNS.

HÄRTEL ET AL. proposed to split space filtered quantities $f$ into a statistically stationary mean value $\langle f\rangle_{H}$ and a fluctuating part $f^{\prime \prime}, f=\langle f\rangle_{H}+f^{\prime \prime}$, see [HKUF94]. Here the operator $\langle\cdot\rangle_{H}$ represents an averaging over wall-parallel planes and over time. Note that $\mathcal{P}_{r}$ is often referred to as SGS dissipation and denoted alternatively by $\epsilon$, although this notation can be somewhat misleading. Then $\mathcal{P}_{r} \equiv \epsilon$ can be decomposed into a statistically stationary mean value $\epsilon^{M S} \equiv \mathcal{P}_{r}^{M S}$ and into a fluctuating part $\epsilon^{F S} \equiv \mathcal{P}_{r}^{F S}$, viz.,

$$
\mathcal{P}_{r}=\mathcal{P}_{r}^{M S}+\mathcal{P}_{r}^{F S}
$$

with

$$
\epsilon^{M S} \equiv \mathcal{P}_{r}^{M S}=-\left\langle\tau^{r}\right\rangle_{H}:\langle\mathbb{S}(\overline{\boldsymbol{u}})\rangle_{H}, \quad \epsilon^{F S} \equiv \mathcal{P}_{r}^{F S}=-\left\langle\tau^{r}: \mathbb{S}(\overline{\boldsymbol{u}})\right\rangle_{H}-\epsilon^{M S} .
$$

Figure 12.16 shows $\left(\epsilon^{M S}\right)^{+} \equiv \epsilon^{M S} \nu / u_{\tau}^{4}$ and $\left(\epsilon^{F S}\right)^{+} \equiv \epsilon^{F S} \nu / u_{\tau}^{4}$ for fully developed pipe flow at $R e_{\tau}=180$, given in [BF99], Figure 1. As shown in [HKUF94], Figure 8, $\epsilon^{M S}$ and $\epsilon^{F S}$ resp. behave very similar in channel flow and in pipe flow. As pointed out in [PYA96], Figure 4, details of the SGS dissipation depend on the type and the size of the filter. Interestingly, HÄRTEL ET AL. found that $\mathcal{P}_{r}^{F S}<0$ in the buffer layer indicating backscattering there. 

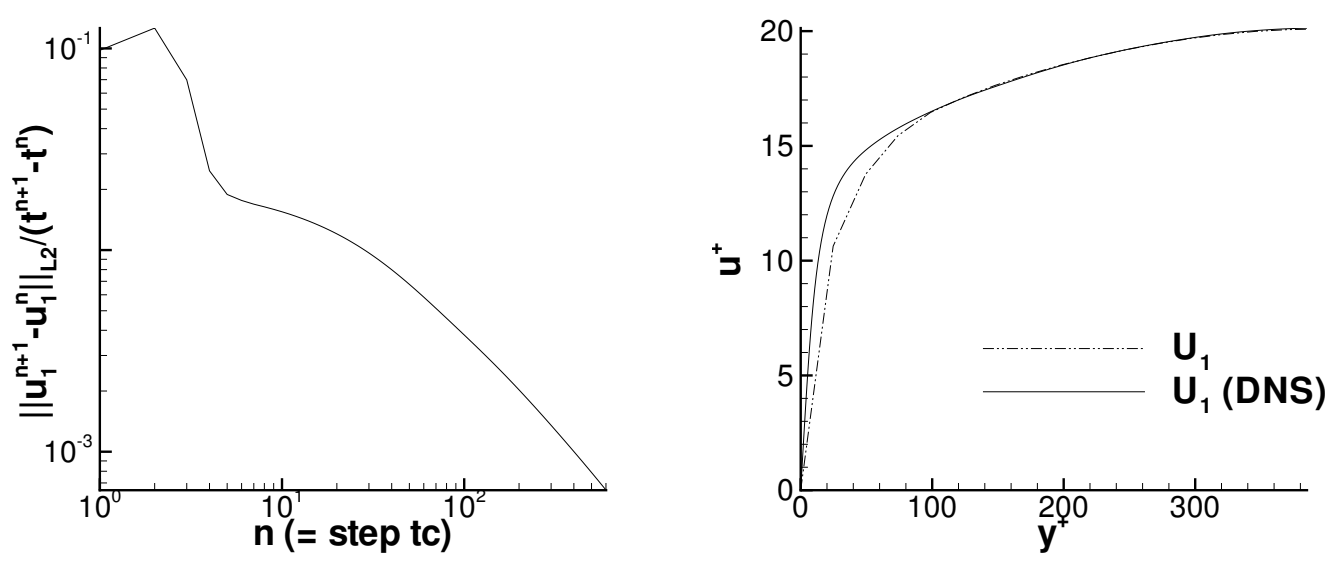

Figure 12.4.: Convergence history for $U_{1}$

Figure 12.5.: Profile for $U_{1}$ at $t=2.0$
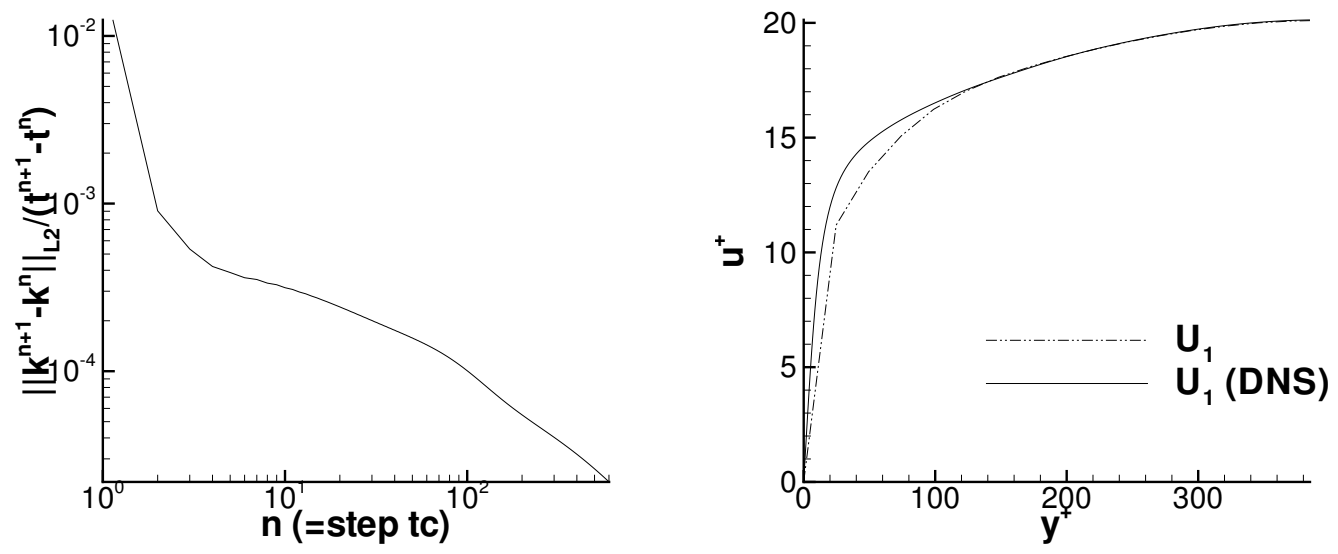

Figure 12.6.: Convergence history for $k$

Figure 12.7.: Profile for $U_{1}$ at $t=4.0$
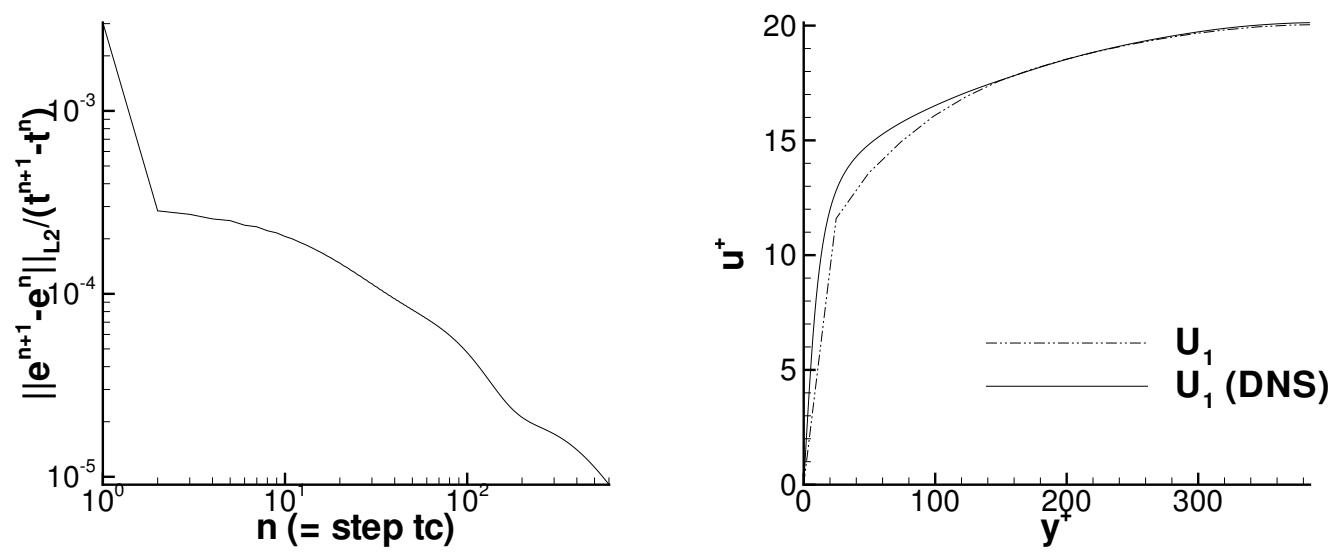

Figure 12.8.: Convergence history for $\epsilon$

Figure 12.9.: Profile for $U_{1}$ at $t=6.0$ 

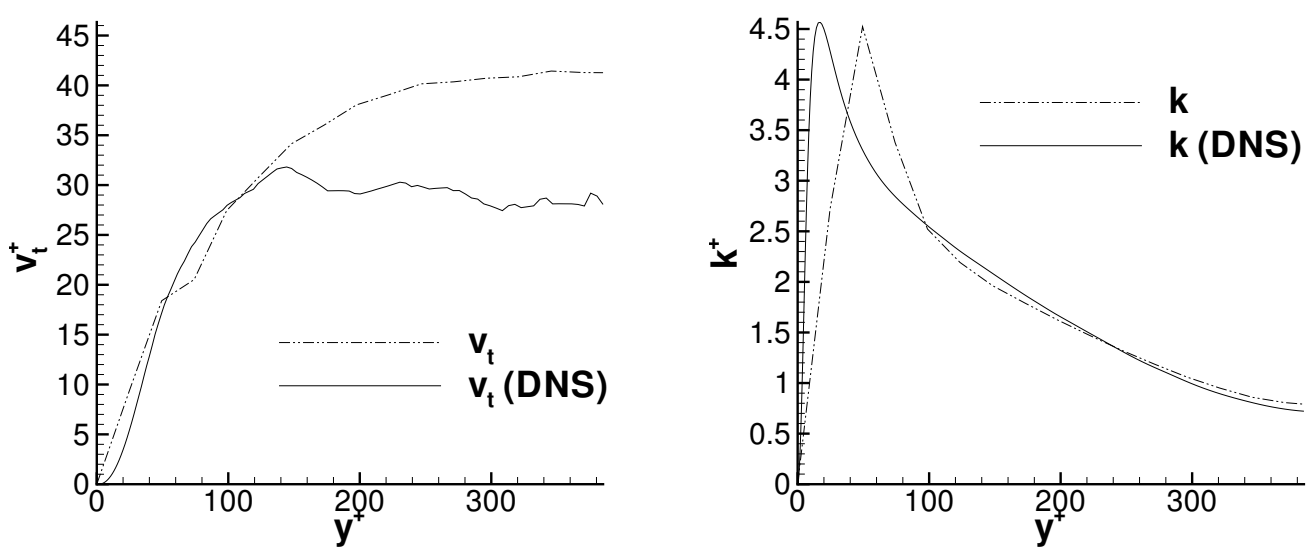

Figure 12.10.: Profile for $\nu_{t}$ at $t=2.0$

Figure 12.11.: Profile for $k$ at $t=2.0$
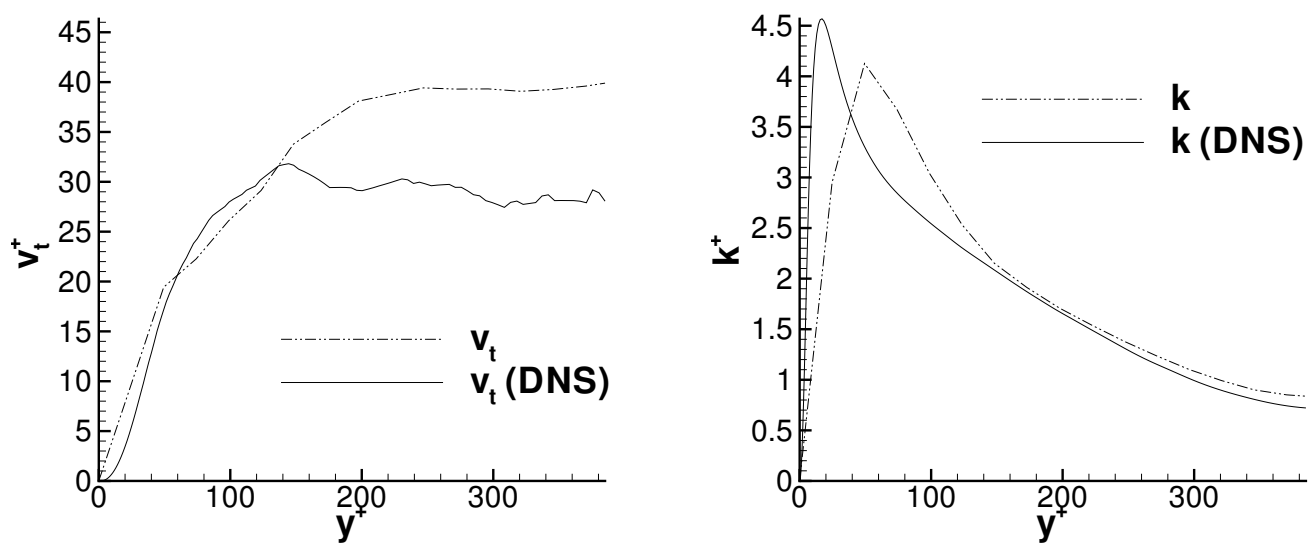

Figure 12.12.: Profile for $\nu_{t}$ at $t=4.0$
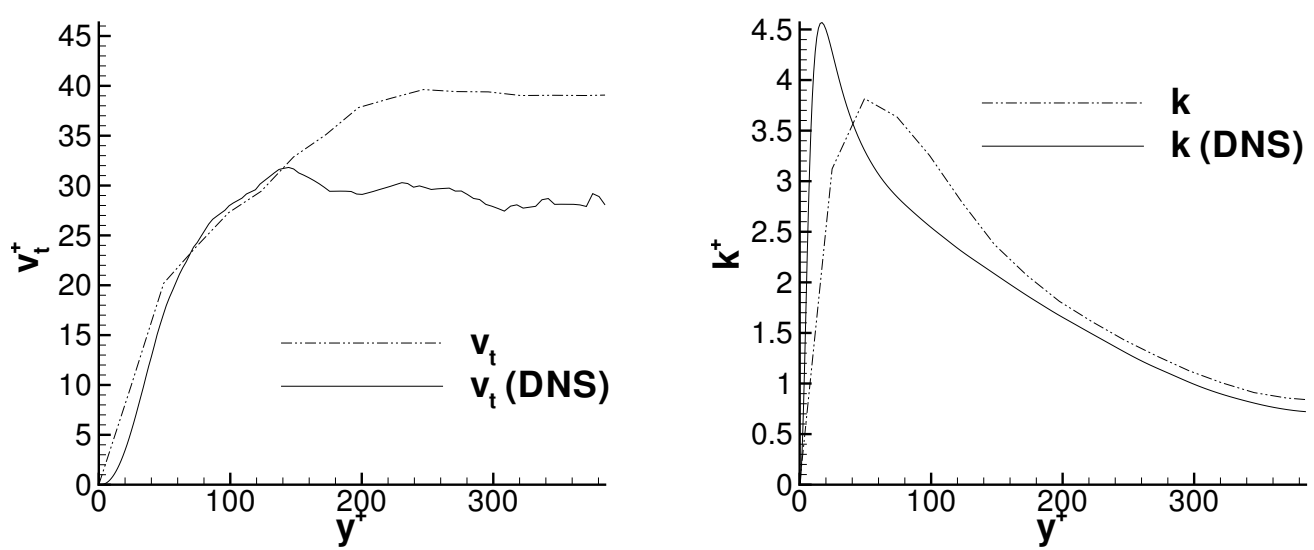

Figure 12.14.: Profile for $\nu_{t}$ at $t=6.0$

Figure 12.15.: Profile for $k$ at $t=6.0$ 


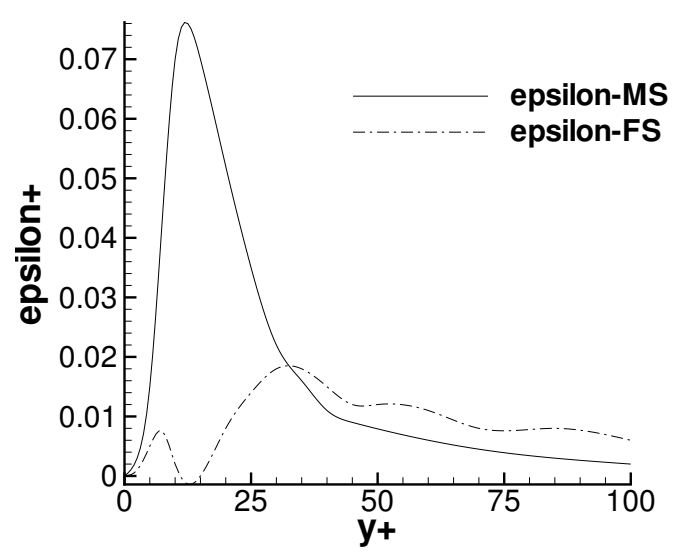

Figure 12.16.: A priori testing for fully developed pipe flow ([BF99] $)$

An a priori testing uses DNS (or experimental) data and is related to a well-resolved LES, i.e., the flow field is resolved in all three directions. In the sequel, we focus on the situation that only the wall-normal direction is properly resolved. The mesh width in streamwise and spanwise direction is assumed to be relatively large. This approach will be referred to as "quasi a priori testing". For details concerning grid requirements for LES on anisotropic meshes see e.g. [ZBK.95]. Such anisotropic meshes are used in applications when the computer resources are limited. The quantity $\epsilon^{M S}$, see (12.14), is related to the mean strain rate, see e.g. [Sag01], p.235. Its dominant contribution stems from $\langle\partial u / \partial y\rangle_{H}$. This quantity is reasonably resolved in a "quasi a priori testing". However, streamwise and spanwise fluctuations which contribute significantly to $\epsilon^{F S}$ cannot be resolved. So the aim is to model $\epsilon^{F S}$ using $\langle u(y)\rangle_{H}$. In our "quasi a priori testing" we study a fully developed channel flow at $R e_{\tau}=395$. We use $\boldsymbol{u}=u_{R} \boldsymbol{e}_{x}$, with $u_{R}$ being the RANS solution of the fully developed channel flow, see (12.9). This can be regarded as the limit case $h_{x} \gg h_{y}$ and $h_{z} \gg h_{y}$, with $h_{x}, h_{y}, h_{z}$ denoting the mesh width in $x, y$, and $z$ direction resp. It will be shown in the following that the Smagorinsky model is a reasonable model for $\mathcal{P}_{r}^{M S}$ whereas the Iliescu-Layton model can be used to model $\mathcal{P}_{r}^{F S}$.

In the sequel the following issues will be addressed.

- The behaviour of the Smagorinsky model (SM model) has been studied thoroughly both in free shear flows and in wall-bounded flows. So far, numerical tests for the Iliescu-Layton model (IL model) are restricted to free shear flows, viz., to the mixing layer, see [Joh02]. Hence we will study the effect of the IL model in a wall-bounded flow by accomplishing a "quasi a priori testing" for a fully developed channel flow.

- A subsequent question is how the predictions for $\mathcal{P}_{r}$ are related to $\epsilon^{M S}$ and $\epsilon^{F S}$, see (12.14). This includes the question whether it is possible to model $\epsilon^{F S}$ in terms $\langle u(y)\rangle_{H}$. This is an issue of practical relevance, since in high Reynolds number applications often only the wall-normal direction can be resolved properly.

- The IL model and also the widespread dynamic SM model use an explicit filtering 
technique for computing $\nu_{t}$. Consequently it is worthwhile investigating the effect of the filtering technique. In particular we are interested in whether the selected filtering technique, viz., filtering by convolution or differential filtering, affects the predictions for $\mathcal{P}_{r}$.

- It is well-known that the SM model has to be damped in the near-wall region. To be more precise, $\nu_{t}$ has to be multiplied with $\mathcal{D}\left(y^{+}\right)^{2}$, with $\mathcal{D}\left(y^{+}\right)$being the van Driest damping function, see (5.33). Generally speaking, if $\nu_{t} \sim \Delta^{\alpha}$, then $\nu_{t}$ has to be multiplied by $\mathcal{D}\left(y^{+}\right)^{\alpha}$. At a first glance, (4.34) suggests that $\nu_{t} \sim \Delta$ for the IL model. However, (A.7), (A.22), and (4.35) suggest that $\nu_{t} \sim \Delta^{3}$. This issue will be studied numerically. Moreover we study whether a suitable damping exponent $\alpha$ depends on the filtering technique.

- Finally we investigate the influence of filter size $\Delta$ and mesh width in wall-normal direction $h$. We also consider the numerical error in differential filtering. In particular we study how this error contaminates the predictions for $\mathcal{P}_{r}$.

For this purpose we introduce

\begin{tabular}{ll}
$g_{\Delta} * u_{R}$ & a very accurate numerical approximation to $g_{\Delta} * u_{R}$ using \\
& the trapezoidal quadrature rule with equidistant grid spacing $h_{c}=0.001$. \\
$\mathcal{R} u_{R}$ & Solution of $(12.15)-(12.16)$. \\
$\mathcal{R}_{h} u_{R}$ & FEM solution of $(12.15)-(12.16)$ on a structured equidistant grid with \\
& $(2 N+1) \times(N+1)$ nodes, $N=64,128,256,512, h=1 / N$. \\
\hline
\end{tabular}

Recall that $u_{R}$ denotes the RANS solution of the fully developed channel flow, see (12.9). For the differential filtering we consider the problem of seeking $\bar{u}$ such that

$$
\begin{aligned}
-\frac{\Delta^{2}}{24} \nabla^{2} \bar{u}+\bar{u} & =u_{R} \quad \text { in } \Omega=(0,6) \times(0,2), \\
\nabla \bar{u} \cdot \boldsymbol{n} & =0 \quad \text { at } y=0 \text { and } y=2 H, \text { and periodic bcds. on } \Gamma_{1}, \Gamma_{2},
\end{aligned}
$$

see Figure 12.1. Motivated by the work of GeurTs and FröHLich, see [Geu(01] we choose $\Delta=1 / 16$ and $\Delta=1 / 32$ for the filter width.

First let us compare both filtering methods by studying $g_{\Delta} * u_{R}$ and $\mathcal{R}_{h} u_{R}$. As the convolution $g_{\Delta} * u_{R}$ is computed on a very fine grid, we can assume that its numerical error is negligible small. Regarding differential filtering, we have to distinguish (i) the numerical error of the finite element scheme, (ii) the error w.r.t $\Delta$ and (iii) a modelling error stemming from the homogeneous Neumann boundary condition. It is worthily explaining (ii) and (iii). Regarding (ii), combining (A.22) and (A.25) reveals that the modelling error of differential filtering in $\mathbb{R}^{d}$ is of formal order $\mathcal{O}\left(\Delta^{4}\right)$. Concerning (iii), recall that for differential filtering in a bounded domain $\Omega$, an artificial homogeneous Neumann boundary condition is imposed, see (4.15). This gives rise to an additional modelling error. To see this, we extend $u_{R}$ by zero onto $\mathbb{R}$ which satisfies $u_{R} \in \mathcal{E}(\mathbb{R})$. Recall that $g_{\Delta} *\left(d u_{R} / d y\right) \in C^{\infty}(\mathbb{R})$ for all $\Delta>0$. Obviously, there exists $K_{0}>0$, s.t. $d u_{R} / d y>K_{0}>0$ in $(0, H / 2)$, with $H$ being the channel half width. In particular, we have $d u_{R} / d y \approx u_{\tau}^{2} / \nu$ for $0<y<10 \nu / u_{\tau}$, 
see (C.14). Thus there exist $K_{1}>0, \Delta_{1}>0$, s.t. $g_{\Delta} *\left(d u_{R} / d y\right) \geq K_{1}$ in $(0, H / 2)$ for all $0<\Delta<\Delta_{1}$. This is in contrast to the homogeneous Neumann boundary condition for $\mathcal{R}\left(u_{R}\right)$ resp. $\mathcal{R}_{h}\left(u_{R}\right)$.

Figures 12.17 and 12.18 show $g_{\Delta} * u_{R}$ and $\mathcal{R}_{h} u_{R}$ for $\Delta=1 / 16$ and $\Delta=1 / 32$ resp. In Figures 12.19 and 12.20 we show $g_{\Delta} * g_{\Delta} * u_{R}$ and $\mathcal{R}_{h} \mathcal{R}_{h} u_{R}$ for $\Delta=1 / 16$ and $\Delta=1 / 32$ resp. Moreover we study the error $e_{h, \Delta}=g_{\Delta} * u_{R}-\mathcal{R}_{h} u_{R}$. In Figures 12.21-12.24 the error in plus-units, i.e., non-dimensionalised by $u_{\tau}$, is given, viz., $e_{h, \Delta}^{+}=e_{h, \Delta} / u_{\tau}$. In the captions of Figures 12.21-12.22 we write $e_{h, \Delta}\left(\overline{u_{R}}\right)$ to indicate that the corresponding figures show $e_{h, \Delta}\left(g_{\Delta} * u_{R}, \mathcal{R}_{h} u_{R}\right)=g_{\Delta} * u_{R}-\mathcal{R}_{h} u_{R}$. Analogously, in the captions of Figures 12.23-12.24 $e_{h, \Delta}\left(\overline{\overline{u_{R}}}\right)$ means that these figures show $e_{h, \Delta}\left(g_{\Delta} * g_{\Delta} * u_{R}, \mathcal{R}_{h} \mathcal{R}_{h} u_{R}\right)=g_{\Delta} * g_{\Delta} * u_{R}-\mathcal{R}_{h} \mathcal{R}_{h} u_{R}$. First we study $e_{h, \Delta}\left(g_{\Delta} * u_{R}, \mathcal{R}_{h} u_{R}\right)$. Keeping in mind that $u_{\tau}$ is small $\left(u_{\tau}=0.0395\right)$, it can be seen that for approximately $y^{+}>70$ the difference $e_{h, \Delta}^{+}$is small. Remote from the wall the approximation is good, i.e., $e_{h, \Delta}$ is small. However, in the near-wall region $y^{+} \lesssim 75(\Delta=1 / 16)$ resp. $y^{+} \lesssim 40(\Delta=1 / 32)$ the error is relatively large. Discernibly, the numerical error w.r.t. $h$ goes to zero as $h \rightarrow 0$, i.e. $\mathcal{R}_{h} u_{R} \rightarrow \mathcal{R} u_{R}$ as $h \rightarrow 0$. Thus as $h \rightarrow 0$ we have $e_{h, \Delta}^{+} \rightarrow e_{r e m, \Delta}^{+} \equiv e_{r e m, \Delta} / u_{\tau}$, with $e_{r e m, \Delta}=g_{\Delta} * u_{R}-\mathcal{R} u_{R}$ being the remaining error. This is the sum of the $\Delta$ - error and the modelling error from the Neumann bcd. As $\Delta$ is decreased by a factor two, so does the sum of $\Delta$ - error and modelling error.

Regarding explicit filtering for LES, the error $e_{h, \Delta}\left(g_{\Delta} * g_{\Delta} * u_{R}, \mathcal{R}_{h} \mathcal{R}_{h} u_{R}\right)$ is even more important. For example, for the Iliescu-Layton model (see (4.34)) we have to evaluate $\nu_{t}=C_{q} \Delta|\overline{\boldsymbol{u}}-\overline{\overline{\boldsymbol{u}}}|$. It can be seen from Figures 12.23-12.24 that the error $e_{h, \Delta}\left(g_{\Delta} * g_{\Delta} *\right.$ $\left.u_{R}, \mathcal{R}_{h} \mathcal{R}_{h} u_{R}\right)$ is largest at the wall, i.e., for $y^{+}=0$. The numerical error w.r.t. $h$ goes to zero as $h \rightarrow 0$, and $e_{h, \Delta}\left(g_{\Delta} * g_{\Delta} * u_{R}, \mathcal{R}_{h} \mathcal{R}_{h} u_{R}\right)$ converges to the sum of $\Delta$-error and modelling error from the Neumann boundary condition. The influence of the Neumann boundary condition on differential filtering can be seen clearly in Figure 12.20, giving rise to a significant modelling error in close proximity of the wall. If $\Delta$ is decreased by a factor two, then the sum of $\Delta$ - error and modelling error is also diminished by a factor close to two.

However, emphasis has to be placed on the fact that we are not interested in the case $\Delta \rightarrow 0$, when applying differential filtering in practical LES computations. We are interested in a "true LES", i.e., $\Delta$ is fixed and $h \rightarrow 0$, see [Geu(0)]. Fig. 12.21-12.24 show that the remaining error is significantly large near the wall. Fig. 12.17 and 12.18 reveal that $\mathcal{R}_{h} u_{R}$ overpredicts $g_{\Delta} * u_{R}$ in the near-wall region. Interestingly, very close to the wall the difference is smallest for the coarse grid $h=1 / 64$. In this case the numerical error counteracts the remaining error.

Next we consider the predictions for $\nu_{t}$ and $\mathcal{P}_{r}$ by the SM model and the IL model. First we explain the captions of Figures 12.25-12.36. For the SM model we introduce the notation $\nu_{t}(w)=\left(C_{S} \Delta\right)^{2}|d w / d y|$ and $\mathcal{P}_{r}(w)=\nu_{t}(w)(d w / d y)^{2}$. We choose $C_{S}=0.1$, see [Pope00], p.602. We write $\nu_{t}\left(\overline{u_{R}}\right)$ indicating that the corresponding figure shows $\nu_{t}\left(g_{\Delta} * u_{R}\right)$ and $\nu_{t}\left(\mathcal{R}_{h} u_{R}\right)$, see Figures 12.25-12.26. Similarly, $\mathcal{P}_{r}\left(\overline{u_{R}}\right)$ denotes $\mathcal{P}_{r}\left(g_{\Delta} * u_{R}\right)$ and $\mathcal{P}_{r}\left(\mathcal{R}_{h} u_{R}\right)$, see Figures 12.29-12.30 and 12.33-12.34. For the IL model we define $\nu_{t}(v, w)=C_{q} \Delta|v-w|$ and $\mathcal{P}_{r}(v, w)=\nu_{t}(v, w)(d v / d y)^{2}$. Here we select $C_{q}=0.1$. We write $\nu_{t}\left(\overline{u_{R}}, \overline{\overline{u_{R}}}\right)$ indicat- 


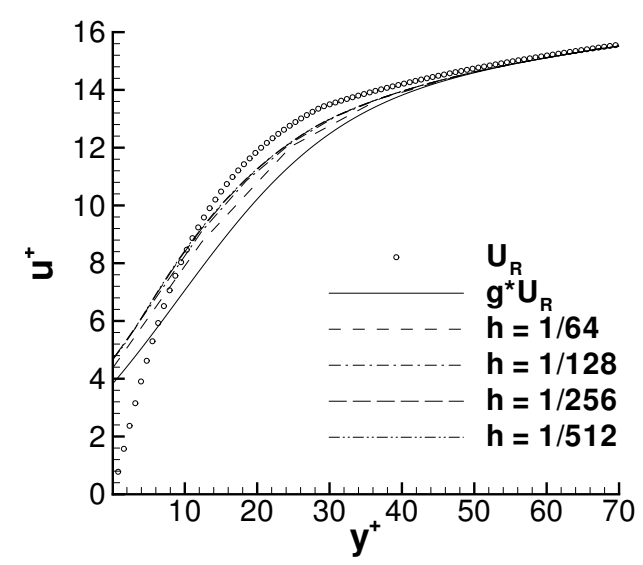

Figure 12.17.: $g_{\Delta} * u_{R}$ and $\mathcal{R}_{h} u_{R}$ for $\Delta=1 / 16$

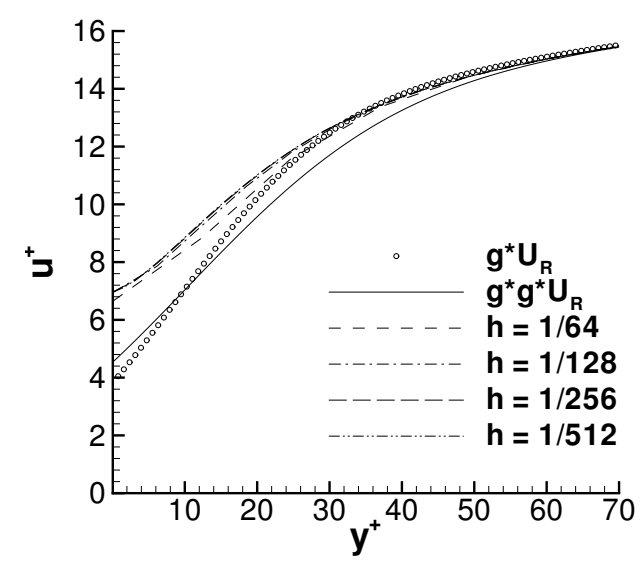

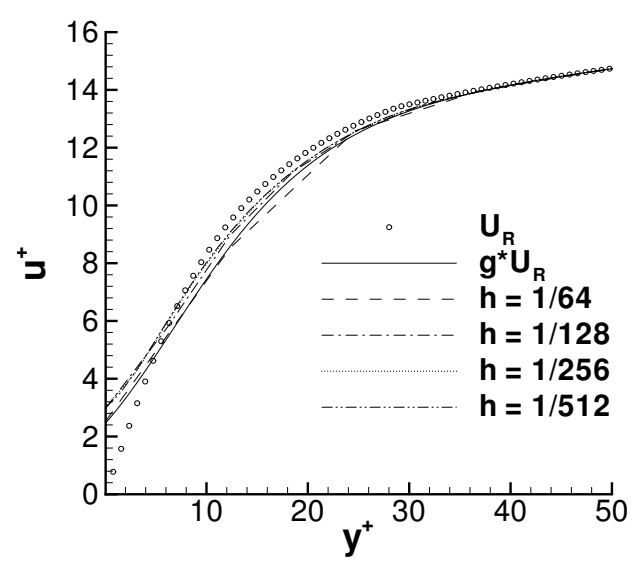

Figure 12.18.: $g_{\Delta} * u_{R}$ and $\mathcal{R}_{h} u_{R}$ for $\Delta=1 / 32$.

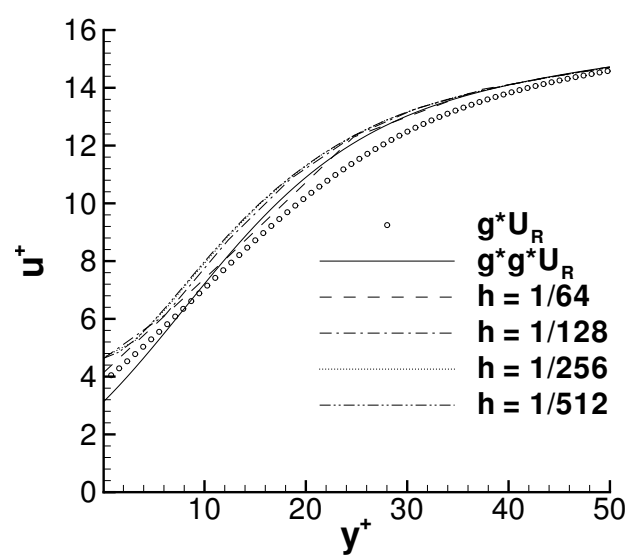

Figure 12.19.: $g_{\Delta} * g_{\Delta} * u_{R}$ and $\mathcal{R}_{h} \mathcal{R}_{h} u_{R}$ Figure 12.20.: $g_{\Delta} * g_{\Delta} * u_{R}$ and $\mathcal{R}_{h} \mathcal{R}_{h} u_{R}$ for $\Delta=1 / 16$. for $\Delta=1 / 32$.
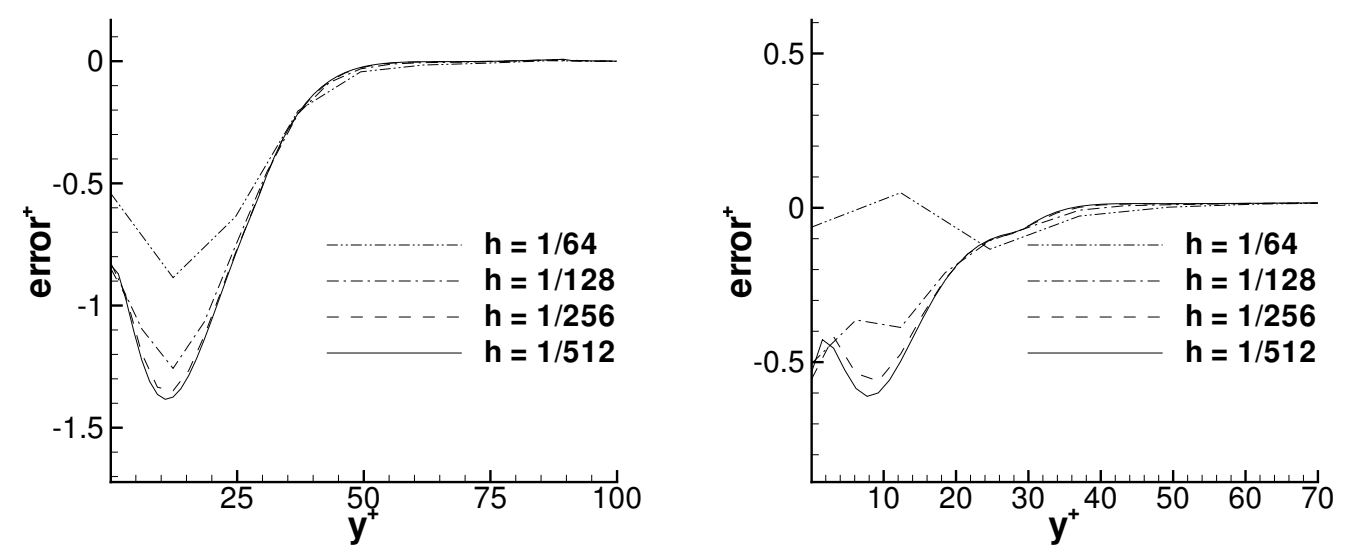

Figure 12.21.: $e_{h, \Delta}\left(\overline{u_{R}}\right)$ for $\Delta=1 / 16$. Figure 12.22.: $e_{h, \Delta}\left(\overline{u_{R}}\right)$ for $\Delta=1 / 32$. 


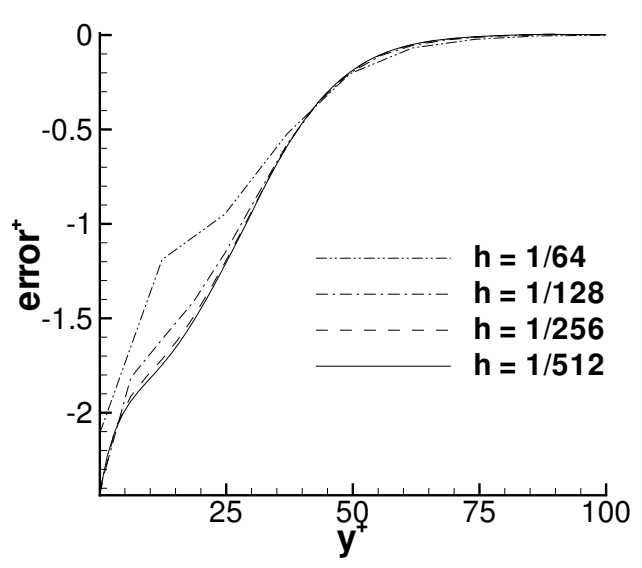

Figure 12.23.: $e_{h, \Delta}\left(\overline{\overline{u_{R}}}\right)$ for $\Delta=1 / 32$.

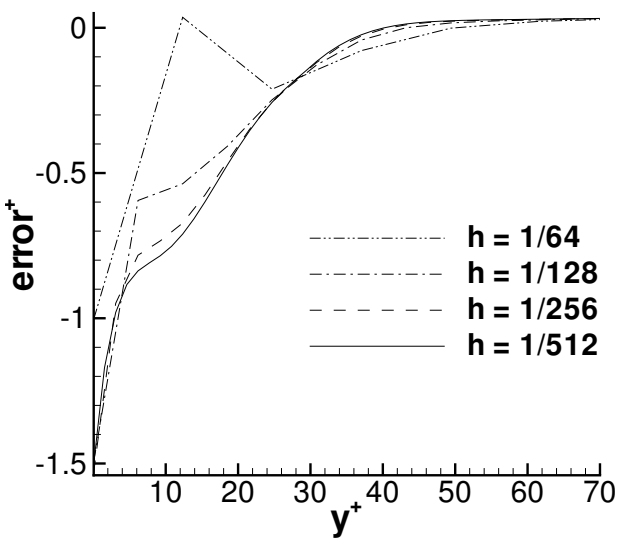

Figure 12.24.: $e_{h, \Delta}\left(\overline{\overline{u_{R}}}\right)$ for $\Delta=1 / 32$.

ing that the corresponding figure shows $\nu_{t}\left(g_{\Delta} * u_{R}, g_{\Delta} * g_{\Delta} * u_{R}\right)$ and $\nu_{t}\left(\mathcal{R}_{h} u_{R}, \mathcal{R}_{h} \mathcal{R}_{h} u_{R}\right)$, cf. Figures 12.27-12.28. Similarly, $\mathcal{P}_{r}\left(u_{R}, \overline{u_{R}}\right)$ and $\mathcal{P}_{r}\left(\overline{u_{R}}, \overline{\overline{u_{R}}}\right)$ denote $\mathcal{P}_{r}\left(u_{R}, g_{\Delta} * u_{R}\right)$, $\mathcal{P}_{r}\left(u_{R}, \mathcal{R}_{h} u_{R}\right)$ resp. $\mathcal{P}_{r}\left(g_{\Delta} * u_{R}, g_{\Delta} * g_{\Delta} * u_{R}\right), \mathcal{P}_{r}\left(\mathcal{R}_{h} u_{R}, \mathcal{R}_{h} \mathcal{R}_{h} u_{R}\right)$, see Figures 12.31-12.32 and 12.35-12.36. As usual, we consider the non-dimensionalised quantities $\nu_{t}^{+}=\nu_{t} u_{\tau}^{-1} H^{-1}$ and $\mathcal{P}_{r}^{+}=\mathcal{P}_{r} \nu / u_{\tau}^{4}$. Without any near-wall modification, for both SGS models $\mathcal{P}_{r}^{+}$is unphysically large in the near-wall region compared to the a priori testing results from DNS data, cf. [BF99]. Damping $\mathcal{P}_{r}$ in the vicinity of the wall is accomplished by using the van Driest damping function $\mathcal{D}\left(y^{+}\right)=1-e^{-y^{+} / 26}$. If $\nu_{t} \sim \Delta^{\alpha}$, then $\nu_{t}$ has to be multiplied by $\mathcal{D}\left(y^{+}\right)^{\alpha}$. For the Smagorinsky model we have $\alpha=2$. Regarding the IL model with convolution we study $\alpha=1$. Additional tests which are not shown here show that $\alpha=2$ for the IL model with convolution is too large. If we choose $\alpha=1$ then the relative magnitude of the two maxima for $\mathcal{P}_{r}$ is similar to the profile of $\epsilon^{F S}$ in Figure 12.16. For the IL model with differential filtering first we study $\alpha=3$. Moreover, for the IL model with filtering by convolution we choose $C_{q}=0.05$ in order to obtain more suitably scaled plots.

First we neglect the effect of the filtering technique and focus on the predictions from filtering by convolution. Thus we study only the solid lines in Figures 12.25 12.36. Obviously the profiles for $\mathcal{P}_{r}\left(g_{\Delta} * u_{R}\right)$ in Figures 12.29 and 12.30 resemble the profile for $\epsilon^{M S}$ plotted in Figure 12.16. However, compared with [BF99], the profile for $\mathcal{P}_{r}$ gives too low values in the log layer and in the core region of the flow. This is at least in parts due to the fact that we can perform only a quasi a priori testing here. The only non-vanishing contribution to $\mathcal{P}_{r}$ is due to $d u_{R} / d y$. In a correct a priori testing all components $\partial u_{i} / \partial x_{j}$ contribute to $\mathcal{P}_{r}$ (including the part linked to velocity fluctuations). Moreover it should be recalled that our "quasi a priori testing" is performed for $R e_{\tau}=395$ whereas $R e_{\tau}=180$ in [BF99]. At a first glance, the predictions for $\mathcal{P}_{r}$ for the IL model, see Figures 12.31-12.32, look at least qualitatively similar to $\epsilon^{F S}$ in Figure 12.16. This is in agreement with the notion in [BF99] that scale similarity models are able to account for backscattering effects in the buffer layer. Although $\nu_{t}$ for the IL model is non-negative, it yields a suprisingly good profile for $\epsilon^{F S}$. Considering the relative magnitude of the two maxima for $\mathcal{P}_{r}$ in Figures (12.31)-(12.32) 
suggests to select $\alpha=1$ for the IL model with filtering by convolution.

At least from a qualitative point of view the idea arises to use a combination of the Smagorinsky model and the Iliescu-Layton model instead of using the former model solely. Denote $\nu_{t, S M}$ and $\nu_{t, I L}$ the predictions for $\nu_{t}$ by the SM resp. IL model. Then $\nu_{t, S M}+\nu_{t, I L}$ yields a quite reasonable prediction for $\mathcal{P}_{r}^{M S}+\mathcal{P}_{r}^{F S}$. Hence supplementing the standard Smagorinsky model with the Iliescu-Layton model seems to be a significant improvement over the standard SM model. This modification benefits from the fact that $\nu_{t} \geq 0$ for the IL model. Hence the well-known numerical stability of the Smagorinsky model is preserved. This can be expressed in an alternative manner: The success of the Smagorinsky model originates in its ability to predict the global SGS dissipation correctly. Supplementing the SM model with the IL model significantly reduces the deficiency of the Smagorinky model to fail at predicting details of the SGS dissipation locally, see e.g. [PYA.96], p.222. The well-known alternative approach, the dynamic Smagorinsky model which allows $\nu_{t}<0$ locally, has the drawback of being numerically instable in some situations. Moreover, it is worth mentioning that the Iliescu-Layton model is much more easy to implement than the dynamic Smagorinsky model.

Now we have to study how the differential filtering technique effects the predictions for $\mathcal{P}_{r}$ by the IL model, see Figures 12.31-12.32 and 12.35-12.36. It will turn out that it is useful to study also $\mathcal{P}_{r}$ by the SM model, see Figures 12.29-12.30 and 12.33-12.34. In a first step we investigate the results for $h=1 / 512$. Then the numerical error w.r.t. $h$ is small enough. Interestingly, the profile for $\mathcal{P}_{r}$ is shifted towards the wall for both the SM and the IL model. This is due to the modelling error stemming from the Neumann boundary condition for differential filtering. As a remedy, we multiply $\mathcal{P}_{r}$ with $\mathcal{D}\left(y^{+}\right)$for both the SM and the IL model, i.e., we choose $\alpha=3$ for the SM model and $\alpha=4$ for the IL model. Then the near-wall error of differential filtering is diminished. Although this is an ad-hoc solution, it works surprisingly well for this test case. Studying Figures 12.21-12.24 gives a motivation for this modification: These figures suggest that the sum of $\Delta$-error and modelling error from the Neumann boundary condition scales linearly with $\Delta$. Therefore we "damp" this error by multiplication with $\mathcal{D}\left(y^{+}\right)$. Of course, the goal of this additional modification is to ensure that the local maximum for $\mathcal{P}_{r}$ for the IL model at $y^{+} \approx 7$ is smaller than the second maximum by a factor of approximately 3.5, cf. Figure 12.16. In a second step we study the influence of the numerical error w.r.t. $h$ on the predictions for $\mathcal{P}_{r}$ by the IL model. The two local maxima and the minimum between both maxima can only be resolved if $h \leq 1 / 256$. Therefore the predictions for $\mathcal{P}_{r}$ become qualitatively wrong if $h \geq 1 / 128$. Recall that in wall-bounded flows the scaled velocity profile $u^{+}\left(y^{+}\right)$ has a universal behaviour. Thus we can infer that LES (with near-wall resolution) using the IL model with differential filtering requires that the first node above the wall is located closer than $y_{\delta}^{+}=y_{\delta} \nu / u_{\tau} \approx 7$ from the wall. Otherwise the modelling error is supplemented by a significant numerical error, i.e., the numerical error is a significant contamination of our scheme. According to Ghosal the numerical error can be interpreted as an additional SGS dissipation due to the numerical scheme. Hence it can be seen from the results that the Iliescu-Layton SGS model is completely or partially masked by the numerical error 

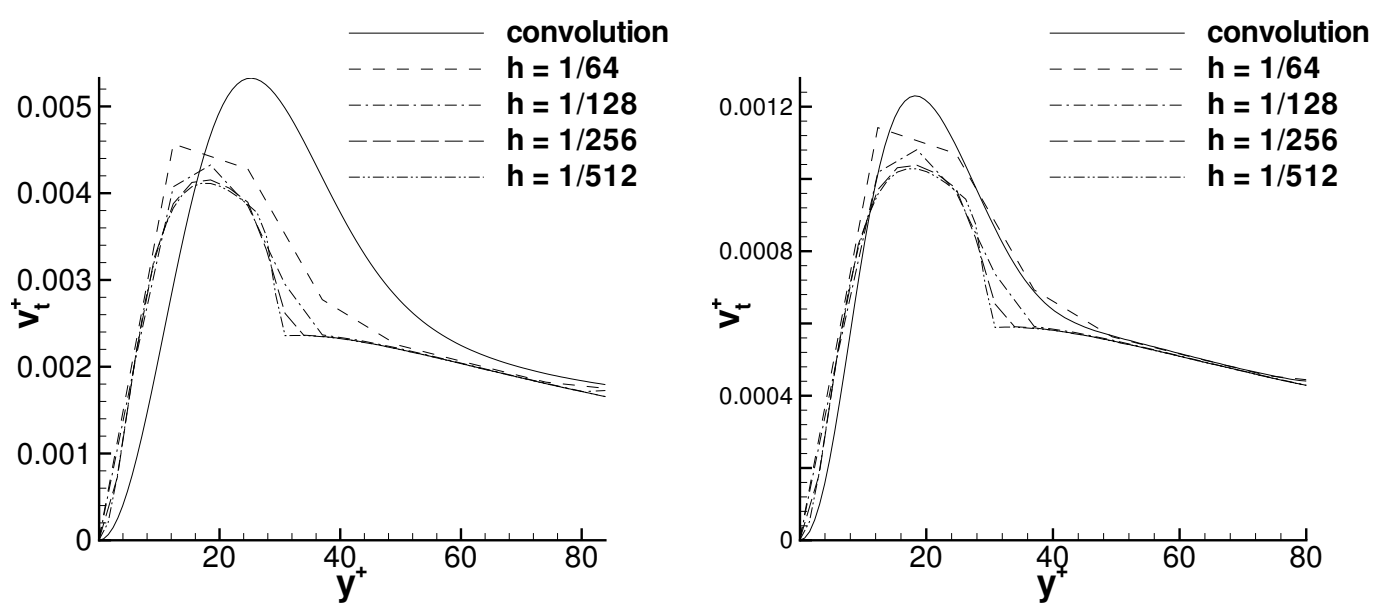

Figure 12.25.: SM, $\nu_{t}\left(\overline{u_{R}}\right), \alpha=2$, $\Delta=1 / 16$.

Figure 12.26.: SM, $\nu_{t}\left(\overline{u_{R}}\right), \alpha=2$,
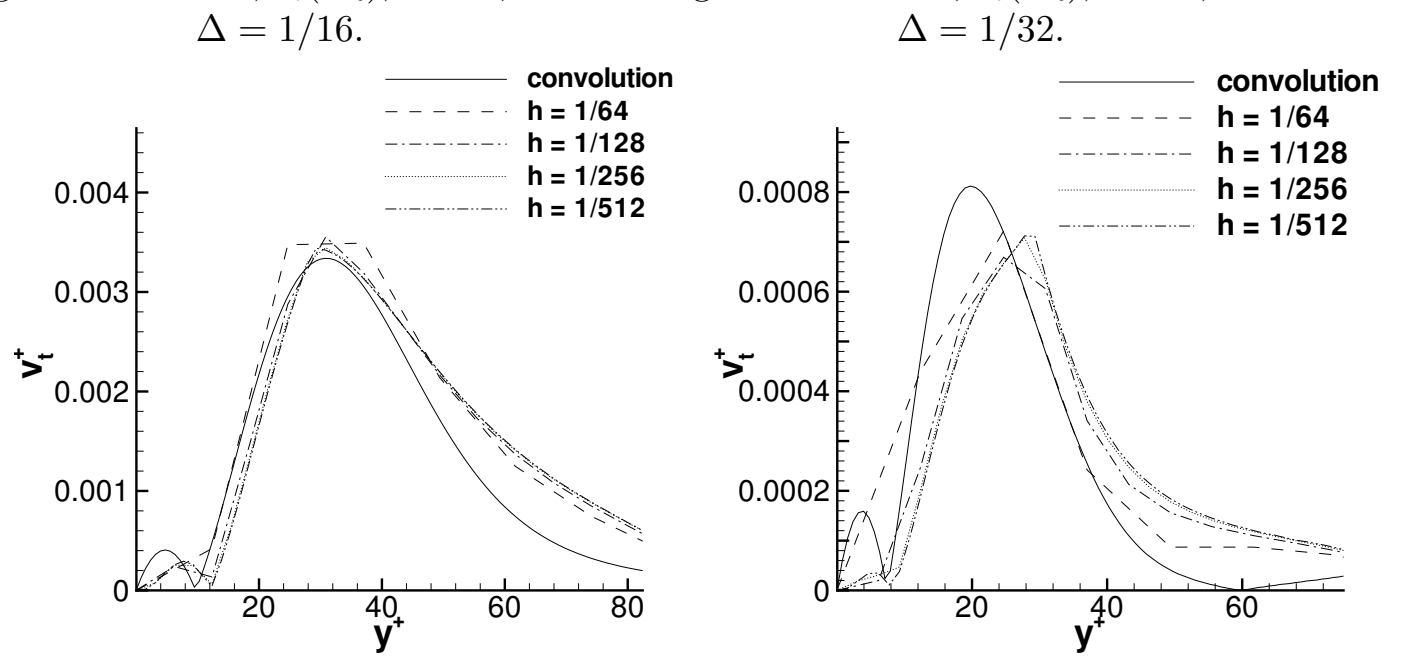

Figure 12.27.: IL, $\nu_{t}\left(\overline{\overline{u_{R}}}, \overline{\overline{u_{R}}}\right), \alpha=3$, $\Delta=1 / 16$.

Figure 12.28.: IL, $\nu_{t}\left(\overline{u_{R}}, \overline{\overline{u_{R}}}\right), \alpha=3$, $\Delta=1 / 32$.
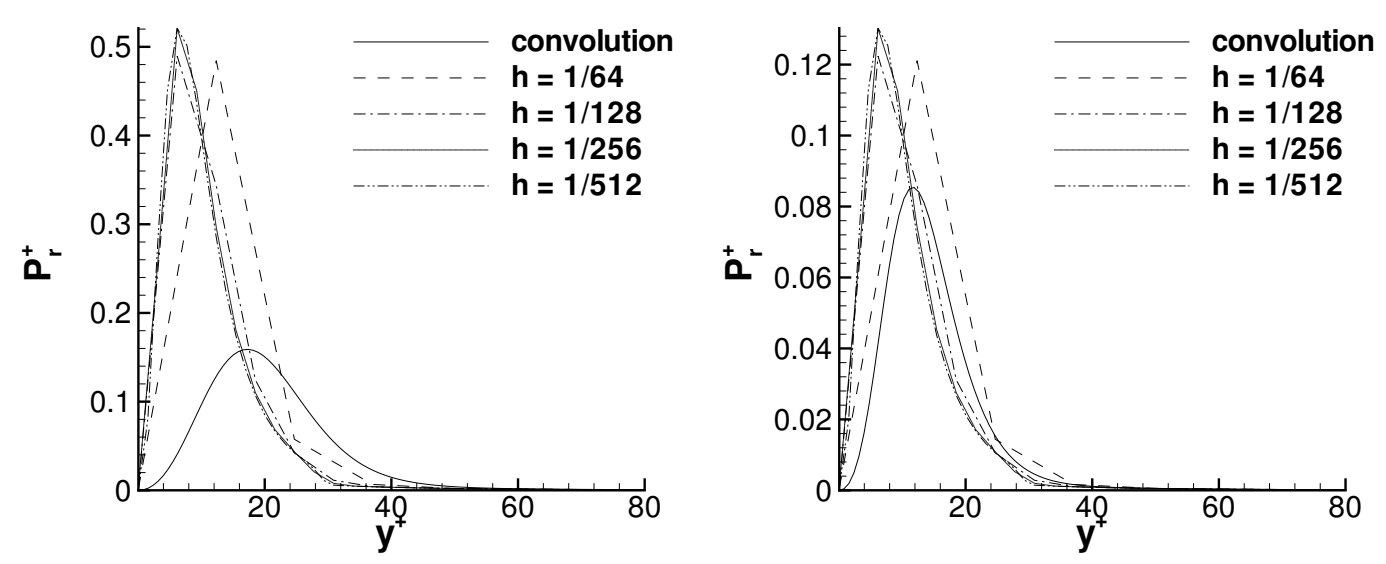

Figure 12.29.: $\mathrm{SM}, P_{r}\left(\overline{u_{R}}\right), \alpha=2$, $\Delta=1 / 16$.

Figure 12.30.: $\mathrm{SM}, P_{r}\left(\overline{u_{R}}\right), \alpha=2$, $\Delta=1 / 32$. 

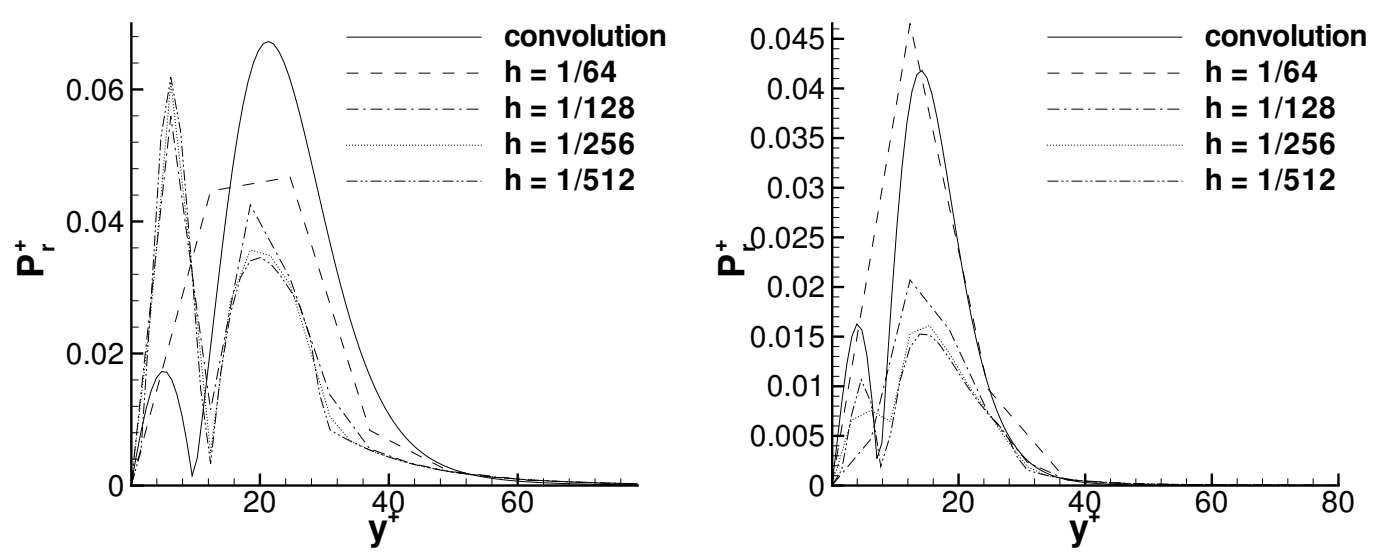

Figure 12.31.: IL, $\mathcal{P}_{r}\left(\overline{u_{R}}, \overline{\overline{u_{R}}}\right), \alpha=3$, $\Delta=1 / 16$.

Figure 12.32.: IL, $\mathcal{P}_{r}\left(\overline{u_{R}}, \overline{\overline{u_{R}}}\right), \alpha=3$, $\Delta=1 / 32$.
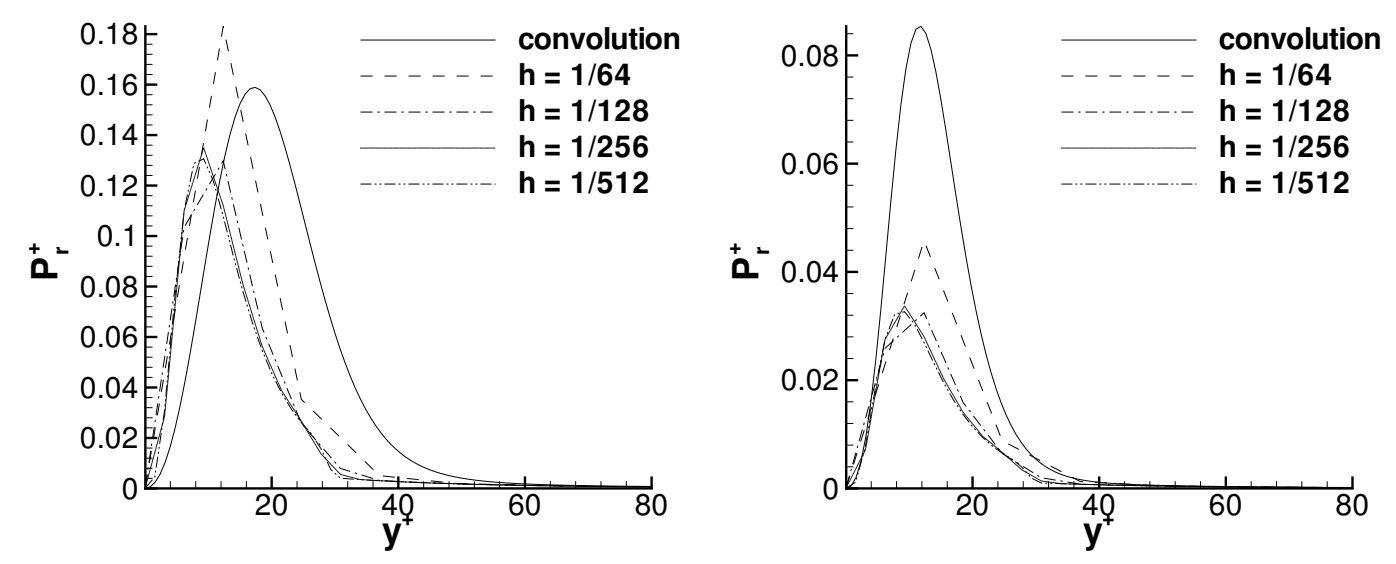

Figure 12.33.: $\mathrm{SM}, \mathcal{P}_{r}\left(\overline{u_{R}}\right), \alpha=3$, $\Delta=1 / 16$.

Figure 12.34.: $\mathrm{SM}, \mathcal{P}_{r}\left(\overline{u_{R}}\right), \alpha=3$, $\Delta=1 / 32$.
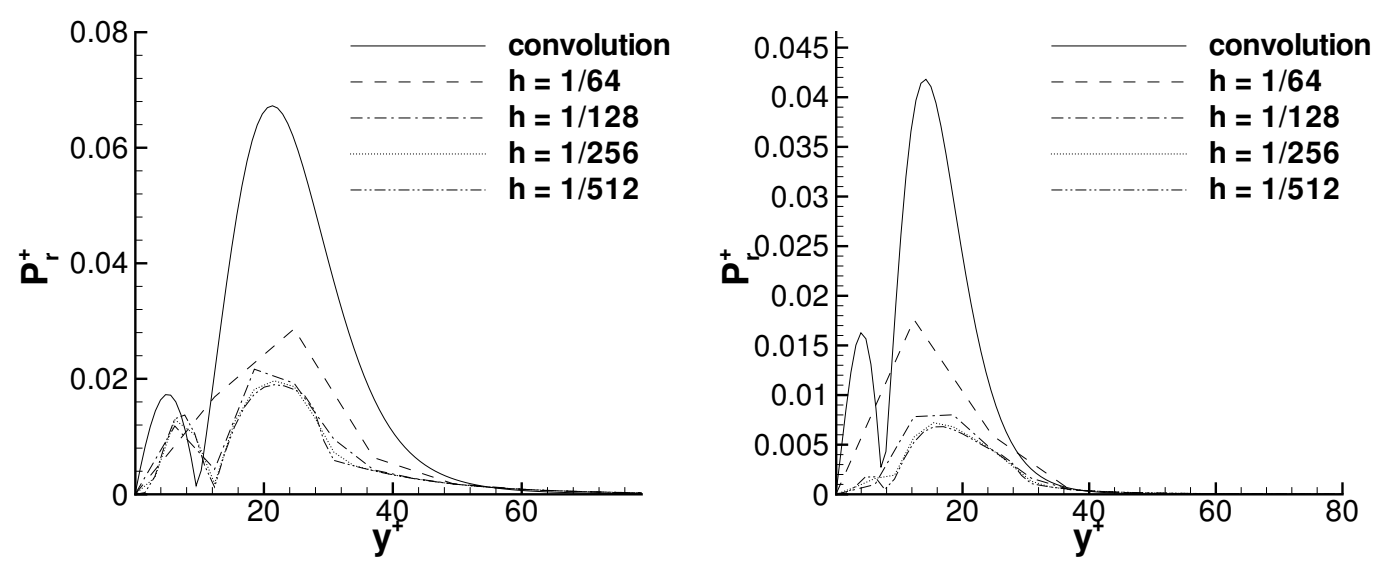

Figure 12.35.: IL, $\mathcal{P}_{r}\left(\overline{u_{R}}, \overline{\overline{u_{R}}}\right), \alpha=4$, $\Delta=1 / 16$.

Figure 12.36.: IL, $\mathcal{P}_{r}\left(\overline{u_{R}}, \overline{\overline{u_{R}}}\right), \alpha=4$, $\Delta=1 / 32$. 


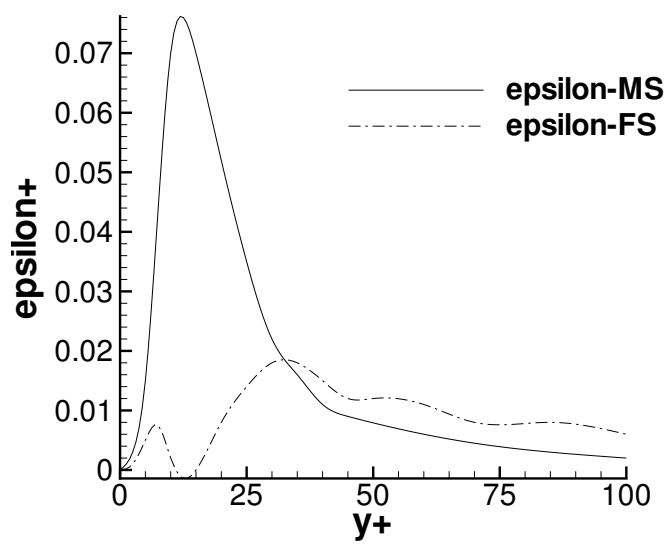

Figure 12.37.: A priori testing ([BF99] $)$.

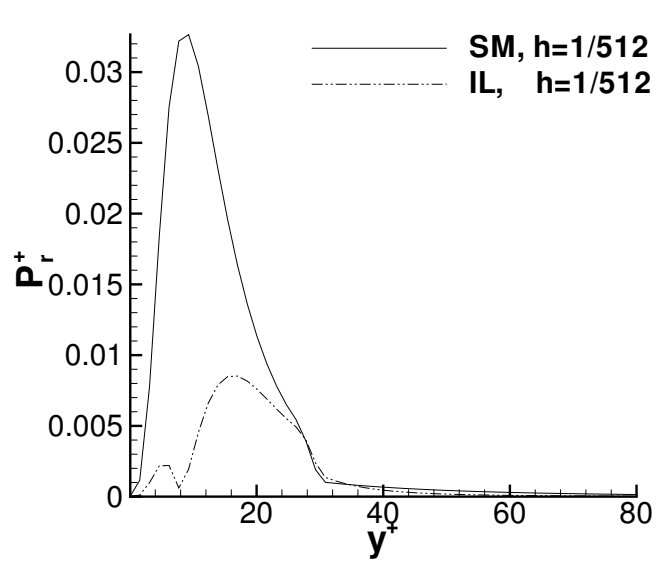

Figure 12.38.: Quasi a priori testing.

unless the grid spacing is sufficiently fine.

Moreover, the results from "quasi a priori testing" provide information regarding the choice of $C_{q}$ in the IL model. The goal is then to balance the relative magnitude of $\mathcal{P}_{r}$ for the SM model and the IL model compared to the profiles for $\epsilon^{M S}$ and $\epsilon^{F S}$ in Figure 12.16. As shown in Figure 12.38, $C_{q}=0.125$ seems a suitable choice for this test case. Moreover, this figure clearly demonstrates the close relation between $\epsilon^{M S}$ and $\epsilon^{F S}$ on the one hand and $\mathcal{P}_{r}$ for the SM model and the IL model on the other.

\section{Remark 12.1}

The so-called dynamic Smagorinsky model also uses an explicit filtering technique called the test-filter. The results of this section indicate that one has to be aware the numerical and modelling error caused by the differential filtering technique for the test-filter.

\section{A priori testing of the wall function concept for LES}

In Chapter 7 we proposed to match the LES solution with the RANS solution in the boundary layer. Now we want to elaborate on this approach. First it can be seen from Figures $12.17-12.18$ that even for the course filter width $\Delta=1 / 16, g_{\Delta} * u_{R} \approx u_{R}$ for $y^{+}>50$. Thus when matching is performed in the log-layer, our concept of matching the global LES with the non-space filtered RANS solution (5.41) is reasonable. For the fine filter width $\Delta=1 / 64$, the approximation is reasonable even for $y^{+}>30$.

The RANS solution is statistically stationary and hence $\tau_{w}$ is statistically stationary. However, LES requires an instantaneous wall shear stress. For $\boldsymbol{x} \in \Gamma_{W}$ the point $\boldsymbol{x}-y_{\delta} \boldsymbol{n}$ resides on $\Gamma_{\delta}$. Since the wall function approach requires $\overline{\boldsymbol{u}} \neq 0$ on $\Gamma_{\delta}$ (which is obviously fulfilled for attached boundary layers), the modeled wall shear stress can be written

$$
\tau_{w}(\boldsymbol{x}, t) \boldsymbol{t}_{j}=\tau_{w} \frac{\overline{\boldsymbol{u}}\left(\boldsymbol{x}-y_{\delta} \boldsymbol{n}, t\right) \cdot \boldsymbol{t}_{j}}{\left\|\left\langle\overline{\boldsymbol{u}}\left(\boldsymbol{x}-y_{\delta} \boldsymbol{n}, t\right)\right\rangle_{T}\right\|} \boldsymbol{t}_{j}, \quad j=1, \ldots, d-1 .
$$

which correlates $\tau_{w}(\boldsymbol{x}, t)$ and $\overline{\boldsymbol{u}}\left(\boldsymbol{x}-y_{\delta} \boldsymbol{n}, t\right)$, i.e., two quantities taken at two different locations in space but at the same point in time are correlated. $\langle\cdot\rangle_{T}$ denotes a long time 
average. Modelling the correlation between wall shear stress and instantaneous space averaged velocity field correctly is still an open problem. For a survey on recent models and a detailed experimental investigation thereon see e.g. [MKP01]. For general problems of merging LES for the outer flow with a RANS for the near-wall region in attached turbulent boundary-layer flows we refer to [Bag98]. Moreover, it is worth pointing out that $\tau_{w}$ and $C_{f}$ are the quantities of interest for engineers. They need $\tau_{w}$ and not a value obtained from filtered quantities like $\left.\overline{\tau_{w}} \equiv \nu \frac{d \bar{U}}{d y}\right|_{y=0}$. Thus the value obtained for $\tau_{w}$ should either come from a RANS (or from a DNS) in the wall adjacent region or from a LES with filter width $\Delta=\Delta(y) \rightarrow 0$ as $y \rightarrow 0$.

\section{Conclusions and implications for the use of LES in a CFD code}

A summary of the previous "quasi a priori testing" for LES closes this chapter. The major result is that a combination of the SM model and the IL model can describe $\mathcal{P}_{r}$, i.e., the transfer of kinetic energy from the filtered scales to the residual scales, in much better agreement with results from a priori testing than the SM model solely. This suggests that this combination is an interesting alternative residual stress model. Compared to the dynamic SM model, it can be expected to have better numerical stability properties. Moreover it can be implemented much easier into an existing CFD code than the dynamic SM model. As a second result we should point out that the homogeneous Neumann boundary condition in differential filtering gives rise to a significant modelling error near the wall for the IL model. The numerical tests suggest that this error can be "damped" with the van Driest damping function $\mathcal{D}\left(y^{+}\right)$. Multiplying $\nu_{t}$ for the IL model with $\mathcal{D}\left(y^{+}\right)^{4}$ instead of $\mathcal{D}\left(y^{+}\right)^{3}$ is appropriate for this test case. However, in an a posteriori test the situation might be different. In an a priori test we evaluate $\mathcal{P}_{r}\left(\mathcal{R}_{h} u_{R}, \mathcal{R}_{h} \mathcal{R}_{h} u_{R}\right)$ with $\mathcal{P}_{r}(v, u)=\nu_{t}(v, u)(d v / d y)^{2}$. Therefore the error due to the differential filtering appears both in $\nu_{t}$ and in $(d v / d y)^{2}$. In an a posteriori test we have to evaluate $\mathcal{P}_{r}\left(w, \mathcal{R}_{h} w\right)$ with $w$ being the solution of the LES momentum equation. Then the error of differential filtering appears only in $\nu_{t}$. (Of course, $w$ might be contaminated by other errors at the wall, but this is a different concern). Finally, balancing the contributions from the SM model and the IL model to $\mathcal{P}_{r}$ suggests $C_{q}=0.125$ for the IL model if $C_{S}=0.1$ is chosen for the SM model. 


\section{Turbulent natural convection in an air filled square cavity}

\subsection{Introduction}

In this chapter we consider natural convection in an air filled closed cavity at a low turbulence level. In this context, the term "low turbulence level" means that the flow does not exhibit any visible transition in the boundary layer along the heated and cooled vertical walls. This type of flow is of interest in several industrial applications like building ventilation, cooling of electrical equipment or films, and safety applications (e.g., heat transfer from fires).

There are two kinds of standard test cases for natural convection, namely, (i) RayleighBénard convection in a cavity which is heated from below and cooled on the top wall and (ii) the flow in a cavity with two differentially heated side walls. Case (i) has been studied, e.g., in [KD00], [WL.94], and [PJ.98].

At the first glance, case (ii) seems to be less challenging compared to case (i). This stems from the fact that the velocity field is relatively large only the proximity of the walls; remote from the walls, the fluid is nearly at rest. However, PENG and DAVIDSON point out that the opposite holds: Regarding the turbulent processes involved, the Rayleigh-Bénard problem seems to be less complicated. In turbulent Rayleigh-Bénard convection, there are two distinct scales of motion, viz., the large convective rolls and turbulence generated primarily in the wall boundary layers and advected by the large-scale motion. This makes the flow configuration amenable to LES studies. Contrarily, in a cavity with two differentially heated side walls, the flow pattern is much richer, see Figure 13.2 (from [TK0na]): In the core region encircled recirculating flows are induced. Further problems arise near the heated side walls: In the vicinity of the side walls temperature gradients normal to the direction of gravity tend to result in the generation of vorticity (according to BVG theory, see Subsection 3.3.2). This is supported by the LES with near-wall resolution performed by Peng And DAVIDSOn, cf. [PD01b]. They report the appearance of coherent structures (streaks), similar to those in a forced convection boundary layer but less elongated. The near-wall small-scale instabilities interact with themselves and with mean flow gradients. Thus a large span of scales occurs and interacts with each other, giving rise to complicated turbulent processes.

Such a cavity with two differentially heated side walls has been studied experimentally by CheEsewright ET AL. in 1986, for details see [CKZ86]. However, it became obvious that their results are not accurate enough, in particular regarding the turbulence quantities. Thus, a decade later, TIAM, KARAYIANNIS, and AMPOFO studied natural convection in a vertical square cavity at a relatively low Rayleigh number $R a=1.58 \times 10^{9}$. The experiment revealed that the flow is overall low-turbulent, and does not exhibit any visible transition in the boundary layer along the heated and cooled vertical walls. The flow configuration used by TIAM et al. has been accepted as new benchmark test case for CFD for natural convection, cf. [PD01b]. It should be appreciated that there are no DNS data for this flow, 
stressing the fact that this is a really challenging flow problem.

We conclude this introduction by pointing out some further difficulties stemming from the complicated physical processes involved. Firstly, during the solution process, the flow undergoes a transition from laminar to turbulent which is computationally difficult, as pointed out in [T(D)B98]. Regarding this issue, it is worth mentioning the corresponding isothermal problem, viz., the so-called impulsively started driven cavity problem: In a twodimensional square cavity with zero body force term and no-slip boundary conditions on bottom and side walls, the fluid motion is induced by sliding the upper side of the cavity to the left at constant velocity in a direction parallel to the bottom wall. For numerical results thereon, see e.g. [APQ02].

A further difficulty is that the eddy-viscosity assumption does not hold in the near-wall region, see [TN98b]. Therefore the prediction for the skin friction coefficient might be not very reliable. Moreover, TIEsZEN reports the presence of an adverse pressure gradient as the flow approaches the corners for the case of a tall cavity, see [T()DB98], p.290. If this observation also holds for the square cavity, then near the corners the boundary-layer approximation can make troubles, see subsection 5.2.4. The flow in the corners of the cavity can be considered as a flow over a surface with concave curvature in the limit of the radius of curvature going to zero. If a flow enters a concave curve, then turbulence is amplified, see Section 5.2. Models based on the eddy-viscosity hypothesis cannot describe this effect. Finally, the low fluid velocities and the strong coupling between momentum, temperature, and turbulence quantities can be expected to result in long iteration times until an at least statistically steady state is reached, see [T()DB98], p.290.

\subsection{Description of the flow configuration}

We focus on a vertical square cavity with two differentially heated side walls, studied experimentally by TIAM and KARAYIANNIS (see [TK00a]), and by AMPOFO and KARAYIANNIS (see [AK02]). Both experiments were carried out in the same cavity; AMPOFO reports that the results for velocity and temperature agree excellently for both experiments, cf. [AKV2], p.6. The flow configuration and a sketch of the velocity field (from [WK00a]) is presented in Figure 13.2. In the following, dimensional quantities are labelled by a tilde.

First we describe the domain $\Omega$ and the partitioning of its boundary $\Gamma$. The dimensions of the cavity are $0.75 m \times 0.75 m \times 1.5 m$, and thus $\Omega=(0,0.75) \times(0,0.75) \times(0,1.5)$.

\begin{tabular}{|l|l|l|}
\hline Part of $\Gamma$ & location & boundary condition \\
\hline$\Gamma_{h}, \Gamma_{c}$ & $\tilde{x}=0$ resp. $\tilde{x}=0.75 \mathrm{~m}$ & $\tilde{T}=50^{\circ} \mathrm{C}$ resp. $\tilde{T}=10^{\circ} \mathrm{C}$ isothermal \\
$\Gamma_{t}, \Gamma_{b}$ & $\tilde{y}=0$ resp. $\tilde{y}=0.75 \mathrm{~m}$ & highly conducting boundaries (see below) \\
$\Gamma_{f}, \Gamma_{r}$ & $\tilde{z}=0$ resp. $\tilde{z}=1.5 \mathrm{~m}$ & insulated \\
\hline
\end{tabular}

Figure 13.1.: Boundary conditions for natural convection in a closed cavity.

Secondly, we specify the boundary conditions, see Table 13.1. First, we regard the temperature equation. The vertical hot and cold wall of the cavity are isothermal at $\tilde{T}_{h} \equiv 50^{\circ} \mathrm{C}$ and $\tilde{T}_{c} \equiv 10^{\circ} \mathrm{C}$. The other boundary conditions require some explanation. Top and bottom 
walls (made from $1.5 \mathrm{~mm}$ mild steel) are highly conducting boundaries. Mathematically this is described by a boundary condition of Robin type, viz.,

$$
\tilde{\lambda} \nabla \tilde{T}=\tilde{\alpha}\left(\tilde{T}_{a}-\tilde{T}\right),
$$

with $\tilde{T}_{a}$ being the ambient temperature $\left(\tilde{T}_{a}=30^{\circ} \mathrm{C}\right)$ and appropriate values for $\tilde{\lambda}$ and $\tilde{\alpha}$. Instead, as suggested by TIAM, the measured temperature distribution on the horizontal walls was used as boundary condition in the numerical tests performed by PENG ET AL., cf. [PD00]. Finally, $\Gamma_{f}$ and $\Gamma_{r}$ are adiabatic.

In the numerical test presented in this thesis, the Dirichlet conditions on $\Gamma_{h} \Gamma_{c}, \Gamma_{t}$, and $\Gamma_{b}$ are replaced by using the wall iteration method for non-isothermal flow problems with significant buoyancy forces.

For the velocity, the no-slip condition is imposed on $\Gamma$, which again is reformulated as a wall stress boundary condition using the wall iteration concept for flows with significant buoyancy forces.

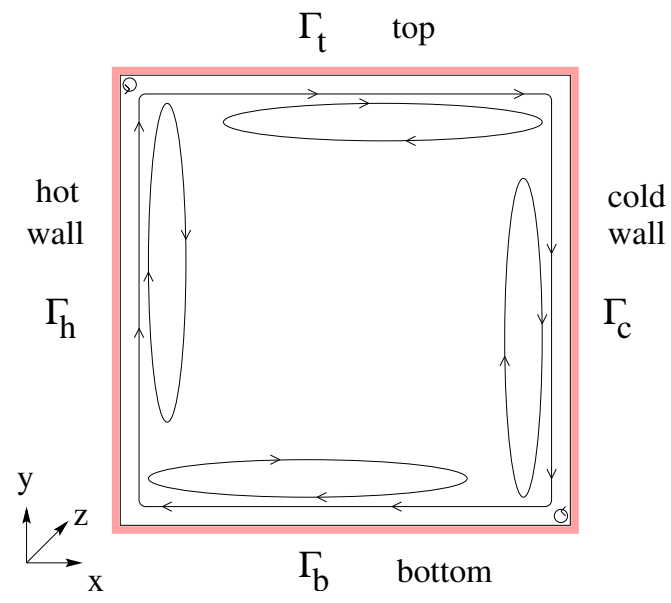

Figure 13.2.: Sketch of cavity and flow.

The Rayleigh number of the flow is given by $R a=\tilde{g} \tilde{\beta}\left(\tilde{T}_{h}-\tilde{T}_{c}\right) \tilde{L}^{3} \operatorname{Pr} / \tilde{\nu}$ with $\tilde{L}=0.75 \mathrm{~m}$. We obtain $R a=1.58 \times 10^{9}$ in agreement with TiAM, using

$$
\tilde{\nu}=15.3 \times 10^{-6}, \tilde{a}=21.86 \times 10^{-6}, \tilde{\lambda}=0.026, \operatorname{Pr}=0.70, \tilde{\beta}=3.192 \times 10^{-3} \text {. }
$$

Note that, strictly speaking, for ideal gases, $\tilde{\beta}$ is temperature dependent according to the relation $\tilde{\beta}=1 / \tilde{T}$ (with $\tilde{T}$ being given in $K$ ), cf. [KC.93], p.398. The constant value chosen for $\tilde{\beta}$ ensures the desired Rayleigh number.

\section{Remark 13.1}

There are several experimental studies with adiabatic horizontal walls. However, as reported by TIAM, their results regarding turbulence quantities differ significantly, because adiabatic boundary conditions on the horizontal walls are very difficult to realise in air filled 
cavities. The large aspect ratio in the $\tilde{z}$ direction ensures that the flow can be assumed to be two-dimensional, except near the side walls $\Gamma_{f}$ and $\Gamma_{r}$. This is confirmed by the experimental investigation by TIAM.

\section{Remark 13.2}

MüLLER [Mue99] considered two-dimensional natural convection at $R a=5.3 \times 10^{10}$ in an air filled vertical tall cavity of dimensions $0.5 m \times 2.5 m$, top and bottom wall being adiabatic. He compared the numerical results from the $k / \epsilon$ model with experimental results by Cheesewright ET AL., see [CKZ86]. GritzKi [Gri01] studied natural convection in a three-dimensional unit cube with top and bottom wall again being adiabatic, without comparing the results with experimental data.

\subsection{Testing the wall iteration concept}

First we study the wall iteration method separately. Given the flow configuration presented above, we consider the flow along the hot wall at $\tilde{y} / \tilde{L}=0.5$. Recall that in this situation the wall-normal direction is the $x$-direction in the global coordinate system. In the local coordinate system, the wall-normal direction is the $y$-direction, as usual. In a similar vein, we denote the streamwise component of the velocity field at the vertical walls with $V$, as it is the $y$-component of $\boldsymbol{u}$ in the global coordinate system. Keeping this in mind should avoid all confusions.

Recall that in the wall iteration procedure, for each grid node on $\Gamma_{W}$, we have to solve (6.7)-(6.10) numerically for given values for velocity $V\left(y_{\delta}\right)$ and temperature $\Theta\left(y_{\delta}\right)$ at a given wall distance $y_{\delta}$. Recall that $y_{\delta}$ is the wall distance of the first mesh node above the wall. Note that $V\left(y_{\delta}\right)$ is denoted by $U\left(y_{\delta}\right)$ in $(6.7)-(6.10)$. The objective of the wall iteration scheme (see Subsection 6.3) is to ensure reasonable predictions for certain surface transfer coefficients even if the first node above the wall is located beyond the velocity maximum, see Subsection 5.4.1. The distance of the velocity maximum from the wall, denoted by $y_{\max }$, is increasing in flow direction. In order to obtain a robust method we have to ensure that the numerical solution of (6.7)-(6.10) yields accurate results if $y_{\delta}$ is varied over an interval around $y_{\max }$.

Thus the aim of this section is to study the behaviour of the numerical solution of (6.7)(6.10) when varying $y_{\delta}$ over an interval around $y_{\max }$. Therefore we consider Table 13.3, whose entries are explained in the sequel. The data for the numerical tests can be found in the first three columns of the following table. The last two columns will be discussed later. The first line containing data, e.g., has to be understood as follows: We choose $y_{\delta}=1.0 \times 10^{-3}, V\left(y_{\delta}\right)=8.43 \times 10^{-2}$, and $\Theta\left(y_{\delta}\right)=0.92$ and then solve (6.7)-(6.10). These values are taken from the experimental data by AMPOFO ET AL., see [AK02].

Thus we solve (6.7)-(6.10) for eight different locations of $y_{\delta}$. The initial value problem (6.12)-(6.15) is solved numerically using the implicit Euler method, see e.g. [Kre.98], Section 10.2. On $\left[0, y_{\delta}\right]$ we use an equidistant grid with 20 nodes. Numerical tests reveal that the results cannot be improved when using more nodes. Note that for $y_{\delta} \leq 0.004$ the wall function concept is used, because the first near-wall point resides in the viscous sublayer. 
13.3. Testing the wall iteration concept

\begin{tabular}{|l|l|l|l|l|}
\hline$y_{\delta}=\tilde{y}_{\delta} / \tilde{L}$ & $\tilde{V}\left(\tilde{y}_{\delta}\right) / \tilde{U}_{0}$ & $\theta=\left(\tilde{T}-\tilde{T}_{c}\right) /\left(\tilde{T}_{h}-\tilde{T}_{c}\right)$ & $\tilde{\tau}_{w} / \tilde{U}_{0}^{2}$ & $N u_{l}$ \\
\hline $1.000 \times 10^{-3}$ & $8.430 \times 10^{-2}$ & $9.200 \times 10^{-1}$ & $2.043027 \times 10^{-3}$ & $6.000 \times 10^{1}$ \\
$2.330 \times 10^{-3}$ & $1.457 \times 10^{-1}$ & $8.373 \times 10^{-1}$ & $1.515480 \times 10^{-3}$ & $5.237 \times 10^{1}$ \\
$4.000 \times 10^{-3}$ & $1.308 \times 10^{-1}$ & $7.635 \times 10^{-1}$ & $1.516995 \times 10^{-3}$ & $4.839 \times 10^{1}$ \\
$6.666 \times 10^{-3}$ & $2.127 \times 10^{-1}$ & $6.793 \times 10^{-1}$ & $1.459557 \times 10^{-3}$ & $5.020 \times 10^{1}$ \\
$9.333 \times 10^{-3}$ & $2.081 \times 10^{-1}$ & $6.186 \times 10^{-1}$ & $1.531031 \times 10^{-3}$ & $5.334 \times 10^{1}$ \\
$1.333 \times 10^{-2}$ & $1.745 \times 10^{-1}$ & $5.655 \times 10^{-1}$ & $1.645618 \times 10^{-3}$ & $5.518 \times 10^{1}$ \\
$2.000 \times 10^{-2}$ & $1.308 \times 10^{-1}$ & $5.270 \times 10^{-1}$ & $2.030134 \times 10^{-3}$ & $5.815 \times 10^{1}$ \\
$2.666 \times 10^{-2}$ & $9.180 \times 10^{-2}$ & $5.171 \times 10^{-1}$ & $2.396009 \times 10^{-3}$ & $6.023 \times 10^{1}$ \\
\hline
\end{tabular}

Figure 13.3.: Data and results for an a priori test of the wall iteration scheme.

We study (i) the predictions for $V$ and $\Theta$ and (ii) the results concerning the wall function method. First we are interested in the predictions for $V$ and $\Theta$, which are shown in Figures 13.4-13.7. The figures show the numerical solution of (6.7)-(6.10) for different locations of $y_{\delta}$, which is denoted with $d$ in the legend. The left figures show all choices for $y_{\delta}$ whereas the right plots focus on the near-wall region. Given $y_{\delta} \leq 0.0133$, the profile for $V$ is good. For $y_{\delta} \in\{0.02,0.0266\}$ the velocity maximum is clearly overpredicted. Concerning the temperature, the solutions for different choices for $y_{\delta}$ are relatively close to each other. Secondly, we study the predictions for $U_{*}$ and $\dot{q}$, see (6.11). Their accuracy is of crucial importance within the wall function approach, see the $k / \epsilon$ MODEL II. Here, we consider $\tau_{w} \equiv \tilde{\tau}_{w} / \tilde{U}_{0}^{2}$ with $\tau_{w}=U_{*}^{2}$ instead of $U_{*}$. Moreover, instead of $\dot{q}$ we study the so-called local Nusselt number $N u_{l}$, being defined as

$$
N u_{l}=-\frac{\tilde{L}}{\left(\tilde{T}_{h}-\tilde{T}_{c}\right)} \frac{\tilde{\dot{q}}}{\tilde{\rho}_{0} \tilde{c}_{p}},
$$

with dimensional quantities being defined in Section 1.1. From their experiments Tiam ET AL. report $\tilde{\tau}_{w} / \tilde{U}_{0}^{2}=0.00164$ and $N u_{l}=58$ at $\tilde{y} / \tilde{L}=0.5$. The numerical results for these quantities are given in the last two columns of Table 13.3. Thus a certain line in the table has to be read as follows: Given $y_{\delta}, V\left(y_{\delta}\right)$ and $\Theta\left(y_{\delta}\right)$ from the first three columns, the numerical solution procedure gives the values for $\tau_{w}$ and $N u_{l}$ specified in the fourth and fifth column resp. The predictions for $\tau_{w}$ are reasonable for $y_{\delta} \in(0.00233,0.0133)$. As $y_{\delta}$ increases, i.e., for $y_{\delta} \in\{0.02,0.0266\}$, the error becomes significantly larger. Concerning $N u_{l}$, the results become better if $y_{\delta}$ is not too close to the wall. Even for $y_{\delta} \in\{0.02,0.0266\}$ the predictions for $N u_{l}$ are good. In the a posteriori testing in the next section we use the a priori information regarding the location of $y_{\max }$ to create a mesh with $y_{\delta} \in\{0.00933,0.0133\}$. Then the a priori testing results of this section suggest that the predictions for both $\tau_{w}$ and $N u_{l}$ are reasonable, at least at the side walls.

Finally we study the convergence behaviour of the Newton's method. The convergence history is given in Figure 13.8. The abscissa gives the iteration step whereas the ordinate shows the following quantity being a measure for the convergence behaviour, viz.,

$$
\sqrt{\frac{\left(\tau_{w}^{n+1}-\tau_{w}^{n}\right)^{2}}{\left(\tau_{w}^{n}\right)^{2}}+\frac{\left(\dot{q}^{n+1}-\dot{q}^{n}\right)^{2}}{\left(\dot{q}^{n}\right)^{2}}} .
$$




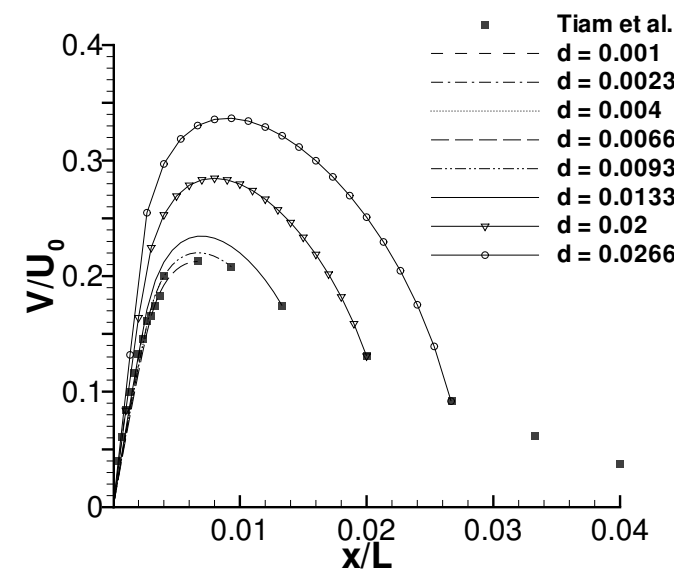

Figure 13.4.: $V / U_{0}$ at $y / L=0.5$

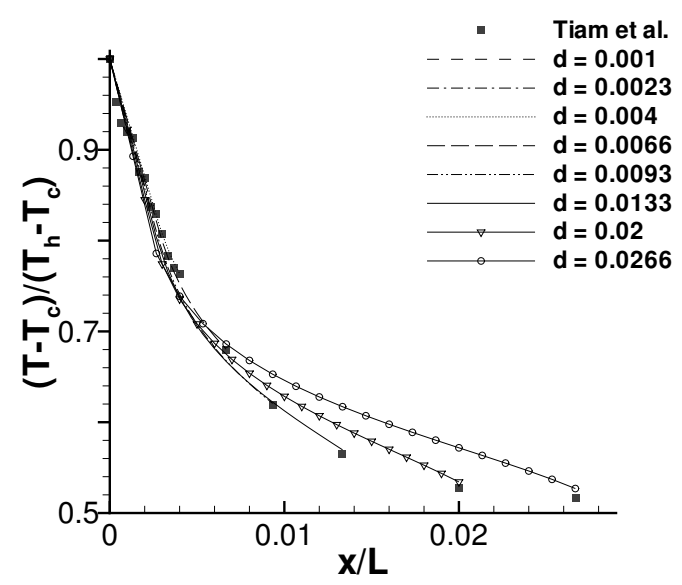

Figure 13.6.: $\frac{T-T_{c}}{T_{h}-T_{c}}$ at $y / L=0.5$

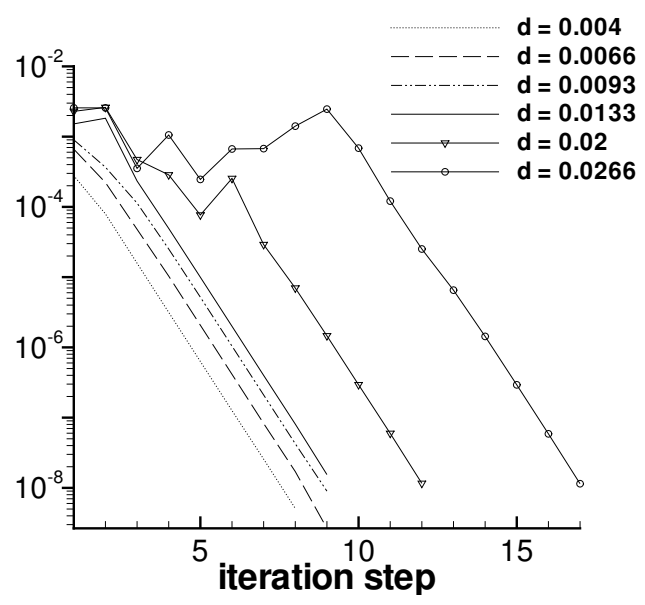

Figure 13.8.: Convergence history

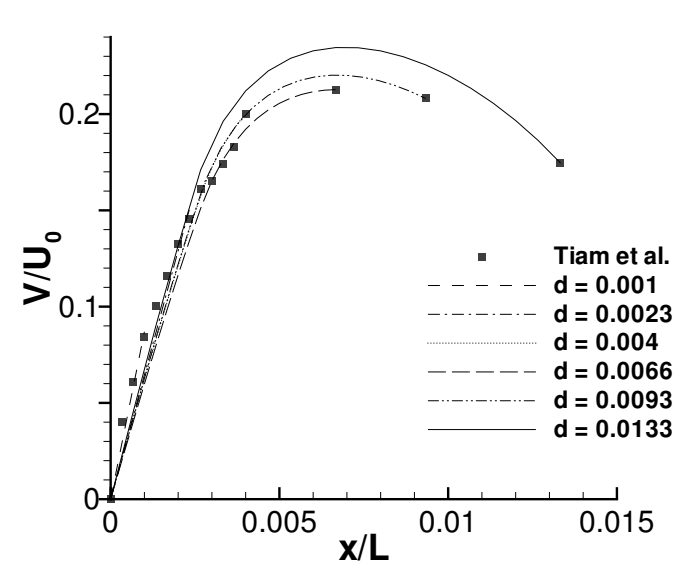

Figure 13.5.: $V / U_{0}$ at $y / L=0.5$

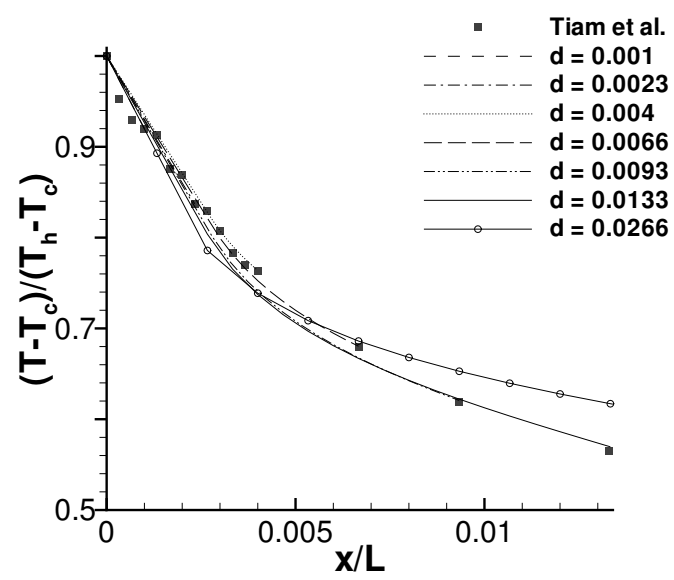

Figure 13.7.: $\frac{T-T_{c}}{T_{h}-T_{c}}$ at $y / L=0.5$

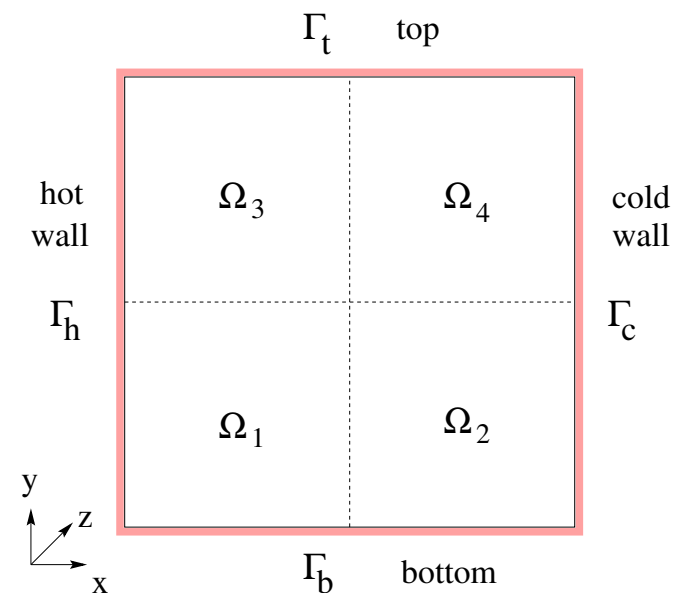

Figure 13.9.: Sketch of the DDM 
Here the upper index $n$ denotes the iteration step. The initial guess for the Newton's method becomes less accurate as the wall distance of the first wall-off node becomes larger. This is the reason why the the iteration scheme takes more cycles when the wall distance of the first wall-off node becomes larger.

\subsection{A posteriori testing for the $k / \epsilon$ model without DDM}

Numerical tests show that the results for the $k / \epsilon$ model do not change if we use the computational domain $\Omega=(0,0.75)^{3}$ instead of $\Omega=(0,0.75) \times(0,0.75) \times(0,1.5)$. The computations are performed on a mesh with $81 \times 65 \times 29$ nodes being distributed equidistantly in each coordinate direction. Hence the discrete problem has approximately $7 \times 1.5 \times 10^{5}$ unknowns. We use a relatively large time step width $\Delta t=1.0$. Note that $\Delta t=1.0$ is approximately the characteristic time scale $\tilde{t}_{0}$ formed from the characteristic length scale $\tilde{H}=0.75 m$ and the characteristic velocity $\tilde{U}_{s c}=\left(\tilde{g} \tilde{\beta}\left(\tilde{T}_{h}-\tilde{T}_{c}\right) \tilde{H}\right)^{1 / 2} \approx 0.9692 \mathrm{~ms}^{-1}$, viz., $\tilde{t}_{0}=\tilde{H}\left(\tilde{g} \tilde{\beta}\left(\tilde{T}_{h}-\tilde{T}_{c}\right) \tilde{H}\right)^{-1 / 2} \approx 0.7738 s$. For their LES, PEng AND Davidson chose $\Delta \tilde{t}=0.013 \tilde{t}_{0} \approx 0.01$, see [PD01b] . Regarding computations with the $k / \epsilon$ model, numerical tests reveal that the results cannot be improved by using a time step width smaller than $\Delta t=1.0$. We use the decoupling and linearisation scheme for the $k / \epsilon$ model described in Section 9.2.2. For the final time we select $T=200.0$ and perform one linearisation cycle per time step. For the temperature equation the shock-capturing scheme, see Section 10.3, is applied.

First we study our scheme without applying the domain decomposition method. We investigate the following two variants. In both cases we select $K_{s}=25.0, R e_{\min }=20.0$.

$\mathrm{V} 1$ We use $\chi=0$ in (5.40), i.e., effects of stratification are neglected in the boundary layer.

V2 We choose $\chi=1$ in (5.40). Then effects of stratification in the boundary layer are taken into account. Moreover we ensure $R e_{\min } \in\left[R e_{\text {min,cut }}, 70.0\right]$ with $R e_{\text {min,cut }}=$ 7.0 using a cut-off technique.

First we focus on variant V1. In order to appraise the accuracy of the solution we start with considering the predictions for the non-dimensional vertical velocity $V / U_{0}$ and for the non-dimensional temperature $\left(T-T_{c}\right) /\left(T_{h}-T_{c}\right)$ at three different positions $y / L=0.2$, $y / L=0.5$, and $y / L=0.8$. As a reference solution we choose the experimental results by AmPOFO and KaraYiannis, see [AKO2]. According to AmPOFO, there is an excellent agreement with the earlier results of TIAM and KARAYIANNIS, see [TKO0)]. In order to appreciate the efforts of the whole research group, the reference data are labelled by "Tiam et al.". Nonetheless it should be pointed out that the reference data have been provided due to the kind support of Felix AMPOFO.

The results are shown in Figures 13.10-13.15. First we investigate the predictions for $V$. At $y / L=0.5$ the prediction is reasonable although the thickness of the velocity boundary layer is slightly overpredicted. The situation at $y / L=0.2$ and $y / L=0.8$ requires some more explanation. It is worth recalling that the fluid motion is in clockwise direction. Thus regarding the hot wall $(x=0)$ the near-wall fluid motion is directed upwards whereas on the cold wall $(x / L=1.0)$ it points downwards. We focus on the cold wall. The discussion for 
the hot wall is analogous. However, as will be discussed later, the predictions for the cold wall are better than those for the hot wall. The reason is that the prediction of the solution in the region near the bottom wall is complicated. Erroneous results near the bottom wall are transported downstream with the fluid motion and deteriorate the results near the hot wall. For $y / L=0.2$ the predictions on the cold wall are good but for $y / L=0.8$ they are more erroneous. The reason is that $y / L=0.8$ is near the upper right corner. As explained before, both the boundary-layer approximation and the eddy-viscosity model fail in the vicinity of corners. The error made in the corner highly affects the solution downstream at $y / L=0.8$. Therefore the recirculating flow in $0.75 \leq x / L \leq 0.9$ at $y / L=0.8$ cannot be predicted correctly. Also details regarding the near-wall temperature profile for $x / L \geq 0.9$ at $y / L=0.8$ cannot be described correctly. One particular aspect of the model's failure in the corners are the two small vortices in the top-hot and in the bottom-cold corner of the cavity, being reported by TIAM, p.855, which cannot be resolved in the present $k / \epsilon$ computation. Downstream at $y / L=0.2$ the situation is much better. The thickness of the velocity boundary layer is predicted very well, but the maximum velocity is significantly underpredicted. The recirculating flow at $0.85 \leq x / L \leq 0.9$ can be resolved. Similarly, details of the near-wall temperature profile are described quite reasonably. It is worthwhile pointing out that the predictions for the mean temperature profiles in the cavity mid are very reasonable.

Next we study the vertical temperature profile, as shown in Figure 13.18. Discernibly the prediction is very satisfying, except the slight underprediction of the temperature maximum resp. minimum near the top and bottom wall resp. It is worthily commenting on the prediction of the vertical temperature profile in [Mue99], p.107. The results in [Mue99] are very similar to those presented in [T(D)B98], Figure 8. This suggests that the reference data by [CK Z86] suffer from experimental deficiencies, i.e., heat loss from the box. Hence the prediction in [Mwe99] can be expected to be much better than the reference data suggest. Next we investigate the predictions for the characteristic surface transfer coefficients, i.e., skin friction coefficient $C_{f}$ and local Nusselt number $N u_{l}$, being defined as

$$
C_{f}=\frac{2 \tilde{\tau_{w}}}{\tilde{U}_{0}^{2}}, \quad N u_{l}=-\frac{\tilde{L}}{\left(\tilde{T}_{h}-\tilde{T}_{c}\right)} \frac{\tilde{\dot{q}}_{w}}{\tilde{\rho}_{0} \tilde{c}_{p}} .
$$

As experimental data for $C_{f}$ are available only for the side walls, the results for top and bottom wall are not shown in the plot. Moreover, in the figures, we have to modify the definition for $C_{f}$ and $N u_{l}$ in (13.5) on the top wall and on the cold wall by using a negative sign there. This is done in order to use the same definition as given in [TK00a]. Note that $\tilde{\tau}_{w}$ and $\tilde{\dot{q}}_{w}$ are provided by the near-wall iteration and do not have to be computed explicitly. The predictions for $C_{f}$ and $N u_{l}$ along the cavity closure surface (clockwise), starting from the lower hot corner, are presented in Figures 13.16-13.17. Therein, the parameter $s$ is a surface parameter, which describes the distance of a certain point at the surface from the lower hot corner in clockwise direction. First we study the results for $C_{f}$. At a certain distance downstream from the lower left corner at $s / H=0.0$ and the upper right corner at $s / H=2.0$ the predictions are reasonable. In particular, the predictions at the cold wall for $2.6 \leq s / H \leq 2.9$ are very good. However near the lower left corner at $s / H=0.0$ and the upper right corner at $s / H=2.0$ discernibly $C_{f}$ is mispredicted: The relatively slow growth 
of $C_{f}$ in downstream direction cannot be described correctly. However, one should recall that according to TsuJI AND NAGANO the eddy-viscosity assumption does not hold in the near-wall region, see [WN98b]. This is an additional indication that we cannot expect the prediction for $C_{f}$ to be accurate due to several modelling errors as explained above.

Now we study the predictions for the local Nusselt number $N u_{l}$. The predictions for the side walls are qualitatively reasonable (except near the lower left corner at $s / H=0.0$ and the upper right corner at $s / H=2.0)$. However, $N u_{l}$ is significantly overpredicted at the side walls. At the top and bottom wall the predictions are poor. At the top wall $(1.0 \leq s / H \leq 2.0) N u_{l}$ is significantly underpredicted and the qualitative behaviour is mispredicted. At the bottom wall $(3.0 \leq s / H \leq 4.0)$ the prediction becomes unreliable, as $N u_{l}$ suffers from oscillations. Therefore the predictions near the bottom wall are erroneous and this error influences the predictions for the hot wall, see above. Regarding the problems near the top and bottom wall we have to recall that we impose a Dirichlet boundary condition instead of a Robin boundary condition there. The problem with Robin boundary conditions is that KARAYIANNIS ET AL. do not specify how to choose $\tilde{\lambda}$ and $\tilde{\alpha}$ in (13.1). A further problem regarding the Robin boundary condition is that then $C_{f}$ and $N u_{l}$ cannot be obtained directly. However, as we shall see in the next section, the reason for the misprediction of $N u_{l}$ is that effects of stratification have to be accounted in the boundary layer.

Finally we look at the turbulent kinetic energy. The predictions are shown in Figures 13.1913.21. For $y / L=0.5$ the prediction is reasonable, although the maximum for $k$ at the cold wall $(x / L=1.0)$ is spuriously overpredicted. Moreover it should be pointed out that the profile for $k$ is wider than the data predict. This is in agreement with the above notion that the thickness of the velocity boundary layer is overpredicted at $y / L=0.5$. Near the top-cold and the bottom-hot corner the prediction is poor. At $y / L=0.2$ the maximum for $k$ at $x=0$ is significantly overpredicted by a factor larger than two. Similarly, at $y / L=0.8$ the prediction for $k$ at the cold wall $(x / L=1.0)$ is much too large. On the other hand, for $y / L=0.2$ the prediction for $k$ near the cold wall $(x / L=1.0)$ is good. Similarly, for $y / L=0.8$ the prediction for $k$ near the hot wall $(x / L=0.0)$ is reasonable. However, the thickness of the profile is slightly overpredicted in both cases.

Next we study the results for variant V2. First we study the results for the vertical velocity component $V$. The difference between the predictions for V2 and V1 is small. At the side walls the velocity boundary layer is slightly wider for V2 than for V1. Regarding the temperature, the difference is significant. It can be seen clearly from Figure 13.30 that the prediction for the vertical velocity profile is not as good as for V1, see Figure 13.18. In particular the predictions near the top and bottom wall are poor .

On the other hand, the results for the surface transfer coefficients $C_{f}$ and $N u_{l}$ for V2 are superior to those for V1. The prediction for $N u_{l}$ is given in Figure 13.29. For the side walls $(0 \leq s / H \leq 1$ and $2 \leq s / H \leq 3)$ the results are similar to V1. On the top wall $(1 \leq s / H \leq 2)$ the prediction is qualitatively and quantitatively reasonable and much better than for V2. Since $\boldsymbol{g} \cdot \boldsymbol{n} \neq 0$ and $\nabla \Theta \cdot \boldsymbol{n} \neq 0$ in (5.40), $R e_{\min }$ is altered in order to take into account effects of stratification. Figure 13.29 reveals that the modification 
(5.40) is a valuable improvement. On the bottom wall, the situation is more complicated. Discernibly the solution exhibits small oscillations. From a qualitative point of view, the prediction is much better than for model V1. However, the result is not satisfying. In order to study the predictions for the bottom wall in more detail, different values for $R e_{\min }, K_{s}$ and $R e_{\text {min,cut }}$ have been tested. The oscillations for $N u_{l}$ are removed only if a larger value for $R e_{\min }$ is chosen. However, then the profile for $N u_{l}$ flatter than for V1. A qualitatively satisfying prediction for $N u_{l}$ is obtained for $R e_{\text {min,cut }}=2.0$ (not shown here) at the cost that the oscillations increase. Surprisingly, when applying a DDM, these oscillations vanish. Thus we postpone the investigation of the predictions for $N u_{l}$ at the bottom wall to the next section. The result for $C_{f}$ is shown in Figure 13.28. In particular, the prediction at the cold wall for $2.4 \leq s / H \leq 2.9$ is remarkably accurate. This is at least in parts due to the reasonable prediction for $N u_{l}$ on the top wall. Finally we study the predictions for $k$, see Figure 13.31-13.33]. The results are similar to those predicted for V1. However, it is noteworthy that the profile for $\mathrm{V} 2$ is wider than for V1.

\subsection{A posteriori testing for the $k / \epsilon$ model with DDM}

In this section we apply the domain decomposition method presented in Chapter 11 to our natural convection problem. For this purpose we divide the domain $\Omega$ into four subdomains as shown in Figure 13.3. Concerning the interface function, instead of (11.9) we choose the simplified variant

$$
z_{k}=\frac{\left|\boldsymbol{b} \cdot \boldsymbol{n}_{k}\right|}{2}+\nu+\sqrt{\nu(c+\lambda)} .
$$

It should be recalled that here $\nu$ denotes a non-constant diffusivity resp. viscosity, see Section 9.2.3. This simplification is based on numerical tests for several advection-diffusionreaction type problems with $\nu$ being constant in $\Omega$. In these tests, the convergence behaviour of the DDM was investigated for $\nu \in\left[10^{-7}, 10^{0}\right]$ for both variants (11.9) and (13.6). These tests reveal that the $\min (. .$.$) term in (11.9) is of crucial importance in order$ to ensure a robust convergence w.r.t. $\nu$ primarily in the case $c=0$. However, for instationary problems $c=1 /(\Delta t)$ with $\Delta t$ being the time step width. For our natural convection problem using the $k / \epsilon$ model we have $\|\nu\|_{m i n, j} \approx 10^{-5}$. Therefore (11.9) becomes

$$
z_{k}=\frac{\left|\boldsymbol{b} \cdot \boldsymbol{n}_{k}\right|}{2}+\max _{j=1,2} \frac{\|\nu\|_{\text {min }, j}}{H_{j}}\left(1+\sqrt{\frac{C_{\infty, j}}{\|\nu\|_{\text {min }, j}}} H_{j}+2 \frac{B_{\infty, j}}{\sqrt{\|c \nu\|_{\text {min }, j}}}\right) .
$$

Comparing (13.7) and (13.6) reveals that $\lambda \approx B_{\infty, j}$. Moveover these tests show that for fixed $\nu$ with $\nu \geq 10^{-2}$ the convergence behaviour is relatively robust w.r.t. $\lambda$, see also [Otto99], Section 4.7.

The aim of a DDM is to improve the performance of the finite-element scheme without introducing an additional error due to the DDM. Thus we have to check whether the DDM solution converges to the solution without DDM. Moreover we have to study the influence of the parameters $\lambda$ and $\theta$. Recall that the relaxation parameter $\theta$ was introduced in (11.4) and in (11.16) resp. It will turn out that the DDM predictions for $N u_{l}$ at the bottom wall do not exhibit oscillations in contrast to the solution without DDM. This provides us the 

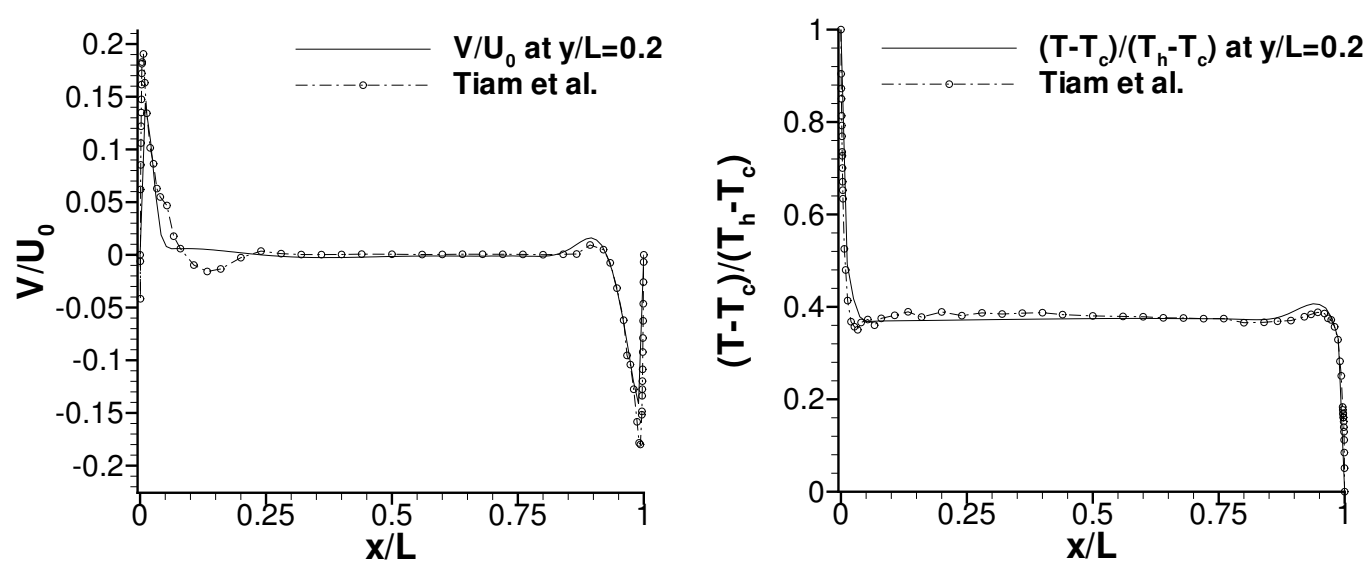

Figure 13.10.: $\frac{V}{U_{0}}$ at $\frac{y}{L}=0.2, \mathrm{~V} 1$.
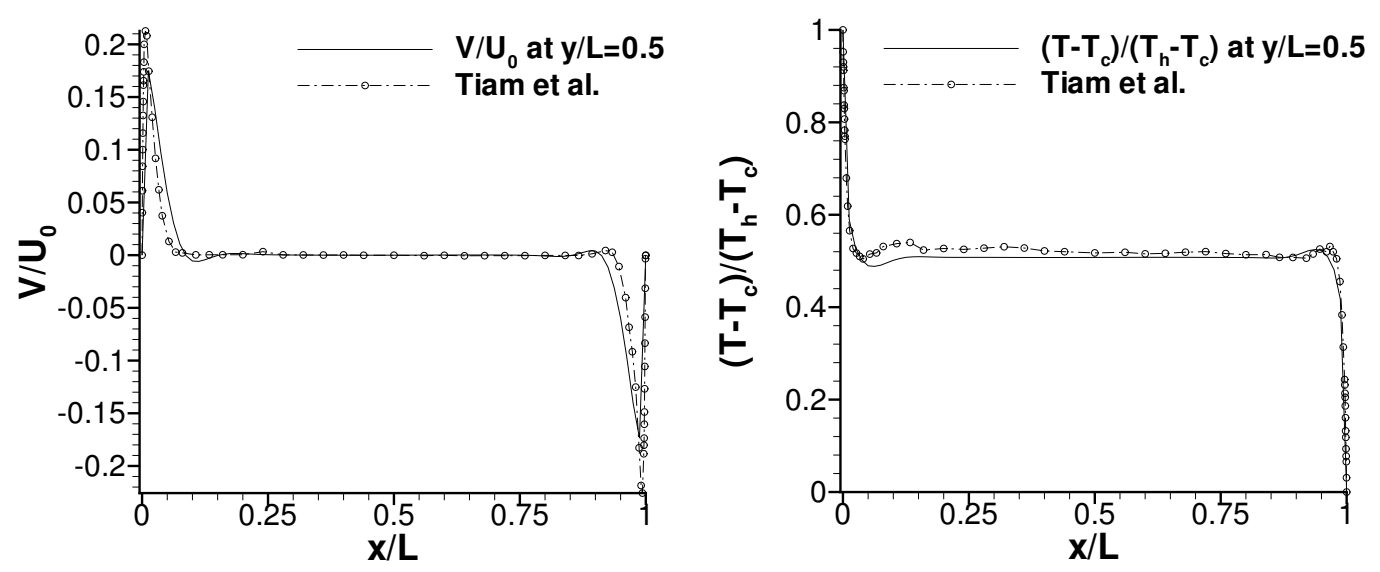

Figure 13.12.: $\frac{V}{U_{0}}$ at $\frac{y}{L}=0.5, \mathrm{~V} 1$.
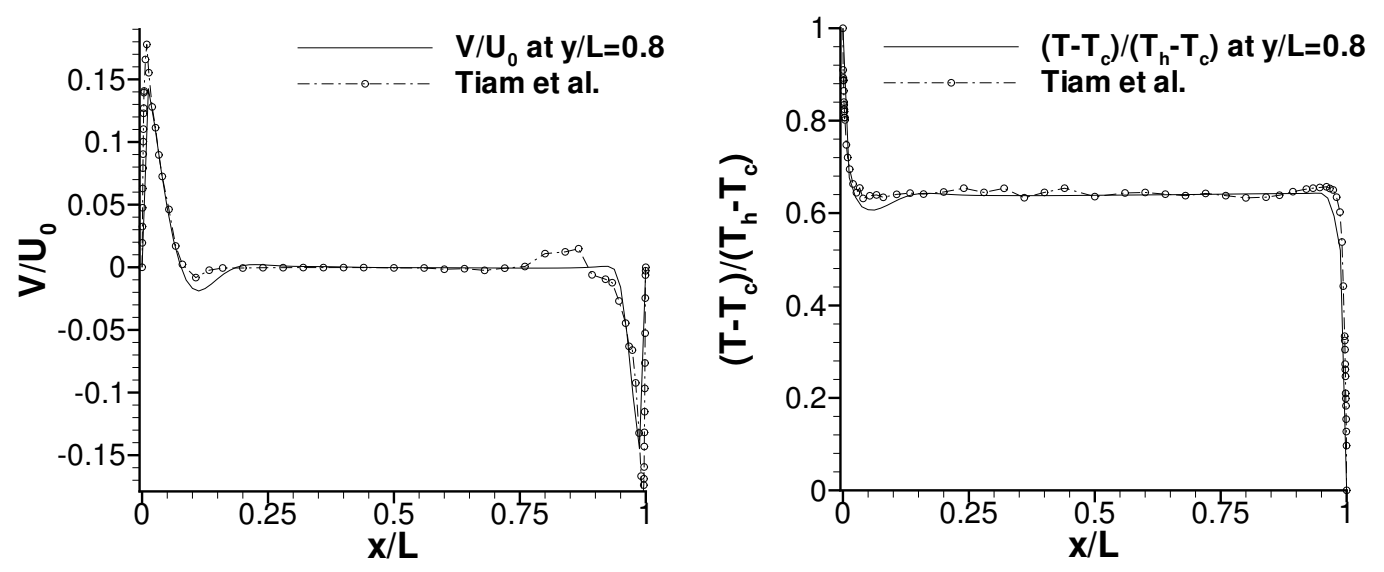

Figure 13.14.: $\frac{V}{U_{0}}$ at $\frac{y}{L}=0.8, \mathrm{~V} 1$.

Figure 13.15.: $\frac{T-T_{c}}{T_{h}-T_{c}}$ at $\frac{y}{L}=0.8, \mathrm{~V} 1$. 


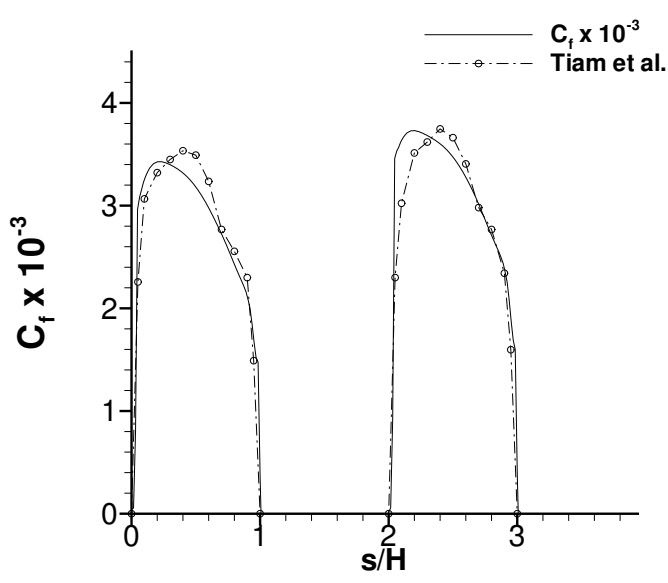

Figure 13.16.: $C_{f}$ from lower top corner along surface clockwise, V1.

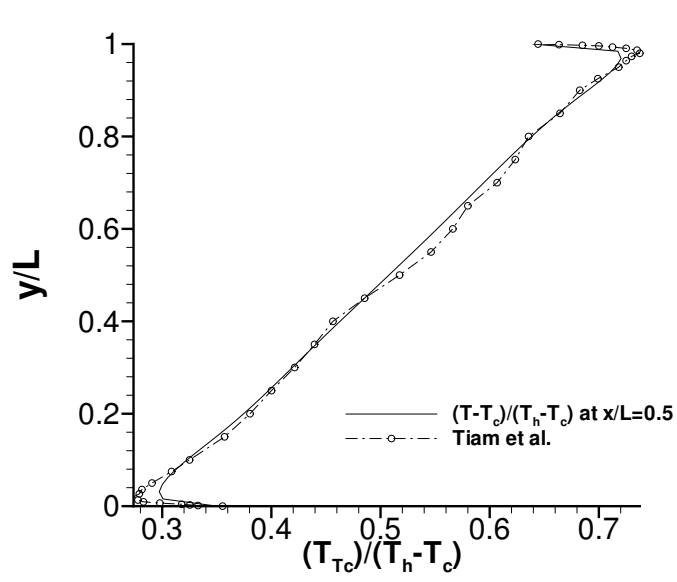

Figure 13.18.: $\frac{T-T_{c}}{T_{h}-T_{c}}$ at $x / L=0.5, \mathrm{~V} 1$.

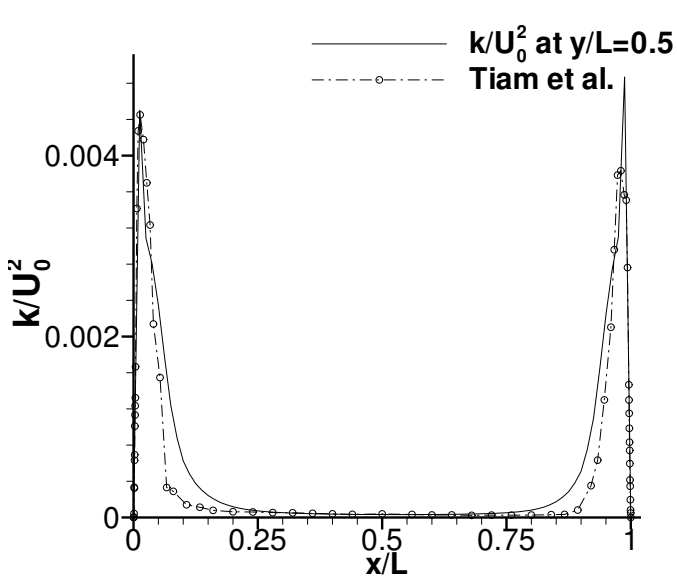

Figure 13.20.: $\frac{k}{U_{0}^{2}}$ at $\frac{y}{L}=0.5, \mathrm{~V} 1$.

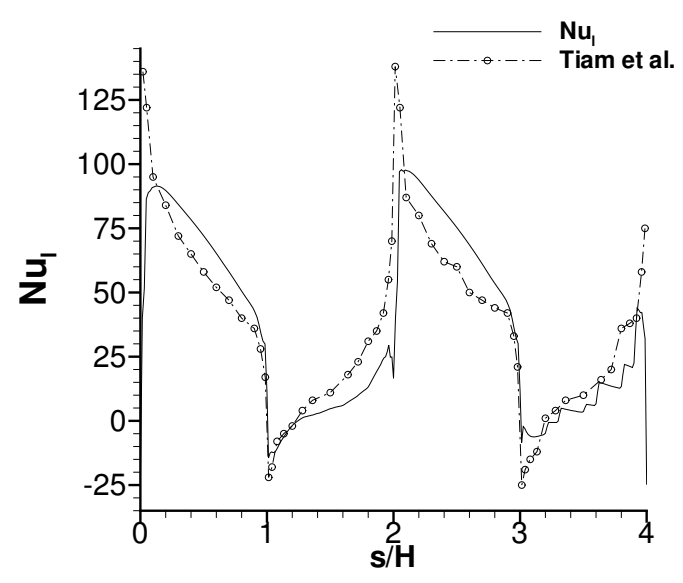

Figure 13.17.: $N u_{l}$ from lower top corner along surface clockwise, V1.

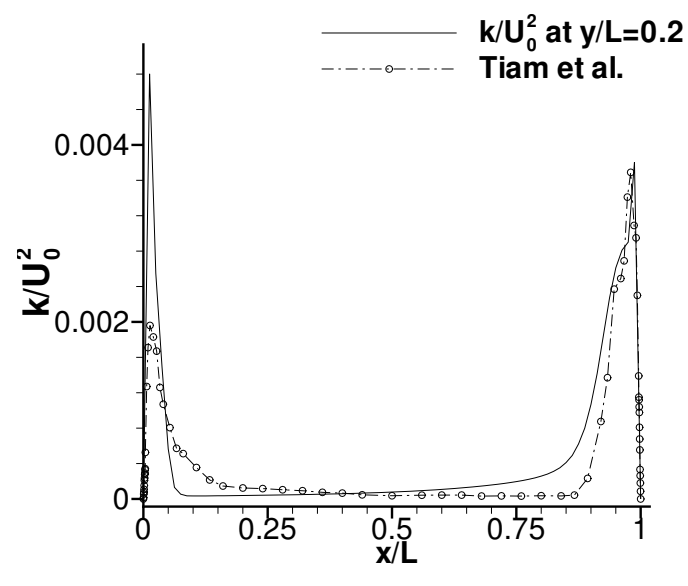

Figure 13.19.: $\frac{k}{U_{0}^{2}}$ at $\frac{y}{L}=0.2, \mathrm{~V} 1$.

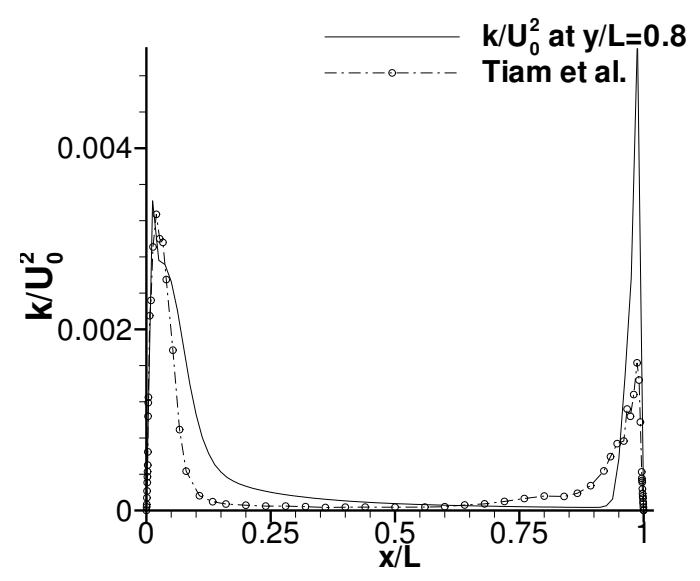

Figure 13.21.: $\frac{k}{U_{0}^{2}}$ at $\frac{y}{L}=0.8, \mathrm{~V} 1$. 

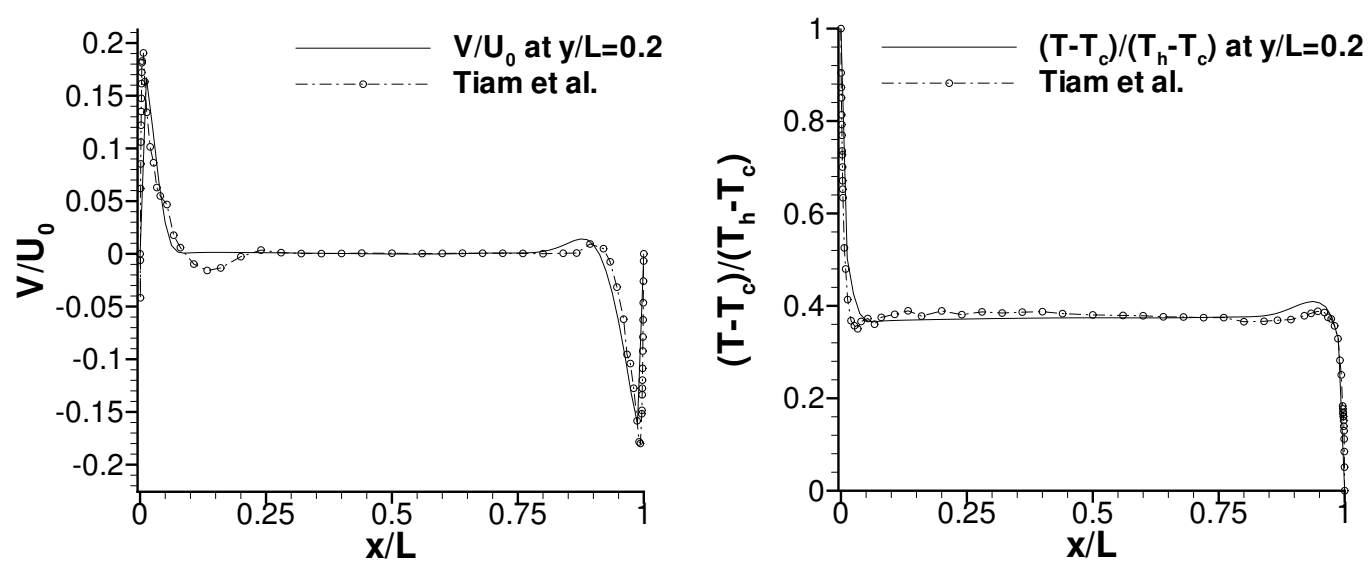

Figure 13.22.: $\frac{V}{U_{0}}$ at $\frac{y}{L}=0.2, \mathrm{~V} 2$.

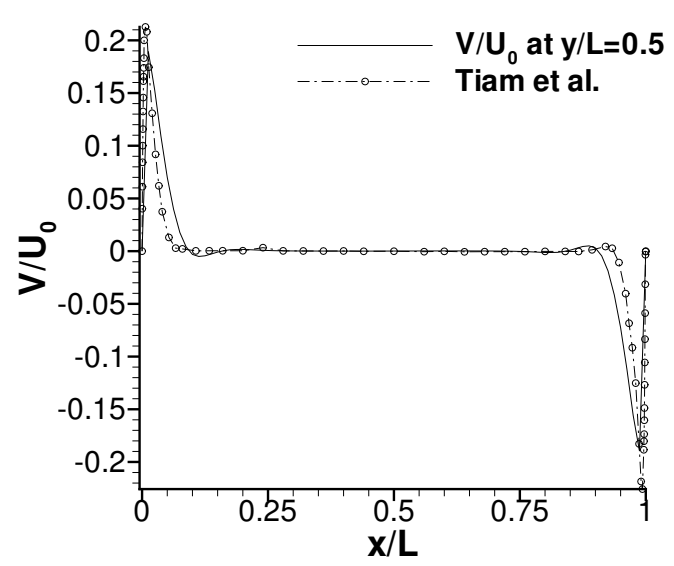

Figure 13.23.: $\frac{T-T_{c}}{T_{h}-T_{c}}$ at $\frac{y}{L}=0.2, \mathrm{~V} 2$.

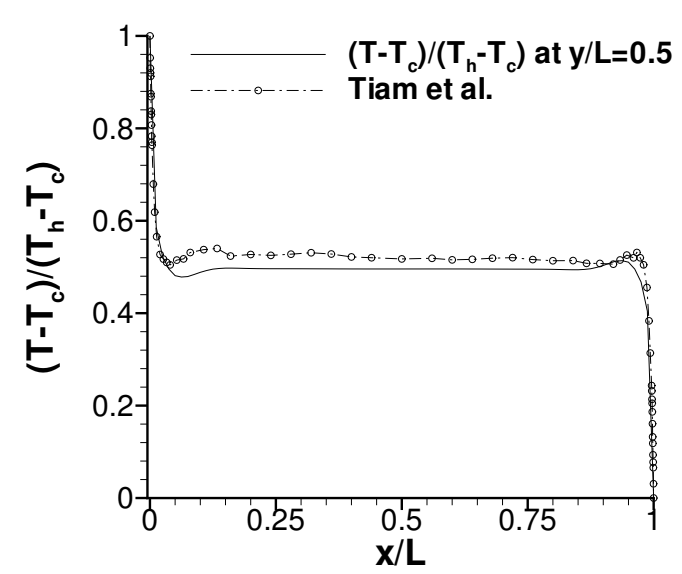

Figure 13.24.: $\frac{V}{U_{0}}$ at $\frac{y}{L}=0.5, \mathrm{~V} 2$.
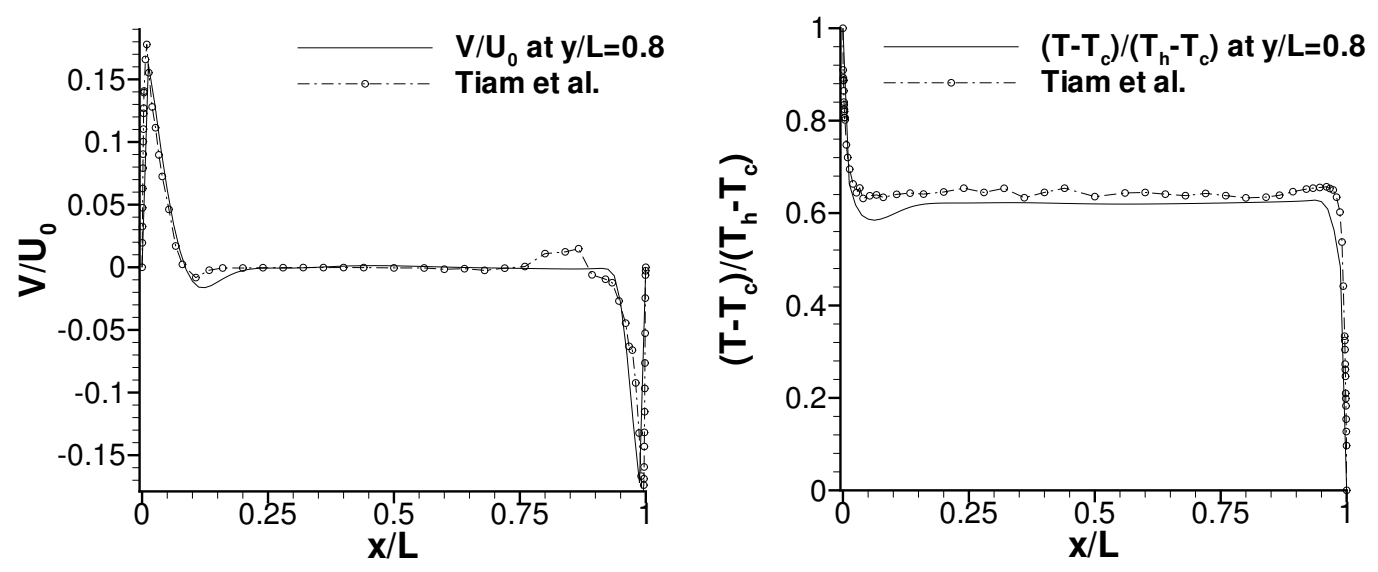

Figure 13.26.: $\frac{V}{U_{0}}$ at $\frac{y}{L}=0.8, \mathrm{~V} 2$.

Figure 13.27.: $\frac{T-T_{c}}{T_{h}-T_{c}}$ at $\frac{y}{L}=0.8, \mathrm{~V} 2$. 


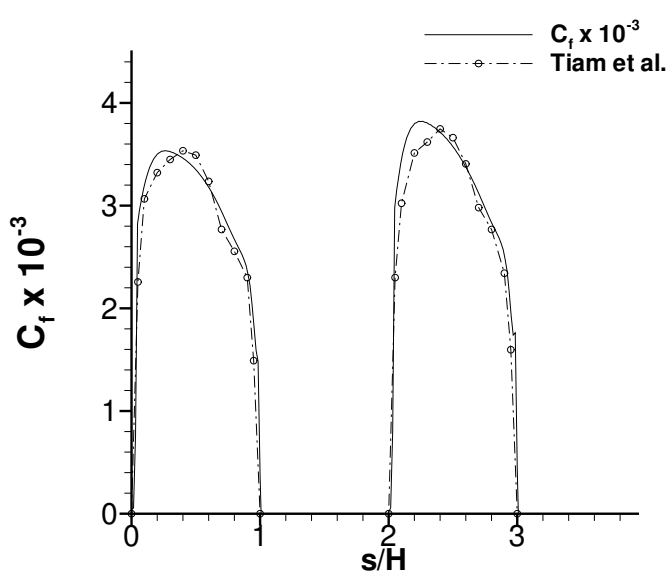

Figure 13.28.: $C_{f}$ from lower top corner along surface clockwise, V2.

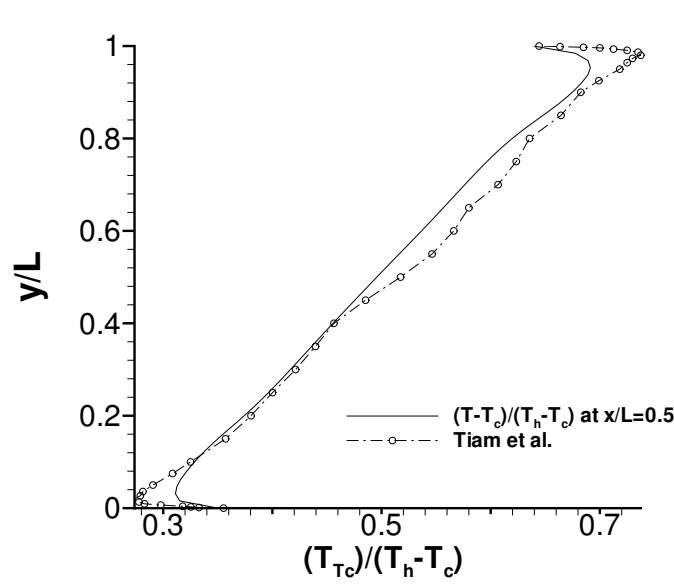

Figure 13.30.: $\frac{T-T_{c}}{T_{h}-T_{c}}$ at $x / L=0.5, \mathrm{~V} 2$.

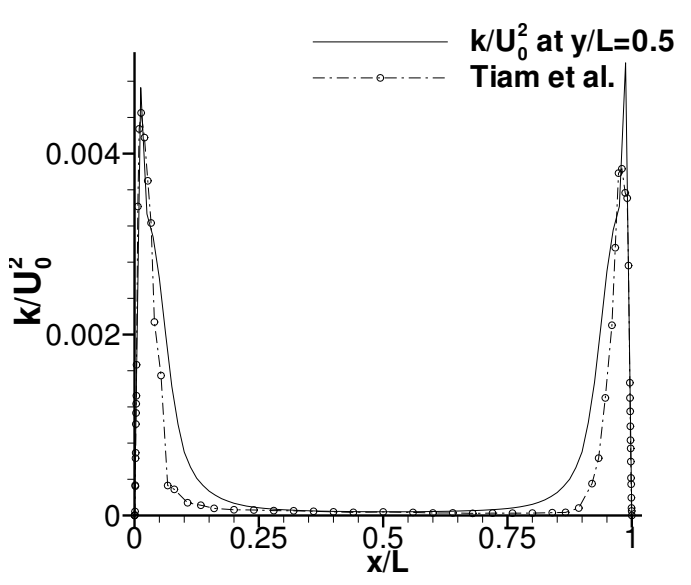

Figure 13.32.: $\frac{k}{U_{0}^{2}}$ at $\frac{y}{L}=0.5, \mathrm{~V} 2$.

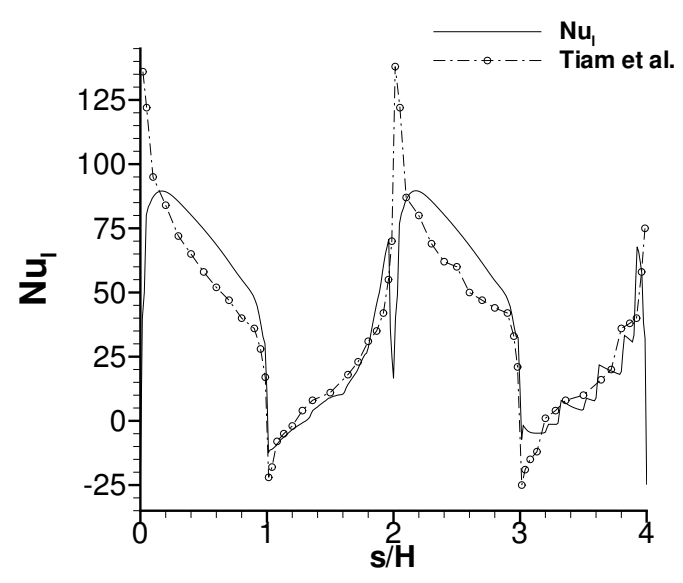

Figure 13.29.: $N u_{l}$ from lower top corner along surface clockwise, V2.

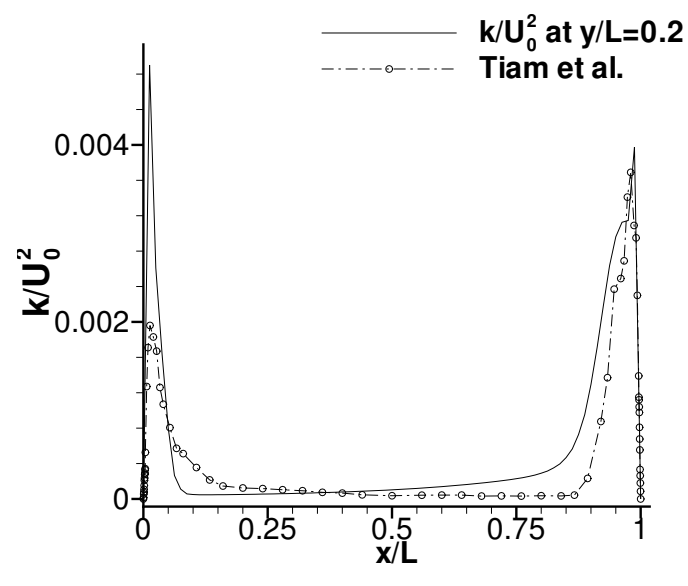

Figure 13.31.: $\frac{k}{U_{0}^{2}}$ at $\frac{y}{L}=0.2, \mathrm{~V} 2$.

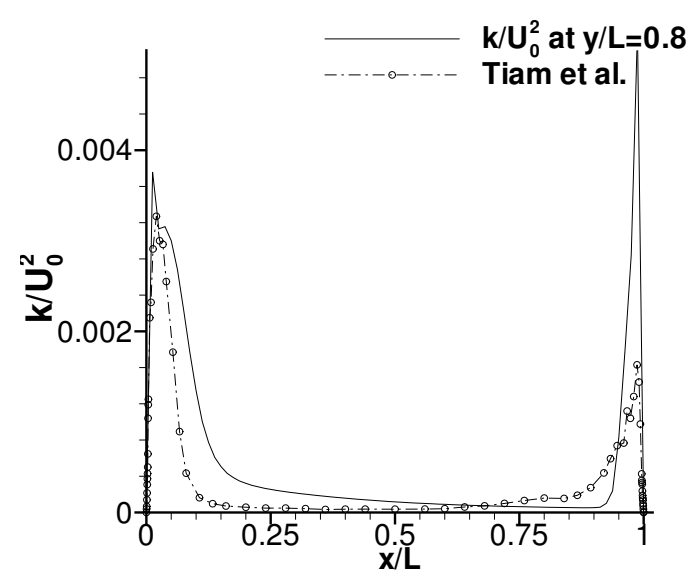

Figure 13.33.: $\frac{k}{U_{0}^{2}}$ at $\frac{y}{L}=0.8, \mathrm{~V} 2$. 
opportunity to study Neitzke's modification (5.40) also for the bottom wall. A suitable choice for $K_{s}$ and $R e_{\min }$ is $K_{s}=25.0, R e_{\min }=20.0$, see also [Sein3]. In order to ensure $R e_{\text {min,cut }} \leq R e_{\min } \leq 70.0$ with $R e_{\text {min }, c u t} \in[2,12]$, we apply a cutoff technique, see Section 9.2 .2 and Section 9.3.2. Since $R e_{m i n, c u t}$ cannot be determined from theoretical reasoning, a suitable choice has to be found numerically. Hence we have to investigate the influence of $R e_{\text {min,cut }}$ on the solution and seek the value which gives the best predictions for this test case. We investigate the following two variants. In all cases we select $K_{s}=25.0$ and $R e_{\min }=20.0$. Within each linearisation cycle (see Section 9.2.2), three iteration cycles for the DDM are performed.

V3a We choose $\chi=0$ in (5.40), i.e., effects of stratification are neglected in the boundary layer. Moreover we choose $\theta=1.0$ and $\lambda=1.0$ for both the momentum equation and the scalar equations, see (13.6).

V3b We select $\chi=0, \theta=1.0$ and $\lambda=100.0$ for the momentum equation resp. $\lambda=10.0$ for the scalar equations.

V4a We choose $\chi=1$ in (5.40). Then effects of stratification in the boundary layer are taken into account. We ensure $R e_{\min } \in\left[R e_{\text {min,cut }}, 70.0\right]$ with $R e_{\text {min,cut }}=2.0$ using a cut-off technique. Moreover we choose $\theta=1.0$ and $\lambda=100.0$ for the momentum equation resp. $\lambda=10.0$ for the scalar equations.

V4b We use $\chi=1$ in (5.40) and $R e_{\text {min,cut }}=7.0$. For the DDM we use $\theta=1.0$ and $\lambda=100.0$ for the momentum equation resp. $\lambda=10.0$ for the scalar equations.

First we investigate the accuracy of the DDM, i.e., we study whether the DDM solution is close to the solution without DDM. We consider V3a and V3b in order to study the influence of the parameters $\theta$ and $\lambda$ on the DDM. Numerical tests which are not shown here show that the profiles for V3a and V3b collapse. Thus we restrict ourselves to considering V3b.

The results for V3b are shown in Figures 13.34-13.45. The plots show the DDM results (solid lines), the results without DDM (dash-dotted lines), and the experimental results from Tiam et al. (dotted lines with symbols). The predictions for $V$ are very satisfying, cf. Figures 13.34,13.36, and 13.38. The results for $k$ are also reasonable, see 13.43-13.45. Concerning the temperature equation, surprisingly the oscillations for the predictions for $N u_{l}$ at the bottom wall vanish, see Figure 13.41. It should be recalled that $N u_{l}$ is related closely to $\dot{q}$ (see (13.5)), which occurs on the right hand side of the Neumann boundary condition for the temperature equation in the wall function procedure (see Section 6.2). Thus it seems reasonable that the predictions for the temperature are altered if the oscillations for $N u_{l}$ are removed. The vertical temperature profile is shown in Figure 13.42. The deviation of the DDM solution from the solution without DDM in the lower part of the cavity is obvious. Near the top wall both solutions collapse. This indicates that the difference is due to the altered predictions for $N u_{l}$ at the bottom wall. This suggests that the modelling error of the wall function procedure (numerical oscillations for $N u_{l}$ ) has a positive influence on the predictions for the vertical temperature profile in the case without DDM and with $\chi=0$ (denoted V1 in the previous section). Thus there is hope that the results for the vertical temperature profile can be improved when accounting for effects of 
stratification in the boundary layer when using the DDM. Finally we study the predictions for $C_{f}$. The agreement is excellent at the top wall and satisfying at the bottom wall, see Figure 13.40. The deviation at the bottom wall stems at least in parts from the different prections for $N u_{l}$ there. The coupling of momentum and temperature in the boundary layer equations (see Section 6.3) implies that modified predictions for the temperature at the bottom wall (see Figure 13.42) influence the predictions for $C_{f}$.

\section{Remark 13.3}

Due to the oscillations for $N u_{l}$ in the case without DDM, the convergence of the DDMsolution to the solution without DDM is somehow slightly less than satisfying. Now homogeneous Neumann boundary conditions are imposed on the top and bottom wall instead of Dirichlet boundary conditions. Then the convergence is excellent, see Figure 13.58 .

Now we activate (5.40) in order to account for effects of stratification in the boundary layer. We start with considering V4a. The results are shown in Figures 13.46-13.57. First we study the predictions for $N u_{l}$, see Figure 13.53. At the top wall the prediction is close to the solution for V2, see Figure 13.29. Recall that V2 is the non-DDM solution with (5.40) but using a different cutoff for $R e_{\min }$. The prediction for $N u_{l}$ along the bottom wall is also very reasonable. It should be pointed out here that the experimental results are inevitably flawed due to inaccuracies and imperfections in the experimental setup. Thus it is not clear how reliable details of the experimental data are. The results indicate that the modification (5.40) gives a valuable, significant improvement regarding the predictions for $N u_{l}$. The profile for $C_{f}$ is shown in Figure 13.40. At the bottom wall $C_{f}$ is slightly increased compared to the results without DDM, see Figure 13.28. The predictions for momentum, temperature and turbulent kinetic energy are close to those for V2 and need not being discussed.

Now we study the effect of the cutoff value for $R e_{\min }$. The results for V4b are also shown in Figures 13.46-13.57. Figure 13.54 reveals that for V4b the prediction for the vertical temperature profile is better than for V4a. On the other hand, the predictions for $C_{f}$ and $N u_{l}$ using V4a are superior to those from V4b, see Figures 13.52 and 13.53]. Thus one has to balance two aspects: If the focus is on the vertical velocity profile, than the results recommend $\mathrm{V} 4 \mathrm{~b}$, whereas more accurate predictions for $C_{f}$ and $N u_{l}$ can be obtained using V4a. Regarding the velocity profile (see Figures 13.46, 13.48, and 13.50) the results suggest choosing V4a.

\subsection{Appraisal of the $k / \epsilon$ model predictions}

It is worthwhile summarising the main results regarding the $k / \epsilon$ model. A comparison of the numerical results with experimental data reveals that the model predictions are very reasonable. The choices for the model parameters, in particular $K_{s}$ and $R e_{m i n}$, can be confirmed. This supports the improved wall function approach presented in this thesis. It should be emphasised that the predictions for $N u_{l}$ can be improved significantly by using Neitzke's modification (5.40). The results for the surface transfer coefficients $C_{f}$ and $N u_{l}$ without resolving the near-wall region are remarkable. Moreover, the accuracy of the DDM is excellent. From these results we can expect that the predictions are accurate also for more complex flow problems including mixed convection, see e.g. [Sei03]. The 
computer ressources needed for real-life applications regarding three-dimensional room-air flow problems are enormous. However, exploiting (i) the improved wall function scheme and (ii) the DDM, there is hope that such problems can be solved with reasonable accuracy on a cluster of workstations.

\subsection{First results for the posteriori testing using LES}

This section is devoted to the presentation of a few first LES results. LES predictions for the fully developed isothermal channel flow using the stabilised finite-element method were presented e.g. in [Jan.99]. Several wall function schemes for LES have been tested for isothermal flow problems, see e.g. [BBP96]. To the author's best knowlegde, combining both a stabilised finite-element method and wall functions for LES has not yet been accomplished for non-isothermal flows. We use Eidson's modification for the Smagorinsky model, see (9.19), with $C_{S}=0.21$. As initial solution we choose the solution of the $k / \epsilon$ model at the final time $T=200.0$. We choose $\Delta t=0.1$ for the time step width (which is relatively large for a LES) and perform three linearisation cycles per time step.

Before studying the numerical results, it is worthwhile to recall some essentials regarding LES and to point out the fundamental differences between LES and the $k / \epsilon$ model. For the closed cavity flow under consideration, the corresponding laminar problem has a stationary solution. In the turbulent case, there is a statistically stationary solution, i.e., statistically averaged quantities become stationary. The $k / \epsilon$ model is a statistical turbulence model; it gives predictions for the statistically averaged quantities. The $k / \epsilon$ model solution is indeed stationary and no ensemble-averaging as a postprocess is necessary. Hence we can study the solution at a certain time instance, viz., at $t=200.0$. For the $k / \epsilon$ model, $\nu_{t}$ is relatively large. Thus, "the tendency for the flow equations to develop chaotic solutions is overcome by the enhanced viscous dissipation" ([DPR01], p. 182). Moreover, as the $k / \epsilon$ model solution is stationary, only little care has to be taken regarding the fixed-point iteration scheme for the non-linear term $\boldsymbol{u} \cdot \nabla \boldsymbol{u}$ in the momentum equation. The simple iteration method $\boldsymbol{u}^{m, i-1} \cdot \nabla \boldsymbol{u}^{m, i}$ (see Section 9.2.2) converges provided $\nu+\nu_{t}$ is large enough (which is true for the $k / \epsilon$ model). Therefore it is reasonable to choose only a few number of fixed-point iteration cycles and to embed the fixed-point iteration in the time-discretisation scheme. In LES, the situation is much more difficult. The aim of an LES is to be much closer to the Navier-Stokes equations by resolving the random motion of the large-scale structures. The solution of the Navier-Stokes equations is only statistically stationary and exhibits random fluctuations. Thus for an LES, $\nu_{t}$ has to be large enough to prevent a blow up of the FEM solution in finite time (see, e.g., [I.L03]), but it has to be small enough to preserve the characteristic turbulent fluctuations. Since in an LES $\nu_{t}$ is significantly smaller than for the $k / \epsilon$ model, it is not clear a priori whether the simple fixed-point iteration scheme $\boldsymbol{u}^{m, i-1} \cdot \nabla \boldsymbol{u}^{m, i}$ is convergent or not. Concerning this issue, numerical tests reveal that we need at least three iteration steps within each time step in order to obtain a statistically stationary solution. However, a detailed investigation of the fixed-point scheme for LES seems to be an important issue for future research.

A correct LES gives accurate predictions for both the statistically averaged quantities and for the turbulent fluctuations. In this thesis we can only give results for the former. We focus on the prediction for the vertical mean velocity profile $V$ at $y / L=0.5$ and for $C_{f}$ 

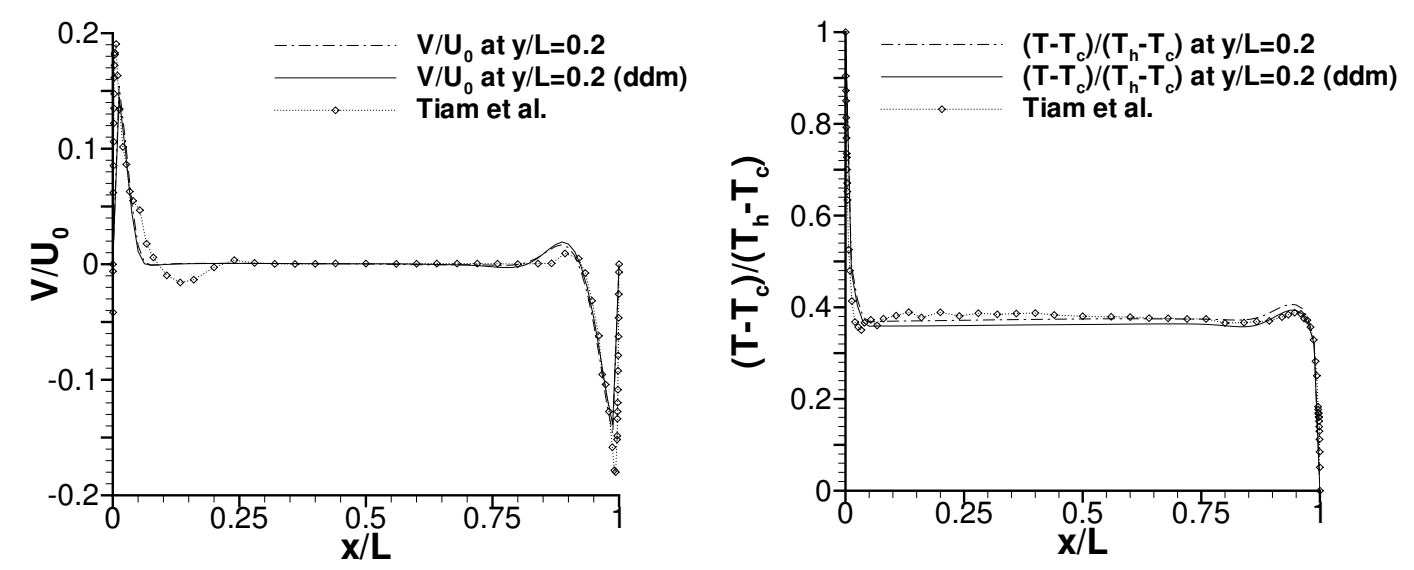

Figure 13.34.: $\frac{V}{U_{0}}$ at $\frac{y}{L}=0.2, \mathrm{~V} 3 \mathrm{~b}$.
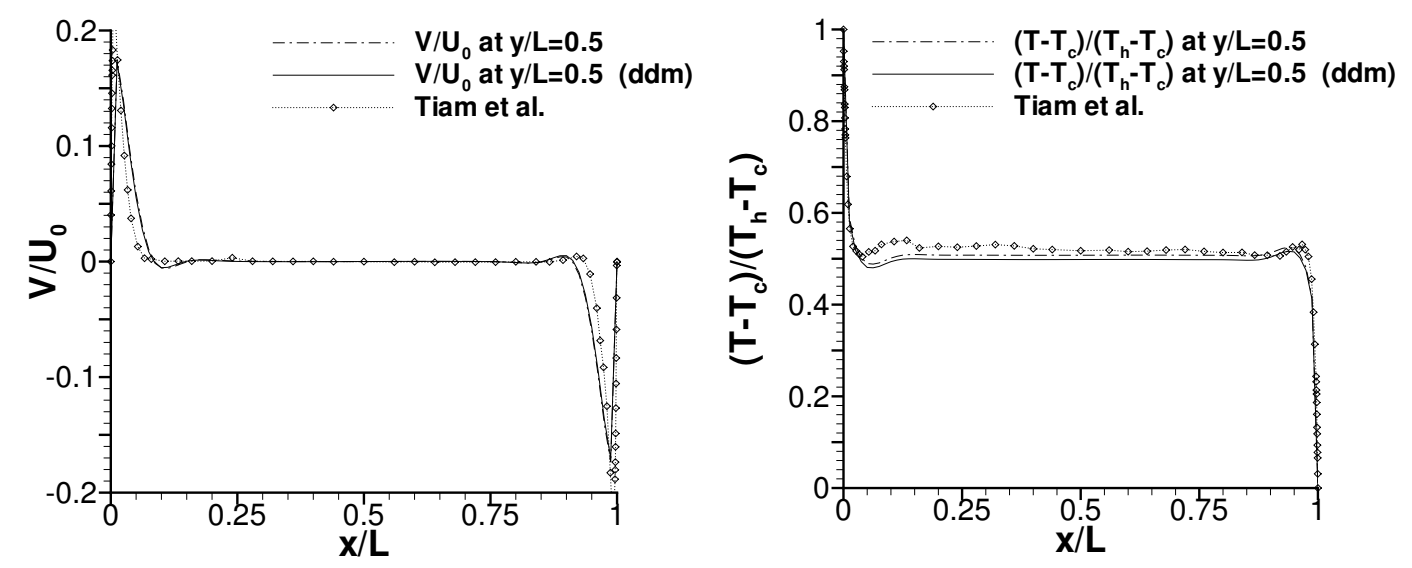

Figure 13.36.: $\frac{V}{U_{0}}$ at $\frac{y}{L}=0.5, \mathrm{~V} 3 \mathrm{~b}$.
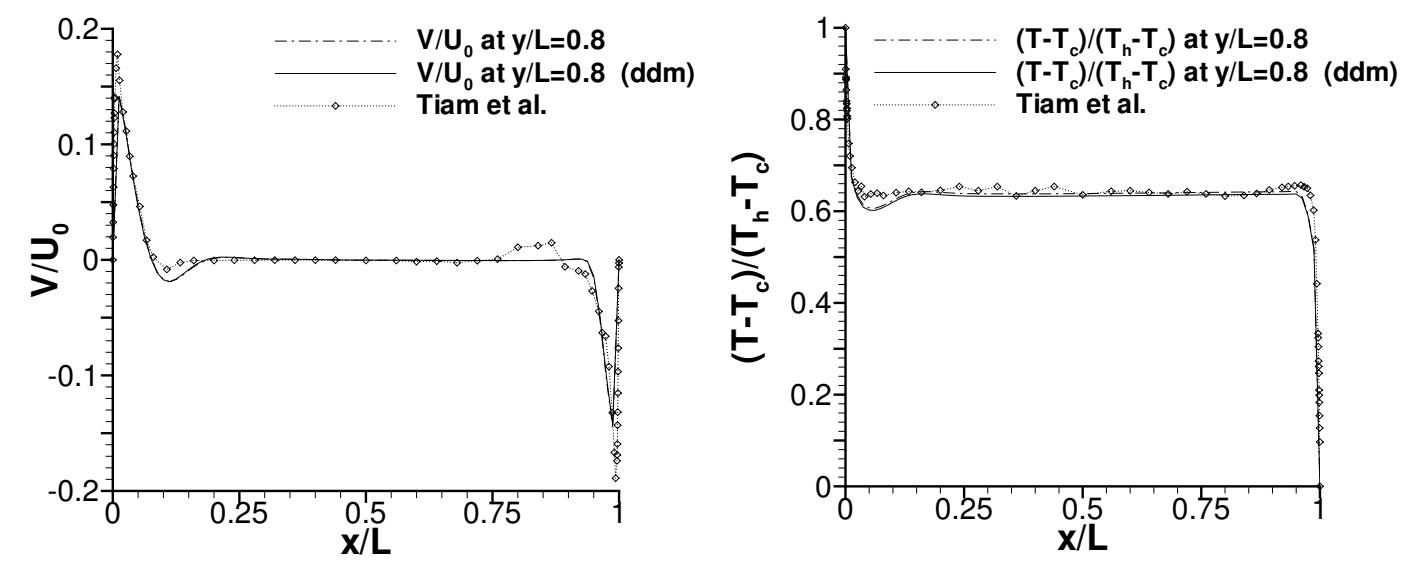

Figure 13.38.: $\frac{V}{U_{0}}$ at $\frac{y}{L}=0.8$, V3b. $\quad$ Figure 13.39.: $\frac{T-T_{c}}{T_{h}-T_{c}}$ at $\frac{y}{L}=0.8$, V3b. 

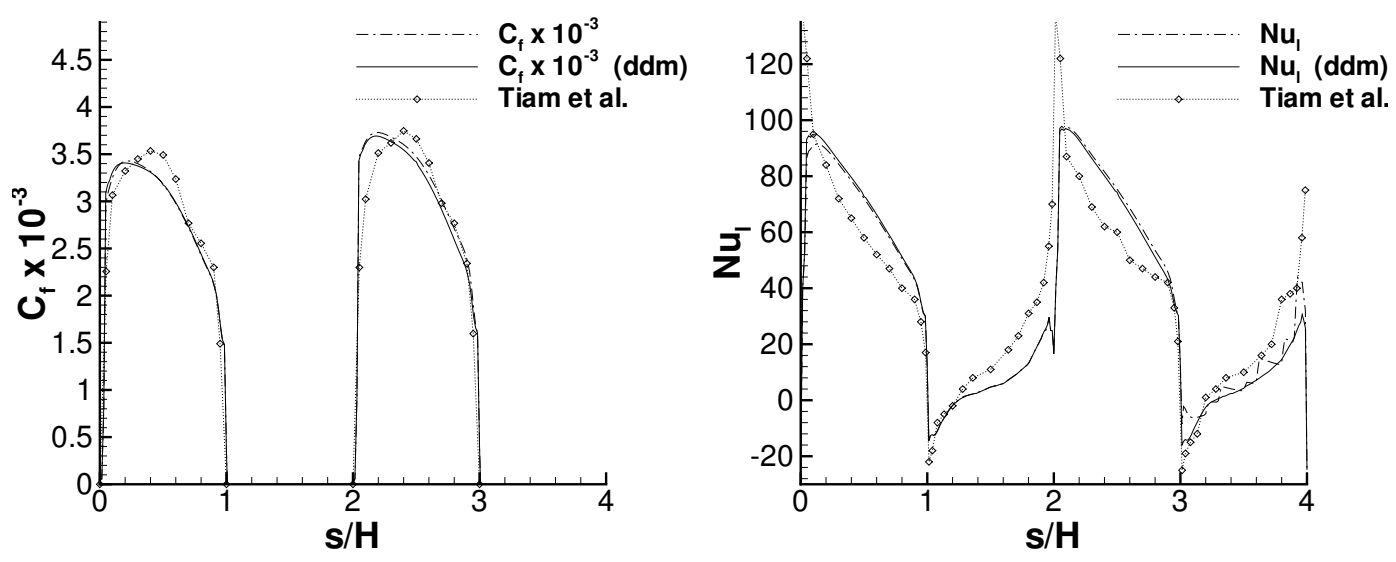

Figure 13.40.: $C_{f}$ (V3b) from lower top corner along surface clock-

Figure 13.41.: $N u_{l}$ (V3b) from lower top wise. corner along surface clockwise.
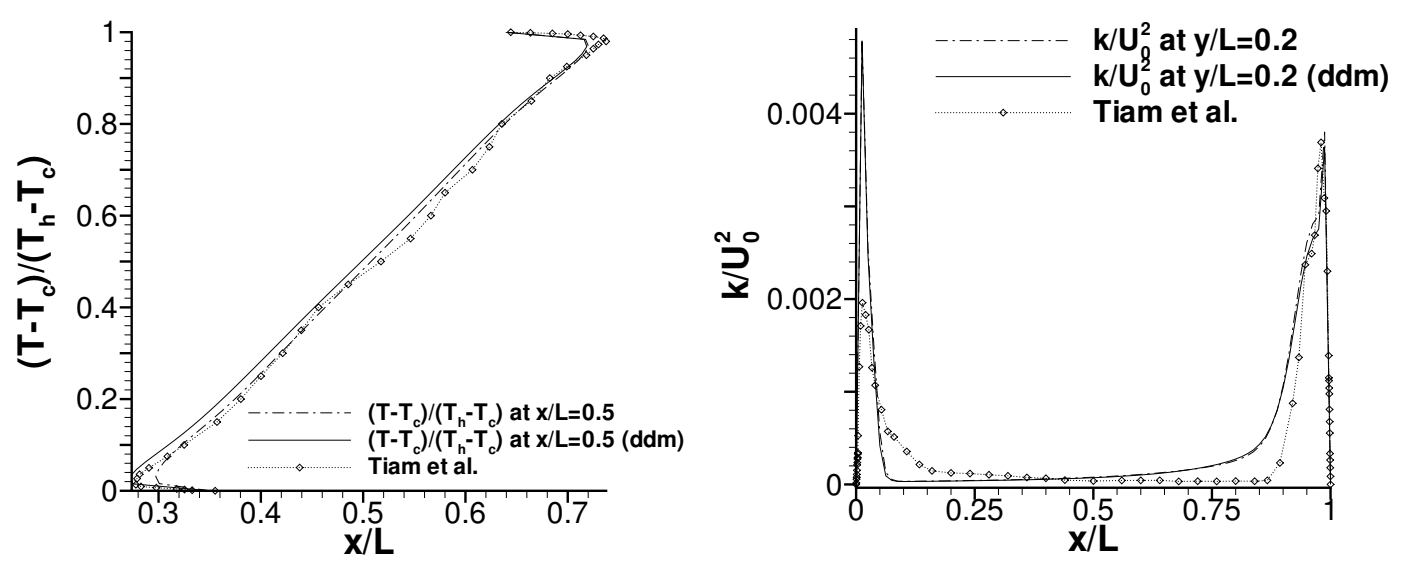

Figure 13.42.: $\frac{T-T_{c}}{T_{h}-T_{c}}$ at $x / L=0.5, \mathrm{~V} 3 \mathrm{~b}$.

Figure 13.43.: $\frac{k}{U_{0}^{2}}$ at $\frac{y}{L}=0.2$, V $3 \mathrm{~b}$.
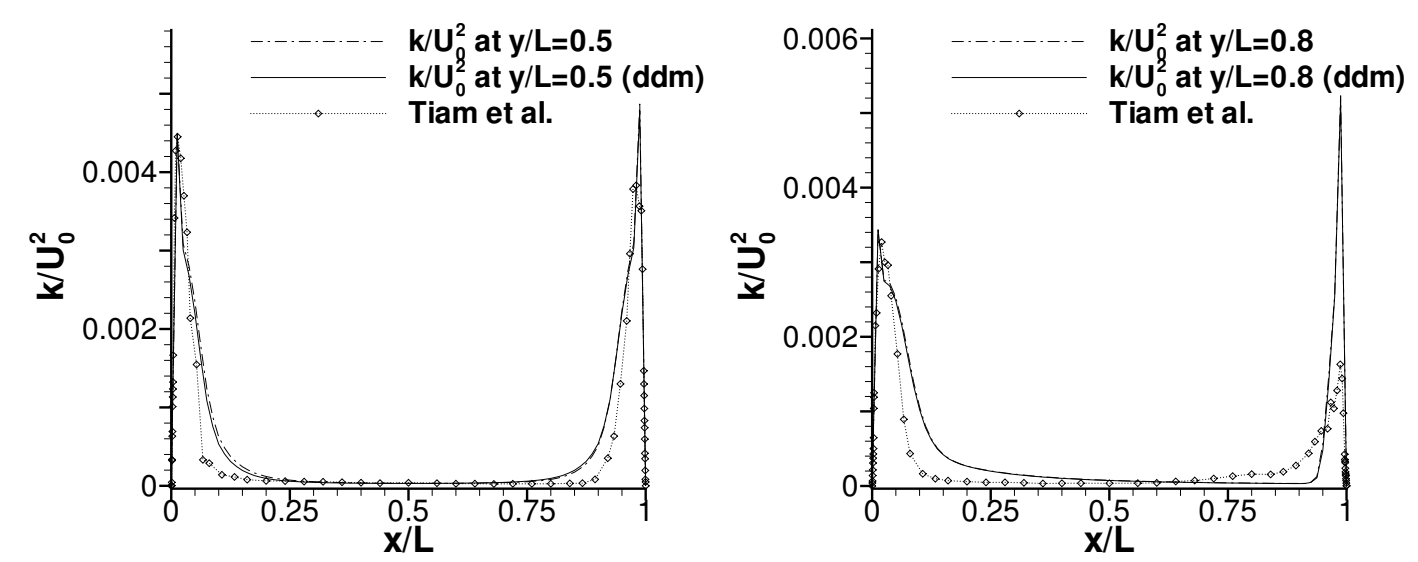

Figure 13.44.: $\frac{k}{U_{0}^{2}}$ at $\frac{y}{L}=0.5$, V3b.

Figure 13.45.: $\frac{k}{U_{0}^{2}}$ at $\frac{y}{L}=0.8, \mathrm{~V} 3 \mathrm{~b}$. 


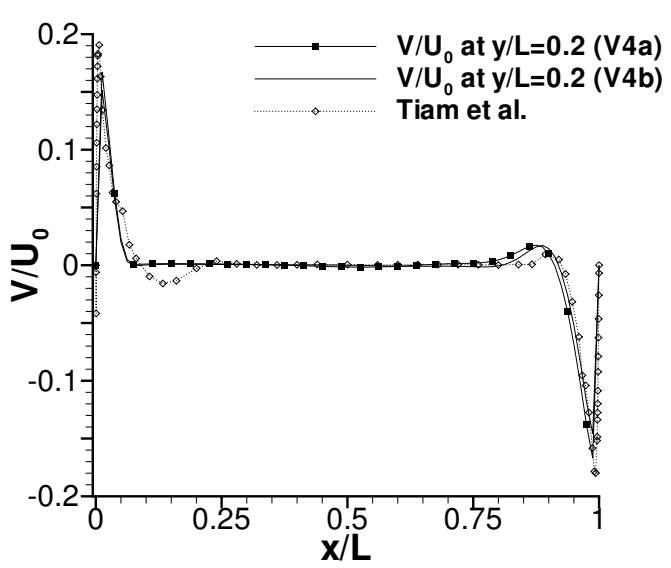

Figure 13.46.: $\frac{V}{U_{0}}$ at $\frac{y}{L}=0.2$.

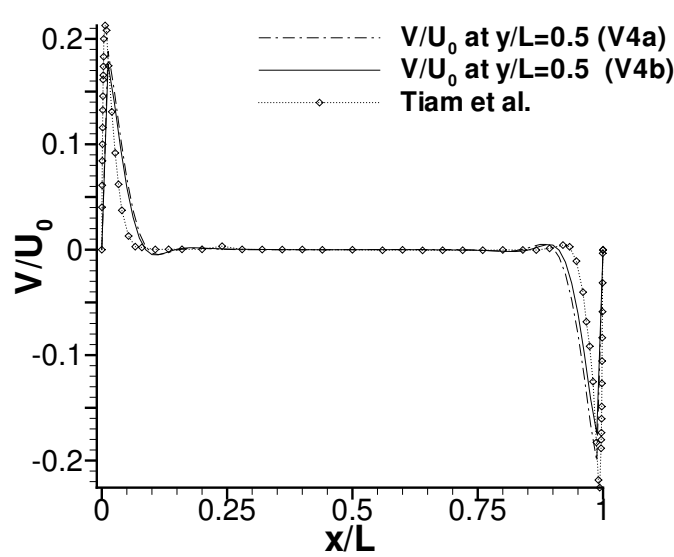

Figure 13.48.: $\frac{V}{U_{0}}$ at $\frac{y}{L}=0.5$.

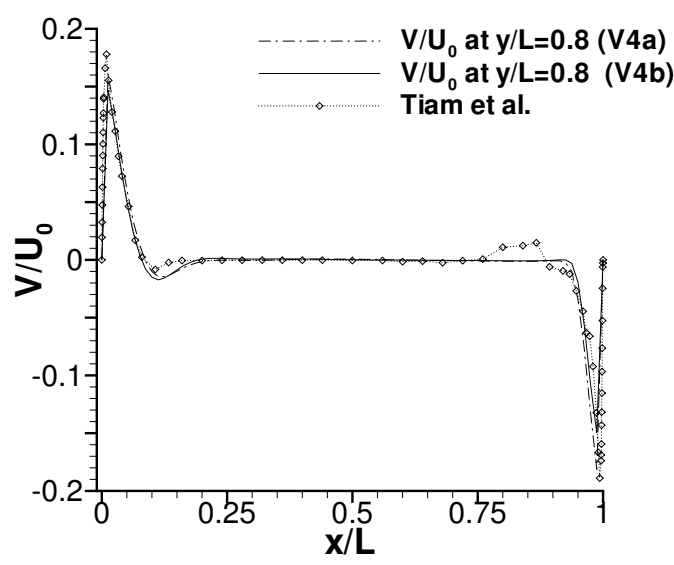

Figure 13.50.: $\frac{V}{U_{0}}$ at $\frac{y}{L}=0.8$.

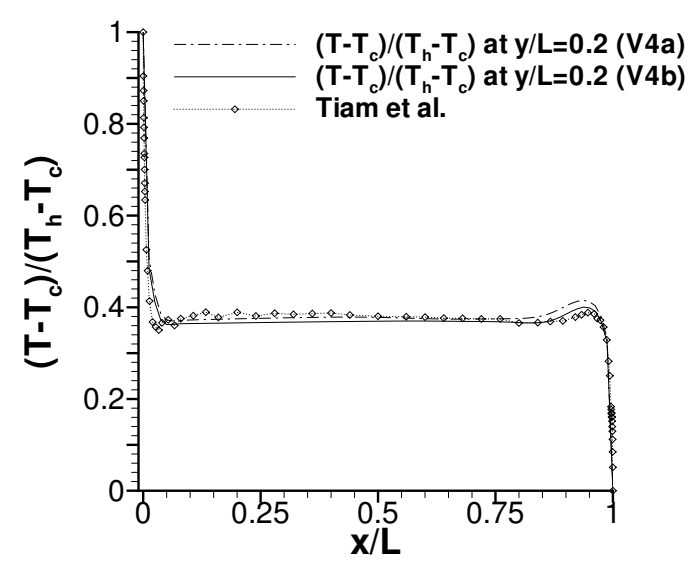

Figure 13.47.: $\frac{T-T_{c}}{T_{h}-T_{c}}$ at $\frac{y}{L}=0.2$.

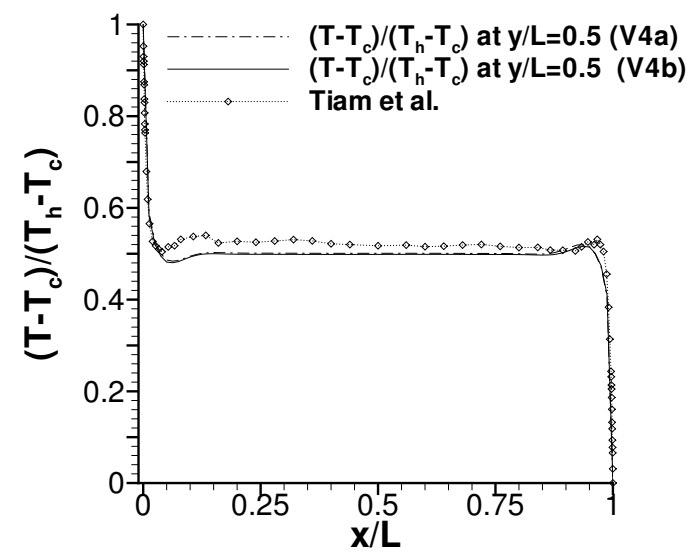

Figure 13.49.: $\frac{T-T_{c}}{T_{h}-T_{c}}$ at $\frac{y}{L}=0.5$.

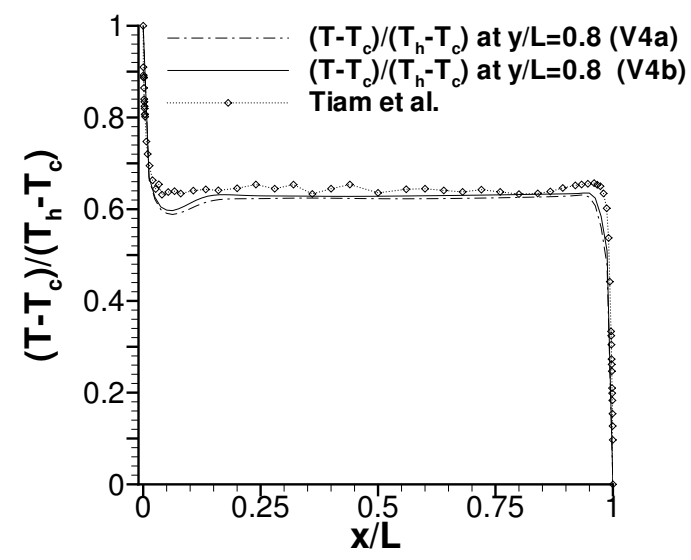

Figure 13.51.: $\frac{T-T_{c}}{T_{h}-T_{c}}$ at $\frac{y}{L}=0.8$. 


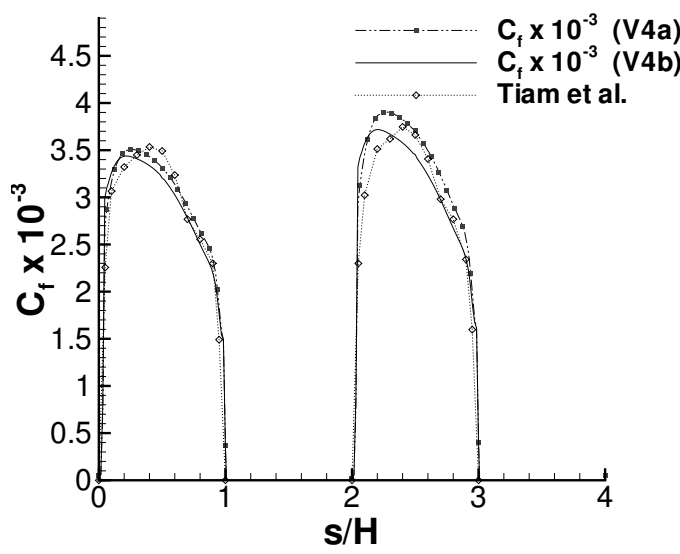

Figure 13.52.: $C_{f}$ from lower top corner along surface clockwise.

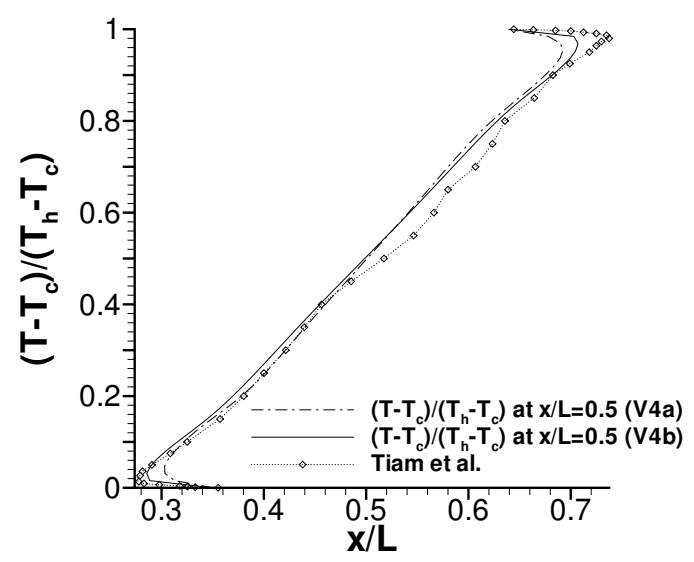

Figure 13.54.: $\frac{T-T_{c}}{T_{h}-T_{c}}$ at $x / L=0.5$.

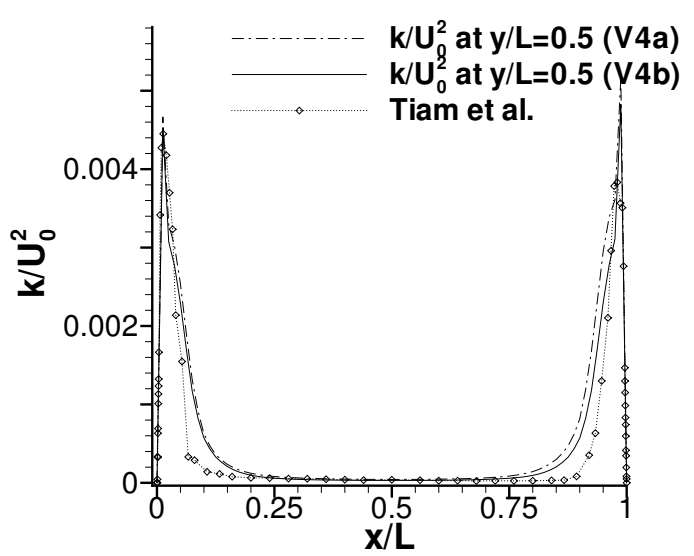

Figure 13.56.: $\frac{k}{U_{0}^{2}}$ at $\frac{y}{L}=0.5$.

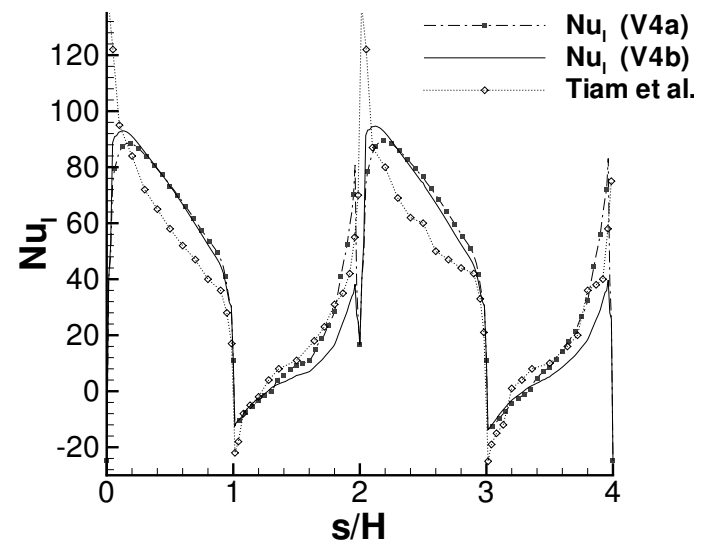

Figure 13.53.: $N u_{l}$ from lower top corner along surface clockwise.

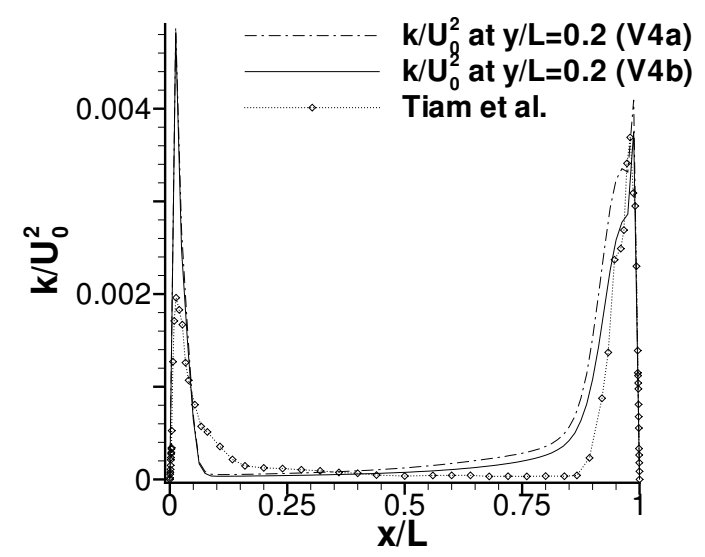

Figure 13.55.: $\frac{k}{U_{0}^{2}}$ at $\frac{y}{L}=0.2$.

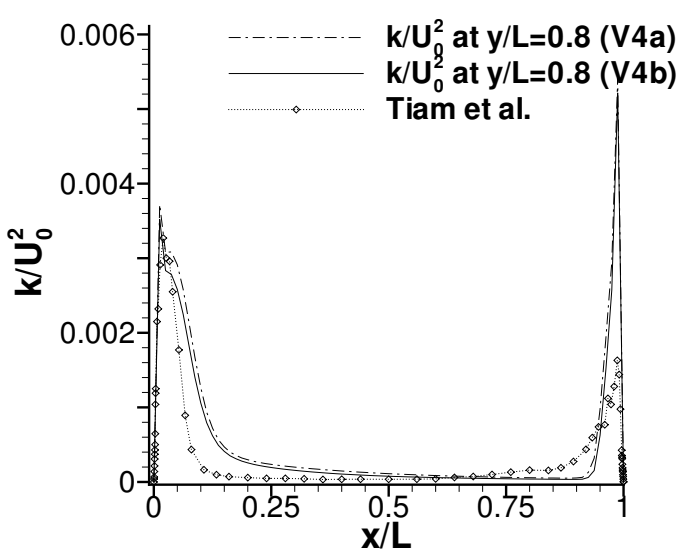

Figure 13.57.: $\frac{k}{U_{0}^{2}}$ at $\frac{y}{L}=0.8$. 

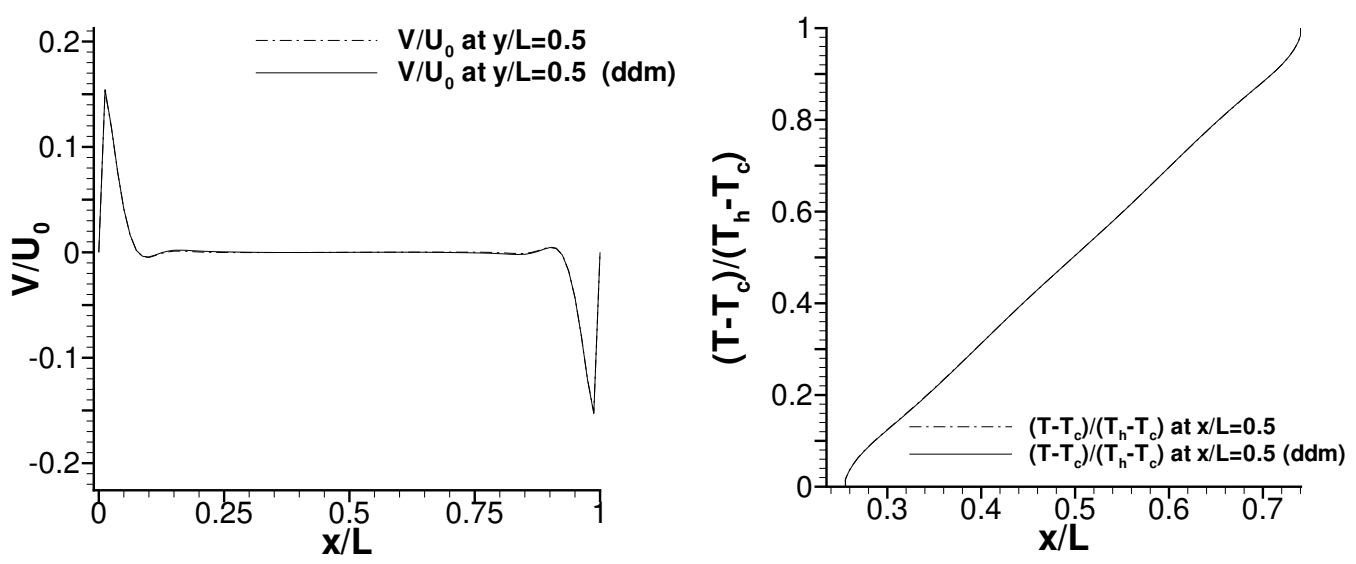

Figure 13.58.: $\frac{V}{U_{0}}$ at $\frac{y}{L}=0.5$ (left) and $\frac{T-T_{c}}{T_{h}-T_{c}}$ at $x / L=0.5$ (right), adiabatic boundary conditions on top and bottom wall.

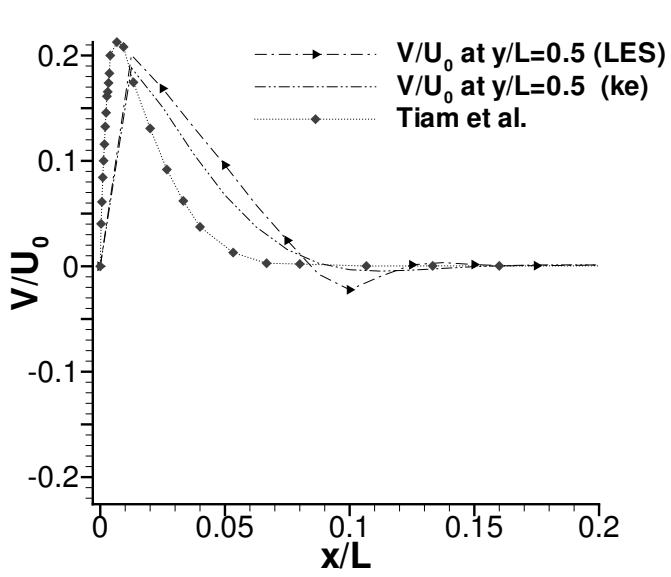

Figure 13.59.: $\frac{V}{U_{0}}$ at $\frac{y}{L}=0.5(k / \epsilon$ model V4a and LES).

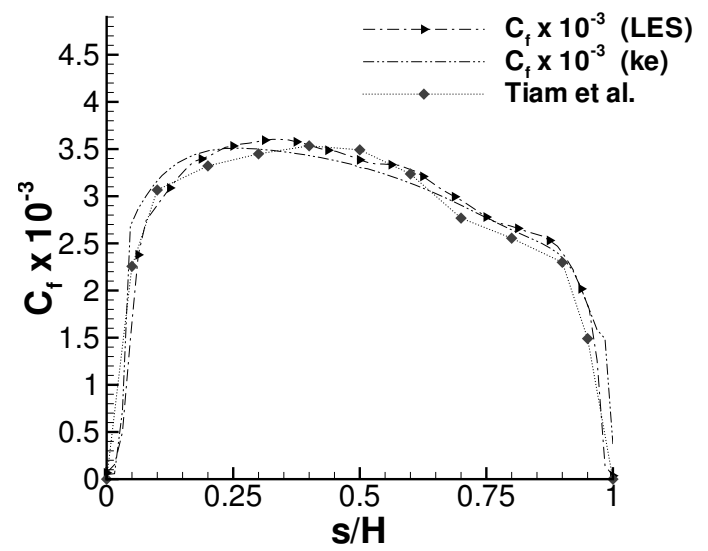

Figure 13.60.: $C_{f}$ from lower top corner along hot wall $(k / \epsilon$ model V4a and LES).

along the hot wall. In order to obtain a statistically mean solution, a time averaging filter is used. Denote $V(t, \cdot)$ the solution for $V$ at time $t$. Then we introduce the long-time average

$$
\langle V\rangle_{t_{0}, N}=\frac{1}{N} \sum_{j=0}^{N-1} V\left(t_{0}+10 j \Delta t, \cdot\right) .
$$

During the first time steps, the LES solution differs widely from the initial $k / \epsilon$ solution (not shown here). After this initial period, at $t \approx 210$ the LES solution comes closer to the $k / \epsilon$ solution (and hence to the experimental data). We study $\langle V\rangle_{216,5}$ and compare the LES results with the $k / \epsilon$ predictions for variant V4a, see Figure 13.59. The maximum 
velocity for LES is closer to the experimental data than for the $k / \epsilon$ model. However, the thickness of the boundary layer is significantly overpredicted and slightly wider than for the $k / \epsilon$ model. This stems at least in parts from the well-known property of the Smagorinsky model of being overdiffusive; the Smagorinsky model produces an excessive damping of the large-scale structures. The non-physical undershoot of the LES solution beyond the boundary layer might vanish for larger times.

Very interestingly, the LES predictions for $C_{f}$ are significantly superior to those of the $k / \epsilon$ model, see Figure 13.60. In particular, the predictions near the corners at $s / H=0$ and $s / H=1$ are much closer to the experimental data by TIAM ET AL. This result is remarkable, since both the complex physical behaviour of the solution near the corners and the numerous modelling approximations have to be appreciated. In contrast, the $k / \epsilon$ model predictions near the corners are less than satisfying. This indicates that the failure near the corners of the $k / \epsilon$ model with wall functions is due to the $k / \epsilon$ model and not due to the wall function procedure. This interpretation is in full agreement with [Wilcox98], p. 174. The LES predictions for $C_{f}$ indicate that the wall-function scheme for LES is a promosing strategy also for non-isothermal flow problems. Moreover the results suggest that LES with the wall-function method does not necessarily suffer from all the flaws of the $k / \epsilon$ model with wall-functions.

The results for the velocity and temperature fluctuations cannot be shown here. This is a major subject of future research. 


\section{Summary and future prospects}

Computational fluid dynamics is an impressive subject to interdisciplinary research. It has stimulated progress in a variety of disciplines, including computer hardware and programming techniques, experimental and theoretical physics, mathematical analysis and numerical algorithms. One major objective of CFD is to provide tools for engineering applications, which (i) make accurate flow predictions (ii) at acceptable computational costs. Current trends in CFD focus on both points. Aspect (i) is to develop turbulence models which yield accurate predictions at least for simple flow configurations. The second aspect is to make these models amenable to more complex flow problems.

In our research code PARALLELNS, the vast majority of the computational costs are needed for assembling the discrete linear systems and for solving them. Our strategy is to pursue both issues (i) and (ii) by combining two domain-decomposition methods, viz., a wallfunction procedure and an iteration-by-subdomains technique. The objective of the improved wall function scheme is to avoid a large number of additional grid points for resolving the near-wall region. The aim of the iteration-by-subdomains method is to divide the flow problem into a set of smaller problems which can be solved in parallel.

In this thesis, two turbulence models have been investigated, viz., the $k / \epsilon$ model and three LES models. The numerical tests for the $k / \epsilon$ model have proved satisfactory, indicating that this approach can be expected to yield reliable results also for more complex flow problems. The application of this scheme to another typical indoor-air flow test case is in progress, see [Sei(13]. On the other hand, regarding LES, there is still a great demand for intensive future research. The present results for the mean velocity field and for $C_{f}$ are promising and fuel optimism. The results indicate that LES with the wall-function scheme does not necessarily suffer from all the deficiencies of the $k / \epsilon$ model with wall-functions and that the wall-function scheme is a suitable tool for LES. In particular, the LES predictions for $C_{f}$ along the heated wall for a natural convection flow are significantly superior to those for the $k / \epsilon$ model. However, it seems necessary to study also simpler test cases like the fully developed channel flow and the mixing-layer in order to achieve a more profound understanding of LES within our computational scheme. Moreover it is worthily studying the natural convection flow in a closed cavity with adiabatic boundary conditions on the top and bottom wall (instead of Dirichlet conditions), which simplifies the physics of the problem considerably. Emphasis has to be placed on the prediction of the fluctuation terms. These are prime objectives for future research.

Finally, it is worthwhile mentioning a few suggestions for improving the existing code. First we consider the accuracy of our method. The boundary conditions for $k$ and $\epsilon$ at the artificial inner boundary $\Gamma_{\delta}$ seem to be not appropriate in any case. A primary objective of our improved wall function concept was to allow that the distance of $\Gamma_{\delta}$ from the wall can be larger than for the classical wall function approach. However, the boundary condition for $k$ and $\epsilon$ on $\Gamma_{\delta}$ (see (5.49)) are valid only if $\Gamma_{\delta}$ resides in the log-layer. Thus the task is to find a system of simple linear differential equations for velocity, temperature and $k$ in order to 
impose a more sophisticated boundary condition at least for $k$ on $\Gamma_{\delta}$. Another aspect for future research is to account for effects of baroclinic vorticity generation theory: If $\Gamma_{\delta}$ is located in the log-layer but close to the buffer layer (see Table 5.7), then it is an interesting idea to use (3.27) instead of (3.26). In a similar vein, a corresponding modification of the LES model seems to be promising. Another interesting improvement is to use the $k-\epsilon-\overline{v^{2}}$ model with a near-wall grid-refinement. However, anisotropic grids in the near-wall region have not been investigated yet using our research code. Some problems might arise due to the stabilisation technique. Finally it is worth studying a more accurate scheme for the semidiscretisation in time. In particular, BDF-schemes seem to be interesting for our purposes.

The other improvements concern the performance of the scheme. Emphasis has to be placed on the fact that the fast solution of the arising linear systems (in particular, for the Oseen problem) still requires strong research. Moreover, the significant computational costs for assembling the PSPG terms recommend to choose finite-element spaces which satisfy the discrete Babuska-Brezzi condition. Finally, it is worthwhile mentioning the strategy of a nodal-based implementation of a stabilized finite-element method, which was devised by Codina, see [Cod00]. 
Part III.

Appendix 



\section{A. Mathematical tools for residual stress modelling in LES}

\section{A.1. Fourier transformation, convolution and distributions}

In this section we summarise some standard results regarding the Fourier transformation.

\section{Definition A.1}

Given $f \in L^{1}\left(\mathbb{R}^{d}\right)$ we define

$$
\mathcal{F}(f(\boldsymbol{x})) \equiv \hat{f}(\boldsymbol{k}) \equiv(2 \pi)^{-\frac{d}{2}} \int_{\mathbb{R}^{d}} f(\boldsymbol{x}) e^{-i \boldsymbol{x} \cdot \boldsymbol{k}} d \boldsymbol{x}, \quad \forall \boldsymbol{k} \in \mathbb{R}^{d} .
$$

The function $\mathcal{F} f \equiv \hat{f}$ is called the Fourier transform of $f$, the mapping $\mathcal{F}: f \mapsto \mathcal{F} f$ is called Fourier transformation.

\section{Theorem A.1}

Given $f \in L^{1}\left(\mathbb{R}^{d}\right)$ we have that $\mathcal{F} f \in C_{0}\left(\mathbb{R}^{d}\right)$. Moreover $\mathcal{F}: L^{1}\left(\mathbb{R}^{d}\right) \mapsto C_{0}\left(\mathbb{R}^{d}\right)$ is a linear and bounded (and consequently continuous) operator satisfying $\|\mathcal{F}\| \leq(2 \pi)^{-d / 2}$.

Proof:

See [Wer95] p.164.

\section{Definition A.2}

The space $\mathcal{S}\left(\mathbb{R}^{d}\right)$ of $C^{\infty}$ functions in $\mathbb{R}^{d}$ rapidly decreasing at infinity is defined as

$$
\mathcal{S}\left(\mathbb{R}^{d}\right)=\left\{f \in C^{\infty}\left(\mathbb{R}^{d}\right): \lim _{|\boldsymbol{x}| \rightarrow \infty} \boldsymbol{x}^{\boldsymbol{\alpha}} D^{\boldsymbol{\beta}} f(\boldsymbol{x})=0 \quad, \quad \forall \boldsymbol{\alpha}, \boldsymbol{\beta} \in \mathbb{N}_{\mathbf{0}}^{\boldsymbol{d}}\right\}
$$

A typical example for a function in $\mathcal{S}\left(\mathbb{R}^{d}\right)$ is $\exp \left(-\boldsymbol{x}^{2}\right)$.

\section{Theorem A.2}

Let $f \in \mathcal{S}\left(\mathbb{R}^{d}\right)$, let $\boldsymbol{\alpha} \in \mathbb{N}_{0}^{d}$. Then

(i) $\mathcal{F} f \in C^{\infty}\left(\mathbb{R}^{d}\right)$ and $D^{\boldsymbol{\alpha}}(\mathcal{F} f)=(-i)^{|\boldsymbol{\alpha}|} \mathcal{F}\left(\boldsymbol{x}^{\boldsymbol{\alpha}} f\right)$.

(ii) $\mathcal{F}\left(D^{\boldsymbol{\alpha}} f\right)=i^{|\boldsymbol{\alpha}|} \boldsymbol{k}^{\alpha} \mathcal{F} f$

(iii) $\mathcal{F}\left(e^{-\boldsymbol{x}^{2} / 2}\right)=e^{-\boldsymbol{k}^{2} / 2}$

(iv) $\mathcal{F}: \mathcal{S}\left(\mathbb{R}^{d}\right) \mapsto \mathcal{S}\left(\mathbb{R}^{d}\right)$ is an isomorphism. Its inverse operator is given by

$$
\mathcal{F}^{-1}(f(\boldsymbol{k}))=(2 \pi)^{-\frac{d}{2}} \int_{\mathbb{R}^{d}} f(\boldsymbol{k}) e^{i \boldsymbol{x} \cdot \boldsymbol{k}} d \boldsymbol{k}, \quad \forall \boldsymbol{x} \in \mathbb{R}^{d} .
$$


(v) $\mathcal{F}$ satisfies the Plancherel equation

$$
\int_{\mathbb{R}^{d}}(\mathcal{F} f)(\mathcal{F} g) d \boldsymbol{k}=\int_{\mathbb{R}^{d}} f g d \boldsymbol{x}, \quad \forall f, g \in \mathcal{S}\left(\mathbb{R}^{d}\right) .
$$

\section{Proof:}

See [Wer9.5]. pp.166 or alternatively [Tre67], pp.267.

Since $\mathcal{S}\left(\mathbb{R}^{d}\right)$ is dense in $L^{2}\left(\mathbb{R}^{d}\right), \mathcal{F}$ can be extended to an isometric operator $\mathcal{F}_{2}$ on $L^{2}\left(\mathbb{R}^{d}\right)$, see [Wer95] p.170., or alternatively, [Tre67], pp.270.

\section{Theorem A.3}

Let $f \in H^{m}\left(\mathbb{R}^{d}\right)$. Then for all $|\boldsymbol{\alpha}| \leq m$

$$
\mathcal{F}\left(D^{\boldsymbol{\alpha}} f\right)(\boldsymbol{k})=(-i)^{|\boldsymbol{\alpha}|} \boldsymbol{k}^{\boldsymbol{\alpha}} \mathcal{F} f .
$$

\section{Proof:}

See [Wer95] p.171.

The second part of this section is devoted to an introduction to the theory of distributions. Denote $\Omega$ an open subset of $\mathbb{R}^{d}$. We introduce the following conventional notation, viz.,

$$
\mathcal{D}(\Omega) \equiv C_{0}^{\infty}(\Omega), \quad \mathcal{E}(\Omega) \equiv C^{\infty}(\Omega) .
$$

\section{Definition A.3}

A continuous linear form on $\mathcal{D}(\Omega)$ is called a distribution. The set of all distributions is denoted by $\mathcal{D}^{\prime}(\Omega)$. Moreover we write $\langle T, \phi\rangle=T \phi$.

As an example, consider the map $L_{l o c}^{1}(\Omega) \rightarrow \mathcal{D}^{\prime}(\Omega), f \rightarrow T_{f}$ given by

$$
\left\langle T_{f}, \phi\right\rangle \equiv \int_{\Omega} f \phi d x
$$

For $f \in L_{\text {loc }}^{1}(\Omega), g \in C^{\infty}(\Omega), \phi \in \mathcal{D}(\Omega)$ we have (at least) $g f \in L_{l o c}^{1}(\Omega)$ and $g \phi \in \mathcal{D}(\Omega)$ and therefore we have the following relation, viz.,

$$
\langle g f, \phi\rangle=\langle f, g \phi\rangle .
$$

This can be regarded as a motivation for defining the multiplication of a distribution by a $C^{\infty}$ function, see e.g. [Tre67], p.250.

\section{Definition A.4}

For $T \in \mathcal{D}^{\prime}(\Omega), g \in C^{\infty}(\Omega)$ we define the distribution $g T$ via

$$
\langle g T, \phi\rangle=\langle T, g \phi\rangle \quad \forall \phi \in \mathcal{D}(\Omega) .
$$


Let $\Omega_{1} \subset \mathbb{R}^{d}$ be an open, bounded domain, let $\boldsymbol{y} \in \mathbb{R}^{d}$ be held fixed, and denote $\Omega_{2}=$ $\boldsymbol{y}+\Omega_{1} \equiv\left\{\boldsymbol{x}+\boldsymbol{y}, \boldsymbol{x} \in \Omega_{1}\right\}$. Let $\tau \boldsymbol{y}: L_{l o c}^{1}\left(\Omega_{1}\right) \mapsto L_{l o c}^{1}\left(\Omega_{2}\right), f \mapsto f(\cdot-\boldsymbol{y})$ denote the translation operator. Then we have

$$
\langle\tau \boldsymbol{y} f, \phi\rangle=\int_{\Omega_{2}} f(\boldsymbol{x}-\boldsymbol{y}) \phi(\boldsymbol{x}) d \boldsymbol{x}=\int_{\Omega_{1}} f(\boldsymbol{z}) \phi(\boldsymbol{x}+\boldsymbol{z}) d \boldsymbol{x}=\left\langle f, \tau_{-} \boldsymbol{y} \phi\right\rangle .
$$

This motivates the following definition (cf. [Tre67], p.296):

Definition A.5

For $T \in \mathcal{D}^{\prime}\left(\Omega_{1}\right)$ we define the distribution $\tau \boldsymbol{y} T \in \mathcal{D}^{\prime}\left(\Omega_{2}\right)$ via

$$
\langle\tau \boldsymbol{y} T, \phi\rangle=\left\langle T, \tau_{-} \boldsymbol{y} \phi\right\rangle \quad \forall \phi \in \mathcal{D}\left(\Omega_{2}\right) .
$$

The derivation of a distribution is defined as follows, cf. [Hor66], pp.323:

Definition A.6

Given $T \in \mathcal{D}^{\prime}(\Omega), g \in C^{\infty}(\Omega)$ we define the distribution $D^{\boldsymbol{\alpha}} T\left(\boldsymbol{\alpha} \in \mathbb{N}^{d}\right)$ as

$$
\left\langle D^{\boldsymbol{\alpha}} T, \phi\right\rangle \equiv(-1)^{|\boldsymbol{\alpha}|}\left\langle T, D^{\boldsymbol{\alpha}} \phi\right\rangle, \quad \forall \phi \in \mathcal{D}(\Omega) .
$$

Next we introduce the restriction of a distribution, see [Hor66], p.317.

\section{Definition A.7}

1. Let $U, \Omega$ be two open subsets of $\mathbb{R}^{2}$ with $U \subset \Omega$. Every function belonging to $\mathcal{D}(U)$ can be considered as a function belonging to $\mathcal{D}(\Omega)$. If $T \in \mathcal{D}^{\prime}(\Omega)$, then its restriction to $\mathcal{D}(U)$ is the distribution $\left.T\right|_{U} \in \mathcal{D}^{\prime}(U)$ defined by

$$
\left\langle\left. T\right|_{U}, \phi\right\rangle \equiv\langle T, \phi\rangle, \quad \forall \phi \in \mathcal{D}(U) .
$$

2. For $T \in \mathcal{D}^{\prime}(\Omega)$ the support of $T$ is defined as

$$
\operatorname{supp}(T) \equiv \Omega \backslash\left\{\boldsymbol{x} \in \Omega \text { : There exists an open set } U \text { with } \boldsymbol{x} \in U \text { and }\left.T\right|_{U}=0\right\} .
$$

An important subset of $\mathcal{D}^{\prime}(\Omega)$ is characterised by the following theorem, see [Hor66] , p.320.

$$
\mathcal{E}^{\prime}(\Omega)=\left\{T \in \mathcal{D}^{\prime}(\Omega): \operatorname{supp}(T) \text { is compact a compact subset of } \Omega\right\} .
$$

Moreover we introduce $\mathcal{S}^{\prime}$, being the (strong) dual space of $\mathcal{S}$. The distributions belonging to $\mathcal{S}^{\prime}$ are referred to as tempered distributions in $\mathbb{R}^{d}$, see [Tre67], p.272. The following inclusion holds: $\mathcal{E}^{\prime} \subset \mathcal{S}^{\prime} \subset \mathcal{D}^{\prime}$. Next we introduce convolution of a distribution and a test function, see [Ure67], p.287.

\section{Definition A.8}

For $f \in L_{\text {loc }}^{1}\left(\mathbb{R}^{d}\right), \phi \in \mathcal{D}\left(\mathbb{R}^{d}\right)$ we define

$$
(f * \phi)(\boldsymbol{x})=\int_{\mathbb{R}^{d}} f(\boldsymbol{x}-\boldsymbol{y}) \phi(\boldsymbol{y}) d \boldsymbol{y}=\int_{\mathbb{R}^{d}} f(\boldsymbol{z}) \phi(\boldsymbol{x}-\boldsymbol{z})=\langle f, \tau \boldsymbol{x} \check{\phi}\rangle .
$$

Here $\tau_{\boldsymbol{x}}$ denotes the translation operator $\tau_{\boldsymbol{x}} f \equiv f(\boldsymbol{x}+\cdot)$ and we use $\check{f}(\boldsymbol{y}) \equiv f(-\boldsymbol{y})$. For (i) $T \in \mathcal{D}^{\prime}\left(\mathbb{R}^{d}\right), \phi \in \mathcal{D}\left(\mathbb{R}^{d}\right)$, (ii) $T \in \mathcal{E}^{\prime}\left(\mathbb{R}^{d}\right), \phi \in \mathcal{E}\left(\mathbb{R}^{d}\right)$ and (iii) $T \in \mathcal{S}^{\prime}\left(\mathbb{R}^{d}\right), \phi \in \mathcal{S}\left(\mathbb{R}^{d}\right)$ resp. we define $T * \phi(\boldsymbol{x}) \equiv\langle T, \tau \boldsymbol{x} \check{\phi}\rangle$. 
The following theorem precises the fact that you can smooth a "rough" function by convolution with a "smooth" function, cf. [Tref7], pp.289,

\section{Theorem A.4}

For $T \in \mathcal{D}^{\prime}\left(\mathbb{R}^{d}\right), \phi \in \mathcal{D}\left(\mathbb{R}^{d}\right)$ we have $T * \phi \in \mathcal{E}\left(\mathbb{R}^{d}\right)$. If $T \in \mathcal{E}^{\prime}\left(\mathbb{R}^{d}\right), \phi \in \mathcal{E}\left(\mathbb{R}^{d}\right)$ then $T * \phi \in \mathcal{E}\left(\mathbb{R}^{d}\right)$. Given $T \in \mathcal{E}^{\prime}\left(\mathbb{R}^{d}\right), \phi \in \mathcal{D}\left(\mathbb{R}^{d}\right)$ then $T * \phi \in \mathcal{D}\left(\mathbb{R}^{d}\right)$. For $S \in \mathcal{S}^{\prime}\left(\mathbb{R}^{d}\right)$, $\phi \in \mathcal{S}\left(\mathbb{R}^{d}\right)$ we have $S * f \in \mathcal{S}\left(\mathbb{R}^{d}\right)$. In all cases we have

$$
D^{\boldsymbol{\alpha}}(S * f)=\left(D^{\boldsymbol{\alpha}} S\right) * f=S *\left(D^{\boldsymbol{\alpha}} f\right) .
$$

Convolution of distributions can be defined in the following cases, see [Tre67], p.293.

\section{Definition A.9}

Let $T \in \mathcal{D}^{\prime}\left(\mathbb{R}^{d}\right), \phi, \psi \in \mathcal{D}\left(\mathbb{R}^{d}\right)$. Then we have $\langle T * \phi, \psi\rangle=\langle T, \check{\phi} \psi\rangle$. For $T \in \mathcal{D}^{\prime}\left(\mathbb{R}^{d}\right)$, $S \in \mathcal{E}^{\prime}\left(\mathbb{R}^{d}\right)$ we define $T * S=S * T$ via

$$
\langle T * S, \phi\rangle=\langle T, \check{S} \phi\rangle, \quad \text { with } \quad\langle\check{T}, \phi\rangle \equiv\langle T, \check{\phi}\rangle .
$$

Given $T \in \mathcal{E}^{\prime}\left(\mathbb{R}^{d}\right), S \in \mathcal{D}^{\prime}\left(\mathbb{R}^{d}\right)$, we define $T * S=S * T$ via

$$
\langle T * S, \phi\rangle=\langle T, \check{S} \phi\rangle .
$$

Finally we define Fourier transformation in the space $\mathcal{S}^{\prime}$, cf. [Wre67], p.275., [Hor66], p.411.

\section{Definition A.10}

Let $T \in \mathcal{S}^{\prime}\left(\mathbb{R}^{d}\right)$. Then we define its Fourier transform $\mathcal{F}(T) \equiv \hat{T} \in \mathcal{S}^{\prime}\left(\mathbb{R}^{d}\right)$ via

$$
\langle\hat{T}, g\rangle=\langle T, \hat{g}\rangle, \quad g \in \mathcal{S}\left(\mathbb{R}^{d}\right) .
$$

Now we can state the inverse Fourier formula for distributions, see e.g. [Hor66], pp.411:

\section{Theorem A.5}

The Fourier transformation is an isomorphism of $\mathcal{S}^{\prime}\left(\mathbb{R}^{d}\right)$ onto $\mathcal{S}^{\prime}\left(\mathbb{R}^{d}\right)$ satisfying $\mathcal{F} \mathcal{F}(T)=$ $\check{T}$. Moreover for $T \in \mathcal{S}^{\prime}\left(\mathbb{R}^{d}\right)$ we have

$$
\mathcal{F}\left(D^{\boldsymbol{\alpha}} T\right)=(-i)^{|\boldsymbol{\alpha}|} \boldsymbol{x}^{\alpha} \mathcal{F} T
$$

\section{Remark A.1}

Since $L^{p}\left(\mathbb{R}^{d}\right) \subset \mathcal{S}^{\prime}\left(\mathbb{R}^{d}\right)$, we can define the Fourier transform for all $L^{p}$-functions. But in the case $2<p \leq \infty$ the Fourier transform in general is not a function, but only a tempered distribution.

Finally we mention the following relation, cf. [Wla.72] p.122. Let $f \in \mathcal{S}^{\prime}\left(\mathbb{R}^{d}\right)$ and $g \in \mathcal{E}^{\prime}\left(\mathbb{R}^{d}\right)$. Then

$$
\mathcal{F}(f * g)=\mathcal{F}(f) \mathcal{F}(g) .
$$




\section{A.2. Closure approximations for LES}

This chapter is devoted to two closure approximations for the momentum and temperature equation. Both approaches are based on the Fourier transform. We start with the term $\langle\boldsymbol{u} \otimes \boldsymbol{u}\rangle_{\Delta}$, and write $\boldsymbol{u}=\overline{\boldsymbol{u}}+\boldsymbol{u}^{\prime}$. Since

$$
\overline{\boldsymbol{u}}=g_{\Delta} * \boldsymbol{u}=g_{\Delta} *\left(\overline{\boldsymbol{u}}+\boldsymbol{u}^{\prime}\right)=g_{\Delta} * \overline{\boldsymbol{u}}+g_{\Delta} * \boldsymbol{u}^{\prime},
$$

Fourier transform gives (using A.4)

$$
\hat{\overline{\boldsymbol{u}}}(\boldsymbol{k})=\hat{g}_{\Delta}(\boldsymbol{k}) \hat{\overline{\boldsymbol{u}}}(\boldsymbol{k})+\hat{g}_{\Delta}(\boldsymbol{k}) \hat{\boldsymbol{u}}^{\prime}(\boldsymbol{k}) .
$$

Therefore $\hat{\boldsymbol{u}}^{\prime}$ is given exactly by

$$
\hat{\boldsymbol{u}}^{\prime}=\left(\frac{1}{\hat{g}_{\Delta}(\boldsymbol{k})}-1\right) \hat{\boldsymbol{u}}(\boldsymbol{k}) .
$$

We introduce the abbreviation $[\hat{\boldsymbol{v}}(\boldsymbol{k}) \star \hat{\boldsymbol{w}}(\boldsymbol{k})]_{i j}=\hat{v}_{i}(\boldsymbol{k}) * \hat{w}_{j}(\boldsymbol{k})$. Thus the Fourier transformed terms in (4.28) read

$$
\begin{aligned}
\mathcal{F}\left(\langle\overline{\boldsymbol{u}} \otimes \overline{\boldsymbol{u}}\rangle_{\Delta}\right) & =\hat{g}_{\Delta}(\boldsymbol{k})[\hat{\overline{\boldsymbol{u}}}(\boldsymbol{k}) \star \hat{\overline{\boldsymbol{u}}}(\boldsymbol{k})] \\
\mathcal{F}\left(\left\langle\overline{\boldsymbol{u}} \otimes \boldsymbol{u}^{\prime}\right\rangle_{\Delta}\right) & =\hat{g}_{\Delta}(\boldsymbol{k})\left[\hat{\overline{\boldsymbol{u}}}(\boldsymbol{k}) \star\left[\left(\frac{1}{\hat{g}_{\Delta}(\boldsymbol{k})}-1\right) \hat{\overline{\boldsymbol{u}}}(\boldsymbol{k})\right]\right], \\
\mathcal{F}\left(\left\langle\boldsymbol{u}^{\prime} \otimes \overline{\boldsymbol{u}}\right\rangle_{\Delta}\right) & =\hat{g}_{\Delta}(\boldsymbol{k})\left[\left[\left(\frac{1}{\hat{g}_{\Delta}(\boldsymbol{k})}-1\right) \hat{\overline{\boldsymbol{u}}}(\boldsymbol{k})\right] \star \hat{\overline{\boldsymbol{u}}}(\boldsymbol{k})\right], \\
\mathcal{F}\left(\left\langle\boldsymbol{u}^{\prime} \otimes \boldsymbol{u}^{\prime}\right\rangle_{\Delta}\right) & =\hat{g}_{\Delta}(\boldsymbol{k})\left[\left[\left(\frac{1}{\hat{g}_{\Delta}(\boldsymbol{k})}-1\right) \hat{\overline{\boldsymbol{u}}}(\boldsymbol{k})\right] \star\left[\left(\frac{1}{\hat{g}_{\Delta}(\boldsymbol{k})}-1\right) \hat{\overline{\boldsymbol{u}}}(\boldsymbol{k})\right]\right] .
\end{aligned}
$$

Similarly, $\hat{\theta}^{\prime}$ is given exactly by

$$
\hat{\theta}^{\prime}=\left(\frac{1}{\hat{g}_{\Delta}(\boldsymbol{k})}-1\right) \hat{\bar{\theta}}(\boldsymbol{k}) .
$$

and hence we get

$$
\begin{aligned}
\mathcal{F}\left(\langle\bar{\theta} \overline{\boldsymbol{u}}\rangle_{\Delta}\right) & =\hat{g}_{\Delta}(\boldsymbol{k})[\hat{\bar{\theta}}(\boldsymbol{k}) * \hat{\overline{\boldsymbol{u}}}(\boldsymbol{k})] \\
\mathcal{F}\left(\left\langle\bar{\theta} \boldsymbol{u}^{\prime}\right\rangle_{\Delta}\right) & =\hat{g}_{\Delta}(\boldsymbol{k})\left[\hat{\bar{\theta}}(\boldsymbol{k}) *\left[\left(\frac{1}{\hat{g}_{\Delta}(\boldsymbol{k})}-1\right) \hat{\overline{\boldsymbol{u}}}(\boldsymbol{k})\right]\right], \\
\mathcal{F}\left(\left\langle\theta^{\prime} \overline{\boldsymbol{u}}\right\rangle_{\Delta}\right) & =\hat{g}_{\Delta}(\boldsymbol{k})\left[\left[\left(\frac{1}{\hat{g}_{\Delta}(\boldsymbol{k})}-1\right) \hat{\bar{\theta}}(\boldsymbol{k})\right] * \hat{\overline{\boldsymbol{u}}}(\boldsymbol{k})\right], \\
\mathcal{F}\left(\left\langle\theta^{\prime} \boldsymbol{u}^{\prime}\right\rangle_{\Delta}\right) & =\hat{g}_{\Delta}(\boldsymbol{k})\left[\left[\left(\frac{1}{\hat{g}_{\Delta}(\boldsymbol{k})}-1\right) \hat{\bar{\theta}}(\boldsymbol{k})\right] *\left[\left(\frac{1}{\hat{g}_{\Delta}(\boldsymbol{k})}-1\right) \hat{\overline{\boldsymbol{u}}}(\boldsymbol{k})\right]\right] .
\end{aligned}
$$

Equations (A.8)-(A.11) and (A.13)- A.16) are the starting point for the two closure approximations that will be presented in the following. The key is how to approximate $g_{\Delta}(\boldsymbol{k})$ and $\left(g_{\Delta}(\boldsymbol{k})-1\right)^{-1}$ by simpler functions. The conventional issue consists in expanding both 
terms in a Taylor series in $\Delta$, see e.g. [Ald90]. GALDI and LAYTON proposed using a rational or Pade approximation, see [GL00].

First we review the conventional strategy, which performs a Taylor approximation, viz.,

$$
g_{\Delta}(\boldsymbol{k})=1-\frac{\Delta^{2}}{4 \gamma} \boldsymbol{k}^{2}+\mathcal{O}^{\text {formal }}\left(\Delta^{4}\right), \quad \frac{1}{g_{\Delta}(\boldsymbol{k})}-1=\frac{\Delta^{2}}{4 \gamma} \boldsymbol{k}^{2}+\mathcal{O}^{\text {formal }}\left(\Delta^{4}\right) .
$$

Using this and (A.3) in (A.8)-(A.11) and taking inverse Fourier transforms yields

$$
\begin{aligned}
\langle\overline{\boldsymbol{u}} \otimes \overline{\boldsymbol{u}}\rangle_{\Delta} & =\overline{\boldsymbol{u}} \otimes \overline{\boldsymbol{u}}+\frac{\Delta^{2}}{4 \gamma} \nabla^{2}(\overline{\boldsymbol{u}} \otimes \overline{\boldsymbol{u}}) \quad+\mathcal{O}^{\text {formal }}\left(\Delta^{4}\right) \\
\left\langle\overline{\boldsymbol{u}} \otimes \boldsymbol{u}^{\prime}\right\rangle_{\Delta} & =-\frac{\Delta^{2}}{4 \gamma} \overline{\boldsymbol{u}} \otimes\left(\nabla^{2} \overline{\boldsymbol{u}}\right)+\mathcal{O}^{\text {formal }}\left(\Delta^{4}\right) \\
\left\langle\boldsymbol{u}^{\prime} \otimes \overline{\boldsymbol{u}}\right\rangle_{\Delta} & =-\frac{\Delta^{2}}{4 \gamma}\left(\nabla^{2} \overline{\boldsymbol{u}}\right) \otimes \overline{\boldsymbol{u}}+\mathcal{O}^{\text {formal }}\left(\Delta^{4}\right) \\
\left\langle\boldsymbol{u}^{\prime} \otimes \boldsymbol{u}^{\prime}\right\rangle_{\Delta} & =\frac{\Delta^{4}}{16 \gamma^{2}}\left(\nabla^{2} \overline{\boldsymbol{u}}\right) \otimes\left(\nabla^{2} \overline{\boldsymbol{u}}\right)+\mathcal{O}^{\text {formal }}\left(\Delta^{6}\right)
\end{aligned}
$$

Applying the relation (with the notation $\left.(\nabla \boldsymbol{V} \bullet \nabla \boldsymbol{W})_{i j}=\sum_{l=1}^{d} \frac{\partial V_{i}}{\partial x_{l}} \frac{\partial W_{j}}{\partial x_{l}}\right)$

$$
\nabla^{2}(\overline{\boldsymbol{u}} \otimes \overline{\boldsymbol{u}})_{i j}=\nabla^{2} \overline{\boldsymbol{u}} \otimes \overline{\boldsymbol{u}}+2 \nabla \overline{\boldsymbol{u}} \bullet \nabla \overline{\boldsymbol{u}}+\overline{\boldsymbol{u}} \otimes \nabla^{2} \overline{\boldsymbol{u}}
$$

we finally get

$$
\langle\boldsymbol{u} \otimes \boldsymbol{u}\rangle_{\Delta}=\overline{\boldsymbol{u}} \otimes \overline{\boldsymbol{u}}+\frac{\Delta^{2}}{2 \gamma} \nabla \overline{\boldsymbol{u}} \bullet \nabla \overline{\boldsymbol{u}}+\mathcal{O}^{\text {formal }}\left(\Delta^{4}\right)
$$

Similarly, for the temperature equation we obtain using (A.3) in (A.13)-(A.16) and taking inverse Fourier transforms

$$
\begin{aligned}
\langle\bar{\theta} \overline{\boldsymbol{u}}\rangle_{\Delta} & =\bar{\theta} \overline{\boldsymbol{u}}+\frac{\Delta^{2}}{4 \gamma} \nabla^{2}(\bar{\theta} \overline{\boldsymbol{u}})+\mathcal{O}^{\text {formal }}\left(\Delta^{4}\right), \\
\left\langle\bar{\theta} \boldsymbol{u}^{\prime}\right\rangle_{\Delta} & =-\frac{\Delta^{2}}{4 \gamma} \bar{\theta}\left(\nabla^{2} \overline{\boldsymbol{u}}\right)+\mathcal{O}^{\text {formal }}\left(\Delta^{4}\right), \\
\left\langle\theta^{\prime} \overline{\boldsymbol{u}}\right\rangle_{\Delta} & =-\frac{\Delta^{2}}{4 \gamma}\left(\nabla^{2} \bar{\theta}\right) \overline{\boldsymbol{u}}+\mathcal{O}^{\text {formal }}\left(\Delta^{4}\right), \\
\left\langle\theta^{\prime} \boldsymbol{u}^{\prime}\right\rangle_{\Delta} & =\frac{\Delta^{4}}{16 \gamma^{2}}\left(\nabla^{2} \bar{\theta}\right)\left(\nabla^{2} \overline{\boldsymbol{u}}\right)+\mathcal{O}^{\text {formal }}\left(\Delta^{6}\right) .
\end{aligned}
$$

Using the relation (where we used the notation $\left.(\nabla \bar{\theta} \circ \nabla \overline{\boldsymbol{u}})_{j}=\sum_{l=1}^{d} \frac{\partial \bar{\theta}}{\partial x_{l}} \frac{\partial U_{j}}{\partial x_{l}}\right)$

$$
\nabla^{2}(\bar{\theta} \overline{\boldsymbol{u}})_{i}=\left(\nabla^{2} \bar{\theta} \overline{\boldsymbol{u}}+2 \nabla \bar{\theta} \circ \nabla \overline{\boldsymbol{u}}+\bar{\theta} \nabla^{2} \overline{\boldsymbol{u}}\right)_{i}
$$

we end up with

$$
\langle\theta \boldsymbol{u}\rangle_{\Delta}=\bar{\theta} \overline{\boldsymbol{u}}+\frac{\Delta^{2}}{2 \gamma} \nabla \bar{\theta} \circ \nabla \overline{\boldsymbol{u}}+\mathcal{O}^{\text {formal }}\left(\Delta^{4}\right)
$$




\section{Remark A.2}

It is worth stressing the fact that in this approach the filter kernel has been expanded into a Taylor series. Although leading to the same results this is in contrast to the approach by LeOnard and Clark, cf. [Ald90]. They expended $\overline{\boldsymbol{u}}$ and $\boldsymbol{u}^{\prime}$ in a Taylor series, which is not correct since $\boldsymbol{u}^{\prime}$ cannot be assumed to be regular enough.

Moreover the following fundamental difference between the Gaussian filter and its Taylor approximation should be underlined: The Gaussian filter attenuates high frequencies (since $\exp \left(\frac{-\Delta^{2}}{4 \gamma}\|\boldsymbol{k}\|_{2}^{2}\right) \rightarrow 0$ as $\left.\|\boldsymbol{k}\| \rightarrow \infty\right)$. The Taylor approximation, however, increases high wave number components due to the term $\Delta^{2}(4 \gamma)^{-1}|| \boldsymbol{k} \|_{2}^{2}$, as $\Delta^{2}(4 \gamma)^{-1}\|\boldsymbol{k}\|_{2}^{2} \rightarrow \infty$ as $\|\boldsymbol{k}\| \rightarrow \infty$.

Therefore Layton et al. looked for an approximation of the same order of accuracy for large eddies (small $|\boldsymbol{k}|$ ) but which attenuates the small eddies. Layton proposed to consider the following Pade approximation to $\hat{g}_{\Delta}(\boldsymbol{k})$ :

$$
\hat{g}_{\Delta}(\boldsymbol{k})=\frac{1}{1+\frac{\Delta^{2}}{4 \gamma}|\boldsymbol{k}|^{2}}+\mathcal{O}^{\text {formal }}\left(\frac{\Delta^{4}}{16 \gamma^{2}}|\boldsymbol{k}|^{4}\right), \quad \frac{1}{\hat{g}_{\Delta}(\boldsymbol{k})}-1=\frac{\Delta^{2}}{4 \gamma}|\boldsymbol{k}|^{2}+\mathcal{O}^{\text {formal }}\left(\frac{\Delta^{4}}{16 \gamma^{2}}|\boldsymbol{k}|^{4}\right) .
$$

This approximation has the same formal accuracy. But it has the major advantage that $\hat{g}_{\Delta}(\boldsymbol{k}) \rightarrow 0$ as $|k| \rightarrow \infty$. Using (A.22) equations (A.8)-(A.11) become

$$
\begin{aligned}
& \mathcal{F}\left(\langle\overline{\boldsymbol{u}} \otimes \overline{\boldsymbol{u}}\rangle_{\Delta}\right)=\left(1+\frac{\Delta^{2}}{4 \gamma}|\boldsymbol{k}|^{2}\right)^{-1}[\hat{\overline{\boldsymbol{u}}}(\boldsymbol{k}) \star \hat{\overline{\boldsymbol{u}}}(\boldsymbol{k})]+\mathcal{O}^{\text {formal }}\left(\Delta^{4}\right) \\
& \mathcal{F}\left(\left\langle\overline{\boldsymbol{u}} \otimes \boldsymbol{u}^{\prime}\right\rangle_{\Delta}\right)=\left(1+\frac{\Delta^{2}}{4 \gamma}|\boldsymbol{k}|^{2}\right)^{-1}\left[\hat{\overline{\boldsymbol{u}}}(\boldsymbol{k}) \star\left[\frac{\Delta^{2}}{4 \gamma}|\boldsymbol{k}|^{2} \hat{\overline{\boldsymbol{u}}}(\boldsymbol{k})\right]\right]+\mathcal{O}^{\text {formal }}\left(\Delta^{4}\right), \\
& \mathcal{F}\left(\left\langle\boldsymbol{u}^{\prime} \otimes \overline{\boldsymbol{u}}\right\rangle_{\Delta}\right)=\left(1+\frac{\Delta^{2}}{4 \gamma}|\boldsymbol{k}|^{2}\right)^{-1}\left[\left[\frac{\Delta^{2}}{4 \gamma}|\boldsymbol{k}|^{2} \hat{\overline{\boldsymbol{u}}}(\boldsymbol{k})\right] \star \hat{\overline{\boldsymbol{u}}}(\boldsymbol{k})\right]+\mathcal{O}^{\text {formal }}\left(\Delta^{4}\right), \\
& \mathcal{F}\left(\left\langle\boldsymbol{u}^{\prime} \otimes \boldsymbol{u}^{\prime}\right\rangle_{\Delta}\right)=\left(1+\frac{\Delta^{2}}{4 \gamma}|\boldsymbol{k}|^{2}\right)^{-1}\left[\left[\frac{\Delta^{4}}{16 \gamma^{2}}|\boldsymbol{k}|^{2} \hat{\overline{\boldsymbol{u}}}(\boldsymbol{k})\right] \star\left[|\boldsymbol{k}|^{2} \hat{\overline{\boldsymbol{u}}}(\boldsymbol{k})\right]\right]+\mathcal{O}^{\text {formal }}\left(\Delta^{6}\right) .
\end{aligned}
$$

Taking inverse Fourier transform gives :

$$
\begin{aligned}
\langle\overline{\boldsymbol{u}} \otimes \overline{\boldsymbol{u}}\rangle_{\Delta} & =\left[-\frac{\Delta^{2}}{4 \gamma} \nabla^{2}+\mathbb{I}\right]^{-1}[\overline{\boldsymbol{u}} \otimes \overline{\boldsymbol{u}}]+\mathcal{O}^{\text {formal }}\left(\Delta^{4}\right) \\
\left\langle\overline{\boldsymbol{u}} \otimes \boldsymbol{u}^{\prime}\right\rangle_{\Delta} & =-\frac{\Delta^{2}}{4 \gamma}\left[-\frac{\Delta^{2}}{4 \gamma} \nabla^{2}+\mathbb{I}\right]^{-1}\left[\overline{\boldsymbol{u}} \otimes\left(\nabla^{2} \overline{\boldsymbol{u}}\right)\right]+\mathcal{O}^{\text {formal }}\left(\Delta^{4}\right), \\
\left\langle\boldsymbol{u}^{\prime} \otimes \overline{\boldsymbol{u}}\right\rangle_{\Delta} & =-\frac{\Delta^{2}}{4 \gamma}\left[-\frac{\Delta^{2}}{4 \gamma} \nabla^{2}+\mathbb{I}\right]^{-1}\left[\left(\nabla^{2} \overline{\boldsymbol{u}}\right) \otimes \overline{\boldsymbol{u}}\right]+\mathcal{O}^{\text {formal }}\left(\Delta^{4}\right), \\
\left\langle\boldsymbol{u}^{\prime} \otimes \boldsymbol{u}^{\prime}\right\rangle_{\Delta} & =\frac{\Delta^{4}}{16 \gamma^{2}}\left[-\frac{\Delta^{2}}{4 \gamma} \nabla^{2}+\mathbb{I}\right]^{-1}\left[\left(\nabla^{2} \overline{\boldsymbol{u}}\right) \otimes\left(\nabla^{2} \overline{\boldsymbol{u}}\right)\right]+\mathcal{O}^{\text {formal }}\left(\Delta^{6}\right) .
\end{aligned}
$$


Thus we obtain

$$
\left\langle\left(\overline{\boldsymbol{u}}+\boldsymbol{u}^{\prime}\right) \otimes\left(\overline{\boldsymbol{u}}+\boldsymbol{u}^{\prime}\right)\right\rangle_{\Delta}=\overline{\boldsymbol{u}} \otimes \overline{\boldsymbol{u}}+\left[-\frac{\Delta^{2}}{4 \gamma} \nabla^{2}+\mathbb{I}\right]^{-1}\left(\frac{\Delta^{2}}{2 \gamma} \nabla \overline{\boldsymbol{u}} \bullet \nabla \overline{\boldsymbol{u}}\right)+\mathcal{O}^{\text {formal }}\left(\Delta^{4}\right) .
$$

For the temperature equation we get (using (A.22)

$$
\begin{aligned}
\mathcal{F}\left(\langle\bar{\theta} \overline{\boldsymbol{u}}\rangle_{\Delta}\right)= & \left(1+\frac{\Delta^{2}}{4 \gamma}|\boldsymbol{k}|^{2}\right)^{-1}[\hat{\bar{\theta}}(\boldsymbol{k}) * \hat{\overline{\boldsymbol{u}}}(\boldsymbol{k})]+\mathcal{O}^{\text {formal }}\left(\Delta^{4}\right) \\
\mathcal{F}\left(\left\langle\bar{\theta} \boldsymbol{u}^{\prime}\right\rangle_{\Delta}\right)= & \left(1+\frac{\Delta^{2}}{4 \gamma}|\boldsymbol{k}|^{2}\right)^{-1}\left[\hat{\bar{\theta}}(\boldsymbol{k}) *\left[\frac{\Delta^{2}}{4 \gamma}|\boldsymbol{k}|^{2} \hat{\overline{\boldsymbol{u}}}(\boldsymbol{k})\right]\right]+\mathcal{O}^{\text {formal }}\left(\Delta^{4}\right) \\
\mathcal{F}\left(\left\langle\theta^{\prime} \overline{\boldsymbol{u}}\right\rangle_{\Delta}\right)= & \left(1+\frac{\Delta^{2}}{4 \gamma}|\boldsymbol{k}|^{2}\right)^{-1}\left[\left[\frac{\Delta^{2}}{4 \gamma}|\boldsymbol{k}|^{2} \hat{\bar{\theta}}(\boldsymbol{k})\right] * \hat{\overline{\boldsymbol{u}}}(\boldsymbol{k})\right]+\mathcal{O}^{\text {formal }}\left(\Delta^{4}\right) \\
\mathcal{F}\left(\left\langle\theta^{\prime} \boldsymbol{u}^{\prime}\right\rangle_{\Delta}\right)= & \left(1+\frac{\Delta^{2}}{4 \gamma}|\boldsymbol{k}|^{2}\right)^{-1}\left[\left[\frac{\Delta^{4}}{16 \gamma^{2}}|\boldsymbol{k}|^{2} \hat{\bar{\theta}}(\boldsymbol{k})\right] *\left[|\boldsymbol{k}|^{2} \hat{\overline{\boldsymbol{u}}}(\boldsymbol{k})\right]\right] \\
& +\mathcal{O}^{\text {formal }}\left(\Delta^{6}\right)
\end{aligned}
$$

Taking inverse Fourier transform gives :

$$
\begin{aligned}
\langle\bar{\theta} \overline{\boldsymbol{u}}\rangle_{\Delta} & =\left[-\frac{\Delta^{2}}{4 \gamma} \nabla^{2}+\mathbb{I}\right]^{-1}[\bar{\theta} \overline{\boldsymbol{u}}]+\mathcal{O}^{\text {formal }}\left(\Delta^{4}\right) \\
\left\langle\bar{\theta} \boldsymbol{u}^{\prime}\right\rangle_{\Delta} & =-\frac{\Delta^{2}}{4 \gamma}\left[-\frac{\Delta^{2}}{4 \gamma} \nabla^{2}+\mathbb{I}\right]^{-1}\left[\bar{\theta}\left(\nabla^{2} \overline{\boldsymbol{u}}\right)\right]+\mathcal{O}^{\text {formal }}\left(\Delta^{4}\right), \\
\left\langle\theta^{\prime} \overline{\boldsymbol{u}}\right\rangle_{\Delta} & =-\frac{\Delta^{2}}{4 \gamma}\left[-\frac{\Delta^{2}}{4 \gamma} \nabla^{2}+\mathbb{I}\right]^{-1}\left[\left(\nabla^{2} \bar{\theta}\right) \overline{\boldsymbol{u}}\right]+\mathcal{O}^{\text {formal }}\left(\Delta^{4}\right), \\
\left\langle\theta^{\prime} \boldsymbol{u}^{\prime}\right\rangle_{\Delta} & =\frac{\Delta^{4}}{16 \gamma^{2}}\left[-\frac{\Delta^{2}}{4 \gamma} \nabla^{2}+\mathbb{I}\right]^{-1}\left[\left(\nabla^{2} \bar{\theta}\right)\left(\nabla^{2} \overline{\boldsymbol{u}}\right)\right]+\mathcal{O}^{\text {formal }}\left(\Delta^{6}\right) .
\end{aligned}
$$

and finally we obtain

$(\mathrm{A} .24)\left\langle\left(\bar{\theta}+\theta^{\prime}\right) \otimes\left(\overline{\boldsymbol{u}}+\boldsymbol{u}^{\prime}\right)\right\rangle_{\Delta}=\bar{\theta} \overline{\boldsymbol{u}}+\left[-\frac{\Delta^{2}}{4 \gamma} \nabla^{2}+\mathbb{I}\right]^{-1}\left(\frac{\Delta^{2}}{2 \gamma} \nabla \bar{\theta} \circ \nabla \overline{\boldsymbol{u}}\right)+\mathcal{O}^{\text {formal }}\left(\Delta^{4}\right)$.

From (A.22) a further motivation for approximating filtering by convolution using a differential filter can be obtained:

$$
\mathcal{F}\left(g_{\Delta} * \boldsymbol{u}\right)=\mathcal{F}\left(g_{\Delta}\right) \mathcal{F}(\boldsymbol{u}) \approx \frac{1}{1+\frac{\|\boldsymbol{k}\|_{2}^{2}}{4 \gamma} \Delta^{2}} \mathcal{F}(\boldsymbol{u})=\mathcal{F}\left(\left[-\frac{\Delta^{2}}{4 \gamma} \nabla^{2}+\mathbb{I}\right]^{-1} \boldsymbol{u}\right)
$$




\section{B. Some mathematical tools for the Navier-Stokes equations}

\section{B.1. Functional analytic fundamentals}

In this section we introduce certain fundamental function spaces and recall some relations between them, which are used in Chapter 8 .

First we consider function spaces for the steady-state case, see e.g. [A]tt.92]. Denote

$$
D^{\boldsymbol{\alpha}} \phi(\boldsymbol{x}) \equiv \frac{\partial^{|\boldsymbol{\alpha}|_{\phi}}}{\partial x_{1}^{\alpha_{1}} \ldots \partial x_{d}^{\alpha_{d}}}(\boldsymbol{x}), \quad D^{(0, \ldots, 0)} \phi(\boldsymbol{x}) \equiv \phi(\boldsymbol{x})
$$

where $\boldsymbol{\alpha}$ is a multiindex, $\boldsymbol{\alpha}=\left(\alpha_{1}, \ldots, \alpha_{d}\right), \alpha_{i} \geq 0,|\boldsymbol{\alpha}|=\alpha_{1}+\ldots+\alpha_{d}$. Then we can define

$$
\begin{aligned}
& C(\Omega) \equiv\{\phi: \Omega \rightarrow \mathbb{R} \mid \phi \text { is continuous }\}, \\
& C^{m}(\Omega) \equiv\left\{\phi \quad: \quad \Omega \rightarrow \mathbb{R}\left|D^{\boldsymbol{\alpha}} \phi \in C(\Omega), \forall \boldsymbol{\alpha}:\right| \boldsymbol{\alpha} \mid \leq m\right\} .
\end{aligned}
$$

$C(\Omega)$ is called space of continuous functions and $C^{m}(\Omega)$ is called space of m-times continuously differentiable functions. $C^{m}(\bar{\Omega})$ is the set of functions in $C^{m}(\Omega)$, whose derivatives can be extended continuously onto the boundary $\partial \Omega$. The norm of $C^{m}(\bar{\Omega})$ is given by

$$
\|\phi\|_{C^{m}(\bar{\Omega})} \equiv \sup _{|\boldsymbol{\alpha}| \leq m} \sup _{\boldsymbol{x} \in \bar{\Omega}}\left|D^{\boldsymbol{\alpha}} \phi(\boldsymbol{x})\right| .
$$

Next we introduce the following spaces of Lebesque integrable functions with their norms:

$$
\begin{aligned}
L^{p}(\Omega) & \equiv\left\{\phi:\left.\Omega \rightarrow \mathbb{R}\left|\int_{\Omega}\right| \phi(\boldsymbol{x})\right|^{p} d \boldsymbol{x}<\infty\right\},\|\phi\|_{L^{p}(\Omega)} \equiv\left(\int_{\Omega}|\phi(\boldsymbol{x})|^{p} d \boldsymbol{x}\right)^{\frac{1}{p}}, \\
L^{\infty}(\Omega) & \equiv\left\{\phi: \Omega \rightarrow \mathbb{R} \mid \text { ess.sup }_{\Omega}|\phi(\boldsymbol{x})|<\infty\right\},\|\phi\|_{L^{\infty}(\Omega)} \equiv\left|\operatorname{ess.sup}_{\Omega}\right| \phi(\boldsymbol{x}) \mid .
\end{aligned}
$$

Moreover, we need the Sobolev spaces $W^{m, p}(\Omega)$, viz.,

$$
W^{m, p}(\Omega) \equiv\left\{\phi \in L^{p}(\Omega)\left|D^{\boldsymbol{\alpha}} \phi \in L^{p}(\Omega), \forall \boldsymbol{\alpha}:\right| \boldsymbol{\alpha} \mid \leq m\right\} .
$$

The spaces $W^{m, p}(\Omega)$ are equipped with the following norms,

$$
\begin{aligned}
\|\phi\|_{m, p, \Omega} \equiv & \|\phi\|_{W^{m, p}(\Omega)} \equiv\left(\sum_{|\boldsymbol{\alpha}| \leq m}\left\|D^{\boldsymbol{\alpha}} \phi\right\|_{L^{p}(\Omega)}^{p}\right)^{\frac{1}{p}}, \\
\|\phi\|_{W^{m, \infty}(\Omega)} & \equiv \sum_{|\boldsymbol{\alpha}| \leq m}\left\|D^{\boldsymbol{\alpha}} \phi\right\|_{L^{\infty}(\Omega)} .
\end{aligned}
$$

In the case $p=2$, we simply write $H^{m}(\Omega)$ instead of $W^{m, p}(\Omega)$ and omit the index $p=2$ in the corresponding norm. The spaces $H^{m-1 / 2}(\Gamma)$ and $W^{m-1 / p, p}(\Gamma), m=1,2,2<p<\infty$ consist of traces of functions in $H^{m}(\Omega)$ and $W^{m, p}(\Omega)$, resp., cf. [Ada75]. Now we summarise some embedding properties and basic inequalities. The following lemma states how $L^{q}$ norms can be bounded by Sobolev norms, cf. [Tem77], p.158. 


\section{Lemma B.1}

Let $\Omega$ be a locally Lipschitz bounded domain in $R^{d}$, d=2,3. Let $u \in W^{m, p}(\Omega), m \leq 1$, $1 \leq p<\infty$.

$$
\begin{array}{r}
\text { If } \frac{1}{p}-\frac{m}{d}=\frac{1}{q}>0 \text {, then }\|u\|_{L^{q}(\Omega)} \leq C_{L S, 1}(m, p, d, \Omega)\|u\|_{W^{m, p}(\Omega)} \\
\text { if } \frac{1}{p}-\frac{m}{d}=0,1 \leq q<\infty, \text { then }\|u\|_{L^{q}(\Omega)} \leq C_{L S, 2}(m, p, d, q, \Omega)\|u\|_{W^{m, p}(\Omega)} \\
\text { if } \frac{1}{p}-\frac{m}{d}<0, \text { then } u \text { is almost everywhere equal to some } \\
u_{c} \in C^{0}(\Omega) \text { s.t. }\left\|u_{c}\right\|_{C^{0}(\Omega)} \leq C_{L S, 3}(m, p, d, \Omega)\|u\|_{W^{m, p}(\Omega)}
\end{array}
$$

In particular, from lemma B.1 we can infer (see [Tem77], p.159) for the case $p=2, m=1$

$$
\begin{aligned}
d=2, & \|u\|_{L^{q}(\Omega)} \leq C(q, \Omega)\|u\|_{1, \Omega}, \quad 1 \leq q<\infty \\
d=3, & \|u\|_{L^{6}(\Omega)} \leq C(\Omega)\|u\|_{1, \Omega} .
\end{aligned}
$$

Next we recall Sobolev's embedding theorem, cf. [Tem77], p. 160.

\section{Lemma B.2}

We assume that $\Omega$ is sufficiently smooth s.t. there exists a continuous linear prolongation operator $\Pi: W^{m, p}(\Omega) \longrightarrow W^{m, p}\left(\mathbb{R}^{d}\right), d=2,3$. Then the embedding

$$
W^{1, p}(\Omega) \longrightarrow L^{q_{1}}(\Omega)
$$

is compact for any $q_{1}, 1 \leq q_{1}<\infty$, if $p \geq d$ and for any $q_{1} \in\left[1 ; \frac{d p}{d-p}[\right.$, if $1 \leq p<d$.

Moreover we will need Rellich's embedding theorem

\section{Lemma B.3}

Let $\Omega$ be a locally Lipschitz bounded domain in $R^{d}$, $d=2,3$. Then the embedding

$$
W^{m, p}(\Omega) \longrightarrow W^{n, p}(\Omega)
$$

is compact for any $m>n$. Then we have

$$
\|u\|_{W^{n, p}(\Omega)} \leq C_{m, n}^{R e l}\|u\|_{W^{m, p}(\Omega)} \quad \forall u \in W^{m, p}(\Omega) .
$$

\section{Proof:}

See Zeidler, IIA, Corollary 21.15, p.239.

Now we introduce the vector valued Lebesgue space $\boldsymbol{L}^{2}(\Omega) \equiv L^{2}(\Omega)^{d}$ with scalar product and norm

$$
(\boldsymbol{u}, \boldsymbol{v}) \equiv \sum_{i=1}^{d} \int_{\Omega} u_{i} v_{i} d \boldsymbol{x}, \quad\|\boldsymbol{u}\|_{\boldsymbol{L}^{2}(\Omega)}=\left(\sum_{i=1}^{d}\left\|u_{i}\right\|_{L^{2}(\Omega)}^{2}\right)^{1 / 2} .
$$


Additionally we define the expressions $(\nabla \boldsymbol{u}, \nabla \boldsymbol{v})$ and $\|\nabla \boldsymbol{u}\|_{\boldsymbol{L}_{(\Omega)}^{2}}$ by

$$
\|\nabla \boldsymbol{u}\|_{\boldsymbol{L}^{2}(\Omega)} \equiv\left(\sum_{i, j=1}^{d}\left\|\frac{\partial u_{i}}{\partial x_{j}}\right\|_{L^{2}(\Omega)}^{2}\right)^{1 / 2}, \quad(\nabla \boldsymbol{u}, \nabla \boldsymbol{v}) \equiv \sum_{i, j=1}^{d} \int_{\Omega} \frac{\partial u_{i}}{\partial x_{j}} \frac{\partial v_{i}}{\partial x_{j}} d \boldsymbol{x} .
$$

\section{Remark B.1}

Some care must be taken, since the interpretation of the scalar product, which is denoted by $(\cdot, \cdot)$ in any case, depends on its arguments. By the way, this is exactly the philosophy of operator overloading in object orient programming languages.

Moreover we need the following vector valued Sobolev spaces

$$
\boldsymbol{H}^{m}(\Omega) \equiv H^{m}(\Omega)^{d}, \quad \text { with norm }\|\boldsymbol{u}\|_{m, \Omega} \equiv\left(\sum_{i=1}^{d} \sum_{|\boldsymbol{\alpha}| \leq m}\left\|D^{\boldsymbol{\alpha}} u_{i}\right\|_{L^{2}(\Omega)}^{2}\right)^{1 / 2} .
$$

Next we consider the following trilinear form

$$
b_{\Omega}(\boldsymbol{u}, \boldsymbol{v}, \boldsymbol{w})=\int_{\Omega} \boldsymbol{u} \nabla \boldsymbol{v} \boldsymbol{w} d \boldsymbol{x}
$$

\section{Lemma B.4}

Let $\Omega$ be a locally Lipschitz bounded domain in $R^{d}, d=2,3$. Then the trilinear form $b_{\Omega}(\cdot, \cdot, \cdot)$ is trilinear continuous on $\boldsymbol{H}^{1}(\Omega) \times \boldsymbol{H}^{1}(\Omega) \times \boldsymbol{H}^{1}(\Omega)$, i.e. we have the following inequalities

$$
\begin{aligned}
\left|b_{\Omega}(\boldsymbol{u}, \boldsymbol{v}, \boldsymbol{w})\right| & \leq C_{b}\|\boldsymbol{u}\|_{1, \Omega}\|\nabla \boldsymbol{v}\|_{\boldsymbol{L}^{2}(\Omega)}\|\boldsymbol{w}\|_{1, \Omega}, \\
\left|b_{\Omega}(\boldsymbol{u}, \boldsymbol{v}, \boldsymbol{w})\right| & \leq C_{b}\|\boldsymbol{u}\|_{1, \Omega}\|\boldsymbol{v}\|_{1, \Omega}\|\boldsymbol{w}\|_{1, \Omega} .
\end{aligned}
$$

\section{Proof:}

See [Tem77], pp.163.

\section{Lemma B.5}

For any $\boldsymbol{u}, \boldsymbol{v}, \boldsymbol{w}$ in $\boldsymbol{H}^{1}(\Omega)$ satisfying (i) $\nabla \cdot \boldsymbol{u}=0$ and $\boldsymbol{u} \cdot \boldsymbol{n}=0$ on $\Gamma$ or (ii) $\nabla \cdot \boldsymbol{u}=0$ and $\boldsymbol{v}=\boldsymbol{w}=\mathbf{0}$ on $\Gamma$ we have

$$
\begin{aligned}
b_{\Omega}(\boldsymbol{u}, \boldsymbol{v}, \boldsymbol{v}) & =0 \\
b_{\Omega}(\boldsymbol{u}, \boldsymbol{v}, \boldsymbol{w}) & =-b_{\Omega}(\boldsymbol{u}, \boldsymbol{w}, \boldsymbol{v}) .
\end{aligned}
$$

\section{Proof:}

For the first equation

$$
\begin{aligned}
b_{\Omega}(\boldsymbol{u}, \boldsymbol{v}, \boldsymbol{v}) & =\sum_{i, j=1}^{d} \int_{\Omega} u_{i} \frac{\partial v_{j}}{\partial x_{i}} v_{j} d x=\sum_{i, j=1}^{d} \int_{\Omega} u_{i} \frac{1}{2} \frac{\partial v_{j}^{2}}{\partial x_{i}} d x \\
& =-\sum_{i, j=1}^{d} \int_{\Omega} \frac{1}{2} \frac{\partial u_{i}}{\partial x_{i}} v_{j}^{2} d x+\sum_{i, j=1}^{d} \int_{\Gamma} \frac{1}{2} u_{i} n_{i} v_{j}^{2} d s=0 .
\end{aligned}
$$


To prove the second equation, we apply the first relation to the following expression:

$$
0=b_{\Omega}(\boldsymbol{u}, \boldsymbol{v}+\boldsymbol{w}, \boldsymbol{v}+\boldsymbol{w})=b_{\Omega}(\boldsymbol{u}, \boldsymbol{v}, \boldsymbol{w})+b_{\Omega}(\boldsymbol{u}, \boldsymbol{w}, \boldsymbol{v}) .
$$

Next we recall the definition and important properties of an evolution triple.

\section{Definition B.1}

An evolution triple $X \subset H \subset X^{*}$ is understood to be the following

1. $\left(X,\|\cdot\|_{X}\right)$ is a real Hilbert-space with dual space being denoted by $\left(X^{*},\|\cdot\|_{X^{*}}\right)$,

2. $\left(H,\|\cdot\|_{H}\right)$ is a real Hilbert-space,

3. the embedding $X \subset H$ is continuous, i.e.

$$
\|v\|_{H} \leq C_{\text {evo }}\|v\|_{X} \quad \forall v \in X
$$

and $X$ is dense in $H$.

According to Riesz' theorem we identify $H$ and $H^{*}$. If $X \subset H$ is compact, then the inclusion $I: X \subset X^{*}$ is also compact. Note that $X \equiv H^{1}(\Omega), H \equiv L^{2}(\Omega)$ and $X^{*} \equiv H^{-1}(\Omega)$ constitute an evolution triple.

Now we introduce the spaces of divergence free vector functions. $\boldsymbol{L}_{\text {div }}(\Omega)$ is the closure of

$$
\left\{\phi \in C_{0}^{\infty}(\Omega): \nabla \cdot \phi=0\right\}
$$

in the topology induced by $\boldsymbol{L}^{2}(\Omega)$. Moreover

$$
\boldsymbol{H}_{\text {div }}(\Omega)=\left\{\boldsymbol{\phi} \in \boldsymbol{H}_{0}^{1}(\Omega): \nabla \cdot \boldsymbol{\phi}=0\right\} .
$$

Then $\boldsymbol{H}_{\text {div }}(\Omega) \subset \boldsymbol{L}_{\text {div }}(\Omega) \subset \boldsymbol{H}_{\text {div }}(\Omega)^{*}$ is an evolution triple, cf. [Zeil $\mathbf{~ ] , ~ S e c t i o n ~ 7 2 . 5 . ~}$

Next we have to study traces of Sobolev functions. For $w \in C^{\infty}(\bar{\Omega})$ denote $\operatorname{Tr}:\left.w \mapsto w\right|_{\Gamma}$. If $\Omega$ is Lipschitz-bounded, then there exists a uniquely determined extension $\operatorname{Tr}: H^{1}(\Omega) \longrightarrow$ $H^{1 / 2}(\Gamma)$, see e.g. [Gal.94]], Chapter II 3. The trace inequality for $w$ in $H^{1}(\Omega)$ reads

$$
\|w\|_{1 / 2,2, \Gamma} \leq C_{T r}\|w\|_{1, \Omega} .
$$

Assume that $\Omega$ is Lipschitz-bounded. Denote $\boldsymbol{n}$ the outer normal vector to $\Gamma$. We study the map $\operatorname{Tr}:\left.\boldsymbol{u} \mapsto \boldsymbol{u}\right|_{\Gamma} \cdot \boldsymbol{n}$ for $\boldsymbol{u} \in C^{\infty}(\bar{\Omega})^{d}$. Then there exists a uniquely determined extension $\operatorname{Tr}: \boldsymbol{H}_{\text {div }}(\Omega) \longrightarrow H^{-1 / 2}(\Gamma)$, see [एem77], pp.9. Given $\boldsymbol{u} \in \boldsymbol{H}_{\text {div }}(\Omega)$ with $\nabla \cdot \boldsymbol{u}=0$ we have the inequality

$$
\|\boldsymbol{u} \cdot \boldsymbol{n}\|_{-1 / 2,2, \Gamma} \leq C_{T r}\|\boldsymbol{u}\|_{1, \Omega} .
$$

Moreover we introduce a bilinear form $\langle\cdot, \cdot\rangle_{\Gamma}$. For $u, v$ in $H^{1}(\Omega)$ the following form is well-defined and bilinear, viz.,

$$
\langle u, v\rangle_{\Gamma} \equiv \int_{\Gamma} u v d s
$$


It follows from Hölder inequality and (B.10) that $\langle\cdot, \cdot\rangle_{\Gamma}$ is bounded.

The Poincare-Friedrich inequality, cf. [Tem77] Ch.1.1, Eq.(1.9) reads

$$
\|\boldsymbol{u}\|_{\boldsymbol{L}_{(\Omega)}^{2}} \leq c_{P o i, 0}(\Omega)\|\nabla \boldsymbol{u}\|_{\boldsymbol{L}_{(\Omega)}^{2}} \quad \forall \boldsymbol{u} \in \boldsymbol{H}_{0}^{1}(\Omega) .
$$

Provided $\Omega$ has no axis of symmetry, the PoInCARE-Morrey inequality (see [Ver87], Inequality (2.6), and references therein)

$$
\|\boldsymbol{u}\|_{\boldsymbol{L}_{(\Omega)}^{2}} \leq c_{P o i}(\Omega)\|\nabla \boldsymbol{u}\|_{\boldsymbol{L}^{2}(\Omega)} \quad \forall \boldsymbol{u} \in \boldsymbol{H}^{1}(\Omega) \quad \text { with } \quad \boldsymbol{u} \cdot \boldsymbol{n}=0 \quad \text { on } \partial \Omega
$$

holds. Then we have the following inequality

$$
\|\boldsymbol{u}\|_{1, \Omega}^{2}=\|\boldsymbol{u}\|_{\boldsymbol{L}^{2}(\Omega)}^{2}+\|\nabla \boldsymbol{u}\|_{\boldsymbol{L}^{2}(\Omega)}^{2} \leq\left(1+c_{P o i}^{2}(\Omega)\right)\|\nabla \boldsymbol{u}\|_{\boldsymbol{L}^{2}(\Omega)}^{2} .
$$

Moreover we have the generalised YounG's inequality (cf. Galdi Cp.II, eq.(1.5))

$$
a b \leq \frac{\epsilon a^{q}}{q}+\frac{\epsilon^{-q^{*} / q} b^{q^{*}}}{q^{*}} \quad, \quad a, b, \epsilon>0, q^{-1}+\left(q^{*}\right)^{-1}=1 .
$$

In the case that $q=q^{*}=2$ we get

$$
a b \leq \frac{\epsilon a^{2}}{2}+\frac{\epsilon^{-1} b^{2}}{2} \quad, \quad a, b, \epsilon>0 .
$$

\section{B.2. Analytical results for some turbulence models}

In the following subsections some analytical results for some turbulence models will we presented. In all cases the corresponding transient problem will be considered.

\section{B.2.1. The $k / \epsilon$ model}

For the $k / \epsilon$ model, there are no results concerning existence and uniqueness available. From a physical and computational point of view, the positivity of $k$ and $\epsilon$ are of prime importance. Therefore Mohammadi proposed to consider another equivalent model, the so called $\phi-\theta$ model with $\theta \equiv k \epsilon^{-1}$ and $\phi \equiv \epsilon^{2} k^{-3}$. For the $\phi-\theta$ model positive initial data and positive Dirichlet boundary data are prescribed, for details see [Col.9.], p.38. Supposing that a solution exists and is continuously differentiable, MoHAMmADI could show that $k$ is stricly positive, see [MP94] pp.65. CoLETTI considered a simplified problem for $\theta$ and $\phi$ by taking $\nu_{t} \equiv 0$ and considering (for the sake of simplicity) homogeneous Dirichlet boundary conditions. Then he could show existence, uniqueness and non-negativity of the solution of the reduced $\phi-\theta$ model, see [Col.9.], theorem 3, p. 40. He also obtains an existence, uniqueness and non-negativity result for the full $\phi-\theta$ model, see [Col.99], Theorem 5, p.41.

\section{B.2.2. The Smagorinsky model}

The Smagorinsky model was studied by LADYZHEnskaya [Lad67] and revised by John [Ioh02]. Existence and uniqueness in $3 \mathrm{~d}$ can be proven even for large data and long time 
intervals. Moreover a stability result is available. PARES [Par92] investigated the existence and uniqueness of a weak solution for a slightly different problem for the Smagorinsky model: Homogeneous Dirichlet boundary conditions are prescribed only on a part of the boundary, whereas on the rest of the boundary, slip with friction and penetration with resistance boundary conditions are imposed.

\section{B.2.3. The Iliescu-Layton model}

ILIESCU AND LAYTON [II.98] proved the existence of at least one weak solution for a certain variant of the Iliescu-Layton model. LAYTON AND LEWANDOWSKI [LLO3] showed the existence of at least one weak solution of the Iliescu-Layton model, when filtering is performed using the differential filtering technique of Stokes type.

\section{B.2.4. The Taylor LES model}

The analysis given by Ladyzhenskaya for the Smagorinsky model was extended to the Taylor LES model augmented with the Smagorinsky model by ColetTi [Col99]. His analysis was revised in [.Joh02]. Under the requirement that the Smagorinsky subgrid scale term dominates the Taylor LES term Coletti could prove existence, uniqueness and stability of a solution (for large data and long time intervals). However, in numerical experiments the Taylor LES model is often not numerically stable unless a dominating Smagorinsky term is added. Thus JoHN points out that an analogous result cannot be expected for a non-dominating Smagorinsky term, see [Joh02], Remark 5.2.2. Indeed, for a vanishing Smagorinsky term Coletti could prove existence, uniqueness and stability of a strong solution only for small data and $\partial \Omega$ being sufficiently smooth.

\section{B.2.5. The Galdi-Layton model}

JoHN showed that the techniques used by Ladyzhenskaya and Coletti cannot be applied to the analysis of the Galdi-Layton model, cf. [Joh02]. Berselli ET AL. [BGLI02] studied the Galdi-Layton model without any additional eddy-viscosity term for the case $\Omega=(0, L)^{3}$ with periodic boundary conditions. They could prove existence and uniqueness of a solution in a certain function space for small time intervals. JoHN mentions that numerical tests suggest that for the Galdi-Layton model without any eddy-viscosity SGS model, stable long-time solutions for small $\nu$ cannot be expected, see [.Joh(2], p.63.

Moreover, Berselli AND GRISANTi [BG(02] obtained a result concerning the modelling error. They could prove that the solution of the Galdi-Layton model converges to that of the Navier-Stokes equations (in some Sobolev spaces) as the filter width $\Delta$ approaches zero. 


\section{Turbulent boundary-layer theory}

This chapter is dedicated to a more detailed view at the turbulent boundary-layer equations.

\section{C.1. Natural convection turbulent boundary layers}

First we consider (5.27)-(5.28). In order to solve these equations, in Subsection 6.3 the corresponding initial value problem (6.12)-(6.14) was introduced, viz. (for simplicity in the sequel we omit the sub- and superscripts and use small letters)

$$
\begin{aligned}
\text { (C.1) }-\frac{d}{d y}\left(\left(\nu+\nu_{t}\right) \frac{d u}{d y}\right) & =-\beta \theta g_{x} \text { in }\left(0, y_{\delta}\right),\left.\quad u\right|_{y=0}=0,\left.\quad \nu \frac{d u}{d y}\right|_{y=0}=r, \\
\text { (C.2) }-\frac{d}{d y}\left(\left(\frac{\nu}{P r}+\frac{\nu_{t}}{P r_{t}}\right) \frac{d \theta}{d y}\right) & =0 \quad \text { in }\left(0, y_{\delta}\right),\left.\quad \theta\right|_{y=0}=\theta w,\left.\quad a \frac{d \theta}{d y}\right|_{y=0}=s .
\end{aligned}
$$

where $(r, s)$ is a parameter in $\mathbb{R} \times \mathbb{R}$. Integrating (C.2) yields

$$
\int_{0}^{y} \frac{d}{d y^{\prime}}\left(a+a_{t}\right) \frac{d \theta}{d y^{\prime}} d y^{\prime}=\left(a+a_{t}\right) \frac{d \theta}{d y}=s .
$$

Thus (C.1)-(C.2) can be equivalently written as the following first order system

$$
\begin{aligned}
-\frac{d \sigma}{d y} & =-\beta g_{x} \theta & & \text { in }\left(0, y_{\delta}\right),\left.\quad \sigma\right|_{y=0}=r, \\
\frac{d u}{d y} & =\frac{1}{\nu+\nu_{t}} \sigma(y) & & \text { in }\left(0, y_{\delta}\right),\left.\quad u\right|_{y=0}=0, \\
\frac{d \theta}{d y} & =\frac{1}{a+a_{t}} s & \text { in }\left(0, y_{\delta}\right), & \left.\theta\right|_{y=0}=\theta_{w} .
\end{aligned}
$$

We assume Neitzke's model in (C.4)-(C.6), i.e., $\nu_{t}=u y / R e_{\min }$ and $a_{t}=\nu_{t} \operatorname{Pr}_{t}^{-1}$. Using

$$
\boldsymbol{z}(y)=\left(\begin{array}{c}
-\sigma(y) \\
u(y) \\
\theta(y)
\end{array}\right), \quad \boldsymbol{h}(y, \boldsymbol{z})=\left(\begin{array}{c}
-\beta g_{x} \theta(y) \\
\left(\nu+\nu_{t}\right)^{-1} \sigma(y) \\
\left(a+a_{t}\right)^{-1} s
\end{array}\right)
$$

(C.4) - (C.6) can be written as

$$
\frac{d \boldsymbol{z}}{d y}=\boldsymbol{h}(y, \boldsymbol{z}) \quad \text { in }\left(0, y_{\delta}\right), \quad \boldsymbol{z}(0)=\left(r, 0, \theta_{w}\right)^{T} .
$$

\section{Remark C.1}


Before proceeding it is worthwhile pointing out a difficulty regarding (C.4)-(C.6) in a CFD code. For this purpose we write (C.1) in dimensional coordinates:

$$
-\tilde{\rho}_{0} \frac{d}{d y}\left[\left(\tilde{\nu}+\tilde{\nu}_{t}\right) \frac{d \tilde{u}}{d y}\right]=-\tilde{\rho}_{0} \tilde{\beta}_{0}\left(\tilde{T}-\tilde{T}_{0}\right) \tilde{\boldsymbol{g}} \cdot \boldsymbol{e}_{x} .
$$

The problem is to select a suitable reference temperature $\tilde{T}_{0}$ for each $\boldsymbol{x} \in \Gamma_{W}$. The theoretical choice is $\tilde{T}_{0}=\tilde{T}_{\infty}$ being the free stream temperature, cf. [KC.93], p.398. However, in many CFD codes only $\left.\tilde{T}\right|_{\Gamma_{W}}$ and $\left.\tilde{T}\right|_{\Gamma_{\delta}}$ can be accessed easily. $T_{\infty}$ is often not available unless the grid data structure is quite sophisticated. Hence there are two choices, viz., (i) $\tilde{T}_{0}=\left.\tilde{T}\right|_{\Gamma_{W}}$ and (ii) $\tilde{T}_{0}=\left.\tilde{T}\right|_{\Gamma_{\delta}}$. Strategy (i) seems to be more robust as $\left.\tilde{T}\right|_{\Gamma_{\delta}}$ in method (ii) depends on the solution process. In the numerical tests we used (i). Additional tests not shown in this thesis revealed that the predictions for variant (i) are significantly superior to those for variant (ii) for the natural convection flow in a closed cavity.

Based on experimental investigations, see e.g. [Nei99] and [TK00a], we can assume that $u>0$ throughout the boundary layer. Now we can state the following lemma.

\section{Lemma C.1}

Suppose $\nu_{t}=u y / R e_{\min }$ and $u>0$ on $\left(0, y_{\delta}\right)$. For every given $(r, s)$ in $\mathbb{R} \times \mathbb{R}$ the initial value problem (C.7) has a uniquely determined solution.

\section{Proof:}

We prove the lemma by showing that (C.7) has a uniquely determined solution for every given $(r, s)$ in $\mathbb{R} \times \mathbb{R}$. The notion that $u>0$ on $\left[0, y_{\delta}\right]$ ensures that $\nu+\nu_{t} \geq \nu>0$ and $a+a_{t} \geq a>0$ in $\left(0, y_{\delta}\right)$. Thus for each $y_{\delta}>0, \boldsymbol{h}(y, \boldsymbol{z})$ is defined on the domain $D:=\left(0, y_{\delta}\right) \times \mathbb{R}_{>0} \times \mathbb{R}^{2} \subset \mathbb{R}^{4}$. Obviously $\boldsymbol{h}(y, \boldsymbol{z})$ is continuous on $D$. Moreover $D$ is convex and for each $s \in \mathbb{R}$ the derivatives $\partial h_{i} / \partial z_{j}$ are continuous and bounded $(1 \leq i, j \leq 3)$. Thus $\boldsymbol{h}$ is Lipschitz-continuous in $D$ w.r.t. $\boldsymbol{z}$ for each $s \in \mathbb{R}$. According to the theory of ordinary differential equations, for each $(r, s) \in \mathbb{R}^{2}$ (C.7) has a uniquely determined solution, which can be extended on $\left[0, y_{\delta}\right]$.

Now the model is simplified by assuming that $\nu+\nu_{t}$ and $a+a_{t}$ are given piecewise as in (5.38)-(5.39). Denote $y_{\text {lam }}^{u}$ and $y_{\text {lam }}^{\theta}$ the thickness of the viscous sublayer for momentum and temperature equation resp., i.e.,

$$
\begin{array}{ll}
y_{\text {lam }}^{u} \equiv \max \left\{y \mid \operatorname{Re}\left(y^{\prime}\right) \leq \operatorname{Re}_{\min }\right. & \left.\forall y^{\prime}<y\right\}, \\
y_{\text {lam }}^{\theta} \equiv \max \left\{y \mid \operatorname{Re}\left(y^{\prime}\right) \leq \frac{P r_{t}}{\operatorname{Pr}} \operatorname{Re} e_{\text {min }} \quad \forall y^{\prime}<y\right\}
\end{array}
$$

with $\operatorname{Re}(y)=U(y) y \nu^{-1}$. Then we can give the following lemma.

\section{Lemma C.2}

Suppose (5.38)-(5.39) and $u>0$ on $\left(0, y_{\delta}\right)$. Then for every given $(r, s)$ in $\mathbb{R} \times \mathbb{R}$ the initial value problem (C.7) has a uniquely determined solution.

\section{Proof:}

We show that for every given $(r, s)$ in $\mathbb{R} \times \mathbb{R}$ (C.7) has a uniquely determined solution, if (5.38)-(5.39) are assumed. Obviously $\nu_{t}$ and $a_{t}$ are piecewise continuously differentiable 
w.r.t. $u$ on $\left(0, y_{\text {lam }}^{u}\right)$ and on $\left(y_{\text {lam }}^{u}, y_{\delta}\right)$ resp. on $\left(0, y_{\text {lam }}^{\theta}\right)$ and on $\left(y_{\text {lam }}^{\theta}, y_{\delta}\right)$. First we consider the following initial value problem: Seek $u^{\text {lam }}, \theta^{\text {lam }}$ s.t.

$$
\begin{aligned}
-\frac{d}{d y}\left(\nu \frac{d u^{\text {lam }}}{d y}\right) & =-\beta \theta g_{x} \text { in }\left(0, y_{\text {lam }}^{u}\right),\left.\quad u^{\text {lam }}\right|_{y=0}=0,\left.\quad \nu \frac{d u^{\text {lam }}}{d y}\right|_{y=0}=r, \\
(\text { C.11 })-\frac{d}{d y}\left(a \frac{d \theta^{\text {lam }}}{d y}\right) & =0 \quad \text { in }\left(0, y_{\text {lam }}^{\theta}\right),\left.\quad \theta^{\text {lam }}\right|_{y=0}=\theta_{w},\left.\quad a \frac{d \theta^{\text {lam }}}{d y}\right|_{y=0}=s .
\end{aligned}
$$

In analogy to the proof of lemma C.1 there exists a uniquely determined solution, that can be extended on $\left[0, y_{\text {lam }}^{u}\right]$ resp. on $\left[0, y_{\text {lam }}^{\theta}\right]$.

Secondly we consider the following initial value problem: Seek $u^{\log }, \theta^{\log }$ s.t.

$$
\begin{aligned}
-\frac{d}{d y}\left(\frac{u^{\log y}}{R e_{\min }} \frac{d u^{\log }}{d y}\right) & =-\beta \theta^{\log g_{x}} & \text { in }\left(y_{\text {lam }}^{u}, y_{\delta}\right) \\
-\frac{d}{d y}\left(\frac{u^{\log y}}{\operatorname{Pr}_{t} \operatorname{Re}_{\min }} \frac{d \theta^{\log }}{d y}\right) & =0 & \text { in }\left(y_{\text {lam }}^{\theta}, y_{\delta}\right)
\end{aligned}
$$

with initial conditions

$$
\begin{aligned}
& \left.u^{l o g}\right|_{y=y_{\text {lam }}^{u}}=\left.u^{\text {lam }}\right|_{y=y_{\text {lam }}^{u}},\left.\quad \frac{u^{\log y}}{R e_{\min }} \frac{d u^{\log }}{d y}\right|_{y=y_{\text {lam }}^{u}}=\left.\nu \frac{d u^{\text {lam }}}{d y}\right|_{y=y_{\text {lam }}^{u}} \\
& \left.\theta^{\log }\right|_{y=y_{\text {lam }}^{\theta}}=\left.\theta^{\operatorname{lam}}\right|_{y=y_{\text {lam }}^{\theta}},\left.\quad \frac{u^{\log y}}{P r_{t} \operatorname{Re} e_{\text {min }}} \frac{d \theta^{\log }}{d y}\right|_{y=y_{\text {lam }}^{\theta}}=\left.\frac{\nu}{\operatorname{Pr}} \frac{d \theta^{\text {lam }}}{d y}\right|_{y=y_{\text {lam }}^{\theta}} .
\end{aligned}
$$

In analogy to the proof of lemma C.1 there exists a uniquely determined solution, that can be extended on $\left[y_{\text {lam }}^{u}, y_{\delta}\right]$ resp. on $\left[y_{\text {lam }}^{\theta}, y_{\delta}\right]$.

Finally, it is worth mentioning that a computational method should exploit the fact that an analytic solution in the viscous sublayer can be found, see e.g. [Nei99], p.49 or (5.43).

\section{C.2. Forced convection boundary-layer equations in non-dimensional form}

In the case of forced convection, we have to solve (C.1)-(C.2) with vanishing buoyancy term, i.e.,

$$
\begin{gathered}
-\frac{d}{d y}\left(\left(\nu+\nu_{t}\right) \frac{d u}{d y}\right)=0 \quad \text { in }\left(0, y_{\delta}\right),\left.\quad u\right|_{y=0}=0,\left.\quad \nu \frac{d u}{d y}\right|_{y=0}=\tau_{w} \\
-\frac{d}{d y}\left(\left(\frac{\nu}{P r}+\frac{\nu_{t}}{P r_{t}}\right) \frac{d \theta}{d y}\right)=0 \quad \text { in }\left(0, y_{\delta}\right),\left.\quad \theta\right|_{y=0}=\theta_{w},\left.\quad a \frac{d \theta}{d y}\right|_{y=0}=\dot{q} / c_{p} .
\end{gathered}
$$

First we derive the non-dimensional form of the forced convection boundary-layer equations. For the velocity we start with

$$
\left(\nu+\nu_{t}\right) \frac{d u}{d y}=\tau_{w}=u_{\tau}^{2}
$$


Using the following scaled variables

$$
u^{+}=\frac{u}{u_{\tau}}, y^{+}=\frac{u_{\tau} y}{\nu}, \nu_{t}^{+}=\frac{\nu_{t}}{\nu}, \theta^{+}=\frac{c_{p} u_{\tau}\left(\theta-\theta_{w}\right)}{\dot{q}}, a_{t}^{+}=\frac{a}{\nu}
$$

we obtain

$$
\left(1+\nu_{t}^{+}\right) \frac{d u^{+}}{d y^{+}}=1 \quad \Longleftrightarrow u^{+}=\int_{0}^{y^{+}} \frac{1}{1+\nu_{t}^{+}} d \tilde{y}^{+} .
$$

Analogously, the temperature equation

$$
\left(a+a_{t}\right) \frac{d \theta}{d y}=\frac{\dot{q}}{c_{p}}
$$

can be rearranged to

$$
\left(\frac{1}{P r}+\frac{\nu_{t}^{+}}{P r_{t}}\right) \frac{d \theta^{+}}{d y^{+}}=1 \quad \Longleftrightarrow \quad \theta^{+}=\int_{0}^{y^{+}} \frac{1}{P r^{-1}+a_{t}^{+}} d \tilde{y}^{+} .
$$

Subtracting (C.18) from (C.16) gives (see also [DPR(01], Eq. (4.4.39))

$$
u^{+}-\theta^{+}=\int_{0}^{y^{+}} \frac{1}{1+\nu_{t}^{+}} d \tilde{y}^{+}-\int_{0}^{y^{+}} \frac{1}{\operatorname{Pr}^{-1}+a_{t}^{+}} d \tilde{y}^{+} .
$$

If $\operatorname{Pr}=1$ and $P r_{t}=1$ then the right hand side of (C.19) vanishes and hence $u^{+}=\theta^{+}$. In indoor-air flow problems we have $\operatorname{Pr}=0.7$ and $P r_{t} \approx 1$ (in the boundary layer, see e.g. [KC.93], pp. 259). The effect of $\operatorname{Pr}=0.7<1$ is noticable only in the viscous sublayer. As $y_{\text {lam }}^{u}$ and $y_{\text {lam }}^{\theta}$ are small, the contribution of the viscous sublayer to the integrals in (C.19) is small. Thus the right hand side of (C.19) in reasonably small in the log-layer and hence in a good approximation $u^{+}=\theta^{+}$. This is also referred to as Reynolds analogy: When suitably normalised, momentum and contaminent transport have the same profile, see [DPR(01], p.80. Therefore we only have to determine the profile for $u$ explicitely and then infer the profile for $\theta$ using the Reynolds analogy.

\section{C.3. The universal log law by Prandtl and van Karman}

The standard approach is to use a two-layer model, i.e., to choose $\nu+\nu_{t}=\nu$ in the viscous sublayer $\left(0, y_{\text {lam }}^{u}\right)$ and $\nu+\nu_{t}=\nu_{t}$ in the log-layer, see e.g. [KC.93], pp. 202. PRANDTL and VAN KARMAN used the following model for $\nu_{t}$ :

$$
\nu_{t}=\kappa^{2} y^{2}\left|\frac{d u}{d y}\right|=u_{\tau} \kappa y \quad \Longleftrightarrow \quad \nu_{t}^{+}=\frac{u_{\tau} \kappa y}{\nu}=\kappa y^{+},
$$

with $\kappa=0.4$ being the van Karman constant. Note that in a forced convection turbulent boundary layer $\frac{d u}{d y}>0$ for all $y \in\left(0, \delta_{\delta}\right)$. In the viscous sublayer integrating (C.16) gives

$$
u^{+}=y^{+} \quad \text { in } \quad\left(0, y_{\text {lam }}^{u,+}\right) .
$$


In agreement with [Nei99] we use $y_{\text {lam }}^{u,+}=11.25$. Alternatively, KAYS and CRAWFORD report $y_{\text {lam }}^{u,+}=10.8$, see [KC.93], p.203. In the log-layer we have to solve

$$
u^{+}=\frac{1}{\kappa y^{+}} \quad \text { in } \quad\left(y_{\text {lam }}^{u,+}, \delta_{\delta}^{+}\right), \quad u^{+}\left(y_{\text {lam }}^{u,+}\right)=y_{\text {lam }}^{u,+}=11.25 .
$$

The solution is given by

$$
u^{+}\left(y^{+}\right)=\frac{1}{\kappa} \ln \left(y^{+}\right)+B \quad \text { in } \quad\left(y_{\text {lam }}^{u,+}, \delta_{\delta}^{+}\right) .
$$

Using the condition that $u^{+}$is continuous at $y^{+}=y_{\text {lam }}^{+}$yields $B=5.2$. To this end, the log law reads

$$
u^{+}\left(y^{+}\right)=2.5 \ln \left(y^{+}\right)+5.2 \quad \text { in }\left(y_{\text {lam }}^{u,+}, \delta_{\delta}^{+}\right) .
$$

The temperature solution can be infered from (C.20) and (C.22) using Reynolds analogy, see [DPR0T] pp.80, or directly by integrating (C.18), see [K(.93], pp.269. The latter approach gives

$$
\begin{aligned}
& u^{+}\left(y^{+}\right)= \begin{cases}y^{+}, & \text {if } y^{+} \leq 10.8 \\
2.44 \ln \left(y^{+}\right)+5.0, & \text { if } y^{+}>10.8\end{cases} \\
& \theta^{+}\left(y^{+}\right)= \begin{cases}\operatorname{Pr} y^{+}, & \text {if } y^{+} \leq 13.2 \\
2.075 \ln \left(y^{+}\right)+3.9, & \text { if } y^{+}>13.2\end{cases}
\end{aligned}
$$

\section{C.4. A non-isothermal wall law for forced convection problems by Neitzke}

\section{C.4.1. The viscous sublayer}

First we consider the viscous sublayer for the momentum equation. According to (5.38)(5.39) we use $\nu+\nu_{t} \approx \nu$ in $\left[0, y_{\text {lam }}^{u}\right]$. Note that the thickness $y_{\text {lam }}^{u}$ of this layer is given implicitely by (C.8)-(C.9). Integrating (C.16) gives (C.20). Then, combining ([C.8)-(C.9) and (C.16) we get

$$
R e=u^{+} y^{+}=y^{+^{2}} \leq R e_{\min } \Longrightarrow y_{\text {lam }}^{u,+}=\sqrt{R e_{\min }}
$$

and thus $y_{\text {lam }}=\nu u_{\tau}^{-1} \sqrt{R e_{\text {min }}}$. This gives the first part of (5.41).

Regarding the heat transfer equation, integrating (C.16) gives the first equation in ([C.24), but with a different value for $y_{\text {lam }}^{\theta,+}$, which is calculated as follows:

$$
a_{t} \leq a \Leftrightarrow \frac{\nu_{t}}{P r_{t}}=\frac{\nu u^{+} y^{+}}{P r_{t} R e_{\min }}=\frac{\nu y^{+2}}{P r_{t} \operatorname{Re}_{\min }} \leq \frac{\nu}{P r} \Leftrightarrow y_{\text {lam }}^{\theta,+}=\sqrt{P r_{t} \operatorname{Pr}^{-1} \operatorname{Re}_{\min }}
$$

Experiments show $y_{\text {lam }}^{\theta,+}=8.0$. Thus we infer $P r_{t}=1.15$ in the boundary layer. 


\section{C.4.2. The log-layer}

Neitzke's model in $\left(y_{\text {lam }}^{u}, \delta_{\delta}\right)$ reads

$$
\nu_{t}=\frac{u y}{R e_{\min }} \quad \Longleftrightarrow \quad \nu_{t}^{+}=\frac{u^{+} y^{+}}{R e_{\min }} .
$$

Substituting this into (C.16), we arrive at the following ordinary differential equation to be solved in $\left(y_{\text {lam }}^{u,+}, \delta_{\delta}^{+}\right)$:

$$
R e_{\min } \frac{1}{y^{+}}=u^{+} \frac{d u^{+}}{d y^{+}} \quad \text { in } \quad\left(\sqrt{R e_{\min }}, \delta_{\delta}^{+}\right),\left.\quad u^{+}\right|_{y^{+}=R e_{\min }^{1 / 2}}=R e_{\min }^{1 / 2}
$$

Integration yields (with $R \equiv \sqrt{R e_{\min }}$ )

$$
u^{+}=R \sqrt{2 \ln \left(\frac{y^{+}}{R}\right)+1}, \quad \text { in } \quad\left(\sqrt{R e_{\min }}, y_{\delta}^{+}\right) .
$$

The log-law for the heat equation (5.42) is derived using the Reynolds analogy. Obviously both profiles are essentially the same but differently scaled. 


\section{Nomenclature}

\section{Upper-case Roman}

\begin{tabular}{|c|c|}
\hline $\mathcal{A}_{u}(\boldsymbol{w})$ & $\rightarrow(4.45)$ \\
\hline $\mathcal{A}_{\theta}(\boldsymbol{w}, \vartheta)$ & $\rightarrow(\overline{4.45})$ \\
\hline$A^{+}$ & van Driest constant, $A^{+}=26 \rightarrow(5.32)$ \\
\hline$B$ & $\log$ law constant $B \in\{5.0,5.2\} \rightarrow(\overrightarrow{\mathrm{C} .21})$ \\
\hline$C_{1}$ & $C_{1}=1.44 \rightarrow(3.35)$ \\
\hline$C_{2}$ & $C_{2}=1.92 \rightarrow(\overline{3.35})$ \\
\hline$C_{S}$ & Smagorinsky coefficient $\rightarrow(4.33)$ \\
\hline$C_{q}$ & coefficient in the Iliescu-Layton model $C_{q}=0.17 \rightarrow(4.33)$ \\
\hline$C_{t}$ & $C_{t}=0.8 \rightarrow(3.26)$ \\
\hline$C_{\theta}$ & coefficient in NEITZKE's law of the wall $C_{\theta}=\operatorname{Pr} R_{\theta} \rightarrow(5.42)$ \\
\hline$C_{\mu}$ & $C_{\mu}=0.09 \rightarrow(3.22)$ \\
\hline $\mathcal{D}(\Omega)$ & $\rightarrow$ Section A.1 \\
\hline $\mathcal{D}^{\prime}(\Omega)$ & $\rightarrow$ Section A.1 \\
\hline $\mathcal{D}\left(y^{+}\right)$ & van Driest damping function $\rightarrow(5.33)$ \\
\hline $\mathcal{E}(\Omega)$ & $\rightarrow$ Section A.1 \\
\hline $\mathcal{E}^{\prime}(\Omega)$ & $\rightarrow$ Section A.1 \\
\hline$E(\kappa)$ & energy-spectrum function $\rightarrow$ (2.4) \\
\hline $\mathcal{F}(f)$ & Fourier transform of $f$ \\
\hline$G$ & $G=C_{t} \beta \frac{\nu_{t}}{P r_{t}} \boldsymbol{g} \nabla \Theta \rightarrow(3.26)$ \\
\hline$G r$ & Grashof number $\rightarrow$ Section 1.1 \\
\hline $\mathbb{I}$ & identity operator \\
\hline$K_{s}$ & parameter in the formula for $R e_{\min , n} \rightarrow$ Section 5.4 .2 \\
\hline$L$ & characteristic lengthscale \\
\hline$L u$ & $L u \equiv-\nabla \cdot(\nu \nabla u)+(\boldsymbol{b} \cdot \boldsymbol{\nabla}) u+c u \rightarrow(9.8)$ \\
\hline$L_{O s}(\boldsymbol{u}, p)$ & $L_{O s}(\boldsymbol{u}, p)=-\boldsymbol{\nabla} \cdot(2 \nu \mathbb{S}(\boldsymbol{u}))+(\boldsymbol{a} \cdot \boldsymbol{\nabla}) \boldsymbol{u}+c \boldsymbol{u}+\boldsymbol{\nabla} p \rightarrow(9.11)$ \\
\hline & characteristic lengthscale of the flow $\rightarrow$ Section 1.1 \\
\hline$L_{11}$ & longitudinal integral lengthscale $\rightarrow$ (2.5) \\
\hline$P$ & non-dimensional mean pressure $P=\overline{\langle p}_{E} \rightarrow$ Section 3.1 \\
\hline$P$ & non-dimensional modified mean pressure $P \equiv P^{*} \rightarrow(3.1)$ \\
\hline$P_{\infty}$ & free stream pressure in the turbulent boundary layer \\
\hline$P^{*}$ & non-dimensional modified mean pressure $P^{*}=P+\frac{2}{3} k \rightarrow(3.1)$ \\
\hline $\mathcal{P}$ & rate of production of turbulent kinetic energy $\mathcal{P}=-\left\langle\boldsymbol{u}^{\prime} \otimes \boldsymbol{u}^{\prime}\right\rangle_{E}: \mathbb{S}(\boldsymbol{U})$ \\
\hline $\mathcal{P}$ & rate of production of turbulent kinetic energy $\mathcal{P}=2 \nu_{t} \mathbb{S}(\boldsymbol{U}): \mathbb{S}(\boldsymbol{U})$ \\
\hline & in RANS models with the eddy viscosity hypothesis \\
\hline $\mathcal{P}_{r}$ & rate of production of residual kinetic energy $\rightarrow(4.25)$ \\
\hline
\end{tabular}


$\mathcal{P}_{r}^{\theta} \quad$ modified rate of production of residual kinetic energy that accounts for buoyancy effects $\rightarrow$ (4.37)

$\operatorname{Pr} \quad$ Prandtl number, $\operatorname{Pr}=0.70$ for air $\rightarrow$ Section 1.1

$\mathrm{Pr}_{t} \quad$ turbulent Prandtl number $\rightarrow$ Section 3.2.1

$\mathrm{Pr}_{t} \quad$ SGS turbulent Prandtl number in LES $\rightarrow$ Section 4.4 .3

$\mathrm{Pr}_{k} \quad$ turbulent Prandtl number for turbulent kinetic energy $\operatorname{Pr}_{k}=1.0 \rightarrow(3.23),(3.35)$

$\operatorname{Pr}_{\epsilon} \quad$ turbulent Prandtl number for dissipation $P r_{\epsilon}=1.3 \rightarrow$ Section 3.4, (3.35)

$\mathcal{R} \quad$ regularization operator in LES $\rightarrow$ (4.15) resp. (7.1) $-(7.2)$

$\mathbb{R}^{+} \quad$ positive real numbers

$R a \quad$ Rayleigh number $\rightarrow$ Section 1.1

Re $\quad$ Reynolds number $\rightarrow$ Section 1.1

Re $\quad$ in the near-wall region $R e=U(y) y \nu^{-1} \rightarrow$ Section 1.1

$R e_{m i n, n} \quad$ coefficient in NeITZKE's algebraic turbulence model

$\rightarrow$ Section 5.4.2, (5.38)-(5.39)

$R e_{\tau} \quad$ Reynolds number based on friction velocity and channel half width $H: R e_{\tau}=u_{\tau} H / \nu$

$R e_{\delta^{*}} \quad R e_{\delta^{*}}=U_{\infty} \delta^{*} / \nu \rightarrow$ Section 5.1

$R i_{f} \quad$ flux Richardson number $\rightarrow(3.19)$

$R i_{g} \quad$ gradient Richardson number $\rightarrow$ Section 5.4 .2

$R_{u} \quad$ coefficient in NeITzKE's law of the wall $R_{u}=\sqrt{R e_{\min , n}}=6.25 \rightarrow(5.41)$

$R_{\theta} \quad$ coefficient in NEITZKE's law of the wall $R_{\theta}=8.0 \rightarrow$ (5.42)

$\mathbb{S}(\boldsymbol{u}) \quad$ rate-of-strain tensor $\mathbb{S}(\boldsymbol{u})=\frac{1}{2}\left(\nabla \boldsymbol{u}+\nabla \boldsymbol{u}^{T}\right) \rightarrow$ Section 1.1

$\mathcal{S}\left(\mathbb{R}^{d}\right) \quad$ see Definition A.2

$\mathcal{S}^{\prime}\left(\mathbb{R}^{d}\right) \quad$ see Section A.1

$\mathcal{S} \quad$ mean strain rate $\mathcal{S}=\sqrt{2 \mathbb{S}(\boldsymbol{U}): \mathbb{S}(\boldsymbol{U})}$

$\overline{\mathcal{S}} \quad \overline{\mathcal{S}}=\sqrt{2 \mathbb{S}(\overline{\boldsymbol{U}}): \mathbb{S}(\overline{\boldsymbol{U}})}$

St $\quad$ Stanton number $\rightarrow$ Section 5.1

$\mathcal{T}(l) \quad$ rate of transfer of energy from eddies larger than $l$ to those smaller than $l \rightarrow$ Section 2.2

$\tilde{T} \quad$ dimensional temperature $\rightarrow$ Section 1.1

$\tilde{T}_{0} \quad$ dimensional reference temperature $\rightarrow$ Section 1.1

$\tilde{T}_{\text {diff }} \quad$ dimensional characteristic temperature difference $\rightarrow$ Section 1.1

$\operatorname{Tr} \quad$ Trace operator, $\rightarrow$ Chapter B.

$T_{u} \quad$ turbulence intensity $\left.T_{u}=\left(u_{\infty}^{\prime}\right)^{2}\right)^{1 / 2} / u_{\infty}$

$\boldsymbol{U} \quad$ non-dimensional mean velocity $\boldsymbol{U}=\langle\boldsymbol{u}\rangle_{E} \rightarrow$ Section 3.1

$\boldsymbol{U}_{\delta} \quad \boldsymbol{U}_{\delta}=\left.\boldsymbol{U}\right|_{\Gamma_{\delta}}$

$\boldsymbol{U}_{\delta, t} \quad \boldsymbol{U}_{\delta, t}=\boldsymbol{U}_{\delta}-\left(\boldsymbol{U}_{\delta} \cdot \boldsymbol{n}\right) \boldsymbol{n}$

$U\left(y_{\delta}\right) \quad U\left(y_{\delta}\right)=\frac{\boldsymbol{U}_{\delta} \cdot \boldsymbol{U}_{\delta, t}}{\left\|\boldsymbol{U}_{\delta, t}\right\|}$

$U_{\infty} \quad$ free stream velocity in a turbulent boundary layer

$\tilde{U}_{s c} \quad$ (dimensional) characteristic velocity $\rightarrow$ Section 1.1

$U, V, W$ (ensemble averaged) mean velocity components in streamwise, wall-normal and spanwise direction 
$U^{B L} \quad$ streamwise velocity in $\Omega_{\delta}$

$U_{*} \quad$ an approximation for $u_{\tau}$ in statistical modelling $\rightarrow$ Chapter 6

$W_{*} \quad$ an approximation for $u_{\tau}$ in LES $\rightarrow$ Section 7

\section{Lower-case Roman}

$\tilde{a} \quad$ dimensional thermal diffusivity $\rightarrow$ Section 1.1

$a \quad$ non-dimensional thermal diffusivity $\rightarrow$ Section 1.1

$a_{t} \quad$ turbulent thermal diffusivity $\rightarrow$ (3.5), (4.36)

$a_{e} \quad$ effective thermal diffusivity $a_{e}=a+a_{t}$

$c_{f} \quad$ skin friction coefficient $c_{f}=2 \tau_{w} / U_{\infty}^{2} \rightarrow$ Section 5.1

$c_{p} \quad \rightarrow$ Section 1.1

$\tilde{c}_{p} \quad$ dimensional specific heat at constant pressure $\rightarrow$ Section 1.1

$d \quad$ space dimension $(d=2,3)$

$d_{99} \quad$ distance to the wall where $U=0.99 U_{\infty} \rightarrow$ Section 5.1

$\boldsymbol{e}_{i} \quad$ unit-vector in the $i$-th coordinate direction

$\boldsymbol{e}_{x}, \boldsymbol{e}_{y}, \boldsymbol{e}_{z}$ unit-vector in the $x, y, z$ (resp.) coordinate direction

$\boldsymbol{g}$ non-dimensional gravitational acceleration $\rightarrow$ Section 1.1

$g_{x} \quad g_{x}=\boldsymbol{g} \cdot \boldsymbol{e}_{x}$ with $\boldsymbol{e}_{x}$ pointing into the streamwise direction

$\tilde{\boldsymbol{g}} \quad$ dimensional gravitational acceleration $\rightarrow$ Section 1.1

$g_{\Delta} \quad$ filter kernel $\rightarrow$ (4.1)

$\boldsymbol{h} \quad \boldsymbol{h} \equiv \overline{\boldsymbol{u} \theta}-\overline{\boldsymbol{u}} \bar{\theta} \rightarrow(4.22)$

$\boldsymbol{h}^{S G S} \quad$ (4.31)

$h \quad$ grid spacing

$k \quad$ turbulent kinetic energy $k=\frac{1}{2}\left\langle\boldsymbol{u}^{\prime 2}\right\rangle_{E} \rightarrow(2.3)$

$k^{+} \quad k^{+} \equiv k u_{\tau}^{-2} \rightarrow(5.49)$

$k_{r} \quad$ residual kinetic energy $k_{r}=\frac{1}{2} \sum_{i=1}^{d} \tau_{i i}^{R} \rightarrow(4.20)$

$l \quad$ characteristic eddy size $\rightarrow$ Section 2.2

$l_{0} \quad$ lengthscale of the largest eddies $\rightarrow$ Section 2.2

$l_{D I} \quad$ demarcation lengthscale between the inertial subrange $\left(l>l_{D I}\right)$

and the dissipation range $\left(l<l_{D I}\right) \rightarrow$ Section 2.2

$l_{E I} \quad$ demarcation lengthscale between the energy-containing range of eddies $\left(l>l_{E I}\right)$ and smaller eddies $\left(l<l_{E I}\right) \rightarrow$ Section 2.2

$l_{m} \quad$ mixing length $\rightarrow(5.30),(3.21)$

$\boldsymbol{n} \quad$ outer unit normal vector to $\Gamma \rightarrow$ Section 1.2

$\tilde{p} \quad$ dimensional pressure $\rightarrow$ Section 1.1

$p^{\prime} \quad$ non-dimensional fluctuating resp. residual pressure $\rightarrow$ Section 2.1 resp. (4.3)

$\tilde{p}_{\text {red }} \quad$ dimensional reduced pressure $\rightarrow$ Section 1.1

$\bar{p}_{\text {mod }} \quad$ reduced mean pressure in LES, for simplicity denoted by $\bar{p} \rightarrow(4.3)$

$q \quad$ solution for pressure in LES, which is an approximation to $\bar{p}$

$\dot{q} \quad$ heat-flux at surface, in $W / m^{2}, \rightarrow$ Section 1.18

$\dot{q}^{V} \rightarrow$ Section 1.1

$\tilde{\tilde{q}}^{V} \quad$ dimensional volume specific (external) heat source, in $W / m^{3}, \rightarrow$ Section 1.1 
$\dot{s} \quad$ heat-flux at surface in LES $\rightarrow$ Section 7.1.1

$\boldsymbol{t}_{j} \quad\left\{\boldsymbol{t}_{\boldsymbol{j}}\right\}_{j=1}^{d-1}$ is a local orthonormal basis of the tangent space of $\Gamma \rightarrow$ Section 1.2

$t \quad$ non-dimensional time variable $\rightarrow$ Section 1.1

$\tilde{t} \quad$ dimensional time variable $\rightarrow$ Section 1.1

$\boldsymbol{u} \quad$ non-dimensional velocity $\rightarrow$ Section 1.1

$\boldsymbol{u}^{\prime} \quad$ non-dimensional fluctuating resp. residual velocity $\rightarrow$ Section 2.1 resp. (4.3)

$\boldsymbol{u}_{i n}, \boldsymbol{u}_{F}$ non-dimensional inflow velocity

$\tilde{\boldsymbol{u}} \quad$ dimensional velocity $\rightarrow$ Section 1.1

$u \quad$ in wall bounded flow: wall-parallel streamwise velocity component

$u^{+} \quad$ mean velocity normalized by the friction velocity $u^{+}=U / u_{\tau} \rightarrow$ Section 5.4 .2

$u_{\tau} \quad$ friction velocity $u_{\tau}=\sqrt{\tau_{w}} \rightarrow$ Section 5.1

$u^{*} \quad$ velocity scale $\rightarrow(5.30)$

$v \quad$ in wall bounded flow: wall-normal velocity component

$\boldsymbol{w} \quad$ solution for velocity in LES, which is an approximation to $\overline{\boldsymbol{u}}$

$w_{\delta}^{+} \quad \rightarrow(7.7)$

$w \quad$ in wall bounded flow: wall-parallel spanwise velocity component

$u^{\prime}, v^{\prime}, w^{\prime}$ fluctuating streamwise, wall-normal resp. spanwise component of the velocity the near wall region

$\boldsymbol{w}_{\delta, t} \quad \rightarrow(7.6),(6.3)$

$\boldsymbol{x} \quad$ non-dimensional position variable $\rightarrow$ Section 1.1

$\tilde{\boldsymbol{x}} \quad$ dimensional position variable $\rightarrow$ Section 1.1

$x \quad$ in wall bounded flows: wall-parallel streamwise coordinate direction

$x_{1} \quad$ in wall bounded flows: $x_{1}=x$

$x_{2} \quad$ in wall bounded flows: $x_{2}=y$

$x_{3} \quad$ in wall bounded flows: $x_{3}=z$

$y \quad$ in wall bounded flows: wall-normal coordinate direction

$y_{\text {lam }}^{u} \rightarrow$ Section C.1

$y_{\text {lam }}^{\theta} \rightarrow$ Section C.1

$y_{\max }$ distance from the wall where $U$ has its maximum

in the natural convection case $\rightarrow$ Section 5.4 .2

$y_{\text {lam }}^{u} \quad$ thickness of the viscous momentum sublayer

$y_{\text {lam }}^{\theta} \quad$ thickness of the viscous temperature sublayer

$y^{+} \quad$ in wall bounded flow: distance from the wall in wall units, $y^{+}=y \delta_{\nu}^{-1}$

$y_{\delta} \quad$ distance of point $\boldsymbol{x}$ in $\Gamma_{\delta}$ from $\Gamma_{W}$

$z \quad$ in wall bounded flows: wall-parallel spanwise coordinate direction

\section{Upper-case Greek}

$\Gamma \quad \Gamma=\partial \Omega \rightarrow$ Section 1.1

$\Gamma_{F} \quad$ forced convection inflow boundary, see $(1.12) \rightarrow$ Section 1.2

$\Gamma_{W} \quad$ represents a solid impermeable wall, see (1.13) $\rightarrow$ Section 1.2

$\Gamma_{W, D}, \Gamma_{W, N} \rightarrow$ Section 1.18

$\Gamma_{N} \quad$ see $(1.14) \rightarrow$ Section 1.2 
$\Gamma_{-}(\boldsymbol{u}) \quad$ see $(1.15) \rightarrow$ Section 1.2

$\Gamma_{0}(\boldsymbol{u}) \quad$ see $(1.16) \rightarrow$ Section 1.2

$\Gamma_{+}(\boldsymbol{u})$ see $(1.17) \rightarrow$ Section 1.2

$\Gamma_{\delta} \quad$ artificial boundary consisting of mesh points with minimal positive distance to $\Gamma_{W}$, located primarily in the log-layer $\rightarrow$ Section 6.1

$\Delta \quad$ filter width

$\Delta x^{+} \quad$ filter width (in wall units) in $\mathrm{x}$ direction

$\Delta y^{+} \quad$ filter width (in wall units) in y direction

$\Delta z^{+} \quad$ filter width (in wall units) in $\mathrm{z}$ direction

$\Delta_{i} \quad$ filter width in the direction $i$

$\Delta y \quad$ filter width in the $y$-direction

$\Delta_{m} \quad$ time step width $\Delta_{m}=t_{m}-t_{m-1}$

$\Delta_{2} \quad$ enthalpy thickness of temperature boundary layer $\rightarrow$ Section 5.1

$\Omega \quad \Omega \subset \mathbb{R}^{d}$ a bounded domain $\rightarrow$ Section 1.1

$\Omega_{\text {layer }}$ boundary layer, $\Omega_{\text {layer }}=\left\{\boldsymbol{x} \in \Omega \mid \operatorname{dist}\left(\boldsymbol{x}, \Gamma_{W}\right)<\delta_{\text {layer }}\right\} \rightarrow$ Section 5.1

$\Omega_{\text {free }} \quad \Omega_{\text {free }}=\Omega \backslash \Omega_{\text {layer }} \rightarrow$ Section 5.1

$\Omega_{\text {outer }} \quad \Omega_{\text {outer }}=\left\{\boldsymbol{x} \in \Omega \mid \operatorname{dist}\left(\boldsymbol{x}, \Gamma_{W}\right)>y_{\delta}\right\} \rightarrow$ Section 6.1

$\Omega_{\delta} \quad \Omega_{\delta}=\left\{\boldsymbol{x} \in \Omega \mid \operatorname{dist}\left(\boldsymbol{x}, \Gamma_{W}\right)<y_{\delta}\right\} \rightarrow$ Section 6.1

$\Theta \quad$ non-dimensional mean temperature $\rightarrow$ Section 3.1

$\Theta \quad$ in near-wall region $\Theta=\Theta(y) \rightarrow$ Section 5.2

$\Theta\left(y_{\delta}\right) \quad \Theta\left(y_{\delta}\right)=\left.\Theta\right|_{\Gamma_{\delta}}$

$\Theta^{B L} \quad$ mean temperature in $\Omega_{\delta}$

$\Theta_{w} \quad$ mean temperature of the wall, $\Theta_{w}=\left.\Theta\right|_{\Gamma_{W}} \rightarrow$ Section 3.1

\section{Lower-case Greek}

$\beta \quad \rightarrow$ Section 1.1

$\beta_{j} \quad$ friction coefficient, see $\rightarrow$ Section 1.1, (1.13) (i)

$\tilde{\beta}_{0} \quad$ dimensional volume expansion coefficient $\rightarrow$ Section 1.1

$\gamma \quad \gamma=6$ constant appearing in the Gaussian filter

$\delta_{\text {layer }} \quad$ boundary layer thickness $\rightarrow$ Section 5.1

$\delta_{\nu} \quad$ viscous lengthscale $\delta_{\nu}=\frac{\nu}{u_{\tau}} \rightarrow$ Section 5.2

$\delta^{*} \quad$ momentum thickness of a boundary layer $\rightarrow$ Section 5.1

$\epsilon \quad$ rate of dissipation of turbulent kinetic energy $\epsilon=2 \nu\left\langle\mathbb{S}\left(\boldsymbol{u}^{\prime}\right): \mathbb{S}\left(\boldsymbol{u}^{\prime}\right)\right\rangle_{E}$

$\rightarrow$ Section 3.3

$\epsilon^{+} \quad \epsilon^{+} \equiv \epsilon \nu u_{\tau}^{-4} \rightarrow$ (5.49)

$\eta \quad$ Kolmogorow lengthscale $\rightarrow$ Section 2.2

$\theta \quad$ non-dimensional temperature $\rightarrow$ Section 1.1

$\theta_{w} \quad$ non-dimensional wall temperature $\rightarrow$ (1.18)

$\theta^{\prime} \quad$ non-dimensional fluctuating resp. residual temperature

$\rightarrow$ Section 2.1 resp. Section 4.4

$\theta_{\text {in }}$ non-dimensional inflow temperature 
$\theta^{+} \quad$ normalized temperature in near-wall region $\theta^{+} \equiv c_{p} u_{\tau}\left(\Theta_{w}-\Theta\right) \dot{q}^{-1}$ $\rightarrow$ Section 5.4 .2

$\vartheta$ solution for temperature in LES, which is an approximation to $\bar{\theta}$

$\boldsymbol{\kappa} \quad$ wavenumber vector

$\kappa \quad$ wavenumber

$\kappa \quad$ von Karman constant $\kappa=0.41$

$\tilde{\lambda} \quad$ dimensional thermal conduction coefficient $\rightarrow$ Section 1.1

$\tilde{\mu} \quad$ dimensional dynamic viscosity coefficient $\rightarrow$ Section 1.1

$\tilde{\nu} \quad$ dimensional kinematic viscosity $\rightarrow$ Section 1.1

$\nu \quad$ non-dimensional kinematic viscosity $\rightarrow$ Section 1.1

$\nu_{t} \quad$ turbulent viscosity $\rightarrow$ (3.4), (4.33)

$\nu_{e} \quad$ effective viscosity $\nu_{e}=\nu+\nu_{t}$

$\tilde{\rho} \quad$ dimensional density $\rightarrow$ Section 1.1

$\tilde{\rho}_{0} \quad$ dimensional reference density $\tilde{\rho}_{0}=\tilde{\rho}\left(\tilde{T}_{0}\right) \rightarrow$ Section 1.1

$\sigma(\boldsymbol{u}, p) \quad$ stress tensor $\sigma(\boldsymbol{u}, p)=-p I+2 \nu \mathbb{S}(\boldsymbol{u})$

$\sigma_{t}(\boldsymbol{u}) \quad$ see $(1.13) \rightarrow$ Section 1.2

$\tau^{R} \quad$ residual (SGS) stress tensor $\tau^{R}=\overline{\boldsymbol{u} \otimes \boldsymbol{u}}-\overline{\boldsymbol{u}} \otimes \overline{\boldsymbol{u}} \rightarrow(4.19)$

$\tau^{r} \quad$ anisotropic residual (SGS) stress tensor $\rightarrow$ (4.21)

$\tau^{S G S} \rightarrow(4.30)$

$\tau_{w} \quad$ wall shear stress $\tau_{w}=\left.\nu \operatorname{Tr}\right|_{\Gamma_{W}} \nabla \boldsymbol{U} \cdot \boldsymbol{n} \rightarrow$ Section 5.1

$\chi \quad \rightarrow(1.13)$

\section{Superscripts}

$\boldsymbol{u}^{\prime} \quad$ non-dimensional fluctuating resp. residual velocity $\rightarrow$ Section 2.1 resp. (4.3)

$p^{\prime} \quad$ non-dimensional fluctuating resp. residual pressure $\rightarrow$ Section 2.1 resp. (4.3)

$\theta^{\prime} \quad$ non-dimensional fluctuating resp. residual temperature

$\rightarrow$ Section 2.1 resp. Section 4.4

$\overline{\boldsymbol{u}} \quad$ non-dimensional space filtered velocity $\rightarrow$ (4.1)

$\bar{p} \quad$ non-dimensional space filtered pressure $\rightarrow$ (4.1)

$\bar{\theta} \quad$ non-dimensional space filtered temperature $\rightarrow$ (4.1)

$\hat{f} \quad$ Fourier transform of $f$

$f^{B L} \quad$ inner solution for $f$ in $\Omega_{\delta}$

\section{Subscripts}

$f_{w} \quad f_{w}=\left.f\right|_{\Gamma_{w}}$ resp. $f_{w}=\left.\operatorname{Tr}\right|_{\Gamma_{w}} f$

$f_{\delta} \quad f_{\delta}=\left.f\right|_{\Gamma_{\delta}}$ resp. $f_{\delta}=\left.\operatorname{Tr}\right|_{\Gamma_{\delta}} f$

\section{Symbols}

$\partial \Omega \quad$ boundary of $\Omega \rightarrow$ Section 1.1

$|\boldsymbol{u}| \quad|\boldsymbol{u}|(\boldsymbol{x})=\sqrt{\sum_{i=1}^{d} u_{i}(\boldsymbol{x})^{2}}$

$\|\phi\|_{\infty, M} \quad \mathcal{L}^{\infty}$ norm $\|\phi\|_{\infty, M}=\operatorname{esssup}_{\boldsymbol{x} \in M}|\phi(\boldsymbol{x})|$

$\|\boldsymbol{u}\|_{\infty, M} \quad \mathcal{L}^{\infty}$ norm $\|\boldsymbol{u}\|_{\infty, M}=\operatorname{esssup}_{\boldsymbol{x} \in M}|\boldsymbol{u}(\boldsymbol{x})|$ 
$\|\mathbb{S}(\boldsymbol{u})\|_{F} \quad\|\mathbb{S}\|_{F}=\sqrt{\mathbb{S}(\boldsymbol{u}): \mathbb{S}(\boldsymbol{u})}$

$\langle\ldots\rangle_{E} \quad$ ensemble average $\rightarrow(2.2)$

$\left\langle\boldsymbol{u}^{\prime} \otimes \boldsymbol{u}^{\prime}\right\rangle_{E} \quad$ Reynolds stress tensor $\rightarrow$ Section 2.1

$\langle\ldots\rangle_{\Delta} \quad$ spatial average $\rightarrow$ (4.1)

$\int \ldots d \sigma \quad(\mathrm{d}-1)$ dimensional surface integral

$\boldsymbol{a} \otimes \boldsymbol{b} \quad(\boldsymbol{a} \otimes \boldsymbol{b})_{i j}=\boldsymbol{a}_{i} \boldsymbol{b}_{j}$

$\mathbb{A}: \mathbb{B} \quad \mathbb{A}: \mathbb{B}=\sum_{i, j=1}^{d} A_{i j} B_{i j}$

$g_{\Delta} * f \quad$ convolution $\rightarrow$ (4.1)

$\nabla \boldsymbol{U} \bullet \nabla \boldsymbol{U} \quad(\nabla \boldsymbol{U} \bullet \nabla \boldsymbol{U})_{i j}=\sum_{l=1}^{d} \frac{\partial U_{i}}{\partial x_{l}} \frac{\partial U_{j}}{\partial x_{l}} \rightarrow(4.32)$

$\nabla \Theta \circ \nabla \boldsymbol{U} \quad(\nabla \Theta \circ \nabla \boldsymbol{U})_{j}=\sum_{l=1}^{d} \frac{\partial \Theta}{\partial x_{l}} \frac{\partial U_{j}}{\partial x_{l}} \rightarrow$ (4.32)

\section{Abbreviations}

DES Detached-eddy simulation

DNS Direct numerical simulation

$k / \epsilon$ MODEL I $\rightarrow$ Section 6.1

$k / \epsilon$ MODEL II $\rightarrow$ Section 6.2

$k / \epsilon$ MODEL III $\rightarrow$ Section 6.3

$k / \epsilon$ MODEL IIIA $\rightarrow$ Section 6.3

$k / \epsilon$ MODEL IIIB $\rightarrow$ Section 6.3

IL $\quad$ IL model : Iliescu-Layton model $\rightarrow$ (4.34)

LES Large-eddy simulation

LES MODEL I $\rightarrow$ Chapter 7

LES MODEL II $\rightarrow$ Section 7.1

LES-NWM Large-eddy simulation with near-wall modelling

LES-NWR Large-eddy simulation with near-wall resolution

RANS Reynolds averaged Navier-Stokes equations

SGS $\quad$ subgrid scale $\rightarrow$ Section 4.3

SM SM model : Smagorinsky model $\rightarrow$ (4.33)

TKE $\quad$ turbulent kinetic energy $\rightarrow$ Section 4.4 .2 


\section{Bibliography}

[Ada75] Adams, R. A. (1975). Sobolev spaces. New York: Academic Press.

[Ald90] Aldama, A. A. (1990). Filtering techniques for turbulent flow simulation. Berlin: Springer-Verlag.

[Alb81] AlBRING, W. (1981). Elementarvorgänge fluider Wirbelbewegungen. Berlin: Akademie-Verlag.

[Alt92] Alt, H. W. (1992). Lineare Funktionalanalysis. Berlin: Springer-Verlag.

[AK02] Ampofo, F., Karayiannis, T. (2002). Experimental benchmark data for turbulent natural convection in an air filled square cavity. To appear in: Int. J. Heat Mass Transfer.

[APQ02] Auteri, F., Parolini, N., Quartapelle, L. (2002). Numerical Investigation of the Stability of Singular Driven Cavity Flow. J. Comp. Phys. 183,1-25.

[Bag98] BAGgett, J. S. (1998). On the feasibility of merging LES with RANS for the near-wall region of attached turbulent flows. In: Annual Research Briefs 1997, 267-277. Stanford: Center for Turbulence Research.

[BBP95] Balaras, E., Benocci, C., Piomelli, U. (1995). Finite- difference computations of high Reynolds number flow using the dynamic subgrid-scale model. Theor. Comput. Fluid Dyn. 7, 207-216.

[BBP96] Balaras, E., Benocci, C., Piomelli, U. (1996). Two-layer approximate boundary conditions for large-eddy simulations. AIAA J.34, 1111-1119.

[BFr80] Bardina, J., Ferziger, J. H., Reynolds, W. C. (1980). Improved subgrid models for large eddy simulation. Paper 80-1357, AIAA.

[BPD98] Behnia, M., Parneix, S., Durbin, P. A. (1998). Prediction of heat transfer in an axisymmetric turbulent jet impinging on a flat plate. Int. J. Heat Mass Transfer 41, 1845-1855.

[BG02] Berselli, L. C., Grisanti, C. R. (2002). On the consistency of the Rational Large Eddy Simulation model. Technical report, Pisa: Quaderno 2001/32 del Dipartimento di Matematica Applicata "U.Dini".

[BGli02] Berselli, L. C., Galdi, G. P., Layton, W. J., Iliescu, T. (2002). Mathematical analysis for the Rational Large Eddy Simulation model. To appear in: Math. Models Methods Appl. Sci. 12 (8), 1-22. 
[BGOK92] Boris, J. P., Grinstein, F. F., Oran, E. S., Kolbe, R. L. (1992). New insights into large eddy simulation. Fluid Dyn. Res. 10, 199-228.

[Tez92] Behr, M., Tezduyar, T. E. (1992). Finite element solution strategies for large-scale flow simulations. Preprint 92-140., University of Minnesota, Minneapolis: Army High-Performance Computing Center.

[BH93] Brooke, J. W., Hanratty, T. J. (1993). Origin of turbulence-producing eddies in a channel flow. Phys. Fluids, 5, 1011-1022.

[BF99] Brun, C., Friedrich, R. (1999). A-priori tests of SGS stress models in fully developed pipe flow and a new local formulation. In: P.R. Voke et al. (editors), Direct and Large-Eddy Simulation III, Kluwer Academic Publishers, 249-262.

[Cab96] Савот, W. (1996). Near-wall models in large eddy simulations of flow behind a backward facing step. In: Annual Research Briefs, 1996, 199-210. Stanford: Center for Turbulence Research.

[Cab97] Савот, W. (1997). Wall models in large eddy simulation of separated flow. In: Annual Research Briefs 1997, 97-106. Stanford: Center for Turbulence Research.

[Cab98] САвот, W. (1998). Large-eddy simulation of a separated boundary layer. In: Annual Research Briefs 1998, 279-288. Stanford: Center for Turbulence Research.

[CJB99] Cавот, W., Jimenez, J., Bagett, J. S. (1999). On wakes and near-wall behaviour in coarse large-eddy simulation of channel flow with wall models and second-order finite-difference methods. In: Annual Research Briefs 1999, 343-354. Stanford: Center for Turbulence Research.

[CM00] CAвот, W., Moin, P. (2000). Approximate wall boundary layer conditions in the large-eddy simulation of high Reynolds number flow. Flow, Turbulence and Combustion, 63, 269-291.

[CM02] Camelli, F. E., Löhner, R. (2002). Combining the Baldwin Lomax and Smagorinsky Turbulence Models to Calculate Flows with Separation Regions. 40th AIAA Aerospace Sciences Meeting \& Exhibit, 14-17 January 2002, Reno, NV.

[CT90] Caussignac, P., Touzani, R. (1990). Solution of three-dimensional boundary layer equations by a discontinuous finite element method, Part I: Numerical analysis of a linear problem. Comput. Methods Appl. Mech. Engrg. 78, 249-271.

[CC99] Cebeci, T., Cousteix, J. (1999). Modeling and Computation of Boundary Layer Flows : Laminar, Turbulent and Transitional Boundary Layers in Incompressible Flows. Berlin: Springer-Verlag 
[CKZ86] Cheesewright, R., King, K. J., Ziai, S. (1986). Experimental data for the evaluation of computer codes for the prediction of two-dimensional buoyant cavity flows. ASME winter annual meeting, pp. 75-81, Anaheim.

[CM94] Choi, H., Morn, P. (1994): Effects of the Computational Time Step on Numerical Solutions of Turbulent Flow. Journal Comp. Physics 113, 1-4.

[Cod93a] Codina, R. (1993). A Finite Element Formulation for the Numerical Solution of the Convection-Diffusion Equation. Monografia (14), Barcelona: Centro Internacional de Metodos Numericos en Ingenieria.

[Cod93b] Codina, R. (1993). A Finite Element Formulation for Viscous Incompressible Flows. Monografia (16), Barcelona: Centro Internacional de Metodos Numericos en Ingenieria.

[Cod00] Codina, R. (2000). A nodal-based implementation of a stabilized finite element method for incompressible flow problems. Int. J. Numer. Meth. Fluids 33, 737766 .

[Col99] Coletti, P. (1998). Analytical and numerical results for $k-\epsilon$ and large eddy turbulence models. PhD.thesis, Univ. of Trento.

[Cod93] Codina, R. (1993). A discontinuity-capturing crosswind-dissipation for the finite element solution of the convection-diffusion equation. Comp. Meth. Appl. Mech. Engrg. 110, 325-342.

[CS99] Codina, R., Soto, O. (1999). Finite element implementation of two-equation and algebraic stress turbulence models for steady incompressible flows. International Journal for Numerical Methods in Fluids 30, 309-334.

[CL98] Comte, P., Lesieur, M. (1998). Large eddy simulation of compressible turbulence. In: Advances in Turbulence Modelling, Lecture Series 1998-05. Rhode Saint Genese: Von Karman Institute for Fluid Dynamics.

[DH70] Daly, B. J., Harlow, F. H. (1970). Transport Equations in Turbulence. Phys. Fluids. 13, 2634-2649.

[Dea76] DeAn, R. B. (1976). A single formula for the complete velocity profile in a turbulent boundary layer. ASMEJ. Fluids Eng 98, 723-727.

[Dur91] Durbin, P. A. (1991). Near-wall turbulence closure modeling without damping functions. Theor. Comput. Fluid Dynamics 3 1-11.

[Dur93] Durbin, P. A. (1993). A Reynolds stress model for near-wall turbulence. J. Fluid Mech. 249, 465-498.

[DPR01] Durbin, P. A., Petterson Reif, B. A. (2001). Statistical theory and modelling for turbulent flows. Chichester: John Wiley \& Sons. 
[DBP01] Diurno, G.V., Balaras, E., Poimelli, U. (2001). Wall-layer models for LES of seperated flows In : Bernard J. Geurts (ed.), Modern Simulation Strategies for Turbulent Flow. Philadelphia: RT Edwards Publishers.

[Eck74] Eckelmann, H. (1974). The structure of the viscous sublayer and the adjacent wall region in a turbulent channel flow J.Fluid Mech. 65, 439-459.

[Eck50] Eckert, E. R. G. (1950). Introduction to the Transfer of Heat and Mass. New York: McGraw-Hill.

[Eid85] Eidson, T. M. (1985). Numerical simulation of the turbulent Rayleigh-Benard problem using subgrid modeling. J. Fluid Mech. 158, 245-268.

[Fis02] Fischer, H. (2002). Das stabilisierte diskontinuierliche Galerkin-Verfahren zur Lösung parabolischer Anfangsrandwertprobleme. Univ. Göttingen, Diplomarbeit.

[Fri96] Frisch, U. (1996). Turbulence. The legacy of A.N.Kolmogorov. Cambridge: Cambridge University Press.

[FR] Fröhlich, J., Rodi, W. (2002). Introduction to Large Eddy Simulation of Turbulent Flows. In B.E. Launder, N.D. Sandham (eds.): Closure Stratigies for Turbulent and Transitional Flows. Cambridge: Cambridge University Press.

[Gal94I] Galdi, G.P. (1994). An Introduction to the Mathematical Theory of the Navier-Stokes Equations Volume I. New York: Springer-Verlag.

[Gal94II] Galdi, G.P. (1994). An Introduction to the Mathematical Theory of the Navier-Stokes Equations Volume II. New York: Springer-Verlag.

[GL00] Galdi, G.P., Layton, W. J. (2000). Approximation of the larger eddies in fluid motion II: A model for space filtered flow. Math. Models and Meth. in Appl. Sciences 10(3), 343-350.

[Gmscd99] Garnier, E., Mossi, M., Sagaut, P., Comte, P., Deville, M. (1999). On the Use of Shock-Capturing Schemes for Large-Eddy Simulation. J. Comp. Phys. 153, 271-311.

[GC79] George, W. K., Capp, S. P. (1979). A Theory for Natural Convection Turbulent Boundary Layer Next to Heated Vertical Surfaces. Int. J. Heat Mass Transfer 22, 813-826.

[Ger86] Germano, M. (1986). Differential filters for the large eddy numerical simulation of turbulent flows. Phys. Fluids 29, 1757-1758.

[GPMC91] Germano, M., Piomelli, U., Moin, C., Cabot, W. H. (1991). A dynamic subgrid-scale eddy-viscosity model. Phys. Fluids A 3, 1760-1765.

[Geu01] GeurTs, B. (2001). Modern simulation strategies for turbulent flows. Philadelphia: RT Edwards Publishers. 
[Gho95] Ghosal, S. (1995). An analysis of numerical errors in large-eddy simulations of turbulence. J. Comp. Phys. 125, 187-206.

[GM95] Ghosal, S., Moin, P. (1995). The basic equations of the large eddy simulation of turbulent flows in complex geometries. J. Comp. Phys. 118, 24-37.

[GR86] Girault, V., Raviart, P.- A. (1986). Finite Element Methods for NavierStokes Equations. Berlin: Springer-Verlag.

[Gri85] Grisvard, P. (1985). Elliptic Problems in Nonsmooth Domains. Boston: Pitman.

[Gro87] Grötzbach, G. (1987): in Encyclopedia of Fluid Mechanics, édité par N.P. Chereminisoff (Gulf, West Orange, NJ), Vol. 6.

[Gri01] Gritzki, R. (2001). Bestimmung der Effektivität nutzerbedingter Fensterlüftung. Dissertation TU Dresden. Osnabrück: Der Andere Verlag.

[HP96] Hennessy, J. L., Patterson, D. A. (1996). Computer architecture. A quantitative approach. San Francisco: Morgan Kaufmann.

[HKUF94] Härtel, C. Kleiser, L., Unger, F., Friedrich, R. (1994). Subgrid-scale energy transfer in the near-wall region of turbulent flows. Phys. Fluids 6, 31303143.

[Hor66] HoRvath, J. (1966). Topological vector spaces and distributions. Volume I. Reading: Addison-Wesley.

[HC01] Houzeaux, G., Codina, R. (1999). A Finite Element Method for the Solution of Incompressible Flows in Rotodynamic Machines.. In: Proceedings of the 2nd ASME Pressure Vessels and Piping Conference, Boston.

[ICASE96] Gatski, T. B., Hussaini, M.Y., Lumley, J. L.(editors) (1996). Simulation and Modeling of Turbulent Flows. Oxford: Oxford University Press.

[iJl03] Iliescu, T., John, V., Layton, W. J., Matthies, G., Tobiska, L. (2003). A numerical study of a class of LES models. Int. J. Comput. Fluid Dyn. 17, 75 -85 .

[IL98] Iliescu, T., Layton, W. J. (1998). Approximating the larger eddies in fluid motion III: The Boussinesq model for turbulent fluctuations. An. St. Univ. "Al. I. Cuza" 44, 245-261.

[IL89] InCE, N. Z., LAUnder, B. E. (1989). On the computation of buoyancy-driven turbulent flows in rectangular enclosures. Int. J. Heat Fluid Flow 10, 110-117.

[Jan99] Jansen, K. E. (1999). A stabilized finite element method for computing turbulence. Computer Methods in Applied Mechanics and Engineering, 174, 299-317. 
[JNR00] Japhet, C., Nataf, F., Rogier, F. (2000). The Optimized Order 2 Method. Application to Convection-Diffusion Problems. Future Generation Computer Systems 18, Elsevier Science.

[John98] John, V. (1998). A posteriori $L^{2}$-error stimates for the nonconforming $P_{1}, P_{0^{-}}$ finite element discretization of the Stokes equations. J. Comp. Appl. Math. 96, 99-116.

[John] John, V. (2002). Slip with friction and penetration with resistance boundary conditions for the Navier-Stokes equations - numerical tests and aspects of the implementation. J. Comp. Appl. Math. 147, 287 - 300.

[JL01] John, V., Layton, W. J. (2001). Approximating Local Averages of Fluid Velocities: The Stokes Problem. Computing 66, 269 - 287.

[JLS02] John, V., Layton, W. J., Sahin, N. (2002). Derivation and Analysis of Near Wall Models for Channel and Recirculation Flows. Preprint 14/02, Fakultt fr Mathematik, Otto-von-Guericke-Universitt Magdeburg.

[Joh02] John, V. (2002). Large Eddy Simulation of Turbulent Incompressible Flows. Analytical and Numerical Results for a Class of LES Models. Habilitationsschrift, Universität Magdeburg.

[KC93] Kays, W. M., Crawford, M. E. (1993). Convective heat and mass transfer. New York: McGraw-Hill.

[KC96] Kevorkian, J., Cole, J. D. (1996). Multiple Scale and Singular Perturbation Methods. Berlin: Springer-Verlag.

[KD00] Kimmel, S. J.,Domaradzki, J. A. (2000). Large eddy simulation of RayleighBenard convection using subgrid scale estimation model. Physics of Fuilds 12, 169-184.

[Key00] Keyes, D. E. (2000). Four Horizons for Enhancing the Performance of Parallel Simulations based on Partial Differential Equations. In: Europar 2000 Parallel Processing, Lecture Notes in Computer Science 1900, 1-17.

[KLR02] Knopp, T., Lube, G., Rapin, G. (2002). Stabilized finite element methods with shock capturing for advection-diffusion problems. Comp. Meths. Appl. Mech. Engrg. 191, 2997-3013.

[Kno99] Knopp, T. (1999). Eine stabilisierte Finite-Elemente-Methode für das $k / \epsilon$ Turbulenzmodell der inkompressiblen und nichtisothermen Navier-StokesGleichungen. Diplom Arbeit, Universität Göttingen.

[KLGR02] Knopp, T., Lube, G., Gritzki, R., Rösler, M. (2002). Iterative substructuring methods for incompressible non-isothermal flows and its application to indoor air flow simulation. Int. J. Numer. Meth. Fluids 40, 1527-1538. 
[KM97] Kravchenko, A. G., Moin, P. (1997). On the effect of numerical errors in large-eddy simulations of turbulent flows. J. Comput. Phys. 131, 310-322.

[Kre89] Kress, R. (1989). Linear Integral Equations. Berlin: Springer-Verlag.

[Kre98] Kress, R.: Numerical Analysis New York: Springer-Verlag.

[Lad67] Ladyzhenskaya, O. A. (1967). New equations for the description of motion of viscous incompressible fluids and solvability in the large of boundary value problems for them. Proc. Steklov Inst. Math. 102,95-118.

[LL03] Layton, W. J., Lewandowski, R. (2003). Analysis of an Eddy Viscosity Model for Large Eddy Simulation of Turbulent Flows. To appear in: Applied Math. Letters.

[Lay02] Layton, W. J. (2002). A Mathematical Introduction to Large Eddy Simulation. To appear in: H. Deconinck (ed.), Computational Fluid DynamicsMultiscale Methods. Rhode-Saint-Genèse: Von Karman Institute for Fluid Dynamics.

[Lay02a] Layton, W. J. (2002). Advanced models in large eddy simulation. To appear in: H. Deconinck (ed.), Computational Fluid Dynamics-Multiscale Methods. Rhode-Saint-Genèse: Von Karman Institute for Fluid Dynamics.

[LTT96] Le Tallec, P., Tidriri, M. D. (1996). Maximum Principles and Application to the Analysis of the explicit time marching algorithm. ICASE Report No. 96-45.

[LTT99] Le Tallec, P., Tidriri, M. D. (1999). Convergence Analysis of Domain Decomposition Algorithms with Full Overlapping for the Advection-Diffusion Problems. Mathematics of Computation 226, 585-606.

[Lio69] Lions, J. (1969). Quelques methodes de resolution des problemes aux limites non lineares. Paris: Dunod.

[Lio90] Lions, P. L. (1990). On the Schwarz alternating method III: a viariant for nonoverlapping subdomains. In: T. Chan et al. (eds.), Third Int. Symp. Domain Decomposition Methods for Partial Differential Equations, 202-223. Philadelphia: SIAM.

[LeR97] LeRoux, C. (1997). Second grade fluids with slip boundary conditions PhD Thesis, University of Pretoria, Pretoria.

[Lia99] Liakos, A. (1999). Weak Imposition of Boundary Conditions in the Stokes and Navier-Stokes Equations PhD thesis, University of Pittsburgh, Pittsburgh.

[Lil62] LiLly, D. K. (1962). On the numerical simulation of buoyant convection. Tellus 14,148 . 
[LMK94] Liu, S., Meneveau, C., Katz, J. (1994). On the properties of similarity subgrid-scale models as deduced from measurement in a turbulent jet. J.Fluid Mech. 275, 83-119.

[LMO00a] Lube, G., Müller, L, Otto, F.C. (2000). A non-overlapping domain decomposition method for the advection-diffusion problem. Computing 64, 49-68.

[LMO00b] Lube, G., Müller, L, Otтo, F.C. (2001). A non-overlapping domain decomposition method for stabilized finite element approximations of the Oseen equations. J.Comp. Appl. Math. 132, 211-236.

[LMO00c] Lube, G., Müller, L, Otтo, F.C. (2000). A new non-overlapping domain decomposition method for stabilized finite element methods applied to the nonstationary Navier-Stokes equations. Numer. Lin Alg. Appl. 7, 449-472.

[LO98] Lube, G., Oтto, F.C. (1998). A non-overlapping domain decomposition method for the Oseen equations. Math. Mod. Meths. Appl. Sc. 8, 1091-1117.

[MAK01] Maeder, T., Adams, N. A.,Kleiser, L. (2001). Direct simulation of turbulent supersonic boundary layers by an extended temporal approach. J. Fluid Mech. 429,187-216.

[MV02] Marsden, A. L., Vasilyev, O. V., and Moin, P. (2002). Construction of Commutative Filters for LES on Unstructured Meshes. J. Comp. Phys. 175, 584-603.

[MKP01] Marusic, I., Kunkel, G. J., Porte-Agel, F. (2001). Experimental study of wall boundary conditions for large-eddy simulations. J.Fluid Mech. 406, 309320 .

[Men94] Menter, F. (1994). Two-equation eddy-viscosity turbulence models for engineering applications. AIAA J. 32, 1598-1605.

[MP94] Mohammadi, B., Pironneau, O. (1994). Analysis of the K-Epsilon Turbulence Model. Chichester: John Wiley \& sons.

[MV01] Morinishi, Y., Vasilyev, O. V. (2001) A recommended modification to the dynamic two-parameter mixed subgrid scale model for large eddy simulation of wall bounded turbulent flow. Phys. Fluids 13, p.3400-3410.

[MKM99] Moser, R. D., Kim, J., Mansour, N. N. (1999). Direct numerical simulation of turbulent channel flow up to $R e_{\tau}=590$. Phys. Fluids 11, 943-946.

[Mue91] MüLleR, U. (1991). Freie Konvektion und Wärmeübertragung. Vorlesung Universität $(\mathrm{TH})$ Karlsruhe.

[Mue97] MÜLlER, L. (1997). Untersuchung einer stabilisierten Finite-ElementeMethode für die Oseen-Gleichungen. Diplomarbeit, Universität Göttingen, Göttingen. 
[Mue00] Müller, L. (2000). Finite Element Solution of Some Time-Dependent Problems Using a Non-Overlapping Domain Decomposition Method. PhD thesis, Universität Göttingen, Göttingen.

[Mue99] MüLLER, H. (1999). Ein Konzept zur numerischen Berechnung inkompressibler Strömungen auf der Grundlage einer diskontinuierlichen Galerkin-Methode in Verbindung mit nichtüberlappender Gebietszerlegung. PhD thesis, TU Dresden, Dresden.

[NR95] Nataf, F. R., Rogier, F. (1995). Factorization of the convection-diffusion operator and the Schwarz algorithm. Math. Mod. Meths. Appl. Sc. 5, 67-93.

[NBMC99] Nicoud, F., Baggett, J., Moin, P., CAвot, W. (1999). LES wall-modeling based on optimal control theory. Phys. Fluids 13, 2968-2984.

[Nei99] NeItzke, K. P. (1999). Experimentelle Untersuchung und numerische Modellierung von wandnahen thermischen Auftriebsströmungen. Dissertation, TU Dresden, Dresden.

[NNWS00] Nikitin, N. V., Nicoud, F., Wasistho, B., Squires, K. D., Spalart, P.R. (2000). An approach to wall-modeling in large-eddy simulations. Physics of Fluids 12, 1629-1632.

[Otto99] Отто, F. C. (1999). A non-overlapping domain decomposition method for elliptic equations. PhD thesis, Universität Göttingen, Göttingen.

[PC96] Piomelli, U., Chasnov, J. R. (1996). Large-eddy simulations: theory and applications. In: M. Hallbäck, D. S. Henningson, A. V. Johansson, P. H. Alfredsson (eds.), Turbulence and Transition Modelling, Chapter 7, 269-336. Dordrecht: Kluwer.

[PFMK89] Piomelli, U., Ferziger, J., Moin, P., Kim, J. (1989). New approximate boundary conditions for large eddy simulation of wall bounded flows. Phys. Fluids A 1, 1061-1068.

[Pio93] Piomellu, U. (1993). High Reynolds number calculations using the dynamic subgrid-scale stress model. Phys. Fluids 7, 839-848.

[PS01] PARK, T. S., Sung, H. J. (2001). Development of a near-wall turbulence model and application to jet impingement heat transfer. Int.J.Heat and Fluid Flow 22, 10-18.

[Pope00] Pope, S.B. (2000). Turbulent Flows. Cambridge: Cambridge University Press.

[OL99] Отто, F. C., Lube, G. (1999). A posteriori estimates for a non-overlapping domain decomposition method for elliptic Problems. Computing 62, 27-43.

[Par92] PARÉs, C. (1992). Existence, uniqueness and regularity of solutions of the equations of a turbulence model for incompressible fluids. Applicable Analysis 43, 245-296. 
[PD01b] Peng, S., Davidson, L. (2001). Large eddy simulation for turbulent buoyant flow in a confined cavity. Int. J. Heat Fluid Flow 22, 323-331.

[PD01a] Peng, S., Davidson, L. (2001). A hybrid LES-RANS model based on a oneequation SGS model and a two-equation $k / \omega$ model. In: The second Int. Symp. on Turbulence and Shear Flow Phenomena, Vol. 2, pp. 175-180. Stockholm.

[PD01] Peng, S., Davidson, L. (2001). Comparative study of LES for turbulent buoyant flow in terms of SGS model and grid resolution. In: The second Int. Symp. on Turbulence and Shear Flow Phenomena, Vol. 2, pp. 455-460, Stockholm.

[PD98] Peng, S., Davidson, L. 1998). Comparison of subgrid-scale models in LES for turbulent convection flow with heat transfer. Turbulent Heat Transfer 2, pp. 5.24-5.35. Manchester.

[PD00] Peng, S., Davidson, L. (2000). Numerical investigation of turbulent buoyant cavity flow using large eddy simulation. In: Y. Nagano, K.Hanjalic, and T.Tsuji (eds.), Turbulence, Heat and Mass Transfer 3, 737-744. Nagoya.

[PYA96] Piomelli, U., Yu, Y., Adrian, R. J. (1996). Subgrid-scale energy transfer and near-wall turbulence structure. Phys. Fluids 8, 215-224.

[QV94] Quarteroni, A., Valli, A. (1994). Numerical Approximation of Partial Differential Equations. Berlin: Springer-Verlag.

[QV99] Quarteroni, A., Valli, A. (1999). Domain Decomposition Methods for Partial Differential Equations. Oxford: Clarendon Press.

[Que01] QuÉmÉRÉ, P. (2001). Une méthode multidomaine/multirésolution avec applications au couplage LES/LES et RANS/LES. PhD thesis, ONERA DSNA, Université Pierre and Marie Curie.

[RST96] Roos, H.-G., Stynes, M., Tobiska, L. (1996). Numerical Methods for Singularly Perturbed Differential Equations. Convection-Diffusion and Flow Problems. Berlin: Springer-Verlag.

[RL00] Rapin, G., Lube, G. (2000). Comparison of two iterative substructuring methods for advection-diffusion problems. Technical Report, Universität Göttingen, 2000. Preprint 2000-17.

[Sag01] Sagaut, P. (2001). Large eddy simulation for incompressible flows. Berlin: Springer-Verlag.

[Sei03] SEIFERT, J. (2003). Berechnung des konvektiven Wärmeübergangs an einem Testfall nach Awbi/Hatton. Technical report, Institut für Thermodynamik und Technische Gebäudeausrüstung, Technische Universität Dresden.

[Soh01] Sohr, H. (2001). The Navier-Stokes Equations. An Elementary Functional Analytic Approach. Basel: Birkhäuser Verlag. 
[SA94] Spalart, P. R., Allmaras, S. R. (1994). A one-equation turbulence model for aerodynamical flows. La recherche Aerospaciale, 1, 5 .

[SJSA97] Spalart, P. R., Jou, W. H., Strelets, M., Allmaras, S. R. (1997). Comments on the Feasibility of LES for Wings, And on a Hybrid RANS/LES Approach. In: C. Liu, and Z. Liu (eds.), First AFOSR International Conference On DNS/LES, Aug. 4-8, 1997, Ruston, Louisiana. Columbus/Ohio: Greyden Press.

[SSST99] Shur, M., Spalart, P. R., Strelets, M., Travin, (1999). DetachedEddy Simulation of an Airfoil at High Angle of Attack Fourth International Symposium on Engineering Turbulence Modeling and Measurements, May 2426, 1999, Corsica.

[SP02] Scotti, A., Piomelli, U. (2002). Turbulence models in pulsating flows. AIAA J. 40, 537-540.

[Tem77] Temam, R.M. (1977). Navier-Stokes-Equations. Theory and Numerical Analysis. Oxford: North-Holland Publishing Company.

[Tem95] Temam, R.M. (1995). Navier-Stokes Equations and Nonlinear Functional Analysis. CBMS-NSF Regional Conference Series in Applied Mathematics SIAM, Philadelphia.

[Tem97] Temam, R.M. (1997). Infinite-dymensional dynamic systems in mechanics and physics. New York: Springer-Verlag.

[Tho97] Thomee, V. (1997). Galerkin Finite Element Methods for Parabolic Problems. Berlin: Springer-Verlag.

[TK00a] Tiam, Y., Karayiannis, T. (2000). Low turbulence natural convection in an air filled square cavity, Part I: The thermal and fluid flow field. Int. J. Heat Mass Transfer 43, 849-866.

[TK00b] Tiam, Y., KaraYiannis, T. (2000). Low turbulence natural convection in an air filled square cavity, Part II: The Turbulence Quantities. Int. J. Heat Mass Transfer 43, 867-884.

[Tid95] TIDriRI, M. D. (1995). Domain decomposition for compressible Navier-Stokes equations with different discretizations and formulations. J. Comp. Phy. 119, 271-282.

[TODB98] Tieszen, S., Ooi, .A., Durbin, P., Behnia, M. (1998). Modeling of natural convection heat transfer Proceedings of the Summer Programm 1998. Stanford: Center for Turbulence Research.

[Tie96] Tieszen, S. R., Nicolette, V. F., Gritzo, L. A., Holen J. K., MurRAY, D., Moya, J. L. (1996). Vortical Structures in Pool Fires: Observation, Speculation, and Simulation. SAND96-2607, November. Albuquerque: Sandia National Laboratories. 
[Tre67] Treves, F. (1967). Topological vector spaces, distributions and kernels. New York: Academic press.

[TN98b] Tsuji, T., Nagano, Y (1998b). Turbulence measurements in a natural convection boundary layer along a vertical flat plate. Int. J. Heat Mass Transfer $31,2101-2111$.

[VGK97] Vreman, B., Geurts, B., Kuerten, H. (1997). Large-eddy simulation of the turbulent mixing layer. J.Fluid Mech. 339, 357-390.

[Ver87] VERFüRTH, R. (1987). Finite element approximation of incompressible NavierStokes equations with slip boundary condition. Numer. Math. 50, 697-721.

[Wan00] WANG, M. (2000). Dynamic wall modeling for LES of complex turbulent flows. In: Annual Research Briefs, 241-250. Stanford: Center for Turbulence Research.

[WCI01] Wang, M., Catalano, P., Iaccarino, G. (2001). Prediction of high Reynolds number flow over a circular cylinder using LES with wall modeling. In: Annual Research Briefs, 45-50. Stanford: Center for Turbulence Research.

[WP96] Wang, W.-P., Pletcher, R. H. (1996). On the large eddy simulation of a turbulent channel flow with significant heat transfer. Phys. Fluids 8, 1996.

[Wer95] Werner, D. (1995). Funktionalanalysis. Springer-Verlag, 1995.

[Wla72] Wladimirow, W. S. (1972). Gleichungen der mathematischen Physik. Berlin: VEB Deutscher Verlag der Wissenschaften.

[Wilcox98] Wilcox, D. C. (1998). Turbulence modeling for CFD. La Canada, CA: DWC Industries.

[WL94] Wong, V. C., Lilly, D. K. (1994). A comparison of two dynamic subgrid closure methods for turbulent thermal convection, Phys. Fluids 6, 1016-1023.

[Yua92] YUAN (1992). New wall functions for the numerical simulation of air flow patterns in rooms. Proceedings ROOMVENT '92, Aalborg.

[ZBK95] Zahrai, S, Bark, F. H., Karlsson, R. I. (1995). On anisotropic subgrid modeling. Eur. J. Mech. B/Fluids 14, 459-486.

[ZeiI] ZeIDler, E. (1993). Nonlinear Functional Analysis and its Applications I. New York: Springer-Verlag.

[ZeiIIa] ZeIdler, E. (1990). Nonlinear Functional Analysis and its Applications II/A. New York: Springer-Verlag.

[ZeiIV] ZeIdler, E. (1994). Nonlinear Functional Analysis and its Applications IV. New York: Springer-Verlag. 


\section{Curriculum vitae - Lebenslauf}

Persönliche Daten:

Name:

Geburtsdatum:

Geburtsort:

Familienstand:

Eltern:

\section{SCHULE:}

Sep. 81 - Jun. 85

Sep. 85 - Jun. 94

24. Juni 94

STUDIUM:

Okt. 94 - Mär. 97

18. Okt 96

Feb. 97 - Sep. 97

17. Okt 97

Okt. 97

Okt. 98 - Aug. 99

Jan. - Mär. 99

28. Okt 99

Nov.- Dez. 99

Jan. - Mär. 00

Promotion:

seit Apr. 00

Apr. 00 - Mär. 03

Aug. - Dez. 01
Tobias Knopp

27. März 1975

Lübeck

ledig

Walter Knopp

Barbara Knopp, geb. Schröder

Kaland-Schule Lübeck (Grundschule)

Oberschule zum Dom Lübeck

Abitur

Studium der Mathematik und Physik an der Georg-

August-Universität Göttingen

Vordiplom Physik

Studium der Physik an der Heinrich-Heine-Universität

Düsseldorf

Vordiplom Mathematik

Studium der Mathematik an der Georg-August-

Universität Göttingen

Diplomarbeit am Institut für Numerische und Angewandte Mathematik bei Prof. Dr. G. Lube

Forschungsaufenthalt an der TU Dresden am Institut für

Thermodynamik und Technische Gebäudeausrüstung

Diplom Mathematik

Wissenschaftliche Hilfskraft am Institut für Numerische und Angewandte Mathematik

Forschungsaufenthalt an der TU Dresden am Institut für

Thermodynamik und Technische Gebäudeausrüstung

Promotion am Institut für Numerische und Angewandte Mathematik bei Prof. Dr. G. Lube

Stipendiat des "Graduiertenkolleg für Strömungsinstabilitäten und Turbulenz"

Forschungsaufenthalt bei Prof. Dr. William J. Layton

an der University of Pittsburgh (USA) 